Supporting Information for:

Highly Enantioselective O-H Bond Insertion of $\alpha$-Alkyl- $\alpha$ diazoacetates and $\alpha$-Alkenyl- $\alpha$-diazoacetates with Water

You Li, Yu-Tao Zhao, Ting Zhou, Meng-Qing Chen, Yi-Pan Li, Ming-Yao Huang, Zhen-Chuang Xu, Shou-Fei Zhu*, and Qi-Lin Zhou

The State Key Laboratory and Institute of Elemento-Organic Chemistry, College of Chemistry, Nankai University, Tianjin 300071, China

Experimental Data

Contents:

1. General Information............................................

2. Analytical Data of New Diazoacetates..............................S3

3. Typical Procedures of $\mathrm{O}-\mathrm{H}$ Bond Insertion Reactions.............S19

4. Analytical Data of O-H Bond Insertion Products.................S23

5. Gram-scale $\mathrm{O}-\mathrm{H}$ Bond Insertion Reactions.....................S43

6. Analytical Data of Side-products................................S45

7. Transformations of $\mathrm{O}-\mathrm{H}$ Bond Insertion Products...............S49

8. NMR Spectra of New Compounds...............................S60

9. HPLC Charts of Chiral Compounds.............................S153

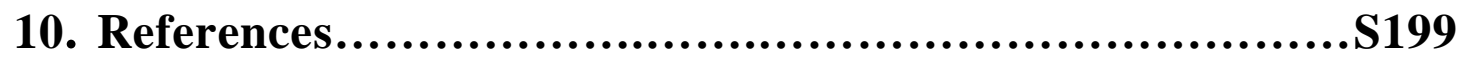




\section{General Information}

All solvents were purified and dried using standard procedures. ${ }^{1}$ The $\mathrm{CHCl}_{3}$ was washed successively by small amount of conc. $\mathrm{H}_{2} \mathrm{SO}_{4}$, water, saturated $\mathrm{NaHCO}_{3}$, washed again with water, then dried over $\mathrm{CaCl}_{2}$ for more than $1 \mathrm{~h}$ before filtrating and distilling, and redistilled over $\mathrm{CaH}_{2}$ prior to use. Water was purified by distillation. Dirhodium (II) carboxylates (Sigma-Aldrich), chiral ligands (Daicel Chiral Technologies) and other commercially available reagents were used without further purification. The chiral spiro phosphoric acids were synthesized according to the reported procedures. ${ }^{2} \alpha$-Alkyl- $\alpha$-diazoesters ${ }^{3}$ and $\alpha$-alkenyl- $\alpha$-diazoesters ${ }^{4}$ were prepared according to the reported procedures. All reactions were performed in an argon-filled glovebox (MBRAUN Labstar or Mikrouna super 1220/750) or using standard Schlenk techniques. Melting points were measured on a RY-I apparatus and uncorrected. NMR spectra were recorded with a Bruker AV 400 spectrometer at 400 $\mathrm{MHz}\left({ }^{1} \mathrm{H} \mathrm{NMR}\right)$ and $100 \mathrm{MHz}\left({ }^{13} \mathrm{C} \mathrm{NMR}\right)$. Chemical shifts ( $\delta$ values $)$ were reported in ppm down field from internal $\mathrm{Me}_{4} \mathrm{Si}\left({ }^{1} \mathrm{H}\right.$ and ${ }^{13} \mathrm{C}$ NMR). High Resolution Mass Spectra (HRMS) were recoreded on an IonSpec FT-ICR mass spectrometer with Electron Spray Ionization (ESI) resource. Enantiomeric excesses (ee) of the $\mathrm{O}-\mathrm{H}$ bond insertion products were determined by High Performance Liquid Chromatography (HPLC). HPLC analyses were performed on a Hewlett Packard Model HP 1100 Series chromatography. Thin Layer Chromatography (TLC) analyses were performed on ZF20D Cabinet UV analyzer (Guang Hao, Shanghai). 


\section{Analytical Data of New Diazoacetates}

\section{2-Phenylpropan-2-yl 2-diazooctanoate (1a)}

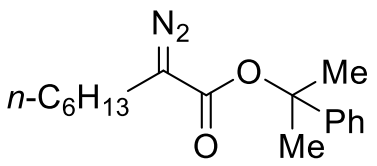

Serial number: 1y-3-151. Yellow oil. TLC $\mathrm{R}_{f}=0.39$ (petroleum ether/ethyl acetate, PE/EA = 50:1 v/v), $68 \%$ yield. ${ }^{1} \mathrm{H}$ NMR $\left(400 \mathrm{MHz}, \mathrm{CDCl}_{3}\right) \delta 7.37-7.29(\mathrm{~m}, 4 \mathrm{H}, \mathrm{Ar}-\mathrm{H}), 7.23(\mathrm{~m}, 1 \mathrm{H}$, Ar-H), 2.33-2.16 (m, 2H, CH 2$), 1.78\left(\mathrm{~s}, 6 \mathrm{H}, \mathrm{C}\left(\mathrm{CH}_{3}\right)_{2}\right), 1.47$ (d, J=6.3 Hz, 2H, $\mathrm{CH}_{2}$ ), $1.34-1.24\left(\mathrm{~m}, 6 \mathrm{H}, \mathrm{CH}_{2}\right), 0.88\left(\mathrm{t}, J=6.7 \mathrm{~Hz}, 3 \mathrm{H}, \mathrm{CH}_{3}\right) .{ }^{13} \mathrm{C} \mathrm{NMR}\left(100 \mathrm{MHz}, \mathrm{CDCl}_{3}\right)$ $\delta 146.0$ (1C, Ar-C), 128.3 (2C, Ar-C), 127.0 (1C, Ar-C), 124.2 (2C, Ar-C), 82.2 (1C, C-O), 31.5 (1C, $\left.\mathrm{CH}_{2}\right), 29.0\left(1 \mathrm{C}, \mathrm{CH}_{2}\right), 28.4\left(2 \mathrm{C}, \mathrm{C}\left(\mathrm{CH}_{3}\right)_{2}\right), 27.5\left(1 \mathrm{C}, \mathrm{CH}_{2}\right), 23.0(1 \mathrm{C}$, $\left.\mathrm{CH}_{2}\right), 22.6\left(1 \mathrm{C}, \mathrm{CH}_{2}\right), 14.1\left(1 \mathrm{C}, \mathrm{CH}_{3}\right)$. IR (neat): 3406, $2079(\mathrm{C}=\mathrm{N}), 1694,1148,1123$, 928, 791, 739, 696, 691, $687 \mathrm{~cm}^{-1}$. HRMS (ESI) Calcd for $\left[\mathrm{C}_{17} \mathrm{H}_{24} \mathrm{~N}_{2} \mathrm{O}_{2} \mathrm{Na}, \mathrm{M}+\mathrm{Na}\right]^{+}$: 311.1730, Found: 311.1733

\section{2-Phenylpropan-2-yl 2-diazobutanoate (1b)}<smiles>CCC(=N)C(=O)OC(C)(C)c1ccccc1</smiles>

Serial number: 1y-4-8. Yellow oil. TLC $R_{f}=0.49$ (PE/EA $=20: 1$ v/v), 73\% yield. ${ }^{1} \mathrm{H}$ NMR $\left(400 \mathrm{MHz}, \mathrm{CDCl}_{3}\right) \delta 7.38-7.29(\mathrm{~m}$, 4H, Ar-H), $7.27-7.20(\mathrm{~m}, 1 \mathrm{H}, \mathrm{Ar}-\mathrm{H}), 2.29(\mathrm{~d}, J=6.7 \mathrm{~Hz}, 2 \mathrm{H}$, $\left.\mathrm{CH}_{2}\right), 1.78\left(\mathrm{~s}, 6 \mathrm{H}, \mathrm{C}\left(\mathrm{CH}_{3}\right)_{2}\right), 1.10\left(\mathrm{t}, J=7.3 \mathrm{~Hz}, 3 \mathrm{H}, \mathrm{CH}_{3}\right) .{ }^{13} \mathrm{C} \mathrm{NMR}\left(100 \mathrm{MHz}, \mathrm{CDCl}_{3}\right)$ $\delta 146.0$ (1C, Ar-C), 128.3 (2C, Ar-C), 127.0 (1C, Ar-C), 124.2 (2C, Ar-C), 82.2 (1C, C-O), 29.0 (2C, $\left.\mathrm{C}\left(\underline{\mathrm{CH}}_{3}\right)_{2}\right), 16.5\left(1 \mathrm{C}, \mathrm{CH}_{2}\right), 11.9\left(1 \mathrm{C}, \mathrm{CH}_{3}\right)$. IR (neat): 3408, $2079(\mathrm{C}=\mathrm{N})$, 1694, $11431121,928,688 \mathrm{~cm}^{-1}$. HRMS (ESI) Calcd for $\left[\mathrm{C}_{13} \mathrm{H}_{16} \mathrm{~N}_{2} \mathrm{O}_{2} \mathrm{Na}, \mathrm{M}+\mathrm{Na}\right]^{+}$: 255.1104, Found: 255.1107

\section{2-Phenylpropan-2-yl 2-diazohexanoate (1c)}

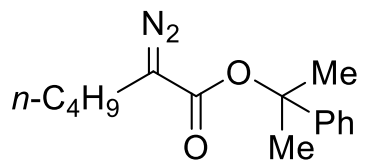

Serial number: ly-3-154. Yellow oil. TLC $R_{f}=0.65(\mathrm{PE} / \mathrm{EA}=$ 20:1 v/v), $77 \%$ yield. ${ }^{1} \mathrm{H}$ NMR (400 MHz, $\left.\mathrm{CDCl}_{3}\right) \delta 7.37-7.31$ (m, 4H, Ar-H), 7.24 (m, 1H, Ar-H), $2.34-2.14\left(\mathrm{~m}, 2 \mathrm{H}, \mathrm{CH}_{2}\right)$, $1.80\left(\mathrm{~s}, 6 \mathrm{H}, \mathrm{C}\left(\mathrm{CH}_{3}\right)_{2}\right), 1.51-1.42\left(\mathrm{~m}, 2 \mathrm{H}, \mathrm{CH}_{2}\right), 1.35\left(\mathrm{~m}, 2 \mathrm{H}, \mathrm{CH}_{2}\right), 0.91(\mathrm{t}, J=7.0 \mathrm{~Hz}$, 
3H, $\left.\mathrm{CH}_{3}\right) .{ }^{13} \mathrm{C} \mathrm{NMR}\left(100 \mathrm{MHz}, \mathrm{CDCl}_{3}\right) \delta 146.0$ (1C, Ar-C), 128.3 (2C, Ar-C), 127.0 (1C, Ar-C), 124.2 (2C, Ar-C), 82.2 (1C, C-O), 29.7 (1C, $\left.\mathrm{CH}_{2}\right), 29.0\left(2 \mathrm{C}, \mathrm{C}\left(\mathrm{CH}_{3}\right)_{2}\right)$, $22.7\left(1 \mathrm{C}, \mathrm{CH}_{2}\right), 21.9\left(1 \mathrm{C}, \mathrm{CH}_{2}\right), 13.7\left(1 \mathrm{C}, \mathrm{CH}_{3}\right)$. IR (neat): 3406, $2080(\mathrm{C}=\mathrm{N}), 1692$, $11431124,931,688 \mathrm{~cm}^{-1}$. HRMS (ESI) Calcd for $\left[\mathrm{C}_{15} \mathrm{H}_{20} \mathrm{~N}_{2} \mathrm{O}_{2} \mathrm{Na}, \mathrm{M}+\mathrm{Na}\right]^{+}: 283.1417$, Found: 283.1421.

\section{2-Phenylpropan-2-yl 2-diazotetradecanoate (1d)}

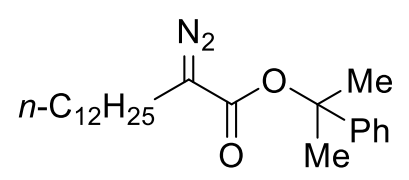

Serial number: 1y-4-17. Yellow oil. TLC $\mathrm{R}_{f}=0.29$ (PE/EA $=$

$50: 1 \mathrm{v} / \mathrm{v}), 86 \%$ yield. ${ }^{1} \mathrm{H}$ NMR $\left(400 \mathrm{MHz}, \mathrm{CDCl}_{3}\right) \delta 7.37-$ 7.27 (m, 4H, Ar-H), 7.25 - 7.19 (m, 1H, Ar-H), 2.40 - 2.05 (m, 2H, $\left.\mathrm{CH}_{2}\right), 1.77$ (s, 6H, C(CH3) $), 1.50-1.42\left(\mathrm{~m}, 2 \mathrm{H}, \mathrm{CH}_{2}\right), 1.26\left(\mathrm{~m}, 18 \mathrm{H}, \mathrm{CH}_{2}\right)$, $0.88\left(\mathrm{t}, J=6.8 \mathrm{~Hz}, 3 \mathrm{H}, \mathrm{CH}_{3}\right) .{ }^{13} \mathrm{C} \mathrm{NMR}\left(100 \mathrm{MHz} \mathrm{CDCl}_{3}\right) \delta 146.1$ (1C, Ar-C), 128.3 (2C, Ar-C), 127.0 (1C, Ar-C), 124.2 (2C, Ar-C), 82.2 (1C, C-O), 32.0 (1C, $\mathrm{CH}_{2}$ ), 29.7 (1C, $\left.\mathrm{CH}_{2}\right), 29.7\left(1 \mathrm{C}, \mathrm{CH}_{2}\right), 29.7\left(1 \mathrm{C}, \mathrm{CH}_{2}\right), 29.7\left(1 \mathrm{C}, \mathrm{CH}_{2}\right), 29.5\left(2 \mathrm{C}, \mathrm{CH}_{2}\right), 29.4(1 \mathrm{C}$, $\left.\mathrm{CH}_{2}\right), 29.0\left(2 \mathrm{C}, \mathrm{C}\left(\underline{\mathrm{CH}}_{3}\right)_{2}\right), 28.8\left(1 \mathrm{C}, \mathrm{CH}_{2}\right), 27.6\left(1 \mathrm{C}, \mathrm{CH}_{2}\right), 22.8\left(1 \mathrm{C}, \mathrm{CH}_{2}\right), 14.2(1 \mathrm{C}$, $\left.\mathrm{CH}_{3}\right)$. IR (neat): 3406, 2079(C=N), 1696, 1162, 928, 825, 786, $687 \mathrm{~cm}^{-1}$. HRMS (ESI) Calcd for $\left[\mathrm{C}_{23} \mathrm{H}_{36} \mathrm{~N}_{2} \mathrm{O}_{2} \mathrm{Na}, \mathrm{M}+\mathrm{Na}\right]^{+}:$395.2669, Found: 395.2674

\section{2-Phenylpropan-2-yl 2-diazo-5-methylhexanoate (1e)}<smiles>CC(C)CCC(=N)C(=O)OC(C)(C)c1ccccc1</smiles>

Serial number: 1y-4-31. Yellow oil. TLC $R_{f}=0.58(\mathrm{PE} / \mathrm{EA}=$

20:1 v/v), $77 \%$ yield. ${ }^{1} \mathrm{H} \mathrm{NMR}\left(400 \mathrm{MHz}, \mathrm{CDCl}_{3}\right) \delta 7.39-$ 7.30 (m, 4H, Ar-H), 7.25 (m, 1H, Ar-H), $2.30-2.18$ (m, 2H, $\left.\mathrm{CH}_{2}\right), 1.80\left(\mathrm{~s}, 6 \mathrm{H}, \mathrm{C}\left(\mathrm{CH}_{3}\right)_{2}\right), 1.64-1.48(\mathrm{~m}, 1 \mathrm{H}, \mathrm{CH}), 1.44-1.30\left(\mathrm{~m}, 2 \mathrm{H}, \mathrm{CH}_{2}\right), 0.90$ $\left(\mathrm{d}, J=6.3 \mathrm{~Hz}, 6 \mathrm{H}, \mathrm{CH}\left(\mathrm{CH}_{3}\right)_{2}\right) .{ }^{13} \mathrm{C} \mathrm{NMR}\left(100 \mathrm{MHz}, \mathrm{CDCl}_{3}\right) \delta 146.0(1 \mathrm{C}, \mathrm{Ar}-\mathrm{C}), 128.3$ (2C, Ar-C), 127.0 (1C, Ar-C), 124.2 (2C, Ar-C), 82.2 (1C, C-O), 36.2 (1C, $\mathrm{CH}_{2}$ ), 29.0 (2C, $\left.\left.\mathrm{C}\left(\underline{\mathrm{CH}}_{3}\right)_{2}\right), 27.4\left(1 \mathrm{C}, \mathrm{CH}_{2}\right), 22.4\left(1 \mathrm{C}, \underline{\mathrm{CH}}\left(\mathrm{CH}_{3}\right)_{2}\right), 20.6\left(2 \mathrm{C}, \mathrm{CH}(\underline{\mathrm{CH}})_{3}\right)_{2}\right)$. IR (neat): 3406, 2079(C=N), 1696, 1162m, 1157, 928m, 825m, 691, $687 \mathrm{~cm}^{-1}$. HRMS (ESI) Calcd for $\left[\mathrm{C}_{16} \mathrm{H}_{22} \mathrm{~N}_{2} \mathrm{O}_{2} \mathrm{Na}, \mathrm{M}+\mathrm{Na}\right]^{+}: 297.1573$, Found: 297.1580 . 


\section{2-Phenylpropan-2-yl 2-diazo-5,5-dimethylhexanoate (1f)}<smiles>CC(C)(C)CCC(=N)C(=O)OC(C)(C)c1ccccc1</smiles>

Serial number: 1y-4-20. Yellow oil. TLC $R_{f}=0.21(\mathrm{PE} / \mathrm{EA}=$

$50: 1 \mathrm{v} / \mathrm{v}), 80 \%$ yield. ${ }^{1} \mathrm{H} \mathrm{NMR}\left(400 \mathrm{MHz}, \mathrm{CDCl}_{3}\right) \delta 7.28-$ $7.22(\mathrm{~m}, 4 \mathrm{H}, \mathrm{Ar}-\mathrm{H}), 7.16-7.11(\mathrm{~m}, 1 \mathrm{H}, \mathrm{Ar}-\mathrm{H}), 2.21-2.06$ (m, 2H, $\left.\mathrm{CH}_{2}\right), 1.71\left(\mathrm{~s}, 6 \mathrm{H}, \mathrm{C}\left(\mathrm{CH}_{3}\right)_{2}\right), 1.34-1.24\left(\mathrm{~m}, 2 \mathrm{H}, \mathrm{CH}_{2}\right), 0.82\left(\mathrm{~s}, 9 \mathrm{H}, \mathrm{C}\left(\mathrm{CH}_{3}\right)_{3}\right)$. ${ }^{13} \mathrm{C}$ NMR (100 MHz, $\mathrm{CDCl}_{3}$ ) $\delta 146.0$ (1C, Ar-C), 128.4 (2C, Ar-C), 127.1 (1C, Ar-C), 124.3 (2C, Ar-C), 82.2 (1C, C-O), $30.2\left(1 \mathrm{C}, \underline{\mathrm{C}}\left(\mathrm{CH}_{3}\right)_{3}\right), 29.2\left(1 \mathrm{C}, \mathrm{CH}_{2}\right), 29.2\left(1 \mathrm{C}, \mathrm{CH}_{2}\right)$, $29.0\left(2 \mathrm{C}, \mathrm{C}\left(\underline{\mathrm{CH}}_{3}\right)_{2}\right), 18.4\left(3 \mathrm{C}, \mathrm{C}\left(\underline{\mathrm{CH}}_{3}\right)_{3}\right) . \mathrm{IR}$ (neat): 3406, $2079(\mathrm{C}=\mathrm{N}), 1696,1162$, 1159, 928, 821, 691, $685 \mathrm{~cm}^{-1}$. HRMS (ESI) Calcd for $\left[\mathrm{C}_{17} \mathrm{H}_{24} \mathrm{~N}_{2} \mathrm{O}_{2} \mathrm{Na}, \mathrm{M}+\mathrm{Na}\right]^{+}$: 311.1730, Found: 311.1733.

\section{2-Phenylpropan-2-yl 4-cyclohexyl-2-diazobutanoate (1g)}<smiles>CC(C)(P)OC(=O)C(=N)CCC1CCCCC1</smiles>

Serial number: ly-3-184. Yellow oil. TLC $R_{f}=0.72$ $(\mathrm{PE} / \mathrm{EA}=10: 1 \mathrm{v} / \mathrm{v}), 87 \%$ yield. ${ }^{1} \mathrm{H}$ NMR $(400 \mathrm{MHz}$, $\left.\mathrm{CDCl}_{3}\right) \delta 7.38-7.28(\mathrm{~m}, 4 \mathrm{H}, \mathrm{Ar}-\mathrm{H}), 7.25-7.19(\mathrm{~m}, 1 \mathrm{H}$, Ar-H), $2.34-2.18\left(\mathrm{~m}, 2 \mathrm{H}, \mathrm{CH}_{2}\right), 1.79\left(\mathrm{~s}, 6 \mathrm{H},\left(\mathrm{CH}_{3}\right)_{2}\right), 1.73-1.62\left(\mathrm{~m}, 5 \mathrm{H}, \mathrm{CH}_{2}, \mathrm{CH}\right)$, $1.42-1.30\left(\mathrm{~m}, 2 \mathrm{H}, \mathrm{CH}_{2}\right), 1.26-1.10\left(\mathrm{~m}, 4 \mathrm{H}, \mathrm{CH}_{2}\right), 0.93-0.85\left(\mathrm{~m}, 2 \mathrm{H}, \mathrm{CH}_{2}\right) .{ }^{13} \mathrm{C}$ NMR (100 MHz, $\left.\mathrm{CDCl}_{3}\right) \delta 146.0$ (1C, Ar-C), 128.3 (2C, Ar-C), 127.0 (1C, Ar-C), 124.2 (2C, Ar-C), 82.2 (1C, C-O), 36.9 (1C, CH), $34.9\left(1 \mathrm{C}, \mathrm{CH}_{2}\right), 33.1\left(2 \mathrm{C}, \mathrm{CH}_{2}\right), 29.0$ (2C, $\left.\mathrm{C}\left(\underline{\mathrm{CH}}_{3}\right)_{2}\right), 26.6$ (1C, $\left.\mathrm{CH}_{2}\right), 26.3$ (2C, $\left.\mathrm{CH}_{2}\right), 20.3$ (1C, $\left.\mathrm{CH}_{2}\right)$. IR (neat): 3403, 2077 $(\mathrm{C}=\mathrm{N}), 1696,1161,928,821,792,748,691,681 \mathrm{~cm}^{-1}$. HRMS (ESI) Calcd for $\left[\mathrm{C}_{19} \mathrm{H}_{26} \mathrm{~N}_{2} \mathrm{O}_{2} \mathrm{Na}, \mathrm{M}+\mathrm{Na}\right]^{+}:$337.1886, Found: 337.1891 .

\section{2-Phenylpropan-2-yl 3-cyclopropyl-2-diazopropanoate (1h)}

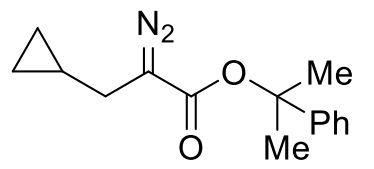

Serial number: 1y-4-140. Yellow oil. TLC $R_{f}=0.43(\mathrm{PE} / \mathrm{EA}=$ 20:1 v/v), 68\% yield. ${ }^{1} \mathrm{H}$ NMR (400 MHz, $\left.\mathrm{CDCl}_{3}\right) \delta 7.43-7.32$ (m, 4H, Ar-H), $7.31-7.21(\mathrm{~m}, 1 \mathrm{H}, \mathrm{Ar}-\mathrm{H}), 2.23(\mathrm{~d}, J=4.0 \mathrm{~Hz}$,

$\left.2 \mathrm{H}, \mathrm{CH}_{2}\right), 1.83\left(\mathrm{~s}, 6 \mathrm{H}, \mathrm{C}\left(\mathrm{CH}_{3}\right)_{2}\right), 1.10-0.71(\mathrm{~m}, 1 \mathrm{H}, \mathrm{CH}), 0.53(\mathrm{q}, J=4.9 \mathrm{~Hz}, 2 \mathrm{H}$, $\left.\mathrm{CH}_{2}\right), 0.18\left(\mathrm{~d}, J=4.6 \mathrm{~Hz}, 2 \mathrm{H}, \mathrm{CH}_{2}\right) .{ }^{13} \mathrm{C} \mathrm{NMR}\left(100 \mathrm{MHz}, \mathrm{CDCl}_{3}\right) \delta 146.1$ (1C, Ar-C), 
128.4 (2C, Ar-C), 127.1 (1C, Ar-C), 124.3 (2C, Ar-C), 82.2 (1C, C-O), 29.0 (2C, $\left.\mathrm{C}\left(\underline{\mathrm{CH}}_{3}\right)_{2}\right), 27.7\left(1 \mathrm{C}, \mathrm{CH}_{2}\right), 9.1(1 \mathrm{C}, \mathrm{CH}), 4.0$ (2C, $\left.\mathrm{CH}_{2}\right)$. IR (neat): 3406, $2079(\mathrm{C}=\mathrm{N})$, 1695, 1161, 1054, 769, 748, $691 \mathrm{~cm}^{-1}$. HRMS (ESI) Calcd for $\left[\mathrm{C}_{15} \mathrm{H}_{18} \mathrm{~N}_{2} \mathrm{O}_{2} \mathrm{Na}, \mathrm{M}+\right.$ $\mathrm{Na}^{+}:$281.1260, Found: 281.1268.

\section{2-Phenylpropan-2-yl 2-diazo-4-methylpentanoate (1i)}<smiles>CC(C)CC(=N)C(=O)OC(C)(C)c1ccccc1</smiles>

Serial number: 1y-4-47. Yellow oil. TLC $R_{f}=0.30(\mathrm{PE} / \mathrm{EA}=20: 1$

v/v), 75\% yield. ${ }^{1} \mathrm{H}$ NMR (400 MHz, $\left.\mathrm{CDCl}_{3}\right) \delta 7.40-7.27(\mathrm{~m}$, 4H, Ar-H), $7.26-7.18$ (m, 1H, Ar-H), $2.11(\mathrm{~d}, J=4.5 \mathrm{~Hz}, 2 \mathrm{H}$, $\left.\mathrm{CH}_{2}\right), 1.81-1.76\left(\mathrm{~m}, 7 \mathrm{H}, \mathrm{CH}, \mathrm{C}\left(\mathrm{CH}_{3}\right)_{2}\right), 0.93\left(\mathrm{~d}, J=6.5 \mathrm{~Hz}, 6 \mathrm{H}, \mathrm{CH}\left(\mathrm{C}_{3}\right)_{2}\right) .{ }^{13} \mathrm{C} \mathrm{NMR}$ $\left(100 \mathrm{MHz} \mathrm{CDCl}_{3}\right) \delta 146.1$ (1C, Ar-C), 128.3 (2C, Ar-C), 127.0 (1C, Ar-C), 124.2 (2C, Ar-C), 82.2 (1C, C-O), 32.1 (1C, $\left.\mathrm{CH}_{2}\right), 29.0$ (2C, $\left.\mathrm{C}\left(\underline{\mathrm{CH}_{3}}\right)_{2}\right), 28.1\left(1 \mathrm{C}, \underline{\mathrm{C}} \mathrm{H}\left(\mathrm{CH}_{3}\right)_{2}\right), 21.9$ (2C, $\left.\mathrm{CH}\left(\underline{\mathrm{CH}}_{3}\right)_{2}\right)$. IR (neat): 3402, $2079(\mathrm{C}=\mathrm{N}), 1695,892,761,691 \mathrm{~cm}^{-1} . \mathrm{HRMS}(\mathrm{ESI})$ Calcd for $\left[\mathrm{C}_{15} \mathrm{H}_{20} \mathrm{~N}_{2} \mathrm{O}_{2} \mathrm{Na}, \mathrm{M}+\mathrm{Na}\right]^{+}: 283.1417$, Found: 283.1420 .

\section{2-Phenylpropan-2-yl 2-diazo-3-phenylpropanoate (1j)}<smiles>CC(C)(OC(=O)C(=N)Cc1ccccc1)c1ccccc1</smiles>

Serial number: 1y-4-35. Yellow oil. TLC $R_{f}=0.28(\mathrm{PE} / \mathrm{EA}=$ $20: 1 \mathrm{v} / \mathrm{v}), 72 \%$ yield. ${ }^{1} \mathrm{H}$ NMR $\left(400 \mathrm{MHz}, \mathrm{CDCl}_{3}\right) \delta 7.46-$ $7.10(\mathrm{~m}, 10 \mathrm{H}, \mathrm{Ar}-\mathrm{H}), 3.56\left(\mathrm{~s}, 2 \mathrm{H}, \mathrm{CH}_{2}\right), 1.79\left(\mathrm{~s}, 6 \mathrm{H}, \mathrm{C}\left(\mathrm{CH}_{3}\right)_{2}\right)$. ${ }^{13} \mathrm{C}$ NMR (100 MHz, $\left.\mathrm{CDCl}_{3}\right) \delta 145.9$ (1C, Ar-C), 137.1 (1C, Ar-C), 128.8 (2C, Ar-C), 128.4 (2C, Ar-C), 128.4 (1C, Ar-C), 127.1 (1C, Ar-C), 127.1 (1C, Ar-C), 124.3 (1C, Ar-C), 82.6 (1C, C-O), 29.0 (2C, C( $\left.\left.\underline{\mathrm{C}}_{3}\right)_{2}\right)$. IR (neat): 3402, $2077(\mathrm{C}=\mathrm{N})$, 1695, 1168, 1051, 761, $691 \mathrm{~cm}^{-1}$. HRMS (ESI) Calcd for $\left[\mathrm{C}_{18} \mathrm{H}_{18} \mathrm{~N}_{2} \mathrm{O}_{2} \mathrm{Na}, \mathrm{M}+\mathrm{Na}\right]^{+}: 317.1260$, Found: 317.1260.

\section{2-Phenylpropan-2-yl 2-diazo-4-phenylbutanoate (1k)}

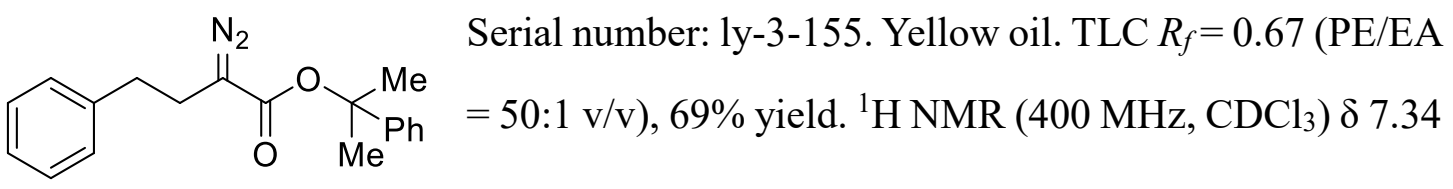


- 7.19 (m, 8H, Ar-H), $7.16(\mathrm{~m}, 2 \mathrm{H}, \mathrm{Ar}-\mathrm{H}), 2.78\left(\mathrm{t}, J=7.0 \mathrm{~Hz}, 2 \mathrm{H}, \mathrm{CH}_{2}\right), 2.62-2.44$ $\left(\mathrm{m}, 2 \mathrm{H}, \mathrm{CH}_{2}\right), 1.76\left(\mathrm{~s}, 6 \mathrm{H}, \mathrm{C}\left(\mathrm{CH}_{3}\right)_{2}\right) .{ }^{13} \mathrm{C} \mathrm{NMR}\left(100 \mathrm{MHz}, \mathrm{CDCl}_{3}\right) \delta 146.0(1 \mathrm{C}, \mathrm{Ar}-\mathrm{C})$, 140.3 (2C, Ar-C), 128.7 (1C, Ar-C), 128.4 (2C, Ar-C), 127.1 (2C, Ar-C), 126.5 (2C, ArC), 124.3 (2C, Ar-C), 82.3 (1C, C-O), 33.9 (1C, $\left.\mathrm{CH}_{2}\right), 29.0\left(2 \mathrm{C}, \mathrm{C}\left(\underline{\mathrm{CH}}_{3}\right)_{2}\right), 25.5$ (1C, $\mathrm{CH}_{2}$ ). IR (neat): 3406, $2076(\mathrm{C}=\mathrm{N}), 1695,1161,1054,769,748,691 \mathrm{~cm}^{-1}$. HRMS (ESI) Calcd for $\left[\mathrm{C}_{19} \mathrm{H}_{20} \mathrm{~N}_{2} \mathrm{O}_{2} \mathrm{Na}, \mathrm{M}+\mathrm{Na}\right]^{+}:$331.1417, Found: 331.1421 .

\section{2-Phenylpropan-2-yl 2-diazo-5-phenylpentanoate (11)}<smiles>CC(C)(OC(=O)C(=N)CCCc1ccccc1)c1ccccc1</smiles>

Serial number: 1y-3-162. Yellow oil. TLC $R_{f}=0.22$ $(\mathrm{PE} / \mathrm{EA}=20: 1 \mathrm{v} / \mathrm{v}), 88 \%$ yield. ${ }^{1} \mathrm{H}$ NMR $(400 \mathrm{MHz}$, $\left.\mathrm{CDCl}_{3}\right) \delta 7.47-7.37$ (m, 4H, Ar-H), $7.37-7.28(\mathrm{~m}, 3 \mathrm{H}$, Ar-H), $7.28-7.19$ (m, 3H, Ar-H), 2.71 (t, $\left.J=7.3 \mathrm{~Hz}, 2 \mathrm{H}, \mathrm{CH}_{2}\right), 2.39-2.32$ (m, 2H, $\left.\mathrm{CH}_{2}\right), 2.01-1.77\left(\mathrm{~m}, 8 \mathrm{H}, \mathrm{CH}_{2}, \mathrm{C}\left(\mathrm{CH}_{3}\right)_{2}\right) .{ }^{13} \mathrm{C} \mathrm{NMR}\left(100 \mathrm{MHz}, \mathrm{CDCl}_{3}\right) \delta 146.1(1 \mathrm{C}$, Ar-C), 141.6 (1C, Ar-C), 128.6 (2C, Ar-C), 128.6 (2C, Ar-C), 128.5 (2C, Ar-C), 127.2 (2C, Ar-C), 126.2 (1C, Ar-C), 124.4 (1C, Ar-C), 82.4 (1C, C-O), 35.1 (1C, $\left.\mathrm{CH}_{2}\right), 29.5$ (1C, $\left.\mathrm{CH}_{2}\right), 29.1\left(2 \mathrm{C}, \mathrm{C}\left(\underline{\mathrm{CH}}_{3}\right)_{2}\right), 22.9\left(1 \mathrm{C}, \mathrm{CH}_{2}\right)$. IR (neat): 3406, $2076(\mathrm{C}=\mathrm{N}), 1695$, 1159, 1121, 769, 748, $688 \mathrm{~cm}^{-1}$. HRMS (ESI) Calcd for $\left[\mathrm{C}_{20} \mathrm{H}_{22} \mathrm{~N}_{2} \mathrm{O}_{2} \mathrm{Na}, \mathrm{M}+\mathrm{Na}\right]^{+}$: 345.1573, Found: 345.1574 .

\section{2-Phenylpropan-2-yl 2-diazopent-4-enoate (1 m)}

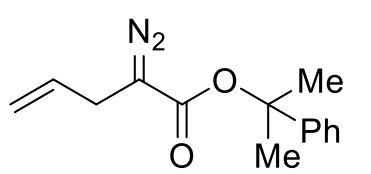

Serial number: 1y-3-172. Yellow oil. TLC $R_{f}=0.24(\mathrm{PE} / \mathrm{EA}=$

$10: 1 \mathrm{v} / \mathrm{v}), 84 \%$ yield. ${ }^{1} \mathrm{H} \mathrm{NMR}\left(400 \mathrm{MHz}, \mathrm{CDCl}_{3}\right) \delta 7.39-7.29$ (m, 4H, Ar-H), $7.28-7.20(\mathrm{~m}, 1 \mathrm{H}$, Ar-H), $5.98-5.64(\mathrm{~m}, 1 \mathrm{H}$, $\mathrm{CH}=), 5.27-5.00\left(\mathrm{~m}, 2 \mathrm{H}, \mathrm{CH}_{2}=\right), 2.99\left(\mathrm{~s}, 2 \mathrm{H}, \mathrm{CH}_{2}\right), 1.80\left(\mathrm{~s}, 6 \mathrm{H}, \mathrm{C}\left(\mathrm{CH}_{3}\right)_{2}\right) .{ }^{13} \mathrm{C} \mathrm{NMR}$ $\left(101 \mathrm{MHz}, \mathrm{CDCl}_{3}\right) \delta 145.9$ (1C, Ar-C), 132.7 (1C, CH=), 128.4 (2C, Ar-C), 127.1 (1C, Ar-C), 124.2 (2C, Ar-C), $117.6\left(1 \mathrm{C}, \mathrm{CH}_{2}=\right), 82.4$ (1C, C-O), $29.0\left(2 \mathrm{C}, \mathrm{C}\left(\underline{\mathrm{C}}_{3}\right)_{2}\right), 26.7$ (1C, $\left.\mathrm{CH}_{2}\right)$. IR (neat): 3403, $2074(\mathrm{C}=\mathrm{N}), 1692,1160,1132,1057,769,748,721,691$ $\mathrm{cm}^{-1}$. HRMS (ESI) Calcd for $\left[\mathrm{C}_{14} \mathrm{H}_{16} \mathrm{~N}_{2} \mathrm{O}_{2} \mathrm{Na}, \mathrm{M}+\mathrm{Na}^{+}:\right.$267.1104, Found: 267.1105. 


\section{2-Phenylpropan-2-yl 2-diazohex-5-enoate (1n)}

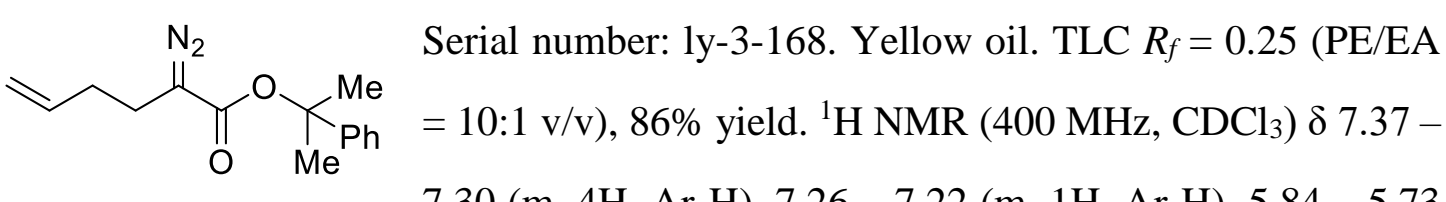

$7.30(\mathrm{~m}, 4 \mathrm{H}, \mathrm{Ar}-\mathrm{H}), 7.26-7.22(\mathrm{~m}, 1 \mathrm{H}, \mathrm{Ar}-\mathrm{H}), 5.84-5.73$ (m, 1H, $\mathrm{CH}=), 5.15-4.94\left(\mathrm{~m}, 2 \mathrm{H}, \mathrm{CH}_{2}=\right), 2.46-2.16\left(\mathrm{~m}, 4 \mathrm{H}, \mathrm{CH}_{2}\right), 1.79(\mathrm{~s}, 6 \mathrm{H}$, $\left.\mathrm{C}\left(\mathrm{CH}_{3}\right)_{2}\right) .{ }^{13} \mathrm{C} \mathrm{NMR}\left(100 \mathrm{MHz}, \mathrm{CDCl}_{3}\right) \delta 146.0$ (1C, $\left.\mathrm{Ar}-\mathrm{C}\right), 136.5(1 \mathrm{C}, \mathrm{CH}=), 128.3$ (2C, Ar-C), 127.0 (1C, Ar-C), 124.2 (2C, Ar-C), 116.5 (1C, $\left.\mathrm{CH}_{2}=\right), 82.3$ (1C, C-O), $31.8\left(1 \mathrm{C}, \mathrm{CH}_{2}\right), 29.0\left(2 \mathrm{C}, \mathrm{C}\left(\underline{\mathrm{CH}}_{3}\right)_{2}\right), 22.7\left(1 \mathrm{C}, \mathrm{CH}_{2}\right)$. IR (neat): 3406, $2073(\mathrm{C}=\mathrm{N}), 1692$, 1154, 1135, 1057, 762, 718, $691 \mathrm{~cm}^{-1}$. HRMS (ESI) Calcd for $\left[\mathrm{C}_{15} \mathrm{H}_{18} \mathrm{~N}_{2} \mathrm{O}_{2} \mathrm{Na}, \mathrm{M}+\right.$ $\mathrm{Na}]^{+}:$281.1260, Found: 281.1265.

\section{2-Phenylpropan-2-yl 2-diazooct-7-enoate (10)}

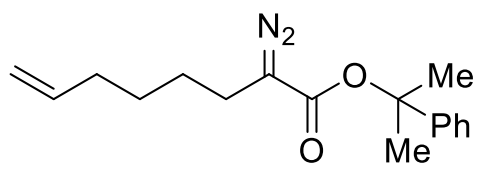

Serial number: ly-3-163. Yellow oil. TLC $R_{f}=0.61$

$(\mathrm{PE} / \mathrm{EA}=8: 1 \mathrm{v} / \mathrm{v}), 80 \%$ yield. ${ }^{1} \mathrm{H}$ NMR $(400 \mathrm{MHz}$, $\left.\mathrm{CDCl}_{3}\right) \delta 7.29-7.18(\mathrm{~m}, 4 \mathrm{H}, \mathrm{Ar}-\mathrm{H}), 7.16-7.09(\mathrm{~m}, 1 \mathrm{H}$, Ar-H), $5.74-5.61(\mathrm{~m}, 1 \mathrm{H}, \mathrm{CH}=), 4.87\left(\mathrm{dd}, J=18.1,14.6 \mathrm{~Hz}, 2 \mathrm{H}, \mathrm{CH}_{2}=\right), 2.18$ (d, $J=$ $\left.17.3 \mathrm{~Hz}, 2 \mathrm{H}, \mathrm{CH}_{2}\right), 1.96\left(\mathrm{~d}, J=6.6 \mathrm{~Hz}, 2 \mathrm{H}, \mathrm{CH}_{2}\right), 1.69\left(\mathrm{~s}, 6 \mathrm{H}, \mathrm{C}\left(\mathrm{CH}_{3}\right)_{2}\right), 1.45-1.24$ (m, 4H, CH $) .{ }^{13} \mathrm{C} \mathrm{NMR}\left(100 \mathrm{MHz}, \mathrm{CDCl}_{3}\right) \delta 146.0(1 \mathrm{C}, \mathrm{Ar}-\mathrm{C}), 138.5(1 \mathrm{C}, \mathrm{CH}=), 128.3$ (2C, Ar-C), 127.0 (1C, Ar-C), 124.2 (2C, Ar-C), 114.8 (1C, $\left.\mathrm{CH}_{2}=\right), 82.2$ (1C, C-O), $33.4\left(1 \mathrm{C}, \mathrm{CH}_{2}\right), 29.0\left(2 \mathrm{C}, \mathrm{C}\left(\mathrm{CH}_{3}\right)_{2}\right), 28.0\left(1 \mathrm{C}, \mathrm{CH}_{2}\right), 27.0\left(1 \mathrm{C}, \mathrm{CH}_{2}\right), 22.9\left(1 \mathrm{C}, \mathrm{CH}_{2}\right)$. IR (neat): 3403, $2075(\mathrm{C}=\mathrm{N}), 1692,1153,1134,1052,761,748,719,690 \mathrm{~cm}^{-1}$. HRMS (ESI) Calcd for $\left[\mathrm{C}_{17} \mathrm{H}_{22} \mathrm{~N}_{2} \mathrm{O}_{2} \mathrm{Na}, \mathrm{M}+\mathrm{Na}\right]^{+}: 309.1573$, Found: 309.1577 .

\section{2-Phenylpropan-2-yl 2-diazohex-5-ynoate (1p)}

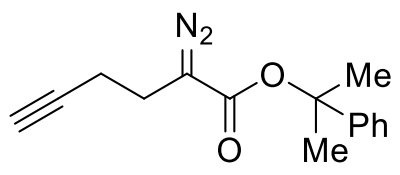

Serial number: ly-4-43. Yellow oil. TLC $R_{f}=0.54(\mathrm{PE} / \mathrm{EA}=$ $20: 1 \mathrm{v} / \mathrm{v}), 66 \%$ yield. ${ }^{1} \mathrm{H}$ NMR (400 $\left.\mathrm{MHz}, \mathrm{CDCl}_{3}\right) \delta 7.38-$ $7.30(\mathrm{~m}, 4 \mathrm{H}, \mathrm{Ar}-\mathrm{H}), 7.28-7.21(\mathrm{~m}, 1 \mathrm{H}, \mathrm{Ar}-\mathrm{H}), 2.44(\mathrm{~m}, 4 \mathrm{H}$, $\left.\mathrm{CH}_{2}\right), 1.98(\mathrm{~s}, 1 \mathrm{H}, \mathrm{CH}), 1.80\left(\mathrm{~s}, 6 \mathrm{H}, \mathrm{C}\left(\mathrm{CH}_{3}\right)_{2}\right) .{ }^{13} \mathrm{C} \mathrm{NMR}\left(100 \mathrm{MHz}, \mathrm{CDCl}_{3}\right) \delta 145.8$ (1C, Ar-C), 128.4 (2C, Ar-C), 127.1 (1C, Ar-C), 124.2 (2C, Ar-C), 82.5 (1C, C-O, 1C, 
$\mathrm{C} \equiv), 69.8(1 \mathrm{C}, \mathrm{CH} \equiv), 29.0\left(2 \mathrm{C}, \mathrm{C}\left(\underline{\mathrm{CH}}_{3}\right)_{2}\right), 23.1\left(1 \mathrm{C}, \mathrm{CH}_{2}\right), 17.9$ (1C, $\left.\mathrm{CH}_{2}\right)$. IR (neat): 3403, 2819, 2264, $2080(\mathrm{C}=\mathrm{N}), 1692,1249,761,748,719,690 \mathrm{~cm}^{-1}$. HRMS (ESI) Calcd for $\left[\mathrm{C}_{15} \mathrm{H}_{16} \mathrm{~N}_{2} \mathrm{O}_{2} \mathrm{Na}, \mathrm{M}+\mathrm{Na}\right]^{+}: 279.1104$, Found: 279.1108 .

\section{2-Phenylpropan-2-yl 2-diazo-5-methoxypentanoate (1q)}

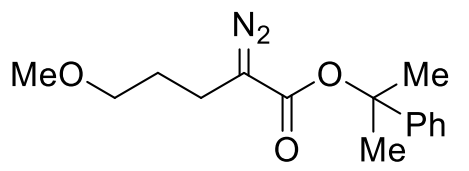

Serial number: ly-3-167. Yellow oil. TLC $R_{f}=0.34$

$(\mathrm{PE} / \mathrm{EA}=5: 1 \mathrm{v} / \mathrm{v}), 81 \%$ yield. ${ }^{1} \mathrm{H} \mathrm{NMR}\left(400 \mathrm{MHz}, \mathrm{CDCl}_{3}\right)$ $\delta 7.46-7.34(\mathrm{~m}, 4 \mathrm{H}, \mathrm{Ar}-\mathrm{H}), 7.31-7.25$ (m, 1H, Ar-H), $3.44\left(\mathrm{t}, J=5.9 \mathrm{~Hz}, 2 \mathrm{H}, \mathrm{CH}_{2}\right), 3.35\left(\mathrm{~s}, 3 \mathrm{H}, \mathrm{OCH}_{3}\right), 2.41$ (t, $\left.J=7.2 \mathrm{~Hz}, 2 \mathrm{H}, \mathrm{CH}_{2}\right), 1.89-$ $1.77\left(\mathrm{~m}, 8 \mathrm{H}, \mathrm{CH}_{2}, \mathrm{C}\left(\mathrm{CH}_{3}\right)_{2}\right) .{ }^{13} \mathrm{C} \mathrm{NMR}\left(100 \mathrm{MHz}, \mathrm{CDCl}_{3}\right) \delta 146.0(1 \mathrm{C}, \mathrm{Ar}-\mathrm{C}), 128.3$ (2C, Ar-C), 127.0 (1C, Ar-C), 124.2 (2C, Ar-C), 82.2 (1C, C-O), $71.2\left(1 \mathrm{C}, \mathrm{OCH}_{2}\right), 58.6$ (1C, $\left.\mathrm{OCH}_{3}\right), 29.0\left(2 \mathrm{C}, \mathrm{C}\left(\underline{\mathrm{CH}}_{3}\right)_{2}\right), 27.8\left(1 \mathrm{C}, \mathrm{CH}_{2}\right), 20.4$ (1C, $\left.\mathrm{CH}_{2}\right)$. IR (neat): 3403, 2080 $(\mathrm{C}=\mathrm{N}), 1692,1151,1143,849,728,691 \mathrm{~cm}^{-1}$. HRMS (ESI) Calcd for $\left[\mathrm{C}_{15} \mathrm{H}_{20} \mathrm{~N}_{2} \mathrm{O}_{3} \mathrm{Na}\right.$, $\mathrm{M}+\mathrm{Na}]^{+}:$299.1366, Found: 299.1370.

\section{2-Phenylpropan-2-yl 5-((tert-butyldimethylsilyl)oxy)-2-diazopentanoate (1r)}<smiles>CC(C)(C)OCCCC(=N)C(=O)OC(C)(C)c1ccccc1</smiles>

Serial number: ly-4-49. Yellow oil. TLC $R_{f}=0.58$

$(\mathrm{PE} / \mathrm{EA}=20: 1 \mathrm{v} / \mathrm{v}), 88 \%$ yield. ${ }^{1} \mathrm{H}$ NMR $(400 \mathrm{MHz}$, $\left.\mathrm{CDCl}_{3}\right) \delta 7.35-7.25(\mathrm{~m}, 4 \mathrm{H}, \mathrm{Ar}-\mathrm{H}), 7.23-7.16(\mathrm{~m}, 1 \mathrm{H}$, Ar-H), $3.74-3.47\left(\mathrm{~m}, 2 \mathrm{H}, \mathrm{CH}_{2}\right), 2.49-2.13\left(\mathrm{~m}, 2 \mathrm{H}, \mathrm{CH}_{2}\right), 1.75\left(\mathrm{~s}, 6 \mathrm{H}, \mathrm{C}\left(\mathrm{CH}_{3}\right)_{2}\right), 1.72$ $-1.60\left(\mathrm{~m}, 2 \mathrm{H}, \mathrm{CH}_{2}\right), 0.84\left(\mathrm{~s}, 9 \mathrm{H}, \mathrm{C}\left(\mathrm{CH}_{3}\right)_{3}\right), 0.03--0.04\left(\mathrm{~m}, 6 \mathrm{H}, \mathrm{Si}\left(\mathrm{CH}_{3}\right)_{2}\right) .{ }^{13} \mathrm{C} \mathrm{NMR}$ $\left(100 \mathrm{MHz}, \mathrm{CDCl}_{3}\right) \delta 146.0$ (1C, Ar-C), 128.3 (2C, Ar-C), 127.0 (1C, Ar-C), 124.2 (2C, Ar-C), 82.2 (1C, C-O), $61.7\left(1 \mathrm{C}, \mathrm{OCH}_{2}\right), 35.7$ (1C, $\left.\underline{\mathrm{C}}\left(\mathrm{CH}_{3}\right)_{3}\right), 29.0\left(2 \mathrm{C}, \mathrm{C}\left(\mathrm{CH}_{3}\right)_{2}\right), 26.0$ (3C, $\left.\mathrm{C}\left(\underline{\mathrm{CH}}_{3}\right)_{3}\right), 19.9\left(1 \mathrm{C}, \mathrm{CH}_{2}\right), 18.3\left(1 \mathrm{C}, \mathrm{CH}_{2}\right),-5.3\left(2 \mathrm{C}, \mathrm{Si}\left(\underline{\mathrm{CH}}_{3}\right)_{2}\right) . \mathrm{IR}$ (neat): 3406, 3208, $2079(\mathrm{C}=\mathrm{N}), 1692,1152,1128,892,789,692 \mathrm{~cm}^{-1}$. HRMS (ESI) Calcd for $\left[\mathrm{C}_{20} \mathrm{H}_{32} \mathrm{~N}_{2} \mathrm{O}_{3} \mathrm{SiNa}, \mathrm{M}+\mathrm{Na}\right]^{+}:$399.2074, Found: 399.2076. 


\section{6-Ethyl 1-(2-phenylpropan-2-yl) 2-diazohexanedioate (1s)}<smiles>CCOC(=O)CCCC(=N)C(=O)OC(C)(C)c1ccccc1</smiles>

Serial number: 1y-3-173. Yellow oil. TLC $R_{f}=0.21$

$(\mathrm{PE} / \mathrm{EA}=5: 1 \mathrm{v} / \mathrm{v}), 82 \%$ yield. ${ }^{1} \mathrm{H} \mathrm{NMR}(400 \mathrm{MHz}$, $\left.\mathrm{CDCl}_{3}\right) \delta 7.37-7.30(\mathrm{~m}, 4 \mathrm{H}, \mathrm{Ar}-\mathrm{H}), 7.27-7.21(\mathrm{~m}, 1 \mathrm{H}$, Ar-H), $4.12\left(\mathrm{q}, J=7.1 \mathrm{~Hz}, 2 \mathrm{H}, \mathrm{CH}_{2}\right), 2.47-2.18\left(\mathrm{~m}, 4 \mathrm{H}, \mathrm{CH}_{2}\right), 1.90-1.71(\mathrm{~m}, 8 \mathrm{H}$, $\left.\mathrm{CH}_{2},\left(\mathrm{CH}_{3}\right)_{2}\right), 1.24\left(\mathrm{t}, J=7.1 \mathrm{~Hz}, 3 \mathrm{H}, \mathrm{CH}_{3}\right) .{ }^{13} \mathrm{C} \mathrm{NMR}\left(100 \mathrm{MHz}, \mathrm{CDCl}_{3}\right) \delta 172.9(1 \mathrm{C}$, $\mathrm{C}=\mathrm{O}), 145.9$ (1C, Ar-C), 128.3 (2C, Ar-C), 127.1 (1C, Ar-C), 124.2 (2C, Ar-C), 82.4 (1C, C-O), $60.5\left(1 \mathrm{C}, \mathrm{OCH}_{2}\right), 33.1\left(1 \mathrm{C}, \mathrm{CH}_{2}\right), 28.9\left(2 \mathrm{C}, \mathrm{C}\left(\mathrm{CH}_{3}\right)_{2}\right), 23.1\left(1 \mathrm{C}, \mathrm{CH}_{2}\right), 22.8$ (1C, $\left.\mathrm{CH}_{2}\right), 14.2\left(1 \mathrm{C}, \mathrm{CH}_{3}\right)$. IR (neat): 3404, $2079(\mathrm{C}=\mathrm{N}), 1692,1690,1151,1139,849$, 728, 719, $690 \mathrm{~cm}^{-1}$. HRMS (ESI) Calcd for $\left[\mathrm{C}_{17} \mathrm{H}_{22} \mathrm{~N}_{2} \mathrm{O}_{4} \mathrm{Na}, \mathrm{M}+\mathrm{Na}\right]^{+}: 341.1472$, Found: 341.1475.

\section{2-Phenylpropan-2-yl 5-cyano-2-diazopentanoate (1t)}

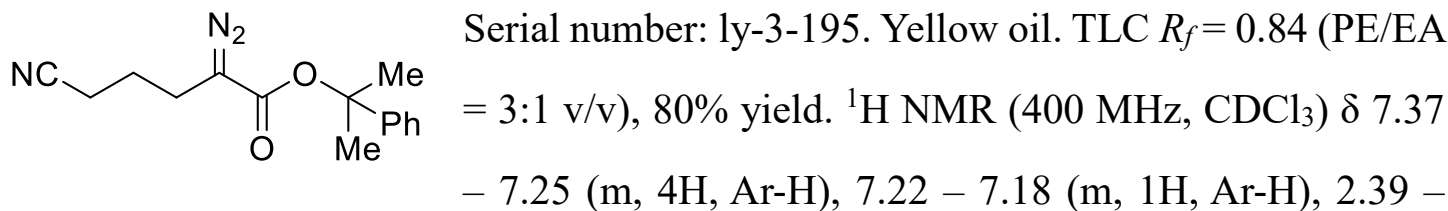

$2.12\left(\mathrm{~m}, 4 \mathrm{H}, \mathrm{CH}_{2}\right), 1.83-1.63\left(\mathrm{~m}, 8 \mathrm{H}, \mathrm{CH}_{2}, \mathrm{C}\left(\mathrm{CH}_{3}\right)_{2}\right) .{ }^{13} \mathrm{C} \mathrm{NMR}\left(100 \mathrm{MHz}, \mathrm{CDCl}_{3}\right)$ $\delta 165.7$ (1C, C=O), 145.9 (1C, Ar-C), 128.4 (2C, Ar-C), 127.1 (1C, Ar-C), 124.3 (2C, Ar-C), $119.3(1 \mathrm{C}, \mathrm{CN}), 82.6$ (1C, C-O), $29.0\left(2 \mathrm{C}, \mathrm{C}\left(\underline{\mathrm{CH}}_{3}\right)_{2}\right), 23.9\left(1 \mathrm{C}, \mathrm{CH}_{2}\right), 22.8(1 \mathrm{C}$, $\left.\mathrm{CH}_{2}\right), 16.1\left(1 \mathrm{C}, \mathrm{CH}_{2}\right)$. IR (neat): 3404, 2188, $2079(\mathrm{C}=\mathrm{N}), 1692,1154,843,712,690$ $\mathrm{cm}^{-1}$. HRMS (ESI) Calcd for $\left[\mathrm{C}_{15} \mathrm{H}_{17} \mathrm{~N}_{3} \mathrm{O}_{2} \mathrm{Na}, \mathrm{M}+\mathrm{Na}\right]^{+}:$294.1213, Found: 294.1217.

\section{(E)-tert-Butyl 2-diazo-4-phenylpent-3-enoate (6a)}<smiles>CCCCOC(=O)/C=C(\C)c1ccccc1</smiles>

Serial number: zt-4-29. Red oil. TLC $\mathrm{R}_{f}=0.49$ (PE/EA $=20: 1$

v/v), 75\% yield. ${ }^{1} \mathrm{H}$ NMR (400 MHz, $\left.\mathrm{CDCl}_{3}\right) \delta 7.44-7.38(\mathrm{~m}$, 2H, Ar-H), 7.32 (t, $J=7.5 \mathrm{~Hz}, 2 \mathrm{H}, \mathrm{Ar}-\mathrm{H}), 7.27-7.24(\mathrm{~m}, 1 \mathrm{H}$, Ar-H), $5.96(\mathrm{~d}, J=1.0 \mathrm{~Hz}, 1 \mathrm{H}, \mathrm{CH}=), 2.08$ (d, $\left.J=1.1 \mathrm{~Hz}, 3 \mathrm{H}, \mathrm{CH}_{3}\right), 1.52(\mathrm{~s}, 9 \mathrm{H}$, $\left.\mathrm{C}\left(\mathrm{CH}_{3}\right)_{3}\right) .{ }^{13} \mathrm{C} \mathrm{NMR}\left(100 \mathrm{MHz}, \mathrm{CDCl}_{3}\right) \delta 165.8(1 \mathrm{C}, \mathrm{C}=\mathrm{O}), 142.7(1 \mathrm{C}, \mathrm{Ar}-\mathrm{C}), 133.6$ $(1 \mathrm{C}, \mathrm{C}=), 128.3$ (2C, Ar-C), 127.1 (1C, Ar-C), 125.7 (2C, Ar-C), $109.6(1 \mathrm{C}, \mathrm{CH}=), 82.0$ 
(1C, C-O), 28.3 (3C, $\left.\mathrm{C}\left(\underline{\mathrm{CH}}_{3}\right)_{3}\right), 16.9$ (1C, $\left.\mathrm{CH}_{3}\right)$. IR (neat): 3057, 3027, 2979, 2073 $(\mathrm{C}=\mathrm{N}), 1699,1247,1164,1114,791,739,696 \mathrm{~cm}^{-1}$. HRMS (ESI) Calcd for $\left[\mathrm{C}_{15} \mathrm{H}_{18} \mathrm{O}_{2} \mathrm{~N}_{2} \mathrm{Na}, \mathrm{M}+\mathrm{Na}\right]^{+}: 281.1260$, Found: 281.1264.

\section{(E)-tert-Butyl 4-(m-tolyl)-2-diazopent-3-enoate (6b)}

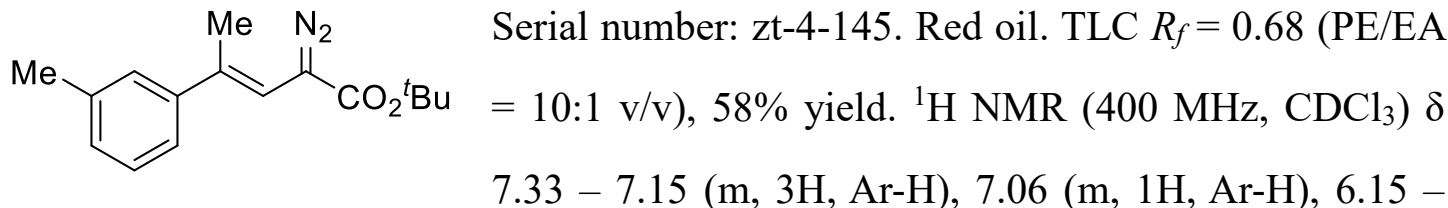

$5.67(\mathrm{~m}, 1 \mathrm{H}, \mathrm{CH}=), 2.35\left(\mathrm{~s}, 3 \mathrm{H}, \mathrm{Ar}-\mathrm{C}_{3}\right), 2.07\left(\mathrm{~d}, J=1.2 \mathrm{~Hz}, 3 \mathrm{H}, \mathrm{CH}_{3}\right), 1.52(\mathrm{~s}, 9 \mathrm{H}$, $\left.\mathrm{C}\left(\mathrm{CH}_{3}\right)_{3}\right) \cdot{ }^{13} \mathrm{C} \mathrm{NMR}\left(100 \mathrm{MHz}, \mathrm{CDCl}_{3}\right) \delta 165.8(1 \mathrm{C}, \mathrm{C}=\mathrm{O}), 142.7(1 \mathrm{C}, \mathrm{Ar}-\mathrm{C}), 137.8$ (1C, Ar-C), 133.8 (1C, C=), 128.2 (1C, Ar-C), 127.9 (1C, Ar-C), 126.5 (1C, Ar-C), $122.8(1 \mathrm{C}, \mathrm{Ar}-\mathrm{C}), 109.3(1 \mathrm{C}, \mathrm{CH}=), 81.9$ (1C, C-O), $28.3\left(3 \mathrm{C}, \mathrm{C}\left(\underline{\mathrm{CH}}_{3}\right)_{3}\right), 21.4(1 \mathrm{C}, \mathrm{Ar}-$ $\left.\underline{\mathrm{CH}}_{3}\right), 16.9\left(1 \mathrm{C}, \mathrm{CH}_{3}\right) . \mathrm{IR}$ (neat): 2979, 2931, $2073(\mathrm{C}=\mathrm{N}), 1698,1369,1252,1162,847$, $780,739 \mathrm{~cm}^{-1}$. HRMS (ESI) Calcd for $\left[\mathrm{C}_{16} \mathrm{H}_{20} \mathrm{~N}_{2} \mathrm{O}_{2} \mathrm{Na}, \mathrm{M}+\mathrm{Na}\right]^{+}:$295.1417, Found: 295.1418.

\section{(E)-tert-Butyl 4-(p-tolyl)-2-diazopent-3-enoate (6c)}

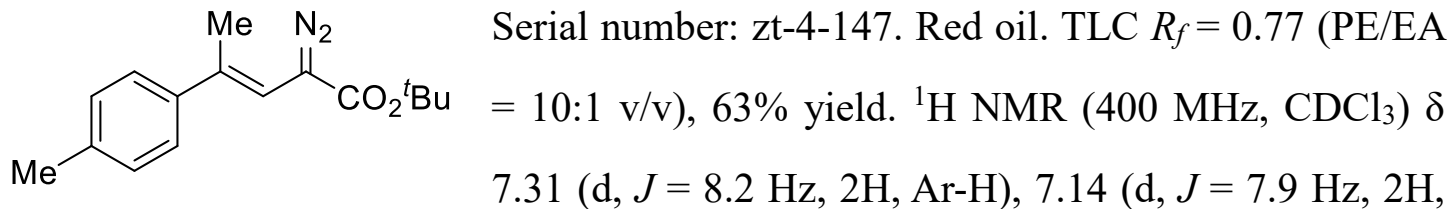
$\operatorname{Ar}-\mathrm{H}), 5.92(\mathrm{q}, J=1.3 \mathrm{~Hz}, 1 \mathrm{H}, \mathrm{CH}=), 2.34$ (s, 3H, $\left.\mathrm{Ar}-\mathrm{CH}_{3}\right), 2.06(\mathrm{~d}, J=1.2 \mathrm{~Hz}, 3 \mathrm{H}$, $\left.\mathrm{CH}_{3}\right), 1.52\left(\mathrm{~s}, 9 \mathrm{H}, \mathrm{C}\left(\mathrm{CH}_{3}\right)_{3}\right) .{ }^{13} \mathrm{C} \mathrm{NMR}\left(100 \mathrm{MHz}, \mathrm{CDCl}_{3}\right) \delta 165.9(1 \mathrm{C}, \mathrm{C}=\mathrm{O}), 139.8$ (1C, Ar-C), 137.0 (1C, Ar-C), 133.7 (1C, C=), 129.0 (2C, Ar-C), 125.6 (2C, Ar-C), $108.7(1 \mathrm{C}, \mathrm{CH}=), 81.9(1 \mathrm{C}, \mathrm{C}-\mathrm{O}), 28.3\left(3 \mathrm{C}, \mathrm{C}\left(\underline{\mathrm{CH}}_{3}\right)_{3}\right), 21.0\left(1 \mathrm{C}, \mathrm{Ar}-\mathrm{CH}_{3}\right), 16.9(1 \mathrm{C}$, $\left.\mathrm{CH}_{3}\right)$. IR (neat): 2978, 2931, $2072(\mathrm{C}=\mathrm{N}), 1698,1369,1249,1164,1114,847,813,739$ $\mathrm{cm}^{-1}$. HRMS (ESI) Calcd for $\left[\mathrm{C}_{16} \mathrm{H}_{20} \mathrm{~N}_{2} \mathrm{O}_{2} \mathrm{Na}, \mathrm{M}+\mathrm{Na}\right]^{+}: 295.1417$, Found: 295.1420 . 
(E)-tert-Butyl 4-(4-methoxyphenyl)-2-diazopent-3-enoate (6d)

$7.35(\mathrm{~d}, J=8.8 \mathrm{~Hz}, 2 \mathrm{H}, \mathrm{Ar}-\mathrm{H}), 6.86(\mathrm{~d}, J=8.8 \mathrm{~Hz}, 2 \mathrm{H}$,
$\mathrm{CO}_{2}{ }^{t} \mathrm{Bu}$
$=20: 1 \mathrm{v} / \mathrm{v}), 66 \%$ yield. ${ }^{1} \mathrm{H} \mathrm{NMR}\left(400 \mathrm{MHz}, \mathrm{CDCl}_{3}\right) \delta$ $\operatorname{Ar}-\mathrm{H}), 5.87(\mathrm{~s}, 1 \mathrm{H}, \mathrm{CH}=), 3.81\left(\mathrm{~s}, 3 \mathrm{H}, \mathrm{OCH}_{3}\right), 2.05\left(\mathrm{~d}, J=0.5 \mathrm{~Hz}, 3 \mathrm{H}, \mathrm{CH}_{3}\right), 1.52$ (s, 9H, $\left.\mathrm{C}\left(\mathrm{CH}_{3}\right)_{3}\right) .{ }^{13} \mathrm{C} \mathrm{NMR}\left(100 \mathrm{MHz}, \mathrm{CDCl}_{3}\right) \delta 166.0(1 \mathrm{C}, \mathrm{C}=\mathrm{O}), 158.9$ (1C, $\left.\mathrm{Ar}-\mathrm{C}\right)$, $135.2(1 \mathrm{C}, \mathrm{Ar}-\mathrm{C}), 133.6(1 \mathrm{C}, \mathrm{C}=), 126.8(2 \mathrm{C}, \mathrm{Ar}-\mathrm{C}), 113.6(2 \mathrm{C}, \mathrm{Ar}-\mathrm{C}), 107.8(1 \mathrm{C}, \mathrm{CH}=)$, 81.9 (1C, C-O), 55.3 (1C, $\left.\mathrm{OCH}_{3}\right), 28.3\left(3 \mathrm{C}, \mathrm{C}\left(\underline{\mathrm{CH}}_{3}\right)_{3}\right), 17.0$ (1C, $\left.\mathrm{CH}_{3}\right)$. IR (neat): 2978, 2934, 2837, $2073(\mathrm{C}=\mathrm{N}), 1696,1513,1250,1167,1112,825,786 \mathrm{~cm}^{-1}$. HRMS (ESI) Calcd for $\left[\mathrm{C}_{16} \mathrm{H}_{20} \mathrm{~N}_{2} \mathrm{O}_{3} \mathrm{Na}, \mathrm{M}+\mathrm{Na}\right]^{+}: 311.1366$, Found: 311.1365 .

\section{(E)-tert-Butyl 4-(4-fluorophenyl)-2-diazopent-3-enoate (6e)}<smiles>CCCOC(=O)/C=C(\C)c1ccc(F)cc1</smiles>

Serial number: zt-3-101. Red oil. TLC $R_{f}=0.52(\mathrm{PE} / \mathrm{EA}=$

$20: 1 \mathrm{v} / \mathrm{v}), 65 \%$ yield. ${ }^{1} \mathrm{H}$ NMR $\left(400 \mathrm{MHz}, \mathrm{CDCl}_{3}\right) \delta 7.36$ (dd, $J=8.7,5.5 \mathrm{~Hz}, 2 \mathrm{H}, \mathrm{Ar}-\mathrm{H}), 7.01(\mathrm{~d}, J=8.7 \mathrm{~Hz}, 2 \mathrm{H}, \mathrm{Ar}-$ $\mathrm{H}), 5.96-5.81(\mathrm{~m}, 1 \mathrm{H}, \mathrm{CH}=), 2.06\left(\mathrm{~d}, J=1.2 \mathrm{~Hz}, 3 \mathrm{H}, \mathrm{CH}_{3}\right), 1.52\left(\mathrm{~s}, 9 \mathrm{H}, \mathrm{C}\left(\mathrm{CH}_{3}\right)_{3}\right)$. ${ }^{13} \mathrm{C} \mathrm{NMR}\left(100 \mathrm{MHz}, \mathrm{CDCl}_{3}\right) \delta 165.7(1 \mathrm{C}, \mathrm{C}=\mathrm{O}), 162.1$ (d, $\left.{ }^{1} J_{\mathrm{C}-\mathrm{F}}=124 \mathrm{~Hz}, 1 \mathrm{C}, \mathrm{Ar}-\mathrm{C}\right)$, $138.8\left(\mathrm{~d},{ }^{4} J_{\mathrm{C}-\mathrm{F}}=3.2 \mathrm{~Hz}, 1 \mathrm{C}, \mathrm{Ar}-\mathrm{C}\right), 132.7(1 \mathrm{C}, \mathrm{C}=), 127.3\left(\mathrm{~d},{ }^{3} J_{\mathrm{C}-\mathrm{F}}=8.0 \mathrm{~Hz}, 2 \mathrm{C}, \mathrm{Ar}-\right.$ C), , $115.1\left(\mathrm{~d},{ }^{2} J_{\mathrm{C}-\mathrm{F}}=21.6 \mathrm{~Hz}, 2 \mathrm{C}, \mathrm{Ar}-\mathrm{C}\right), 109.6(1 \mathrm{C}, \mathrm{CH}=), 82.1(1 \mathrm{C}, \mathrm{C}-\mathrm{O}), 28.3(3 \mathrm{C}$, $\left.\mathrm{C}\left(\mathrm{CH}_{3}\right)_{3}\right), 17.0\left(1 \mathrm{C}, \mathrm{CH}_{3}\right)$. IR (neat): 2979, 2933, $2074(\mathrm{C}=\mathrm{N}), 1697,1510,1245,1161$, 1114, 828, 788, $739 \mathrm{~cm}^{-1}$. HRMS (ESI) Calcd for $\left[\mathrm{C}_{15} \mathrm{H}_{17} \mathrm{FN}_{2} \mathrm{O}_{2} \mathrm{Na}, \mathrm{M}+\mathrm{Na}\right]^{+}: 299.1166$, Found: 299.1168.

\section{(E)-tert-Butyl 4-(2-chlorophenyl)-2-diazopent-3-enoate (6f)}<smiles>CCCOC(=O)/C=C(\C)c1ccccc1Cl</smiles>

Serial number: zt-4-82. Red oil. TLC $R_{f}=0.83$ (PE/EA $=10: 1$ v/v), 65\% yield. ${ }^{1} \mathrm{H}$ NMR (400 MHz, $\left.\mathrm{CDCl}_{3}\right) \delta 7.40-7.31(\mathrm{~m}$, 1H, Ar-H), $7.24-7.18$ (m, 3H, Ar-H), $5.66(\mathrm{~s}, 1 \mathrm{H}, \mathrm{CH}=), 2.05$ (s, 3H, $\left.\mathrm{CH}_{3}\right), 1.51$ (s, 9H, $\left.\mathrm{C}\left(\mathrm{CH}_{3}\right)_{3}\right) .{ }^{13} \mathrm{C} \mathrm{NMR}\left(100 \mathrm{MHz}, \mathrm{CDCl}_{3}\right) \delta 165.4(1 \mathrm{C}, \mathrm{C}=\mathrm{O})$, 143.4 (1C, Ar-C), 133.0 (1C, Ar-C), 132.1 (1C, C=), 130.1 (1C, Ar-C), 129.6 (1C, Ar- 
C), 128.3 (1C, Ar-C), 126.7 (1C, Ar-C), 113.1 (1C, $\mathrm{CH}=), 81.9$ (1C, C-O), 28.3 (3C, $\left.\mathrm{C}\left(\underline{\mathrm{CH}}_{3}\right)_{3}\right), 18.5\left(1 \mathrm{C}, \mathrm{CH}_{3}\right)$. IR (neat): 2980, 2934, $2078(\mathrm{C}=\mathrm{N}), 1699,1370,1166,1116$, 849, 754, $741 \mathrm{~cm}^{-1}$. HRMS (ESI) Calcd for $\left[\mathrm{C}_{15} \mathrm{H}_{17} \mathrm{ClN}_{2} \mathrm{O}_{2} \mathrm{Na}, \mathrm{M}+\mathrm{Na}\right]^{+}: 315.0871$, Found: 315.0869 .

\section{(E)-tert-Butyl 4-(3-chlorophenyl)-2-diazopent-3-enoate (6g)}<smiles>CCCOC(=O)C=C(C)c1cccc(Cl)c1</smiles>

Serial number: zt-4-79. Red oil. TLC $R_{f}=0.84(\mathrm{PE} / \mathrm{EA}=$

10:1 v/v), $70 \%$ yield. ${ }^{1} \mathrm{H}$ NMR (400 MHz, $\left.\mathrm{CDCl}_{3}\right) \delta 7.38$ (t, $J=2.0 \mathrm{~Hz}, 1 \mathrm{H}, \mathrm{Ar}-\mathrm{H}), 7.29-7.20$ (m, 3H, Ar-H), 6.01 $-5.94(\mathrm{~m}, 1 \mathrm{H}, \mathrm{CH}=), 2.06\left(\mathrm{~d}, J=1.6 \mathrm{~Hz}, 3 \mathrm{H}, \mathrm{CH}_{3}\right), 1.52\left(\mathrm{~s}, 9 \mathrm{H}, \mathrm{C}\left(\mathrm{CH}_{3}\right)_{3}\right) .{ }^{13} \mathrm{C} \mathrm{NMR}$ $\left(100 \mathrm{MHz} \mathrm{CDCl}_{3}\right) \delta 165.5(1 \mathrm{C}, \mathrm{C}=\mathrm{O}), 144.6(1 \mathrm{C}, \mathrm{Ar}-\mathrm{C}), 134.3(1 \mathrm{C}, \mathrm{Ar}-\mathrm{C}), 131.8(1 \mathrm{C}$, $\mathrm{C}=), 129.5$ (1C, Ar-C), 127.0 (1C, Ar-C), 125.9 (1C, Ar-C), 123.8 (1C, Ar-C), 111.0 $(1 \mathrm{C}, \mathrm{CH}=), 82.2(1 \mathrm{C}, \mathrm{C}-\mathrm{O}), 28.3\left(3 \mathrm{C}, \mathrm{C}\left(\underline{\mathrm{CH}}_{3}\right)_{3}\right), 16.7$ (1C, $\left.\mathrm{CH}_{3}\right)$. IR (neat): 2980, 2934, $2076(\mathrm{C}=\mathrm{N}), 1698,1296,1246,1163,1117,780,741 \mathrm{~cm}^{-1}$. HRMS (ESI) Calcd for $\left[\mathrm{C}_{15} \mathrm{H}_{17} \mathrm{ClN}_{2} \mathrm{O}_{2} \mathrm{Na}, \mathrm{M}+\mathrm{Na}\right]^{+}: 315.0871$, Found: 315.0871 .

\section{(E)-tert-Butyl 4-(4-chlorophenyl)-2-diazopent-3-enoate (6h)}<smiles>CCCOC(=O)C(C#N)=CC(C)c1ccc(Cl)cc1</smiles>

Serial number: zt-3-117. Red oil. TLC $R_{f}=0.43(\mathrm{PE} / \mathrm{EA}=$

$20: 1 \mathrm{v} / \mathrm{v}), 68 \%$ yield. ${ }^{1} \mathrm{H}$ NMR (400 MHz, $\left.\mathrm{CDCl}_{3}\right) \delta 7.39$ - $7.20(\mathrm{~m}, 4 \mathrm{H}, \mathrm{Ar}-\mathrm{H}), 5.94(\mathrm{~s}, 1 \mathrm{H}, \mathrm{CH}=), 2.06(\mathrm{~d}, J=0.9$ $\left.\mathrm{Hz}, 3 \mathrm{H}, \mathrm{CH}_{3}\right), 1.52\left(\mathrm{~s}, 9 \mathrm{H}, \mathrm{C}\left(\mathrm{CH}_{3}\right)_{3}\right) .{ }^{13} \mathrm{C} \mathrm{NMR}\left(100 \mathrm{MHz}, \mathrm{CDCl}_{3}\right) \delta 165.6(1 \mathrm{C}, \mathrm{C}=\mathrm{O})$, $141.1(1 \mathrm{C}, \mathrm{C}=), 132.9$ (1C, Ar-C), 132.2 (1C, Ar-C), 128.4 (2C, Ar-C), 126.9 (2C, ArC), $110.2(1 \mathrm{C}, \mathrm{CH}=), 82.2(1 \mathrm{C}, \mathrm{C}-\mathrm{O}), 28.3\left(3 \mathrm{C}, \mathrm{C}\left(\underline{\mathrm{CH}}_{3}\right)_{3}\right), 16.8\left(1 \mathrm{C}, \mathrm{CH}_{3}\right)$. IR (neat): 2979, 2931, $2073(\mathrm{C}=\mathrm{N}), 1697,1454,1368,1245,1162,1166,979,845,820,740 \mathrm{~cm}^{-}$ 1. HRMS (ESI) Calcd for $\left[\mathrm{C}_{15} \mathrm{H}_{17} \mathrm{ClN}_{2} \mathrm{O}_{2} \mathrm{Na}, \mathrm{M}+\mathrm{Na}\right]^{+}: 315.0871$, Found: 315.0875. 


\section{(E)-tert-Butyl 4-(3,4-dimethylphenyl)-2-diazopent-3-enoate (6i)}

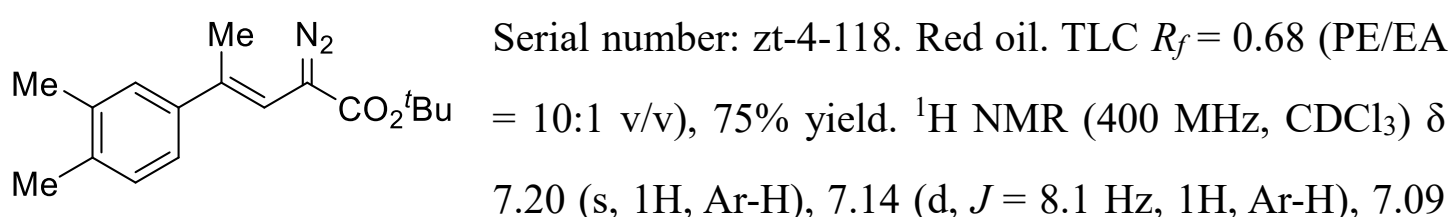

$(\mathrm{d}, J=7.9 \mathrm{~Hz}, 1 \mathrm{H}, \mathrm{Ar}-\mathrm{H}), 5.91(\mathrm{~s}, 1 \mathrm{H}, \mathrm{CH}=), 2.26\left(\mathrm{~d}, J=4.8 \mathrm{~Hz}, 6 \mathrm{H}, \mathrm{Ar}-\left(\mathrm{CH}_{3}\right)_{2}\right), 2.05$ $\left(\mathrm{s}, 3 \mathrm{H}, \mathrm{CH}_{3}\right), 1.52\left(\mathrm{~s}, 9 \mathrm{H}, \mathrm{C}\left(\mathrm{CH}_{3}\right)_{3}\right) .{ }^{13} \mathrm{C} \mathrm{NMR}\left(100 \mathrm{MHz}, \mathrm{CDCl}_{3}\right) \delta 166.1(1 \mathrm{C}, \mathrm{C}=\mathrm{O})$, 140.3 (1C, Ar-C), 136.4 (1C, Ar-C), 135.7 (1C, Ar-C), 133.9 (1C, C=), 129.6 (1C, ArC), 127.1 (1C, Ar-C), 123.1 (1C, Ar-C), 108.5 (1C, $\mathrm{CH}=), 81.9$ (1C, C-O), 28.3 (3C, $\left.\mathrm{C}\left(\underline{\mathrm{CH}}_{3}\right)_{3}\right), 19.8\left(1 \mathrm{C}, \mathrm{CH}_{3}\right), 19.4\left(1 \mathrm{C}, \mathrm{CH}_{3}\right), 16.9$ (1C, $\left.\mathrm{CH}_{3}\right)$. IR (neat): 2978, 2934, 2082 $(\mathrm{C}=\mathrm{N}), 1728,1369,1224,1161,739 \mathrm{~cm}^{-1}$. HRMS (ESI) Calcd for $\left[\mathrm{C}_{17} \mathrm{H}_{22} \mathrm{~N}_{2} \mathrm{O}_{2} \mathrm{Na}, \mathrm{M}\right.$ $+\mathrm{Na}]^{+}:$309.1573, Found: 309.1575.

\section{(E)-tert-Butyl 4-(benzo[d][1,3]dioxol-5-yl)-2-diazopent-3-enoate (6j)}

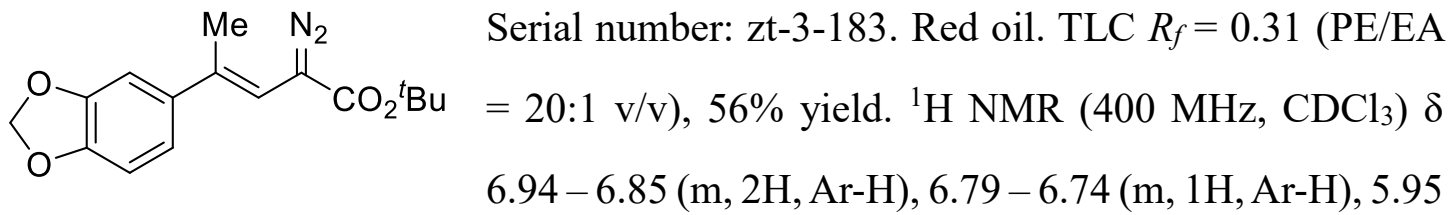

$\left(\mathrm{s}, 2 \mathrm{H}, \mathrm{CH}_{2}\right), 5.84(\mathrm{~s}, 1 \mathrm{H}, \mathrm{CH}=), 2.02\left(\mathrm{~s}, 3 \mathrm{H}, \mathrm{CH}_{3}\right), 1.51\left(\mathrm{~s}, 9 \mathrm{H}, \mathrm{C}\left(\mathrm{CH}_{3}\right)_{3}\right) .{ }^{13} \mathrm{C} \mathrm{NMR}$ $\left(100 \mathrm{MHz}, \mathrm{CDCl}_{3}\right) \delta 165.8(1 \mathrm{C}, \mathrm{C}=\mathrm{O}), 147.7$ (1C, Ar-C-O), 146.8 (1C, Ar-C-O), 137.1 $(1 \mathrm{C}, \mathrm{C}=), 133.5$ (1C, Ar-C), 119.3 (1C, Ar-C), 108.5 (1C, CH=), 107.9 (1C, Ar-C), 106.3 (1C, Ar-C), 101.0 (1C, O- $\left.\mathrm{CH}_{2}-\mathrm{O}\right), 81.9$ (1C, C-O), 28.3 (3C, $\left.\mathrm{C}\left(\underline{\mathrm{C}} \mathrm{H}_{3}\right)_{3}\right), 17.1(1 \mathrm{C}$, $\mathrm{CH}_{3}$ ). IR (neat): 3069, 2978, 2933, 2900, $2079(\mathrm{C}=\mathrm{N}), 1728,1697,1368,1319,1297$, 1253, 1159, 847, 816, $746 \mathrm{~cm}^{-1}$. HRMS (ESI) Calcd for $\left[\mathrm{C}_{16} \mathrm{H}_{18} \mathrm{~N}_{2} \mathrm{O}_{4} \mathrm{Na}, \mathrm{M}+\mathrm{Na}\right]^{+}$: 325.1159, Found: 325.1162.

\section{(E)-tert-Butyl 4-(naphthalen-2-yl)-2-diazopent-3-enoate (6k)}

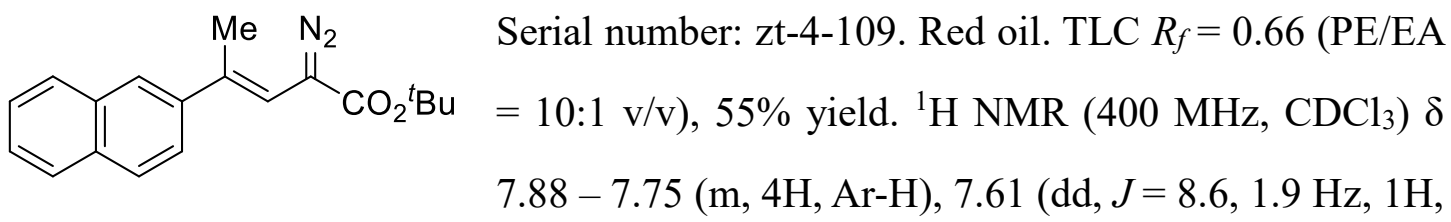


Ar-H), $7.55-7.39$ (m, 2H, Ar-H), 6.13 (d, J=1.2 Hz, 1H, CH=), 2.19 (d, J=1.2 Hz, $\left.3 \mathrm{H}, \mathrm{CH}_{3}\right), 1.54\left(\mathrm{~s}, 9 \mathrm{H}, \mathrm{C}\left(\mathrm{CH}_{3}\right)_{3}\right) .{ }^{13} \mathrm{C} \mathrm{NMR}\left(100 \mathrm{MHz}, \mathrm{CDCl}_{3}\right) \delta 165.0(1 \mathrm{C}, \mathrm{C}=\mathrm{O})$, 139.9 (1C, Ar-C), 133.4 (1C, C=), 133.3 (1C, Ar-C), 132.5 (1C, Ar-C), 128.1 (1C, ArC), 127.8 (1C, Ar-C), 127.5 (1C, Ar-C), 126.2 (1C, Ar-C), 125.8 (1C, Ar-C), 124.2 (1C, Ar-C), 124.1 (1C, Ar-C), $110.1(1 \mathrm{C}, \mathrm{CH}=), 82.1$ (1C, C-O), 28.3 (3C, $\left.\mathrm{C}\left(\underline{\mathrm{CH}}_{3}\right)_{3}\right), 16.9$ (1C, $\left.\mathrm{CH}_{3}\right)$. IR (neat): 3056, 2977, 2932, $2073(\mathrm{C}=\mathrm{N}), 1697,1368,1253,1159,1112$, $847,816,788,746,476 \mathrm{~cm}^{-1}$. HRMS (ESI) Calcd for $\left[\mathrm{C}_{19} \mathrm{H}_{20} \mathrm{~N}_{2} \mathrm{O}_{2} \mathrm{Na}, \mathrm{M}+\mathrm{Na}\right]^{+}$: 331.1417, Found: 331.1421.

\section{(E)-tert-Butyl 4-(thiophen-2-yl)-2-diazopent-3-enoate (6l)}

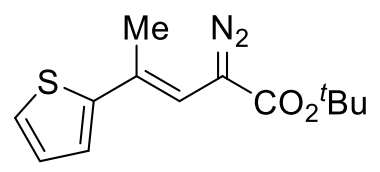

Serial number: zt-4-6. Red oil. TLC $R_{f}=0.89$ (PE/EA $=10: 1$

v/v), 66\% yield. ${ }^{1} \mathrm{H} \mathrm{NMR}\left(400 \mathrm{MHz}, \mathrm{CDCl}_{3}\right) \delta 7.17(\mathrm{~d}, J=5.0$ $\mathrm{Hz}, 1 \mathrm{H}, \mathrm{Ar}-\mathrm{H}), 7.03-7.00$ (m, 1H, Ar-H), $6.99-6.95$ (t, $J=$ $3.8 \mathrm{~Hz}, 1 \mathrm{H}, \mathrm{Ar}-\mathrm{H}), 6.06(\mathrm{~s}, 1 \mathrm{H}, \mathrm{CH}=), 2.08\left(\mathrm{~s}, 3 \mathrm{H}, \mathrm{CH}_{3}\right), 1.52\left(\mathrm{~s}, 9 \mathrm{H}, \mathrm{C}\left(\mathrm{CH}_{3}\right)_{3}\right) .{ }^{13} \mathrm{C}$ NMR (100 MHz, $\left.\mathrm{CDCl}_{3}\right) \delta 165.5(1 \mathrm{C}, \mathrm{C}=\mathrm{O}), 146.8$ (1C, Ar-C), 127.4 (1C, Ar-C), 127.3 $(1 \mathrm{C}, \mathrm{C}=), 124.3$ (1C, Ar-C), 122.6 (1C, Ar-C), 107.9 (1C, $\mathrm{CH}=), 82.1$ (1C, C-O), 28.3 (3C, $\left.\mathrm{C}\left(\underline{\mathrm{CH}}_{3}\right)_{3}\right), 17.0\left(1 \mathrm{C}, \mathrm{CH}_{3}\right) . \mathrm{IR}$ (neat): 3107, 3074, 2978, 2932, 2873, $2075(\mathrm{C}=\mathrm{N})$, 1727, 1704, 1392, 1369, 1294, 1245, 849, $699 \mathrm{~cm}^{-1}$. HRMS (ESI) Calcd for $\left[\mathrm{C}_{13} \mathrm{H}_{16} \mathrm{SN}_{2} \mathrm{O}_{2} \mathrm{Na}, \mathrm{M}+\mathrm{Na}\right]^{+}:$287.0825, Found: 287.0829 .

\section{tert-Butyl-4,4-Diphenyl-2-diazobut-3-enoate (6m)}

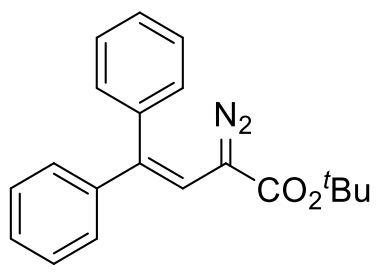

Serial number: 1y-5-60. Red oil. TLC $R_{f}=0.77$ (PE/EA = 10:1 v/v), 46\% yield. ${ }^{1} \mathrm{H}$ NMR (400 MHz, $\left.\mathrm{CDCl}_{3}\right) \delta 7.51-7.17(\mathrm{~m}$,

$10 \mathrm{H}, \mathrm{Ar}-\mathrm{H}), 6.27(\mathrm{~s}, 1 \mathrm{H}, \mathrm{CH}=), 1.50\left(\mathrm{~s}, 9 \mathrm{H}, \mathrm{C}\left(\mathrm{CH}_{3}\right)_{3}\right) .{ }^{13} \mathrm{C}$ NMR (100 MHz, $\left.\mathrm{CDCl}_{3}\right) \delta 165.6(1 \mathrm{C}, \mathrm{C}=\mathrm{O}), 142.0$ (1C, ArC), 138.5 (1C, Ar-C), 135.9 (1C, $\mathrm{CH}=), 130.3$ (2C, Ar-C), 128.2 (2C, Ar-C), 128.2 (2C, Ar-C), 127.8 (1C, Ar-C), 127.1 (1C, Ar-C), 126.9 (2C, Ar-C), 110.3 (1C, $\mathrm{CH}=), 82.1$ (1C, C-O), 28.3 (3C, $\left.\mathrm{C}\left(\underline{\mathrm{CH}}_{3}\right)_{3}\right)$. IR (neat): 3057, 3027, 2979, $2073(\mathrm{C}=\mathrm{N}), 1699,1247$, 
1164, 1114, 791, 739, $696 \mathrm{~cm}^{-1}$. HRMS (ESI) Calcd for $\left[\mathrm{C}_{20} \mathrm{H}_{20} \mathrm{~N}_{2} \mathrm{O}_{2} \mathrm{Na}, \mathrm{M}+\mathrm{Na}\right]^{+}$: 343.1417, Found: 343.1422.

\section{(E)-tert-Butyl 2-diazo-4-phenylbut-3-enoate (6n)}<smiles>CCOC(=O)/C=C/c1ccccc1</smiles>
Serial number: 1y-5-62. Red oil. TLC $R_{f}=0.85$ (PE/EA $=30: 1$ v/v), 89\% yield. ${ }^{1} \mathrm{H}$ NMR (400 MHz, $\left.\mathrm{CDCl}_{3}\right) \delta 7.36-7.15(\mathrm{~m}$, 5H, Ar-H), $6.46(\mathrm{~d}, J=16.3 \mathrm{~Hz}, 1 \mathrm{H}, \mathrm{CH}=), 6.16(\mathrm{~d}, J=16.3$ $\mathrm{Hz}, 1 \mathrm{H}, \mathrm{CH}=), 1.53\left(\mathrm{~s}, 9 \mathrm{H}, \mathrm{C}\left(\mathrm{CH}_{3}\right)_{3}\right) .{ }^{13} \mathrm{C} \mathrm{NMR}\left(100 \mathrm{MHz}, \mathrm{CDCl}_{3}\right) \delta 164.4(1 \mathrm{C}, \mathrm{C}=\mathrm{O})$, 137.0 (1C, Ar-C), 128.7 (2C, Ar-C), 126.9 (1C, Ar-C), 125.7 (2C, Ar-C), 122.5 (1C, $\mathrm{CH}=), 111.9(1 \mathrm{C}, \mathrm{CH}=), 82.3(1 \mathrm{C}, \mathrm{C}-\mathrm{O}), 28.3\left(3 \mathrm{C}, \mathrm{C}\left(\underline{\mathrm{CH}}_{3}\right)_{3}\right)$. IR (neat): 3416, 2959, $2078(\mathrm{C}=\mathrm{N}), 1702,1640,1136,951,620 \mathrm{~cm}^{-1}$. HRMS (ESI) Calcd for $\left[\mathrm{C}_{14} \mathrm{H}_{16} \mathrm{~N}_{2} \mathrm{O}_{2} \mathrm{Na}\right.$, $\left.\mathrm{M}^{+} \mathrm{Na}\right]^{+}: 267.1104$, Found: 267.1108.

\section{Benzhydryl (E)-2-diazopent-3-enoate (6o)}<smiles>C/C=C/C(=N)C(=O)OC(c1ccccc1)c1ccccc1</smiles>

Serial number: 1y-5-116. Red oil. TLC $R_{f}=0.60(\mathrm{PE} / \mathrm{EA}=$ $20: 1 \mathrm{v} / \mathrm{v}), 78 \%$ yield. ${ }^{1} \mathrm{H}$ NMR (400 $\left.\mathrm{MHz}, \mathrm{CDCl}_{3}\right) \delta 7.33-$ 7.24 (m, 10H, Ar-H), 7.01 (s, 1H, C$\left.(\mathrm{Ph})_{2}\right), 5.75$ (d, J=15.7 Hz, 1H, CH=), 5.33 $5.24(\mathrm{~m}, 1 \mathrm{H}, \mathrm{CH}=), 1.76\left(\mathrm{~d}, J=6.0 \mathrm{~Hz}, 3 \mathrm{H}, \mathrm{CH}_{3}\right) .{ }^{13} \mathrm{C} \mathrm{NMR}\left(100 \mathrm{MHz}, \mathrm{CDCl}_{3}\right) \delta 164.3$ (1C, C=O), 140.2 (2C, Ar-C), 128.7 (4C, Ar-C), 128.2 (2C, Ar-C), 127.2 (4C, Ar-C), $120.8(1 \mathrm{C}, \mathrm{CH}=), 112.7(1 \mathrm{C}, \mathrm{CH}=), 77.4$ (1C, C-O), 18.4 (1C, $\left.\mathrm{CH}_{3}\right)$. IR (neat): 3109, 3044, 2818, $2077(\mathrm{C}=\mathrm{N}), 1698,1421,1388,1284,1256,1189,696 \mathrm{~cm}^{-1}$. HRMS (ESI) Calcd for $\left[\mathrm{C}_{18} \mathrm{H}_{16} \mathrm{~N}_{2} \mathrm{O}_{2} \mathrm{Na}, \mathrm{M}+\mathrm{Na}\right]^{+}:$315.1104, Found: 315.1108 .

\section{Benzhydryl (E)-2-diazohex-3-enoate (6p)}<smiles>CCC=CC(=N)C(=O)OC(c1ccccc1)c1ccccc1</smiles>

Serial number: 1y-5-137. Red oil. TLC $R_{f}=0.59$ (PE/EA = $20: 1 \mathrm{v} / \mathrm{v}), 75 \%$ yield. ${ }^{1} \mathrm{H} \mathrm{NMR}\left(400 \mathrm{MHz}, \mathrm{CDCl}_{3}\right) \delta 7.36-$ $7.21(\mathrm{~m}, 10 \mathrm{H}, \mathrm{Ar}-\mathrm{H}), 7.00\left(\mathrm{~s}, 1 \mathrm{H}, \mathrm{C} \underline{\mathrm{H}}(\mathrm{Ph})_{2}\right), 5.74(\mathrm{~d}, J=15.9$

$\mathrm{Hz}, 1 \mathrm{H}, \mathrm{CH}=), 5.37(\mathrm{dt}, J=15.8,6.5 \mathrm{~Hz}, 1 \mathrm{H}, \mathrm{CH}=), 2.31-2.01\left(\mathrm{~m}, 2 \mathrm{H}, \mathrm{CH}_{2}\right), 1.00(\mathrm{t}$, $\left.J=7.4 \mathrm{~Hz}, 3 \mathrm{H}, \mathrm{CH}_{3}\right) .{ }^{13} \mathrm{C} \mathrm{NMR}\left(100 \mathrm{MHz}, \mathrm{CDCl}_{3}\right) \delta 164.8(1 \mathrm{C}, \mathrm{C}=\mathrm{O}), 140.1(1 \mathrm{C}$, $\mathrm{CH}=), 128.6$ (4C, Ar-C), 128.1 (2C, Ar-C), 127.6 (2C, Ar-C), 127.1 (4C, Ar-C), 110.8 
(1C, $\mathrm{CH}=), 77.4$ (1C, C-O), $26.0\left(1 \mathrm{C}, \mathrm{CH}_{2}\right), 13.9$ (1C, $\left.\mathrm{CH}_{3}\right) . \mathrm{IR}$ (neat): 3110, 3044, 2818, $2077(\mathrm{C}=\mathrm{N})$, 1698, 1421, 1388, 1284, 1256,1189, $696 \mathrm{~cm}^{-1}$. HRMS (ESI) Calcd for $\left[\mathrm{C}_{19} \mathrm{H}_{18} \mathrm{~N}_{2} \mathrm{O}_{2} \mathrm{Na}, \mathrm{M}+\mathrm{Na}\right]^{+}: 329.1260$, Found: 329.1264 .

\section{Benzhydryl (E)-2-diazohept-3-enoate (6q)}

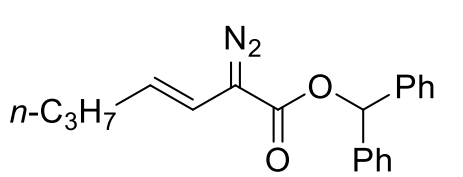

Serial number: 1y-5-138. Red oil. TLC $R_{f}=0.56(\mathrm{PE} / \mathrm{EA}=$ $20: 1 \mathrm{v} / \mathrm{v}), 78 \%$ yield. ${ }^{1} \mathrm{H}$ NMR $\left(400 \mathrm{MHz}, \mathrm{CDCl}_{3}\right) \delta 7.48$ - 7.32 (m, 10H, Ar-H), $7.12-7.07\left(\mathrm{~m}, 1 \mathrm{H}, \mathrm{CH}(\mathrm{Ph})_{2}\right), 5.85$ (dd, $J=16.1,4.7 \mathrm{~Hz}, 1 \mathrm{H}, \mathrm{CH}=), 5.43$ (dt, $J=15.1,7.1 \mathrm{~Hz}, 1 \mathrm{H}, \mathrm{CH}=), 2.27-2.17$ (m, $\left.2 \mathrm{H}, \mathrm{CH}_{2}\right), 1.50\left(\mathrm{q}, J=7.3 \mathrm{~Hz}, 2 \mathrm{H}, \mathrm{CH}_{2}\right), 0.99\left(\mathrm{t}, J=7.3 \mathrm{~Hz}, 3 \mathrm{H}, \mathrm{CH}_{3}\right) .{ }^{13} \mathrm{C} \mathrm{NMR}(100$ $\left.\mathrm{MHz}, \mathrm{CDCl}_{3}\right) \delta 164.7(1 \mathrm{C}, \mathrm{C}=\mathrm{O}), 140.1(1 \mathrm{C}, \mathrm{CH}=), 128.6$ (4C, Ar-C), 128.1 (2C, ArC), 127.1 (4C, Ar-C), 126.0 (2C, Ar-C), 111.7 (1C, CH=), 77.3 (1C, C-O), 35.0 (1C, $\left.\mathrm{CH}_{2}\right), 22.8$ (1C, $\left.\mathrm{CH}_{2}\right), 13.7\left(1 \mathrm{C}, \mathrm{CH}_{3}\right)$. IR (neat): 3116, 3019, 2821, $2076(\mathrm{C}=\mathrm{N}), 1699$, $1418,1377,1281,1256,693 \mathrm{~cm}^{-1}$. HRMS (ESI) Calcd for $\left[\mathrm{C}_{20} \mathrm{H}_{20} \mathrm{~N}_{2} \mathrm{O}_{2} \mathrm{Na}, \mathrm{M}+\mathrm{Na}\right]^{+}$: 343.1417, Found: 343.1422.

\section{Benzhydryl (E)-2-diazodec-3-enoate (6r)}

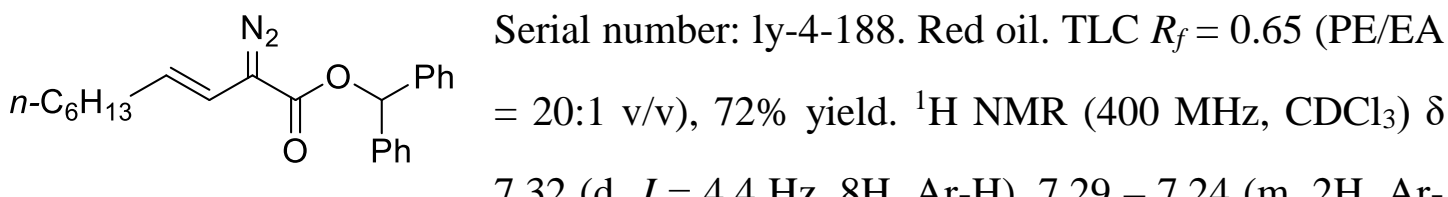
H), $7.00\left(\mathrm{~s}, 1 \mathrm{H}, \mathrm{C} \underline{\mathrm{H}}(\mathrm{Ph})_{2}\right), 5.74(\mathrm{~d}, J=15.8 \mathrm{~Hz}, 1 \mathrm{H}, \mathrm{CH}=), 5.43-5.26\left(\mathrm{~m}, 1 \mathrm{H}, \mathrm{CH}_{2}\right)$, $2.15\left(\mathrm{q}, J=6.8 \mathrm{~Hz}, 1 \mathrm{H}, \mathrm{CH}_{2}\right), 1.41-1.34\left(\mathrm{~m}, 2 \mathrm{H}, \mathrm{CH}_{2}\right), 1.28-1.26\left(\mathrm{~m}, 4 \mathrm{H}, \mathrm{CH}_{2}\right)$, $0.91-0.85\left(\mathrm{~m}, 2 \mathrm{H}, \mathrm{CH}_{2}\right) .{ }^{13} \mathrm{C} \mathrm{NMR}\left(100 \mathrm{MHz}, \mathrm{CDCl}_{3}\right) \delta 164.7(1 \mathrm{C}, \mathrm{C}=\mathrm{O}), 140.1(1 \mathrm{C}$, $\mathrm{CH}=), 128.6$ (4C, Ar-C), 128.4 (2C, Ar-C), 128.1 (2C, Ar-C), 127.1 (4C, Ar-C), 111.5 (1C, $\mathrm{CH}=), 77.3(1 \mathrm{C}, \mathrm{C}-\mathrm{O}), 32.9\left(1 \mathrm{C}, \mathrm{CH}_{2}\right), 31.8\left(1 \mathrm{C}, \mathrm{CH}_{2}\right), 29.5\left(1 \mathrm{C}, \mathrm{CH}_{2}\right), 28.8(1 \mathrm{C}$, $\left.\mathrm{CH}_{2}\right), 22.7$ (1C, $\left.\mathrm{CH}_{2}\right), 14.2$ (1C, $\left.\mathrm{CH}_{2}\right)$. IR (neat): 3112, 3033, 2865, $2077(\mathrm{C}=\mathrm{N}), 1699$, 1288, 1265, 1242, 1185, 1132, 1104, 748, 721, 708, $694 \mathrm{~cm}^{-1}$. HRMS (ESI) Calcd for $\left[\mathrm{C}_{23} \mathrm{H}_{26} \mathrm{~N}_{2} \mathrm{O}_{2} \mathrm{Na}, \mathrm{M}+\mathrm{Na}\right]^{+}:$385.1886, Found: 385.1890 . 


\section{Benzhydryl (E)-2-diazo-5-phenylpent-3-enoate (6s)}

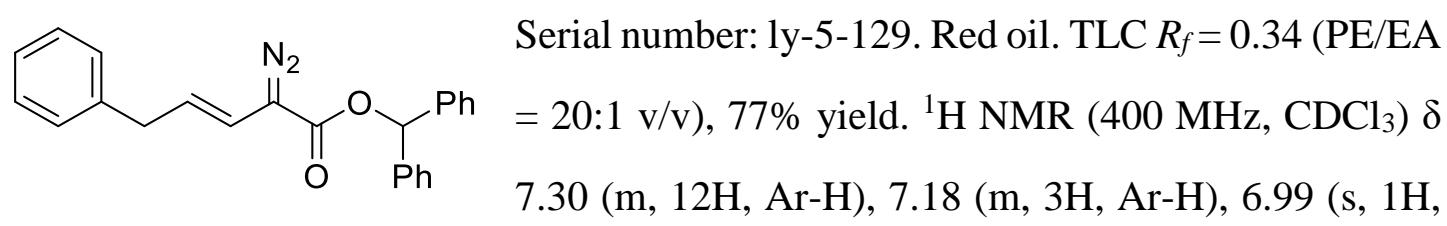

$\left.\mathrm{CH}(\mathrm{Ph})_{2}\right), 5.84(\mathrm{~d}, J=15.8 \mathrm{~Hz}, 1 \mathrm{H}, \mathrm{CH}=), 5.48(\mathrm{dt}, J=15.7,7.0 \mathrm{~Hz}, 1 \mathrm{H}, \mathrm{CH}=), 3.47$ $\left(\mathrm{d}, J=6.8 \mathrm{~Hz}, 2 \mathrm{H}, \mathrm{CH}_{2}\right) .{ }^{13} \mathrm{C} \mathrm{NMR}\left(100 \mathrm{MHz}, \mathrm{CDCl}_{3}\right) \delta 163.9(1 \mathrm{C}, \mathrm{C}=\mathrm{O}), 140.1(1 \mathrm{C}$, $\mathrm{CH}=), 139.9$ (1C, Ar-C), 128.7 (2C, Ar-C), 128.6 (2C, Ar-C), 128.6 (2C, Ar-C), 128.1 (2C, Ar-C), 128.0 (2C, Ar-C), 127.2 (2C, Ar-C), 127.1 (2C, Ar-C), 126.4 (2C, Ar-C), 124.3 (1C, Ar-C), 113.2 (1C, $\mathrm{CH}=), 77.0$ (1C, C-O), 38.4 (1C, $\mathrm{CH}_{2}$ ). IR (neat): 3109, 3060, 2820, 2077 (C=N), 1698, 1248, 1232, 1209, 1184, 1165, 1134, $841 \mathrm{~cm}^{-1}$. HRMS (ESI) Calcd for $\left[\mathrm{C}_{24} \mathrm{H}_{20} \mathrm{~N}_{2} \mathrm{O}_{2} \mathrm{Na}, \mathrm{M}+\mathrm{Na}\right]^{+}:$391.1417, Found: 391.1423. 


\section{Typical Procedures of $\mathrm{O}-\mathrm{H}$ Bond Insertion Reactions}

\subsection{Typical procedure for $\mathrm{O}-\mathrm{H}$ bond insertion with $\alpha$-alkyl- $\alpha$-diazoesters}
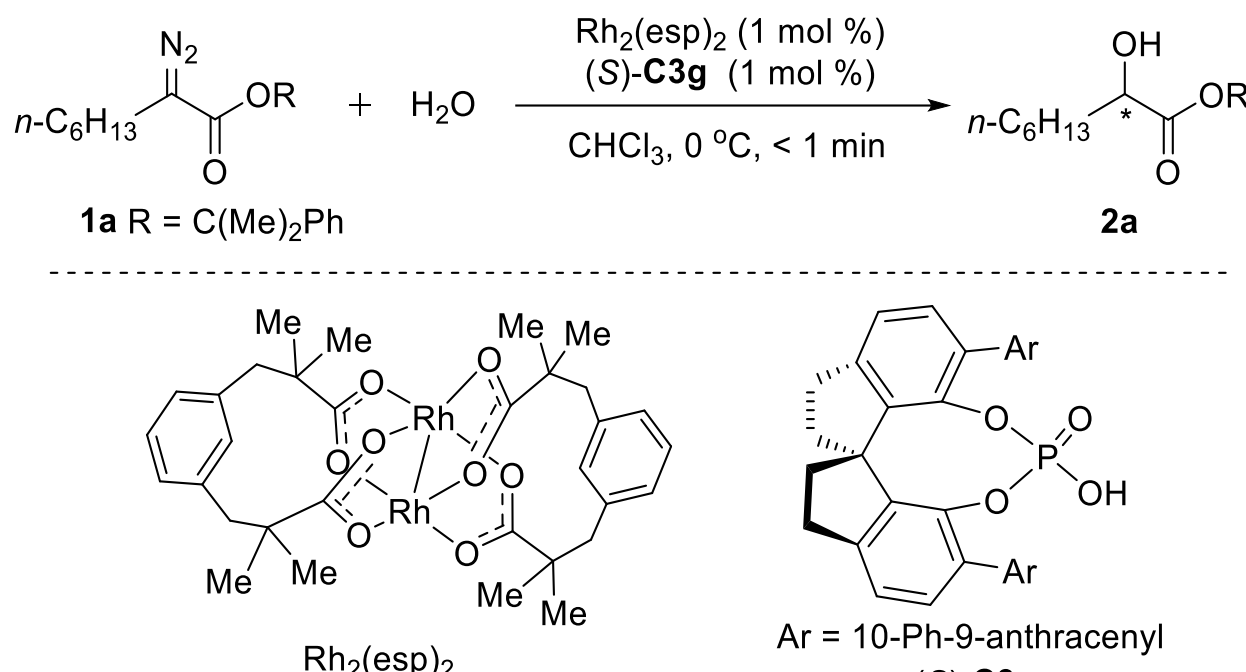

$(S)-\mathbf{C} \mathbf{g}$

The $\mathrm{Rh}_{2}(\mathrm{esp})_{2}(1.5 \mathrm{mg}, 0.002 \mathrm{mmol}, 1 \mathrm{~mol} \%)$ and $(S)-\mathbf{C 3 g}(1.6 \mathrm{mg}, 0.002 \mathrm{mmol}$, $1 \mathrm{~mol} \%$ ) were introduced into an oven-dried Schlenk tube in an argon-filled glovebox. After $1 \mathrm{~mL} \mathrm{CHCl}_{3}$ was injected into the Schlenk tube, the mixture was stirred at $0{ }^{\circ} \mathrm{C}$. Then $\mathrm{H}_{2} \mathrm{O}(18 \mu \mathrm{L}, 1 \mathrm{mmol})$ was added by a micro syringe in one portion and a solution of 2-phenylpropan-2-yl 2-diazooctanoate (1a, $58 \mathrm{mg}, 0.2 \mathrm{mmol})$ in $2 \mathrm{~mL} \mathrm{CHCl}_{3}$ was added by a syringe in one portion, subsequently. The reaction finished in $1 \mathrm{~min}$ after the addition (judged by the discoloration of diazo compound and confirmed by TLC monitoring). Then the reaction mixture was concentrated and purified by a flash chromatography on silica gel $(\mathrm{PE} / \mathrm{EA}=20: 1 \mathrm{v} / \mathrm{v})$ to give 2-phenylpropan-2-yl 2hydroxyoctanoate (2a) as a colorless oil. The analysis data for $\mathrm{O}-\mathrm{H}$ insertion products were listed below. 


\subsection{Typical procedure for $\mathrm{O}-\mathrm{H}$ bond insertion with $\alpha$-alkenyl- $\alpha$-diazoesters}

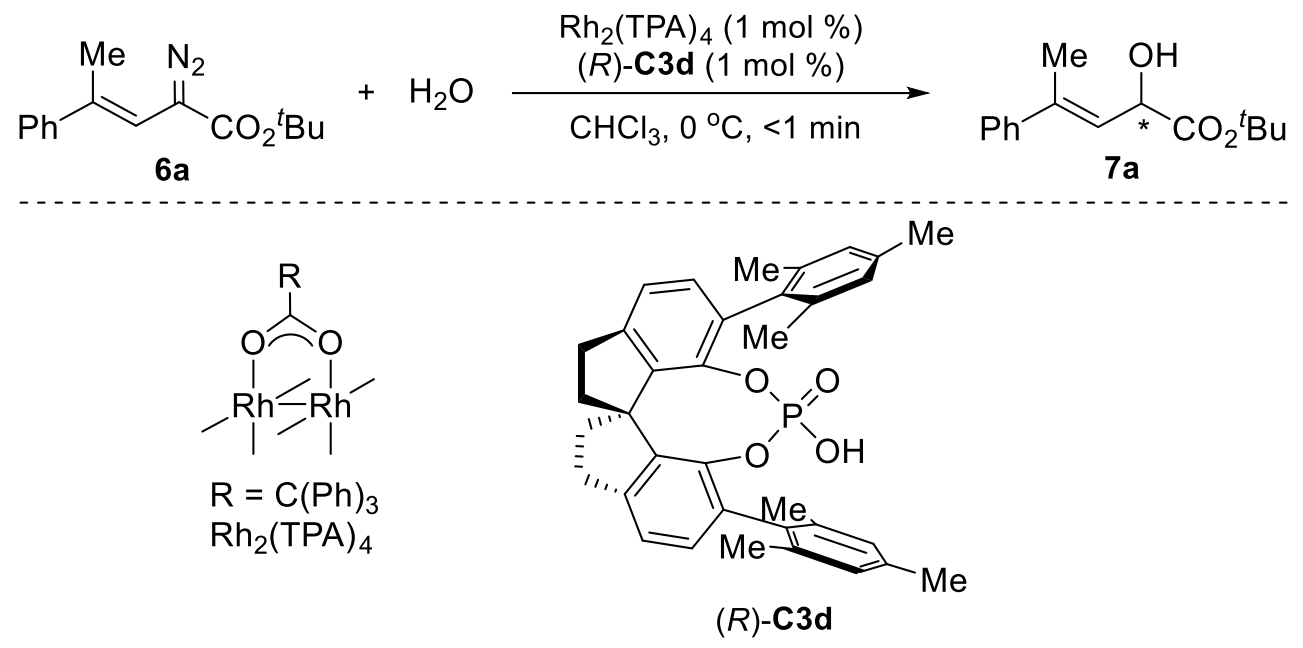

The $\mathrm{Rh}_{2}(\mathrm{TPA})_{4}(2.7 \mathrm{mg}, 0.002 \mathrm{mmol}, 1 \mathrm{~mol} \%)$ and $(R)$-C3d (1.1 mg, $0.002 \mathrm{mmol}$, $1 \mathrm{~mol} \%)$ were introduced into an oven-dried Schlenk tube in an argon-filled glovebox. After $1 \mathrm{~mL} \mathrm{CHCl}{ }_{3}$ was injected into the Schlenk tube, the mixture was stirred at $0{ }^{\circ} \mathrm{C}$. Then $\mathrm{H}_{2} \mathrm{O}(18 \mu \mathrm{L}, 1 \mathrm{mmol})$ was added by a micro syringe in one portion and solution of (E)-tert-butyl 2-diazo-4-phenylpent-3-enoate 6a (52 $\mathrm{mg}, 0.2 \mathrm{mmol})$ in $2 \mathrm{~mL} \mathrm{CHCl}_{3}$ was added by a syringe in one portion, subsequently. The reaction finished in $1 \mathrm{~min}$ after the addition (judged by the discoloration of diazo compound and confirmed by TLC monitoring). Then the reaction mixture was concentrated and purified by a flash chromatography on silica gel $(\mathrm{PE} / \mathrm{EA}=10: 1 \mathrm{v} / \mathrm{v})$ to give $(E)$-tert-butyl 2-hydroxy-4phenylpent-3-enoate 7a as a white solid. The analysis data for $\mathrm{O}-\mathrm{H}$ insertion products were listed below. 


\subsection{O-H bond insertion with $\alpha$-alkenyl- $\alpha$-diazoesters: optimization of reaction}

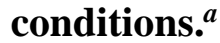

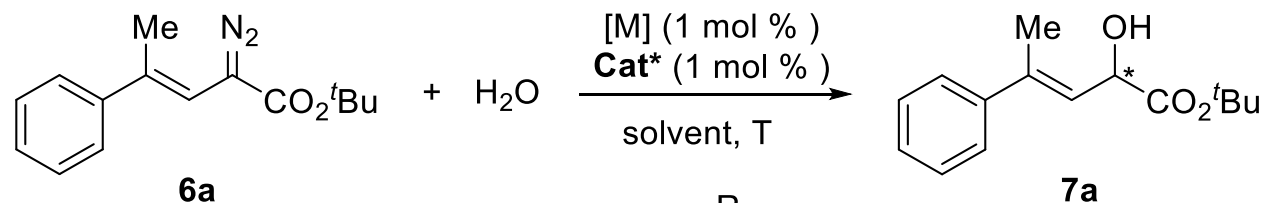

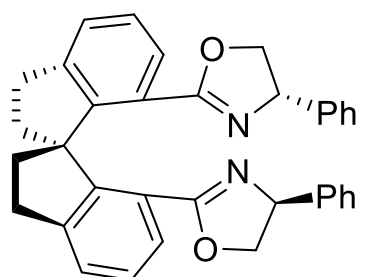

L1

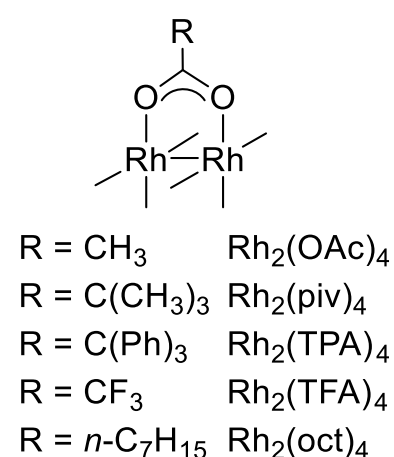

$7 \mathbf{a}$

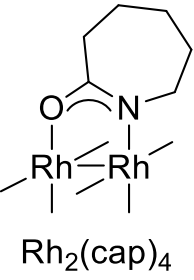<smiles>[R]c1ccc2c3c1OP(=O)(O)Oc1c([R])ccc(c1-3)CC2</smiles>

C3a: $\mathrm{R}=\mathrm{Ph}$

C3b: $\mathrm{R}=4-\mathrm{PhC}_{6} \mathrm{H}_{4}$

C3c: $\mathrm{R}=3,5-(\mathrm{Ph})_{2} \mathrm{C}_{6} \mathrm{H}_{3}$

C3d: $\mathrm{R}=2,4,6-(\mathrm{Me}){ }_{3} \mathrm{C}_{6} \mathrm{H}_{2}$

C3e: $R=2$-naphthyl

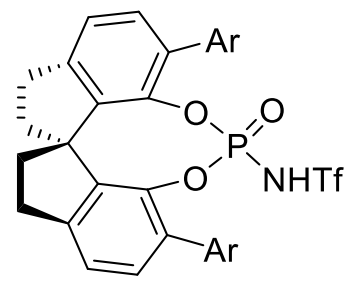

C3f: $R=9$-phenanthryl

C5

C3g: $\mathrm{R}=10-\mathrm{Ph}-9$-anthracenyl $\mathrm{Ar}=10-\mathrm{Ph}-9$-anthracenyl

\begin{tabular}{|c|c|c|c|c|c|c|c|}
\hline entry & {$[\mathrm{M}]$} & Cat* & solvent & $\begin{array}{l}\mathrm{T} \\
\left({ }^{\circ} \mathrm{C}\right)\end{array}$ & time & $\begin{array}{l}\text { yield } \\
(\%)^{b}\end{array}$ & $\begin{array}{l}\text { ee } \\
(\%)^{c}\end{array}$ \\
\hline $1^{d}$ & $\mathrm{Pd}(\mathrm{PhCN})_{2} \mathrm{Cl}_{2} / \mathbf{L} \mathbf{1}$ & none & $\mathrm{CHCl}_{3}$ & 40 & $12 \mathrm{~h}$ & $<2$ & -- \\
\hline $2^{d}$ & $\mathrm{CuSO}_{4} / \mathbf{L 1}$ & none & $\mathrm{CHCl}_{3}$ & 40 & $12 \mathrm{~h}$ & $<2$ & -- \\
\hline 3 & $\mathrm{Rh}_{2}(\mathrm{TPA})_{4}$ & $(R)-\mathbf{C} \mathbf{3 a}$ & $\mathrm{CHCl}_{3}$ & 25 & $5 \mathrm{~min}$ & 38 & 31 \\
\hline 4 & $\mathrm{Rh}_{2}(\mathrm{TPA})_{4}$ & $(R)-\mathbf{C} 3 \mathbf{b}$ & $\mathrm{CHCl}_{3}$ & 25 & $5 \mathrm{~min}$ & 68 & 49 \\
\hline 5 & $\mathrm{Rh}_{2}(\mathrm{TPA})_{4}$ & $(R)-\mathbf{C} 3 \mathbf{c}$ & $\mathrm{CHCl}_{3}$ & 25 & $2 \mathrm{~min}$ & 84 & 66 \\
\hline 6 & $\mathrm{Rh}_{2}(\mathrm{TPA})_{4}$ & $(R)-\mathbf{C} 3 \mathbf{d}$ & $\mathrm{CHCl}_{3}$ & 25 & $<1 \min$ & 97 & 90 \\
\hline 7 & $\mathrm{Rh}_{2}(\mathrm{TPA})_{4}$ & $(R)-\mathbf{C} 3 \mathbf{f}$ & $\mathrm{CHCl}_{3}$ & 25 & $5 \mathrm{~min}$ & 82 & 62 \\
\hline 8 & $\mathrm{Rh}_{2}(\mathrm{TPA})_{4}$ & $(R)-\mathbf{C} 3 \mathbf{g}$ & $\mathrm{CHCl}_{3}$ & 25 & $5 \mathrm{~min}$ & 85 & 73 \\
\hline 9 & $\mathrm{Rh}_{2}(\mathrm{TPA})_{4}$ & $(S)-\mathbf{C 5}$ & $\mathrm{CHCl}_{3}$ & 25 & $<1 \min$ & 48 & 46 \\
\hline 10 & $\mathrm{Rh}_{2}(\mathrm{OAc})_{4}$ & $(R)-\mathbf{C} 3 \mathbf{d}$ & $\mathrm{CHCl}_{3}$ & 25 & $2 \mathrm{~min}$ & 74 & 93 \\
\hline 11 & $\mathrm{Rh}_{2}(\text { piv })_{4}$ & $(R)-\mathbf{C} 3 \mathbf{d}$ & $\mathrm{CHCl}_{3}$ & 25 & $10 \mathrm{~min}$ & 51 & 72 \\
\hline 12 & $\mathrm{Rh}_{2}(\mathrm{TFA})_{4}$ & $(R)-\mathbf{C} 3 \mathbf{d}$ & $\mathrm{CHCl}_{3}$ & 25 & $2 \mathrm{~h}$ & 40 & 62 \\
\hline
\end{tabular}




\begin{tabular}{llllllll}
13 & $\mathrm{Rh}_{2}(\text { oct })_{4}$ & $(R)-\mathbf{C 3 d}$ & $\mathrm{CHCl}_{3}$ & 25 & $2 \mathrm{~h}$ & 65 & 75 \\
14 & $\mathrm{Rh}_{2}(\text { cap })_{4}$ & $(R)-\mathbf{C 3 d}$ & $\mathrm{CHCl}_{3}$ & 25 & $2 \mathrm{~h}$ & 60 & 82 \\
15 & $\mathrm{Rh}_{2}(\mathrm{TPA})_{4}$ & $(R)-\mathbf{C 3 d}$ & $\mathrm{DCM}$ & 25 & $<1 \mathrm{~min}$ & 96 & 88 \\
16 & $\mathrm{Rh}_{2}(\mathrm{TPA})_{4}$ & $(R)-\mathbf{C 3 d}$ & $\mathrm{DCE}$ & 25 & $<1 \mathrm{~min}$ & 91 & 89 \\
17 & $\mathrm{Rh}_{2}(\mathrm{TPA})_{4}$ & $(R)-\mathbf{C 3 d}$ & $c$-hexane & 25 & $30 \mathrm{~min}$ & 57 & 81 \\
18 & $\mathrm{Rh}_{2}(\mathrm{TPA})_{4}$ & $(R)-\mathbf{C 3 d}$ & toluene & 25 & $2 \mathrm{~min}$ & 89 & 84 \\
19 & $\mathrm{Rh}_{2}(\mathrm{TPA})_{4}$ & $(R)-\mathbf{C 3 d}$ & $\mathrm{CHCl}_{3}$ & 0 & $<1 \mathrm{~min}$ & 97 & 94 \\
20 & $\mathrm{Rh}_{2}(\mathrm{TPA})_{4}$ & $(R)-\mathbf{C 3 d}$ & $\mathrm{CHCl}_{3}$ & 40 & $<1 \mathrm{~min}$ & 85 & 89 \\
\hline
\end{tabular}

${ }^{a}$ Reaction conditions: $[\mathrm{Rh}] / \mathbf{C a t} * / \mathbf{6 a} / \mathrm{H}_{2} \mathrm{O}=0.002: 0.002: 0.2: 1.0(\mathrm{mmol})$ in $3 \mathrm{~mL}$ solvent. ${ }^{b}$ Isolated yield. ${ }^{c}$ Determined by HPLC using Chiralcel OD-H column. ${ }^{d}$ Using 5 mol \% [M] and 6 mol \% L1. 


\section{Analytical Data of O-H Bond Insertion Products}

\section{2-Phenylpropan-2-yl 2-hydroxyoctanoate (2a)}

n- $\left.\mathrm{C}_{6} \mathrm{H}_{13} \prod_{\mathrm{O}}^{\mathrm{O}} \mathrm{Me}_{\mathrm{Ph}}^{\mathrm{Me}}=10: 1 \mathrm{v} / \mathrm{v}\right), 80 \%$ yield, 95\% ee [HPLC condition: Chiralpak AD-H column, $n$-hexane/2-propanol $=96: 4$, flow rate $=0.8$ $\mathrm{mL} / \mathrm{min}$, wavelength $=210 \mathrm{~nm}, t_{\mathrm{R}}=14.58$ min for major isomer, $t_{\mathrm{R}}=15.61$ min for minor isomer], $[\alpha]_{\mathrm{D}}{ }^{25}=11.1\left(c 1.0, \mathrm{CHCl}_{3}\right) .{ }^{1} \mathrm{H} \mathrm{NMR}\left(400 \mathrm{MHz}, \mathrm{CDCl}_{3}\right) \delta 7.39-7.31$ (m, 4H, Ar-H), 7.31 - 7.25 (m, 1H, Ar-H), 4.14 (dd, J= 7.2, 4.2 Hz, 1H, C$(\mathrm{OH})$ ), 2.71 (s, 1H, OH), $1.81\left(\mathrm{~s}, 6 \mathrm{H}, \mathrm{C}\left(\mathrm{CH}_{3}\right)_{2}\right), 1.65\left(\mathrm{~m}, 2 \mathrm{H}, \mathrm{CH}_{2}\right), 1.40-1.22\left(\mathrm{~m}, 8 \mathrm{H}, \mathrm{CH}_{2}\right), 0.90$ $\left(\mathrm{t}, J=6.8 \mathrm{~Hz}, 3 \mathrm{H}, \mathrm{CH}_{3}\right) .{ }^{13} \mathrm{C} \mathrm{NMR}\left(100 \mathrm{MHz}, \mathrm{CDCl}_{3}\right) \delta 174.2(1 \mathrm{C}, \mathrm{C}=\mathrm{O}), 145.0(1 \mathrm{C}$, Ar-C), 128.4 (2C, Ar-C), 127.4 (2C, Ar-C), 124.3 (1C, Ar-C), 83.4 (1C, C-O), 70.6 (1C, $\mathrm{CH}(\mathrm{OH})), 34.4\left(1 \mathrm{C}, \mathrm{CH}_{2}\right), 31.7\left(1 \mathrm{C}, \mathrm{CH}_{2}\right), 29.1\left(1 \mathrm{C}, \mathrm{CH}_{2}\right), 28.5\left(1 \mathrm{C},\left(\mathrm{CH}_{3}\right)_{2}\right), 28.4(1 \mathrm{C}$, $\left.\left(\mathrm{CH}_{3}\right)_{2}\right), 24.7\left(1 \mathrm{C}, \mathrm{CH}_{2}\right), 22.6\left(1 \mathrm{C}, \mathrm{CH}_{2}\right), 14.1\left(1 \mathrm{C}, \mathrm{CH}_{3}\right)$. HRMS (ESI) Calcd for $\left[\mathrm{C}_{17} \mathrm{H}_{26} \mathrm{O}_{3} \mathrm{Na}, \mathrm{M}+\mathrm{Na}\right]^{+}:$301.1774, Found: 301.1778 .

\section{2-Phenylpropan-2-yl 2-hydroxybutanoate (2b)}<smiles>CCCCCC(O)C(=O)OC(C)(C)[N+](C)(C)c1ccccc1</smiles>

Serial number: 1y-3-147. Colorless oil. TLC $R_{f}=0.16(\mathrm{PE} / \mathrm{EA}=$ 20:1 v/v), 82\% yield, 97\% ee [HPLC condition: Chiralpak AD-H column, $n$-hexane $/ 2$-propanol $=90: 10$, flow rate $=1 \mathrm{~mL} / \mathrm{min}$, wavelength $=210 \mathrm{~nm}, t_{\mathrm{R}}=7.04 \mathrm{~min}$ for minor isomer, $t_{\mathrm{R}}=7.84$ min for major isomer], $[\alpha]_{\mathrm{D}}^{25}=10.0\left(c 1.0, \mathrm{CHCl}_{3}\right) .{ }^{1} \mathrm{H} \mathrm{NMR}\left(400 \mathrm{MHz}, \mathrm{CDCl}_{3}\right) \delta 7.44-7.34(\mathrm{~m}, 4 \mathrm{H}, \mathrm{Ar}-\mathrm{H})$, $7.35-7.28(\mathrm{~m}, 1 \mathrm{H}, \mathrm{Ar}-\mathrm{H}), 4.16(\mathrm{dd}, J=6.6,4.4 \mathrm{~Hz}, 1 \mathrm{H}, \mathrm{C} \underline{\mathrm{H}}(\mathrm{OH})), 2.83(\mathrm{~s}, 1 \mathrm{H}, \mathrm{OH})$, $2.00-1.89\left(\mathrm{~m}, 1 \mathrm{H}, \mathrm{CH}_{2}\right), 1.86\left(\mathrm{~d}, J=3.4 \mathrm{~Hz}, 6 \mathrm{H}, \mathrm{C}\left(\mathrm{CH}_{3}\right)_{2}\right), 1.80-1.70\left(\mathrm{~m}, 1 \mathrm{H}, \mathrm{CH}_{2}\right)$, $1.02\left(\mathrm{t}, J=7.4 \mathrm{~Hz}, 3 \mathrm{H}, \mathrm{CH}_{3}\right) .{ }^{13} \mathrm{C}$ NMR $\left(100 \mathrm{MHz}, \mathrm{CDCl}_{3}\right) \delta 174.0(1 \mathrm{C}, \mathrm{C}=\mathrm{O}), 145.0$ (1C, Ar-C), 128.4 (2C, Ar-C), 127.4 (1C, Ar-C), 124.3 (2C, Ar-C), 83.4 (1C, C-O), $71.5(1 \mathrm{C}, \mathrm{CH}), 28.6\left(1 \mathrm{C}, \mathrm{C}\left(\underline{\mathrm{CH}}_{3}\right)_{2}\right), 28.4\left(1 \mathrm{C}, \mathrm{C}\left(\underline{\mathrm{CH}}_{3}\right)_{2}\right), 27.5\left(1 \mathrm{C}, \mathrm{CH}_{2}\right), 8.9\left(1 \mathrm{C}, \mathrm{CH}_{3}\right)$. HRMS (ESI) Calcd for $\left[\mathrm{C}_{13} \mathrm{H}_{18} \mathrm{O}_{3} \mathrm{Na}, \mathrm{M}+\mathrm{Na}\right]^{+}:$245.1148, Found: 245.1150. 


\section{2-Phenylpropan-2-yl 2-hydroxyhexanoate (2c)}<smiles>CCCCCCCCCCC(O)C(=O)OC(C)(C)c1ccccc1</smiles>

Serial number: ly-3-157. Colorless oil. TLC $R_{f}=0.20(\mathrm{PE} / \mathrm{EA}=$

20:1 v/v), 82\% yield, 95\% ee [HPLC condition: Chiralpak AS$\mathrm{H}$ column, $n$-hexane $/ 2$-propanol $=95: 5$, flow rate $=1.0 \mathrm{~mL} / \mathrm{min}$, wavelength $=210 \mathrm{~nm}, t_{\mathrm{R}}=5.03 \mathrm{~min}$ for major isomer, $t_{\mathrm{R}}=5.92 \mathrm{~min}$ for minor isomer], $[\alpha]_{\mathrm{D}}^{25}=10.5\left(c 1.0, \mathrm{CHCl}_{3}\right) .{ }^{1} \mathrm{H} \mathrm{NMR}\left(400 \mathrm{MHz}, \mathrm{CDCl}_{3}\right) \delta 7.39-7.31(\mathrm{~m}, 4 \mathrm{H}, \mathrm{Ar}-\mathrm{H})$, $7.30-7.26(\mathrm{~m}, 1 \mathrm{H}, \mathrm{Ar}-\mathrm{H}), 4.21-4.06(\mathrm{~m}, 1 \mathrm{H}, \mathrm{C} \underline{\mathrm{H}}(\mathrm{OH})), 2.71(\mathrm{~d}, J=4.5 \mathrm{~Hz}, 1 \mathrm{H}, \mathrm{OH})$, $1.82\left(\mathrm{~s}, 6 \mathrm{H}, \mathrm{C}\left(\mathrm{CH}_{3}\right)_{2}\right), 1.70-1.56\left(\mathrm{~m}, 2 \mathrm{H}, \mathrm{CH}_{2}\right), 1.47-1.32\left(\mathrm{~m}, 4 \mathrm{H}, \mathrm{CH}_{2}\right), 0.93(\mathrm{t}, J=$ $\left.7.1 \mathrm{~Hz}, 3 \mathrm{H}, \mathrm{CH}_{3}\right) .{ }^{13} \mathrm{C} \mathrm{NMR}\left(100 \mathrm{MHz}, \mathrm{CDCl}_{3}\right) \delta 174.2(1 \mathrm{C}, \mathrm{C}=\mathrm{O}), 144.9$ (1C, Ar-C), 128.4 (2C, Ar-C), 127.4 (1C, Ar-C), 124.3 (2C, Ar-C), 83.4 (1C, C-O), 70.6 (1C, $\mathrm{CH}(\mathrm{OH})), 34.1\left(1 \mathrm{C}, \mathrm{CH}_{2}\right), 28.5\left(1 \mathrm{C}, \mathrm{C}\left(\underline{\mathrm{CH}}_{3}\right)_{2}\right), 28.4\left(1 \mathrm{C}, \mathrm{C}\left(\underline{\mathrm{CH}}_{3}\right)_{2}\right), 26.9\left(1 \mathrm{C}, \mathrm{CH}_{2}\right)$, $22.5\left(1 \mathrm{C}, \mathrm{CH}_{2}\right), 14.0\left(1 \mathrm{C}, \mathrm{CH}_{3}\right)$. HRMS (ESI) Calcd for $\left[\mathrm{C}_{15} \mathrm{H}_{22} \mathrm{O}_{3} \mathrm{Na}, \mathrm{M}+\mathrm{Na}\right]^{+}$: 273.1461, Found: 273.1464.

\section{2-Phenylpropan-2-yl 2-hydroxytetradecanoate（2d）}

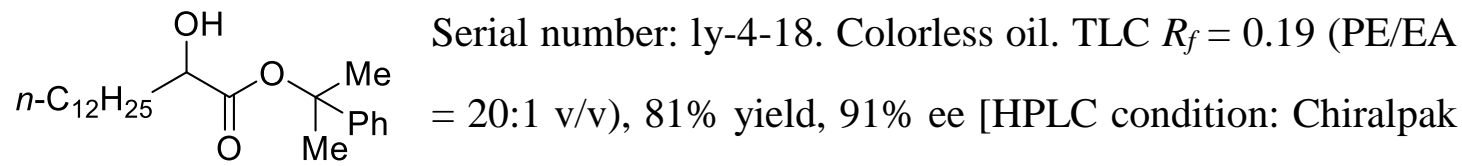
AS-H column, $n$-hexane/2-propanol $=95: 5$, flow rate $=1$ $\mathrm{mL} / \mathrm{min}$, wavelength $=210 \mathrm{~nm}, t_{\mathrm{R}}=3.81 \mathrm{~min}$ for major isomer, $t_{\mathrm{R}}=4.57 \mathrm{~min}$ for minor isomer], $[\alpha]_{\mathrm{D}}{ }^{25}=13.3\left(c 1.0, \mathrm{CHCl}_{3}\right) .{ }^{1} \mathrm{H} \mathrm{NMR}\left(400 \mathrm{MHz}, \mathrm{CDCl}_{3}\right) \delta 7.40-7.31(\mathrm{~m}$, 4H, Ar-H), $7.30-7.25$ (m, 1H, Ar-H), 4.14 (dt, J=7.2, 5.3 Hz, 1H, C$(\mathrm{OH})), 2.71(\mathrm{~d}$, $J=5.6 \mathrm{~Hz}, 1 \mathrm{H}, \mathrm{OH}), 1.81\left(\mathrm{~s}, 6 \mathrm{H}, \mathrm{C}\left(\mathrm{CH}_{3}\right)_{2}\right), 1.72-1.57(\mathrm{~m}, 1 \mathrm{H}, \mathrm{CH}), 1.48-1.16(\mathrm{~m}$, $\left.21 \mathrm{H}, \mathrm{CH}_{2}\right), 0.88\left(\mathrm{t}, J=6.8 \mathrm{~Hz}, 3 \mathrm{H}, \mathrm{CH}_{3}\right) .{ }^{13} \mathrm{C} \mathrm{NMR}\left(100 \mathrm{MHz}, \mathrm{CDCl}_{3}\right) \delta 174.2(1 \mathrm{C}$, $\mathrm{C}=\mathrm{O}), 144.9$ (1C, Ar-C), 128.4 (2C, Ar-C), 127.4 (1C, Ar-C), 124.3 (2C, Ar-C), 83.4 (1C, C-O), 70.5 (1C, $\mathrm{CH}(\mathrm{OH})$ ), 34.4 (1C, $\left.\mathrm{CH}_{2}\right), 32.0$ (1C, $\left.\mathrm{CH}_{2}\right), 29.7$ (1C, $\left.\mathrm{CH}_{2}\right), 29.7$ (2C, $\left.\mathrm{CH}_{2}\right), 29.6\left(1 \mathrm{C}, \mathrm{CH}_{2}\right), 29.6\left(1 \mathrm{C}, \mathrm{CH}_{2}\right), 29.5\left(1 \mathrm{C}, \mathrm{CH}_{2}\right), 29.4\left(1 \mathrm{C}, \mathrm{CH}_{2}\right), 28.5(1 \mathrm{C}$, $\left.\mathrm{C}\left(\underline{\mathrm{CH}}_{3}\right)_{2}\right), 28.4\left(1 \mathrm{C}, \mathrm{C}\left(\underline{\mathrm{CH}}_{3}\right)_{2}\right), 24.7\left(1 \mathrm{C}, \mathrm{CH}_{2}\right), 22.7\left(1 \mathrm{C}, \mathrm{CH}_{2}\right), 14.2\left(1 \mathrm{C}, \mathrm{CH}_{3}\right) . \mathrm{HRMS}$ (ESI) Calcd for $\left[\mathrm{C}_{23} \mathrm{H}_{38} \mathrm{O}_{3} \mathrm{Na}, \mathrm{M}+\mathrm{Na}\right]^{+}:$385.2713, Found: 385.2721. 


\section{2-Phenylpropan-2-yl 2-hydroxy-5-methylhexanoate(2e)}<smiles>CC(C)CCC(O)C(=O)OC(C)(C)c1ccccc1</smiles>

Serial number: 1y-4-32. Colorless oil. TLC $R_{f}=0.11$

$(\mathrm{PE} / \mathrm{EA}=20: 1 \mathrm{v} / \mathrm{v}), 80 \%$ yield, 95\% ee $[\mathrm{HPLC}$ condition: Chiralpak AS-H column, n-hexane/2propanol $=95: 5$, flow rate $=1 \mathrm{~mL} / \mathrm{min}$, wavelength $=210 \mathrm{~nm}, t_{\mathrm{R}}=4.63 \mathrm{~min}$ for major isomer, $t_{\mathrm{R}}=5.18 \mathrm{~min}$ for minor isomer $],[\alpha]_{\mathrm{D}}{ }^{25}=18.4\left(c\right.$ 1.0, $\left.\mathrm{CHCl}_{3}\right) .{ }^{1} \mathrm{H} \mathrm{NMR}(400$ $\left.\mathrm{MHz}, \mathrm{CDCl}_{3}\right) \delta 7.40-7.31(\mathrm{~m}, 4 \mathrm{H}, \mathrm{Ar}-\mathrm{H}), 7.30-7.24(\mathrm{~m}, 1 \mathrm{H}, \mathrm{Ar}-\mathrm{H}), 4.14(\mathrm{dd}, J=$ 11.0, 4.7 Hz, 1H, $\mathrm{C} \underline{\mathrm{H}}(\mathrm{OH})), 2.74(\mathrm{~d}, J=5.0 \mathrm{~Hz}, 1 \mathrm{H}, \mathrm{OH}), 1.91-1.77(\mathrm{~m}, 7 \mathrm{H}, \mathrm{CH}$, $\left.\mathrm{C}\left(\mathrm{CH}_{3}\right)_{2}\right), 1.72-1.51\left(\mathrm{~m}, 2 \mathrm{H}, \mathrm{CH}_{2}\right), 1.43-1.32\left(\mathrm{~m}, 1 \mathrm{H}, \mathrm{CH}_{2}\right), 1.29-1.17(\mathrm{~m}, 1 \mathrm{H}$, $\left.\mathrm{CH}_{2}\right), 0.92\left(\mathrm{~d}, J=6.6 \mathrm{~Hz}, 6 \mathrm{H}, \mathrm{CH}\left(\mathrm{CH}_{3}\right)_{2}\right) .{ }^{13} \mathrm{C} \mathrm{NMR}\left(100 \mathrm{MHz}, \mathrm{CDCl}_{3}\right) \delta 174.2(1 \mathrm{C}$, $\mathrm{C}=\mathrm{O}), 144.9$ (1C, Ar-C), 128.4 (2C, Ar-C), 127.4 (1C, Ar-C), 124.3 (2C, Ar-C), 83.4 (1C, C-O), $70.7(1 \mathrm{C}, \mathrm{CH}(\mathrm{OH})), 33.6\left(1 \mathrm{C}, \mathrm{CH}_{2}\right), 32.3\left(1 \mathrm{C}, \mathrm{CH}_{2}\right), 28.5\left(1 \mathrm{C}, \mathrm{C}\left(\underline{\mathrm{CH}}_{3}\right)_{2}\right)$, $28.4\left(1 \mathrm{C}, \mathrm{C}\left(\underline{\mathrm{C}}_{3}\right)_{2}\right), 28.0\left(1 \mathrm{C}, \underline{\mathrm{C}} \mathrm{H}\left(\mathrm{CH}_{3}\right)_{2}\right), 22.7\left(1 \mathrm{C}, \mathrm{CH}\left(\underline{\mathrm{CH}}_{3}\right)_{2}\right), 22.4\left(1 \mathrm{C}, \mathrm{CH}\left(\underline{\mathrm{CH}}_{3}\right)_{2}\right)$. HRMS (ESI) Calcd for $\left[\mathrm{C}_{16} \mathrm{H}_{24} \mathrm{O}_{3} \mathrm{Na}, \mathrm{M}+\mathrm{Na}\right]^{+}:$287.1618, Found: 287.1621.

\section{2-Phenylpropan-2-yl 2-hydroxy-5,5-dimethylhexanoate (2f)}<smiles>CC(C)(C)CCC(O)C(=O)OC(C)(C)c1ccccc1</smiles>

Serial number: 1y-4-39. Colorless oil. TLC $R_{f}=0.14$ $(\mathrm{PE} / \mathrm{EA}=20: 1 \mathrm{v} / \mathrm{v}), 80 \%$ yield, $91 \%$ ee $[$ HPLC condition: Chiralpak AS-H column, $n$-hexane/2-propanol = 95:5, flow rate $=1 \mathrm{~mL} / \mathrm{min}$, wavelength $=210 \mathrm{~nm}, t_{\mathrm{R}}=4.35 \mathrm{~min}$ for major isomer, $t_{\mathrm{R}}=5.00 \mathrm{~min}$ for minor isomer], $[\alpha]_{\mathrm{D}}{ }^{25}=16.2\left(c\right.$ 1.0, $\left.\mathrm{CHCl}_{3}\right) .{ }^{1} \mathrm{H} \mathrm{NMR}\left(400 \mathrm{MHz}, \mathrm{CDCl}_{3}\right) \delta 7.41-$ $7.32(\mathrm{~m}, 4 \mathrm{H}, \mathrm{Ar}-\mathrm{H}), 7.31-7.26(\mathrm{~m}, 1 \mathrm{H}, \mathrm{Ar}-\mathrm{H}), 4.15(\mathrm{dd}, J=10.7,5.5 \mathrm{~Hz}, 1 \mathrm{H}, \mathrm{C} \underline{\mathrm{H}}(\mathrm{OH}))$, $2.72(\mathrm{~d}, J=5.5 \mathrm{~Hz}, 1 \mathrm{H}, \mathrm{OH}), 1.90-1.76\left(\mathrm{~m}, 1 \mathrm{H}, \mathrm{CH}_{2}, 6 \mathrm{H}, \mathrm{C}\left(\mathrm{CH}_{3}\right)_{2}\right), 1.70-1.58(\mathrm{~m}$, $\left.1 \mathrm{H}, \mathrm{CH}_{2}\right), 1.29\left(\mathrm{dtd}, J=49.2,13.0,4.4 \mathrm{~Hz}, 2 \mathrm{H}, \mathrm{CH}_{2}\right), 0.91\left(\mathrm{~s}, 9 \mathrm{H}, \mathrm{C}\left(\mathrm{CH}_{3}\right)_{3}\right) .{ }^{13} \mathrm{C} \mathrm{NMR}$ $\left(100 \mathrm{MHz}, \mathrm{CDCl}_{3}\right) \delta 174.1(1 \mathrm{C}, \mathrm{C}=\mathrm{O}), 144.9$ (1C, Ar-C), 128.4 (2C, Ar-C), $127.4(1 \mathrm{C}$, Ar-C), 124.3 (2C, Ar-C), 83.4 (1C, C-O), 70.9 (1C, $\mathrm{CH}(\mathrm{OH})), 38.3\left(1 \mathrm{C}, \mathrm{CH}_{2}\right), 30.0$ (1C, $\left.\underline{\mathrm{C}}\left(\mathrm{CH}_{3}\right)_{3}\right), 29.4\left(1 \mathrm{C}, \mathrm{C}\left(\underline{\mathrm{CH}}_{3}\right)_{2}\right), 29.3\left(3 \mathrm{C}, \mathrm{C}\left(\underline{\mathrm{CH}}_{3}\right)_{3}\right), 28.7\left(1 \mathrm{C}, \mathrm{CH}_{2}\right), 28.3(1 \mathrm{C}$, $\left.\mathrm{C}\left(\underline{\mathrm{CH}}_{3}\right)_{2}\right)$. HRMS (ESI) Calcd for $\left[\mathrm{C}_{17} \mathrm{H}_{26} \mathrm{O}_{3} \mathrm{Na}, \mathrm{M}+\mathrm{Na}\right]^{+}: 301.1774$, Found: 301.1777. 


\section{2-Phenylpropan-2-yl 4-cyclohexyl-2-hydroxybutanoate (2g)}<smiles>CC(C)(OC(=O)C(O)CCC1CCCCC1)c1ccccc1</smiles>

Serial number: 1y-4-190. Colorless oil. TLC $R_{f}=0.22$ $(\mathrm{PE} / \mathrm{EA}=20: 1 \mathrm{v} / \mathrm{v}), 82 \%$ yield, $91 \%$ ee $[$ HPLC condition:

Chiralpak AS-H column, $n$-hexane/2-propanol = 95:5, flow rate $=1 \mathrm{~mL} / \mathrm{min}$, wavelength $=210 \mathrm{~nm}, t_{\mathrm{R}}=4.73 \mathrm{~min}$ for major isomer, $t_{\mathrm{R}}=6.59$ min for minor isomer], $[\alpha]_{\mathrm{D}}{ }^{25}=20.3\left(c 1.0, \mathrm{CHCl}_{3}\right) .{ }^{1} \mathrm{H} \mathrm{NMR}\left(400 \mathrm{MHz}, \mathrm{CDCl}_{3}\right) \delta 7.40$ - 7.14 (m, 5H, Ar-H), 4.12 (s, 1H, C$(\mathrm{OH})$ ), 2.74 (s, 1H, OH), $1.92-1.42$ (m, 13H, $\left.\mathrm{C}\left(\mathrm{CH}_{3}\right)_{2}, \mathrm{CH}_{2}, \mathrm{C} \underline{\mathrm{H}}\left(\mathrm{CH}_{2}\right)_{2}\right), 1.34-1.07\left(\mathrm{~m}, 6 \mathrm{H}, \mathrm{CH}_{2}\right), 0.89$ (d, J=4.8 Hz, 2H, $\left.\mathrm{CH}_{2}\right)$. ${ }^{13} \mathrm{C}$ NMR $\left(100 \mathrm{MHz}, \mathrm{CDCl}_{3}\right) \delta 174.2$ (1C, C=O), 144.9 (1C, Ar-C), 128.4 (2C, Ar-C), 127.4 (1C, Ar-C), 124.3 (2C, Ar-C), 83.4 (1C,C-O), 70.8 (1C, $\mathrm{CH}(\mathrm{OH})), 37.6$ (1C, $\left.\mathrm{CH}_{2}\right), 33.5\left(1 \mathrm{C}, \mathrm{CH}_{2}\right), 33.2\left(1 \mathrm{C}, \mathrm{CH}_{2}\right), 32.1\left(1 \mathrm{C}, \mathrm{CH}_{2}\right), 31.8\left(1 \mathrm{C}, \mathrm{CH}_{2}\right), 28.5\left(1 \mathrm{C}, \mathrm{CH}_{2}\right)$, $28.4(1 \mathrm{C}, \mathrm{CH}), 26.7\left(1 \mathrm{C}, \mathrm{CH}_{2}\right), 26.4\left(2 \mathrm{C}, \mathrm{C}\left(\underline{\mathrm{CH}}_{3}\right)_{2}\right)$. HRMS (ESI) Calcd for $\left[\mathrm{C}_{19} \mathrm{H}_{28} \mathrm{O} 3 \mathrm{Na}, \mathrm{M}+\mathrm{Na}\right]^{+}:$327.1931, Found: 327.1935.

\section{2-Phenylpropan-2-yl 3-cyclopropyl-2-hydroxypropanoate (2h)}

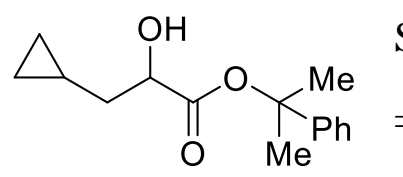

Serial number: 1y-4-141. Colorless oil. TLC $R_{f}=0.22(\mathrm{PE} / \mathrm{EA}$

$=20: 1 \mathrm{v} / \mathrm{v}), 74 \%$ yield, $91 \%$ ee [HPLC condition: Chiralpak AS-H column, $n$-hexane/2-propanol $=95: 5$, flow rate $=1$ $\mathrm{mL} / \mathrm{min}$, wavelength $=210 \mathrm{~nm}, t_{\mathrm{R}}=5.82 \mathrm{~min}$ for major isomer, $t_{\mathrm{R}}=6.29 \mathrm{~min}$ for minor isomer], $[\alpha]_{\mathrm{D}}{ }^{25}=29.3\left(c 1.0, \mathrm{CHCl}_{3}\right) .{ }^{1} \mathrm{H} \mathrm{NMR}\left(400 \mathrm{MHz}, \mathrm{CDCl}_{3}\right) \delta 7.43-7.32(\mathrm{~m}$, $4 \mathrm{H}, \mathrm{Ar}-\mathrm{H}), 7.31-7.25(\mathrm{~m}, 1 \mathrm{H}, \mathrm{Ar}-\mathrm{H}), 4.23(\mathrm{td}, J=6.4,4.6 \mathrm{~Hz}, 1 \mathrm{H}, \mathrm{C} \underline{\mathrm{H}}(\mathrm{OH})), 2.85(\mathrm{~d}$, $J=6.0 \mathrm{~Hz}, 1 \mathrm{H}, \mathrm{OH}), 1.83\left(\mathrm{~s}, 6 \mathrm{H}, \mathrm{C}\left(\mathrm{CH}_{3}\right)_{2}\right), 1.76-1.65\left(\mathrm{~m}, 2 \mathrm{H}, \mathrm{CH}_{2}\right), 0.94-0.83(\mathrm{~m}$, $1 \mathrm{H}, \mathrm{CH}), 0.57-0.45\left(\mathrm{~m}, 2 \mathrm{H}, \mathrm{CH}_{2}\right), 0.21-0.11\left(\mathrm{~m}, 2 \mathrm{H}, \mathrm{CH}_{2}\right) .{ }^{13} \mathrm{C} \mathrm{NMR}(100 \mathrm{MHz}$, $\left.\mathrm{CDCl}_{3}\right) \delta 174.0(1 \mathrm{C}, \mathrm{C}=\mathrm{O}), 145.0(1 \mathrm{C}, \mathrm{Ar}-\mathrm{C}), 128.4$ (2C, Ar-C), 127.4 (1C, Ar-C), 124.3 (2C, Ar-C), 83.5 (1C, C-O), 71.2 (1C, $\mathrm{CH}(\mathrm{OH})), 39.4\left(1 \mathrm{C}, \mathrm{CH}_{2}\right), 28.7(1 \mathrm{C}$, $\left.\mathrm{C}\left(\underline{\mathrm{CH}}_{3}\right)_{2}\right), 28.1\left(1 \mathrm{C}, \mathrm{C}\left(\underline{\mathrm{CH}}_{3}\right)_{2}\right), 6.9(1 \mathrm{C}, \mathrm{CH}), 4.7\left(1 \mathrm{C}, \mathrm{CH}_{2}\right), 4.1\left(1 \mathrm{C}, \mathrm{CH}_{2}\right) . \mathrm{HRMS}(\mathrm{ESI})$ Calcd for $\left[\mathrm{C}_{15} \mathrm{H}_{20} \mathrm{O}_{3} \mathrm{Na}, \mathrm{M}+\mathrm{Na}\right]^{+}:$271.1305, Found: 271.1305. 


\section{2-Phenylpropan-2-yl 2-hydroxy-4-methylpentanoate (2i)}

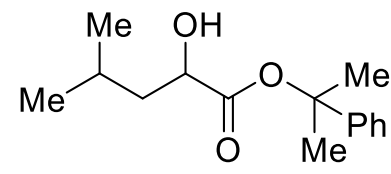

Serial number: 1y-4-12. Colorless oil. TLC $R_{f}=0.26(\mathrm{PE} / \mathrm{EA}=$ 20:1 v/v), 76\% yield, 88\% ee [HPLC condition: Chiralpak AS-

H column, $n$-hexane $/ 2$-propanol $=95: 5$, flow rate $=1 \mathrm{~mL} / \mathrm{min}$, wavelength $=210 \mathrm{~nm}, t_{\mathrm{R}}=4.69 \mathrm{~min}$ for major isomer, $t_{\mathrm{R}}=5.29 \mathrm{~min}$ for minor isomer], $[\alpha]_{\mathrm{D}}^{25}=25.4\left(c\right.$ 1.0, $\left.\mathrm{CHCl}_{3}\right) .{ }^{1} \mathrm{H}$ NMR $\left(400 \mathrm{MHz}, \mathrm{CDCl}_{3}\right) \delta 7.35(\mathrm{~d}, J=3.5 \mathrm{~Hz}, 4 \mathrm{H}$, Ar-H), $7.31-7.23(\mathrm{~m}, 1 \mathrm{H}, \mathrm{Ar}-\mathrm{H}), 4.34-3.92(\mathrm{~m}, 1 \mathrm{H}, \mathrm{C} \underline{\mathrm{H}}(\mathrm{OH})), 2.66(\mathrm{~d}, J=4.6 \mathrm{~Hz}$, $1 \mathrm{H}, \mathrm{OH}), 1.98-1.86(\mathrm{~m}, 1 \mathrm{H}, \mathrm{CH}), 1.81\left(\mathrm{~s}, 6 \mathrm{H}, \mathrm{C}\left(\mathrm{CH}_{3}\right)_{2}\right), 1.68-1.47\left(\mathrm{~m}, 2 \mathrm{H}, \mathrm{CH}_{2}\right)$, $1.05-0.92\left(\mathrm{~m}, 6 \mathrm{H}, \mathrm{CH}\left(\mathrm{CH}_{3}\right)_{2}\right) .{ }^{13} \mathrm{C} \mathrm{NMR}\left(100 \mathrm{MHz}, \mathrm{CDCl}_{3}\right) \delta 174.6(1 \mathrm{C}, \mathrm{C}=\mathrm{O}), 145.0$ (1C, Ar-C), 128.4 (2C, Ar-C), 127.4 (1C, Ar-C), 124.2 (2C, Ar-C), 83.3 (1C, C-O), $69.2(1 \mathrm{C}, \underline{\mathrm{CH}}(\mathrm{OH})), 43.5\left(1 \mathrm{C}, \mathrm{CH}_{2}\right), 28.6\left(1 \mathrm{C}, \mathrm{C}\left(\underline{\mathrm{CH}}_{3}\right)_{2}\right), 28.4\left(1 \mathrm{C}, \mathrm{C}\left(\underline{\mathrm{CH}}_{3}\right)_{2}\right), 24.6(1 \mathrm{C}$, $\left.\underline{\mathrm{CH}}\left(\mathrm{CH}_{3}\right)_{2}\right), 23.5\left(1 \mathrm{C}, \mathrm{C}\left(\underline{\mathrm{CH}}_{3}\right)_{2}\right), 21.5\left(1 \mathrm{C}, \mathrm{C}\left(\underline{\mathrm{CH}}_{3}\right)_{2}\right)$. HRMS (ESI) Calcd for $\left[\mathrm{C}_{15} \mathrm{H}_{22} \mathrm{O}_{3} \mathrm{Na}, \mathrm{M}+\mathrm{Na}\right]^{+}:$273.1461, Found:273.1466.

\section{2-Phenylpropan-2-yl 2-hydroxy-3-phenylpropanoate (2j)}

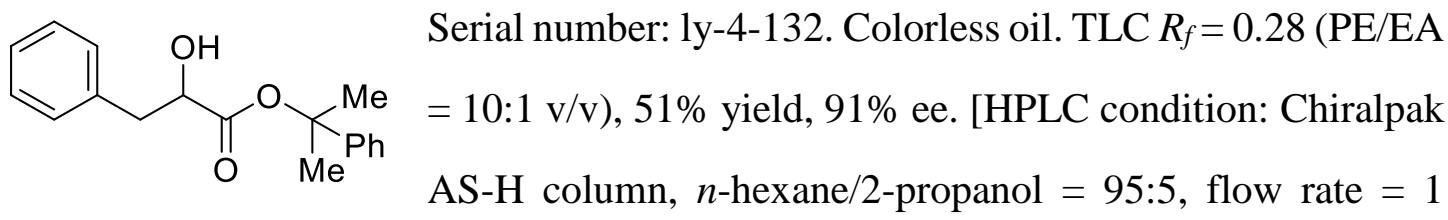
$\mathrm{mL} / \mathrm{min}$, wavelength $=210 \mathrm{~nm}, t_{\mathrm{R}}=4.17 \mathrm{~min}$ for major isomer, $t_{\mathrm{R}}=4.94 \mathrm{~min}$ for minor isomer], $[\alpha]_{\mathrm{D}}{ }^{25}=22.2\left(c 1.0, \mathrm{CHCl}_{3}\right) .{ }^{1} \mathrm{H} \mathrm{NMR}\left(400 \mathrm{MHz}, \mathrm{CDCl}_{3}\right) \delta 7.50-7.46(\mathrm{~m}$, 1H, Ar-H), $7.33-7.24$ (m, 9H, Ar-H), 4.40 (ddd, $J=7.0,5.8,4.7 \mathrm{~Hz}, 1 \mathrm{H}, \mathrm{C} \underline{\mathrm{H}}(\mathrm{OH}))$, $3.17\left(\mathrm{dd}, J=14.0,4.6 \mathrm{~Hz}, 1 \mathrm{H}, \mathrm{CH}_{2}\right), 2.96\left(\mathrm{dd}, J=14.0,7.1 \mathrm{~Hz}, 1 \mathrm{H}, \mathrm{CH}_{2}\right), 2.85(\mathrm{~d}, J=$ $5.8 \mathrm{~Hz}, 1 \mathrm{H}, \mathrm{OH}), 1.76\left(\mathrm{~s}, 6 \mathrm{H}, \mathrm{C}\left(\mathrm{CH}_{3}\right)_{2}\right) .{ }^{13} \mathrm{C} \mathrm{NMR}\left(100 \mathrm{MHz}, \mathrm{CDCl}_{3}\right) \delta 173.0(1 \mathrm{C}$, $\mathrm{C}=\mathrm{O}), 149.1$ (1C, Ar-C), 144.9 (1C, Ar-C), 136.8 (1C, Ar-C), 129.7 (1C, Ar-C), 128.4 (1C, Ar-C), 128.4 (1C, Ar-C), 128.3 (1C, Ar-C), 127.4 (1C, Ar-C), 126.8 (1C, Ar-C), 126.7 (1C, Ar-C), 124.4 (1C, Ar-C), 124.4 (1C, Ar-C), 83.8 (1C, C-O), 71.4 (1C, $\mathrm{CH}(\mathrm{OH})), 40.5\left(1 \mathrm{C}, \mathrm{CH}_{2}\right), 28.8\left(1 \mathrm{C}, \mathrm{CH}\left(\underline{\mathrm{CH}}_{3}\right)_{2}\right), 28.0\left(1 \mathrm{C}, \mathrm{CH}\left(\underline{\mathrm{CH}}_{3}\right)_{2}\right) . \mathrm{HRMS}(\mathrm{ESI})$ Calcd for $\left[\mathrm{C}_{18} \mathrm{H}_{20} \mathrm{O}_{3} \mathrm{Na}, \mathrm{M}+\mathrm{Na}\right]^{+}:$307.1305, Found: 307.1309. 


\section{2-Phenylpropan-2-yl 2-hydroxy-4-phenylbutanoate (2k)}<smiles>CC(C)(OC(=O)C(O)CCc1ccccc1)c1ccccc1</smiles>

Serial number: 1y-3-158. Colorless oil. TLC $R_{f}=0.16$

$(\mathrm{PE} / \mathrm{EA}=20: 1 \mathrm{v} / \mathrm{v}), 65 \%$ yield, $88 \%$ ee $[\mathrm{HPLC}$ condition:

Chiralpak AS-H column, $n$-hexane/2-propanol $=95: 5$, flow rate $=1 \mathrm{~mL} / \mathrm{min}$, wavelength $=210 \mathrm{~nm}, t_{\mathrm{R}}=7.59$ min for major isomer, $t_{\mathrm{R}}=10.01$ min for minor isomer], $[\alpha]_{\mathrm{D}}{ }^{25}=30.1\left(c 1.0, \mathrm{CHCl}_{3}\right) .{ }^{1} \mathrm{H} \mathrm{NMR}\left(400 \mathrm{MHz}, \mathrm{CDCl}_{3}\right) \delta 7.35$ - $7.16(\mathrm{~m}, 10 \mathrm{H}, \mathrm{Ar}-\mathrm{H}), 4.14$ (ddd, $J=8.1,5.2,4.1 \mathrm{~Hz}, 1 \mathrm{H}, \mathrm{C} \underline{\mathrm{H}}(\mathrm{OH})), 2.92(\mathrm{~d}, J=5.3$ $\mathrm{Hz}, 1 \mathrm{H}, \mathrm{OH}), 2.85-2.63\left(\mathrm{~m}, 2 \mathrm{H}, \mathrm{CH}_{2}\right), 2.16\left(\mathrm{tdd}, J=14.0,7.0,4.0 \mathrm{~Hz}, 1 \mathrm{H}, \mathrm{CH}_{2}\right), 2.02$ $-1.88\left(\mathrm{~m}, 1 \mathrm{H}, \mathrm{CH}_{2}\right), 1.79$ (s, 6H, $\left.\mathrm{C}\left(\mathrm{CH}_{3}\right)_{2}\right) .{ }^{13} \mathrm{C} \mathrm{NMR}\left(100 \mathrm{MHz}, \mathrm{CDCl}_{3}\right) \delta 174.0(1 \mathrm{C}$, $\mathrm{C}=\mathrm{O}), 144.9$ (1C, Ar-C), 141.5 (1C, Ar-C), 128.7 (2C, Ar-C), 128.6 (2C, Ar-C), 128.5 (2C, Ar-C), 127.5 (1C, Ar-C), 126.1 (1C, Ar-C), 124.4 (2C, Ar-C), 83.6 (1C, C-O), $70.0(1 \mathrm{C}, \mathrm{CH}(\mathrm{OH})), 36.2\left(1 \mathrm{C}, \mathrm{CH}_{2}\right), 31.2\left(1 \mathrm{C}, \mathrm{CH}_{2}\right), 28.6\left(1 \mathrm{C}, \mathrm{C}\left(\underline{\mathrm{CH}}_{3}\right)_{2}\right), 28.5(1 \mathrm{C}$, $\left.\mathrm{C}\left(\underline{\mathrm{CH}}_{3}\right)_{2}\right)$. HRMS (ESI) Calcd for $\left[\mathrm{C}_{19} \mathrm{H}_{22} \mathrm{O}_{3} \mathrm{Na}, \mathrm{M}+\mathrm{Na}\right]^{+}: 321.1461$, Found: 321.1465 .

\section{2-Phenylpropan-2-yl 2-hydroxy-5-phenylpentanoate (2l)}<smiles>CC(C)(OC(=O)C(O)CCCc1ccccc1)P(C)(C)(C)C</smiles>
Serial number: 1y-3-164. Colorless oil. TLC $R_{f}=0.18$

$(\mathrm{PE} / \mathrm{EA}=10: 1 \mathrm{v} / \mathrm{v}), 78 \%$ yield, $95 \%$ ee $[\mathrm{HPLC}$ condition: Chiralpak AD-H column, n-hexane/2propanol $=90: 10$, flow rate $=1 \mathrm{~mL} / \mathrm{min}$, wavelength $=210 \mathrm{~nm}, t_{\mathrm{R}}=7.59 \mathrm{~min}$ for minor isomer, $t_{\mathrm{R}}=9.52 \mathrm{~min}$ for major isomer $],[\alpha]_{\mathrm{D}}{ }^{25}=38.8\left(c 1.0, \mathrm{CHCl}_{3}\right) .{ }^{1} \mathrm{H} \mathrm{NMR}(400$ $\left.\mathrm{MHz}, \mathrm{CDCl}_{3}\right) \delta 7.31-7.16(\mathrm{~m}, 7 \mathrm{H}, \mathrm{Ar}-\mathrm{H}), 7.12(\mathrm{~m}, 3 \mathrm{H}, \mathrm{Ar}-\mathrm{H}), 4.17-3.99(\mathrm{~m}, 1 \mathrm{H}$, $\mathrm{C} \underline{\mathrm{H}}(\mathrm{OH})), 2.77-2.48\left(\mathrm{~m}, 1 \mathrm{H}, \mathrm{OH}, 2 \mathrm{H}, \mathrm{CH}_{2}\right), 1.86-1.75\left(\mathrm{~m}, 2 \mathrm{H}, \mathrm{CH}_{2}\right), 1.71(\mathrm{~s}, 6 \mathrm{H}$, $\left.\mathrm{C}\left(\mathrm{CH}_{3}\right)_{2}\right), 1.68-1.57\left(\mathrm{~m}, 2 \mathrm{H}, \mathrm{CH}_{2}\right) .{ }^{13} \mathrm{C} \mathrm{NMR}\left(100 \mathrm{MHz}, \mathrm{CDCl}_{3}\right) \delta 174.0(1 \mathrm{C}, \mathrm{C}=\mathrm{O})$, 144.9 (1C, Ar-C), 142.0 (1C, Ar-C), 128.5 (2C, Ar-C), 128.4 (2C, Ar-C), 128.4 (2C, Ar-C), 127.4 (1C, Ar-C), 125.9 (1C, Ar-C), 124.3 (2C, Ar-C), 83.5 (1C, C-O), 70.4 (1C, $\mathrm{CH}(\mathrm{OH})), 35.6\left(1 \mathrm{C}, \mathrm{CH}_{2}\right), 33.9\left(1 \mathrm{C}, \mathrm{CH}_{2}\right), 28.5\left(1 \mathrm{C}, \mathrm{C}\left(\underline{\mathrm{CH}}_{3}\right)_{2}\right), 28.3\left(1 \mathrm{C}, \mathrm{C}\left(\underline{\mathrm{CH}}_{3}\right)_{2}\right)$, $26.5\left(1 \mathrm{C}, \mathrm{CH}_{2}\right)$. HRMS (ESI) Calcd for $\left[\mathrm{C}_{20} \mathrm{H}_{24} \mathrm{O}_{3} \mathrm{Na}, \mathrm{M}+\mathrm{Na}\right]^{+}: 335.1618$, Found: 335.1620. 


\section{2-Phenylpropan-2-yl 2-hydroxypent-4-enoate (2m)}

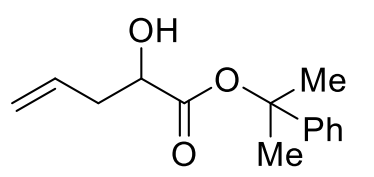

Serial number: 1y-3-183. Colorless oil. TLC $R_{f}=0.16(\mathrm{PE} / \mathrm{EA}$ $=10: 1 \mathrm{v} / \mathrm{v}), 54 \%$ yield, $95 \%$ ee [HPLC condition: Chiralcel OJ3 column, $n$-hexane $/ 2$-propanol $=95: 5$, flow rate $=1 \mathrm{~mL} / \mathrm{min}$, wavelength $=210 \mathrm{~nm}, t_{\mathrm{R}}=13.71 \mathrm{~min}$ for major isomer, $t_{\mathrm{R}}=18.40 \mathrm{~min}$ for minor isomer], $[\alpha]_{\mathrm{D}}{ }^{25}=62.3\left(c 1.0, \mathrm{CHCl}_{3}\right) .{ }^{1} \mathrm{H} \mathrm{NMR}\left(400 \mathrm{MHz}, \mathrm{CDCl}_{3}\right) \delta 7.43-7.18(\mathrm{~m}$, $5 \mathrm{H}, \mathrm{Ar}-\mathrm{H}), 5.82(\mathrm{ddt}, J=17.1,10.2,7.0 \mathrm{~Hz}, 1 \mathrm{H}, \mathrm{CH}=), 5.34-5.03\left(\mathrm{~m}, 2 \mathrm{H}, \mathrm{CH}_{2}=\right)$, $4.22(\mathrm{dd}, J=10.7,5.9 \mathrm{~Hz}, 1 \mathrm{H}, \mathrm{C} \underline{\mathrm{H}}(\mathrm{OH})), 2.83(\mathrm{~d}, J=5.8 \mathrm{~Hz}, 1 \mathrm{H}, \mathrm{OH}), 2.67-2.57(\mathrm{~m}$, $\left.1 \mathrm{H}, \mathrm{CH}_{2}\right), 2.56-2.38\left(\mathrm{~m}, 1 \mathrm{H}, \mathrm{CH}_{2}\right), 1.81\left(\mathrm{~s}, 6 \mathrm{H}, \mathrm{C}\left(\mathrm{CH}_{3}\right)_{2}\right) .{ }^{13} \mathrm{C} \mathrm{NMR}(100 \mathrm{MHz}$, $\left.\mathrm{CDCl}_{3}\right) \delta 173.2(1 \mathrm{C}, \mathrm{C}=\mathrm{O}), 144.9(1 \mathrm{C}, \mathrm{Ar}-\mathrm{C}), 132.7$ (1C, $\left.\mathrm{CH}=\right), 128.4$ (2C, Ar-C), 127.5 (1C, Ar-C), 124.3 (2C, Ar-C), 118.6 (1C, $\left.\mathrm{CH}_{2}=\right), 83.7$ (1C, C-O), 70.0 (1C, $\mathrm{CH}(\mathrm{OH})), 38.8\left(1 \mathrm{C}, \mathrm{CH}_{2}\right), 28.7\left(1 \mathrm{C}, \mathrm{C}\left(\underline{\mathrm{CH}}_{3}\right)_{2}\right), 28.2\left(1 \mathrm{C}, \mathrm{C}\left(\underline{\mathrm{CH}}_{3}\right)_{2}\right)$. HRMS (ESI) Calcd for $\left[\mathrm{C}_{14} \mathrm{H}_{18} \mathrm{O}_{3} \mathrm{Na}, \mathrm{M}+\mathrm{Na}\right]^{+}:$257.1148, Found: 257.1151 .

\section{2-Phenylpropan-2-yl 2-hydroxyhex-5-enoate (2n)}<smiles>C=CCCC(O)C(=O)OC(C)(C)c1ccccc1</smiles>

Serial number: ly-3-182. Colorless oil. TLC $R_{f}=0.21(\mathrm{PE} / \mathrm{EA}$ $=10: 1 \mathrm{v} / \mathrm{v}), 78 \%$ yield, $94 \%$ ee [HPLC condition: Chiralpak AS-H column, $n$-hexane/2-propanol $=95: 5$, flow rate $=1$

$\mathrm{mL} / \mathrm{min}$, wavelength $=210 \mathrm{~nm}, t_{\mathrm{R}}=5.26 \mathrm{~min}$ for major isomer, $t_{\mathrm{R}}=6.34 \mathrm{~min}$ for minor isomer $],[\alpha]_{\mathrm{D}}{ }^{25}=64.5\left(c 1.0, \mathrm{CHCl}_{3}\right) .{ }^{1} \mathrm{H} \mathrm{NMR}\left(400 \mathrm{MHz}, \mathrm{CDCl}_{3}\right) \delta 7.28-7.19(\mathrm{~m}$, 4H, Ar-H), 7.19-7.11 (m, 1H, Ar-H), 5.74 (ddt, $J=16.9,10.2,6.7 \mathrm{~Hz}, 1 \mathrm{H}, \mathrm{CH}=), 5.12$ $-4.79\left(\mathrm{~m}, 2 \mathrm{H}, \mathrm{CH}_{2}=\right), 4.03(\mathrm{dt}, J=7.8,5.3 \mathrm{~Hz}, 1 \mathrm{H}, \mathrm{C} \underline{\mathrm{H}}(\mathrm{OH})), 2.61(\mathrm{~d}, J=5.5 \mathrm{~Hz}, 1 \mathrm{H}$, $\mathrm{OH}), 2.18-2.01\left(\mathrm{~m}, 2 \mathrm{H}, \mathrm{CH}_{2}\right), 1.92-1.77\left(\mathrm{~m}, 1 \mathrm{H}, \mathrm{CH}_{2}\right), 1.70\left(\mathrm{~s}, 6 \mathrm{H}, \mathrm{C}\left(\mathrm{CH}_{3}\right)_{2}\right), 1.66$ $-1.57\left(\mathrm{~m}, 1 \mathrm{H}, \mathrm{CH}_{2}\right) .{ }^{13} \mathrm{C} \mathrm{NMR}\left(100 \mathrm{MHz}, \mathrm{CDCl}_{3}\right) \delta 174.0(1 \mathrm{C}, \mathrm{C}=\mathrm{O}), 144.9(1 \mathrm{C}, \mathrm{Ar}-$ C), 137.7 (1C, $\mathrm{CH}=), 128.4$ (2C, Ar-C), 127.4 (1C, Ar-C), 124.3 (2C, Ar-C), 115.4 (1C, $\left.\mathrm{CH}_{2}=\right), 83.5(1 \mathrm{C}, \mathrm{C}-\mathrm{O}), 69.9$ (1C, $\left.\mathrm{CH}(\mathrm{OH})\right), 33.6\left(1 \mathrm{C}, \mathrm{CH}_{2}\right), 29.1\left(1 \mathrm{C}, \mathrm{CH}_{2}\right), 28.5(1 \mathrm{C}$, $\left.\mathrm{C}\left(\underline{\mathrm{CH}}_{3}\right)_{2}\right), 28.5\left(1 \mathrm{C}, \mathrm{C}\left(\underline{\mathrm{CH}}_{3}\right)_{2}\right)$. HRMS (ESI) Calcd for $\left[\mathrm{C}_{15} \mathrm{H}_{20} \mathrm{O}_{3} \mathrm{Na}, \mathrm{M}+\mathrm{Na}\right]^{+}$: 271.1305, Found: 271.1306. 
2-Phenylpropan-2-yl 2-hydroxyoct-7-enoate (2o)

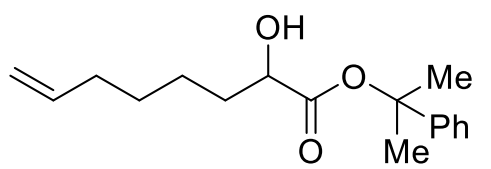

Serial number: 1y-3-165. Colorless oil. TLC $R_{f}=0.21$

$(\mathrm{PE} / \mathrm{EA}=10: 1 \mathrm{v} / \mathrm{v}), 76 \%$ yield, $93 \%$ ee $[\mathrm{HPLC}$ condition: Chiralpak AS-H column, n-hexane/2propanol $=95: 5$, flow rate $=1 \mathrm{~mL} / \mathrm{min}$, wavelength $=210 \mathrm{~nm}, t_{\mathrm{R}}=4.97 \mathrm{~min}$ for major isomer, $t_{\mathrm{R}}=6.73 \mathrm{~min}$ for minor isomer $],[\alpha]_{\mathrm{D}}{ }^{25}=69.3\left(c\right.$ 1.0, $\left.\mathrm{CHCl}_{3}\right) .{ }^{1} \mathrm{H}$ NMR $(400$ $\left.\mathrm{MHz}, \mathrm{CDCl}_{3}\right) \delta 7.27-7.19(\mathrm{~m}, 4 \mathrm{H}, \mathrm{Ar}-\mathrm{H}), 7.18-7.12(\mathrm{~m}, 1 \mathrm{H}, \mathrm{Ar}-\mathrm{H}), 5.70$ (ddt, $J=$ $16.9,10.2,6.7 \mathrm{~Hz}, 1 \mathrm{H}, \mathrm{CH}=), 4.95-4.76\left(\mathrm{~m}, 2 \mathrm{H}, \mathrm{CH}_{2}=\right), 4.05-3.98(\mathrm{~m}, 1 \mathrm{H}, \mathrm{C} \underline{\mathrm{H}}(\mathrm{OH}))$, $2.62(\mathrm{~s}, 1 \mathrm{H}, \mathrm{OH}), 1.96\left(\mathrm{q}, J=6.9 \mathrm{~Hz}, 2 \mathrm{H}, \mathrm{CH}_{2}\right), 1.78-1.66\left(\mathrm{~m}, 7 \mathrm{H}, \mathrm{CH}_{2}, \mathrm{C}\left(\mathrm{CH}_{3}\right)_{2}\right)$, $1.60-1.48\left(\mathrm{~m}, 1 \mathrm{H}, \mathrm{CH}_{2}\right), 1.43-1.23\left(\mathrm{~m}, 4 \mathrm{H}, \mathrm{CH}_{2}\right) .{ }^{13} \mathrm{C} \mathrm{NMR}\left(100 \mathrm{MHz}, \mathrm{CDCl}_{3}\right) \delta$ 174.1 (1C, C=O), 144.9 (1C, Ar-C), 138.7 (1C, CH=), 128.4 (2C, Ar-C), 127.4 (1C, Ar-C), 124.3 (2C, Ar-C), 114.6 (1C, $\left.\mathrm{CH}_{2}=\right), 83.4$ (1C, C-O), 70.5 (1C, $\left.\mathrm{CH}(\mathrm{OH})\right), 34.3$ (1C, $\left.\mathrm{CH}_{2}\right), 33.6\left(1 \mathrm{C}, \mathrm{CH}_{2}\right), 28.7\left(1 \mathrm{C}, \mathrm{CH}_{2}\right), 28.5\left(1 \mathrm{C}, \mathrm{C}\left(\underline{\mathrm{CH}}_{3}\right)_{2}\right), 28.4\left(1 \mathrm{C}, \mathrm{C}\left(\underline{\mathrm{CH}}_{3}\right)_{2}\right)$, $24.2\left(1 \mathrm{C}, \mathrm{CH}_{2}\right)$. HRMS (ESI) Calcd for $\left[\mathrm{C}_{17} \mathrm{H}_{24} \mathrm{O}_{3} \mathrm{Na}, \mathrm{M}+\mathrm{Na}\right]^{+}:$299.1618, Found: 299.1620 .

\section{2-Phenylpropan-2-yl 2-hydroxyhex-5-ynoate (2p)}

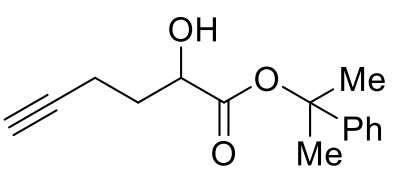

Serial number: ly-3-44. Colorless oil. TLC $R_{f}=0.51(\mathrm{PE} / \mathrm{EA}=$

10:1 v/v), 80\% yield, 90\% ee [HPLC condition: Chiralpak ASH column, $n$-hexane $/ 2$-propanol $=95: 5$, flow rate $=1 \mathrm{~mL} / \mathrm{min}$, wavelength $=210 \mathrm{~nm}, t_{\mathrm{R}}=9.00 \mathrm{~min}$ for major isomer, $t_{\mathrm{R}}=11.10 \mathrm{~min}$ for minor isomer $]$, $[\alpha]_{\mathrm{D}}^{25}=62.3\left(c\right.$ 1.0, $\left.\mathrm{CHCl}_{3}\right) .{ }^{1} \mathrm{H}$ NMR $\left(400 \mathrm{MHz}, \mathrm{CDCl}_{3}\right) \delta 7.36(\mathrm{~d}, J=4.3 \mathrm{~Hz}, 4 \mathrm{H}$, Ar-H), $7.31-7.26$ (m, 1H, Ar-H), 4.24 (ddd, $J=8.8,5.2,3.8 \mathrm{~Hz}, 1 \mathrm{H}, \mathrm{C} \underline{\mathrm{H}}(\mathrm{OH})), 2.75$ $(\mathrm{d}, J=5.3 \mathrm{~Hz}, 1 \mathrm{H}, \mathrm{OH}), 2.46-2.27\left(\mathrm{~m}, 2 \mathrm{H}, \mathrm{CH}_{2}\right), 2.15-2.06(\mathrm{~m}, 1 \mathrm{H}, \mathrm{CH} \equiv), 2.01(\mathrm{t}$, $\left.J=2.6 \mathrm{~Hz}, 1 \mathrm{H}, \mathrm{CH}_{2}\right), 1.88-1.78\left(\mathrm{~m}, 1 \mathrm{H}, \mathrm{CH}_{2}, 6 \mathrm{H}, \mathrm{C}\left(\mathrm{CH}_{3}\right)_{2}\right) .{ }^{13} \mathrm{C} \mathrm{NMR}(100 \mathrm{MHz}$, $\left.\mathrm{CDCl}_{3}\right) \delta 172.7(1 \mathrm{C}, \mathrm{C}=\mathrm{O}), 144.5$ (1C, Ar-C), 128.4 (2C, Ar-C), 127.5 (1C, Ar-C), 124.3 (2C, Ar-C), 83.8 (1C, C $\equiv), 83.2$ (1C, C-O), 69.2 (1C, $\mathrm{CH} \equiv), 69.1(1 \mathrm{C}, \mathrm{CH}(\mathrm{OH}))$, 33.2 (1C, $\left.\mathrm{CH}_{2}\right), 28.5\left(1 \mathrm{C}, \mathrm{C}\left(\underline{\mathrm{CH}}_{3}\right)_{2}\right), 28.4\left(1 \mathrm{C}, \mathrm{C}\left(\underline{\mathrm{CH}}_{3}\right)_{2}\right), 14.5$ (1C, $\left.\mathrm{CH}_{2}\right) . \mathrm{HRMS}$ (ESI) Calcd for $\left[\mathrm{C}_{15} \mathrm{H}_{18} \mathrm{O}_{3} \mathrm{Na}, \mathrm{M}+\mathrm{Na}\right]^{+}:$269.1148, Found: 269.1152 . 


\section{2-Phenylpropan-2-yl 2-hydroxy-5-methoxypentanoate (2q)}<smiles>COCCCC(O)C(=O)OC(C)(C)c1ccccc1</smiles>

Serial number: 1y-3-176. Colorless oil. TLC $R_{f}=0.19$ $(\mathrm{PE} / \mathrm{EA}=20: 1 \mathrm{v} / \mathrm{v}), 77 \%$ yield, 90\% ee [HPLC condition:

Chiralpak AS-H column, $n$-hexane/2-propanol = 95:5, flow rate $=1 \mathrm{~mL} / \mathrm{min}$, wavelength $=210 \mathrm{~nm}, t_{\mathrm{R}}=8.81$ min for major isomer, $t_{\mathrm{R}}=11.18$ min for minor isomer], $[\alpha]_{\mathrm{D}}{ }^{25}=24.2\left(c 1.0, \mathrm{CHCl}_{3}\right) .{ }^{1} \mathrm{H} \mathrm{NMR}\left(400 \mathrm{MHz}, \mathrm{CDCl}_{3}\right) \delta 7.39$ - 7.30 (m, 4H, Ar-H), 7.29 - $7.23(\mathrm{~m}, 1 \mathrm{H}, \mathrm{Ar}-\mathrm{H}), 4.20-3.12(\mathrm{~m}, 1 \mathrm{H}, \mathrm{C} \underline{\mathrm{H}}(\mathrm{OH})), 3.43$ (tt, $\left.J=5.2,2.6 \mathrm{~Hz}, 2 \mathrm{H}, \mathrm{CH}_{2}\right), 3.35\left(\mathrm{~s}, 3 \mathrm{H}, \mathrm{OCH}_{3}\right), 3.04(\mathrm{~d}, J=5.7 \mathrm{~Hz}, 1 \mathrm{H}, \mathrm{OH}), 2.00-$ $1.87\left(\mathrm{~m}, 1 \mathrm{H}, \mathrm{CH}_{2}\right), 1.81\left(\mathrm{~s}, 6 \mathrm{H}, \mathrm{C}\left(\mathrm{CH}_{3}\right)_{2}\right), 1.78-1.66\left(\mathrm{~m}, 3 \mathrm{H}, \mathrm{CH}_{2}\right) .{ }^{13} \mathrm{C} \mathrm{NMR}$ $\left(100 \mathrm{MHz}, \mathrm{CDCl}_{3}\right) \delta 173.8(1 \mathrm{C}, \mathrm{C}=\mathrm{O}), 145.0$ (1C, Ar-C), 128.4 (2C, Ar-C), $127.4(1 \mathrm{C}$, Ar-C), 124.3 (2C, Ar-C), 83.4 (1C, C-O), 72.4 (1C, $\left.\mathrm{OCH}_{2}\right), 70.4$ (1C, $\left.\mathrm{CH}(\mathrm{OH})\right), 58.6$ (1C, $\left.\mathrm{OCH}_{3}\right), 31.4\left(1 \mathrm{C}, \mathrm{CH}_{2}\right), 28.6\left(1 \mathrm{C}, \mathrm{C}\left(\underline{\mathrm{CH}}_{3}\right)_{2}\right), 28.4\left(1 \mathrm{C}, \mathrm{C}\left(\underline{\mathrm{CH}}_{3}\right)_{2}\right), 25.1\left(1 \mathrm{C}, \mathrm{CH}_{2}\right)$. HRMS (ESI) Calcd for $\left[\mathrm{C}_{15} \mathrm{H}_{22} \mathrm{O}_{4} \mathrm{Na}, \mathrm{M}+\mathrm{Na}\right]^{+}:$289.1410, Found: 289.1415.

\section{2-Phenylpropan-2-yl 5-((tert-butyldimethylsilyl)oxy)-2-hydroxypentanoate (2r)}<smiles>CCCCOCCCC(O)C(=O)OC(C)(C)c1ccccc1</smiles>

Serial number: ly-4-50. Colorless oil. TLC $R_{f}=0.31$

$(\mathrm{PE} / \mathrm{EA}=10: 1 \mathrm{v} / \mathrm{v}), 82 \%$ yield, $94 \%$ ee $[\mathrm{HPLC}$ condition: Chiralpak AS-H column, $n$-hexane/2propanol $=99: 1$, flow rate $=1 \mathrm{~mL} / \mathrm{min}$, wavelength $=210 \mathrm{~nm}, t_{\mathrm{R}}=4.06 \mathrm{~min}$ for major isomer, $t_{\mathrm{R}}=4.42 \mathrm{~min}$ for minor isomer $],[\alpha]_{\mathrm{D}}{ }^{25}=22.4\left(c\right.$ 1.0, $\left.\mathrm{CHCl}_{3}\right) .{ }^{1} \mathrm{H}$ NMR $(400$ $\left.\mathrm{MHz}, \mathrm{CDCl}_{3}\right) \delta 7.39$ - $7.32(\mathrm{~m}, 4 \mathrm{H}, \mathrm{Ar}-\mathrm{H}), 7.29$ - 7.24 (m, 1H, Ar-H), 4.17 (dt, J = 7.2, $4.8 \mathrm{~Hz}, 1 \mathrm{H}, \mathrm{C} \underline{\mathrm{H}}(\mathrm{OH})), 3.68\left(\mathrm{t}, J=5.8 \mathrm{~Hz}, 2 \mathrm{H}, \mathrm{CH}_{2}\right), 2.98(\mathrm{~d}, J=5.5 \mathrm{~Hz}, 1 \mathrm{H}, \mathrm{OH}), 2.01$ - 1.90 (m, 1H, $\left.\mathrm{CH}_{2}\right), 1.81$ (s, 6H, $\left.\mathrm{C}\left(\mathrm{CH}_{3}\right)_{2}\right), 1.76-1.60$ (m, 2H, $\left.\mathrm{CH}_{2}, 1 \mathrm{H}, \mathrm{CH}_{2}\right), 0.91$ (s, 9H, $\left.\mathrm{Si}\left(\mathrm{CH}_{3}\right)_{3}\right), 0.07$ (s, 6H, $\left.\mathrm{Si}\left(\mathrm{CH}_{3}\right)_{2}\right) .{ }^{13} \mathrm{C} \mathrm{NMR}\left(100 \mathrm{MHz}, \mathrm{CDCl}_{3}\right) \delta 174.0(1 \mathrm{C}$, $\mathrm{C}=\mathrm{O}), 145.0$ (1C, Ar-C), 128.4 (2C, Ar-C), 127.4 (1C, Ar-C), 124.3 (2C, Ar-C), 83.3 (1C, C-O), $70.4(1 \mathrm{C}, \mathrm{CH}(\mathrm{OH})), 62.7\left(1 \mathrm{C}, \mathrm{OCH}_{2}\right), 31.3\left(1 \mathrm{C}, \underline{\mathrm{C}}\left(\mathrm{CH}_{3}\right)_{3}\right), 28.5(1 \mathrm{C}$, $\left.\mathrm{C}\left(\underline{\mathrm{CH}}_{3}\right)_{2}\right), 28.4\left(1 \mathrm{C}, \underline{\mathrm{C}}\left(\mathrm{CH}_{3}\right)_{2}\right), 28.1\left(1 \mathrm{C}, \mathrm{CH}_{2}\right), 26.0$ (3C, $\left.\mathrm{C}\left(\underline{\mathrm{CH}}_{3}\right)_{3}\right), 18.4\left(1 \mathrm{C}, \mathrm{CH}_{2}\right),-$ $5.3\left(2 \mathrm{C}, \mathrm{Si}\left(\mathrm{CH}_{3}\right)_{2}\right)$. HRMS (ESI) Calcd for $\left[\mathrm{C}_{20} \mathrm{H}_{34} \mathrm{O}_{4} \mathrm{SiNa}, \mathrm{M}+\mathrm{Na}\right]^{+}: 389.2119$, Found: 389.2120. 


\section{6-Ethyl 1-(2-phenylpropan-2-yl) 2-hydroxyhexanedioate (2s)}<smiles>CCOC(=O)CCCC(O)C(=O)OC(C)(C)c1ccccc1</smiles>

Serial number: 1y-3-175. Colorless oil. TLC $R_{f}=0.19$ $(\mathrm{PE} / \mathrm{EA}=5: 1 \mathrm{v} / \mathrm{v}), 69 \%$ yield, $89 \%$ ee $[\mathrm{HPLC}$ condition:

Chiralpak AS-H column, $n$-hexane/2-propanol = 95:5, flow rate $=1 \mathrm{~mL} / \mathrm{min}$, wavelength $=210 \mathrm{~nm}, t_{\mathrm{R}}=9.53 \mathrm{~min}$ for major isomer, $t_{\mathrm{R}}=11.42$ min for minor isomer], $[\alpha]_{\mathrm{D}}{ }^{25}=31.1\left(c 1.0, \mathrm{CHCl}_{3}\right) .{ }^{1} \mathrm{H} \mathrm{NMR}\left(400 \mathrm{MHz}, \mathrm{CDCl}_{3}\right) \delta 7.40$ $-7.31(\mathrm{~m}, 4 \mathrm{H}, \mathrm{Ar}-\mathrm{H}), 7.31-7.23(\mathrm{~m}, 1 \mathrm{H}, \mathrm{Ar}-\mathrm{H}), 4.36-4.05\left(\mathrm{~m}, 2 \mathrm{H}, \mathrm{CH}_{2}, 1 \mathrm{H}\right.$, $\mathrm{C} \underline{\mathrm{H}}(\mathrm{OH})), 3.78-3.72\left(\mathrm{~m}, 1 \mathrm{H}, \mathrm{CH}_{2}\right), 2.79(\mathrm{~d}, J=5.4 \mathrm{~Hz}, 1 \mathrm{H}, \mathrm{OH}), 2.37(\mathrm{td}, J=7.0,1.8$ $\left.\mathrm{Hz}, 2 \mathrm{H}, \mathrm{CH}_{2}\right), 1.95-1.64\left(\mathrm{~m}, 12 \mathrm{H}, \mathrm{CH}_{2}, \mathrm{CH}_{2}, \mathrm{CH}_{2}, \mathrm{C}\left(\mathrm{CH}_{3}\right)_{2}\right), 1.26(\mathrm{t}, J=7.1 \mathrm{~Hz}, 3 \mathrm{H}$, $\left.\mathrm{CH}_{3}\right) .{ }^{13} \mathrm{C}$ NMR (100 MHz, $\left.\mathrm{CDCl}_{3}\right) \delta 173.8(1 \mathrm{C}, \mathrm{C}=\mathrm{O}), 173.3(1 \mathrm{C}, \mathrm{C}=\mathrm{O}), 144.8(1 \mathrm{C}$, Ar-C), 128.4 (2C, Ar-C), 127.4 (1C, Ar-C), 124.3 (2C, Ar-C), 83.6 (1C, C-O), 70.2 (1C, $\mathrm{CH}(\mathrm{OH})), 60.4\left(1 \mathrm{C}, \mathrm{OCH}_{2}\right), 33.9\left(1 \mathrm{C}, \mathrm{CH}_{2}\right), 28.5\left(1 \mathrm{C}, \mathrm{C}\left(\underline{\mathrm{CH}}_{3}\right)_{2}\right), 28.3\left(1 \mathrm{C}, \mathrm{C}\left(\underline{\mathrm{CH}}_{3}\right)_{2}\right)$, $25.6\left(1 \mathrm{C}, \mathrm{CH}_{2}\right), 20.4\left(1 \mathrm{C}, \mathrm{CH}_{2}\right), 14.3\left(1 \mathrm{C}, \mathrm{CH}_{3}\right)$. HRMS (ESI) Calcd for $\left[\mathrm{C}_{17} \mathrm{H}_{24} \mathrm{O}_{5} \mathrm{Na}\right.$, $\mathrm{M}+\mathrm{Na}]^{+}:$331.1516, Found:331.1519.

\section{2-Phenylpropan-2-yl 5-cyano-2-hydroxypentanoate (2t)}<smiles>CC(C)(OC(=O)C(O)CCCC#N)P(C)(C)(C)C</smiles>

Serial number: ly-3-197. Colorless oil. TLC $R_{f}=0.17$ $(\mathrm{PE} / \mathrm{EA}=3: 1 \mathrm{v} / \mathrm{v}), 84 \%$ yield, $91 \%$ ee $[$ HPLC condition: Chiralpak AS-H column, $n$-hexane/2-propanol = 95:5, flow rate $=1 \mathrm{~mL} / \mathrm{min}$, wavelength $=210 \mathrm{~nm}, t_{\mathrm{R}}=14.19 \mathrm{~min}$ for major isomer, $t_{\mathrm{R}}=15.61$ min for minor isomer $],[\alpha]_{\mathrm{D}}{ }^{25}=22.4\left(c 1.0, \mathrm{CHCl}_{3}\right) .{ }^{1} \mathrm{H} \mathrm{NMR}\left(400 \mathrm{MHz}, \mathrm{CDCl}_{3}\right) \delta 7.39$ - 7.33 (m, 4H, Ar-H), $7.32-7.26(\mathrm{~m}, 1 \mathrm{H}, \mathrm{Ar}-\mathrm{H}), 4.28-4.07$ (m, 1H, $\mathrm{C} \underline{\mathrm{H}}(\mathrm{OH})), 2.82$ $(\mathrm{d}, J=4.3 \mathrm{~Hz}, 1 \mathrm{H}, \mathrm{OH}), 2.51-2.33\left(\mathrm{~m}, 2 \mathrm{H}, \mathrm{CH}_{2}\right), 2.08-1.93\left(\mathrm{~m}, 1 \mathrm{H}, \mathrm{CH}_{2}\right), 1.88-$ $1.66\left(\mathrm{~m}, 1 \mathrm{H}, \mathrm{CH}_{2}, 2 \mathrm{H}, \mathrm{CH}_{2}, 6 \mathrm{H}, \mathrm{C}\left(\mathrm{CH}_{3}\right)_{2}\right) .{ }^{13} \mathrm{C} \mathrm{NMR}\left(100 \mathrm{MHz}, \mathrm{CDCl}_{3}\right) \delta 173.3(1 \mathrm{C}$, $\mathrm{C}=\mathrm{O}), 144.5$ (1C, Ar-C), 128.5 (2C, Ar-C), 127.6 (1C, Ar-C), 124.3 (2C, Ar-C), 119.4 $(1 \mathrm{C}, \mathrm{C} \equiv \mathrm{N}), 84.0(1 \mathrm{C}, \mathrm{C}-\mathrm{O}), 69.7(1 \mathrm{C}, \mathrm{CH}(\mathrm{OH})), 32.8\left(1 \mathrm{C}, \mathrm{CH}_{2}\right), 28.4\left(1 \mathrm{C}, \mathrm{C}\left(\underline{\mathrm{CH}}_{3}\right)_{2}\right)$, $28.4\left(1 \mathrm{C}, \mathrm{C}\left(\mathrm{CH}_{3}\right)_{2}\right), 21.2\left(1 \mathrm{C}, \mathrm{CH}_{2}\right), 17.0\left(1 \mathrm{C}, \mathrm{CH}_{2}\right)$. HRMS (ESI) Calcd for $\left[\mathrm{C}_{15} \mathrm{H}_{19} \mathrm{NO}_{3} \mathrm{Na}, \mathrm{M}+\mathrm{Na}\right]^{+}:$284.1257, Found: 284.1260. 


\section{(E)-tert-Butyl 2-hydroxy-4-phenylpent-3-enoate (7a)}

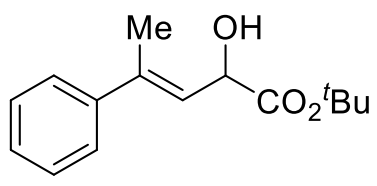

Serial number: zt-3-95. White solid, mp: $74 \sim 75^{\circ} \mathrm{C}$. TLC $R_{f}=$ $0.72(\mathrm{PE} / \mathrm{EA}=2: 1 \mathrm{v} / \mathrm{v}), 97 \%$ yield, $94 \%$ ee $[\mathrm{HPLC}$ condition:

Chiralcel OD-H column, $n$-hexane/2-propanol = 90:10, flow rate $=1.0 \mathrm{~mL} / \mathrm{min}$, wavelength $=254 \mathrm{~nm}, t_{\mathrm{R}}=5.38 \mathrm{~min}$ for major isomer, $t_{\mathrm{R}}=6.03$ min for minor isomer], $[\alpha]_{\mathrm{D}}{ }^{25}=-62.0\left(c\right.$ 1.0, $\left.\mathrm{CHCl}_{3}\right) .{ }^{1} \mathrm{H} \mathrm{NMR}\left(400 \mathrm{MHz}, \mathrm{CDCl}_{3}\right) \delta$ $7.42-7.38(\mathrm{~m}, 2 \mathrm{H}, \mathrm{Ar}-\mathrm{H}), 7.33(\mathrm{t}, J=7.3 \mathrm{~Hz}, 2 \mathrm{H}, \mathrm{Ar}-\mathrm{H}), 7.30-7.25$ (m, 1H, Ar-H), $5.66(\mathrm{dd}, J=8.5,1.1 \mathrm{~Hz}, 1 \mathrm{H}, \mathrm{CH}=), 4.91(\mathrm{dd}, J=8.4,5.7 \mathrm{~Hz}, 1 \mathrm{H}, \mathrm{C} \underline{\mathrm{H}}(\mathrm{OH})), 3.14(\mathrm{~d}$, $J=5.8 \mathrm{~Hz}, 1 \mathrm{H}, \mathrm{O}-\mathrm{H}), 2.18\left(\mathrm{~d}, J=0.8 \mathrm{~Hz}, 3 \mathrm{H}, \mathrm{CH}_{3}\right), 1.48\left(\mathrm{~s}, 9 \mathrm{H}, \mathrm{C}_{\left.\left(\mathrm{CH}_{3}\right)_{3}\right) .{ }^{13} \mathrm{C} \mathrm{NMR}}\right.$ $\left(100 \mathrm{MHz}, \mathrm{CDCl}_{3}\right) \delta 173.2(1 \mathrm{C}, \mathrm{C}=\mathrm{O}), 142.6(1 \mathrm{C}, \mathrm{Ar}-\mathrm{C}), 140.3(1 \mathrm{C}, \mathrm{C}=), 128.3(2 \mathrm{C}$, Ar-C), 127.5 (1C, Ar-C), 125.9 (2C, Ar-C), 125.1 (1C, $\mathrm{CH}=), 82.8$ (1C, C-O), 68.8 (1C, $\mathrm{CH}(\mathrm{OH})), 28.0\left(3 \mathrm{C}, \mathrm{C}\left(\underline{\mathrm{CH}}_{3}\right)_{3}\right), 16.9\left(1 \mathrm{C}, \mathrm{CH}_{3}\right)$. HRMS (ESI) Calcd for $\left[\mathrm{C}_{15} \mathrm{H}_{20} \mathrm{O}_{3} \mathrm{Na}\right.$, $\mathrm{M}+\mathrm{Na}]^{+}:$271.1305, Found: 271.1308.

\section{(E)-tert-Butyl 2-hydroxy-4-(m-tolyl)pent-3-enoate (7b)}<smiles>CCCCC(O)C(O)C=C(C)c1cccc(C)c1</smiles>

Serial number: zt-4-146. White solid, mp: $70 \sim 72{ }^{\circ} \mathrm{C}$. TLC $R_{f}=0.19(\mathrm{PE} / \mathrm{EA}=10: 1 \mathrm{v} / \mathrm{v}), 97 \%$ yield, $91 \%$ ee $[\mathrm{HPLC}$ condition: Chiralcel OD-H column, $n$-hexane/2-propanol $=90: 10$, flow rate $=1.0 \mathrm{~mL} / \mathrm{min}$, wavelength $=254 \mathrm{~nm}, t_{\mathrm{R}}=5.16 \mathrm{~min}$ for minor isomer, $t_{\mathrm{R}}=5.57 \mathrm{~min}$ for major isomer], $[\alpha]_{\mathrm{D}}{ }^{28}=-108.3\left(c 1.0, \mathrm{CHCl}_{3}\right) .{ }^{1} \mathrm{H} \mathrm{NMR}(400 \mathrm{MHz}$, $\left.\mathrm{CDCl}_{3}\right) \delta 7.13(\mathrm{~m}, 3 \mathrm{H}, \mathrm{Ar}-\mathrm{H}), 7.02(\mathrm{~m}, 1 \mathrm{H}, \mathrm{Ar}-\mathrm{H}), 5.86-5.38(\mathrm{~m}, 1 \mathrm{H}, \mathrm{CH}=), 4.83(\mathrm{dd}$, $J=8.5,5.4 \mathrm{~Hz}, 1 \mathrm{H}, \mathrm{C} \underline{\mathrm{H}}(\mathrm{OH})), 3.09(\mathrm{~d}, J=5.7 \mathrm{~Hz}, 1 \mathrm{H}, \mathrm{O}-\mathrm{H}), 2.28\left(\mathrm{~s}, 3 \mathrm{H}, \mathrm{CH}_{3}\right), 2.09$ $\left(\mathrm{s}, 3 \mathrm{H}, \mathrm{CH}_{3}\right), 1.40\left(\mathrm{~s}, 9 \mathrm{H}, \mathrm{C}\left(\mathrm{CH}_{3}\right)_{3}\right) .{ }^{13} \mathrm{C} \mathrm{NMR}\left(100 \mathrm{MHz}, \mathrm{CDCl}_{3}\right) \delta 173.3(1 \mathrm{C}, \mathrm{C}=\mathrm{O})$, 142.6 (1C, Ar-C), 140.4 (1C, Ar-C), 137.8 (1C, C=), 128.2 (1C, Ar-C), 128.1 (1C, ArC), 126.7 (1C, Ar-C), 124.9 (1C, Ar-C), 123.0 (1C, $\mathrm{CH}=), 82.7$ (1C, C-O), 68.8 (1C, $\mathrm{CH}(\mathrm{OH})), 28.0$ (3C, $\left.\mathrm{C}\left(\underline{\mathrm{CH}}_{3}\right)_{3}\right), 21.4$ (1C, $\left.\mathrm{CH}_{3}-\mathrm{Ar}\right), 16.9$ (1C, $\left.\mathrm{CH}_{3}\right)$. HRMS (ESI) Calcd for $\left[\mathrm{C}_{16} \mathrm{H}_{22} \mathrm{O}_{3} \mathrm{Na}, \mathrm{M}+\mathrm{Na}\right]^{+}: 285.1461$, Found: 285.1462 . 


\section{(E)-tert-Butyl 2-hydroxy-4-(p-tolyl)pent-3-enoate (7c)}<smiles>CCCCC(O)C(O)C=C(C)c1ccc(C)cc1</smiles>

Serial number: zt-4-148. White solid, mp: $78 \sim 79^{\circ} \mathrm{C}$. TLC

$R_{f}=0.21(\mathrm{PE} / \mathrm{EA}=10: 1 \mathrm{v} / \mathrm{v}), 97 \%$ yield, $93 \%$ ee $[\mathrm{HPLC}$ condition: Chiralcel OD-H column, $n$-hexane/2-propanol $=90: 10$, flow rate $=1.0 \mathrm{~mL} / \mathrm{min}$, wavelength $=254 \mathrm{~nm}, t_{\mathrm{R}}=5.51 \mathrm{~min}$ for major isomer, $t_{\mathrm{R}}=6.58 \mathrm{~min}$ for minor isomer $],[\alpha]_{\mathrm{D}}{ }^{28}=-109.6\left(c 1.0, \mathrm{CHCl}_{3}\right) .{ }^{1} \mathrm{H} \mathrm{NMR}(400 \mathrm{MHz}$, $\left.\mathrm{CDCl}_{3}\right) \delta 7.23(\mathrm{~d}, J=8.2 \mathrm{~Hz}, 2 \mathrm{H}, \mathrm{Ar}-\mathrm{H}), 7.06(\mathrm{~d}, J=7.9 \mathrm{~Hz}, 2 \mathrm{H}, \mathrm{Ar}-\mathrm{H}), 5.56(\mathrm{dd}, J=$ 8.4, 1.6 Hz, $1 \mathrm{H}, \mathrm{CH}=), 4.83(\mathrm{dd}, J=8.5,5.6 \mathrm{~Hz}, 1 \mathrm{H}, \mathrm{C} \underline{\mathrm{H}}(\mathrm{OH})), 3.12(\mathrm{~d}, J=5.7 \mathrm{~Hz}, 1 \mathrm{H}$, $\mathrm{OH}), 2.27\left(\mathrm{~s}, 3 \mathrm{H}, \mathrm{CH}_{3}\right), 2.09\left(\mathrm{~d}, J=1.4 \mathrm{~Hz}, 3 \mathrm{H}, \mathrm{CH}_{3}\right), 1.40\left(\mathrm{~s}, 9 \mathrm{H}, \mathrm{C}\left(\mathrm{CH}_{3}\right)_{3}\right) .{ }^{13} \mathrm{C} \mathrm{NMR}$ $\left(100 \mathrm{MHz}, \mathrm{CDCl}_{3}\right) \delta 173.3(1 \mathrm{C}, \mathrm{C}=\mathrm{O}), 140.1,(1 \mathrm{C}, \mathrm{Ar}-\mathrm{C}) 139.7$ (1C, Ar-C), $137.3(1 \mathrm{C}$, $\mathrm{C}=), 128.9(2 \mathrm{C}, \mathrm{Ar}-\mathrm{C}), 125.8$ (2C, Ar-C), 124.3 (1C, CH=), 82.7 (1C, C-O), 68.9 (1C, $\mathrm{CH}(\mathrm{OH})), 28.0\left(3 \mathrm{C}, \mathrm{C}\left(\underline{\mathrm{CH}}_{3}\right)_{3}\right), 21.0$ (1C, $\left.\mathrm{CH}_{3}-\mathrm{Ar}\right), 16.8$ (1C, $\left.\mathrm{CH}_{3}\right)$. HRMS (ESI) Calcd for $\left[\mathrm{C}_{16} \mathrm{H}_{22} \mathrm{O}_{3} \mathrm{Na}, \mathrm{M}+\mathrm{Na}\right]^{+}:$285.1461, Found: 285.1462 .

\section{(E)-tert-Butyl 2-hydroxy-4-(4-methoxyphenyl)pent-3-enoate (7d)}

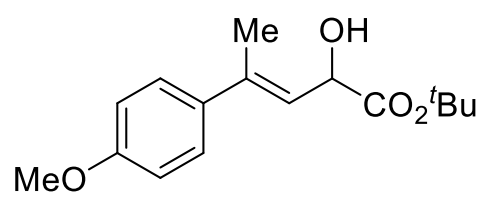

Serial number: zt-3-115. White solid, mp: $74 \sim 75{ }^{\circ} \mathrm{C}$.

TLC $R_{f}=0.32(\mathrm{PE} / \mathrm{EA}=5: 1 \mathrm{v} / \mathrm{v}), 87 \%$ yield, $90 \%$ ee [HPLC condition: Chiralcel OD-H column, $n$-hexane/2propanol $=90: 10$, flow rate $=1.0 \mathrm{~mL} / \mathrm{min}$, wavelength $=254 \mathrm{~nm}, t_{\mathrm{R}}=6.58 \mathrm{~min}$ for major isomer, $t_{\mathrm{R}}=7.60 \mathrm{~min}$ for minor isomer $],[\alpha]_{\mathrm{D}}{ }^{27}=-88.0\left(c 1.0, \mathrm{CHCl}_{3}\right) .{ }^{1} \mathrm{H} \mathrm{NMR}$ $\left(400 \mathrm{MHz}, \mathrm{CDCl}_{3}\right) \delta 7.40-7.30(\mathrm{~m}, 2 \mathrm{H}, \mathrm{Ar}-\mathrm{H}), 6.92-6.83(\mathrm{~m}, 2 \mathrm{H}, \mathrm{Ar}-\mathrm{H}), 5.60(\mathrm{dd}, J$ $=8.5,1.1 \mathrm{~Hz}, 1 \mathrm{H}, \mathrm{CH}=), 4.89(\mathrm{dd}, J=8.5,5.8 \mathrm{~Hz}, 1 \mathrm{H}, \mathrm{C} \underline{\mathrm{H}}(\mathrm{OH})), 3.81\left(\mathrm{~s}, 3 \mathrm{H}, \mathrm{OCH}_{3}\right)$, $3.11(\mathrm{~d}, J=5.8 \mathrm{~Hz}, 1 \mathrm{H}, \mathrm{OH}), 2.16\left(\mathrm{~d}, J=1.0 \mathrm{~Hz}, 3 \mathrm{H}, \mathrm{CH}_{3}\right), 1.48\left(\mathrm{~s}, 9 \mathrm{H}, \mathrm{C}\left(\mathrm{CH}_{3}\right)_{3}\right) .{ }^{13} \mathrm{C}$ NMR (100 MHz, $\left.\mathrm{CDCl}_{3}\right) \delta 173.4$ (1C, C=O), 159.1 (1C, Ar-C), 139.7 (1C, Ar-C), 135.0 (1C, $\mathrm{C}=), 127.0$ (2C, Ar-C), 123.5 (1C, $\mathrm{CH}=), 113.6$ (2C, Ar-C), 82.7 (1C, C-O), 68.9 $(1 \mathrm{C}, \mathrm{CH}(\mathrm{OH})), 55.3\left(1 \mathrm{C}, \mathrm{OCH}_{3}\right), 28.0\left(3 \mathrm{C}, \mathrm{C}\left(\mathrm{CH}_{3}\right)_{3}\right), 16.8\left(1 \mathrm{C}, \mathrm{CH}_{3}\right) . \mathrm{HRMS}(\mathrm{ESI})$ Calcd for $\left[\mathrm{C}_{16} \mathrm{H}_{22} \mathrm{O}_{4} \mathrm{Na}, \mathrm{M}+\mathrm{Na}\right]^{+}: 301.1410$, Found: 301.1414 . 


\section{(E)-tert-Butyl 2-hydroxy-4-(4-fluorophenyl)pent-3-enoate (7e)}<smiles>CCCCOC(O)C=C(C)c1ccc(F)cc1</smiles>

Serial number: zt-3-107. White solid, mp: $78 \sim 80^{\circ} \mathrm{C}$. TLC

$R_{f}=0.41(\mathrm{PE} / \mathrm{EA}=5: 1 \mathrm{v} / \mathrm{v}), 96 \%$ yield, $94 \%$ ee $[\mathrm{HPLC}$ condition: Chiralcel OD-H column, $n$-hexane/2-propanol = 99:1, flow rate $=1.0 \mathrm{~mL} / \mathrm{min}$, wavelength $=254 \mathrm{~nm}, t_{\mathrm{R}}=13.45 \mathrm{~min}$ for minor isomer, $t_{\mathrm{R}}=14.91 \mathrm{~min}$ for major isomer], $[\alpha]_{\mathrm{D}}{ }^{27}=-99.4\left(c 1.0, \mathrm{CHCl}_{3}\right) .{ }^{1} \mathrm{H} \mathrm{NMR}(400 \mathrm{MHz}$, $\left.\mathrm{CDCl}_{3}\right) \delta 7.39-7.32(\mathrm{~m}, 2 \mathrm{H}, \mathrm{Ar}-\mathrm{H}), 7.06-6.97(\mathrm{~m}, 2 \mathrm{H}, \mathrm{Ar}-\mathrm{H}), 5.60(\mathrm{dd}, J=8.5,1.0$ $\mathrm{Hz}, 1 \mathrm{H}, \mathrm{CH}=), 4.88(\mathrm{dd}, J=8.4,5.7 \mathrm{~Hz}, 1 \mathrm{H}, \mathrm{C} \underline{\mathrm{H}}(\mathrm{OH})), 3.16(\mathrm{~d}, J=5.7 \mathrm{~Hz}, 1 \mathrm{H}, \mathrm{OH})$, $2.16\left(\mathrm{~d}, J=0.9 \mathrm{~Hz}, 3 \mathrm{H}, \mathrm{CH}_{3}\right), 1.48\left(\mathrm{~s}, 9 \mathrm{H}, \mathrm{C}\left(\mathrm{CH}_{3}\right)_{3}\right) .{ }^{13} \mathrm{C} \mathrm{NMR}\left(100 \mathrm{MHz}, \mathrm{CDCl}_{3}\right) \delta$ $173.1(1 \mathrm{C}, \mathrm{C}=\mathrm{O}), 162.3$ (d, $J=246.6 \mathrm{~Hz}, 1 \mathrm{C}, \mathrm{Ar}-\mathrm{C}), 139.3(1 \mathrm{C}, \mathrm{C}=), 138.7(\mathrm{~d}, J=3.1$ $\mathrm{Hz}, 1 \mathrm{C}, \mathrm{Ar}-\mathrm{C}), 127.5$ (d, J=7.9 Hz, 2C, Ar-C), 125.1 (1C, CH=), 115.1 (d, J=21.4 Hz, 2C, Ar-C), 82.9 (1C, C-O), $68.8(1 \mathrm{C}, \mathrm{CH}(\mathrm{OH})), 28.0\left(3 \mathrm{C}, \mathrm{C}\left(\mathrm{CH}_{3}\right)_{3}\right), 17.0\left(1 \mathrm{C}, \mathrm{CH}_{3}\right)$. HRMS (ESI) Calcd for $\left[\mathrm{C}_{15} \mathrm{H}_{19} \mathrm{FO}_{3} \mathrm{Na}, \mathrm{M}+\mathrm{Na}\right]^{+}:$289.1210, Found: 289.1215.

\section{(E)-tert-Butyl 2-hydroxy-4-(2-chlorophenyl)pent-3-enoate (7f)}<smiles>CCCCCC(O)C(O)C=C(C)c1ccccc1Cl</smiles>

Serial number: zt-3-93. Colorless oil. TLC $R_{f}=0.22(\mathrm{PE} / \mathrm{EA}=$ 10:1 v/v), 91\% yield, 89\% ee [HPLC condition: Chiralcel OD$\mathrm{H}$ column, $n$-hexane/2-propanol $=98: 2$, flow rate $=1.0$ $\mathrm{mL} / \mathrm{min}$, wavelength $=254 \mathrm{~nm}, t_{\mathrm{R}}=8.23 \mathrm{~min}$ for minor isomer, $t_{\mathrm{R}}=8.96 \mathrm{~min}$ for major isomer], $[\alpha]_{\mathrm{D}}{ }^{28}=-93.0\left(c 1.0, \mathrm{CHCl}_{3}\right) .{ }^{1} \mathrm{H} \mathrm{NMR}\left(400 \mathrm{MHz}, \mathrm{CDCl}_{3}\right) \delta 7.38-7.31(\mathrm{~m}$, 1H, Ar-H), $7.25-7.12$ (m, 3H, Ar-H), $5.59-5.13$ (m, 1H, CH=), 4.89 (dd, $J=8.5,5.5$ $\mathrm{Hz}, 1 \mathrm{H}, \mathrm{CH}(\mathrm{OH})), 3.23(\mathrm{~d}, J=5.8 \mathrm{~Hz}, 1 \mathrm{H}, \mathrm{OH}), 2.12\left(\mathrm{~d}, J=1.5 \mathrm{~Hz}, 3 \mathrm{H}, \mathrm{CH}_{3}\right), 1.51(\mathrm{~s}$, 9H, $\left.\mathrm{C}\left(\mathrm{CH}_{3}\right)_{3}\right) .{ }^{13} \mathrm{C}$ NMR (100 MHz, $\left.\mathrm{CDCl}_{3}\right) \delta 173.0(1 \mathrm{C}, \mathrm{C}=\mathrm{O}), 143.0(1 \mathrm{C}, \mathrm{Ar}-\mathrm{C})$, 140.1 (1C, Ar-C), 132.0 (1C, C=), 129.7 (1C, Ar-C), 129.5 (1C, Ar-C), 128.3 (1C, ArC), 128.2 (1C, Ar-C), $126.7(1 \mathrm{C}, \mathrm{CH}=), 82.8$ (1C, C-O), 68.4 (1C, $\mathrm{CH}(\mathrm{OH})), 28.0$ (3C, $\left.\mathrm{C}\left(\underline{\mathrm{CH}}_{3}\right)_{3}\right), 18.4\left(1 \mathrm{C}, \mathrm{CH}_{3}\right)$. HRMS (ESI) Calcd for $\left[\mathrm{C}_{15} \mathrm{H}_{19} \mathrm{ClO}_{3} \mathrm{Na}, \mathrm{M}+\mathrm{Na}\right]^{+}:$305.0915, Found: 305.0916. 


\section{(E)-tert-Butyl 2-hydroxy-4-(3-chlorophenyl)pent-3-enoate (7g)}<smiles>CCCCC(O)C(O)C=C(C)c1cccc(Cl)c1</smiles>

Serial number: zt-4-50. White solid, mp: $64 \sim 65^{\circ} \mathrm{C}$. TLC

$R_{f}=0.23(\mathrm{PE} / \mathrm{EA}=10: 1 \mathrm{v} / \mathrm{v}), 90 \%$ yield, $92 \%$ ee $[\mathrm{HPLC}$ condition: Chiralcel OD-H column, $n$-hexane/2-propanol $=90: 10$, flow rate $=1.0 \mathrm{~mL} / \mathrm{min}$, wavelength $=254 \mathrm{~nm}, t_{\mathrm{R}}=4.93 \mathrm{~min}$ for minor isomer, $t_{\mathrm{R}}=5.94$ min for major isomer $],[\alpha]_{\mathrm{D}}^{28}=-84.9\left(c 1.0, \mathrm{CHCl}_{3}\right) .{ }^{1} \mathrm{H} \mathrm{NMR}(400 \mathrm{MHz}$, $\left.\mathrm{CDCl}_{3}\right) \delta 7.42-7.33(\mathrm{~m}, 1 \mathrm{H}, \mathrm{Ar}-\mathrm{H}), 7.30-7.20(\mathrm{~m}, 3 \mathrm{H}, \mathrm{Ar}-\mathrm{H}), 5.99-5.45(\mathrm{~m}, 1 \mathrm{H}$, $\mathrm{CH}=), 4.89(\mathrm{dd}, J=8.4,5.5 \mathrm{~Hz}, 1 \mathrm{H}, \mathrm{C} \underline{\mathrm{H}}(\mathrm{OH})), 3.20(\mathrm{~d}, J=5.7 \mathrm{~Hz}, 1 \mathrm{H}, \mathrm{OH}), 2.16(\mathrm{~d}$, $\left.J=1.3 \mathrm{~Hz}, 3 \mathrm{H}, \mathrm{CH}_{3}\right), 1.48\left(\mathrm{~s}, 9 \mathrm{H}, \mathrm{C}\left(\mathrm{CH}_{3}\right)_{3}\right) .{ }^{13} \mathrm{C} \mathrm{NMR}\left(100 \mathrm{MHz}, \mathrm{CDCl}_{3}\right) \delta 172.9(1 \mathrm{C}$, $\mathrm{C}=\mathrm{O}), 144.4$ (1C, Ar-C), 139.1 (1C, Ar-C), 134.2 (1C, C=), 129.5 (1C, Ar-C), 127.5 (1C, Ar-C), 126.2 (1C, Ar-C), 126.1 (1C, Ar-C), 124.1 (1C, $\mathrm{CH}=), 83.0$ (1C, C-O), 68.7 $(1 \mathrm{C}, \mathrm{CH}(\mathrm{OH})), 28.0 \quad\left(3 \mathrm{C}, \mathrm{C}\left(\underline{\mathrm{CH}}_{3}\right)_{3}\right), 16.8 \quad\left(1 \mathrm{C}, \mathrm{CH}_{3}\right)$. HRMS (ESI) Calcd for $\left[\mathrm{C}_{15} \mathrm{H}_{19} \mathrm{ClO}_{3} \mathrm{Na}, \mathrm{M}+\mathrm{Na}\right]^{+}:$305.0915, Found: 305.0914 .

\section{(E)-tert-Butyl 2-hydroxy-4-(4-chlorophenyl)pent-3-enoate (7h)}<smiles>CCCCC(O)C(O)C=C(C)c1ccc(Cl)cc1</smiles>

Serial number: zt-3-119. White solid, mp: $83 \sim 85^{\circ} \mathrm{C}$. TLC $R_{f}=0.39(\mathrm{PE} / \mathrm{EA}=5: 1 \mathrm{v} / \mathrm{v}), 96 \%$ yield, $91 \%$ ee $[\mathrm{HPLC}$ condition: Chiralcel OD-H column, $n$-hexane/2-propanol $=90: 10$, flow rate $=1.0 \mathrm{~mL} / \mathrm{min}$, wavelength $=254 \mathrm{~nm}, t_{\mathrm{R}}=15.75 \mathrm{~min}$ for major isomer, $t_{\mathrm{R}}=17.35 \mathrm{~min}$ for minor isomer $],[\alpha]_{\mathrm{D}}{ }^{27}=-65.0\left(c 1.0, \mathrm{CHCl}_{3}\right) .{ }^{1} \mathrm{H} \mathrm{NMR}(400 \mathrm{MHz}$, $\left.\mathrm{CDCl}_{3}\right) \delta 7.35-7.27(\mathrm{~m}, 4 \mathrm{H}, \mathrm{Ar}-\mathrm{H}), 5.64(\mathrm{dd}, J=8.5,1.1 \mathrm{~Hz}, 1 \mathrm{H}, \mathrm{CH}=), 4.88(\mathrm{dd}, J=$ 8.4, $5.7 \mathrm{~Hz}, 1 \mathrm{H}, \mathrm{CH}(\mathrm{OH})), 3.14(\mathrm{~d}, J=5.6 \mathrm{~Hz}, 1 \mathrm{H}, \mathrm{OH}), 2.15$ (s, 3H, $\left.\mathrm{CH}_{3}\right), 1.48(\mathrm{~s}, 9 \mathrm{H}$, $\left.\mathrm{C}\left(\mathrm{CH}_{3}\right)_{3}\right) .{ }^{13} \mathrm{C} \mathrm{NMR}\left(100 \mathrm{MHz}, \mathrm{CDCl}_{3}\right) \delta 173.0(1 \mathrm{C}, \mathrm{C}=\mathrm{O}), 141.0(1 \mathrm{C}, \mathrm{Ar}-\mathrm{C}), 139.2$ (1C, Ar-C), $133.3(1 \mathrm{C}, \mathrm{C}=), 128.4(2 \mathrm{C}, \mathrm{Ar}-\mathrm{C}), 127.2(2 \mathrm{C}, \mathrm{Ar}-\mathrm{C}), 125.6(1 \mathrm{C}, \mathrm{CH}=), 83.0$ (1C, C-O), $68.7(1 \mathrm{C}, \mathrm{CH}(\mathrm{OH})), 28.0\left(3 \mathrm{C}, \mathrm{C}\left(\underline{\mathrm{CH}}_{3}\right)_{3}\right), 16.8\left(1 \mathrm{C}, \mathrm{CH}_{3}\right)$. HRMS (ESI) Calcd for $\left[\mathrm{C}_{15} \mathrm{H}_{19} \mathrm{ClO}_{3} \mathrm{Na}, \mathrm{M}+\mathrm{Na}\right]^{+}:$305.0915, Found: 305.0910 . 


\section{(E)-tert-Butyl 4-(3,4-dimethylphenyl)-2-hydroxypent-3-enoate (7i)}<smiles>C/C(=C\C(O)C(=O)OCC(C)(C)C)c1ccc(C)c(C)c1</smiles>

Serial number: zt-4-119. White solid, mp: $80 \sim 82^{\circ} \mathrm{C}$. TLC

$R_{f}=0.17(\mathrm{PE} / \mathrm{EA}=10: 1 \mathrm{v} / \mathrm{v}), 94 \%$ yield, 93\% ee $[\mathrm{HPLC}$ condition: Chiralcel OD-H column, $n$-hexane/2-propanol $=90: 10$, flow rate $=1.0 \mathrm{~mL} / \mathrm{min}$, wavelength $=254 \mathrm{~nm}, t_{\mathrm{R}}=5.49 \mathrm{~min}$ for major isomer, $t_{\mathrm{R}}=7.10 \mathrm{~min}$ for minor isomer $],[\alpha]_{\mathrm{D}}{ }^{28}=-106.2\left(c 1.0, \mathrm{CHCl}_{3}\right) .{ }^{1} \mathrm{H} \mathrm{NMR}(400 \mathrm{MHz}$, $\left.\mathrm{CDCl}_{3}\right) \delta 7.19(\mathrm{~s}, 1 \mathrm{H}, \mathrm{Ar}-\mathrm{H}), 7.17-7.07(\mathrm{~m}, 2 \mathrm{H}, \mathrm{Ar}-\mathrm{H}), 5.63(\mathrm{dd}, J=8.5,1.4 \mathrm{~Hz}, 1 \mathrm{H}$, $\mathrm{CH}=), 4.90(\mathrm{dd}, J=8.5,5.7 \mathrm{~Hz}, 1 \mathrm{H}, \mathrm{C} \underline{\mathrm{H}}(\mathrm{OH})), 3.10(\mathrm{~d}, J=5.8 \mathrm{~Hz}, 1 \mathrm{H}, \mathrm{OH}), 2.27$ (d, $\left.J=5.5 \mathrm{~Hz}, 3 \mathrm{H}, \mathrm{Ar}-\mathrm{CH}_{3}, 3 \mathrm{H}, \mathrm{Ar}-\mathrm{CH}_{3}\right), 2.16\left(\mathrm{~d}, J=1.4 \mathrm{~Hz}, 3 \mathrm{H}, \mathrm{CH}_{3}\right), 1.48(\mathrm{~s}, 9 \mathrm{H}$, $\left.\mathrm{C}\left(\mathrm{CH}_{3}\right)_{3}\right) .{ }^{13} \mathrm{C} \mathrm{NMR}\left(100 \mathrm{MHz}, \mathrm{CDCl}_{3}\right) \delta 173.3(1 \mathrm{C}, \mathrm{C}=\mathrm{O}), 140.2(1 \mathrm{C}, \mathrm{Ar}-\mathrm{C}), 136.3$ (1C, Ar-C), 136.0 (1C, C=), 129.5 (1C, Ar-C), 127.2 (1C, Ar-C), 124.2 (1C, Ar-C), $123.3(1 \mathrm{C}, \mathrm{CH}=), 82.7(1 \mathrm{C}, \mathrm{C}-\mathrm{O}), 68.9(1 \mathrm{C}, \mathrm{CH}(\mathrm{OH})), 28.0\left(3 \mathrm{C}, \mathrm{C}\left(\underline{\mathrm{CH}}_{3}\right)_{3}\right), 19.8(1 \mathrm{C}$, Ar- $\left.\mathrm{CH}_{3}\right), 19.4\left(1 \mathrm{C}, \mathrm{Ar}-\mathrm{CH}_{3}\right), 16.8\left(1 \mathrm{C}, \mathrm{CH}_{3}\right)$. HRMS (ESI) Calcd for $\left[\mathrm{C}_{17} \mathrm{H}_{24} \mathrm{O}_{3} \mathrm{Na}, \mathrm{M}\right.$ $+\mathrm{Na}]^{+}: 299.1618$, Found: 299.1618

\section{(E)-tert-Butyl 4-(benzo[d][1,3]dioxol-5-yl)-2-hydroxypent-3-enoate (7j)}<smiles>CCCCC(O)C(O)C=C(C)c1ccc2c(c1)OCO2</smiles>

Serial number: zt-3-185, White solid, mp: $77 \sim 78^{\circ} \mathrm{C}$. TLC $R_{f}=0.35(\mathrm{PE} / \mathrm{EA}=5: 1 \mathrm{v} / \mathrm{v}), 93 \%$ yield, $91 \%$ ee $[\mathrm{HPLC}$ condition: Chiralpak AS-H column, $n$-hexane/2-propanol $=90: 10$, flow rate $=1.0 \mathrm{~mL} / \mathrm{min}$, wavelength $=254 \mathrm{~nm}, t_{\mathrm{R}}=15.90$ min for major isomer, $t_{\mathrm{R}}=17.95 \mathrm{~min}$ for minor isomer $],[\alpha]_{\mathrm{D}}{ }^{27}=-98.4\left(c 1.0, \mathrm{CHCl}_{3}\right) .{ }^{1} \mathrm{H} \mathrm{NMR}(400 \mathrm{MHz}$, $\left.\mathrm{CDCl}_{3}\right) \delta 6.92-6.85(\mathrm{~m}, 2 \mathrm{H}, \mathrm{Ar}-\mathrm{H}), 6.76(\mathrm{~d}, J=8.0 \mathrm{~Hz}, 1 \mathrm{H}, \mathrm{Ar}-\mathrm{H}), 5.95\left(\mathrm{~s}, 2 \mathrm{H}, \mathrm{CH}_{2}\right)$, $5.56(\mathrm{dd}, J=8.5,1.2 \mathrm{~Hz}, 1 \mathrm{H}, \mathrm{CH}=), 4.87(\mathrm{dd}, J=8.4,5.8 \mathrm{~Hz}, 1 \mathrm{H}, \mathrm{C} \underline{\mathrm{H}}(\mathrm{OH})), 3.13(\mathrm{~d}$, $J=5.8 \mathrm{~Hz}, 1 \mathrm{H}, \mathrm{OH}), 2.13\left(\mathrm{~d}, J=1.0 \mathrm{~Hz}, 3 \mathrm{H}, \mathrm{CH}_{3}\right), 1.47\left(\mathrm{~s}, 9 \mathrm{H}, \mathrm{C}\left(\mathrm{CH}_{3}\right)_{3}\right) .{ }^{13} \mathrm{C} \mathrm{NMR}$ $\left(100 \mathrm{MHz}, \mathrm{CDCl}_{3}\right) \delta 173.2(1 \mathrm{C}, \mathrm{C}=\mathrm{O}), 147.6$ (1C, Ar-C), 147.0 (1C, Ar-C), $139.8(1 \mathrm{C}$, Ar-C), 137.0 (1C, C=), 124.1 (1C, Ar-C), 119.5 (1C, Ar-C), 107.9 (1C, Ar-C), 106.5 $(1 \mathrm{C}, \mathrm{CH}=), 101.0\left(1 \mathrm{C}, \mathrm{CH}_{2}\right), 82.7(1 \mathrm{C}, \mathrm{C}-\mathrm{O}), 68.8(1 \mathrm{C}, \mathrm{CH}(\mathrm{OH})), 28.0\left(3 \mathrm{C}, \mathrm{C}\left(\underline{\mathrm{CH}}_{3}\right)_{3}\right)$, $17.0\left(1 \mathrm{C}, \mathrm{CH}_{3}\right)$. HRMS (ESI) Calcd for $\left[\mathrm{C}_{16} \mathrm{H}_{20} \mathrm{O}_{5} \mathrm{Na}, \mathrm{M}+\mathrm{Na}\right]^{+}: 315.1203$, Found: 315.1206. 


\section{(E)-tert-Butyl 2-hydroxy-4-(naphthalen-2-yl)pent-3-enoate (7k)}

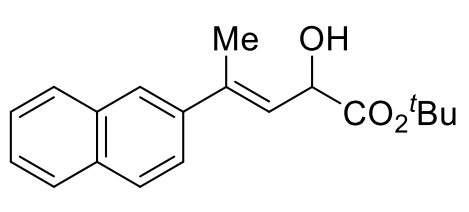

Serial number: zt-3-169. White solid, mp: $156 \sim 158^{\circ} \mathrm{C}$. TLC $R_{f}=0.38(\mathrm{PE} / \mathrm{EA}=5: 1 \mathrm{v} / \mathrm{v}), 94 \%$ yield, $94 \%$ ee [HPLC condition: Chiralcel OD-H column, $n$-hexane/2propanol $=90: 10$, flow rate $=1.0 \mathrm{~mL} / \mathrm{min}$, wavelength $=254 \mathrm{~nm}, t_{\mathrm{R}}=6.03 \mathrm{~min}$ for major isomer, $t_{\mathrm{R}}=7.09 \mathrm{~min}$ for minor isomer $],[\alpha]_{\mathrm{D}}{ }^{27}=-98.4\left(c 1.0, \mathrm{CHCl}_{3}\right) .{ }^{1} \mathrm{H} \mathrm{NMR}$ $\left(400 \mathrm{MHz}, \mathrm{CDCl}_{3}\right) \delta 7.87-7.78(\mathrm{~m}, 4 \mathrm{H}, \mathrm{Ar}-\mathrm{H}), 7.58(\mathrm{dd}, J=8.6,1.3 \mathrm{~Hz}, 1 \mathrm{H}, \mathrm{Ar}-\mathrm{H})$, $7.51-7.43(\mathrm{~m}, 2 \mathrm{H}, \mathrm{Ar}-\mathrm{H}), 5.83(\mathrm{~d}, J=8.4 \mathrm{~Hz}, 1 \mathrm{H}, \mathrm{CH}=), 4.98(\mathrm{dd}, J=8.3,5.7 \mathrm{~Hz}, 1 \mathrm{H}$, $\mathrm{C} \underline{\mathrm{H}}(\mathrm{OH})), 3.19(\mathrm{~d}, J=5.7 \mathrm{~Hz}, 1 \mathrm{H}, \mathrm{OH}), 2.30\left(\mathrm{~s}, 3 \mathrm{H}, \mathrm{CH}_{3}\right), 1.50\left(\mathrm{~s}, 9 \mathrm{H}, \mathrm{C}\left(\mathrm{CH}_{3}\right)_{3}\right) .{ }^{13} \mathrm{C}$ NMR (100 MHz, $\left.\mathrm{CDCl}_{3}\right) \delta 173.2$ (1C, C=O), 140.1 (1C, Ar-C), 139.7 (1C, Ar-C), 133.3 (1C, Ar-C), 132.8 (1C, C=), 128.1 (1C, Ar-C), 127.8 (1C, Ar-C), 127.5 (1C, Ar-C), 126.2 (1C, Ar-C), 125.9 (1C, Ar-C), 125.6 (1C, Ar-C), 124.7 (1C, Ar-C), 124.2 (1C, $\mathrm{CH}=), 82.9(1 \mathrm{C}, \mathrm{C}-\mathrm{O}), 68.9(1 \mathrm{C}, \mathrm{CH}(\mathrm{OH})), 28.0\left(3 \mathrm{C}, \mathrm{C}\left(\mathrm{C}_{3}\right)_{3}\right), 16.9\left(1 \mathrm{C}, \mathrm{CH}_{3}\right) . \mathrm{HRMS}$ (ESI) Calcd for $\left[\mathrm{C}_{19} \mathrm{H}_{22} \mathrm{O}_{3} \mathrm{Na}, \mathrm{M}+\mathrm{Na}\right]^{+}: 321.1461$, Found: 321.1462.

\section{(E)-tert-Butyl 2-hydroxy-4-(thiophen-2-yl)pent-3-enoate (7l)}

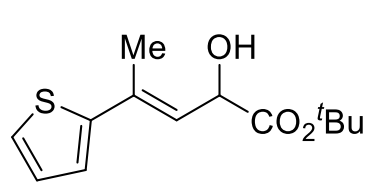

Serial number: zt-4-8. White solid, mp: $97 \sim 99{ }^{\circ} \mathrm{C}$. TLC $R_{f}=$ $0.45(\mathrm{PE} / \mathrm{EA}=5: 1 \mathrm{v} / \mathrm{v}), 93 \%$ yield, 91\% ee [HPLC condition: Chiralcel OD-H column, $n$-hexane/2-propanol $=90: 10$, flow rate $=1.0 \mathrm{~mL} / \mathrm{min}$, wavelength $=254 \mathrm{~nm}, t_{\mathrm{R}}=5.42 \mathrm{~min}$ for minor isomer, $t_{\mathrm{R}}=6.76$ $\min$ for major isomer], $[\alpha]_{\mathrm{D}}{ }^{27}=-79.6\left(c 1.0, \mathrm{CHCl}_{3}\right) .{ }^{1} \mathrm{H}$ NMR $\left(400 \mathrm{MHz}, \mathrm{CDCl}_{3}\right) \delta$ 7.18 (d, $J=4.8 \mathrm{~Hz}, 1 \mathrm{H}, \mathrm{Ar}-\mathrm{H}), 7.07$ (d, $J=2.7 \mathrm{~Hz}, 1 \mathrm{H}, \mathrm{Ar}-\mathrm{H}), 6.99$ (d, $J=4.3 \mathrm{~Hz}, 1 \mathrm{H}$, Ar-H), $5.80(\mathrm{~d}, J=8.2 \mathrm{~Hz}, 1 \mathrm{H}, \mathrm{CH}=), 4.93-4.78(\mathrm{~m}, 1 \mathrm{H}, \mathrm{C} \underline{\mathrm{H}}(\mathrm{OH})), 3.12(\mathrm{~d}, J=5.3$ $\mathrm{Hz}, 1 \mathrm{H}, \mathrm{OH}), 2.19\left(\mathrm{~s}, 3 \mathrm{H}, \mathrm{CH}_{3}\right), 1.48\left(\mathrm{~s}, 9 \mathrm{H}, \mathrm{C}\left(\mathrm{CH}_{3}\right)_{3}\right) .{ }^{13} \mathrm{C} \mathrm{NMR}\left(100 \mathrm{MHz}, \mathrm{CDCl}_{3}\right) \delta$ $173.0(1 \mathrm{C}, \mathrm{C}=\mathrm{O}), 146.3$ (1C, Ar-C), 133.8 (1C, C=), 127.3 (1C, Ar-C), 124.3 (1C, ArC), 123.6 (1C, Ar-C), $123.3(1 \mathrm{C}, \mathrm{CH}=), 83.0$ (1C, C-O), $68.6(1 \mathrm{C}, \mathrm{CH}(\mathrm{OH})), 28.0$ (3C, $\left.\mathrm{C}\left(\underline{\mathrm{CH}}_{3}\right)_{3}\right), 16.7\left(1 \mathrm{C}, \mathrm{CH}_{3}\right)$. HRMS (ESI) Calcd for $\left[\mathrm{C}_{13} \mathrm{H}_{18} \mathrm{SO}_{3} \mathrm{Na}, \mathrm{M}+\mathrm{Na}\right]^{+}:$277.0869, Found: 277.0870. 


\section{(E)-tert-Butyl 2-hydroxy-4,4-diphenylbut-3-enoate (7 m)}

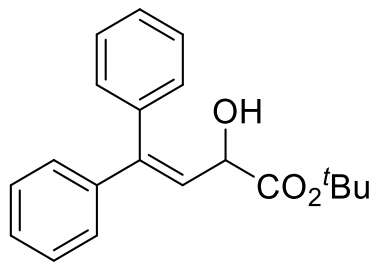

Serial number: 1y-5-61. White solid, $167 \sim 168^{\circ} \mathrm{C}$. TLC $R_{f}=$ $0.17(\mathrm{PE} / \mathrm{EA}=10: 1 \mathrm{v} / \mathrm{v}), 78 \%$ yield, $91 \%$ ee $[\mathrm{HPLC}$ condition: Chiralpak AS-H column, $n$-hexane/2-propanol = 90:10, flow rate $=1.0 \mathrm{~mL} / \mathrm{min}$, wavelength $=254 \mathrm{~nm}, t_{\mathrm{R}}=5.73 \mathrm{~min}$ for minor isomer, $t_{\mathrm{R}}=10.94 \mathrm{~min}$ for major isomer $],[\alpha]_{\mathrm{D}}{ }^{25}=84.3\left(c 1.0, \mathrm{CHCl}_{3}\right) .{ }^{1} \mathrm{H} \mathrm{NMR}$ $\left(400 \mathrm{MHz}, \mathrm{CDCl}_{3}\right) \delta 7.47-7.21(\mathrm{~m}, 10 \mathrm{H}, \mathrm{Ar}-\mathrm{H}), 5.94(\mathrm{~d}, J=9.8 \mathrm{~Hz}, 1 \mathrm{H}, \mathrm{CH}=), 4.58$ $(\mathrm{d}, J=9.8 \mathrm{~Hz}, 1 \mathrm{H}, \mathrm{CH}), 3.16(\mathrm{~s}, 1 \mathrm{H}, \mathrm{O}-\mathrm{H}), 1.50\left(\mathrm{~s}, 9 \mathrm{H}, \mathrm{C}\left(\mathrm{CH}_{3}\right)_{3}\right) .{ }^{13} \mathrm{C} \mathrm{NMR}(100 \mathrm{MHz}$, $\left.\mathrm{CDCl}_{3}\right) \delta 173.4(1 \mathrm{C}, \mathrm{C}=\mathrm{O}), 146.8(1 \mathrm{C}, \mathrm{Ar}-\mathrm{C}), 141.6(1 \mathrm{C}, \mathrm{Ar}-\mathrm{C}), 138.7(1 \mathrm{C}, \mathrm{C}=), 130.2$ (2C, Ar-C), 128.2 (2C, Ar-C), 128.2 (2C, Ar-C), 128.0 (1C, Ar-C), 127.8 (2C, Ar-C), 127.7 (1C, Ar-C), 124.8 (1C, Ar-C), 83.1 (1C, $\mathrm{CH}(\mathrm{OH})), 69.2$ (1C, C-O), 28.0 (3C, $\left.\mathrm{C}\left(\mathrm{CH}_{3}\right)_{3}\right)$. HRMS (ESI) Calcd for $\left[\mathrm{C}_{20} \mathrm{H}_{22} \mathrm{O}_{3} \mathrm{Na}, \mathrm{M}+\mathrm{Na}\right]^{+}: 333.1461$, Found: 333.1461 .

\section{(E)-tert-Butyl 2-hydroxy-4-phenylbut-3-enoate (7n)}

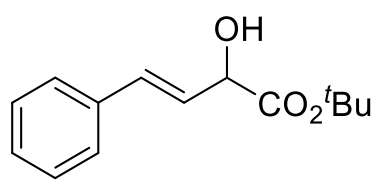

Serial number: 1y-5-63. Colorless oil. TLC $R_{f}=0.27(\mathrm{PE} / \mathrm{EA}=$

10:1 v/v), 82\% yield, 90\% ee [HPLC condition: Chiralpak AD$\mathrm{H}$ column, $n$-hexane/2-propanol $=90: 10$, flow rate $=1.0$ $\mathrm{mL} / \mathrm{min}$, wavelength $=210 \mathrm{~nm}, t_{\mathrm{R}}=3.81 \mathrm{~min}$ for major isomer, $t_{\mathrm{R}}=4.57 \mathrm{~min}$ for minor isomer], $[\alpha]_{\mathrm{D}}{ }^{25}=84.9\left(c 1.0, \mathrm{CHCl}_{3}\right) .{ }^{1} \mathrm{H} \mathrm{NMR}\left(400 \mathrm{MHz}, \mathrm{CDCl}_{3}\right) \delta 7.40(\mathrm{~d}, J=8.3$, 2H, Ar-H), 7.33 (t, $J=7.4$ Hz, 2H, Ar-H), 7.27 (d, $J=1.5 \mathrm{~Hz}, 1 \mathrm{H}, \mathrm{Ar}-\mathrm{H}), 6.80$ (dd, $J=$ $15.7,1.8 \mathrm{~Hz}, 1 \mathrm{H}, \mathrm{CH}=), 6.23(\mathrm{dd}, J=15.9,5.3 \mathrm{~Hz}, 1 \mathrm{H}, \mathrm{CH}=), 4.70(\mathrm{dd}, J=5.3,1.8 \mathrm{~Hz}$, $1 \mathrm{H}, \mathrm{CH}), 3.12(\mathrm{~s}, 1 \mathrm{H}, \mathrm{OH}), 1.51\left(\mathrm{~s}, 9 \mathrm{H}, \mathrm{C}\left(\mathrm{CH}_{3}\right)_{3}\right) .{ }^{13} \mathrm{C} \mathrm{NMR}\left(100 \mathrm{MHz}, \mathrm{CDCl}_{3}\right) \delta 172.5$ $(1 \mathrm{C}, \mathrm{C}=\mathrm{O}), 136.4$ (1C, Ar-C), 131.5 (1C, Ar-C), 128.5 (2C, Ar-C), 127.8 (1C, $\mathrm{CH}=)$, $126.6(2 \mathrm{C}, \mathrm{Ar}-\mathrm{C}), 126.0(1 \mathrm{C}, \mathrm{CH}=), 83.2(1 \mathrm{C}, \mathrm{C}-\mathrm{O}), 71.4(1 \mathrm{C}, \mathrm{CH}(\mathrm{OH})), 28.0$ (3C, $\left.\mathrm{C}\left(\underline{\mathrm{CH}}_{3}\right)_{3}\right)$. HRMS (ESI) Calcd for $\left[\mathrm{C}_{14} \mathrm{H}_{18} \mathrm{O}_{3} \mathrm{Na}, \mathrm{M}+\mathrm{Na}\right]^{+}:$257.1148, Found: 257.1144 .

\section{Benzhydryl (E)-2-hydroxypent-3-enoate (7o)}

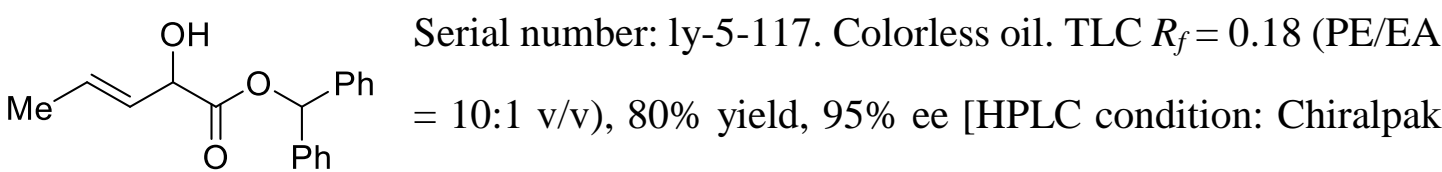


AD-H column, $n$-hexane/2-propanol $=90: 10$, flow rate $=1.0 \mathrm{~mL} / \mathrm{min}$, wavelength $=$ $210 \mathrm{~nm}, t_{\mathrm{R}}=8.35 \mathrm{~min}$ for minor isomer, $t_{\mathrm{R}}=10.61 \mathrm{~min}$ for major isomer $],[\alpha]_{\mathrm{D}}{ }^{25}=$ 54.6 (c 1.0, $\left.\mathrm{CHCl}_{3}\right) .{ }^{1} \mathrm{H}$ NMR (400 MHz, $\left.\mathrm{CDCl}_{3}\right) \delta 7.50-7.10(\mathrm{~m}, 10 \mathrm{H}, \mathrm{Ar}-\mathrm{H}), 6.92$ $\left(\mathrm{s}, 1 \mathrm{H}, \mathrm{C} \underline{\mathrm{H}}(\mathrm{Ph})_{2}\right), 5.86(\mathrm{dqd}, J=13.1,6.5,1.2 \mathrm{~Hz}, 1 \mathrm{H}, \mathrm{CH}=), 5.65-5.44(\mathrm{~m}, 1 \mathrm{H}, \mathrm{CH}=)$, $4.67(\mathrm{t}, J=6.0 \mathrm{~Hz}, 1 \mathrm{H}, \mathrm{C} \underline{\mathrm{H}}(\mathrm{OH})), 3.30(\mathrm{~d}, J=6.1 \mathrm{~Hz}, 1 \mathrm{H}, \mathrm{OH}), 1.66(\mathrm{~d}, J=6.6 \mathrm{~Hz}$, $\left.3 \mathrm{H}, \mathrm{CH}_{3}\right) .{ }^{13} \mathrm{C} \mathrm{NMR}\left(100 \mathrm{MHz}, \mathrm{CDCl}_{3}\right) \delta 173.0(1 \mathrm{C}, \mathrm{C}=\mathrm{O}), 139.7(1 \mathrm{C}, \mathrm{Ar}-\mathrm{H}), 139,7$ (1C, Ar-C), 130.0 (1C, CH=), 128.7 (2C, Ar-C), 128.6 (2C, Ar-C), 128.4 (1C, Ar-C), 128.2 (1C, Ar-C), 127.4 (2C, Ar-C), 127.4 (1C, CH=), 127.0 (2C, Ar-C), 78.3 (1C, CO), $71.7(1 \mathrm{C}, \mathrm{CH}(\mathrm{OH})), 17.8\left(1 \mathrm{C}, \mathrm{CH}_{3}\right)$. HRMS (ESI) Calcd for $\left[\mathrm{C}_{18} \mathrm{H}_{18} \mathrm{O}_{3} \mathrm{Na}, \mathrm{M}+\right.$ $\mathrm{Na}^{+}:$305.1148, Found: 305.1151.

\section{Benzhydryl (E)-2-hydroxyhex-3-enoate (7p)}<smiles>CCC=CC(O)C(=O)OC(c1ccccc1)c1ccccc1</smiles>

Serial number: 1y-5-138. Colorless oil. TLC $R_{f}=0.24$ $(\mathrm{PE} / \mathrm{EA}=10: 1 \mathrm{v} / \mathrm{v}), 84 \%$ yield, 89\% ee [HPLC condition: Chiralpak AD-H column, $n$-hexane/2-propanol = 90:10, flow rate $=1.0 \mathrm{~mL} / \mathrm{min}$, wavelength $=210 \mathrm{~nm}, t_{\mathrm{R}}=10.16 \mathrm{~min}$ for monor isomer, $t_{\mathrm{R}}$ $=10.77 \mathrm{~min}$ for major isomer], $[\alpha]_{\mathrm{D}}{ }^{25}=77.2\left(c 1.0, \mathrm{CHCl}_{3}\right) .{ }^{1} \mathrm{H} \mathrm{NMR}\left(400 \mathrm{MHz}, \mathrm{CDCl}_{3}\right)$ $\delta 7.36-7.25$ (m, 10H, Ar-H), 6.93 (s, 1H, $\left.\mathrm{C} \underline{\mathrm{H}}(\mathrm{Ph})_{2}\right), 5.96$ (dtd, $J=15.2,6.3,1.2 \mathrm{~Hz}$, $1 \mathrm{H}, \mathrm{CH}=), 5.54$ (ddt, $J=15.4,6.3,1.5 \mathrm{~Hz}, 1 \mathrm{H}, \mathrm{CH}=), 4.71(\mathrm{t}, J=5.8 \mathrm{~Hz}, 1 \mathrm{H}, \mathrm{C} \underline{\mathrm{H}}(\mathrm{OH})$ ), $2.91(\mathrm{~d}, J=6.1 \mathrm{~Hz}, 1 \mathrm{H}, \mathrm{OH}), 2.17-1.93\left(\mathrm{~m}, 2 \mathrm{H}, \mathrm{CH}_{2}\right), 1.00\left(\mathrm{t}, J=7.5 \mathrm{~Hz}, 3 \mathrm{H}, \mathrm{CH}_{3}\right)$. ${ }^{13} \mathrm{C}$ NMR (100 MHz, $\left.\mathrm{CDCl}_{3}\right) \delta 173.0(1 \mathrm{C}, \mathrm{C}=\mathrm{O}), 139.5$ (1C, Ar-C), 139.4 (1C, Ar-C), $136.8(1 \mathrm{C}, \mathrm{CH}=), 128.6$ (2C, Ar-C), 128.5 (2C, Ar-C), 128.3 (1C, Ar-C), $128.11 \mathrm{C}, \mathrm{Ar}-$ C), 127.3 (2C, Ar-C), 126.8 (2C, Ar-C), 125.1 (1C, $\mathrm{CH}=), 78.3$ (1C, C-O), 71.6 (1C, $\mathrm{CH}(\mathrm{OH})), 25.2\left(1 \mathrm{C}, \mathrm{CH}_{2}\right), 13.2\left(1 \mathrm{C}, \mathrm{CH}_{3}\right)$. HRMS (ESI) Calcd for $\left[\mathrm{C}_{19} \mathrm{H}_{20} \mathrm{O}_{3} \mathrm{Na}, \mathrm{M}+\right.$ $\mathrm{Na}]^{+}: 319.1305$, Found: 319.1309 .

\section{Benzhydryl (E)-2-hydroxyhept-3-enoate (7q)}

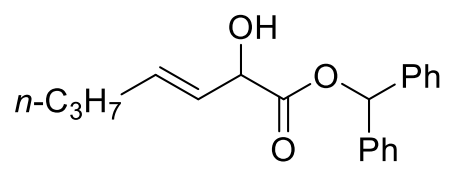

Serial number: ly-5-139. Colorless oil. TLC $R_{f}=0.24$ $(\mathrm{PE} / \mathrm{EA}=10: 1 \mathrm{v} / \mathrm{v}), 82 \%$ yield, $90 \%$ ee $[$ HPLC condition: Chiralpak AD-H column, $n$-hexane/2-propanol = 90:10, 
flow rate $=0.5 \mathrm{~mL} / \mathrm{min}$, wavelength $=210 \mathrm{~nm}, t_{\mathrm{R}}=33.0 \mathrm{~min}$ for monor isomer, $t_{\mathrm{R}}$ $=35.3 \mathrm{~min}$ for major isomer $],[\alpha]_{\mathrm{D}}{ }^{25}=79.4\left(c 1.0, \mathrm{CHCl}_{3}\right) .{ }^{1} \mathrm{H} \mathrm{NMR}\left(400 \mathrm{MHz}, \mathrm{CDCl}_{3}\right)$ $\delta 7.33-7.13(\mathrm{~m}, 10 \mathrm{H}, \mathrm{Ar}-\mathrm{H}), 6.89\left(\mathrm{~s}, 1 \mathrm{H}, \mathrm{C} \underline{\mathrm{H}}(\mathrm{Ph})_{2}\right), 5.84(\mathrm{dtd}, J=15.3,6.9,1.4 \mathrm{~Hz}$, $1 \mathrm{H}, \mathrm{CH}=), 5.50(\mathrm{ddt}, J=15.3,6.2,1.5 \mathrm{~Hz}, 1 \mathrm{H}, \mathrm{CH}=), 4.72-4.61(\mathrm{~m}, 1 \mathrm{H}, \mathrm{C} \underline{\mathrm{H}}(\mathrm{OH}))$, $3.14(\mathrm{~d}, J=6.1 \mathrm{~Hz}, 1 \mathrm{H}, \mathrm{OH}), 2.07-1.92\left(\mathrm{~m}, 2 \mathrm{H}, \mathrm{CH}_{2}\right), 1.39-1.29\left(\mathrm{~m}, 2 \mathrm{H}, \mathrm{CH}_{2}\right), 0.84$ $-0.81\left(\mathrm{~m}, 3 \mathrm{H}, \mathrm{CH}_{3}\right) .{ }^{13} \mathrm{C} \mathrm{NMR}\left(100 \mathrm{MHz}, \mathrm{CDCl}_{3}\right) \delta 173.0(1 \mathrm{C}, \mathrm{C}=\mathrm{O}), 139.6(1 \mathrm{C}, \mathrm{Ar}-$ C), 139.6 (1C, Ar-C), 135.0 (1C, CH=), 128.7 (2C, Ar-C), 128.6 (2C, Ar-C), 128.3 (1C, Ar-C), 128.1 (1C, Ar-C), 127.4 (2C, Ar-C), 126.9 (1C, Ar-C), 126.3 (1C, CH=), 78.3 (1C, C-O), $71.7(1 \mathrm{C}, \mathrm{CH}(\mathrm{OH})), 34.3\left(1 \mathrm{C}, \mathrm{CH}_{2}\right), 22.1$ (1C, $\left.\mathrm{CH}_{2}\right), 13.7$ (1C, $\left.\mathrm{CH}_{3}\right) . \mathrm{HRMS}$ (ESI) Calcd for $\left[\mathrm{C}_{20} \mathrm{H}_{22} \mathrm{O}_{3} \mathrm{Na}, \mathrm{M}+\mathrm{Na}\right]^{+}$: 333.1461, Found: 333.1464.

\section{Benzhydryl (E)-2-hydroxydec-3-enoate (7r)}

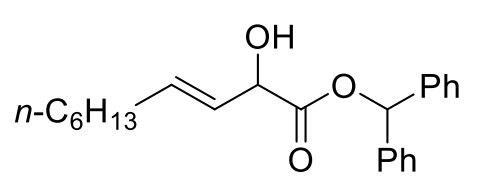

Serial number: ly-4-190. Colorless oil. TLC $R_{f}=0.21$

$(\mathrm{PE} / \mathrm{EA}=10: 1 \mathrm{v} / \mathrm{v}), 84 \%$ yield, $91 \%$ ee $[$ HPLC condition:

Chiralpak AD-H column, $n$-hexane/2-propanol = 90:10, flow rate $=1.0 \mathrm{~mL} / \mathrm{min}$, wavelength $=210 \mathrm{~nm}, t_{\mathrm{R}}=6.20 \mathrm{~min}$ for major isomer, $t_{\mathrm{R}}=$ $7.57 \mathrm{~min}$ for minor isomer], $[\alpha]_{\mathrm{D}}{ }^{25}=68.8\left(c 1.0, \mathrm{CHCl}_{3}\right) .{ }^{1} \mathrm{H} \mathrm{NMR}\left(400 \mathrm{MHz}, \mathrm{CDCl}_{3}\right)$ $\delta 7.28-7.20(\mathrm{~m}, 10 \mathrm{H}, \mathrm{Ar}-\mathrm{H}), 6.88\left(\mathrm{~s}, 1 \mathrm{H}, \mathrm{C} \underline{\mathrm{H}}(\mathrm{Ph})_{2}\right), 5.97-5.75(\mathrm{~m}, 1 \mathrm{H}, \mathrm{CH}=), 5.49$ $(\mathrm{dd}, J=15.4,6.3 \mathrm{~Hz}, 1 \mathrm{H}, \mathrm{CH}=), 4.64(\mathrm{t}, J=6.0 \mathrm{~Hz}, 1 \mathrm{H}, \mathrm{C} \underline{\mathrm{H}}(\mathrm{OH})), 3.05(\mathrm{~d}, J=6.1 \mathrm{~Hz}$, $1 \mathrm{H}, \mathrm{OH}), 2.00\left(\mathrm{q}, J=7.0 \mathrm{~Hz}, 2 \mathrm{H}, \mathrm{CH}_{2}\right), 1.35-1.26\left(\mathrm{~m}, 2 \mathrm{H}, \mathrm{CH}_{2}\right), 1.25-1.17(\mathrm{~m}, 6 \mathrm{H}$, $\left.\mathrm{CH}_{2}\right), 0.83\left(\mathrm{t}, J=6.5 \mathrm{~Hz}, 3 \mathrm{H}, \mathrm{CH}_{3}\right) .{ }^{13} \mathrm{C} \mathrm{NMR}\left(100 \mathrm{MHz}, \mathrm{CDCl}_{3}\right) \delta 173.0(1 \mathrm{C}, \mathrm{C}=\mathrm{O})$, 139.6 (1C, Ar-C ), 139.6 (1C, Ar-C), 135.4 (1C, CH=), 128.7 (2C, Ar-C), 128.6 (2C, Ar-C), 128.3 (1C, Ar-C), 128.1 (1C, Ar-C), 127.4 (2C, Ar-C), 126.9 (2C, Ar-C), 126.1 $(1 \mathrm{C}, \mathrm{CH}=), 78.3(1 \mathrm{C}, \mathrm{C}-\mathrm{O}), 71.7(1 \mathrm{C}, \mathrm{CH}(\mathrm{OH})), 32.3\left(1 \mathrm{C}, \mathrm{CH}_{2}\right), 31.8\left(1 \mathrm{C}, \mathrm{CH}_{2}\right), 29.0$ (1C, $\left.\mathrm{CH}_{2}\right), 28.9\left(1 \mathrm{C}, \mathrm{CH}_{2}\right), 22.7\left(1 \mathrm{C}, \mathrm{CH}_{2}\right), 14.2\left(1 \mathrm{C}, \mathrm{CH}_{3}\right)$. HRMS (ESI) Calcd for $\left[\mathrm{C}_{23} \mathrm{H}_{28} \mathrm{O}_{3} \mathrm{Na}, \mathrm{M}+\mathrm{Na}\right]^{+}:$375.1931, Found: 375.1935 .

\section{Benzhydryl (E)-2-hydroxy-5-phenylpent-3-enoate (7s)}<smiles>O=C(OC(c1ccccc1)c1ccccc1)C(O)C=CCc1ccccc1</smiles>

Serial number: ly-5-131. White solid, mp: $197 \sim 198{ }^{\circ} \mathrm{C}$.

TLC $R_{f}=0.21(\mathrm{PE} / \mathrm{EA}=10: 1 \mathrm{v} / \mathrm{v}), 68 \%$ yield, $90 \%$ ee 
[HPLC condition: Chiralpak AD-H column, $n$-hexane/2-propanol = 90:10, flow rate $=$ $1.0 \mathrm{~mL} / \mathrm{min}$, wavelength $=210 \mathrm{~nm}, t_{\mathrm{R}}=3.54 \mathrm{~min}$ for major isomer, $t_{\mathrm{R}}=4.16 \mathrm{~min}$ for minor isomer $],[\alpha]_{\mathrm{D}}{ }^{25}=118.7\left(c 1.0, \mathrm{CHCl}_{3}\right) .{ }^{1} \mathrm{H} \mathrm{NMR}\left(400 \mathrm{MHz}, \mathrm{CDCl}_{3}\right) \delta 7.34-7.22$ (m, 12H, Ar-H), 7.19 (m, 1H, Ar-H), 7.13 (m, 2H, Ar-H), 6.91 (s, 1H, C $\left.\underline{H}(\mathrm{Ph})_{2}\right), 6.07$ (ddd, $J=7.7,6.6,3.3 \mathrm{~Hz}, 1 \mathrm{H}, \mathrm{CH}=), 5.59(\mathrm{dd}, J=15.3,6.1 \mathrm{~Hz}, 1 \mathrm{H}, \mathrm{CH}=), 4.72$ (d, $J=$ $5.0 \mathrm{~Hz}, 1 \mathrm{H}, \mathrm{C} \underline{\mathrm{H}}(\mathrm{OH})), 3.38\left(\mathrm{~d}, J=6.5 \mathrm{~Hz}, 2 \mathrm{H}, \mathrm{CH}_{2}\right), 3.07(\mathrm{~s}, 1 \mathrm{H}, \mathrm{OH}) .{ }^{13} \mathrm{C} \mathrm{NMR}(100$ $\left.\mathrm{MHz}, \mathrm{CDCl}_{3}\right) \delta 172.8$ (1C, C=O), 139.5 (1C, Ar-C), 139.4 (1C, Ar-C ), 139.4 (1C, ArC), $133.6(1 \mathrm{C}, \mathrm{CH}=), 128.8$ (2C, Ar-C), 128.7 (2C, Ar-C), 128.6 (2C, Ar-C), 128.6 (2C, Ar-C), 128.4 (1C, Ar-C), 128.2 (1C, Ar-C), 127.4 (1C, Ar-C), 127.4 (2C, Ar-C), 126.9 (2C, Ar-C), $126.3(1 \mathrm{C}, \mathrm{CH}=), 78.6(1 \mathrm{C}, \mathrm{C}-\mathrm{O}), 71.5(1 \mathrm{C}, \mathrm{CH}(\mathrm{OH})), 38.5\left(1 \mathrm{C}, \mathrm{CH}_{2}\right)$. HRMS (ESI) Calcd for $\left[\mathrm{C}_{24} \mathrm{H}_{22} \mathrm{O}_{3} \mathrm{Na}, \mathrm{M}+\mathrm{Na}\right]^{+}: 381.1461$, Found: 381.1465 . 


\section{Gram-scale O-H Bond Insertion Reactions}

\subsection{Gram-scale $\mathrm{O}-\mathrm{H}$ bond inserion with $1 \mathrm{~b}$}

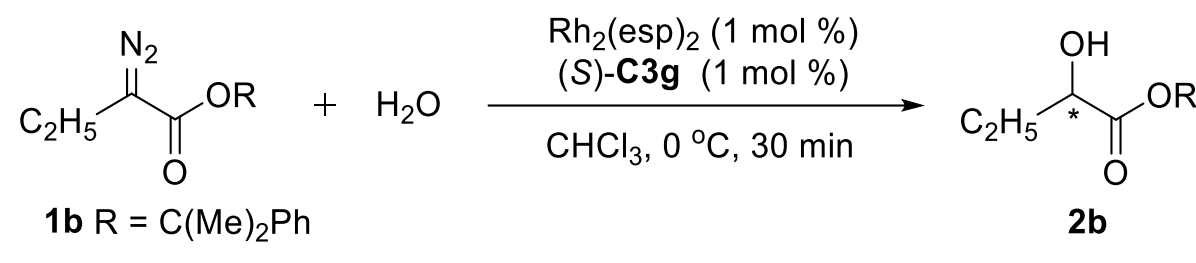

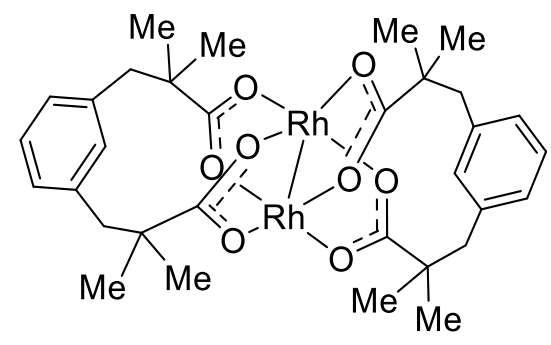

$\mathrm{Rh}_{2}(\mathrm{esp})_{2}$

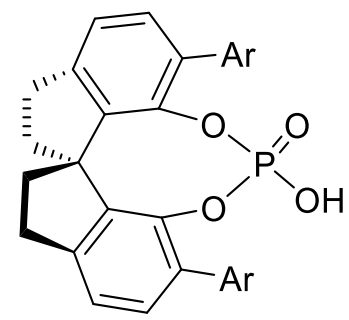

$\mathrm{Ar}=10-\mathrm{Ph}-9$-anthracenyl

$(S)-\mathbf{C} \mathbf{g}$

The $\mathrm{Rh}_{2}(\mathrm{esp})_{2}(75 \mathrm{mg}, 0.1 \mathrm{mmol}, 1 \mathrm{~mol} \%)$ and $(S)-\mathbf{C 3 g}(80 \mathrm{mg}, 0.1 \mathrm{mmol}, 1$ mol \%) were introduced into an oven-dried Schlenk tube in an argon-filled glovebox. After $50 \mathrm{~mL} \mathrm{CHCl}_{3}$ was injected into the Schlenk tube, the mixture was stirred at $0{ }^{\circ} \mathrm{C}$ for $10 \mathrm{~min}$. Then $\mathrm{H}_{2} \mathrm{O}(900 \mu \mathrm{L}, 50 \mathrm{mmol})$ was added by a micro syringe in one portion and a solution of $\mathbf{1 b}(2.32 \mathrm{~g}, 10 \mathrm{mmol})$ in $100 \mathrm{~mL} \mathrm{CHCl} 3$ was added in one portion, subsequently. The reaction finished in $30 \mathrm{~min}$ after the addition (judged by the discoloration of diazo compound and confirmed by TLC monitoring). The reaction mixture was concentrated and purified by flash chromatography on silica gel $(\mathrm{PE} / \mathrm{EA}=$ $20: 1 \mathrm{v} / \mathrm{v})$ to give $\mathbf{2 b}(1.7 \mathrm{~g}, 78 \%$ yield, $97 \%$ ee) as a colorless oil. We recovered $\mathrm{Rh}_{2}(\mathrm{esp})_{2}$ (63 $\mathrm{mg}, 84 \%$ recovery yield) and $(S)-\mathbf{C 3 g}(66 \mathrm{mg}, 83 \%$ recovery yield) through column chromatography after the reaction. 


\subsection{Gram-scale $\mathrm{O}-\mathrm{H}$ bond inserion with $6 \mathrm{a}$}<smiles>CCCCOC(=N)/C=C(\C)c1ccccc1</smiles>

$6 a$

$$
\mathrm{H}_{2} \mathrm{O} \underset{\mathrm{Rh}_{2}(\mathrm{TPA})_{4}(0.5 \mathrm{~mol} \%)}{(R)-\mathrm{C} 3 \mathrm{~d}(0.5 \mathrm{~mol} \%)} \stackrel{\stackrel{\mathrm{CHCl}_{3}, 0^{\circ} \mathrm{C}, 30 \mathrm{~min}}{\longrightarrow}}{\longrightarrow}
$$<smiles>CCCCOC(=O)C(O)C=C(C)c1ccccc1</smiles>

$7 a$
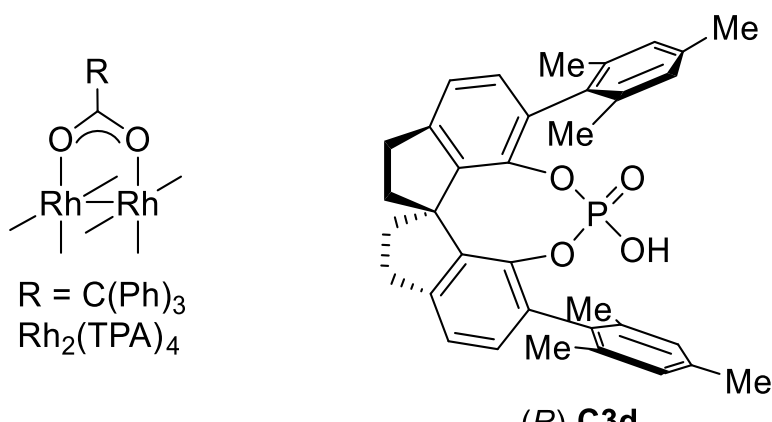

$(R)-\mathbf{C} 3 \mathbf{d}$

The $\mathrm{Rh}_{2}(\mathrm{TPA})_{4}(25.7 \mathrm{mg}, 0.019 \mathrm{mmol}, 0.5 \mathrm{~mol} \%)$ and $(R)-\mathbf{C 3 d}(11 \mathrm{mg}, 0.019$ mmol, $0.5 \mathrm{~mol} \%$ ) were introduced into an oven-dried Schlenk tube in an argon-filled glovebox. After $10 \mathrm{~mL} \mathrm{CHCl}_{3}$ was injected into the Schlenk tube, the resulted reaction mixture was stirred at $0{ }^{\circ} \mathrm{C}$ for $10 \mathrm{~min}$. Then $\mathrm{H}_{2} \mathrm{O}(333 \mu \mathrm{L}, 18.5 \mathrm{mmol})$ was added by a micro syringe in one portion and a solution of $(E)$-tert-butyl 2-diazopent-3-enoate 6a (955 $\mathrm{mg}, 3.7 \mathrm{mmol}$ ) in $20 \mathrm{~mL} \mathrm{CHCl}_{3}$ was added by a syringe in one portion, subsequently. The reaction finished in $30 \mathrm{~min}$ after the addition (judged by the discoloration of diazo compound and confirmed by TLC monitoring). Then the reaction mixture was concentrated and purified by flash chromatography on silica gel $(\mathrm{PE} / \mathrm{EA}=$ 10:1 v/v) to give $(E)$-tert-butyl 2-hydroxy-4-phenylpent-3-enoate $7 \mathbf{a}(890 \mathrm{mg}, 97 \%$ yield, $94 \%$ ee) as a white solid. 


\section{Analytical Data of Side-products}

\subsection{The $\mathrm{O}-\mathrm{H}$ bond insertion reaction of $1 \mathrm{a}$ and water (Table 1 )}

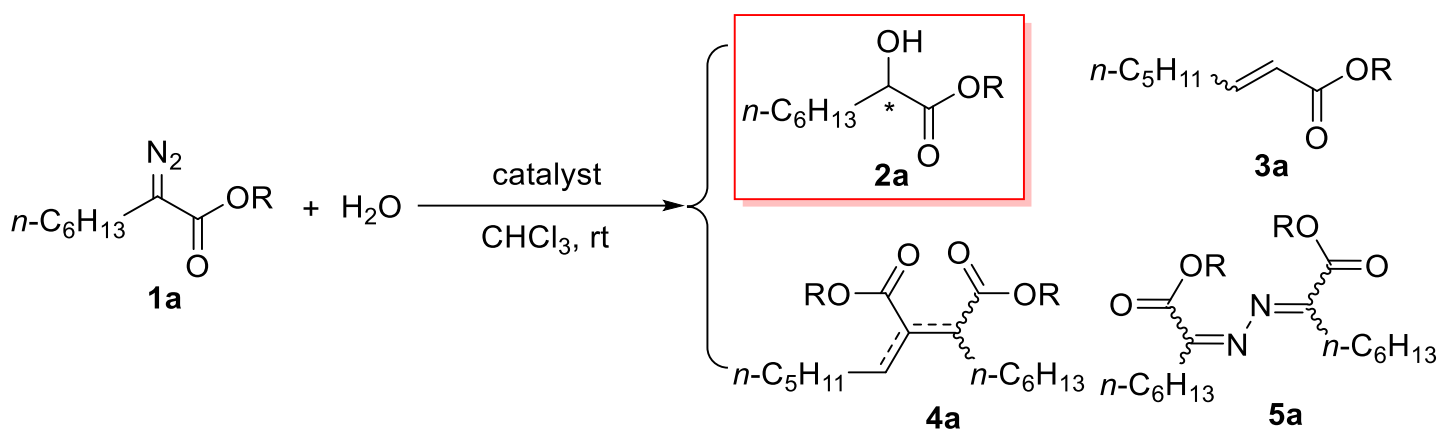

The $\mathrm{Rh}_{2}(\mathrm{OAc})_{4}(22 \mathrm{mg}, 0.05 \mathrm{mmol}, 1 \mathrm{~mol} \%)$ was introduced into an oven-dried Schlenk tube in an argon-filled glovebox. After $25 \mathrm{~mL} \mathrm{CHCl}_{3}$ was injected into the Schlenk tube, the mixture was stirred at room temperature for 5 min. Then $\mathrm{H}_{2} \mathrm{O}(450$ $\mu \mathrm{L}, 25 \mathrm{mmol}$ ) was added by a micro syringe in one portion and a solution of $1 \mathbf{a}$ (920 $\mathrm{mg}, 5 \mathrm{mmol}$ ) in $50 \mathrm{~mL} \mathrm{CHCl}_{3}$ was added in one portion, subsequently. The reaction finished in 10 min after the addition (judged by the discoloration of diazo compound and confirmed by TLC monitoring). The reaction mixture was concentrated and purified by flash chromatography on silica gel $(\mathrm{PE} / \mathrm{EA}=20: 1 \mathrm{v} / \mathrm{v})$ to give all products.

\section{Methyl (Z)-oct-2-enoat, $(Z)-(3 a)^{5}$}

$n-\mathrm{C}_{5} \mathrm{H}_{11} \mathrm{CO}_{2} \mathrm{Me}$

Colorless oil. ${ }^{1} \mathrm{H}$ NMR $\left(400 \mathrm{MHz}, \mathrm{CDCl}_{3}\right) \delta 6.24(\mathrm{dt}, J=11.5,7.5$

$\mathrm{Hz}, 1 \mathrm{H}, \mathrm{CH}=), 5.77(\mathrm{dt}, J=11.5,1.7 \mathrm{~Hz}, 1 \mathrm{H}, \mathrm{CH}=), 3.71(\mathrm{~s}, 3 \mathrm{H}$,

$\left.\mathrm{OCH}_{3}\right), 2.65\left(\mathrm{ddd}, J=15.0,7.5,1.7 \mathrm{~Hz}, 2 \mathrm{H}, \mathrm{CH}_{2}\right), 1.50-1.39\left(\mathrm{~m}, 2 \mathrm{H}, \mathrm{CH}_{2}\right), 1.37-$

$1.24\left(\mathrm{~m}, 4 \mathrm{H}, \mathrm{CH}_{2}\right), 0.93-0.84\left(\mathrm{~m}, 3 \mathrm{H}, \mathrm{CH}_{3}\right) .{ }^{13} \mathrm{C} \mathrm{NMR}\left(100 \mathrm{MHz}, \mathrm{CDCl}_{3}\right) \delta 166.9$

$(1 \mathrm{C}, \mathrm{C}=\mathrm{O}), 151.1(1 \mathrm{C}, \mathrm{CH}=), 119.1(1 \mathrm{C}, \mathrm{CH}=), 51.0(1 \mathrm{C}, \mathrm{C}-\mathrm{O}), 31.5\left(1 \mathrm{C}, \mathrm{CH}_{2}\right), 29.0$

(1C, $\left.\mathrm{CH}_{2}\right), 28.7\left(1 \mathrm{C}, \mathrm{CH}_{2}\right), 22.5\left(1 \mathrm{C}, \mathrm{CH}_{2}\right), 14.0\left(1 \mathrm{C}, \mathrm{CH}_{3}\right)$. HRMS (ESI) Calcd for

$\left[\mathrm{C}_{9} \mathrm{H}_{16} \mathrm{O}_{2} \mathrm{Na}, \mathrm{M}+\mathrm{Na}\right]^{+}:$179.1043, Found: 179.1046 . 
Dimethyl 2,3-dihexylbut-2-enedioate and (E)-2-hexyl-3-hexylidenesuccinate (4a) ${ }^{6}$

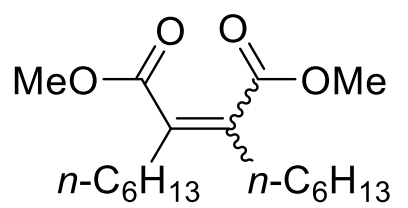<smiles></smiles>

This dimer product is easy converted to dimethyl (E)-2-hexyl-3hexylidenesuccinate in room temperature. ${ }^{6 \mathrm{~b}}$ The ratio of two compounds is nearly to 36:64. Colorless oil. ${ }^{1} \mathrm{H}$ NMR $\left(400 \mathrm{MHz}, \mathrm{CDCl}_{3}\right) \delta 6.92(\mathrm{t}, J=7.6 \mathrm{~Hz}, 1 \mathrm{H}, \mathrm{CH}=), 3.75$ (s, 3.34H, $\left.\mathrm{OCH}_{3}\right), 3.72\left(\mathrm{~s}, 3.01 \mathrm{H}, \mathrm{OCH}_{3}\right), 3.65\left(\mathrm{~s}, 3.0 \mathrm{H}, \mathrm{OCH}_{3}\right), 3.48(\mathrm{dd}, J=9.1,5.5$ $\mathrm{Hz}, 1.01 \mathrm{H}, \mathrm{CH}), 2.38-2.29\left(\mathrm{~m}, 2.21 \mathrm{H}, \mathrm{CH}_{2}\right), 2.29-2.02\left(\mathrm{~m}, 3.43 \mathrm{H}, \mathrm{CH}_{2}\right), 1.74-1.57$ $\left(\mathrm{m}, 2.03 \mathrm{H}, \mathrm{CH}_{2}\right), 1.52-1.17\left(\mathrm{~m}, 26.54 \mathrm{H}, \mathrm{CH}_{2}\right), 0.93-0.84\left(\mathrm{~m}, 10.22 \mathrm{H}, \mathrm{CH}_{3}\right) .{ }^{13} \mathrm{C}$ NMR (100 MHz, $\left.\mathrm{CDCl}_{3}\right) \delta 173.7(\mathrm{C}=\mathrm{O}), 169.3(\mathrm{C}=\mathrm{O}), 167.1(\mathrm{C}=\mathrm{O}), 145.3(\mathrm{C}=), 137.9$ $(\mathrm{C}=), 130.3(\mathrm{CH}=), 52.0\left(\mathrm{OCH}_{3}\right), 51.8\left(\mathrm{OCH}_{3}\right), 51.7\left(\mathrm{OCH}_{3}\right), 43.1(\mathrm{CH}), 31.7\left(\mathrm{CH}_{2}\right)$, $31.5\left(\mathrm{CH}_{2}\right), 31.5\left(\mathrm{CH}_{2}\right), 29.9\left(\mathrm{CH}_{2}\right), 29.5\left(\mathrm{CH}_{2}\right), 29.2\left(\mathrm{CH}_{2}\right), 29.1\left(\mathrm{CH}_{2}\right), 28.8\left(\mathrm{CH}_{2}\right)$, $28.3\left(\mathrm{CH}_{2}\right), 28.2\left(\mathrm{CH}_{2}\right), 27.6\left(\mathrm{CH}_{2}\right), 22.6\left(\mathrm{CH}_{2}\right), 22.5\left(\mathrm{CH}_{2}\right), 22.4\left(\mathrm{CH}_{2}\right), 14.0\left(\mathrm{CH}_{3}\right)$, 14.0 $\left(\mathrm{CH}_{3}\right), 13.9\left(\mathrm{CH}_{3}\right)$. HRMS (ESI) Calcd for $\left[\mathrm{C}_{18} \mathrm{H}_{32} \mathrm{O}_{4}, \mathrm{M}+\mathrm{H}\right]^{+}:$313.2373, Found: 313.2377.

\section{Dimethyl 2,2'-(hydrazine-1,2-diylidene) -dioctanoate (5a)}<smiles>COC(=O)C(=N/N=C(/PC(=O)c1ccccc1)C(=O)OC)C(=O)c1ccccc1</smiles>
The ratio of $\left(E, E^{\prime}\right) /(Z, E) /\left(Z, Z^{\prime}\right)$ diastereoisomers is nearly to 77:15:8. ${ }^{1} \mathrm{H} \mathrm{NMR}\left(400 \mathrm{MHz}, \mathrm{CDCl}_{3}\right) \delta 3.89$ (s, $\left.6 \mathrm{H}, \mathrm{OCH}_{3}\right), 3.86\left(\mathrm{~s}, 0.64 \mathrm{H}, \mathrm{OCH}_{3}\right), 3.78(\mathrm{~s}, 0.56 \mathrm{H}$, $\left.\mathrm{OCH}_{3}\right), 3.73\left(\mathrm{~s}, 0.56 \mathrm{H}, \mathrm{OCH}_{3}\right), 2.58(\mathrm{t}, J=7.5 \mathrm{~Hz}, 0.43 \mathrm{H}$,

$\left.\mathrm{CH}_{2}\right), 2.51-2.37\left(\mathrm{~m}, 4.78 \mathrm{H}, \mathrm{CH}_{2}\right), 1.80-1.57\left(\mathrm{~m}, 1.42 \mathrm{H}, \mathrm{CH}_{2}\right), 1.53-1.39(\mathrm{~m}, 5.41 \mathrm{H}$, $\left.\mathrm{CH}_{2}\right), 1.38-1.19\left(\mathrm{~m}, 17.95 \mathrm{H}, \mathrm{CH}_{2}\right), 0.87\left(\mathrm{t}, J=6.8 \mathrm{~Hz}, 8.65 \mathrm{H}, \mathrm{CH}_{3}\right) .{ }^{13} \mathrm{C} \mathrm{NMR}(100$ $\left.\mathrm{MHz}, \mathrm{CDCl}_{3}\right) \delta 164.1(2 \mathrm{C}, \mathrm{C}=\mathrm{O}), 151.7(2 \mathrm{C}, \mathrm{C}=\mathrm{N}), 52.8\left(2 \mathrm{C}, \mathrm{OCH}_{3}\right), 31.3\left(2 \mathrm{C}, \mathrm{CH}_{2}\right)$, $29.2\left(2 \mathrm{C}, \mathrm{CH}_{2}\right), 28.6\left(2 \mathrm{C}, \mathrm{CH}_{2}\right), 25.6\left(2 \mathrm{C}, \mathrm{CH}_{2}\right), 22.4\left(2 \mathrm{C}, \mathrm{CH}_{2}\right), 14.0\left(2 \mathrm{C} \mathrm{CH}_{3}\right)$. HRMS (ESI) Calcd for $\left[\mathrm{C}_{18} \mathrm{H}_{32} \mathrm{O}_{4} \mathrm{~N}_{2}, \mathrm{M}+\mathrm{H}\right]^{+}: 341.2435$, Found: 340.2440. 


\subsection{The $\mathrm{O}-\mathrm{H}$ bond insertion reaction of methyl $\alpha$-diazobutyrate and}

water

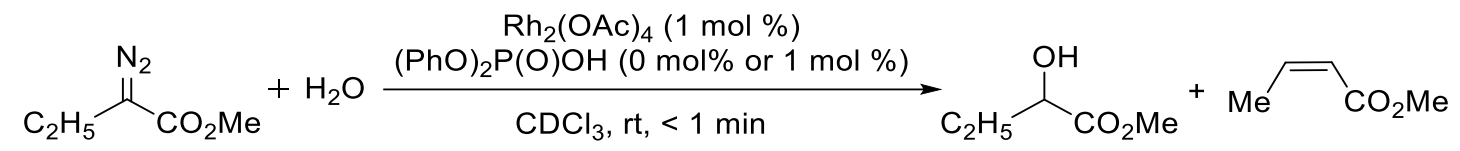

The $\mathrm{Rh}_{2}(\mathrm{OAc})_{4}(1.78 \mathrm{mg}, 0.004 \mathrm{mmol}, 1 \mathrm{~mol} \%)$ with or without $(\mathrm{PhO})_{2} \mathrm{P}(\mathrm{O}) \mathrm{OH}$ (1.0 mg, $0.004 \mathrm{mmol}, 1 \mathrm{~mol} \%)$ was introduced into an oven-dried Schlenk tube in an argon-filled glovebox. After $2 \mathrm{~mL} \mathrm{CDCl}_{3}$ was introduced, the resulted reaction mixture was stirred at room temperature for $2 \mathrm{~min}$. Then $\mathrm{H}_{2} \mathrm{O}(36 \mu \mathrm{L}, 2 \mathrm{mmol})$ was added by a micro syringe in one portion and a solution of methyl 2-diazobutanoate (51 mg, 0.4 mmol) in $4 \mathrm{~mL} \mathrm{CDCl}_{3}$ was added in one portion, subsequently. The reaction finished in 1 min after the addition (judged by the discoloration of diazo compound and confirmed by TLC monitoring). We analyzed the reaction mixture through ${ }^{1} \mathrm{H}$ NMR using 1,3,5trimethoxybenzene as an internal standard. In the presence of $(\mathrm{PhO})_{2} \mathrm{P}(\mathrm{O}) \mathrm{OH}(1 \mathrm{~mol} \%)$, the reaction gave insertion product methyl 2-hydroxybutanoate in $85 \%$ yield along with $\beta$-H migration by-product methyl (Z)-but-2-enoate in $9 \%$ yield; in the absence of the phosphoric acid, the corresponding yields were $30 \%$ and $40 \%$.

Analysis data for methyl 2-hydroxybutanoate (Figure 1). ${ }^{1} \mathrm{H}$ NMR (400 MHz, $\left.\mathrm{CDCl}_{3}\right) \delta 3.86(\mathrm{t}, J=6.3 \mathrm{~Hz}, 1 \mathrm{H}, \mathrm{CH}(\mathrm{OH})), 3.80(\mathrm{~s}, 1 \mathrm{H}, \mathrm{OH}), 3.74\left(\mathrm{~s}, 3 \mathrm{H}, \mathrm{OCH}_{3}\right), 1.81$ $\left(\mathrm{p}, J=7.3 \mathrm{~Hz}, 2 \mathrm{H}, \mathrm{CH}_{2}\right), 0.99\left(\mathrm{t}, J=7.4 \mathrm{~Hz}, 3 \mathrm{H}, \mathrm{CH}_{3}\right) .{ }^{13} \mathrm{C} \mathrm{NMR}\left(100 \mathrm{MHz}, \mathrm{CDCl}_{3}\right) \delta$ $172.4(1 \mathrm{C}, \mathrm{C}=\mathrm{O}), 81.0(1 \mathrm{C}, \mathrm{C}-\mathrm{O}), 51.8(1 \mathrm{C}, \mathrm{CH}(\mathrm{OH})), 25.9\left(1 \mathrm{C}, \mathrm{CH}_{2}\right), 9.6\left(1 \mathrm{C}, \mathrm{CH}_{3}\right)$. 

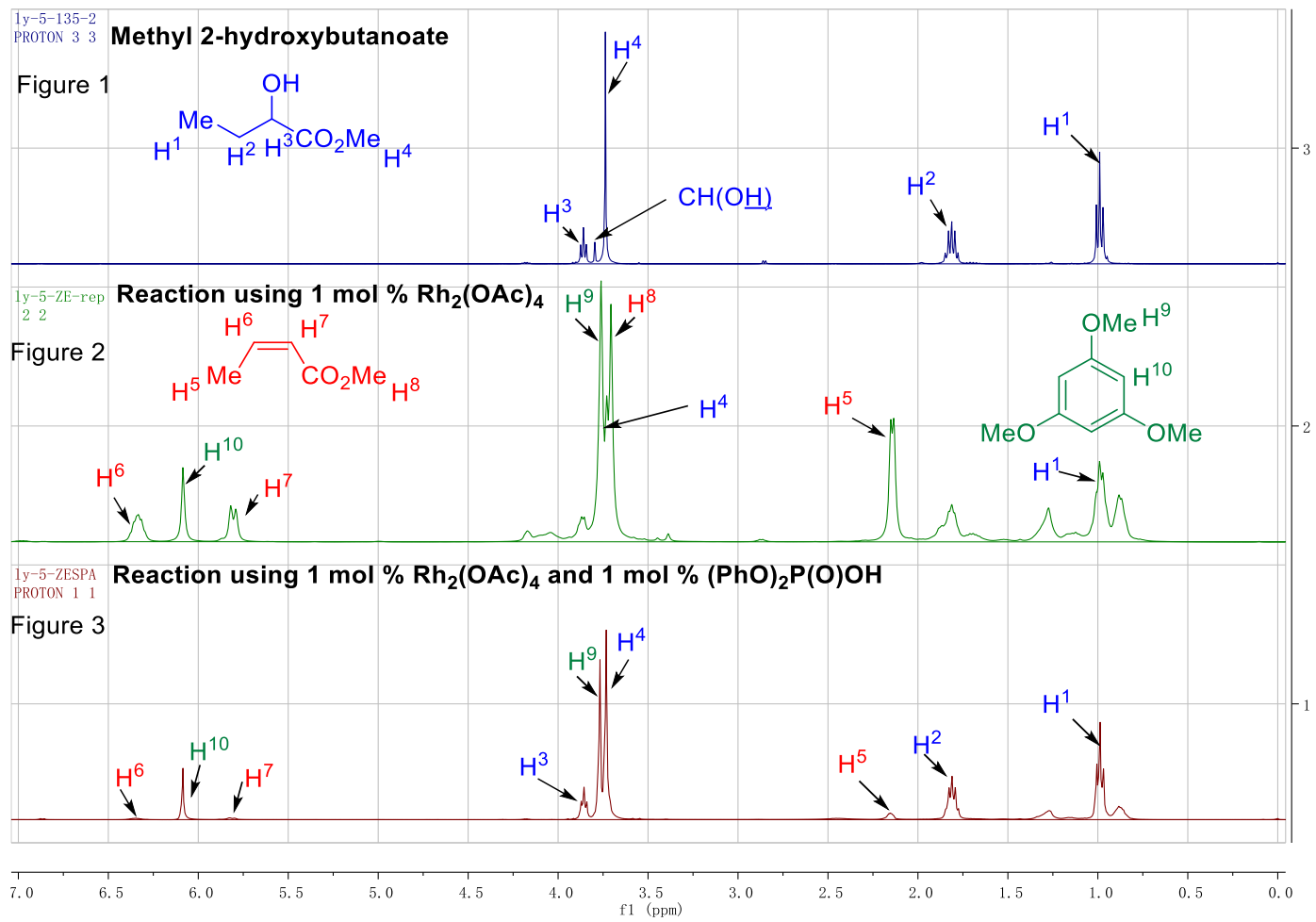

S48 


\section{Transformations of $\mathbf{O}-\mathbf{H}$ Bond Insertion Products}

\subsection{Synthesis of compounds $(S)-8$ and $(S)-9$}

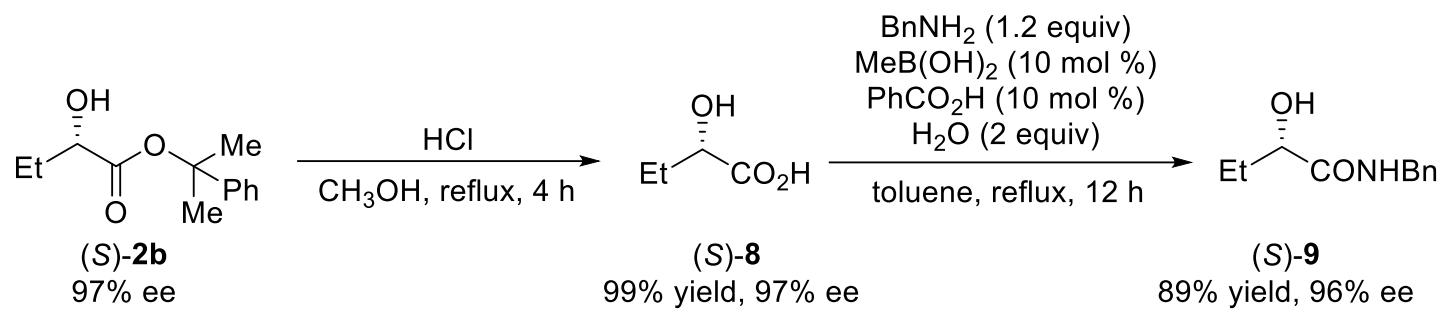

Compound $(S)-\mathbf{2 b}(89 \mathrm{mg}, 0.4 \mathrm{mmol})$ was dissolved in $\mathrm{CH}_{3} \mathrm{OH}(2 \mathrm{~mL})$ and $6 \mathrm{~N}$ $\mathrm{HCl}(4 \mathrm{~mL})$. The resulted solution was heated to reflux for $4 \mathrm{~h}$ and then evaporated under vacuo at $40{ }^{\circ} \mathrm{C}$. The resulted mixture was quenched with $\mathrm{H}_{2} \mathrm{O}$ and extracted with EA. The combined organic layers were dried over $\mathrm{MgSO}_{4}$ and evaporated. The residue was purified by flash column chromatography $(\mathrm{PE} / \mathrm{EA}=5: 1 \mathrm{v} / \mathrm{v})$ to afford the corresponding compound $(S)-8(41 \mathrm{mg}, 99 \%$ yield $)$ as a colorless oil. $[\alpha]_{\mathrm{D}}{ }^{25}=-4.3(c$ 3.0, $\left.\mathrm{H}_{2} \mathrm{O}\right)\left\{[\alpha]_{\mathrm{D}}{ }^{25}=-4.6\left(c 1.0, \mathrm{H}_{2} \mathrm{O}\right)\right.$ for $(S)-8$ with $99.2 \%$ ee according to the literature $\left.{ }^{7}\right\} .{ }^{1} \mathrm{H}$ NMR (400 MHz, $\left.\mathrm{CDCl}_{3}\right) \delta 4.25(\mathrm{~m}, 1 \mathrm{H}, \mathrm{CH}), 2.71(\mathrm{~s}, 1 \mathrm{H}, \mathrm{OH}), 1.84-$ $1.99\left(\mathrm{~m}, 1 \mathrm{H}, \mathrm{CH}_{2}\right), 1.75\left(\mathrm{~m}, 1 \mathrm{H}, \mathrm{CH}_{2}\right), 1.02\left(\mathrm{t}, J=7.5 \mathrm{~Hz}, 3 \mathrm{H}, \mathrm{CH}_{3}\right) .{ }^{13} \mathrm{C}$ NMR (100 $\left.\mathrm{MHz}, \mathrm{CDCl}_{3}\right) \delta 178.4(1 \mathrm{C}, \mathrm{C}=\mathrm{O}), 71.3(1 \mathrm{C}, \mathrm{CH}(\mathrm{OH})), 27.3\left(1 \mathrm{C}, \mathrm{CH}_{2}\right), 8.9\left(1 \mathrm{C}, \mathrm{CH}_{3}\right)$.

A mixture of $(S)-8(41 \mathrm{mg}, 0.4 \mathrm{mmol})$, benzylamine (43 $\mathrm{mg}, 0.4 \mathrm{mmol})$, $\mathrm{MeB}(\mathrm{OH})_{2}$ (2.4 mg, $\left.0.04 \mathrm{mmol}\right), \mathrm{PhCO}_{2} \mathrm{H}$ (4.9 mg, $\left.0.04 \mathrm{mmol}\right)$, and $\mathrm{H}_{2} \mathrm{O}$ (15 $\mu \mathrm{L}, 0.8$ $\mathrm{mmol})$ in toluene $(2 \mathrm{~mL})$ was heat at $115^{\circ} \mathrm{C}(12 \mathrm{~h})$ before cooling to room temperature and then the solvent was evaporated in vacuo. The crude product was purified by flash column chromatography $(\mathrm{PE} / \mathrm{EA}=1: 1 \mathrm{v} / \mathrm{v})$ to give the desired compound $(S)-9(69 \mathrm{mg}$, $89 \%$ yield $)$. White solid, $63 \sim 64^{\circ} \mathrm{C} .[\alpha]_{\mathrm{D}}^{25}=-28.0\left(c 1.0, \mathrm{CHCl}_{3}\right)\left\{[\alpha]_{\mathrm{D}}^{28.5}=+28.3(c\right.$ 2.2, $\left.\mathrm{CHCl}_{3}\right)$ for optical pure $(R)-9$ according to the literature $\left.{ }^{8}\right\}$. Serial number: ly-4-161. ${ }^{1} \mathrm{H}$ NMR (400 MHz, $\left.\mathrm{CDCl}_{3}\right) \delta 7.36-7.08(\mathrm{~m}, 6 \mathrm{H}, \mathrm{Ar}-\mathrm{H}, 1 \mathrm{H}, \mathrm{NH}), 4.39(\mathrm{qd}, J=14.8$, $\left.5.9 \mathrm{~Hz}, 2 \mathrm{H}, \mathrm{CH}_{2}\right), 4.14-3.82\left(\mathrm{~m}, 2 \mathrm{H}, \mathrm{CH}_{2}\right), 1.89-1.77(\mathrm{~m}, 1 \mathrm{H}), 1.65$ (td, $J=14.3,7.2$ $\mathrm{Hz}, 1 \mathrm{H}), 0.98-0.87\left(\mathrm{~m}, 3 \mathrm{H}, \mathrm{CH}_{3}\right) .{ }^{13} \mathrm{C} \mathrm{NMR}\left(100 \mathrm{MHz}, \mathrm{CDCl}_{3}\right) \delta 174.4(1 \mathrm{C}, \mathrm{C}=\mathrm{O})$, 137.9 (1C, Ar-C), 128.7 (2C, Ar-C), 127.6 (2C, Ar-C), 127.5 (1C, Ar-C), 73.0 (1C, $\mathrm{CH}(\mathrm{OH})), 43.1\left(1 \mathrm{C}, \mathrm{CH}_{2}\right), 27.8\left(1 \mathrm{C}, \mathrm{CH}_{2}\right), 9.2\left(1 \mathrm{C}, \mathrm{CH}_{3}\right)$. 
7.2 Synthesis of compound $(S)-10$<smiles>CCC(O)C(=O)OC(C)(C)c1ccccc1</smiles>

$(S)-\mathbf{2 b}$

$97 \%$ ee<smiles>CC[C@H](O)CO</smiles>

(S)-10

$98 \%$ yield, $96 \%$ ee

The insertion product $(S)-\mathbf{2 b}(44 \mathrm{mg}, 0.2 \mathrm{mmol})$ and $\mathrm{LiAlH}_{4}(23 \mathrm{mg}, 0.6 \mathrm{mmol})$ were introduced into an oven-dried Schlenk tube in nitrogen atmosphere. After $2 \mathrm{~mL}$ THF was injected into the Schlenk tube at $0{ }^{\circ} \mathrm{C}$, the mixture was stirred for $1 \mathrm{~h}$ at room temperature. The TLC showed that the reaction finished. EtOAc $(2 \mathrm{~mL})$ was added and stirred for $2 \mathrm{~h}$. The reaction was quenched by water. The suspension was extracted with EtOAc. The combined organic solutions were dried over $\mathrm{MgSO}_{4}$ and filtered. The filtrate was concentrated and purified by a flash chromatography on silica gel (PE/EA $=1: 2 \mathrm{v} / \mathrm{v})$ to afford diol $(S)-\mathbf{1 0}(35 \mathrm{mg}, 98 \%$ yield $)$ as a colorless oil. $[\alpha]_{\mathrm{D}}{ }^{20}=-14.7(c$ 1.0, EtOH $).\left\{[\alpha]_{\mathrm{D}}{ }^{20}=-15.4(c 1.0, \mathrm{EtOH})\right.$ for optically pure $(S)-\mathbf{1 0}$ according to the literature ${ }^{9 b}$ \}. ${ }^{1} \mathrm{H}$ NMR (400 MHz, $\left.\mathrm{CDCl}_{3}\right) \delta 3.99-3.78(\mathrm{~m}, 2 \mathrm{H}, \mathrm{OH}), 3.67-3.54(\mathrm{~m}$, $\left.2 \mathrm{H}, \mathrm{CH}_{2}\right), 3.49-3.35(\mathrm{~m}, 1 \mathrm{H}, \mathrm{CH}), 1.54-1.37\left(\mathrm{~m}, 2 \mathrm{H}, \mathrm{CH}_{2}\right), 0.96(\mathrm{t}, J=7.5 \mathrm{~Hz}, 3 \mathrm{H}$, $\left.\mathrm{CH}_{3}\right) .{ }^{13} \mathrm{C} \mathrm{NMR}\left(100 \mathrm{MHz}, \mathrm{CDCl}_{3}\right) \delta 73.7(1 \mathrm{C}, \mathrm{CH}), 66.4\left(1 \mathrm{C}, \mathrm{CH}_{2}\right), 26.0\left(1 \mathrm{C}, \mathrm{CH}_{2}\right)$, $10.0\left(1 \mathrm{C}, \mathrm{CH}_{3}\right)$. 


\subsection{Synthesis of compound $(S)-11$}

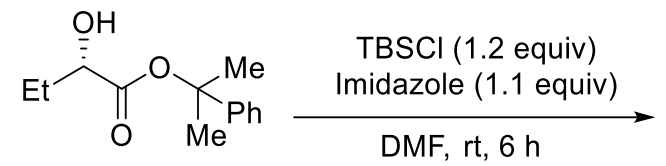

$(S)-\mathbf{2 b}$

$97 \%$ ee

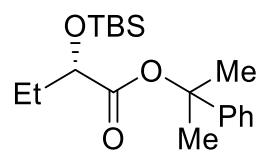

(S)-2ba

$80 \%$ yield, $96 \%$ ee

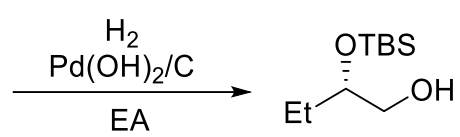

(S)-11

$96 \%$ yield, $96 \%$ ee

A solution of $(S)-\mathbf{2 b}(89 \mathrm{mg}, 0.4 \mathrm{mmol}), \mathrm{TBSCl}(72 \mathrm{mg}, 0.48 \mathrm{mmol})$, and imidazole (30 mg, 0.44mmol) in DMF (4mL) was stirred at room temperature for $6 \mathrm{~h}$. The resulted mixture was quenched with $\mathrm{H}_{2} \mathrm{O}$ and extracted with EA. The combined organic layers were dried over $\mathrm{MgSO}_{4}$ and evaporated. The residue was purified by column chromatography $(\mathrm{PE} / \mathrm{EA}=50: 1 \mathrm{v} / \mathrm{v})$ to afford $(S)$-2 ba $(108 \mathrm{mg}, 80 \%$ yield $)$ as a colorless oil. ${ }^{10}$ Serial number: ly-4-125. $[\alpha]_{\mathrm{D}}{ }^{25}=23.48\left(c 1.0, \mathrm{CHCl}_{3}\right) .{ }^{1} \mathrm{H}$ NMR $(400$ $\left.\mathrm{MHz}, \mathrm{CDCl}_{3}\right) \delta 7.27(\mathrm{dd}, J=8.2,0.9 \mathrm{~Hz}, 2 \mathrm{H}, \mathrm{Ar}-\mathrm{H}), 7.20(\mathrm{dd}, J=10.4,4.9 \mathrm{~Hz}, 2 \mathrm{H}$, Ar-H), $7.14-7.07$ (m, 1H, Ar-H), 4.01 (dd, J = 7.2, 4.6 Hz, 1H, CH), $1.74-1.60$ (m, $\left.8 \mathrm{H}, \mathrm{CH}_{2}, \mathrm{C}\left(\mathrm{CH}_{3}\right)_{2}\right), 0.92-0.82\left(\mathrm{~m}, 12 \mathrm{H}, \mathrm{CH}_{3}, \mathrm{Si}\left(\mathrm{CH}_{3}\right)_{3}\right), 0.00$ (s, 3H, $\left.\mathrm{Si}\left(\mathrm{CH}_{3}\right)\right),-0.03(\mathrm{~s}$, 3H, $\left.\mathrm{Si}\left(\mathrm{CH}_{3}\right)\right) .{ }^{13} \mathrm{C}$ NMR (100 MHz, $\left.\mathrm{CDCl}_{3}\right) \delta 172.0(1 \mathrm{C}, \mathrm{C}=\mathrm{O}), 145.7$ (1C, Ar-C), 128.3 (2C, Ar-C), 127.1 (1C, Ar-C), 124.4 (2C, Ar-C), 81.9 (1C, C-O), 73.7 (1C, CH), 28.8 (1C, $\left.\underline{\mathrm{C}}\left(\mathrm{CH}_{3}\right)_{3}\right), 28.4\left(1 \mathrm{C}, \mathrm{C}\left(\underline{\mathrm{CH}}_{3}\right)\right), 28.4\left(1 \mathrm{C}, \mathrm{C}\left(\underline{\mathrm{CH}}_{3}\right)_{2}\right), 25.9$ (1C, $\left.\mathrm{C}\left(\underline{\mathrm{C}} \mathrm{H}_{3}\right)_{3}\right), 25.9$ (1C, $\left.\mathrm{C}\left(\underline{\mathrm{CH}}_{3}\right)_{3}\right), 25.8\left(1 \mathrm{C}, \mathrm{C}\left(\underline{\mathrm{CH}}_{3}\right)_{3}\right), 18.4\left(1 \mathrm{C}, \mathrm{CH}_{2}\right), 9.8\left(1 \mathrm{C}, \mathrm{CH}_{3}\right),-4.6\left(1 \mathrm{C}, \mathrm{Si}\left(\underline{\mathrm{CH}}_{3}\right)_{2}\right),-5.3$ (1C, $\left.\mathrm{Si}\left(\underline{\mathrm{CH}}_{3}\right)_{2}\right)$. HRMS (ESI) Calcd for $\left[\mathrm{C}_{19} \mathrm{H}_{32} \mathrm{O}_{3} \mathrm{SiNa}, \mathrm{M}+\mathrm{Na}\right]^{+}:$359.2013, Found: 359.2014 .

The compound (S)-2ba $(67.2 \mathrm{mg}, 0.2 \mathrm{mmol})$ was dissolved in EA and treated with $\mathrm{H}_{2}$ balloon over $\mathrm{Pd}(\mathrm{OH})_{2} / \mathrm{C}$ for $1.5 \mathrm{~h}$ at room temperature. The residue was purified by column chromatography $(\mathrm{PE} / \mathrm{EA}=1: 1 \mathrm{v} / \mathrm{v})$ to afford $(S)-\mathbf{1 1}(39 \mathrm{mg}, \mathbf{9 6 \%}$ yield $)$ as a colorless oil. ${ }^{11}$ Serial number: ly-4-130, $[\alpha]_{\mathrm{D}}{ }^{25}=19.47\left(c\right.$ 1.0, $\left.\mathrm{CHCl}_{3}\right) .{ }^{1} \mathrm{H}$ NMR $(400$ $\left.\mathrm{MHz}, \mathrm{CDCl}_{3}\right) \delta 3.54\left(\mathrm{dd}, J=9.6,3.5 \mathrm{~Hz}, 1 \mathrm{H}, \mathrm{CH}_{2}\right), 3.51-3.41(\mathrm{~m}, 1 \mathrm{H}, \mathrm{CH}), 3.35(\mathrm{dd}$, $\left.J=9.6,7.1 \mathrm{~Hz}, 1 \mathrm{H}, \mathrm{CH}_{2}\right), 2.61(\mathrm{~d}, J=3.6 \mathrm{~Hz}, 1 \mathrm{H}, \mathrm{OH}), 1.38\left(\mathrm{p}, J=7.3 \mathrm{~Hz}, 2 \mathrm{H}, \mathrm{CH}_{2}\right)$, $0.97-0.73\left(\mathrm{~m}, 12 \mathrm{H}, \mathrm{CH}_{3}, \mathrm{C}\left(\mathrm{CH}_{3}\right)_{3}\right),-0.00\left(\mathrm{~s}, 6 \mathrm{H}, \mathrm{Si}\left(\mathrm{CH}_{3}\right)_{2}\right) .{ }^{13} \mathrm{C} \mathrm{NMR}(101 \mathrm{MHz}$, $\left.\mathrm{CDCl}_{3}\right) \delta 73.1(1 \mathrm{C}, \mathrm{C}-\mathrm{O}), 66.9\left(1 \mathrm{C}, \mathrm{CH}_{2}\right), 25.8\left(3 \mathrm{C}, \mathrm{C}\left(\underline{\mathrm{CH}_{3}}\right)_{3}, 25.7\left(1 \mathrm{C}, \underline{\mathrm{C}}\left(\mathrm{CH}_{3}\right)_{3}\right), 18.2\right.$ (1C, $\left.\mathrm{CH}_{2}\right), 9.9\left(1 \mathrm{C}, \mathrm{CH}_{3}\right),-5.5\left(1 \mathrm{C}, \mathrm{Si}\left(\underline{\mathrm{CH}}_{3}\right)\right),-5.5\left(1 \mathrm{C}, \mathrm{Si}\left(\underline{\mathrm{CH}}_{3}\right)\right)$. 


\subsection{Synthesis of compound $(R)-12$}<smiles>[M]C([Y10])([R])OC(=O)C(O)CC</smiles>

$(S)-\mathbf{2 b}$

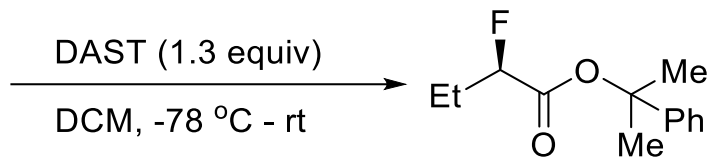

$(R)-12$

$68 \%$ yield, $94 \%$ ee<smiles>CCN(CC)S(F)(F)F</smiles>

DAST

A modified literature procedure ${ }^{12}$ was used to prepare compound $(R)-\mathbf{1 2}$. Compound (S)-2b (89 mg, $0.4 \mathrm{mmol})$ was added to a solution of DAST (53 $\mu \mathrm{L}, 0.52$ $\mathrm{mmol})$ in DCM $(2 \mathrm{~mL})$ at $-78^{\circ} \mathrm{C}$. After stirring for $2 \mathrm{~h}$ at $-78^{\circ} \mathrm{C}$ and another $3 \mathrm{~h}$ at room temperature, the reaction mixture was diluted with EA and quenched with saturated aqueous $\mathrm{NaHCO}_{3}(2 \mathrm{~mL})$. The organic phase was then washed with brine and dried over anhydrated $\mathrm{Na}_{2} \mathrm{SO}_{4}$. The solvent was evaporated under reduced pressure, and the residue was purified by column chromatography $(\mathrm{PE} / \mathrm{EA}=50: 1)$ to afford $(R)-\mathbf{1 2}(61 \mathrm{mg}$, $68 \%$ yield). Serial number: ly-4-155, colorless oil. TLC $R_{f}=0.48(\mathrm{PE} / \mathrm{EA}=50: 1 \mathrm{v} / \mathrm{v})$, 94\% ee [HPLC condition: Chiralcel OJ-3 column, $n$-hexane/2-propanol $=90: 10$, flow rate $=1 \mathrm{~mL} / \mathrm{min}$, wavelength $=210 \mathrm{~nm}, t_{\mathrm{R}}=13.02 \mathrm{~min}$ for minor isomer, $t_{\mathrm{R}}=13.80 \mathrm{~min}$ for major isomer, $[\alpha]_{\mathrm{D}}{ }^{25}=13.29\left(c 1.0, \mathrm{CHCl}_{3}\right) .{ }^{1} \mathrm{H} \mathrm{NMR}\left(400 \mathrm{MHz}, \mathrm{CDCl}_{3}\right) \delta 7.40-$ 7.29 (m, 4H, Ar-H), $7.29-7.21$ (m, 1H, Ar-H), 4.79 (ddd, $J=49.3,7.0,4.5 \mathrm{~Hz}, 1 \mathrm{H}$, $\mathrm{CH}), 2.02-1.86\left(\mathrm{~m}, 2 \mathrm{H}, \mathrm{CH}_{2}\right), 1.82\left(\mathrm{~s}, 6 \mathrm{H}, \mathrm{C}\left(\mathrm{CH}_{3}\right)_{2}\right), 1.02\left(\mathrm{t}, J=7.5 \mathrm{~Hz}, 3 \mathrm{H}, \mathrm{CH}_{3}\right)$. ${ }^{13} \mathrm{C}$ NMR $\left(100 \mathrm{MHz}, \mathrm{CDCl}_{3}\right) \delta 168.4\left(\mathrm{~d},{ }^{2} J_{\mathrm{C}-\mathrm{F}}=23.7 \mathrm{~Hz}, 1 \mathrm{C}, \mathrm{C}=\mathrm{O}\right), 145.0$ (1C, Ar-C), 128.4 (2C, Ar-C), 127.4 (1C, Ar-C), 124.3 (2C, Ar-C), 90.9 (d, ${ }^{1} J_{\mathrm{C}-\mathrm{F}}=183.5 \mathrm{~Hz}, 1 \mathrm{C}, \mathrm{C}-$ F), 89.0 (1C, C-F), $83.4(1 \mathrm{C}, \mathrm{C}-\mathrm{O}), 28.5\left(1 \mathrm{C}, \mathrm{C}\left(\mathrm{CH}_{3}\right)_{2}\right), 28.4\left(1 \mathrm{C}, \mathrm{C}\left(\mathrm{CH}_{3}\right)_{2}\right), 25.8(\mathrm{~d}$, $\left.{ }^{2} J_{\mathrm{C}-\mathrm{F}}=23.7 \mathrm{~Hz}, 1 \mathrm{C}, \mathrm{CH}_{2}\right), 8.7\left(\mathrm{~d},{ }^{3} J_{\mathrm{C}-\mathrm{F}}=23.7 \mathrm{~Hz}, 1 \mathrm{C}, \mathrm{CH}_{3}\right) .{ }^{19} \mathrm{~F} \mathrm{NMR}\left(376 \mathrm{MHz}, \mathrm{CDCl}_{3}\right)$ $\delta$-192.46 (dt, $J=49.4,24.8 \mathrm{~Hz}$ ). HRMS (ESI) Calcd for $\left[\mathrm{C}_{13} \mathrm{H}_{17} \mathrm{FO}_{2} \mathrm{Na}, \mathrm{M}+\mathrm{Na}\right]^{+}$: 247.1105, Found: 247.1110. 


\subsection{Synthesis of compound 13}<smiles>CCCCC(=O)C(O)C=C(C)c1ccccc1</smiles>

$93 \%$ ee<smiles>COCc1ccccc1</smiles>

$\mathrm{MeOH}$, reflux, $6 \mathrm{~h}$<smiles>CC(=CC(O)C(=O)O)c1ccccc1</smiles>

$98 \%$ yield, $92 \%$ ee

A modified literature procedure ${ }^{6}$ was used to prepare compound 13. Compound 7a (50 mg, $0.2 \mathrm{mmol})$ was dissolved in $\mathrm{CH}_{3} \mathrm{OH}(2 \mathrm{~mL})$ and $6 \mathrm{~N} \mathrm{HCl}(4 \mathrm{~mL})$. The resulted solution was heated to reflux for $6 \mathrm{~h}$ and then evaporated under reduced pressure at 40 ${ }^{\circ} \mathrm{C}$. The resulted mixture was quenched with $\mathrm{H}_{2} \mathrm{O}$ and extracted with EA. The combined organic layers were dried over $\mathrm{MgSO}_{4}$ and evaporated. The residue was purified by flash column chromatography $(\mathrm{PE} / \mathrm{EA}=1: 5 \mathrm{v} / \mathrm{v})$ to afford the corresponding compound 13 (38 mg, 98\% yield) as a white solid. Serial number: ly-5-69. White solid, mp: $106 \sim 108^{\circ} \mathrm{C} . \mathrm{TLC} R_{f}=0.51(\mathrm{PE} / \mathrm{EA}=1: 2 \mathrm{v} / \mathrm{v}), 92 \%$ ee [HPLC condition: Chiralcel OD$\mathrm{H}$ column, $n$-hexane $/ 2$-propanol $=50: 50$, flow rate $=1.0 \mathrm{ml} / \mathrm{min}$, wavelength $=254 \mathrm{~nm}$, $t_{\mathrm{R}}=6.54 \mathrm{~min}$ for major isomer, $t_{\mathrm{R}}=9.72 \mathrm{~min}$ for minor isomer $],[\alpha]_{\mathrm{D}}{ }^{25}=-62.0(c 1.0$, $\left.\mathrm{CHCl}_{3}\right) .{ }^{1} \mathrm{H} \mathrm{NMR}\left(400 \mathrm{MHz}, \mathrm{CDCl}_{3}\right) \delta 7.38-7.32$ (m, 2H, Ar-H), 7.29 (t, J=7.3 Hz, 2H, Ar-H), $7.30-7.26(\mathrm{~m}, 1 \mathrm{H}, \mathrm{Ar}-\mathrm{H}), 5.69(\mathrm{dd}, J=8.5,1.1 \mathrm{~Hz}, 1 \mathrm{H}, \mathrm{CH}=), 4.98(\mathrm{~m}$, 1H, $\mathrm{CH}(\mathrm{OH})), 3.19(\mathrm{~d}, J=5.9 \mathrm{~Hz}, 1 \mathrm{H}, \mathrm{O}-\mathrm{H}), 2.18\left(\mathrm{~d}, J=0.9 \mathrm{~Hz}, 3 \mathrm{H}, \mathrm{CH}_{3}\right) .{ }^{13} \mathrm{C} \mathrm{NMR}$ $\left(100 \mathrm{MHz}, \mathrm{CDCl}_{3}\right) \delta 172.9(1 \mathrm{C}, \mathrm{C}=\mathrm{O}), 142.4(1 \mathrm{C}, \mathrm{Ar}-\mathrm{C}), 140.1(1 \mathrm{C}, \mathrm{C}=), 128.1(2 \mathrm{C}$, Ar-C), 127.2 (1C, Ar-C), 125.4 (2C, Ar-C), 125.0 (1C, CH=), 68.3 (1C, $\mathrm{CH}(\mathrm{OH})), 16.5$ (1C, $\left.\mathrm{CH}_{3}\right)$. HRMS (ESI) Calcd for $\left[\mathrm{C}_{11} \mathrm{H}_{12} \mathrm{O}_{3} \mathrm{Na}, \mathrm{M}+\mathrm{Na}\right]^{+}: 215.0679$, Found: 215.0682.

\subsection{Synthesis of compound 14}

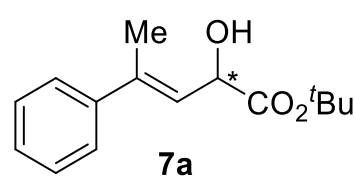

$93 \%$ ee

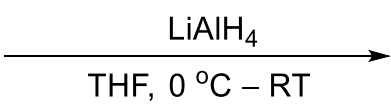

$-\mathrm{RT}$

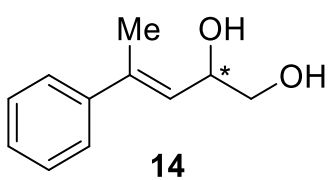

$>99 \%$ yield, $92 \%$ ee

A modified procedure ${ }^{13}$ was used to prepare compound $\mathbf{1 4}$. The compound $7 \mathbf{a}$ (30 $\mathrm{mg}, 0.12 \mathrm{mmol})$ and $\mathrm{LiAlH}_{4}(14 \mathrm{mg}, 0.36 \mathrm{mmol})$ were introduced into an oven-dried Schlenk tube in nitrogen atmosphere. After $2 \mathrm{~mL}$ THF was injected into the Schlenk tube at $0{ }^{\circ} \mathrm{C}$, the resulted mixture was stirred at room temperature for $1 \mathrm{~h}$. EA $(2 \mathrm{~mL})$ was added and stirred for a while. The reaction was quenched by a small amount of 
water. The suspension was extracted with EA. The combined organic solutions were dried over $\mathrm{MgSO}_{4}$ and filtered. The filtrate was concentrated and purified by a flash chromatography on silica gel $(\mathrm{PE} / \mathrm{EA}=1: 2 \mathrm{v} / \mathrm{v})$ to afford $14(21 \mathrm{mg},>99 \%$ yield $)$ as a white solid. Mp: $42 \sim 44{ }^{\circ} \mathrm{C}$, TLC $R_{f}=0.37(\mathrm{PE} / \mathrm{EA}=1: 2 \mathrm{v} / \mathrm{v}), 100 \%$ yield, $92 \%$ ee [HPLC condition: Chiralpak AS-H column, $n$-hexane/2-propanol $=90: 10$, flow rate $=$ $1.0 \mathrm{~mL} / \mathrm{min}$, wavelength $=254 \mathrm{~nm}, t_{\mathrm{R}}=11.85 \mathrm{~min}$ for minor isomer, $t_{\mathrm{R}}=15.50 \mathrm{~min}$ for major isomer], $[\alpha]_{\mathrm{D}}{ }^{28}=-17.2\left(c 0.5, \mathrm{CHCl}_{3}\right) .{ }^{1} \mathrm{H} \mathrm{NMR}\left(400 \mathrm{MHz}, \mathrm{CDCl}_{3}\right) \delta 7.40$ (d, $J=7.5 \mathrm{~Hz}, 2 \mathrm{H}, \mathrm{Ar}-\mathrm{H}), 7.37-7.23$ (m, 3H, Ar-H), 5.74 (d, $J=8.2 \mathrm{~Hz}, 1 \mathrm{H}, \mathrm{CH}=$ ), $4.95-4.43$ (m, 1H, CH), $4.14-3.17$ (m, 2H, $\left.\mathrm{CH}_{2}\right), 2.54$ (s, 2H, dr, 2O-H), $2.12(\mathrm{~s}, 3 \mathrm{H}$, $\left.\mathrm{CH}_{3}\right) .{ }^{13} \mathrm{C}$ NMR $\left(101 \mathrm{MHz}, \mathrm{CDCl}_{3}\right) \delta 142.5$ (1C, Ar-C), 139.4 (1C, C), 128.3 (2C, ArC), 127.5 (1C, Ar-C), 125.8 (1C, $\mathrm{CH}=), 125.8$ (2C, Ar-C), 69.9 (1C, C-O), 66.2 (1C, CO), $16.5\left(1 \mathrm{C}, \mathrm{CH}_{3}\right)$. HRMS (ESI) Calcd for $\left[\mathrm{C}_{13} \mathrm{H}_{18} \mathrm{SO}_{3} \mathrm{Na}, \mathrm{M}+\mathrm{Na}\right]^{+}:$201.0886, Found: 201.0887 .

\subsection{Synthesis of compound $15^{14}$}

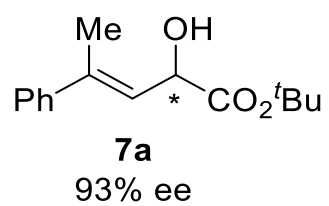

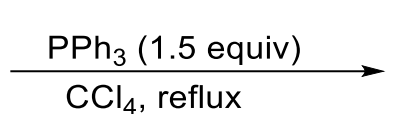

$\mathrm{CCl}_{4}$, reflux

$93 \%$ ee<smiles>CCCCOC(=O)C(Cl)/C=C(\C)c1ccccc1</smiles>

15

$81 \%$ yield, $93 \%$ ee

The compound 7a (50 mg, $0.2 \mathrm{mmol})$ and $\mathrm{PPh}_{3}(80 \mathrm{mg}, 1.5$ equiv) were introduced into an oven-dried Schlenk tube in nitrogen atmosphere, and then $4 \mathrm{~mL}$ dried $\mathrm{CCl}_{4}$ was injected into the Schlenk tube. The resulted mixture was stirred at $100{ }^{\circ} \mathrm{C}$ overnight. The reaction mixture was concentrated and the residue was purified by column chromatography $\left(\mathrm{PE} / \mathrm{Et}_{2} \mathrm{O}=10: 1 \mathrm{v} / \mathrm{v}\right)$ to afford $15(43 \mathrm{mg}, 81 \%$ yield $)$ as a yellow oil. [HPLC condition: Chiralpak AS-H column, $n$-hexane/2-propanol $=90: 10$, flow rate $=$ $1.0 \mathrm{~mL} / \mathrm{min}$, wavelength $=254 \mathrm{~nm}, t_{\mathrm{R}}=8.31 \mathrm{~min}$ for minor isomer, $t_{\mathrm{R}}=10.56 \mathrm{~min}$ for major isomer], $[\alpha]_{\mathrm{D}}{ }^{25}=-43.4\left(c 0.5, \mathrm{CHCl}_{3}\right) .{ }^{1} \mathrm{H} \mathrm{NMR}\left(400 \mathrm{MHz}, \mathrm{CDCl}_{3}\right) \delta 7.42-7.38$ (m, 2H, Ar-H), 7.33 (t, $J=7.3 \mathrm{~Hz}, 2 \mathrm{H}, \mathrm{Ar}-\mathrm{H}), 7.30-7.25$ (m, 1H, Ar-H), 5.63 (dd, $J=$ 8.5, $1.1 \mathrm{~Hz}, 1 \mathrm{H}, \mathrm{CH}=), 4.93(\mathrm{~m}, \mathrm{C} \underline{\mathrm{H}}(\mathrm{Cl})), 2.15\left(\mathrm{~d}, J=0.8 \mathrm{~Hz}, 3 \mathrm{H}, \mathrm{CH}_{3}\right), 1.48(\mathrm{~d}, J=6.0$ $\left.\mathrm{Hz}, 9 \mathrm{H}, \mathrm{C}\left(\mathrm{CH}_{3}\right)_{3}\right) .{ }^{13} \mathrm{C} \mathrm{NMR}\left(100 \mathrm{MHz}, \mathrm{CDCl}_{3}\right) \delta 173.0(1 \mathrm{C}, \mathrm{C}=\mathrm{O}), 142.4(1 \mathrm{C}, \mathrm{Ar}-\mathrm{C})$, $140.3(1 \mathrm{C}, \mathrm{C}=), 128.1$ (2C, Ar-C), 127.3 (1C, Ar-C), 125.6 (2C, Ar-C), $125.0(1 \mathrm{C}, \mathrm{CH}=)$, 82.7 (1C, C-O), 68.3(1C, $\mathrm{CH}(\mathrm{Cl})), 28.3\left(3 \mathrm{C}, \mathrm{C}\left(\underline{\mathrm{CH}}_{3}\right)_{3}\right), 16.5\left(1 \mathrm{C}, \mathrm{CH}_{3}\right) . \mathrm{HRMS}(\mathrm{ESI})$ 
Calcd for $\left[\mathrm{C}_{15} \mathrm{H}_{19} \mathrm{O}_{2} \mathrm{ClNa}, \mathrm{M}+\mathrm{Na}\right]^{+}:$289.0966, Found: 289.0969.

\subsection{Synthesis of compound 16}<smiles>CCCCC(O)C(O)=CC(=O)c1ccccc1</smiles>

$93 \%$ ee

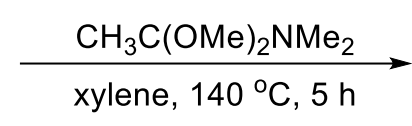

xylene, $140{ }^{\circ} \mathrm{C}, 5 \mathrm{~h}$

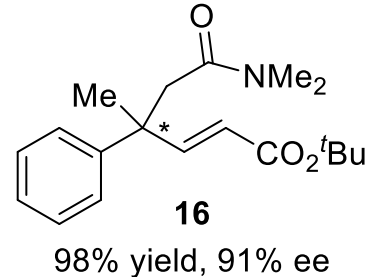

$98 \%$ yield, $91 \%$ ee

A modified literature procedure ${ }^{15}$ was used to prepare compound 16. The insertion product $7 \mathbf{a}(20 \mathrm{mg}, 0.08 \mathrm{mmol})$ were introduced into an oven-dried Schlenk tube in nitrogen atmosphere. After $3 \mathrm{~mL}$ xylene and 1,1-dimethoxy- $N, N$ dimethylethan-1-amine $(0.1 \mathrm{~mL}, 0.8 \mathrm{mmol})$ was injected into the Schlenk tube, the resulted mixture was refluxed at $140{ }^{\circ} \mathrm{C}$ for $5 \mathrm{~h}$. Then the reaction mixture was concentrated and purified by a flash chromatography on silica gel $(\mathrm{PE} / \mathrm{EA}=1: 1 \mathrm{v} / \mathrm{v})$ to afford compound 16 (24 mg, 98\% yield). White solid, mp: 76 78 ${ }^{\circ} \mathrm{C}$, TLC $R_{f}=0.74$ pure EA), 91\% ee [HPLC condition: Chiralcel OD-H column, $n$-hexane/2-propanol = $85: 15$, flow rate $=1.0 \mathrm{~mL} / \mathrm{min}$, wavelength $=210 \mathrm{~nm}, t_{\mathrm{R}}=5.77 \mathrm{~min}$ for minor isomer, $t_{\mathrm{R}}=6.93 \mathrm{~min}$ for major isomer], $[\alpha]_{\mathrm{D}}{ }^{28}=-6.0\left(c 1.0, \mathrm{CHCl}_{3}\right) .{ }^{1} \mathrm{H} \mathrm{NMR}(400 \mathrm{MHz}$, $\left.\mathrm{CDCl}_{3}\right) \delta 7.37-7.23(\mathrm{~m}, 5 \mathrm{H}, \mathrm{Ar}-\mathrm{H}), 7.20(\mathrm{~d}, J=15.9 \mathrm{~Hz}, 1 \mathrm{H}, \mathrm{CH}=), 5.74(\mathrm{~d}, J=16.0$ $\mathrm{Hz}, 1 \mathrm{H}, \mathrm{CH}=), 2.93-2.74$ (m, 5H, NMe, $\mathrm{CH}_{2}$ ), 2.72 (s, 3H, NMe), 1.66 (s, 3H, CH 3 ), $1.49\left(\mathrm{~s}, 9 \mathrm{H}, \mathrm{C}\left(\mathrm{CH}_{3}\right)_{3}\right) .{ }^{13} \mathrm{C} \mathrm{NMR}\left(100 \mathrm{MHz}, \mathrm{CDCl}_{3}\right) \delta 169.8(1 \mathrm{C}, \mathrm{C}=\mathrm{O}), 166.2(1 \mathrm{C}$, $\mathrm{C}=\mathrm{O}), 154.4$ (1C, Ar-C), 145.1 (1C, Ar-C), 128.3 (2C, Ar-C), 126.6 (1C, $\mathrm{CH}=), 126.4$ (2C, Ar-C), 120.7 (1C, CH=), 80.3 (1C, C-O), 43.6 (1C, N-C), 43.0 (1C, N-C), 37.6 (1C, $\left.\mathrm{CH}_{2}\right), 35.4$ (1C, C), 28.1 (3C, $\left.\mathrm{C}\left(\underline{\mathrm{CH}}_{3}\right)_{3}\right), 25.2$ (1C, $\left.\mathrm{CH}_{3}\right)$. HRMS (ESI) Calcd for $\left[\mathrm{C}_{13} \mathrm{H}_{18} \mathrm{SO}_{3} \mathrm{H}, \mathrm{M}+\mathrm{H}\right]^{+}:$318.2064, Found: 318.2070. 


\subsection{Synthesis of compound 17}<smiles>CCCCOC(=O)[C@H](O)/C=C/c1ccccc1</smiles>

$(S)-7 p$

$97 \%$ ee

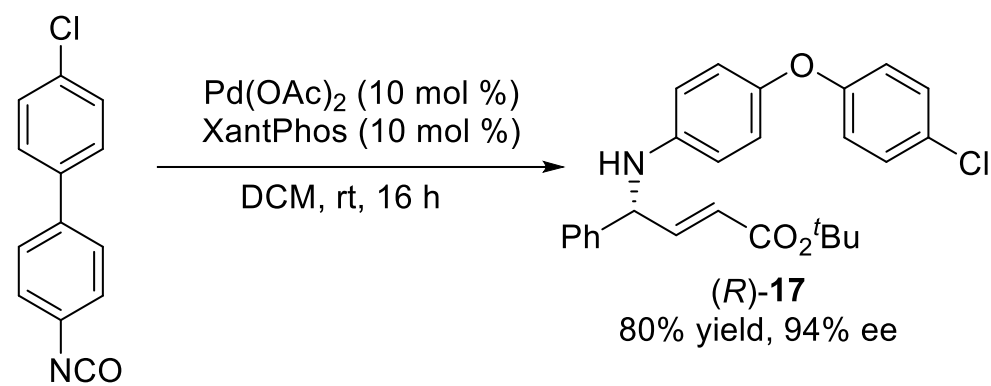

A modified literature procedure ${ }^{16}$ was used to prepare compound $(R)-\mathbf{1 7}$. The insertion product $(S)-7 \mathbf{p}(45 \mathrm{mg}, 0.2 \mathrm{mmol})$ and 4-chloro-4'-isocyanato-1,1'-biphenyl (46 mg, $0.2 \mathrm{mmol}$ ) were introduced into an oven-dried Schlenk tube in nitrogen atmosphere. After introduced DCM (3 mL) into the Schlenk tube, the mixture was refluxed overnight. Then $\mathrm{Pd}(\mathrm{OAc})_{2}$ (4.5 mg, $2 \mathrm{~mol} \%$ ) and XantPhos (12 mg, $2 \mathrm{~mol} \%$ ) was added to the reaction mixture and further stirred at room temperature for $16 \mathrm{~h}$. The resulted mixture was concentrated and the residue was purified by column chromatography $(\mathrm{PE} / \mathrm{EA}=10: 1 \mathrm{v} / \mathrm{v})$ to afford $(R)-17(70 \mathrm{mg}, 80 \%$ yield $)$ as a yellow solid. Serial number: ly-5-108, mp: $146-147^{\circ} \mathrm{C}$. TLC $R_{f}=0.37(\mathrm{PE} / \mathrm{EA}=10: 1 \mathrm{v} / \mathrm{v}), 94 \%$ ee [HPLC condition: Chiralcel OJ-3 column, $n$-hexane/2-propanol $=90: 10$, flow rate $=$ $1 \mathrm{ml} / \mathrm{min}$, wavelength $=210 \mathrm{~nm}, t_{\mathrm{R}}=13.02 \mathrm{~min}$ for minor isomer, $t_{\mathrm{R}}=13.80 \mathrm{~min}$ for major isomer], $[\alpha]_{\mathrm{D}}^{25}=13.29\left(c 1.0, \mathrm{CHCl}_{3}\right) .{ }^{1} \mathrm{H} \mathrm{NMR}\left(400 \mathrm{MHz}, \mathrm{CDCl}_{3}\right) \delta 7.39(\mathrm{~d}, J$ $=7.2 \mathrm{~Hz}, 2 \mathrm{H}, \mathrm{Ar}-\mathrm{H}), 7.32(\mathrm{t}, J=7.4 \mathrm{~Hz}, 2 \mathrm{H}, \mathrm{Ar}-\mathrm{H}), 7.27-7.17(\mathrm{~m}, 3 \mathrm{H}, \mathrm{Ar}-\mathrm{H}), 6.83$ (m, 5H, Ar-H), $6.64(\mathrm{~m}, 1 \mathrm{H}, \mathrm{Ar}-\mathrm{H}, 1 \mathrm{H}, \mathrm{CH}=), 6.28(\mathrm{~m}, 1 \mathrm{H}, \mathrm{CH}=), 4.66-4.52(\mathrm{~m}, 1 \mathrm{H}$, $\mathrm{NH}), 1.49$ (s, 9H, $\left.\mathrm{C}\left(\mathrm{CH}_{3}\right)_{3}\right) .{ }^{13} \mathrm{C} \mathrm{NMR}\left(100 \mathrm{MHz}, \mathrm{CDCl}_{3}\right) \delta 170.7(1 \mathrm{C}, \mathrm{C}=\mathrm{O}), 157.7$ (1C, Ar-C), 147.9 (1C, Ar-C), 143.4 (1C, Ar-C), 136.4 (1C, Ar-C), 132.6 (1C, CH=), 129.4 (2C, Ar-C), 128.7 (2C, Ar-C), 128.0 (1C, Ar-C), 126.9 (1C, Ar-C), 126.7 (2C, Ar-C), 125.5 (1C, $\mathrm{CH}=), 121.1$ (2C, Ar-C), 118.4 (2C, Ar-C), 114.7 (2C, Ar-C), 82.7 (1C, $\left.\underline{\mathrm{C}}\left(\mathrm{CH}_{3}\right)_{3}\right), 59.8(1 \mathrm{C}, \mathrm{CH}), 28.1$ (3C, $\left.\mathrm{C}\left(\underline{\mathrm{CH}}_{3}\right)_{3}\right)$. HRMS (ESI) Calcd for $\left[\mathrm{C}_{26} \mathrm{H}_{26} \mathrm{ClNO}_{3}, \mathrm{M}+\mathrm{Na}\right]^{+}:$458.1493, Found: 458.1496 . 


\subsection{Synthesis of compound $18^{16}$}<smiles>CC(C)(C)OC(=O)/C=C/[C@H](Nc1ccc(Cl)cc1)c1ccccc1</smiles>

(R)-17

$94 \%$ ee

$$
\begin{aligned}
& \text { 1) } \mathrm{H}_{2}, \mathrm{Pd} / \mathrm{C}(20 \mathrm{~mol} \%) \\
& \text { 2) }{ }^{t} \mathrm{BuOK}(2 \text { equiv }) \\
& \mathrm{THF}
\end{aligned}
$$

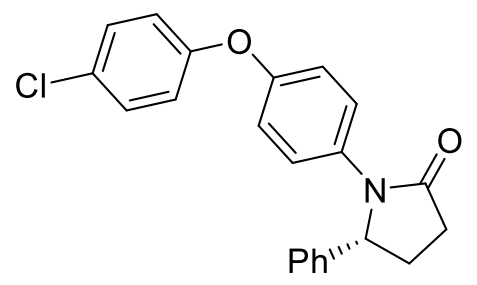

$(R)-18$

$72 \%$ yield, $91 \%$ ee

The compound $(R)-17(87 \mathrm{mg}, 0.2 \mathrm{mmol})$ was dissolved in $\mathrm{MeOH}$ and treated with $\mathrm{H}_{2}$ balloon over $\mathrm{Pd} / \mathrm{C}$ for $2 \mathrm{~h}$ at room temperature. Then ${ }^{t} \mathrm{BuOK}$ was put into this system at room temperature for $5 \mathrm{~h}$. The reaction mixture was concentrated and purified by a flash chromatography on silica gel $(\mathrm{PE} / \mathrm{EA}=5: 1 \mathrm{v} / \mathrm{v})$ to afford compound $(R)-\mathbf{1 8}(58$ mg, $72 \%$ yield). White solid, mp: $174 \sim 175^{\circ} \mathrm{C}$, TLC $R_{f}=0.21(\mathrm{PE} / \mathrm{EA}=1: 1 \mathrm{v} / \mathrm{v}), 91 \%$ ee [HPLC condition: Chiralcel OD-H column, $n$-hexane/2-propanol $=50: 50$, flow rate $=1.0 \mathrm{~mL} / \mathrm{min}$, wavelength $=210 \mathrm{~nm}, t_{\mathrm{R}}=10.64 \mathrm{~min}$ for minor isomer, $t_{\mathrm{R}}=11.32 \mathrm{~min}$ for major isomer $],[\alpha]_{\mathrm{D}}^{28}=-128.6\left(c 1.0, \mathrm{CHCl}_{3}\right) .{ }^{1} \mathrm{H} \mathrm{NMR}\left(400 \mathrm{MHz}, \mathrm{CDCl}_{3}\right) \delta 7.36$ (d, $J=7.1 \mathrm{~Hz}, 2 \mathrm{H}, \mathrm{Ar}-\mathrm{H}), 7.29$ (t, $J=7.3 \mathrm{~Hz}, 2 \mathrm{H}, \mathrm{Ar}-\mathrm{H}), 7.26-7.15(\mathrm{~m}, 4 \mathrm{H}, \mathrm{Ar}-\mathrm{H})$, 6.81-6.62 (m, 5H, Ar-H), 5.01 (d, J=14.3 Hz, 1H, CH), 4.29 (t, J = 6.3 Hz, 1H, $\mathrm{CH}_{2}$ ), 2.49-2.31 (m, 2H, $\left.\mathrm{CH}_{2}\right), 1.84-1.75\left(\mathrm{~m}, 2 \mathrm{H}, \mathrm{CH}_{2}\right) ;{ }^{13} \mathrm{C} \mathrm{NMR}\left(100 \mathrm{MHz}, \mathrm{CDCl}_{3}\right) \delta 174.9$ (1C, C=O), 157.4 (1C, Ar-C), 147.6 (1C, Ar-C), 143.1 (1C, Ar-C), 136.2 (2C, Ar-C), 132.3 (2C, Ar-C), 129.1 (2C, Ar-C), 128.4 (2C, Ar-C), 128.0 (2C, Ar-C), 126.3 (2C, Ar-C), 126.4 (1C, Ar-C), 125.2 (1C, Ar-C), 121.0 (1C, Ar-C), 60.6 (1C, C-O), 30.2 (1C, $\mathrm{CH}_{2}$ ), 28.1 (1C, $\left.\mathrm{CH}_{2}\right)$. HRMS (ESI) Calcd for $\left[\mathrm{C}_{22} \mathrm{H}_{18} \mathrm{ClNO}_{2}, \mathrm{M}+\mathrm{Na}\right]^{+}$: 386.0918, Found: 386.0921.

\subsection{Synthesis of compound 19}

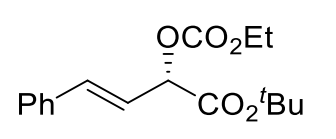

(S)-7na $97 \%$ ee<smiles>Clc1ccc(Br)c(Cl)c1</smiles>

1,10-phenanthroline (10 mol \%) $\mathrm{Pd}(\mathrm{OAc})_{2}(10 \mathrm{~mol} \%)$ $\operatorname{AgOTf}(10 \mathrm{~mol} \%)$

chlorobenzene, reflux, $10 \mathrm{~h}$

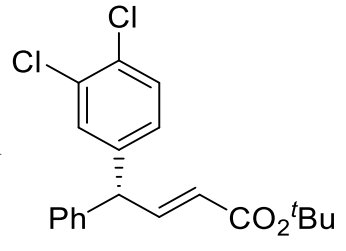

$(R)-19$

$70 \%$ yield, $96 \%$ ee

A modified literature procedure ${ }^{18}$ was used to prepare compound $(R)-\mathbf{1 9}$. Compound 7na (122 mg, 0.4 mmol), 3,4-dichlorophenylboronic acid (76 mg, 0.4 
mmol), $\mathrm{Pd}(\mathrm{OAc})_{2}(9 \mathrm{mg}, 4 \mathrm{~mol} \%)$, 1,10-phenanthroline (8 mg, $4 \mathrm{~mol} \%$ ), and AgOTf (10 mg, $4 \mathrm{~mol} \%$ ) were introduced into an oven-dried Schlenk tube in nitrogen atmosphere. After introducing $6 \mathrm{~mL}$ of chlorobenzene into the Schlenk tube, the resulted mixture was refluxed for $10 \mathrm{~h}$. The reaction was washed by water and extracted with EA. The combined organic solutions were dried over $\mathrm{MgSO}_{4}$ and filtered. The filtrate was concentrated and purified by a flash chromatography on silica gel (PE/EA $=10: 1 \mathrm{v} / \mathrm{v})$ to afford $(R)-19$. Serial number: 1y-5-106. Yellow solid, mp: $117-118^{\circ} \mathrm{C}$, TLC $R_{f}=0.66(\mathrm{PE} / \mathrm{EA}=10: 1 \mathrm{v} / \mathrm{v}), 70 \%$ yield, $96 \%$ ee [HPLC condition: Chiralcel OJ3 column, $n$-hexane/2-propanol $=98: 2$, flow rate $=0.7 \mathrm{ml} / \mathrm{min}$, wavelength $=210 \mathrm{~nm}$, $t_{\mathrm{R}}=5.07 \mathrm{~min}$ for minor isomer, $t_{\mathrm{R}}=5.50 \mathrm{~min}$ for major isomer, $[\alpha]_{\mathrm{D}}^{25}=57.92(c 1.0$, $\left.\mathrm{CHCl}_{3}\right) .{ }^{1} \mathrm{H}$ NMR $\left(400 \mathrm{MHz}, \mathrm{CDCl}_{3}\right) \delta 7.49$ - 7.45 (m, 2H, Ar-H), 7.41 - 7.38 (m, 2H, Ar-H), $7.30-7.25$ (m, 3H, Ar-H), $7.18-7.15$ (m, 1H, Ar-H), 6.82 (s, 1H, CH=), 3.79 $(\mathrm{s}, 1 \mathrm{H}, \mathrm{CH}=, 1 \mathrm{H}, \mathrm{CH}), 1.29\left(\mathrm{~s}, 9 \mathrm{H}, \mathrm{C}\left(\mathrm{CH}_{3}\right)_{3}\right) .{ }^{13} \mathrm{C} \mathrm{NMR}\left(100 \mathrm{MHz}, \mathrm{CDCl}_{3}\right) \delta 164.1(1 \mathrm{C}$, $\mathrm{C}=\mathrm{O}), 154.9$ (1C, Ar-C), 150.2 (1C, Ar-C), 136.3 (1C, $\mathrm{CH}=), 135.0$ (1C, Ar-C), 131.3 (1C, Ar-C), 130.3 (1C, Ar-C), 128.8 (1C, Ar-C), 128.7 (1C, Ar-C), 128.6 (1C, Ar-C), 128.2 (1C, Ar-C), 128.1 (1C, Ar-C), 127.9 (1C, Ar-C), 125.7 (1C, Ar-C), 125.0 (1C, $\mathrm{CH}=), 80.1(1 \mathrm{C}, \mathrm{C}-\mathrm{O}), 43.5(1 \mathrm{C}, \mathrm{CH}), 28.2\left(3 \mathrm{C}, \mathrm{C}\left(\underline{\mathrm{CH}}_{3}\right)_{3}\right)$. HRMS (ESI) Calcd for $\left[\mathrm{C}_{20} \mathrm{H}_{20} \mathrm{Cl}_{2} \mathrm{O}_{2}, \mathrm{M}+\mathrm{Na}\right]^{+}:$385.0733, Found: 385.0737.

\subsection{Synthesis of compound 20}

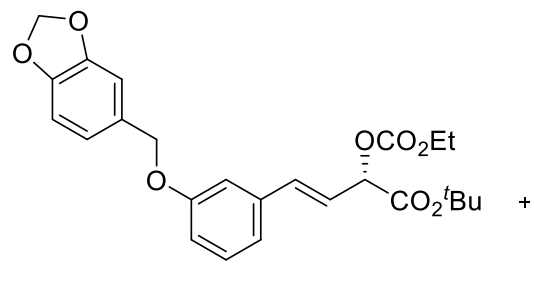

7 ta $(95 \%$ ee $)$
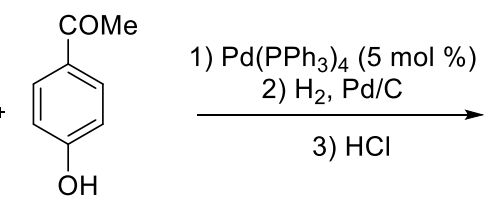

3) $\mathrm{HCl}$

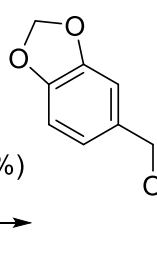

$(90 \%$ yield, $94 \%$ ee $)$

A modified literature procedure ${ }^{17}$ was used to prepare compound 20. The compound 7ta (91 mg, $0.2 \mathrm{mmol}$ ), 1-(4-hydroxyphenyl)ethan-1-one (33 mg, $0.24 \mathrm{mmol}$ ) and $\mathrm{Pd}\left(\mathrm{PPh}_{3}\right)_{4}(12 \mathrm{mg}, 5 \mathrm{~mol} \%)$ were introduced into an oven-dried Schlenk tube in nitrogen atmosphere. After introducing $3 \mathrm{~mL}$ of DCM into the Schlenk tube, the resulted mixture was reacted for $8 \mathrm{~h}$. The reaction was washed by water and extracted with EA. The combined organic solutions were dried over $\mathrm{MgSO}_{4}$ and filtered. The 
filtrate was concentrated and purified by a flash chromatography on silica gel (PE/EA $=10: 1 \mathrm{v} / \mathrm{v}$ ) to afford the etherification product as a pale yellow oil. This oil product was dissolved in $\mathrm{MeOH}$ and treated with $\mathrm{H}_{2}$ balloon over $\mathrm{Pd} / \mathrm{C}$ for $2 \mathrm{~h}$ at room temperature. Then $6 \mathrm{~N} \mathrm{HCl}(2 \mathrm{~mL})$ was put into this system at room temperature and stirred for $4 \mathrm{~h}$. The reaction mixture was concentrated and purified by a flash chromatography on silica gel to afford compound $\mathbf{2 0}$ as a yellow solid (81 mg, 90\% yield). Serial number: ly-3182-2, $\mathrm{TLC} \mathrm{R}_{f}=0.21\left(\mathrm{PE} / \mathrm{Et}_{2} \mathrm{O}=1: 1 \mathrm{v} / \mathrm{v}\right)$, white solid, $\mathrm{mp}: 186 \sim 188^{\circ} \mathrm{C}, 94 \%$ ee [HPLC condition: Chiralpak AD-H column, $n$-hexane/2-propanol $=50: 50$, flow rate $=1$ $\mathrm{mL} / \mathrm{min}$, wavelength $=210 \mathrm{~nm}, t_{\mathrm{R}}=5.26 \mathrm{~min}$ for major isomer, $t_{\mathrm{R}}=6.34 \mathrm{~min}$ for minor isomer, $[\alpha]_{\mathrm{D}}{ }^{25}=79.82\left(c 1.0, \mathrm{CHCl}_{3}\right) .{ }^{1} \mathrm{H} \mathrm{NMR}\left(400 \mathrm{MHz}, \mathrm{CDCl}_{3}\right) \delta 7.68-7.21(\mathrm{~m}$, 6H, Ar-H), 6.97 (s, 1H, Ar-H), $6.82-6.59$ (m, 4H, Ar-H), 5.97 (s, 2H, $\left.\mathrm{OCH}_{2} \mathrm{O}\right), 5.16$ (s, 2H, $\left.\mathrm{CH}_{2}\right), 4.83$ (dd, $\left.J=8.5,5.6 \mathrm{~Hz}, 1 \mathrm{H}, \mathrm{CH}\right), 2.5\left(\mathrm{~s}, 3 \mathrm{H}, \mathrm{CH}_{3}\right), 2.33-2.17$ (m, 4H, $\left.\mathrm{CH}_{2}\right) .{ }^{13} \mathrm{C}$ NMR $\left(100 \mathrm{MHz}, \mathrm{CDCl}_{3}\right) \delta 197.0(1 \mathrm{C}, \mathrm{C}=\mathrm{O}), 160.7$ (1C, Ar-C-O), 157.3 (1C, Ar-C), 149.0 (1C, Ar-C), 147.7 (1C, Ar-C), 139.5 (1C, Ar-C), 137.3 (2C, Ar-C), 128.4 (2C, Ar-C), 127.3 (2C, Ar-C), 125.6 (2C, Ar-C), 124.5 (1C, CH=), 123.2 (2C, Ar-C), 118.6 (1C, Ar-C), 114.6 (1C, Ar-C), 100.9 (1C, $\left.\mathrm{OCH}_{2} \mathrm{O}\right), 83.8$ (1C, $\left.\mathrm{CH}\right), 71.2(1 \mathrm{C}$, $\left.\mathrm{OCH}_{2}\right), 31.5\left(1 \mathrm{C}, \mathrm{CH}_{2}\right), 31.0\left(1 \mathrm{C}, \mathrm{CH}_{2}\right), 26.5\left(1 \mathrm{C}, \mathrm{CH}_{3}\right)$. 


\section{NMR Spectra for New Compounds}

\section{2-Phenylpropan-2-yl 2-diazooctanoate (1a)}
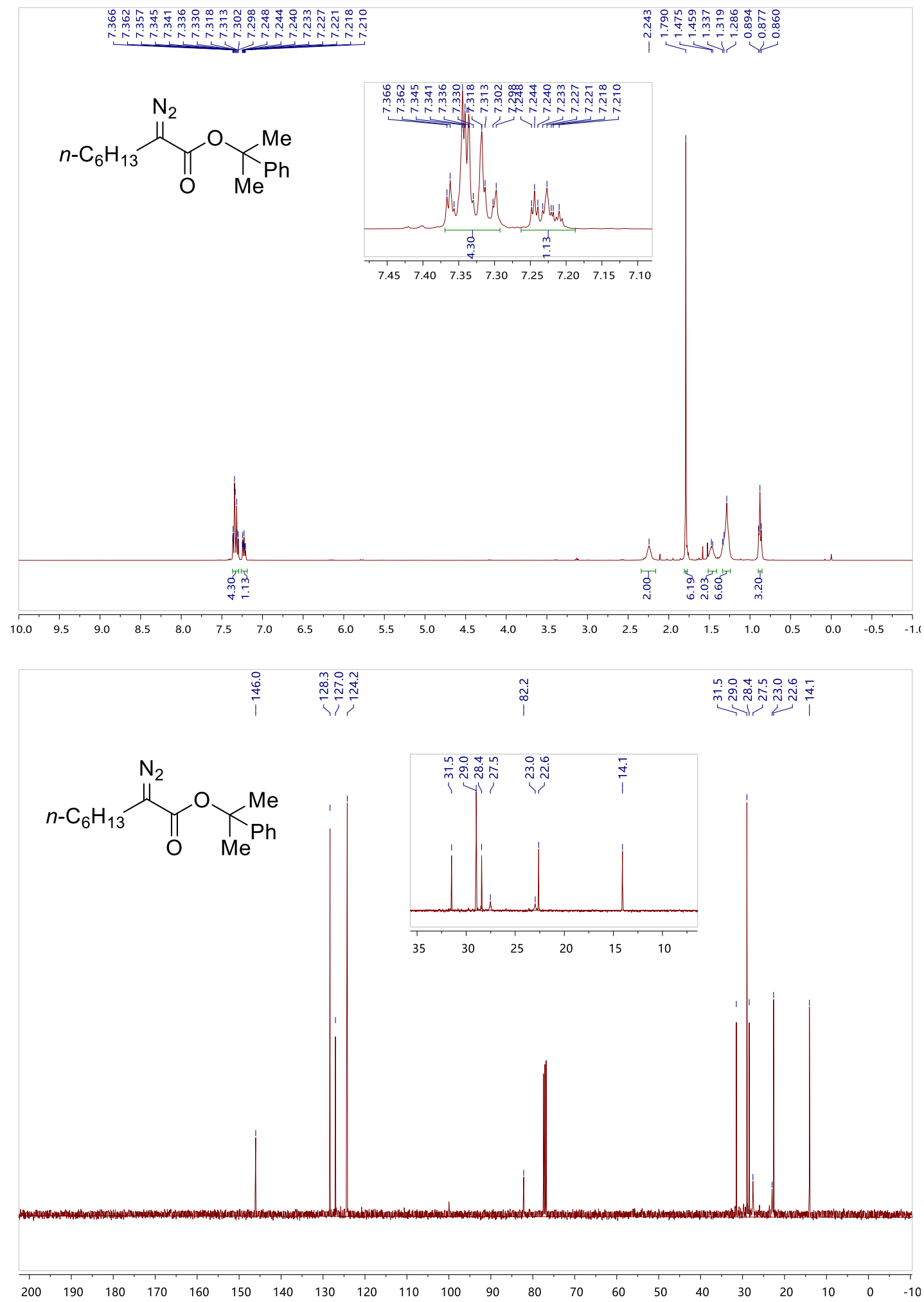
2-Phenylpropan-2-yl 2-diazobutanoate (1b)
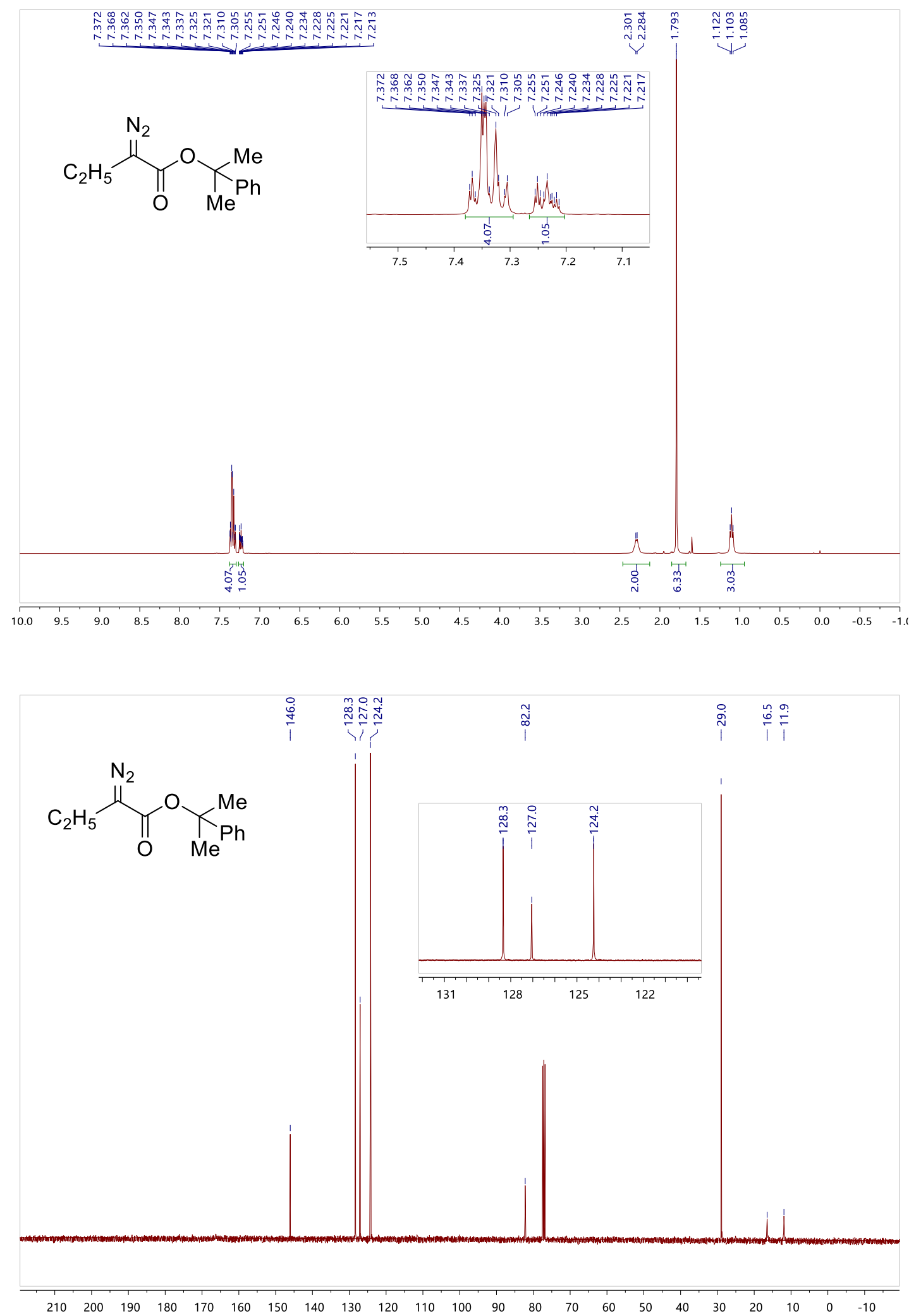
2-Phenylpropan-2-yl 2-diazohexanoate (1c)
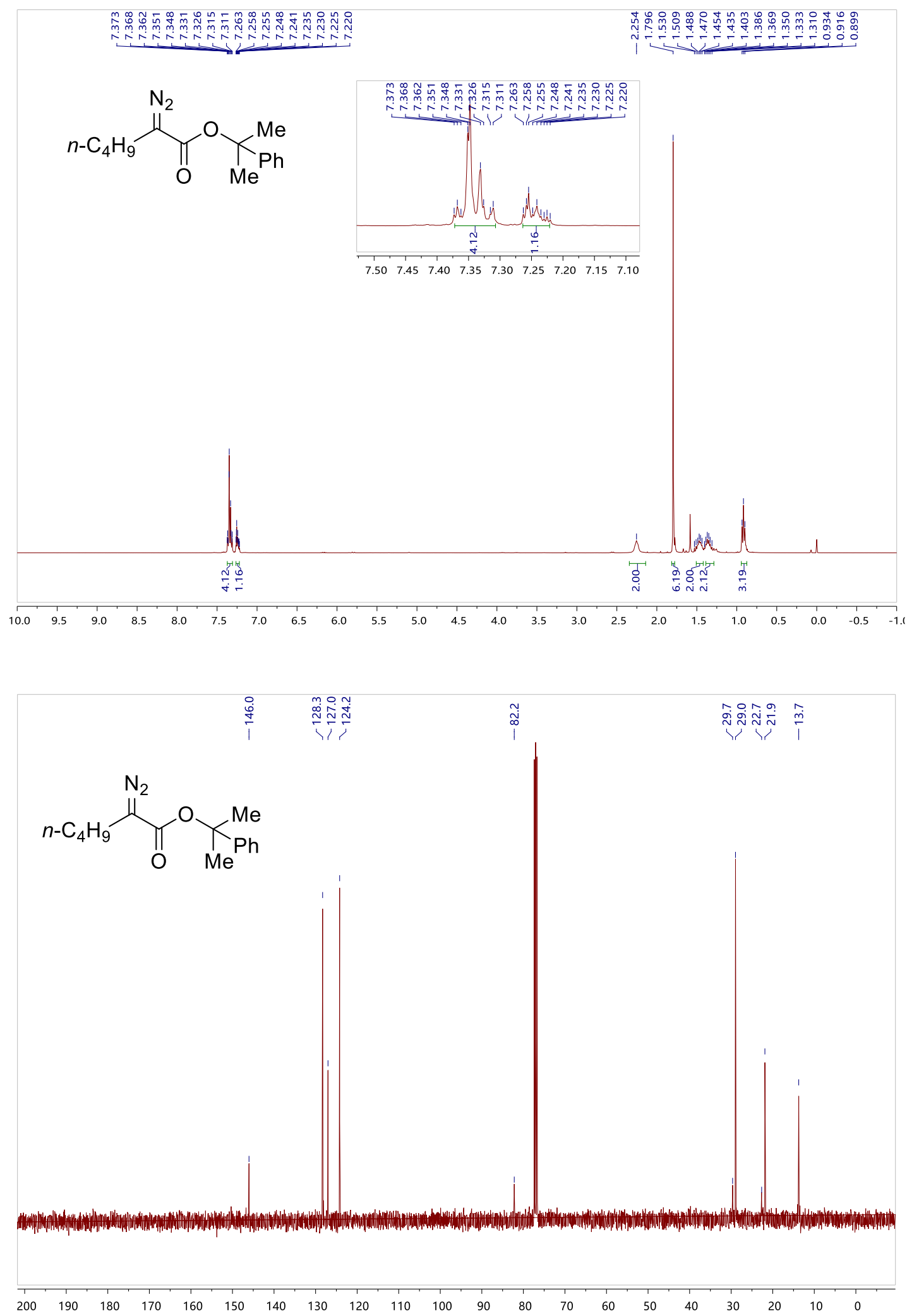
2-Phenylpropan-2-yl 2-diazotetradecanoate (1d)
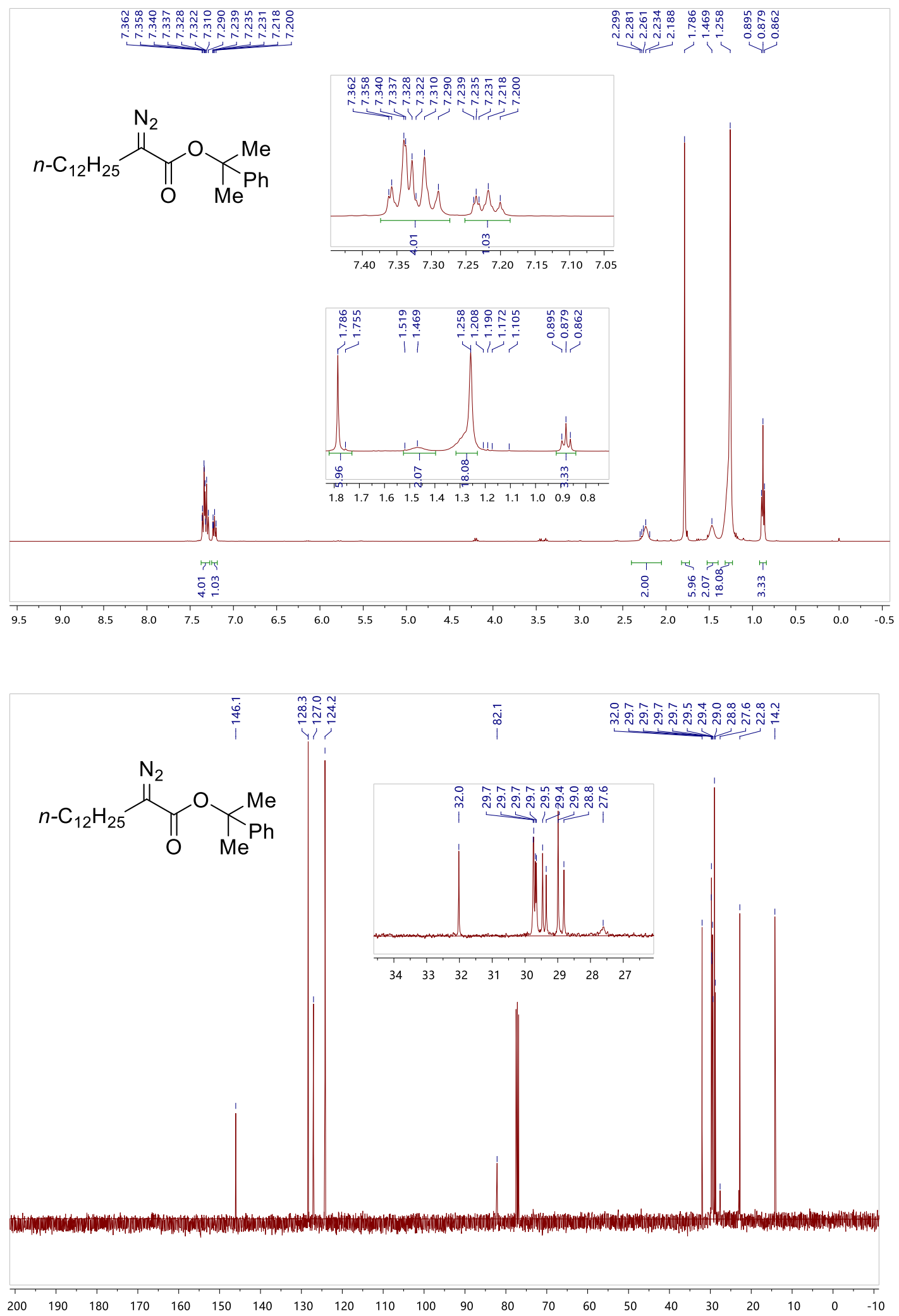
2-Phenylpropan-2-yl 2-diazo-5-methylhexanoate (1e)
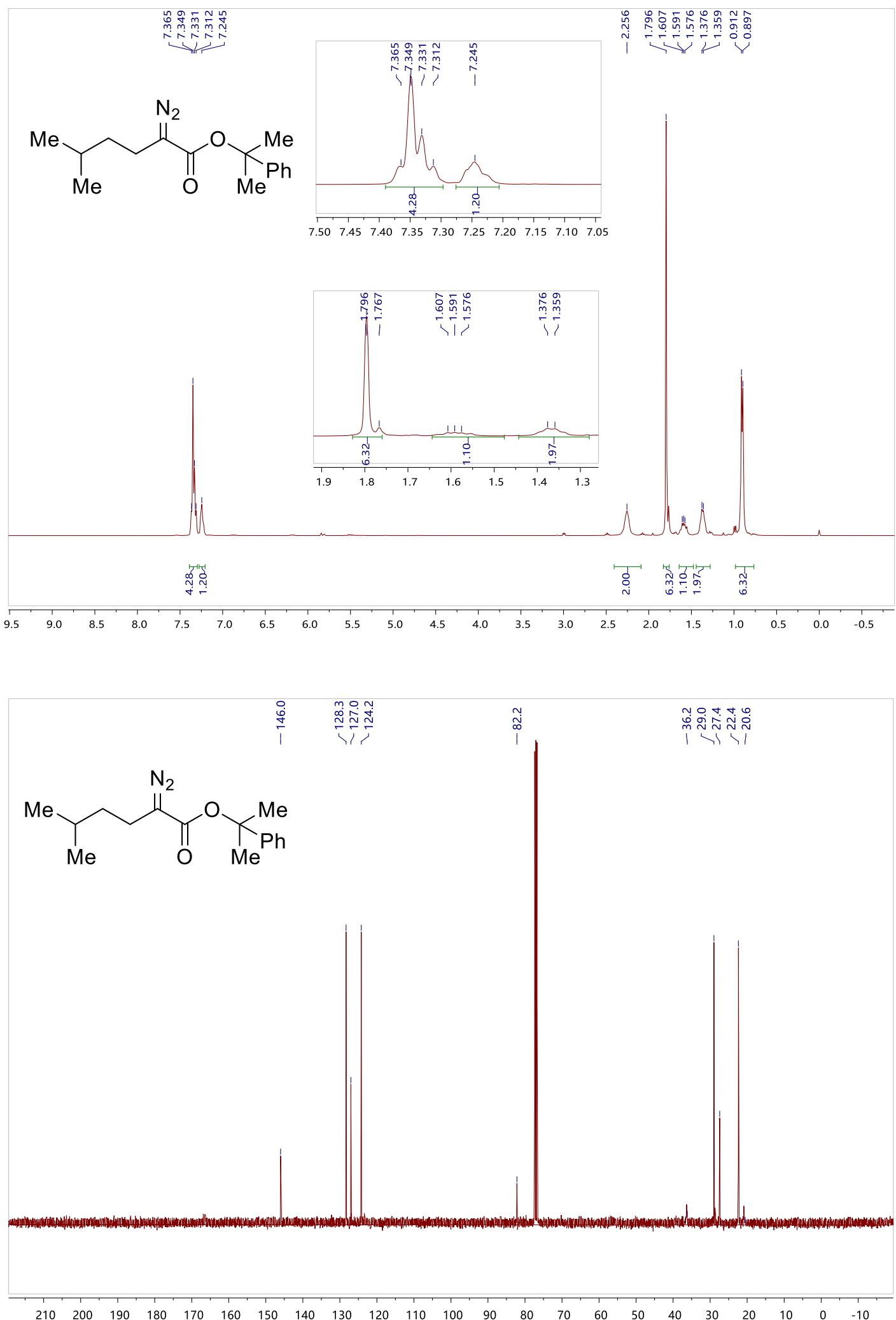
2-Phenylpropan-2-yl 2-diazo-5,5-dimethylhexanoate (1f)
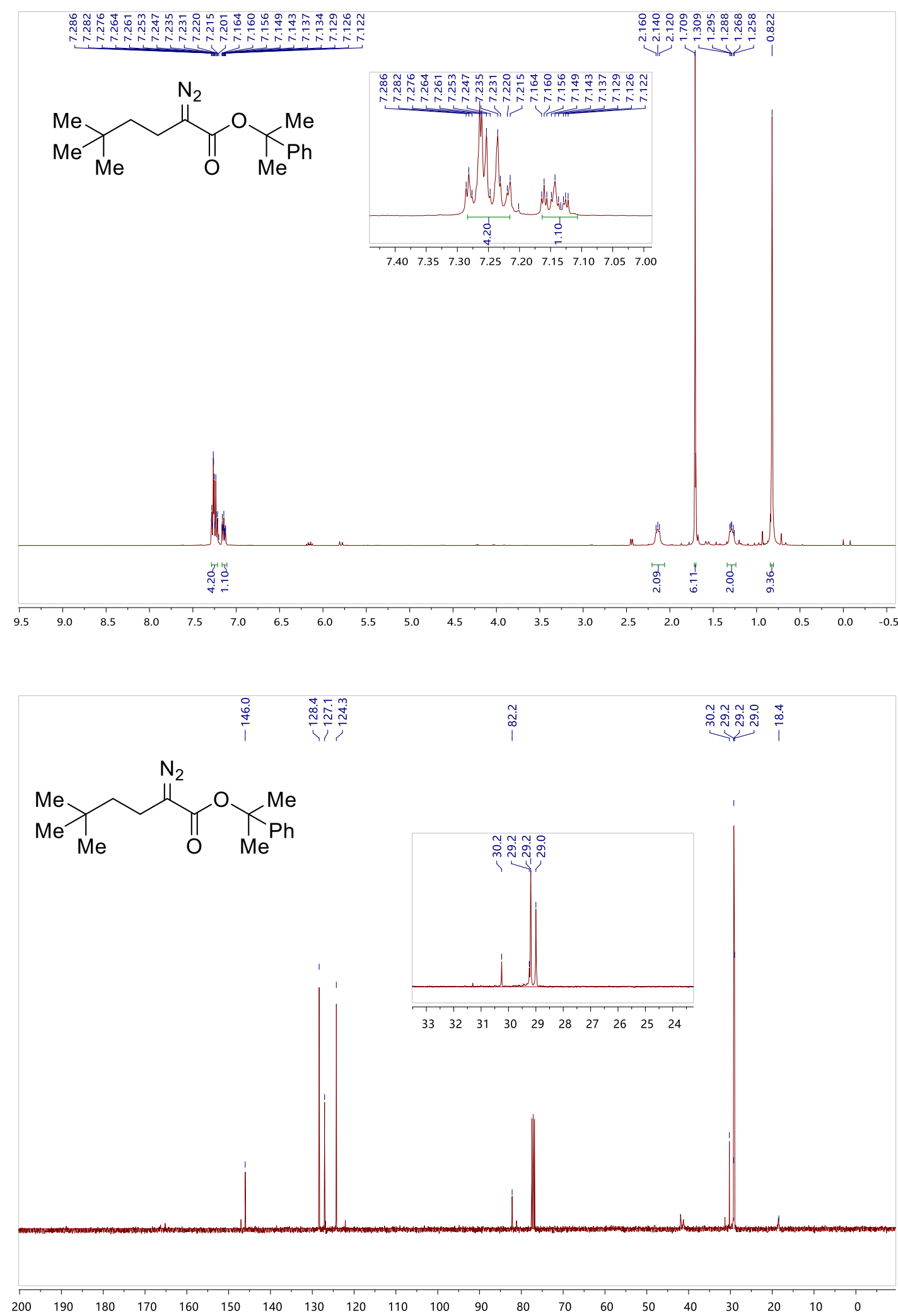
2-Phenylpropan-2-yl 4-cyclohexyl-2-diazobutanoate (1g)
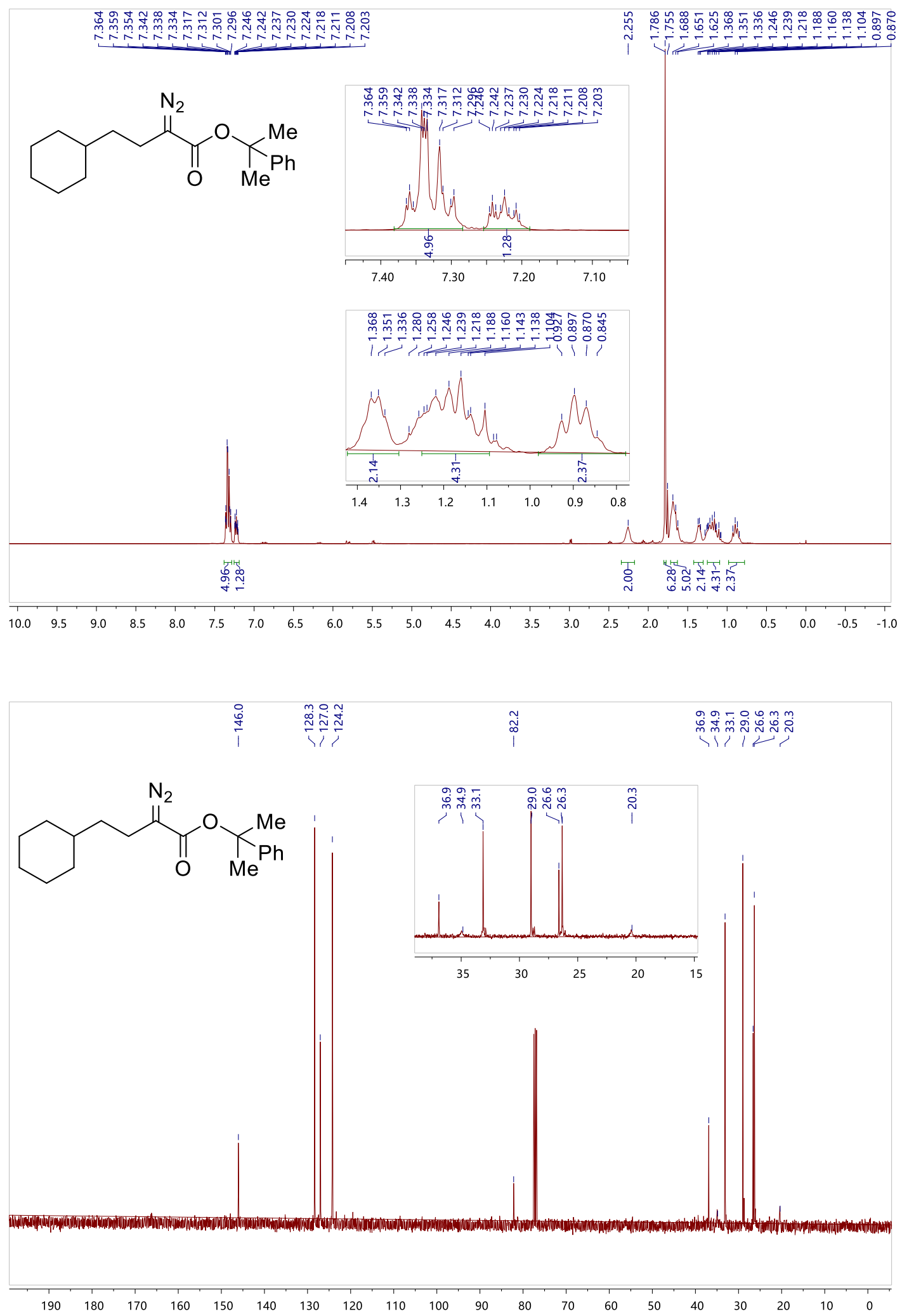
2-Phenylpropan-2-yl 3-cyclopropyl-2-diazopropanoate (1h)
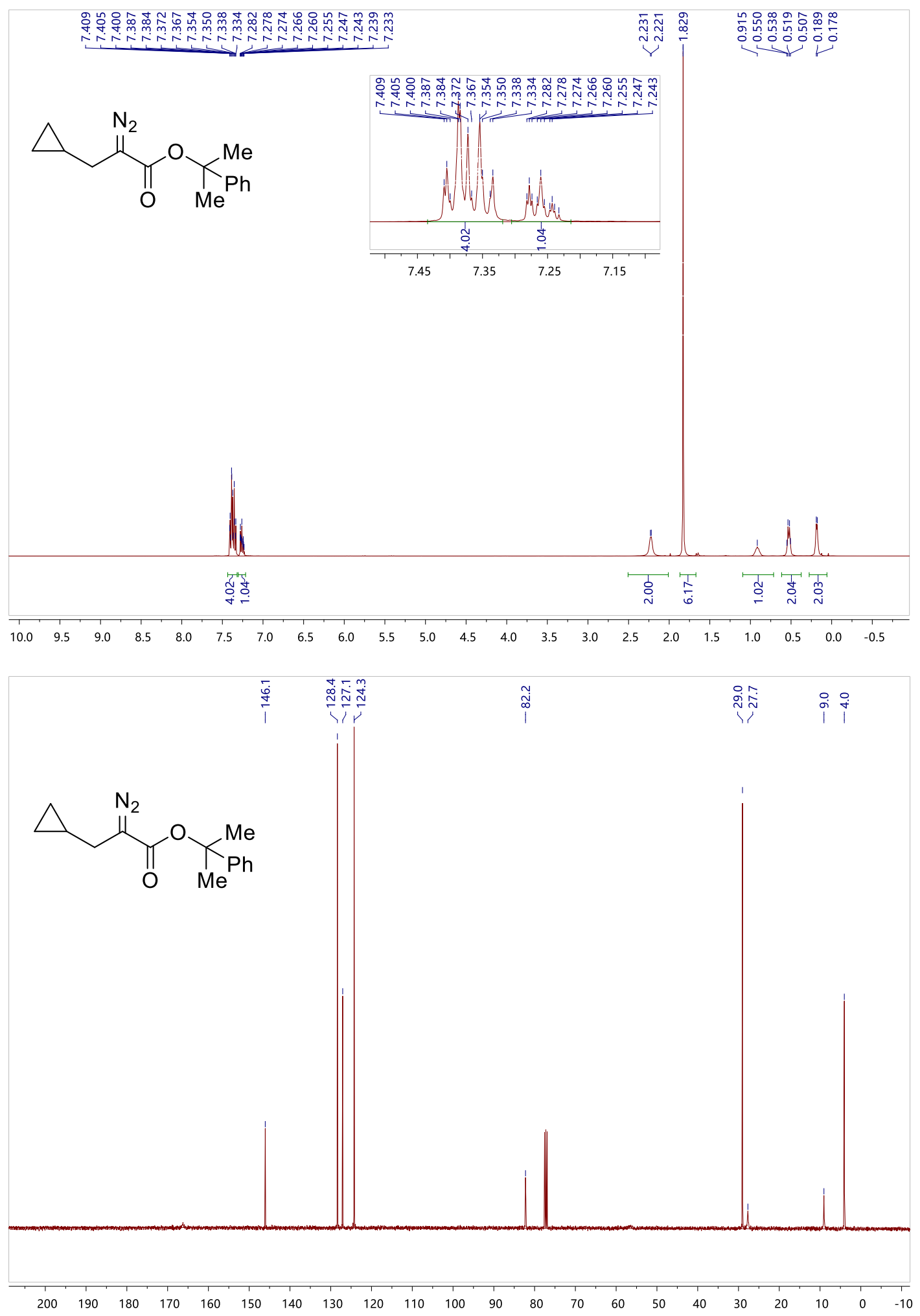
2-Phenylpropan-2-yl 2-diazo-4-methylpentanoate (1i)
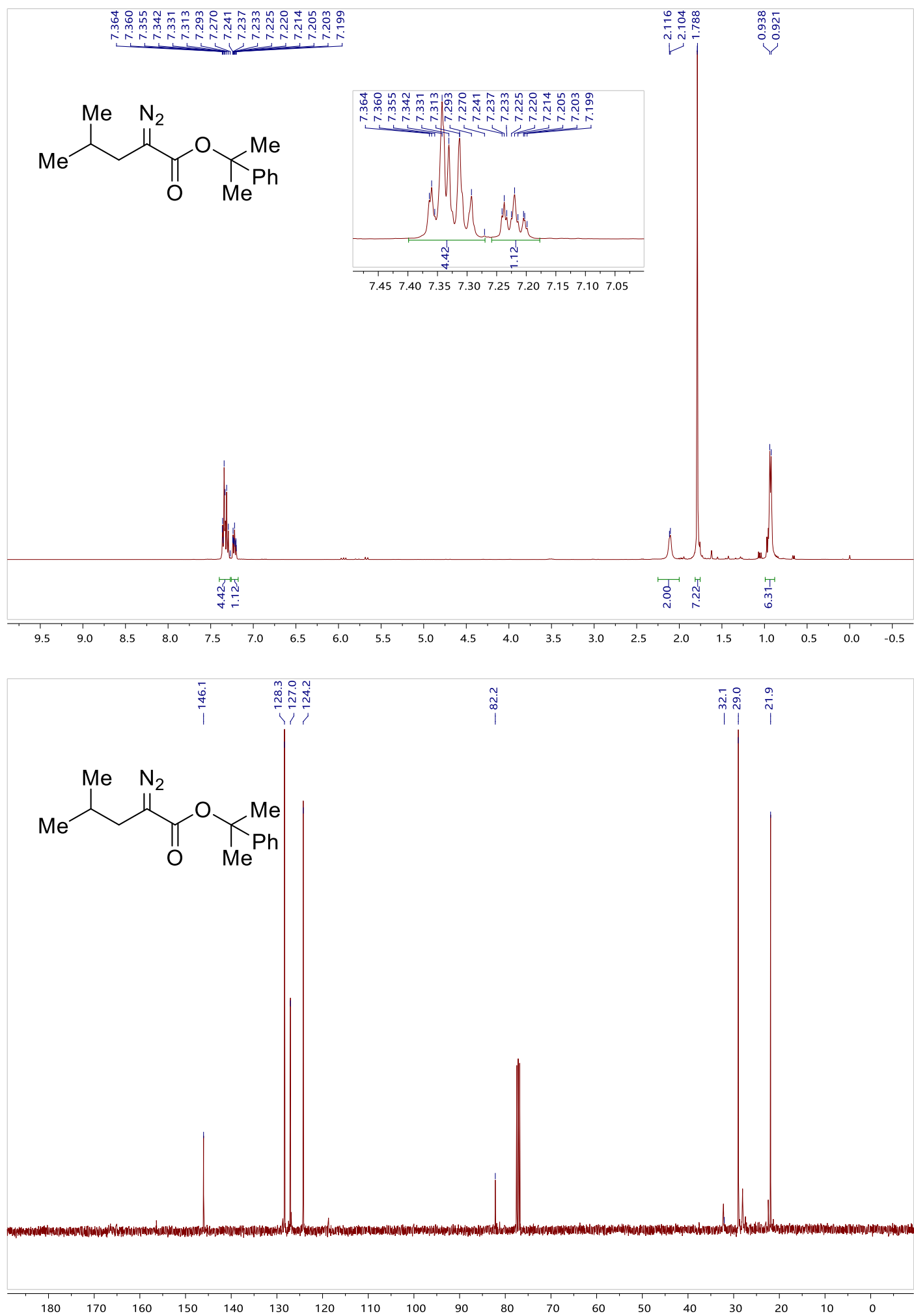
2-Phenylpropan-2-yl 2-diazo-3-phenylpropanoate (1j)
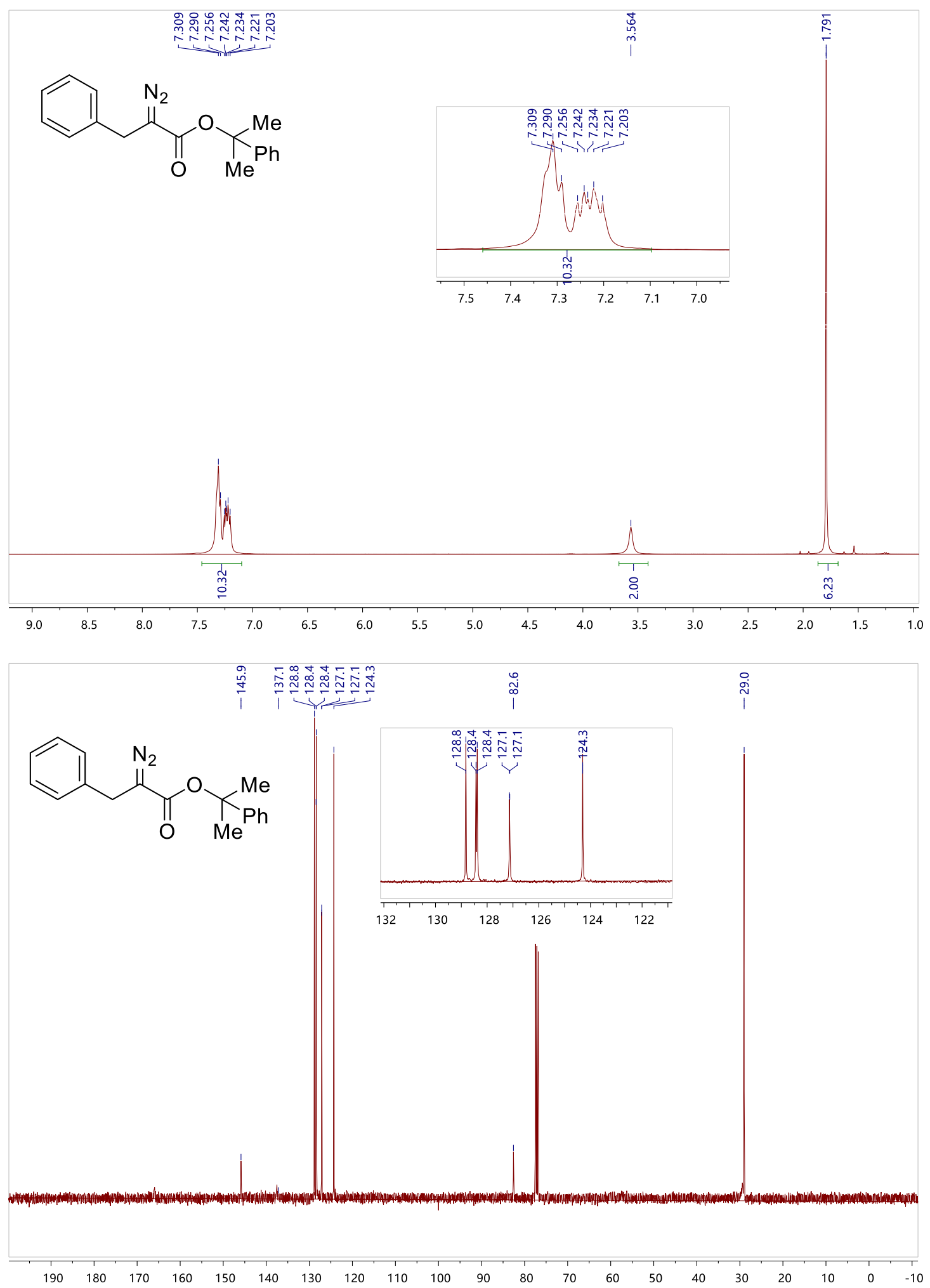
2-Phenylpropan-2-yl 2-diazo-4-phenylbutanoate (1k)
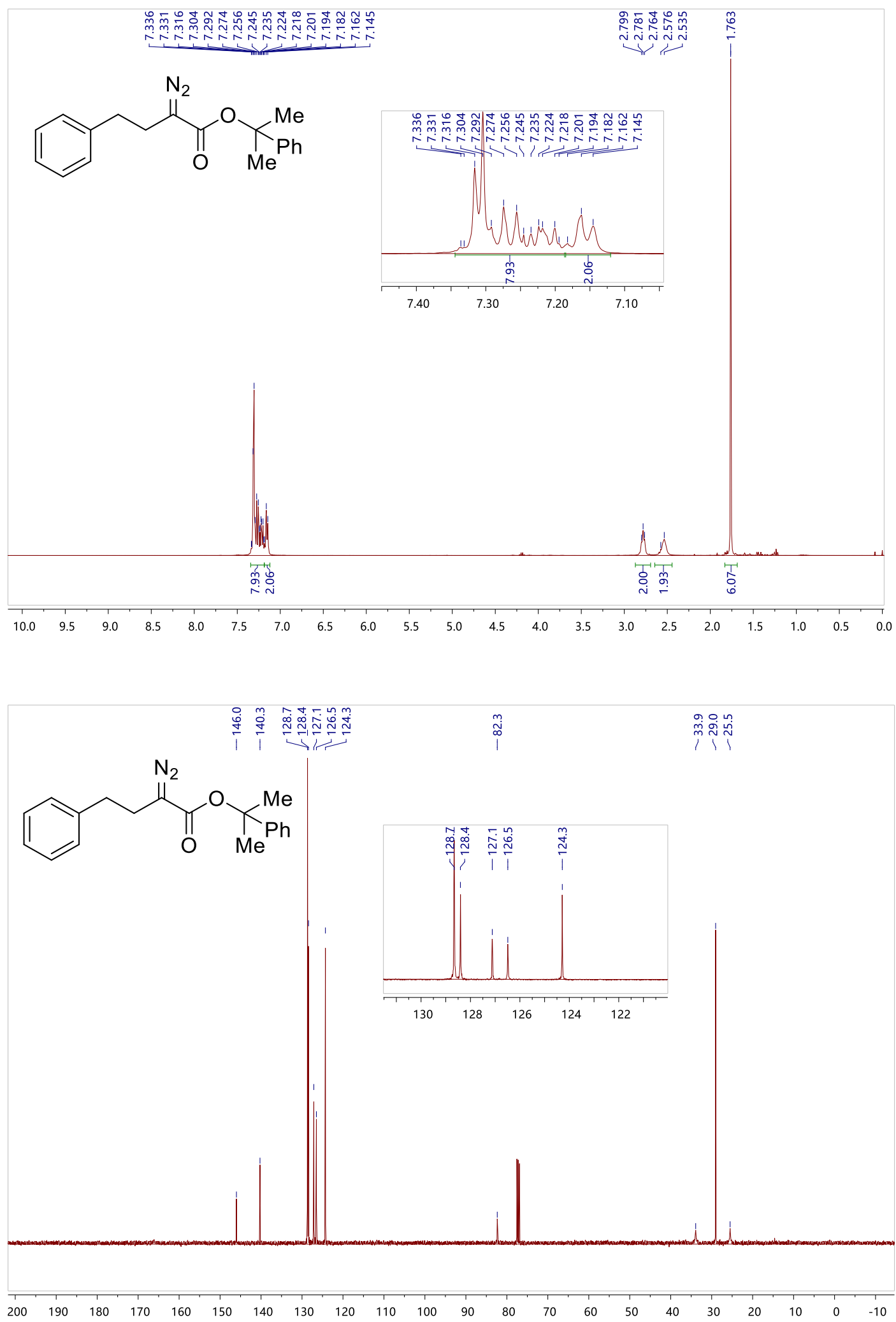
2-Phenylpropan-2-yl 2-diazo-5-phenylpentanoate (11)
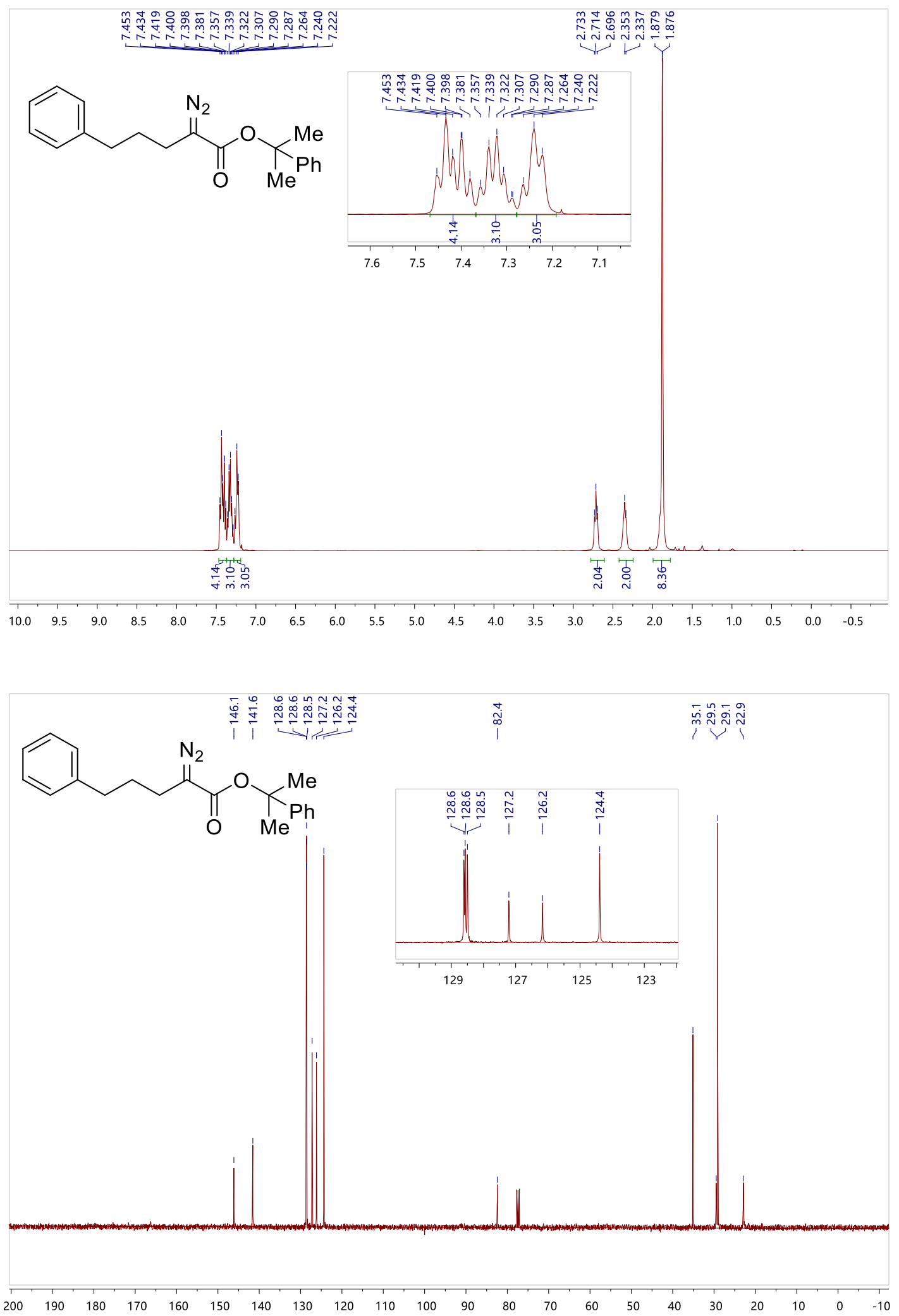
2-Phenylpropan-2-yl 2-diazopent-4-enoate (1 $\mathrm{m})$
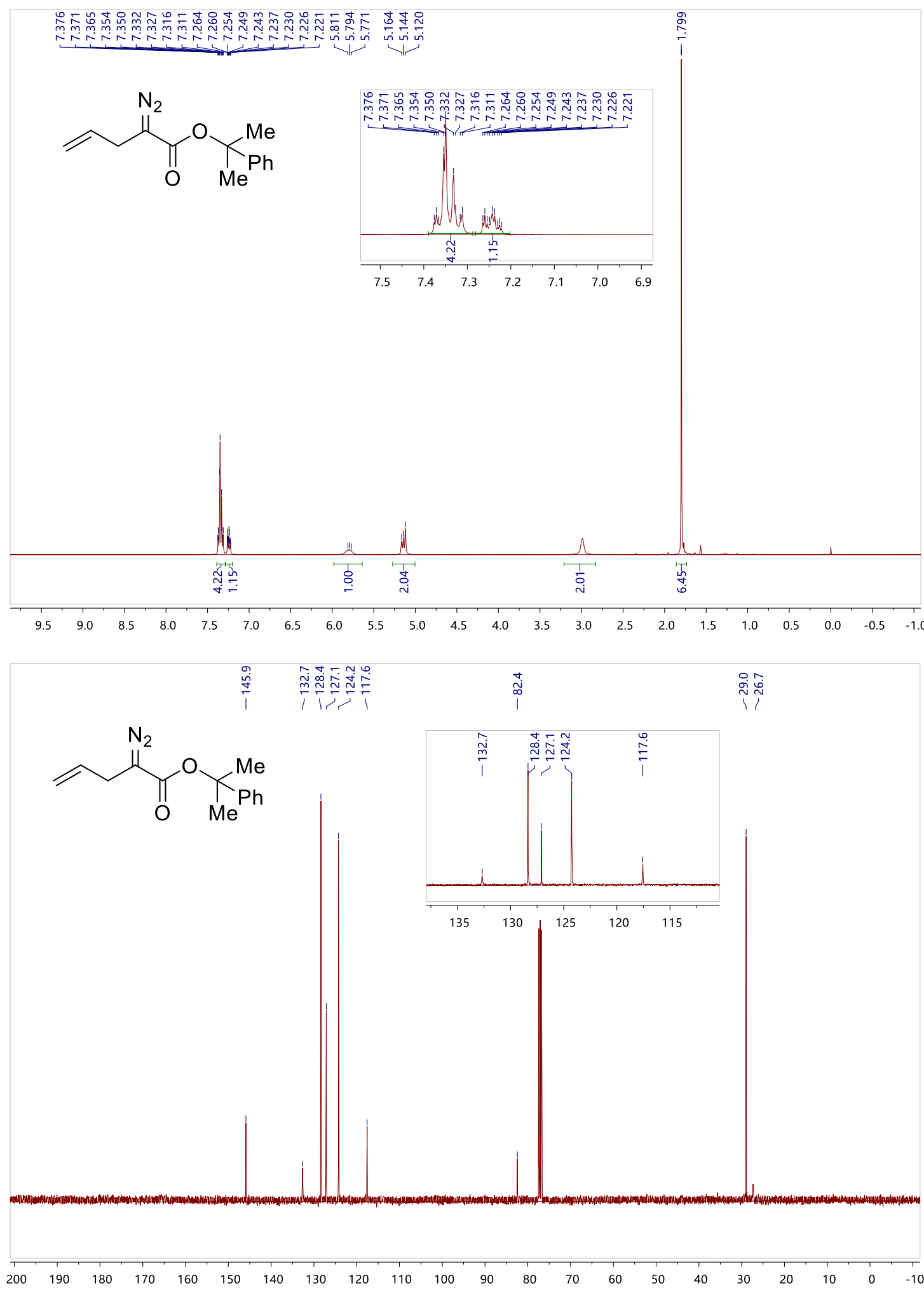
2-Phenylpropan-2-yl 2-diazohex-5-enoate (1n)
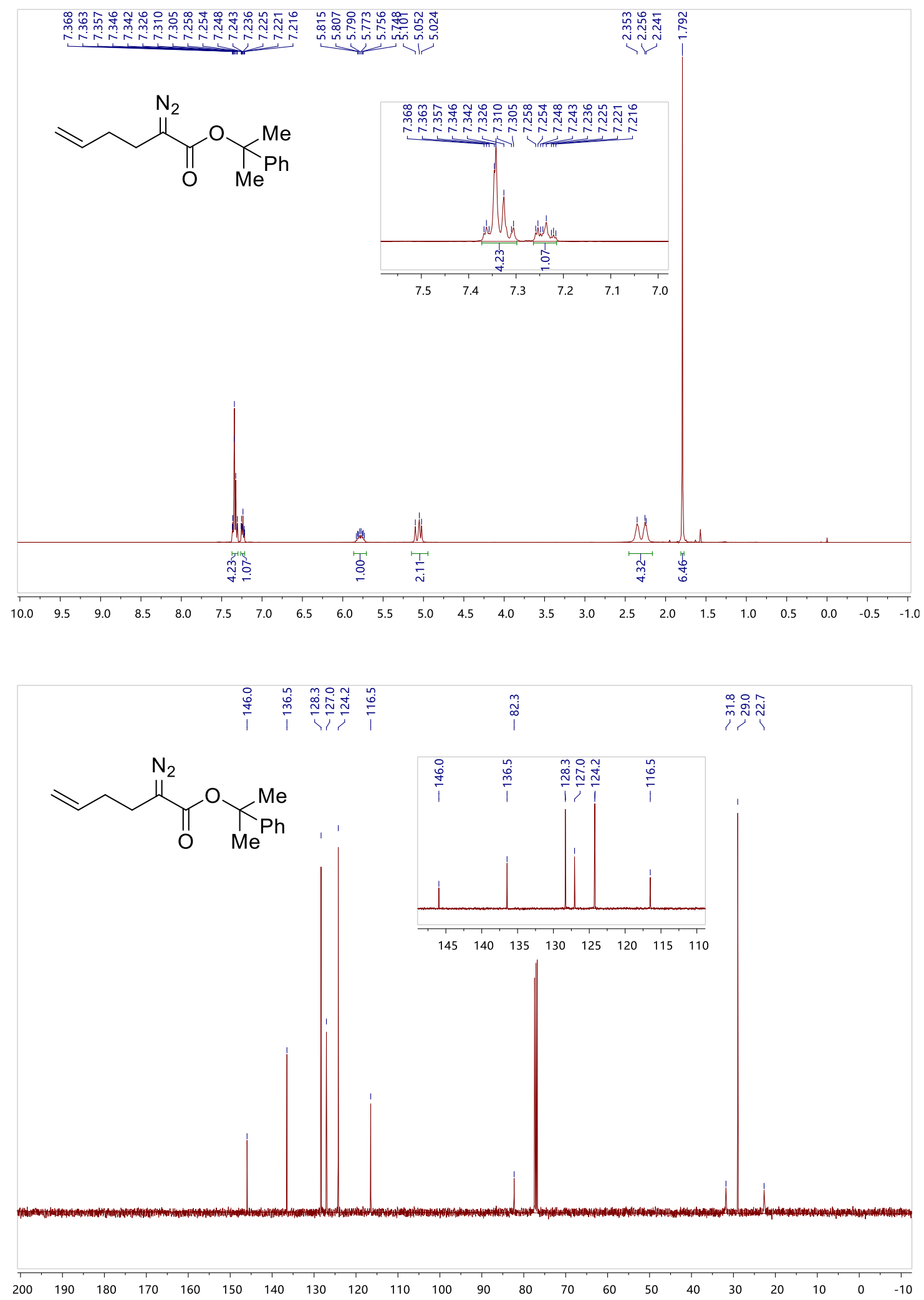
2-Phenylpropan-2-yl 2-diazooct-7-enoate (10)
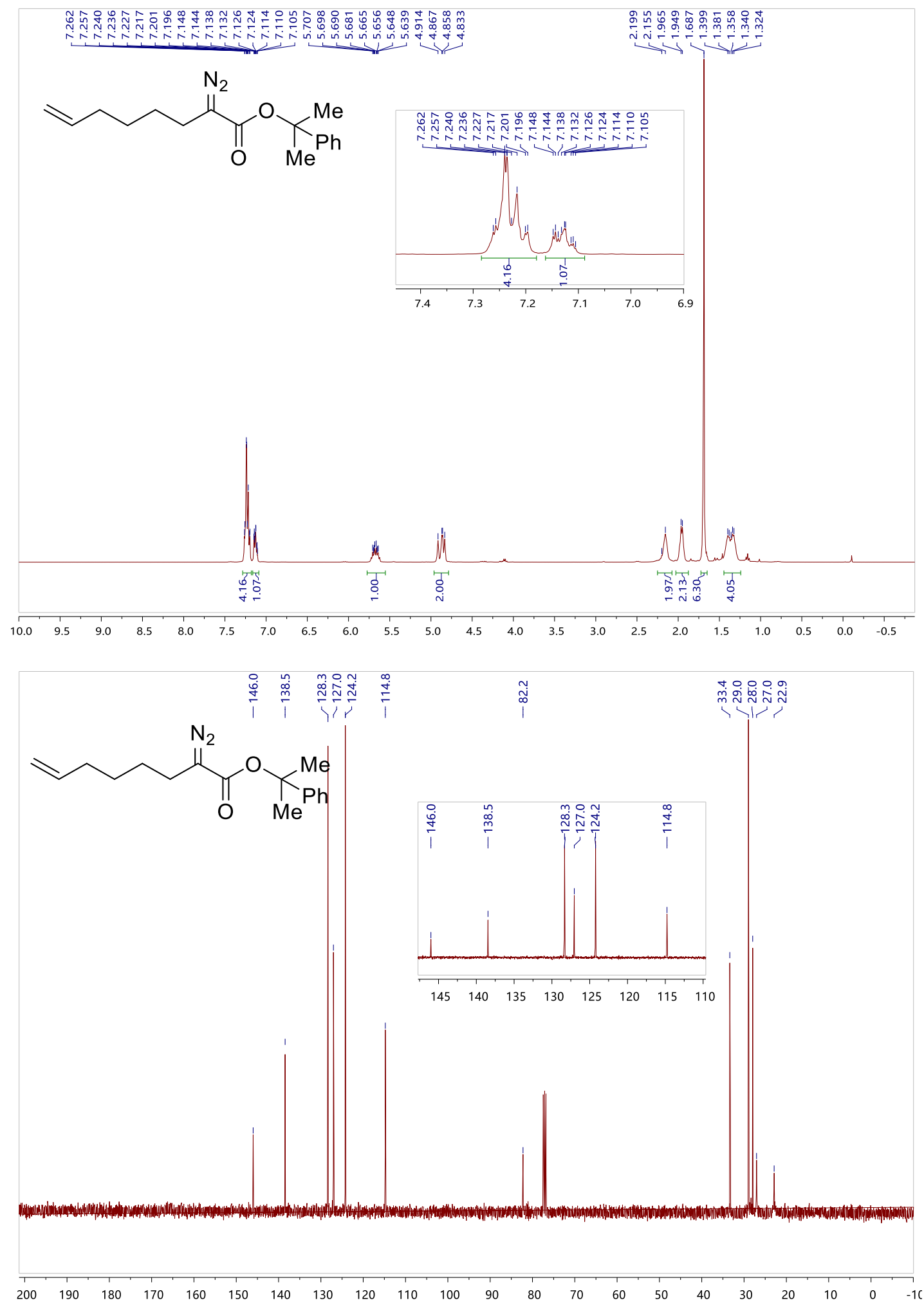
2-Phenylpropan-2-yl 2-diazohex-5-ynoate (1p)
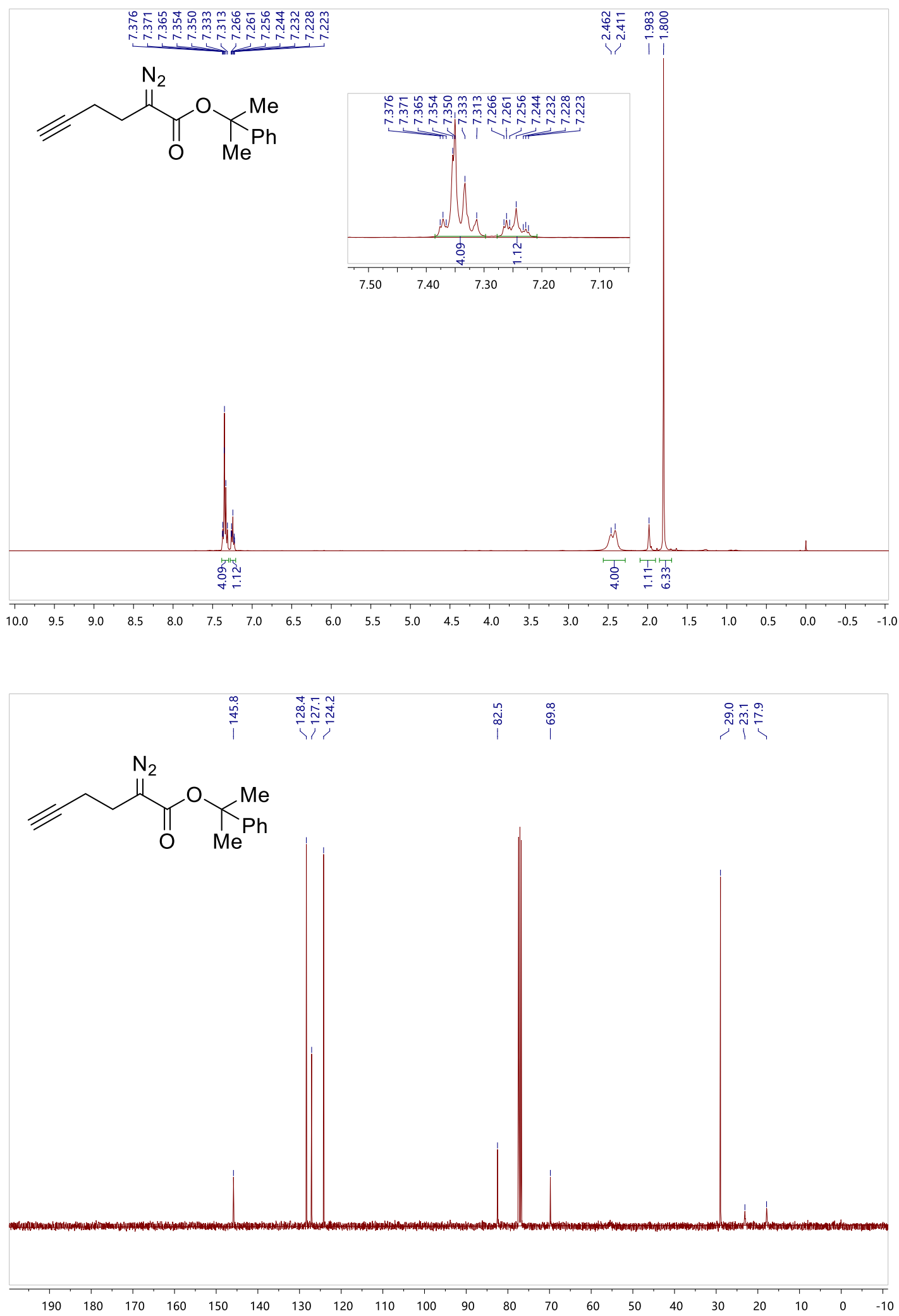
2-Phenylpropan-2-yl 2-diazo-5-methoxypentanoate (1q)
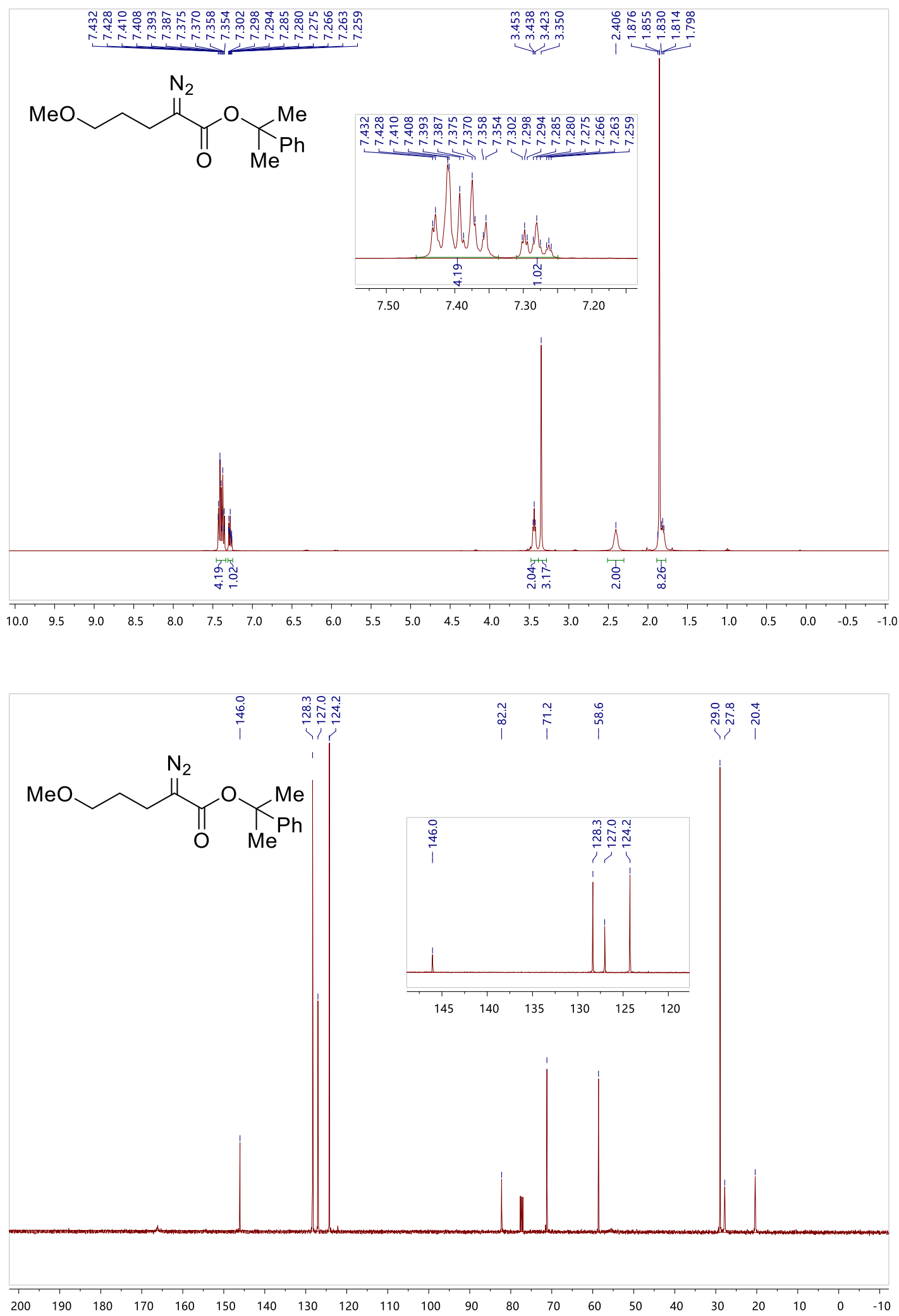
6-Ethyl 1-(2-phenylpropan-2-yl) 2-diazohexanedioate (1r)
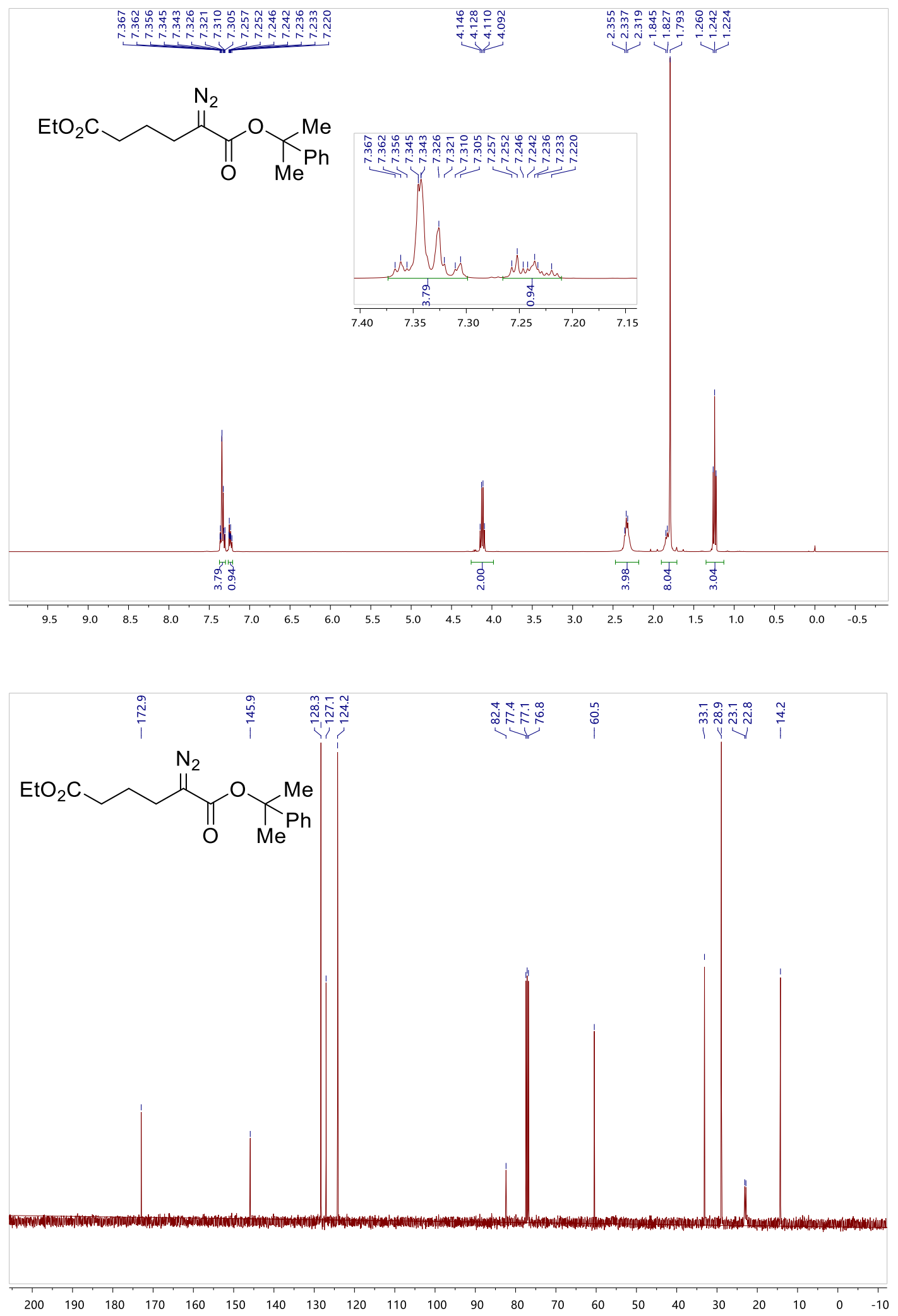
2-Phenylpropan-2-yl 5-cyano-2-diazopentanoate (1s)
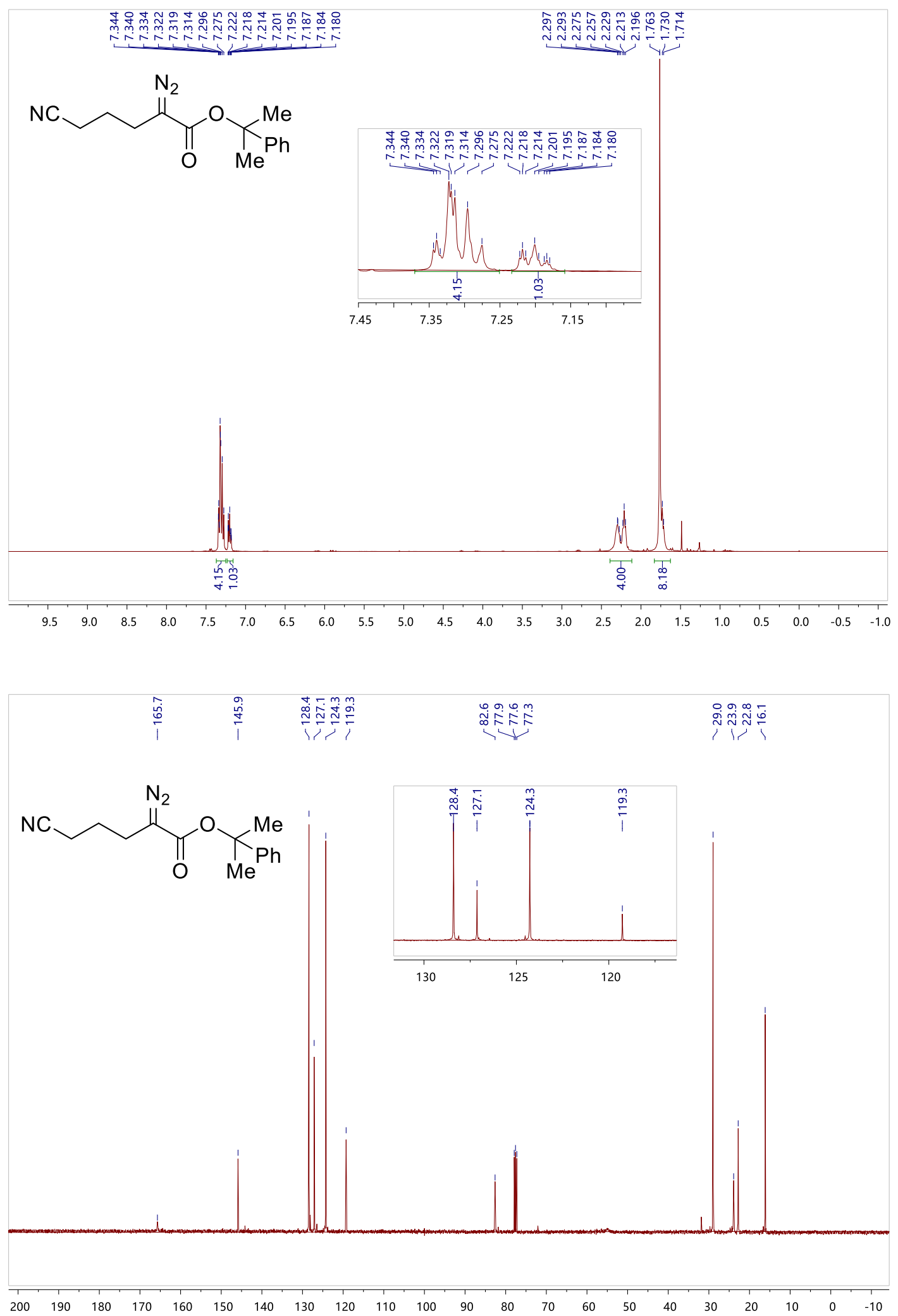
2-Phenylpropan-2-yl 5-((tert-butyldimethylsilyl)oxy)-2-diazopentanoate (1t)
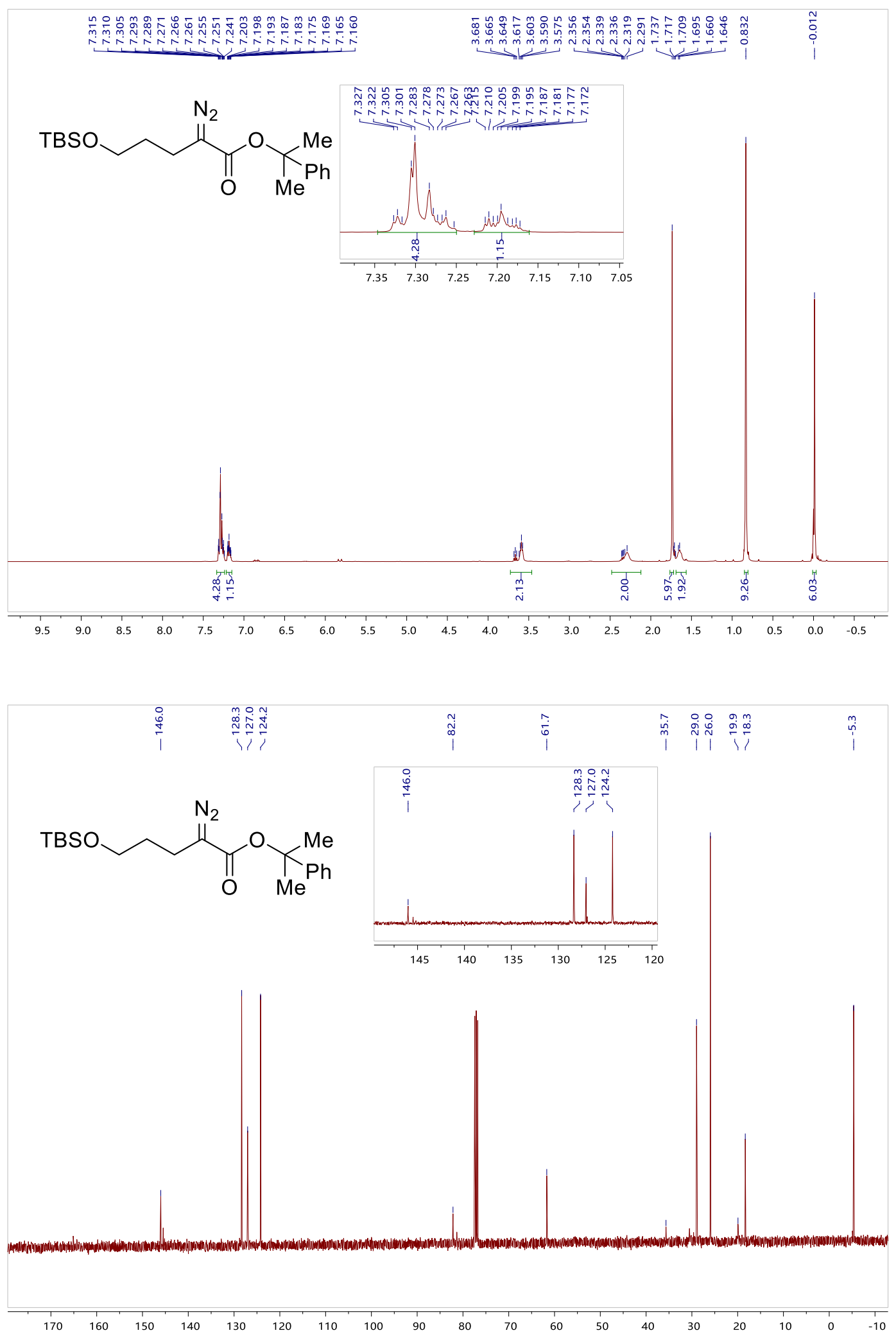
(E)-tert-Butyl 2-diazo-4-phenylpent-3-enoate (6a)
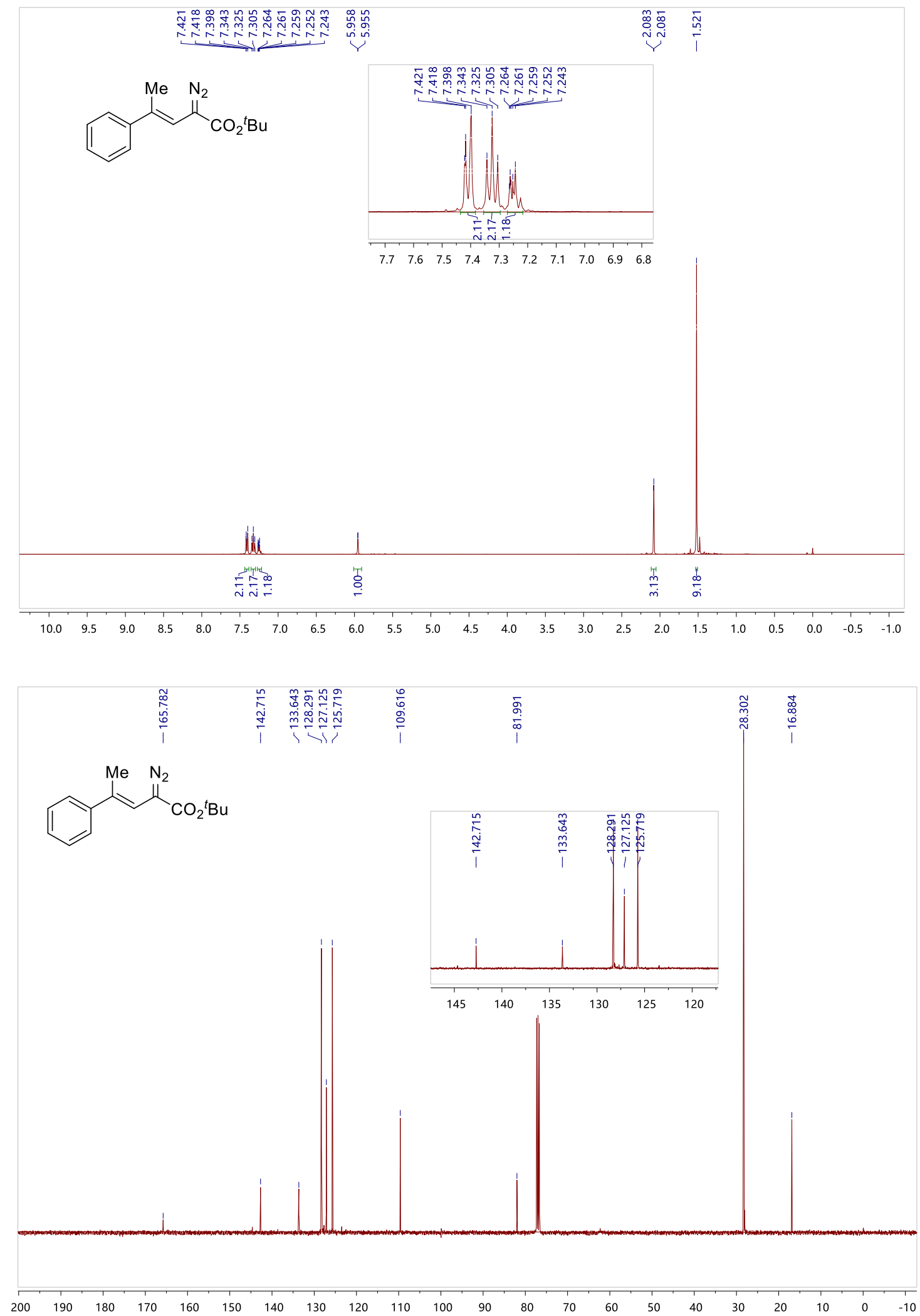
(E)-tert-Butyl 4-(m-tolyl)-2-diazopent-3-enoate (6b)
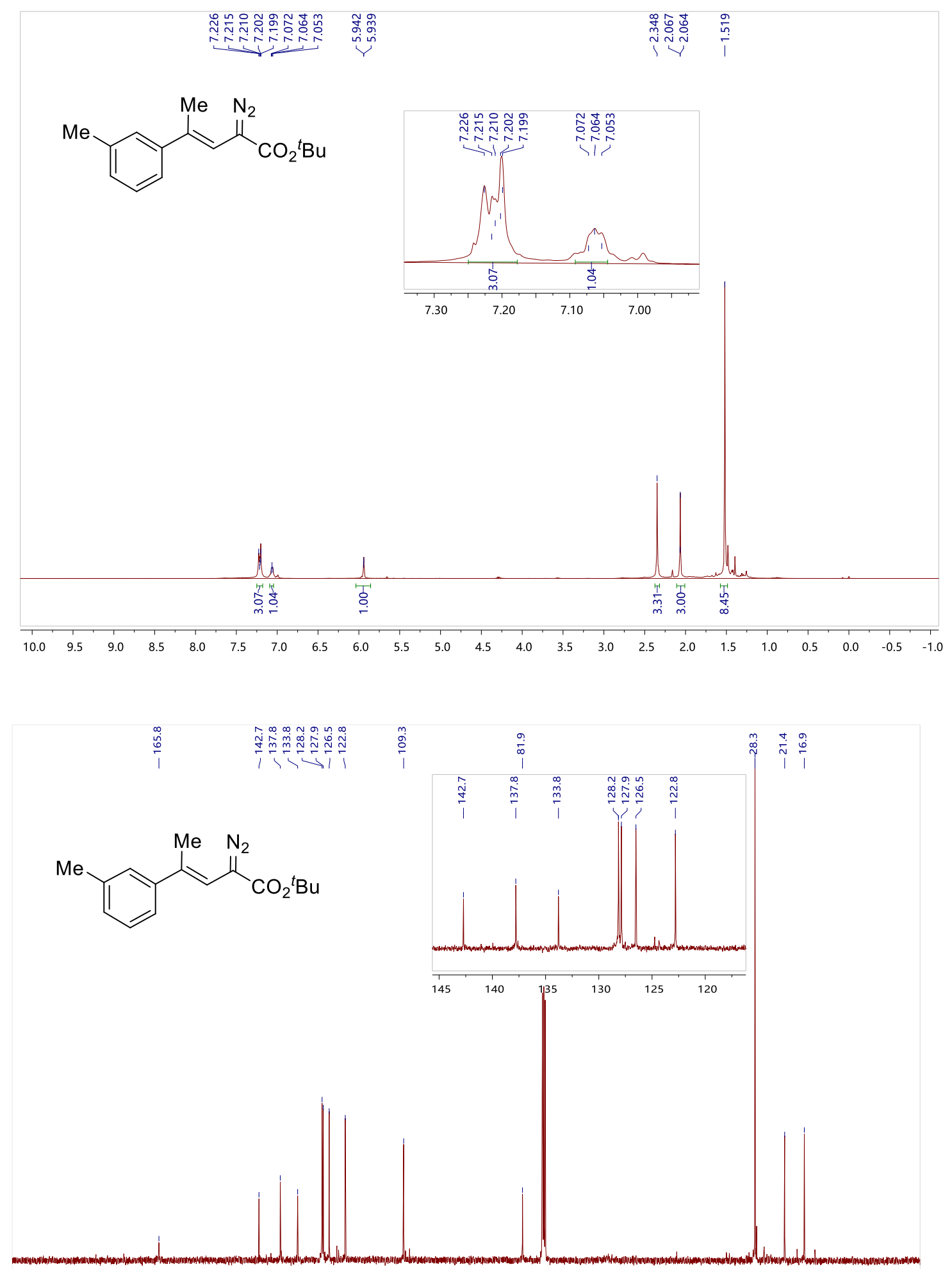

$\begin{array}{llllllllll}190 & 180 & 170 & 160 & 150 & 140 & 130 & 120 & 110 & 100\end{array}$ 
(E)-tert-Butyl 4-(p-tolyl)-2-diazopent-3-enoate (6c)
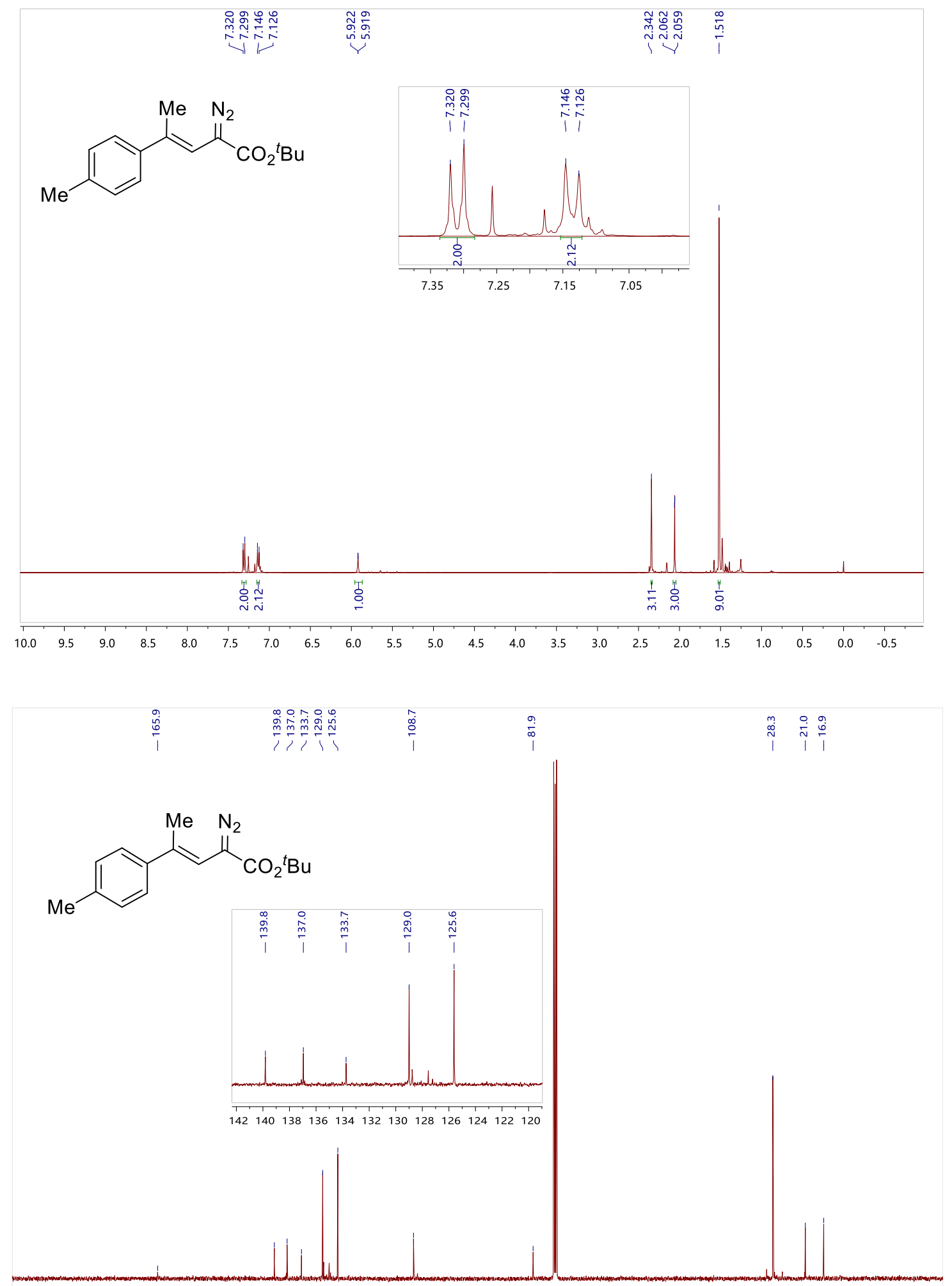
(E)-tert-Butyl 4-(4-methoxyphenyl)-2-diazopent-3-enoate (6d)
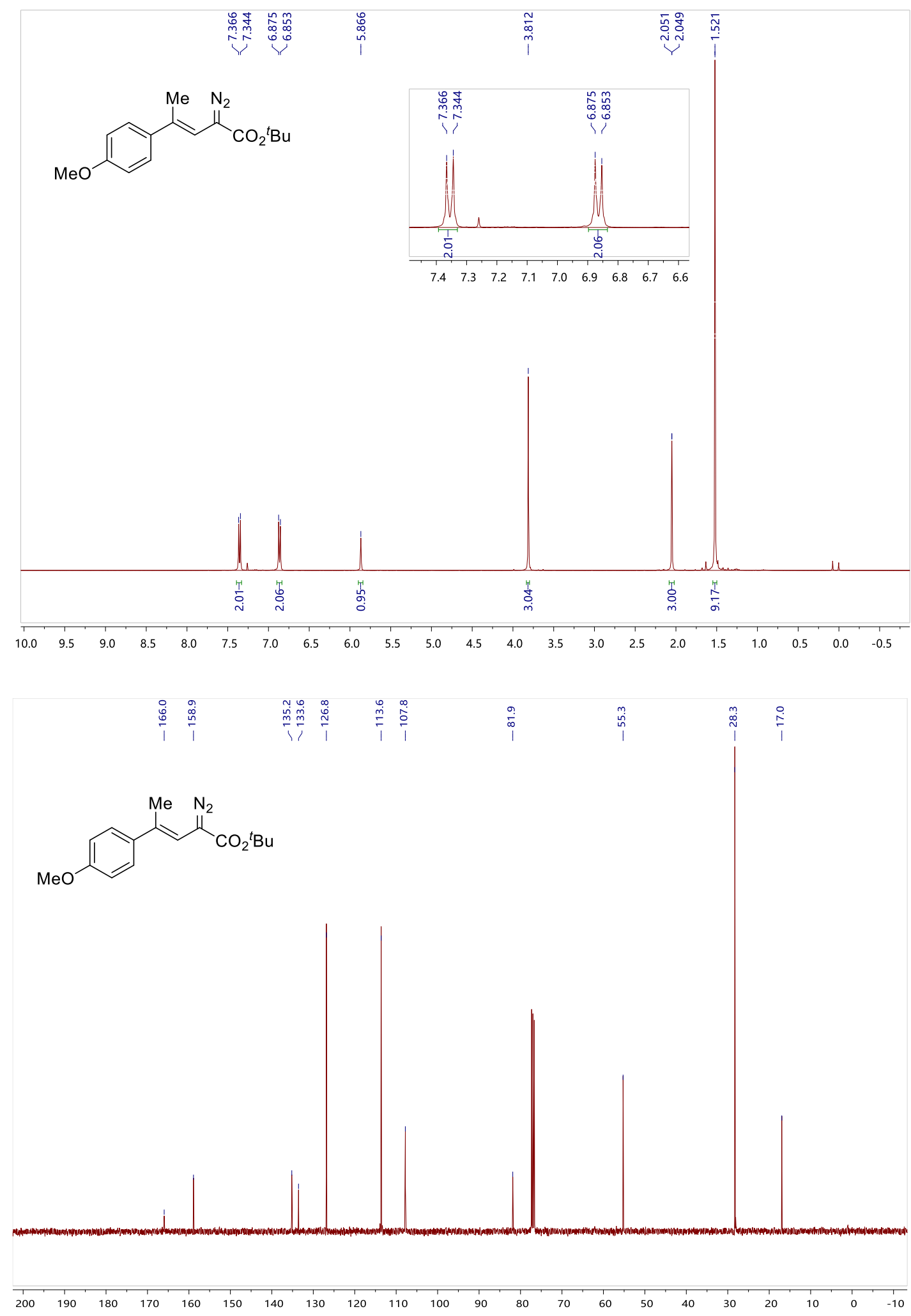
(E)-tert-Butyl 4-(4-fluorophenyl)-2-diazopent-3-enoate (6e)
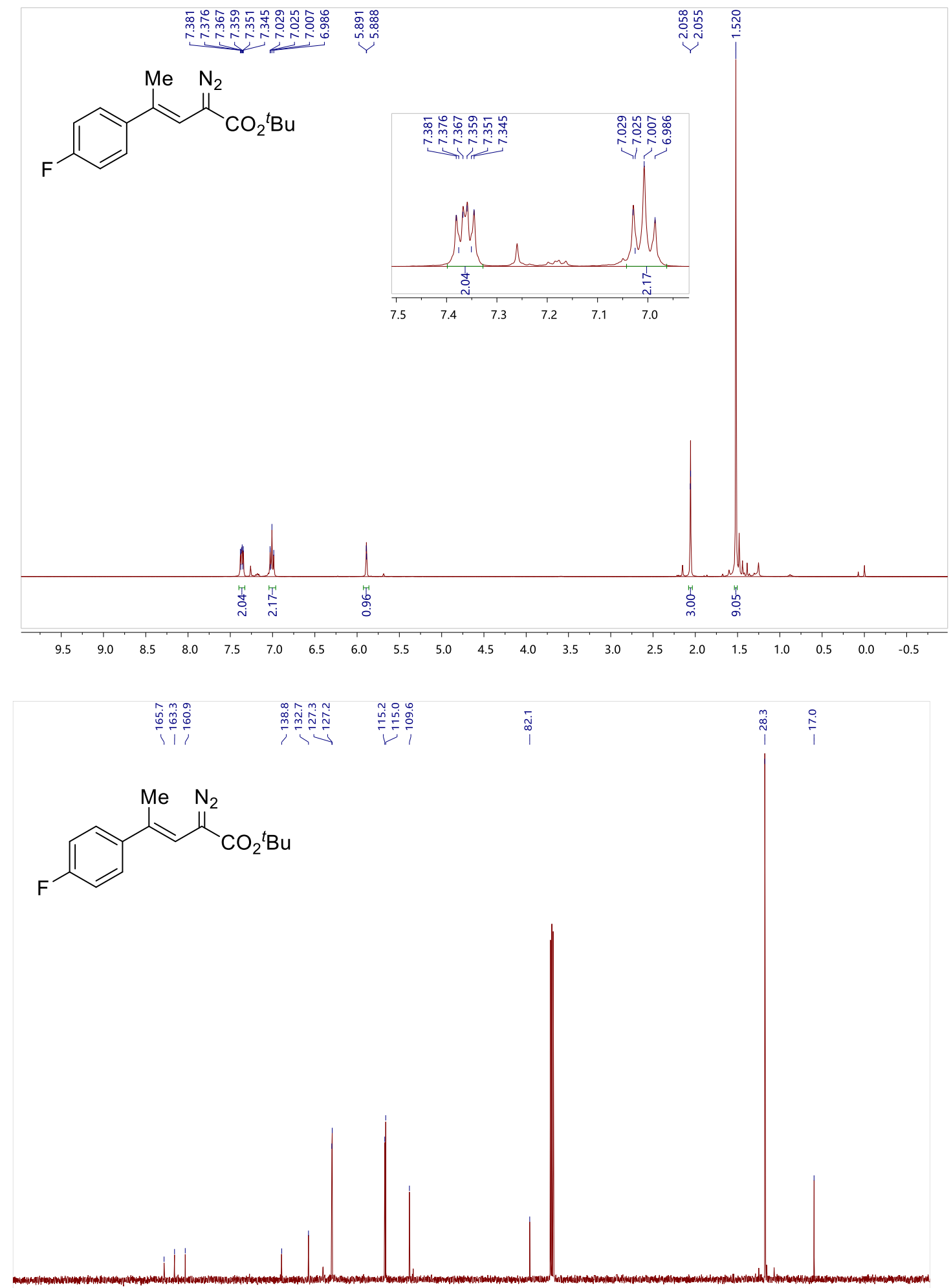

\begin{tabular}{llllllllllll}
\hline 00 & 190 & 180 & 170 & 160 & 150 & 140 & 130 & 120 & 110 & 100 & 90
\end{tabular} 
(E)-tert-Butyl 4-(2-chlorophenyl)-2-diazopent-3-enoate (6f)
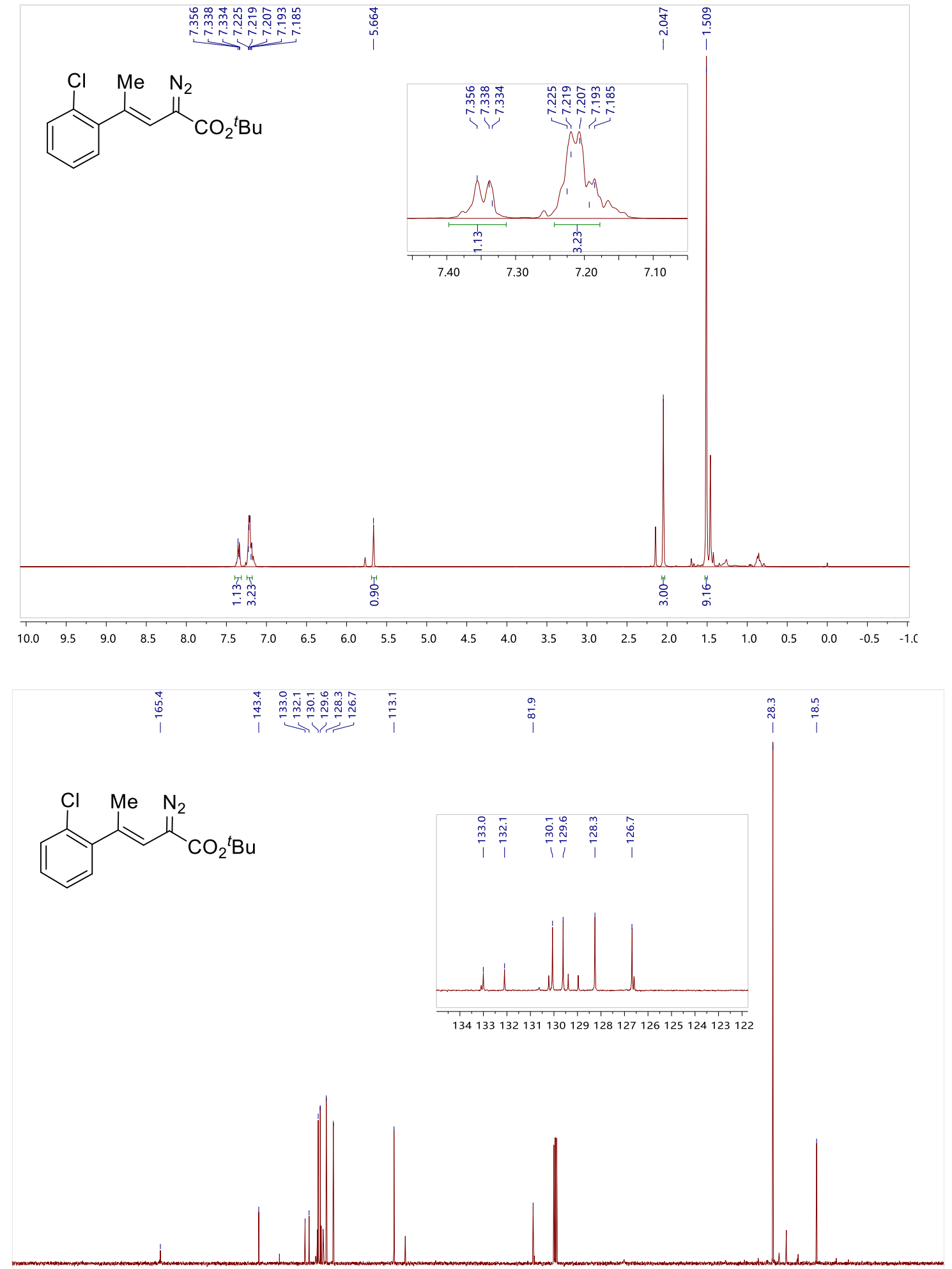

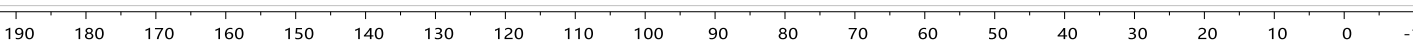


(E)-tert-Butyl 4-(3-chlorophenyl)-2-diazopent-3-enoate (6g)
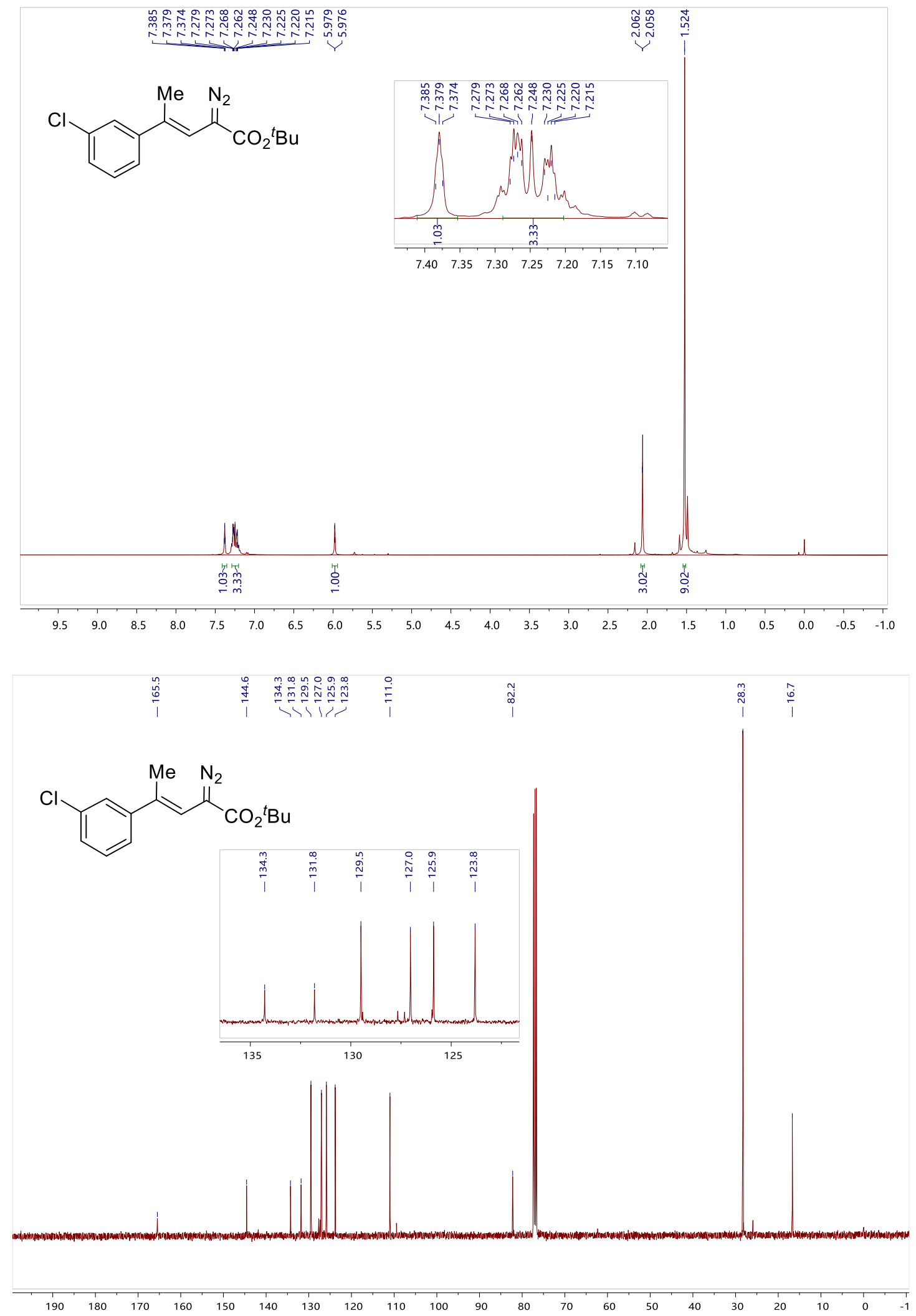
(E)-tert-Butyl 4-(4-chlorophenyl)-2-diazopent-3-enoate (6h)
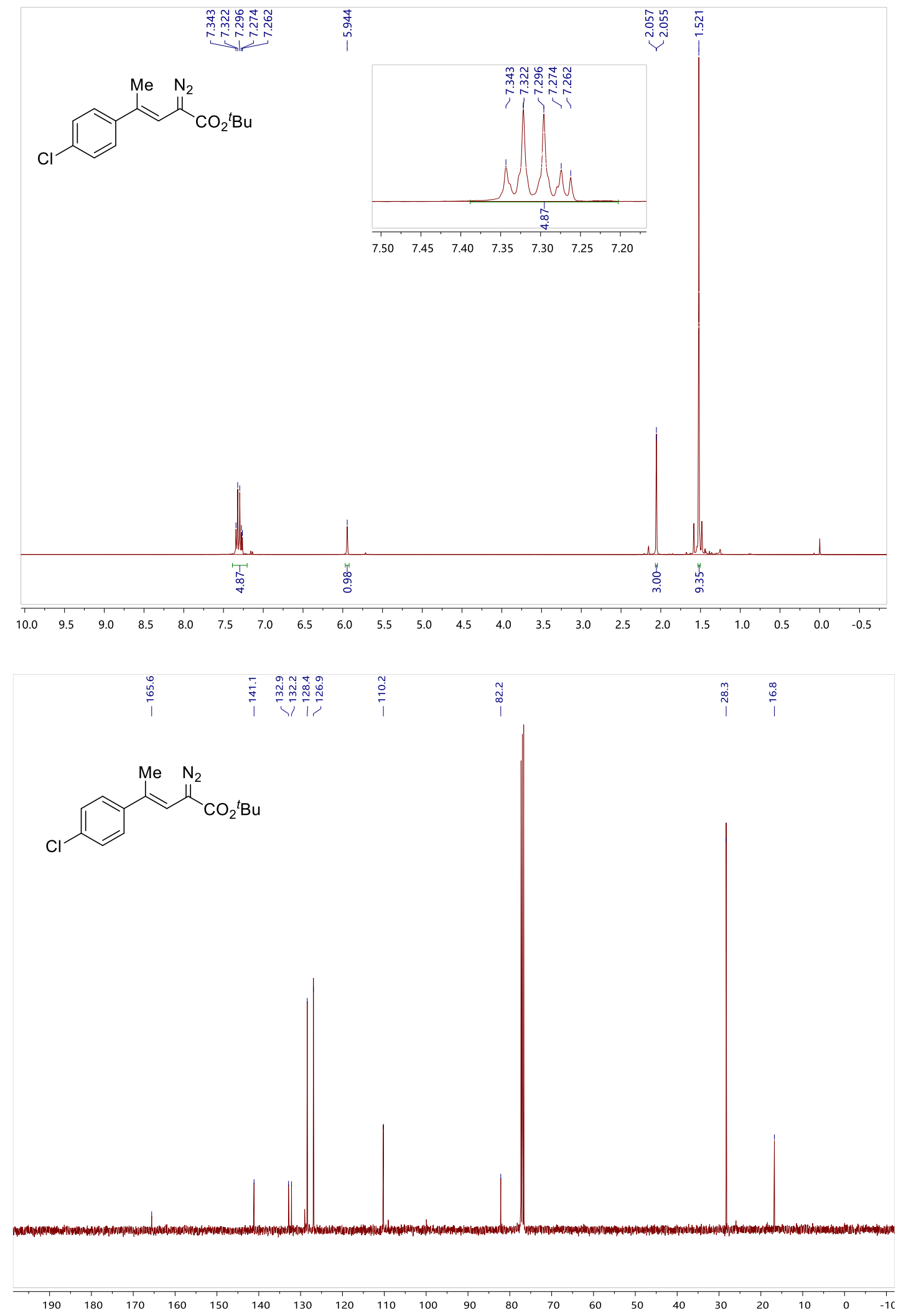
(E)-tert-Butyl 4-(3,4-dimethylphenyl)-2-diazopent-3-enoate (6i)
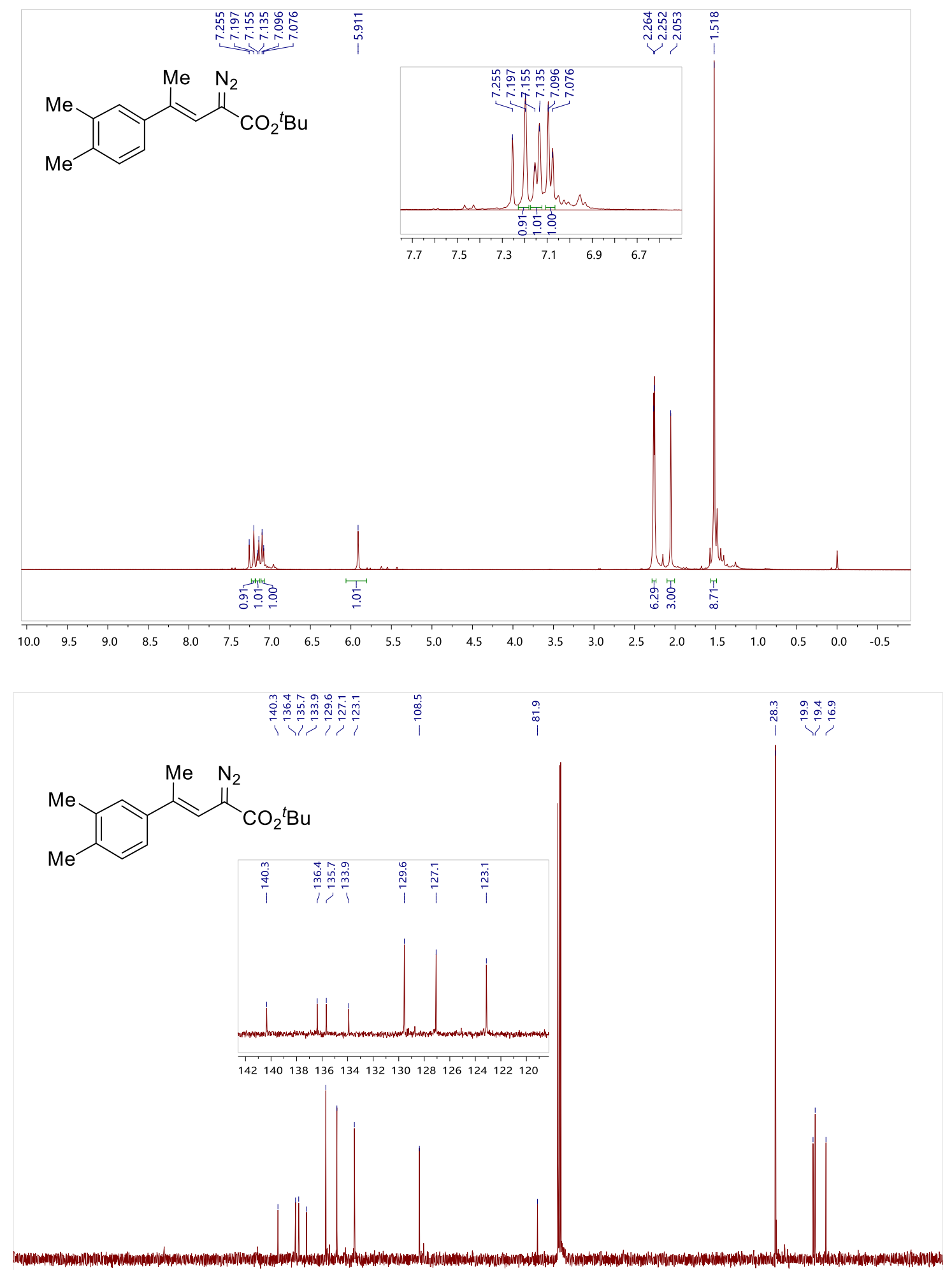

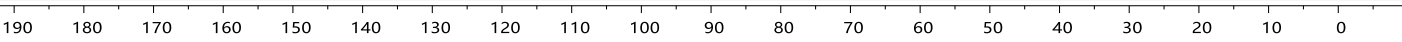


(E)-tert-Butyl 4-(benzo[d][1,3]dioxol-5-yl)-2-diazopent-3-enoate (6j)
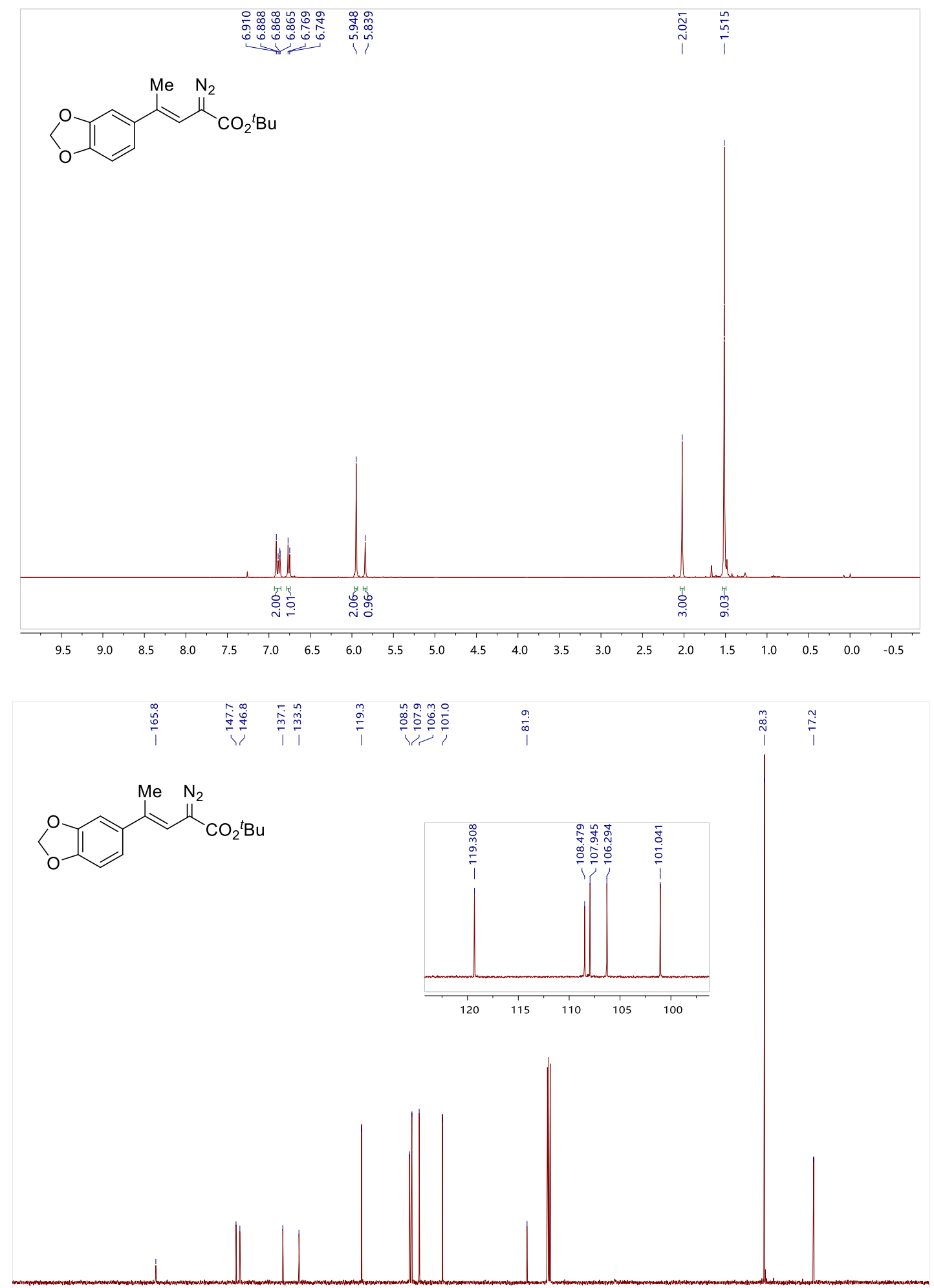

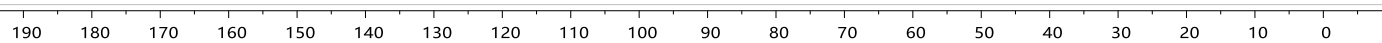


(E)-tert-Butyl 4-(naphthalen-2-yl)-2-diazopent-3-enoate (6k)
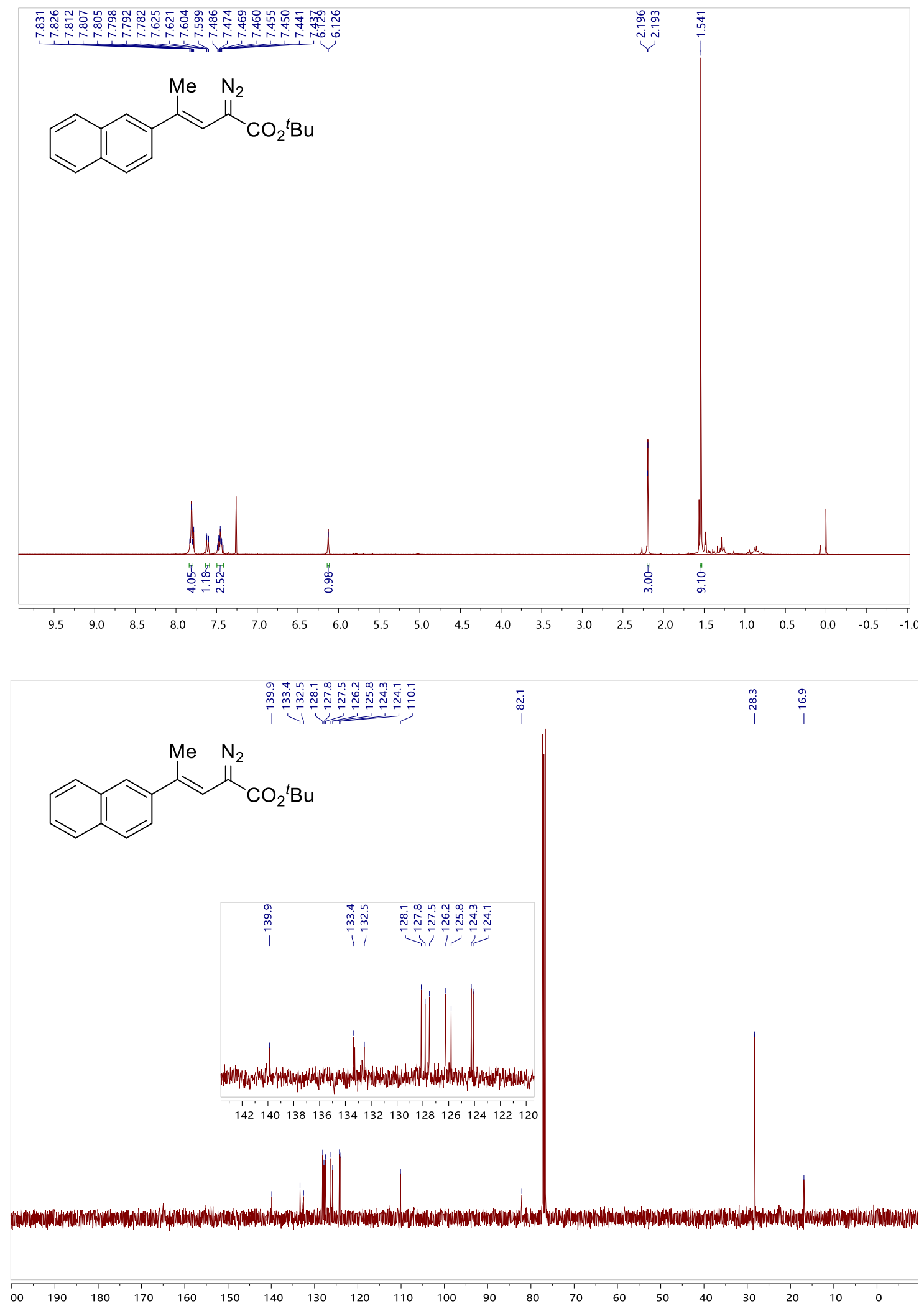
(E)-tert-Butyl 4-(thiophen-2-yl)-2-diazopent-3-enoate (61)
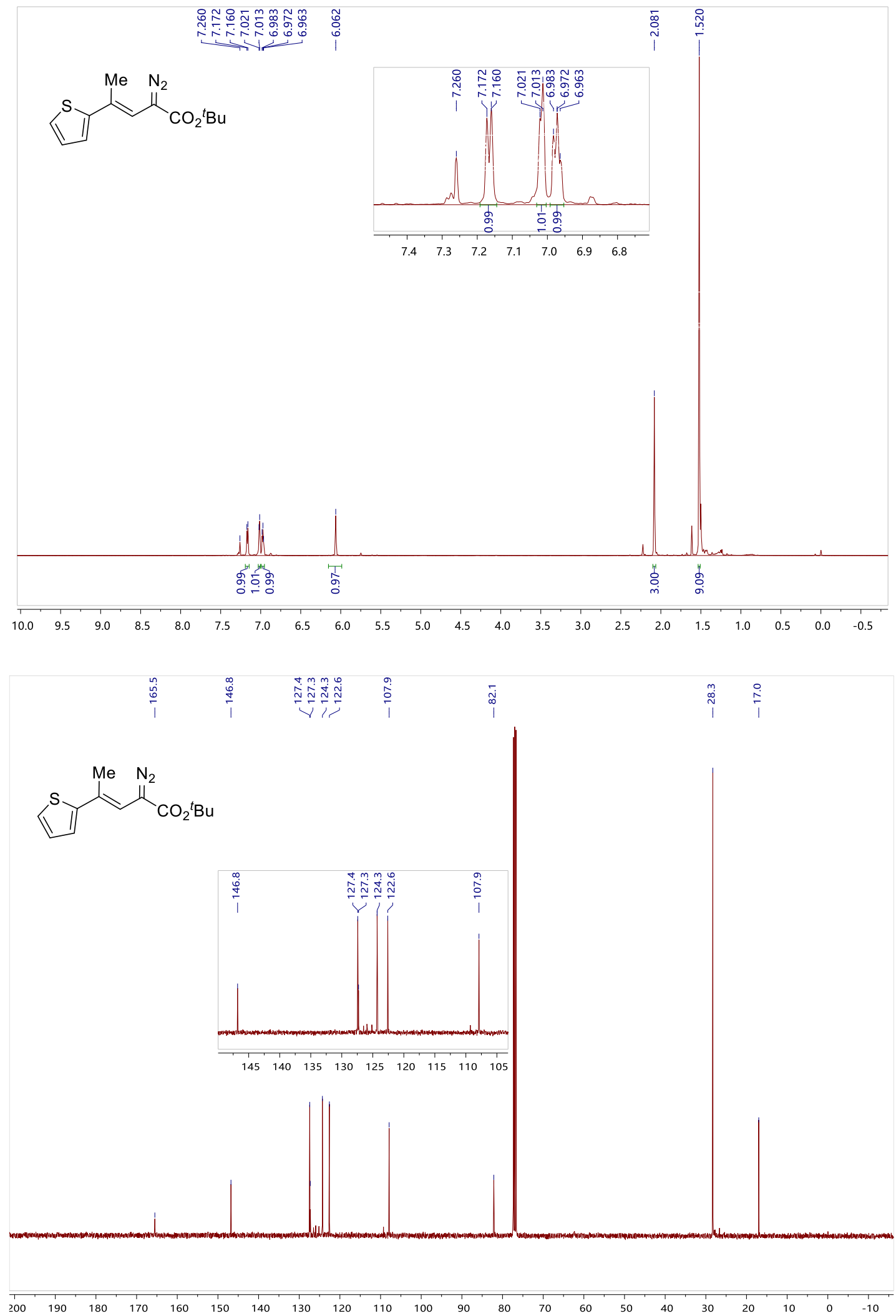
tert-Butyl 4,4-diphenyl-2-diazobut-3-enoate (6m)
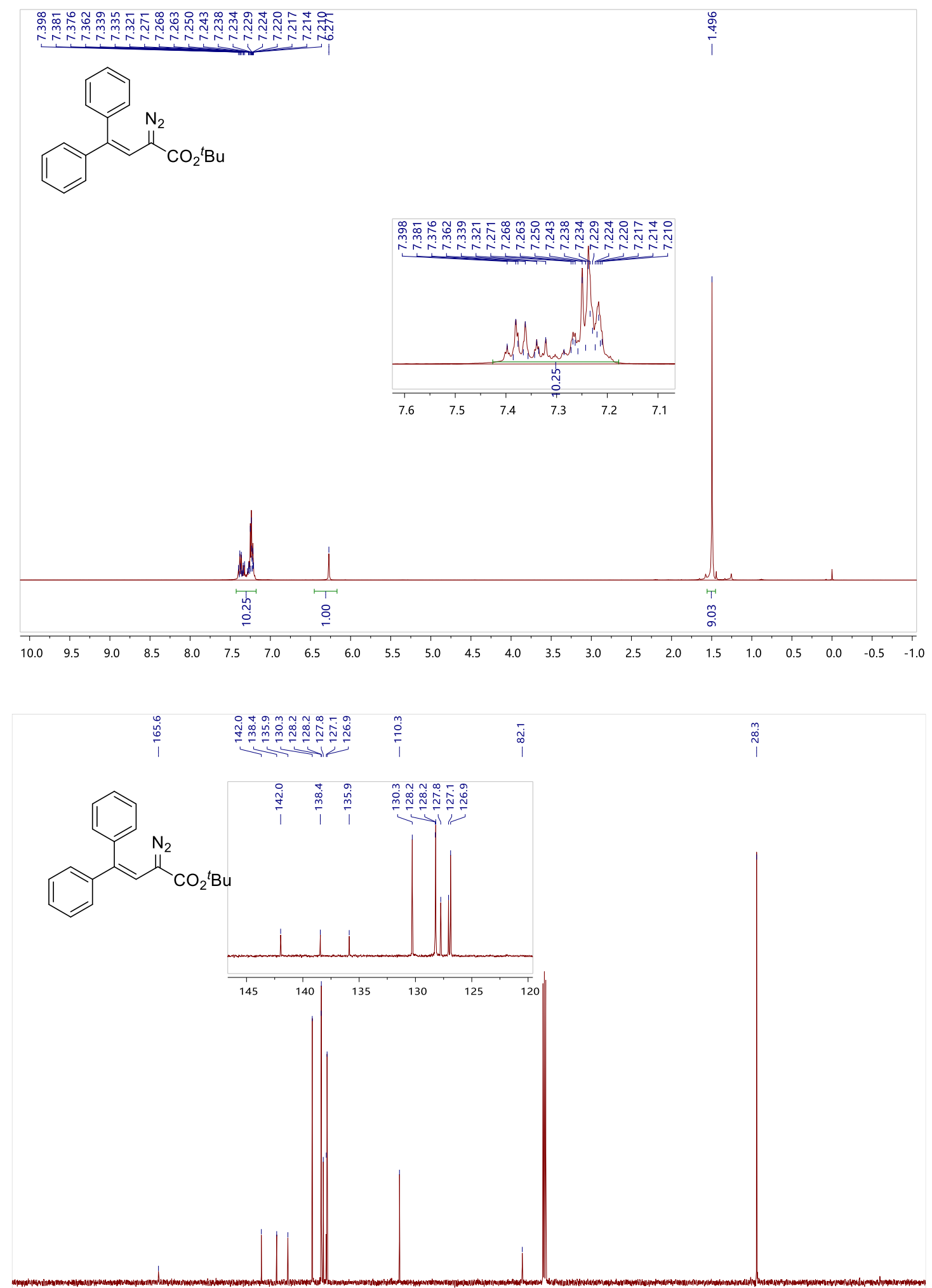
(E)-tert-Butyl 2-diazo-4-phenylbut-3-enoate (6n)
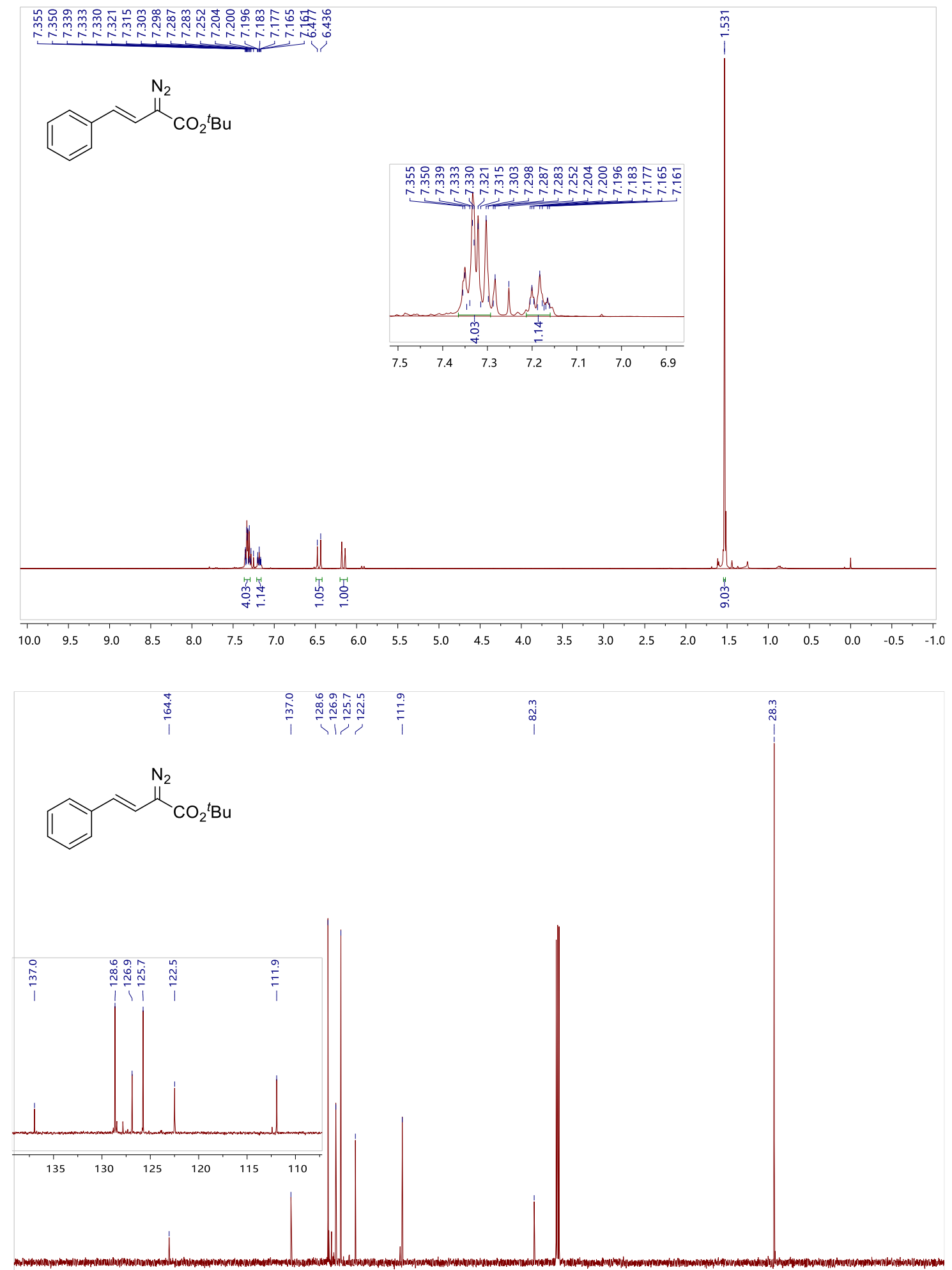
Benzhydryl (E)-2-diazopent-3-enoate (6o)
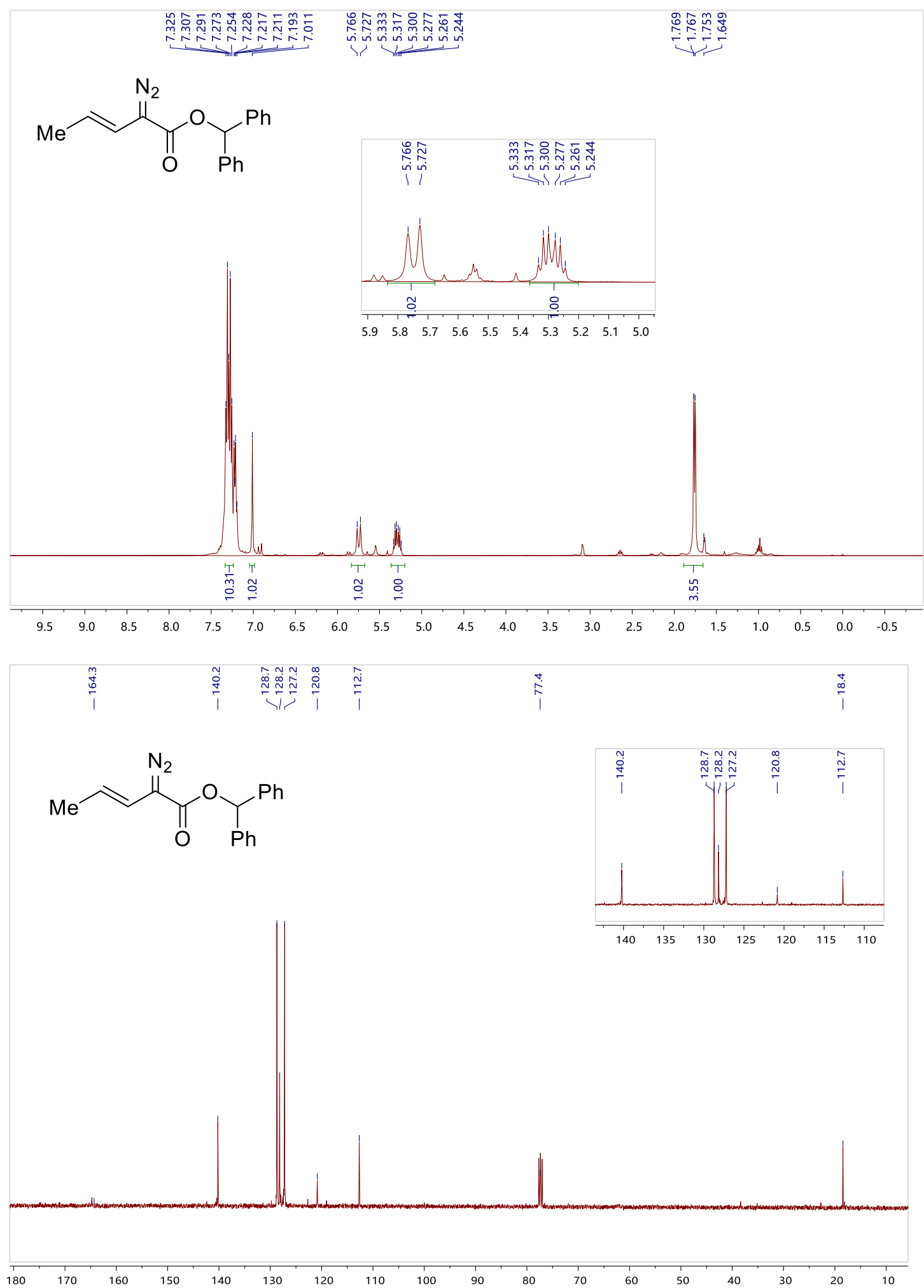
Benzhydryl (E)-2-diazohex-3-enoate (6p)
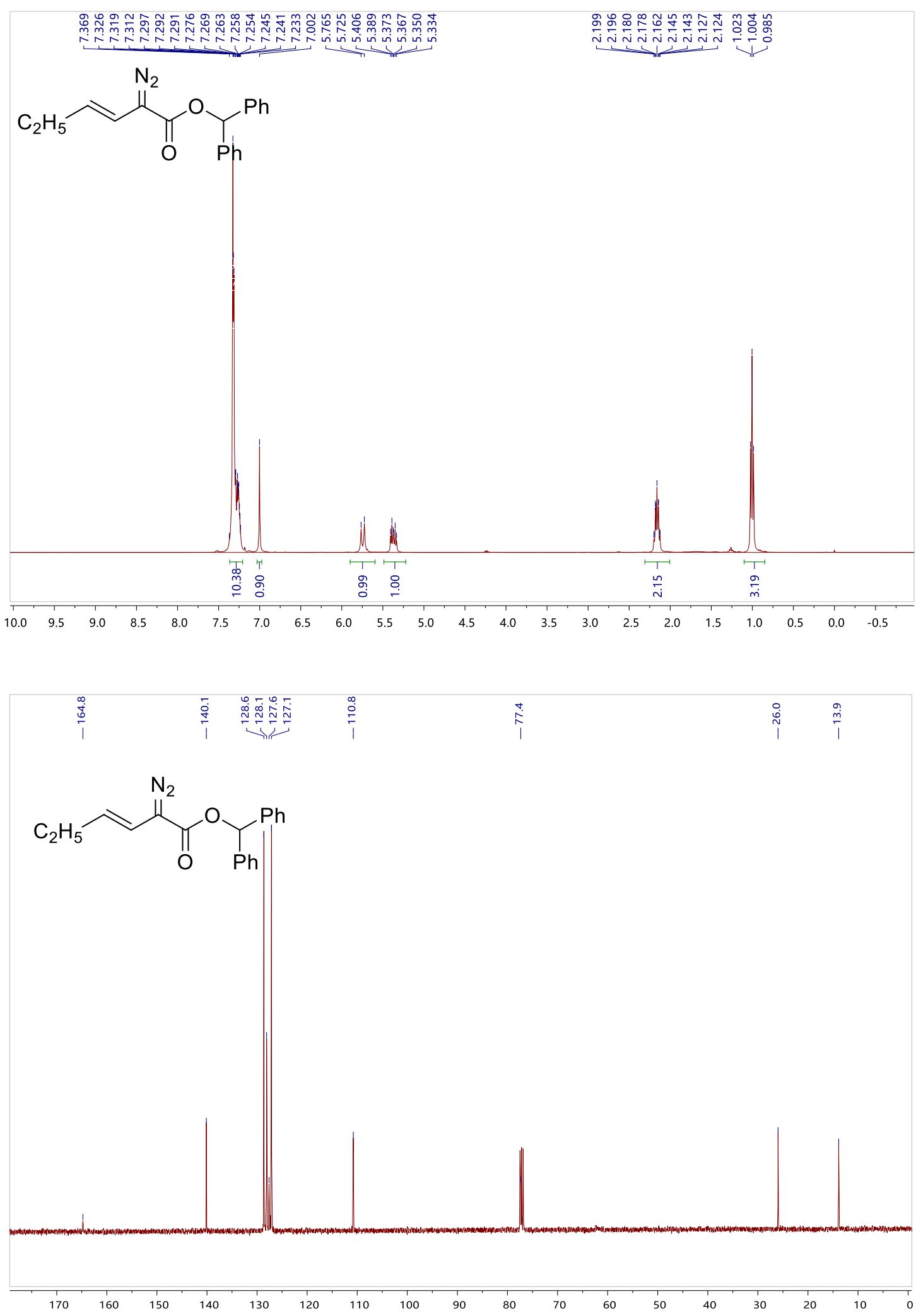
Benzhydryl (E)-2-diazohept-3-enoate (6q)

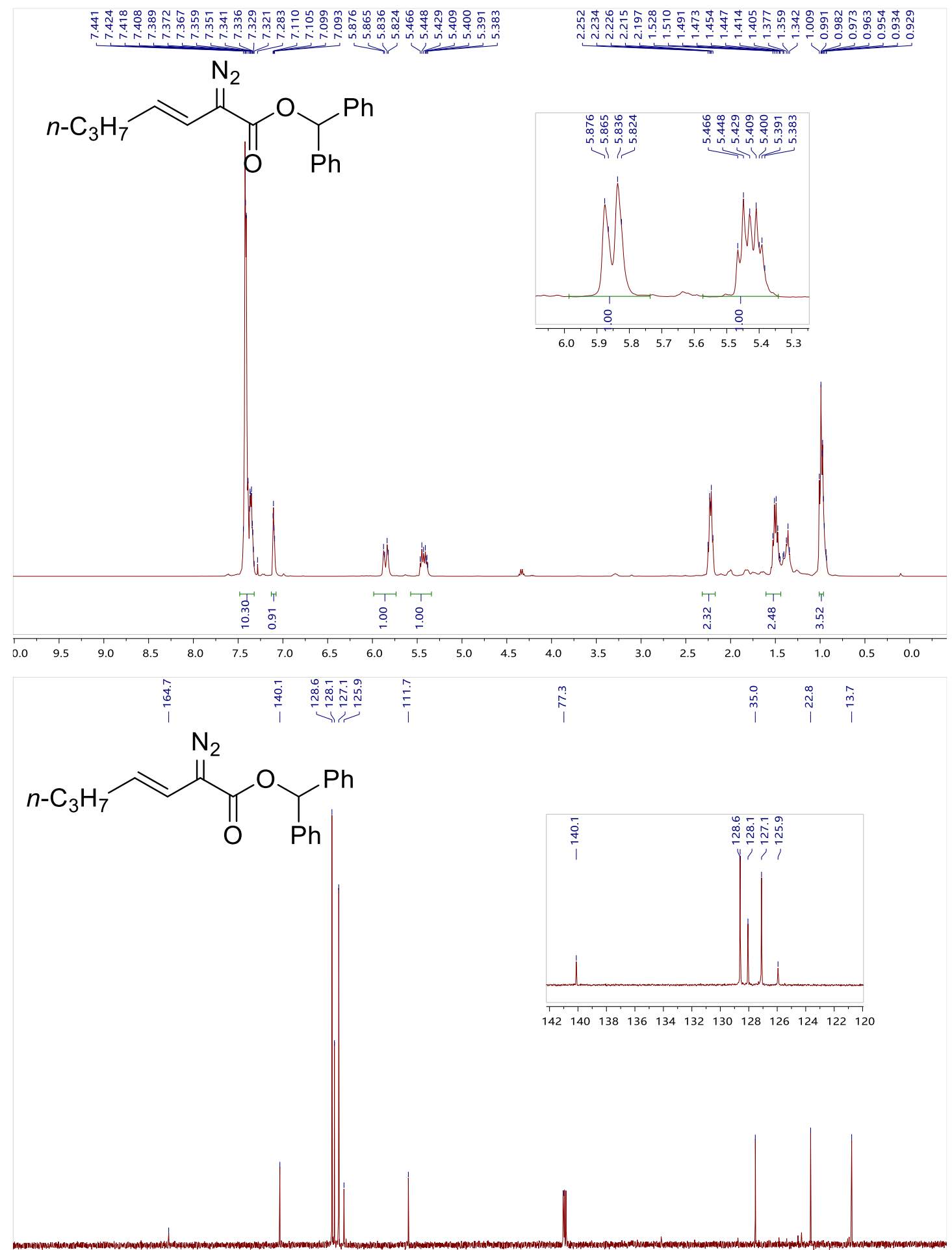

$\begin{array}{lllllllllllllllllllll}1 & 190 & 180 & 170 & 160 & 150 & 140 & 130 & 120 & 110 & 100 & 90 & 80 & 70 & 60 & 50 & 40 & 30 & 20 & 10 & 0\end{array}$ 
Benzhydryl (E)-2-diazodec-3-enoate (6r)
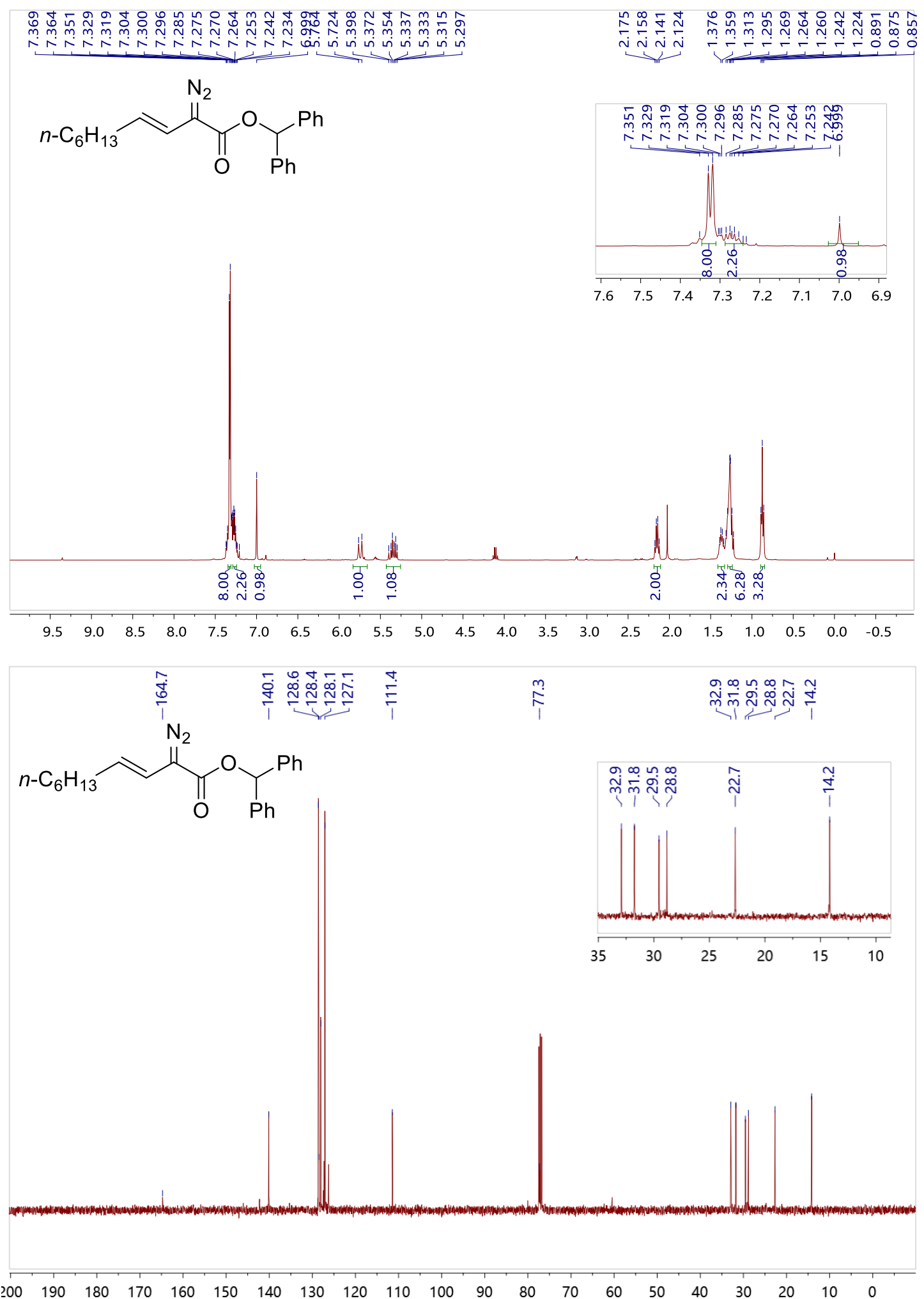
Benzhydryl (E)-2-diazo-5-phenylpent-3-enoate (6s)
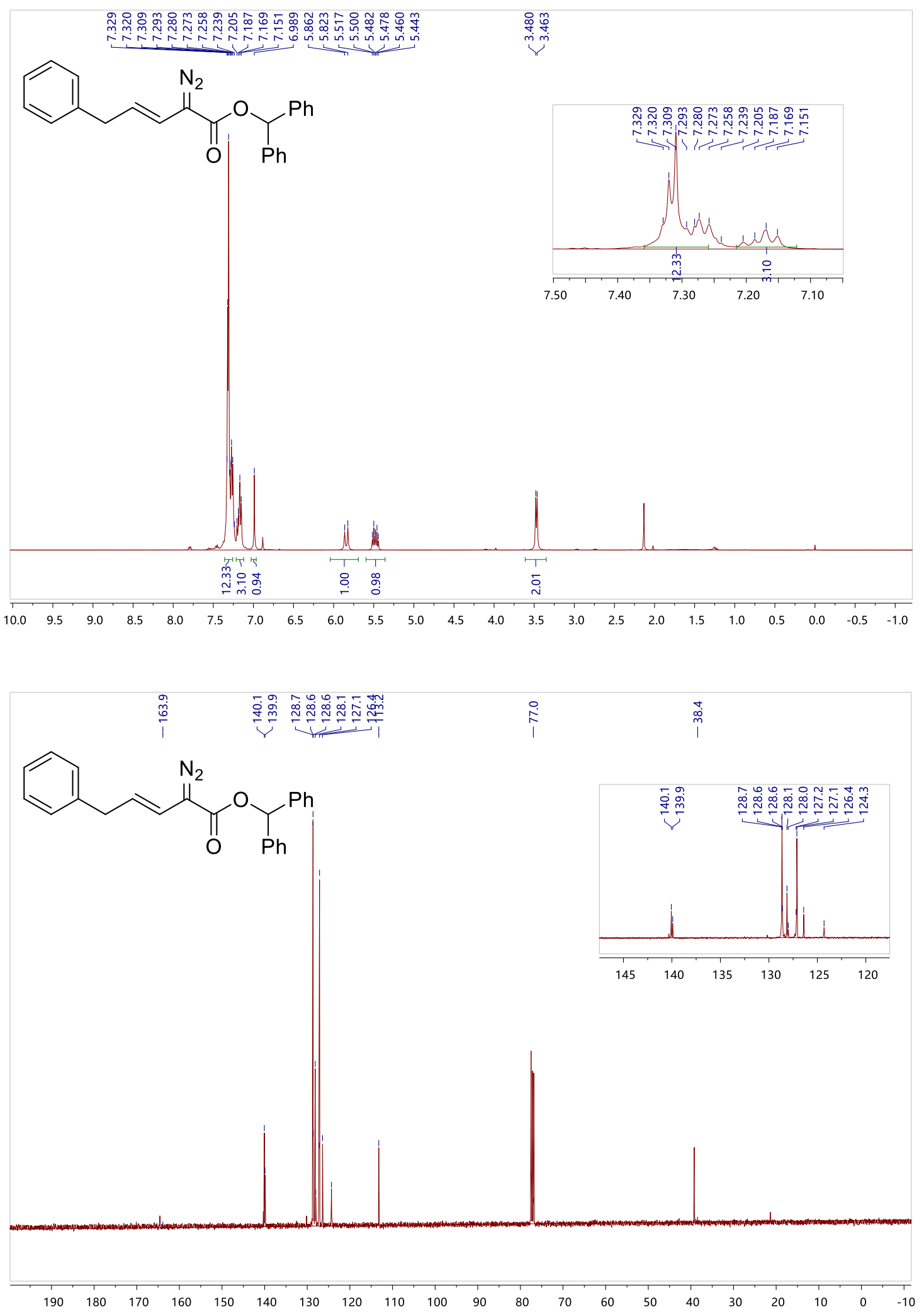
2-Phenylpropan-2-yl 2-hydroxyoctanoate (2a)
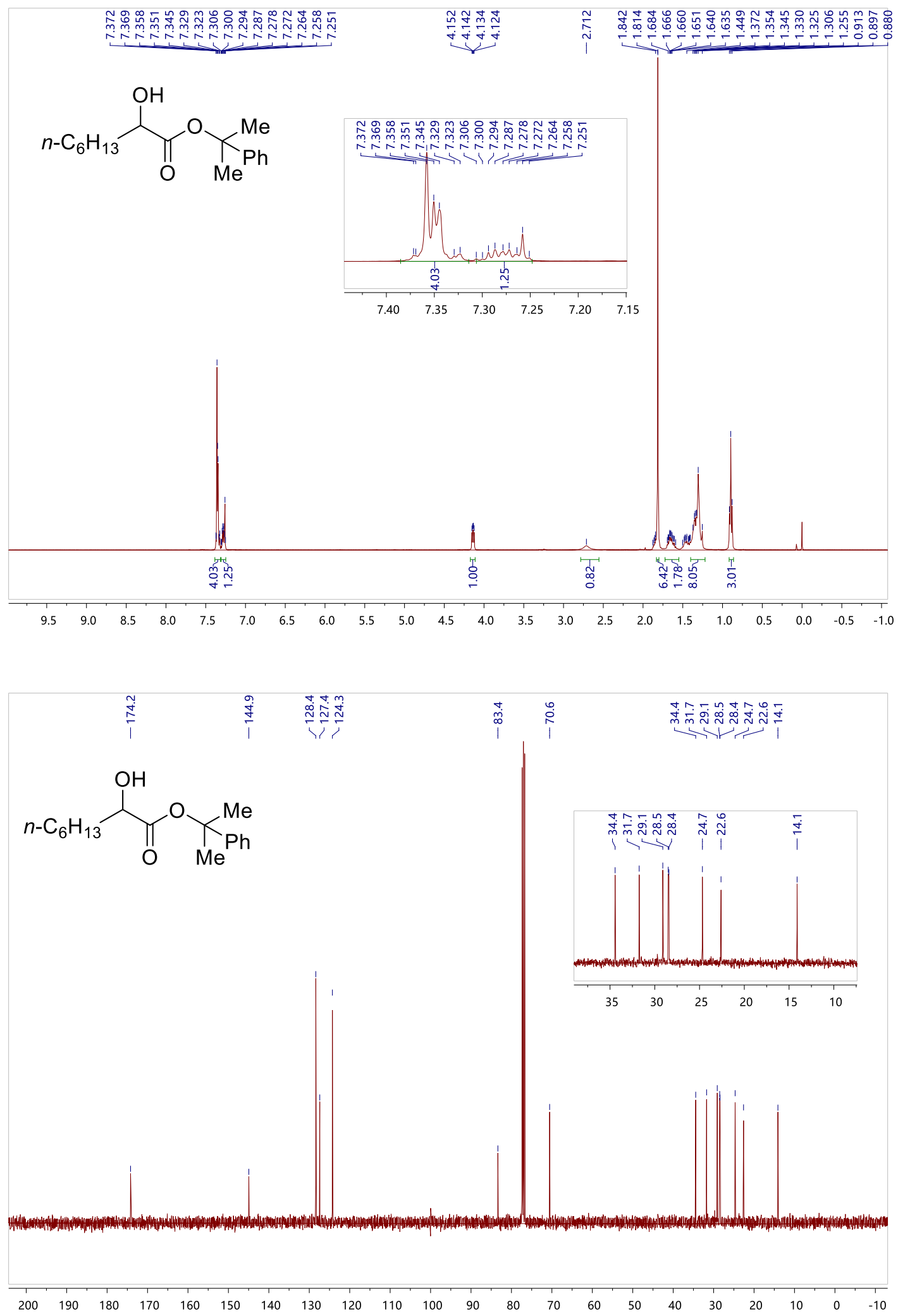
2-Phenylpropan-2-yl 2-hydroxybutanoate (2b)

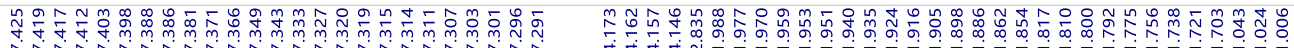<smiles>CCCCC(O)C(=O)OC(C)(C)c1ccccc1</smiles>

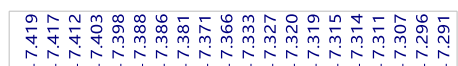
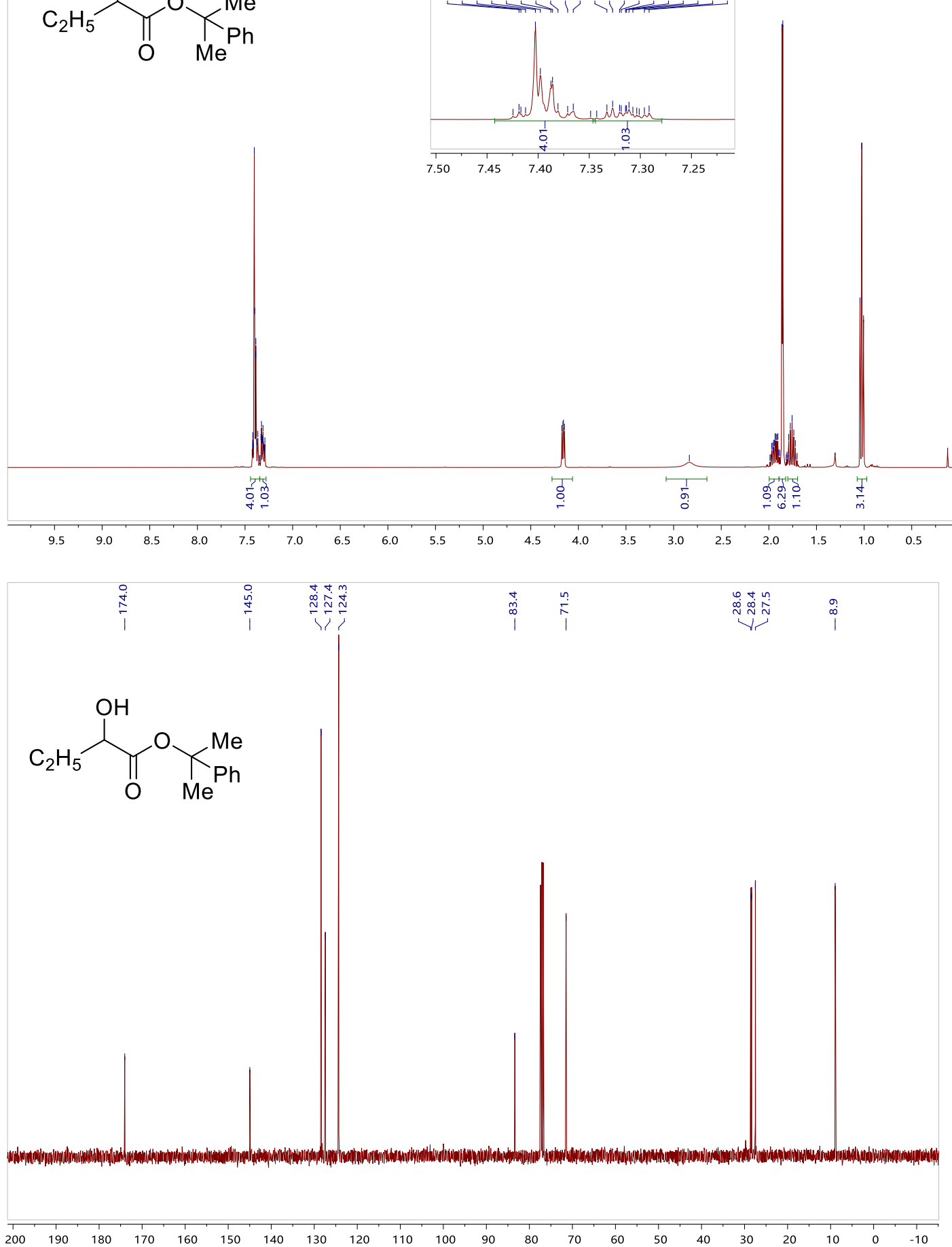
2-Phenylpropan-2-yl 2-hydroxyhexanoate (2c)

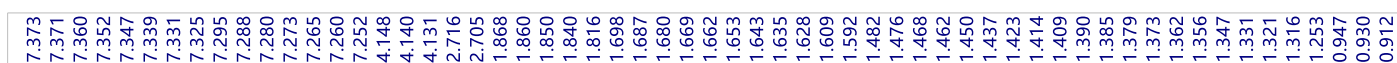<smiles>CCCCCCCCCC(O)C(=O)OC(C)(c1ccccc1)c1ccccc1</smiles>
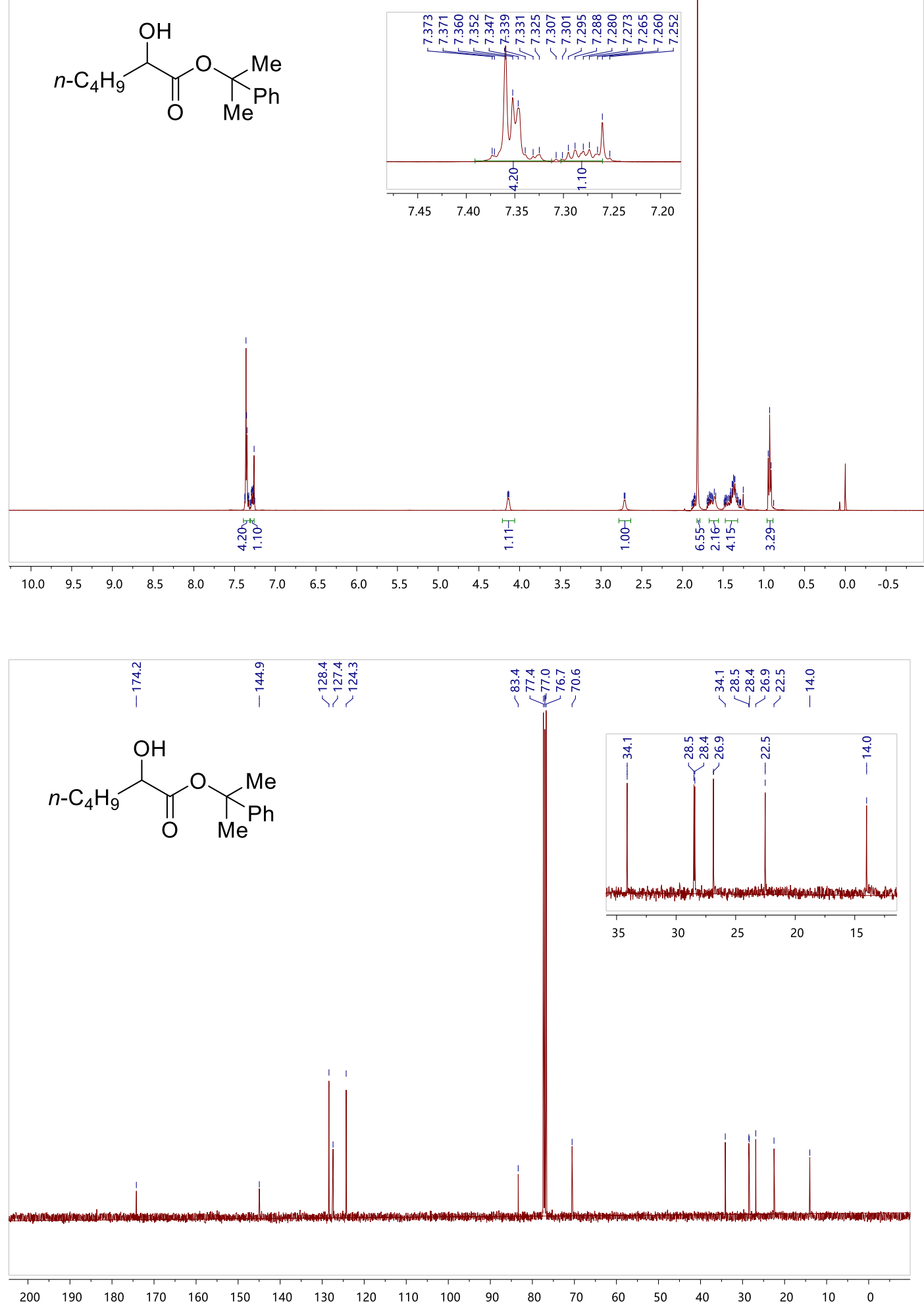
2-Phenylpropan-2-yl 2-hydroxytetradecanoate（2d）
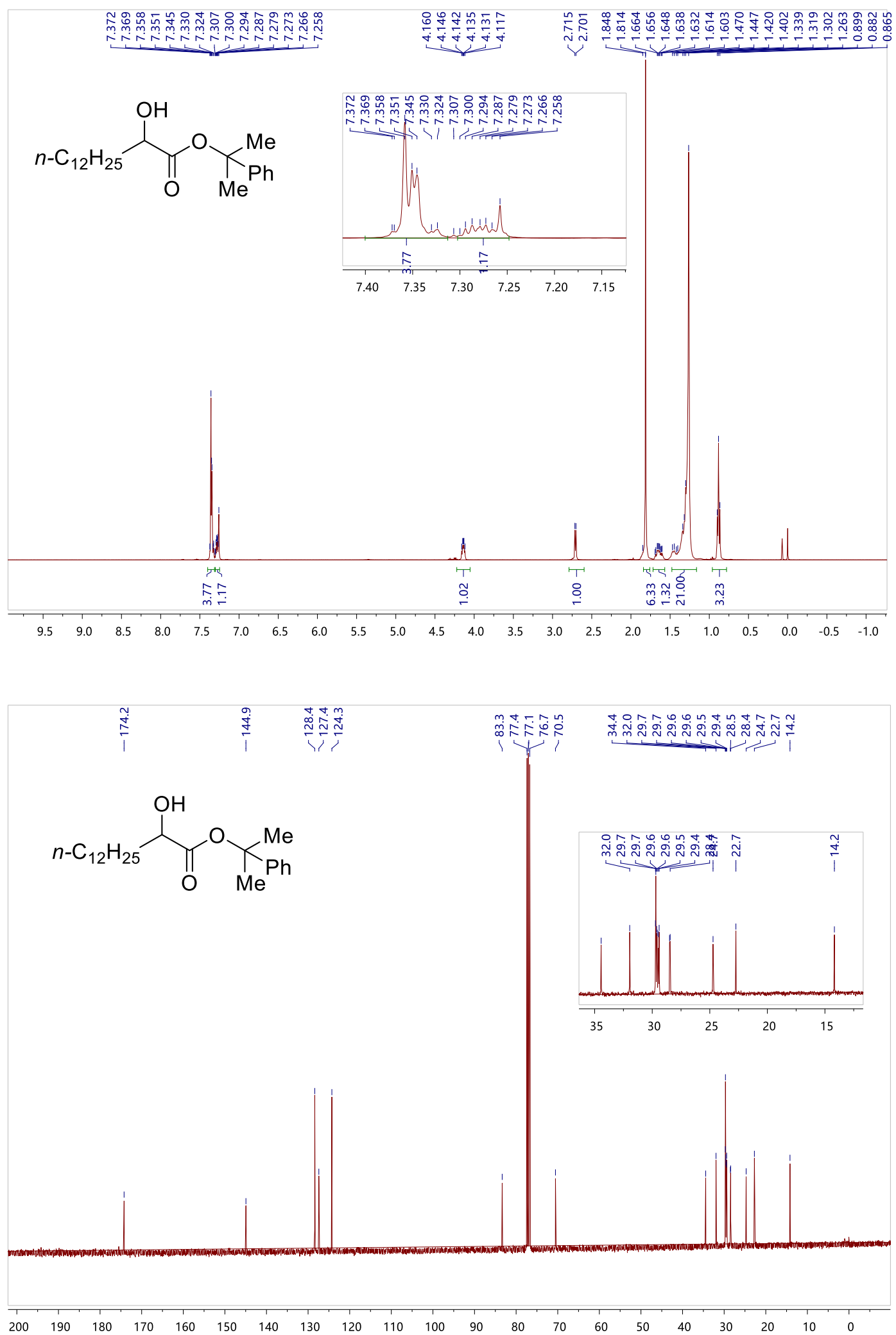
2-Phenylpropan-2-yl 2-hydroxy-5-methylhexanoate (2e)
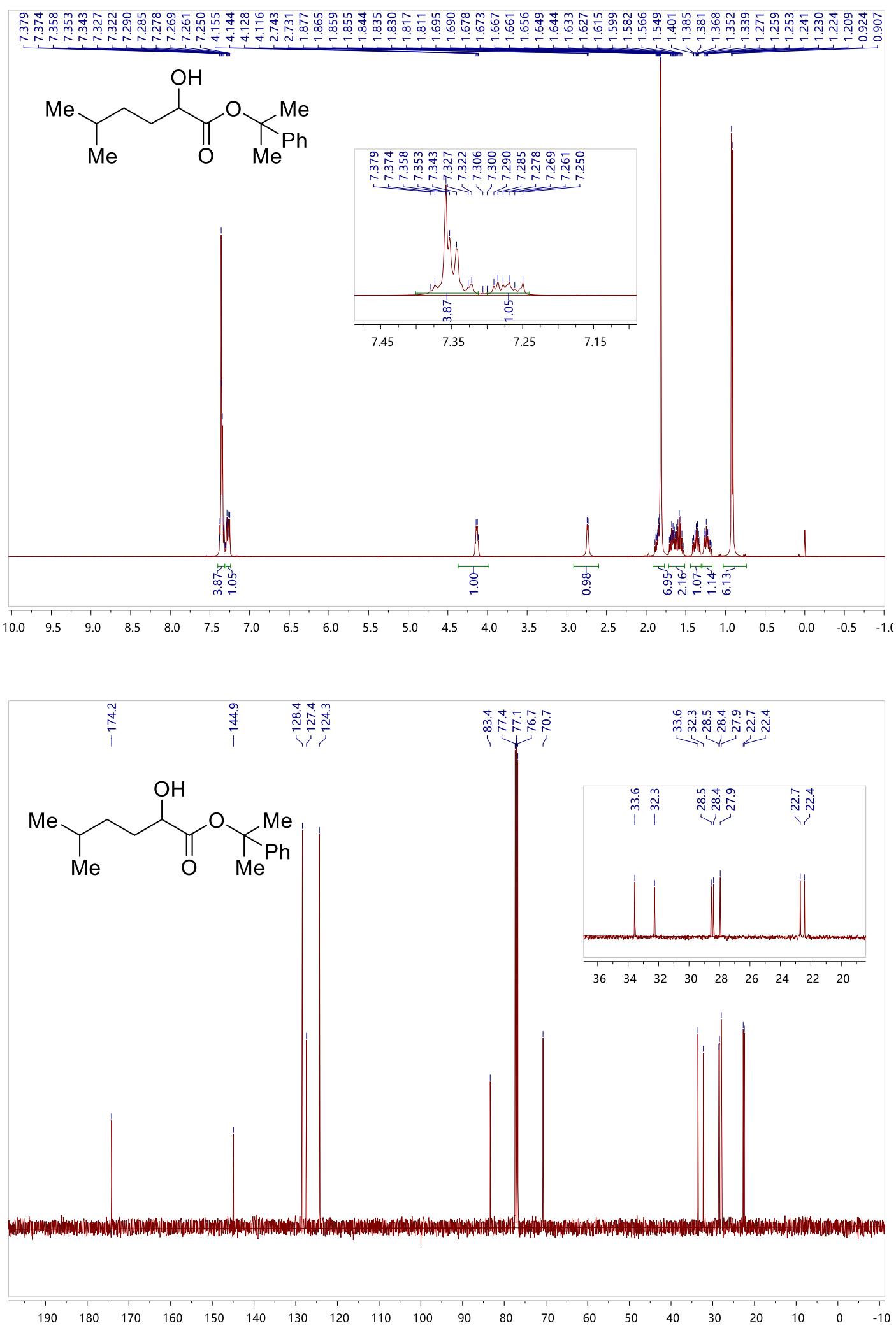
2-Phenylpropan-2-yl 2-hydroxy-5,5-dimethylhexanoate (2f)
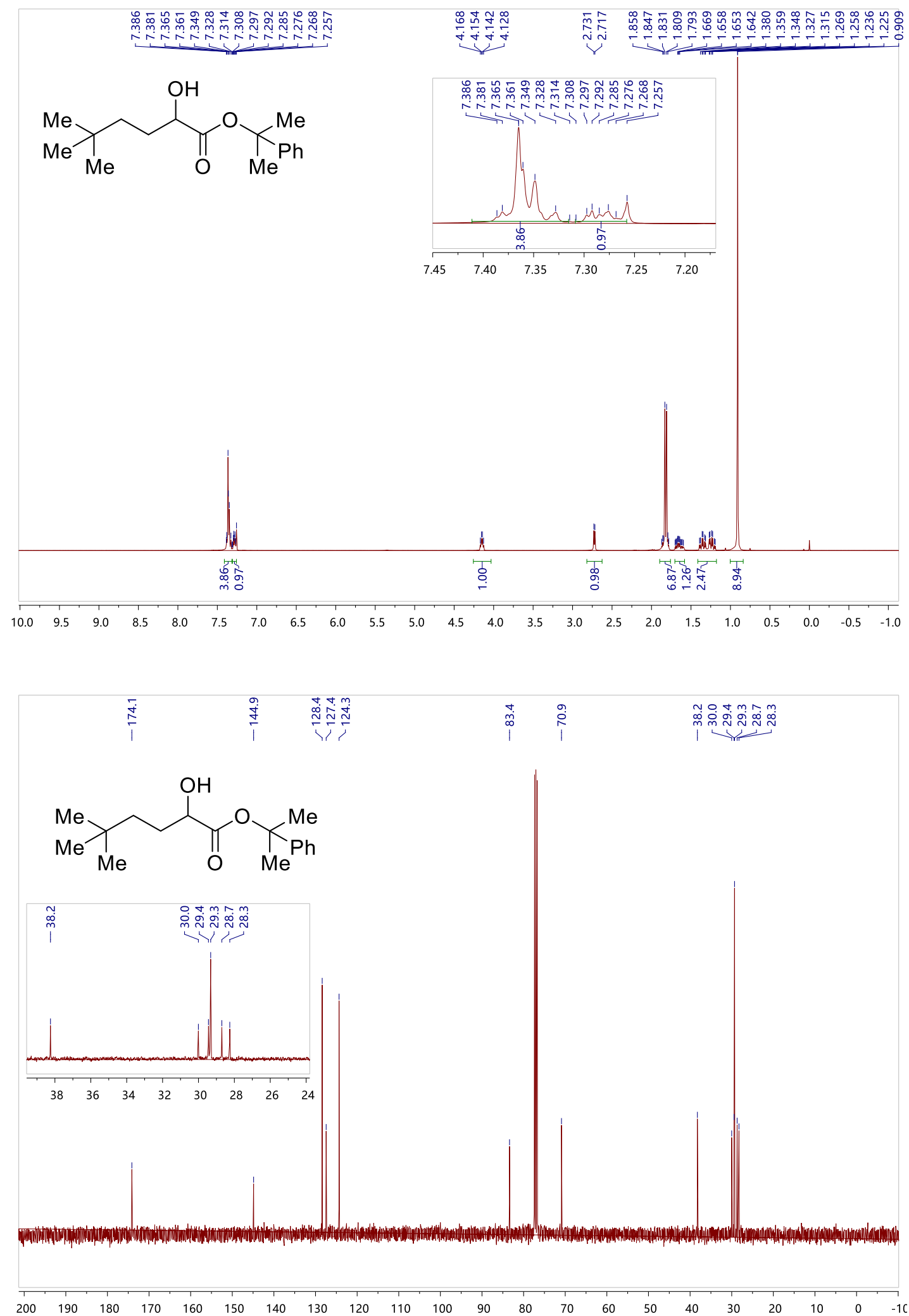
2-Phenylpropan-2-yl 4-cyclohexyl-2-hydroxybutanoate (2g)
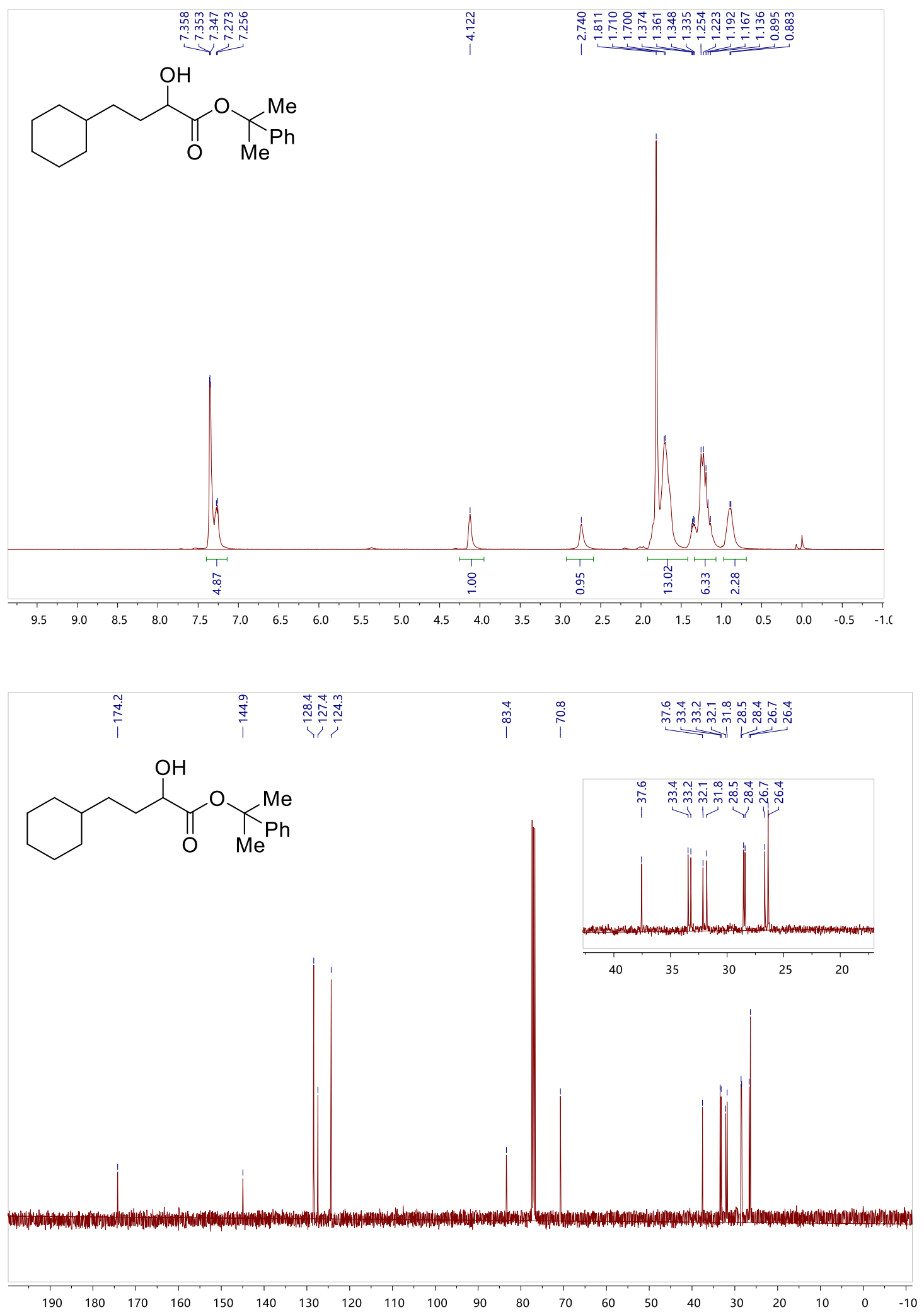
2-Phenylpropan-2-yl 3-cyclopropyl-2-hydroxypropanoate (2h)
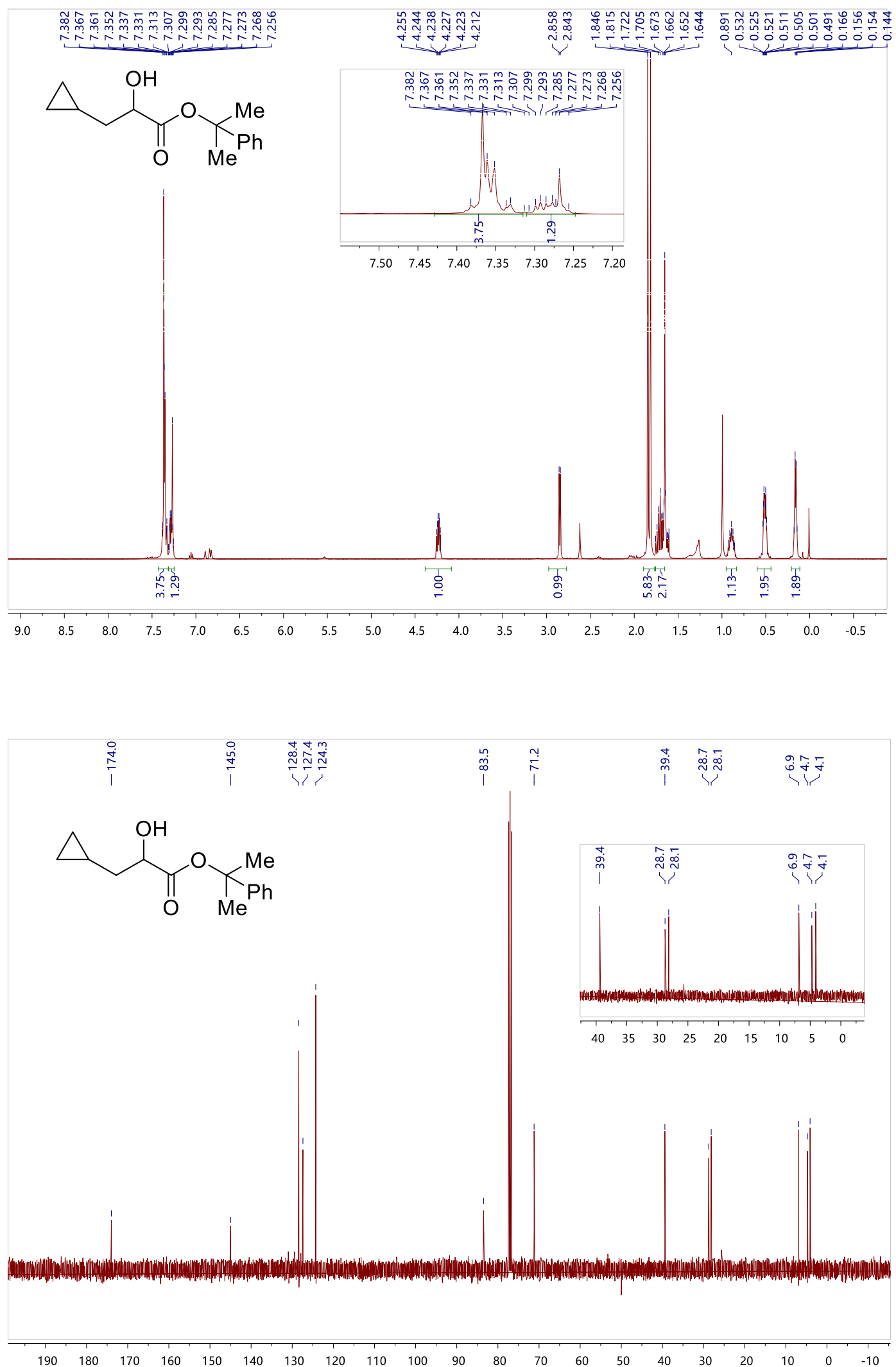
2-Phenylpropan-2-yl 2-hydroxy-4-methylpentanoate (2i)
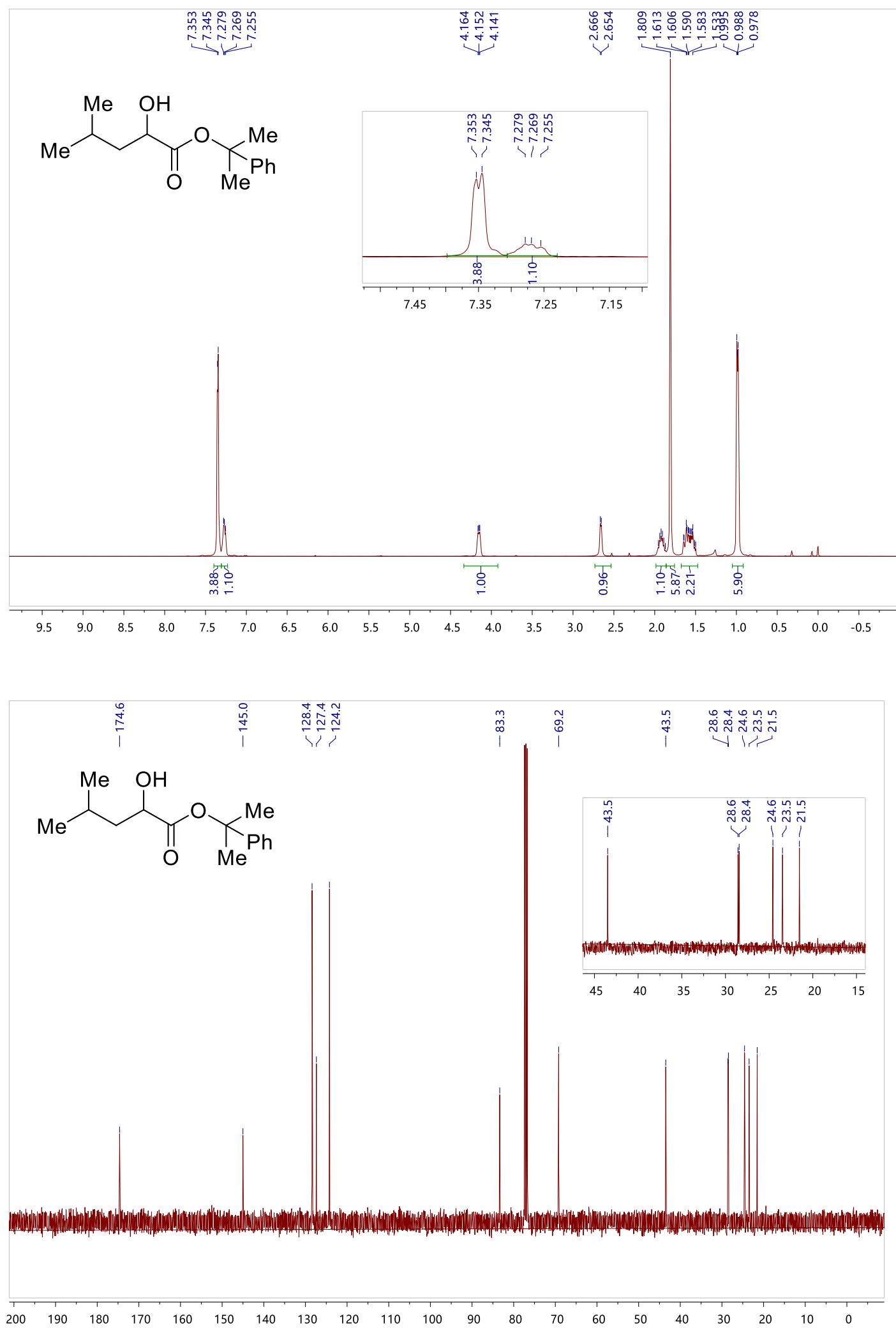
2-Phenylpropan-2-yl 2-hydroxy-3-phenylpropanoate (2j)
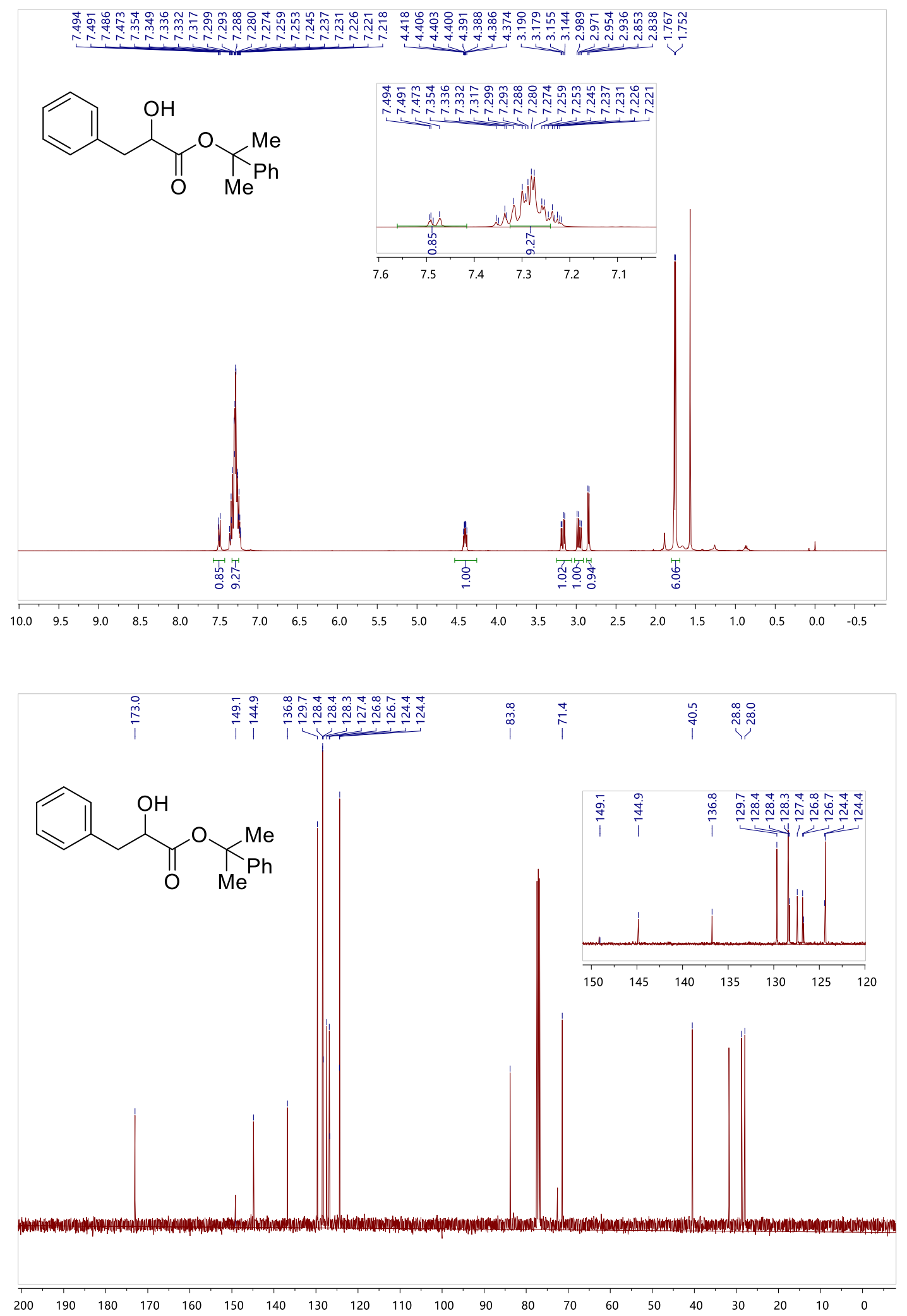
2-Phenylpropan-2-yl 2-hydroxy-4-phenylbutanoate (2k)

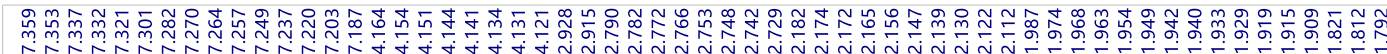<smiles>CC(C)(OC(=O)C(O)CCc1ccccc1)c1ccccc1</smiles>
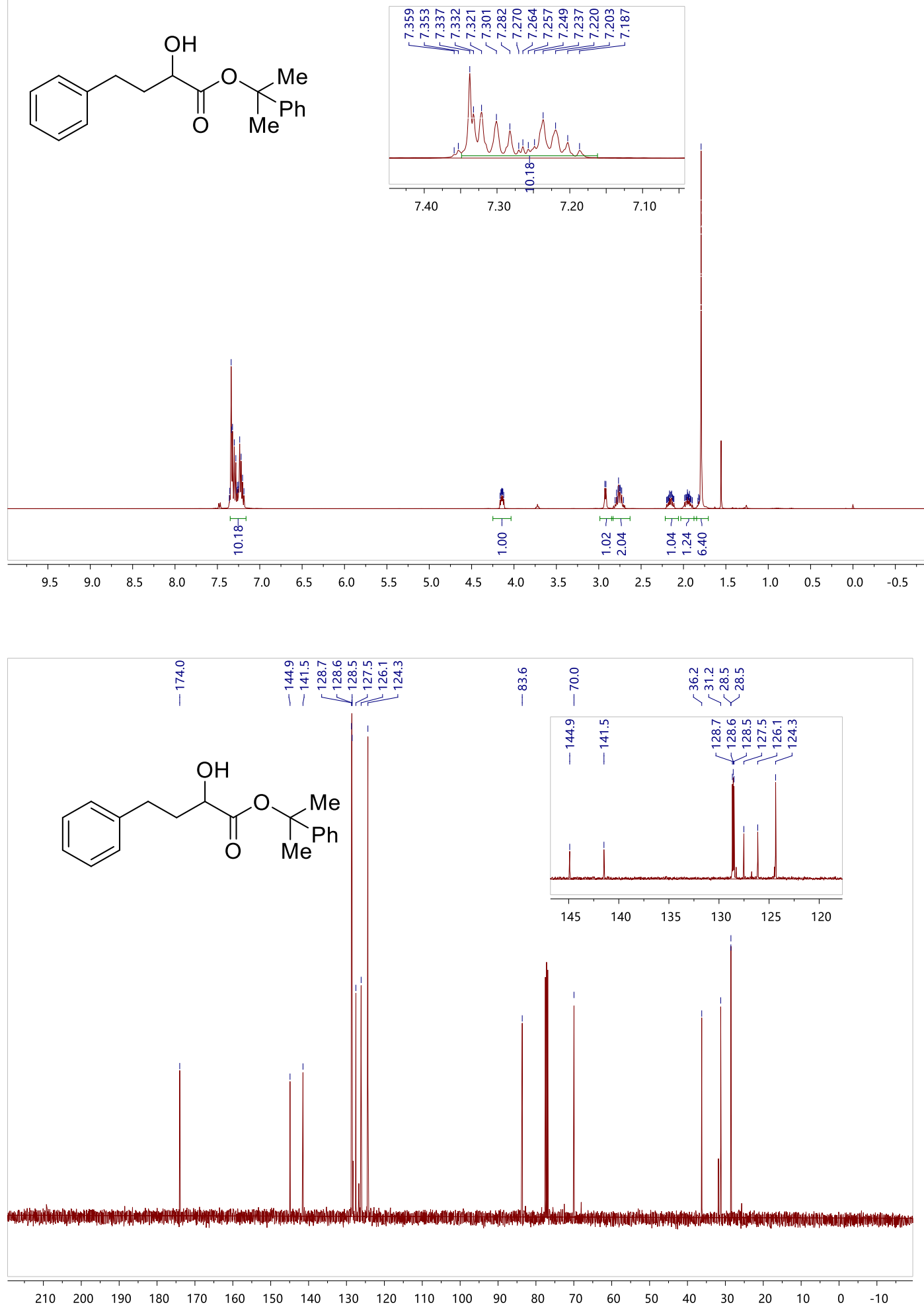
2-Phenylpropan-2-yl 2-hydroxy-5-phenylpentanoate (2l)

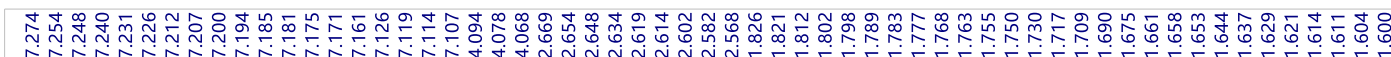
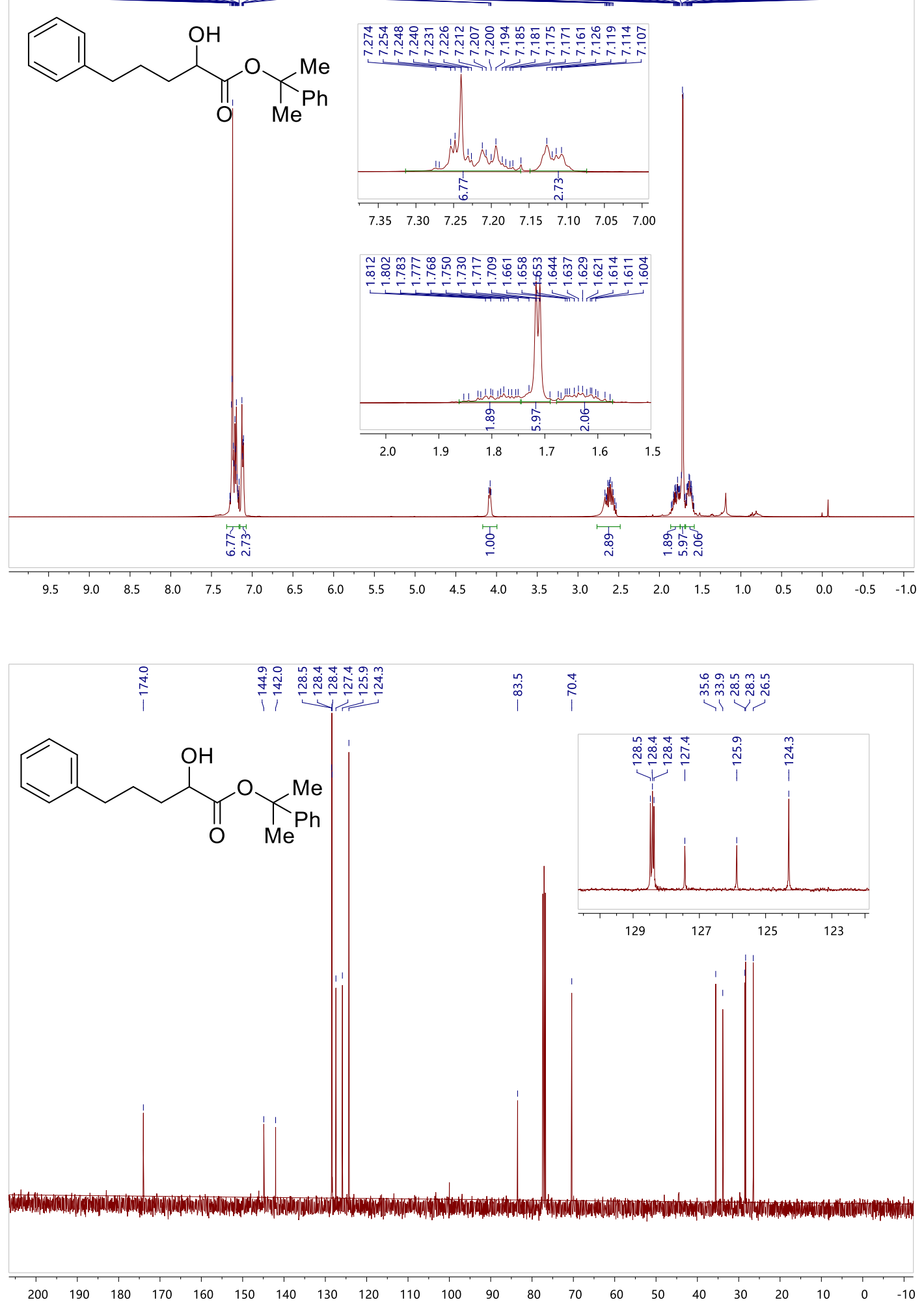
2-Phenylpropan-2-yl 2-hydroxypent-4-enoate (2m)
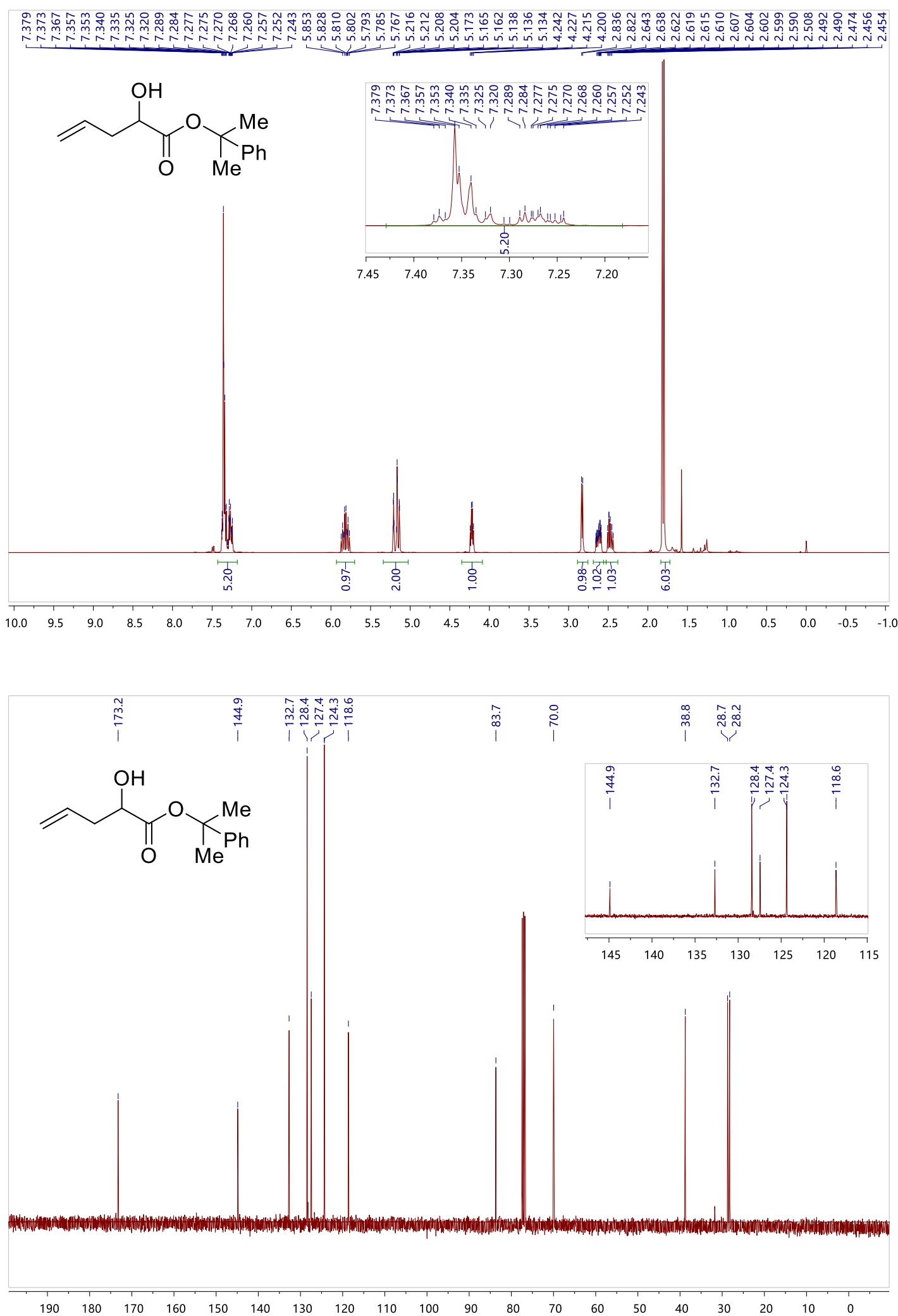
2-Phenylpropan-2-yl 2-hydroxyhex-5-enoate (2n)

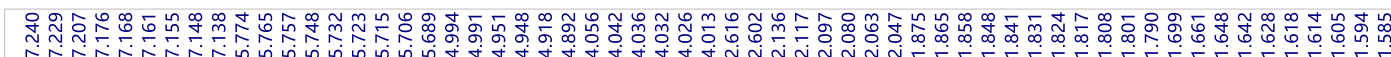<smiles>C=CCCC(O)C(=O)OC(C)(C)[R6]([Na])([Na])c1ccccc1</smiles>
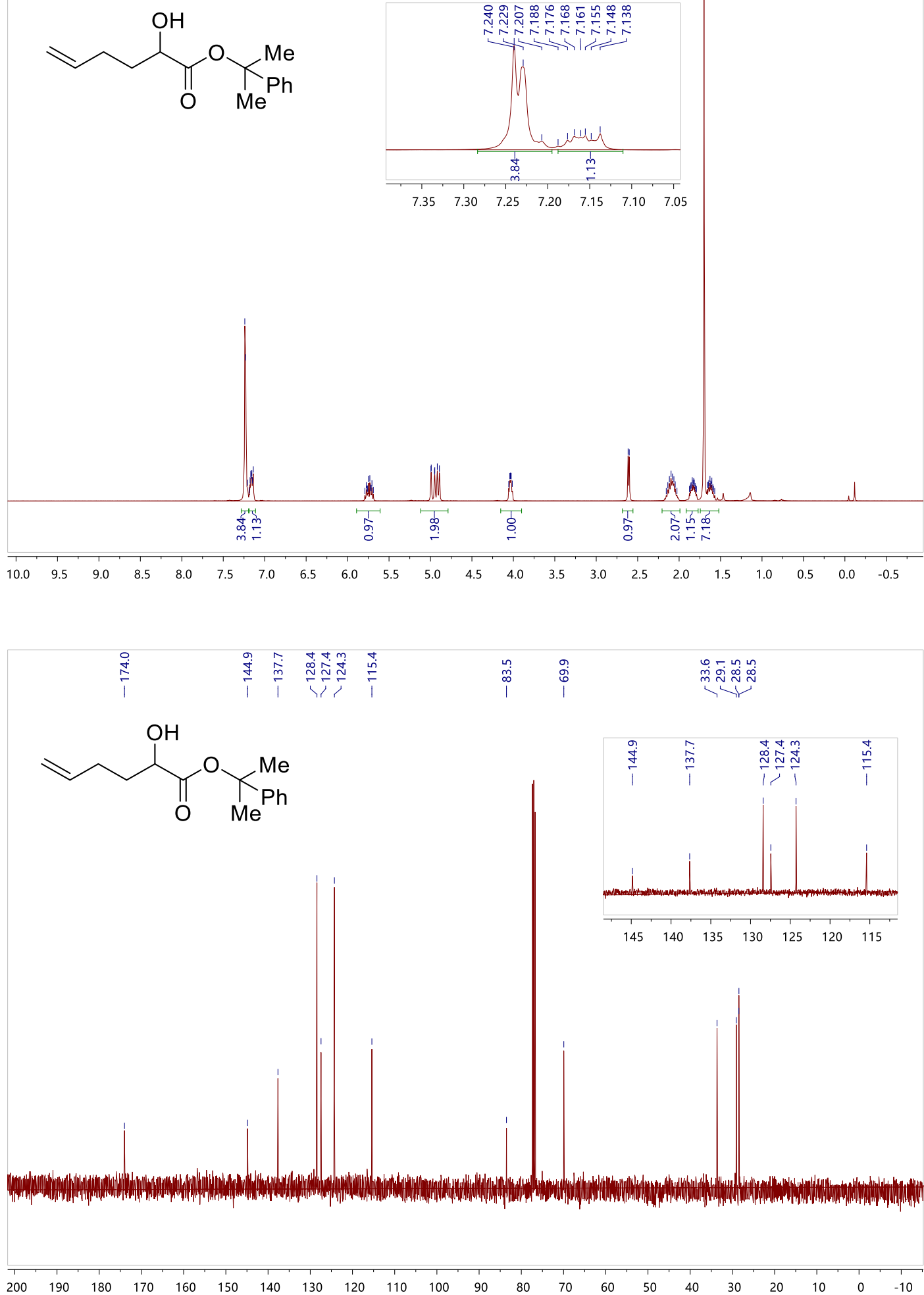
2-Phenylpropan-2-yl 2-hydroxyoct-7-enoate (20)
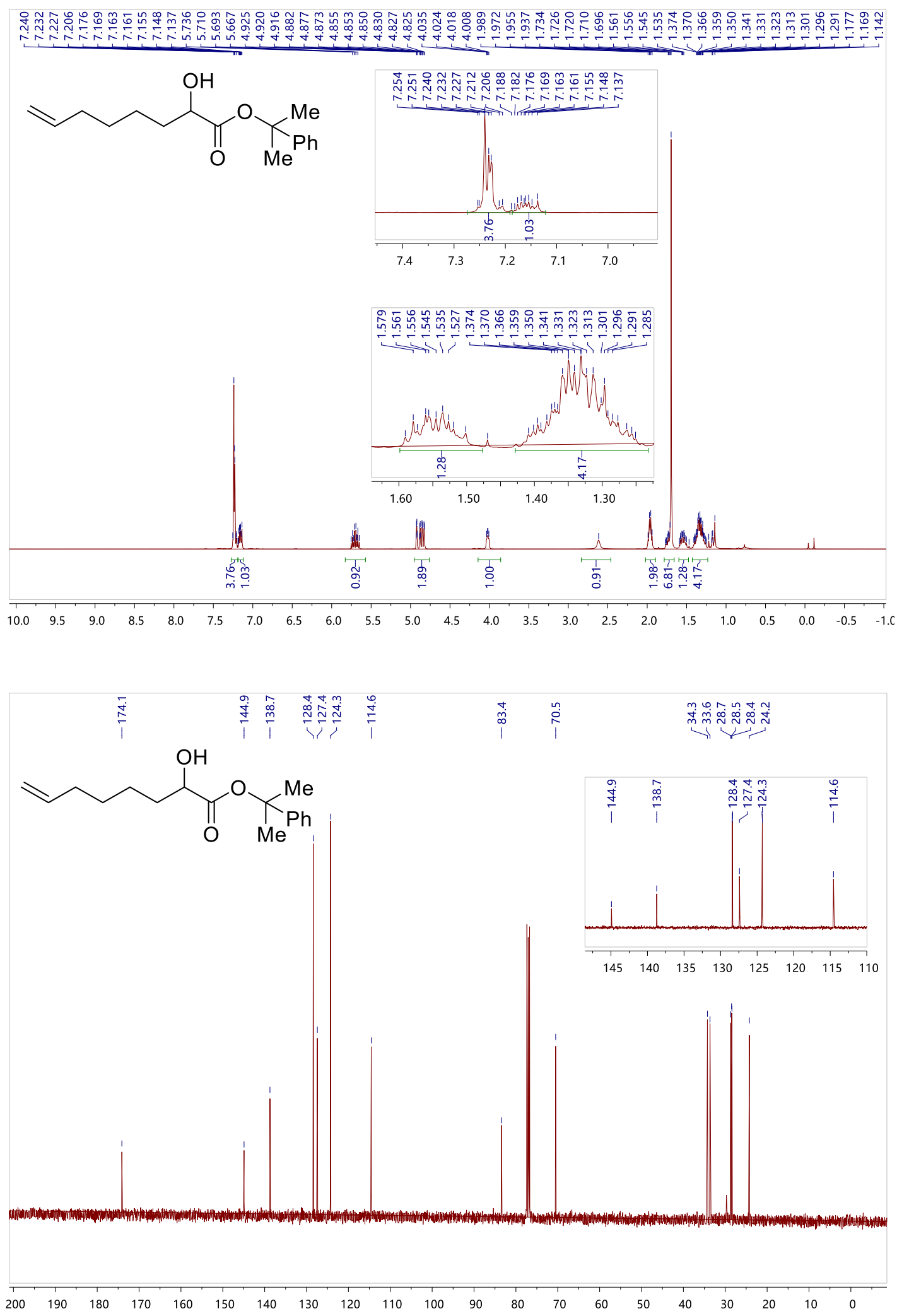
2-Phenylpropan-2-yl 2-hydroxyhex-5-ynoate (2p)
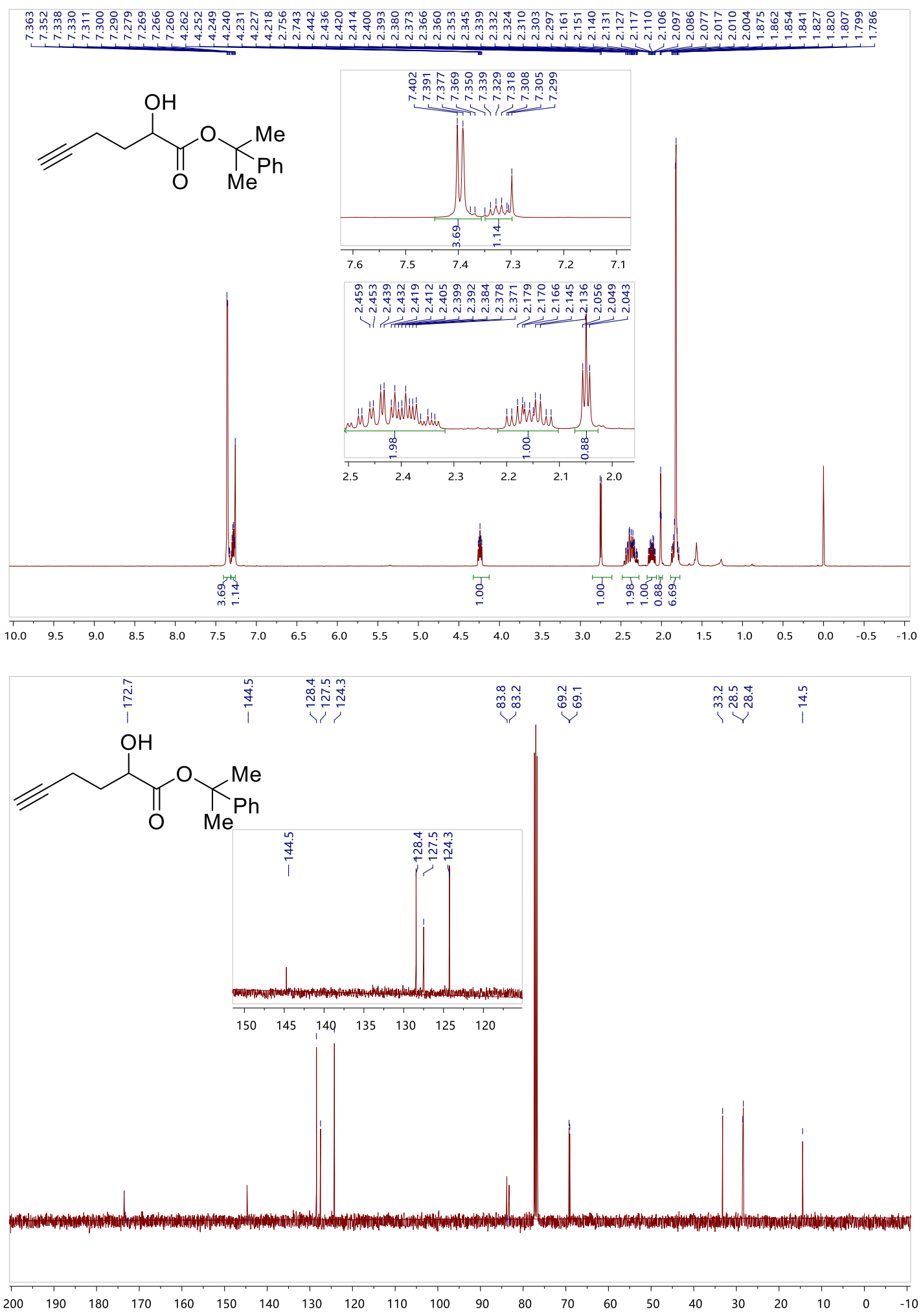
2-Phenylpropan-2-yl 2-hydroxy-5-methoxypentanoate (2q)
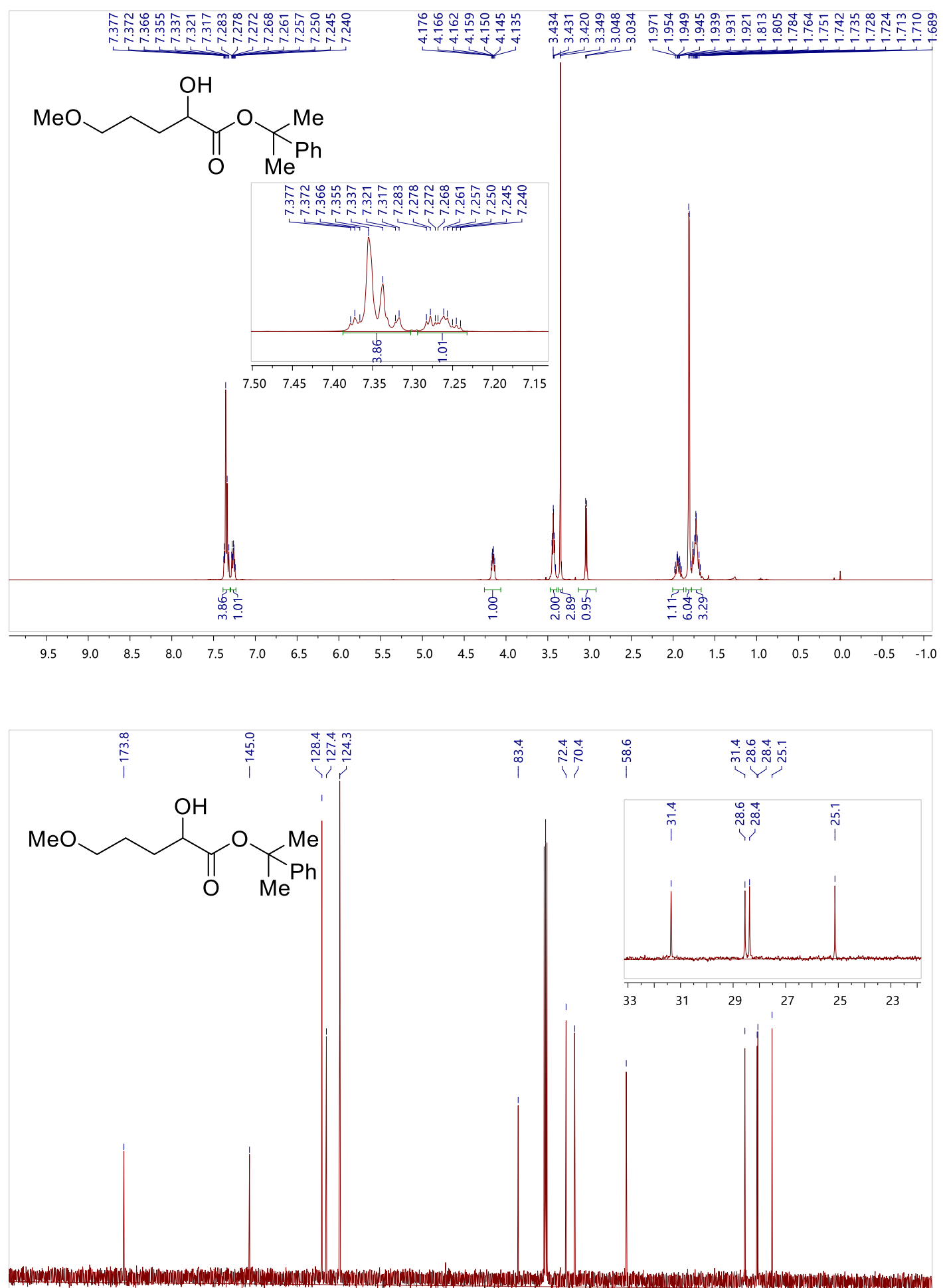

$\begin{array}{lllllllllllllllllllllllllll}200 & 190 & 180 & 170 & 160 & 150 & 140 & 130 & 120 & 110 & 100 & 90 & 80 & 70 & 60 & 50 & 40 & 30 & 20 & 10 & 0 & -10\end{array}$ 
2-Phenylpropan-2-yl 5-((tert-butyldimethylsilyl)oxy)-2-hydroxypentanoate (2r)

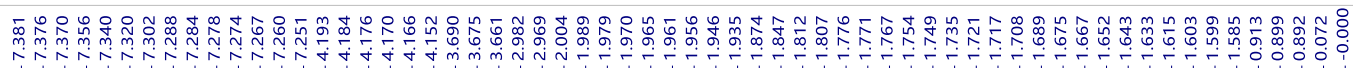<smiles>CC(C)(C)OCCCC(O)C(=O)OC(C)(C)c1ccccc1</smiles>
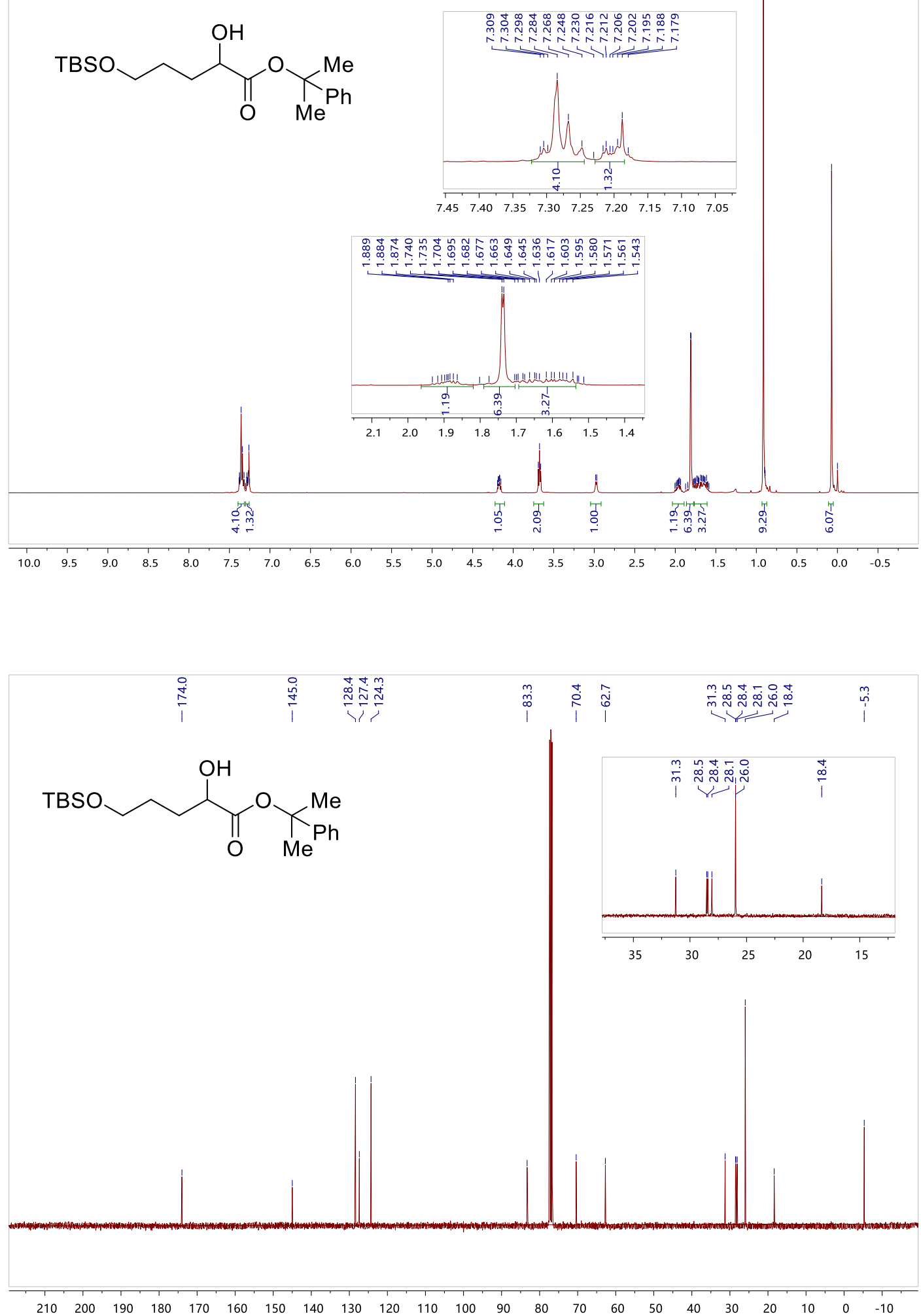
2-Phenylpropan-2-yl 2-hydroxy-5-(propionyloxy)pentanoate (2s)

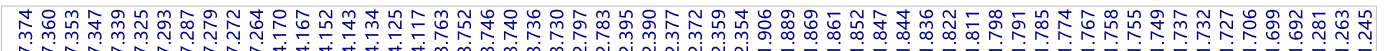
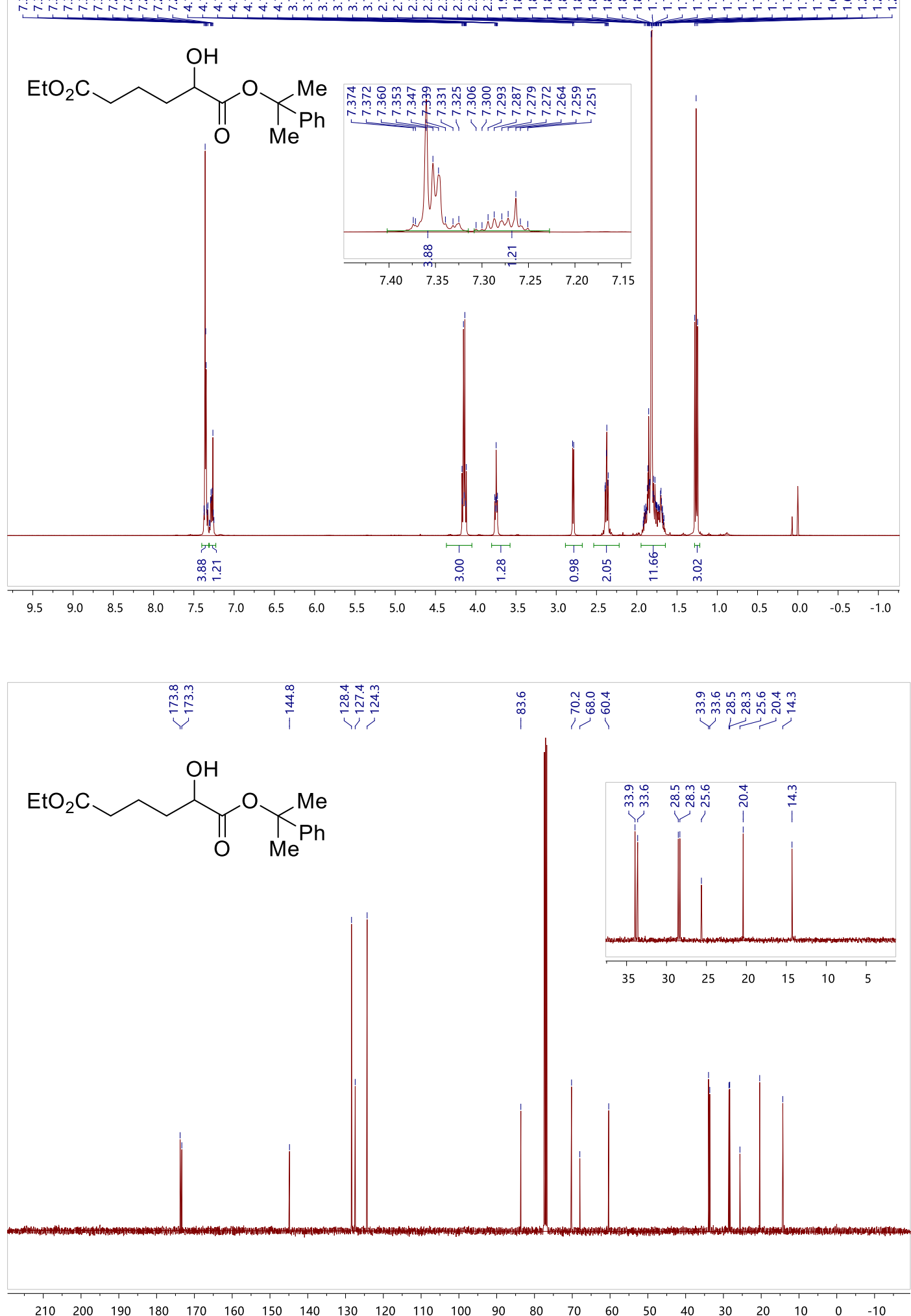
2-Phenylpropan-2-yl 5-cyano-2-hydroxypentanoate (2t)
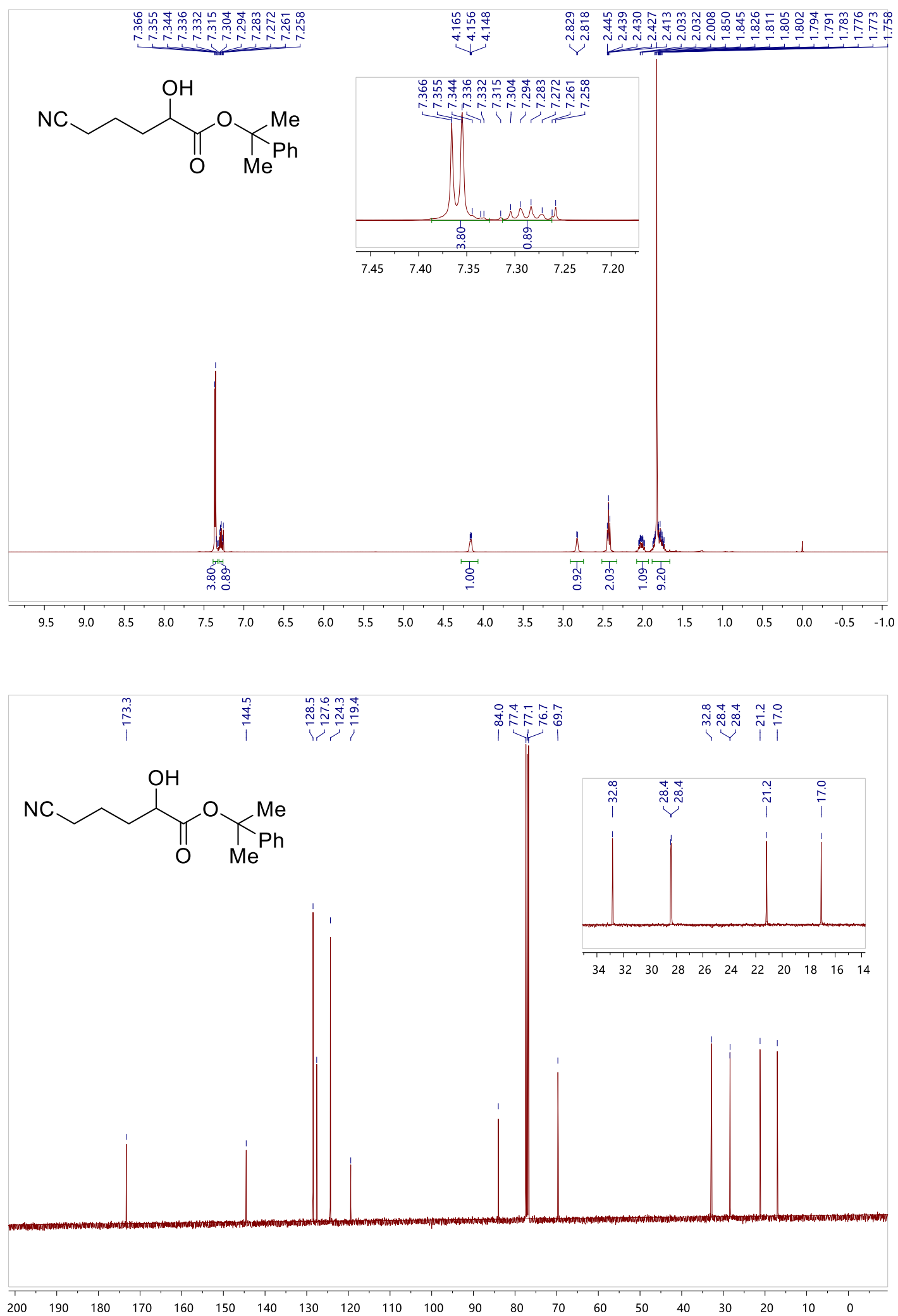
(E)-tert-Butyl 2-hydroxy-4-phenylpent-3-enoate (7a)
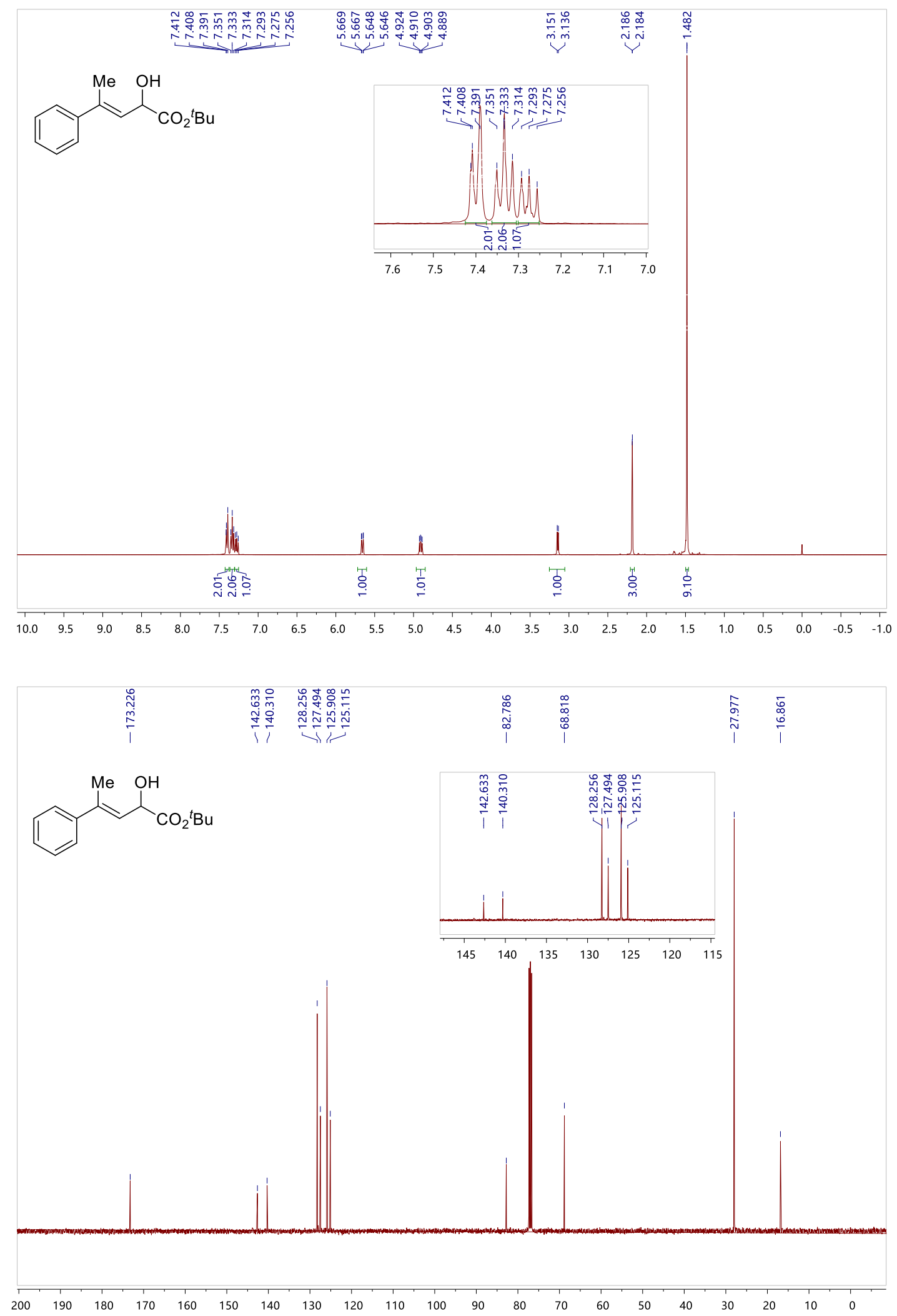
(E)-tert-Butyl 2-hydroxy-4-(m-tolyl)pent-3-enoate (7b)
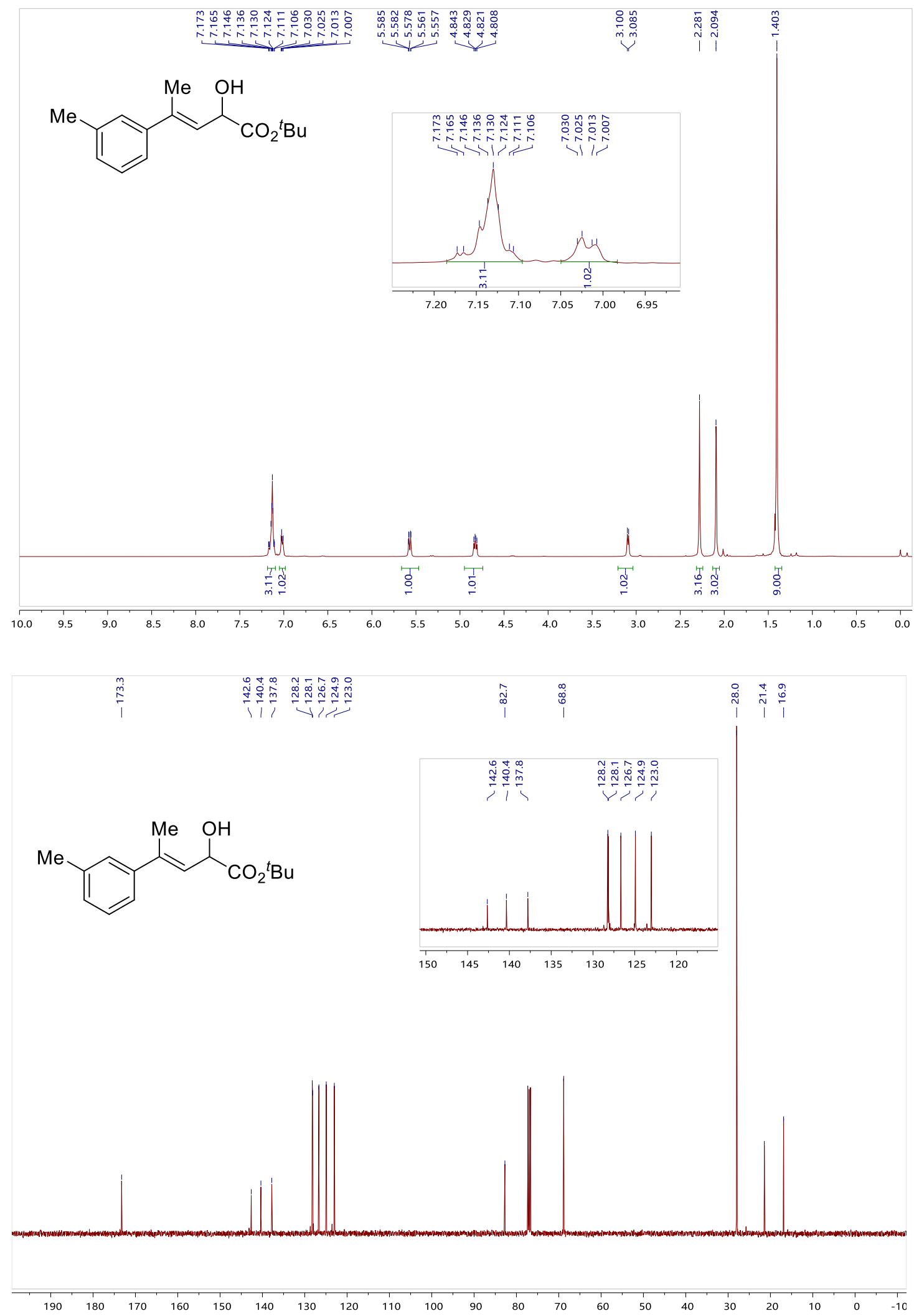
(E)-tert-Butyl 2-hydroxy-4-(p-tolyl)pent-3-enoate (7c)
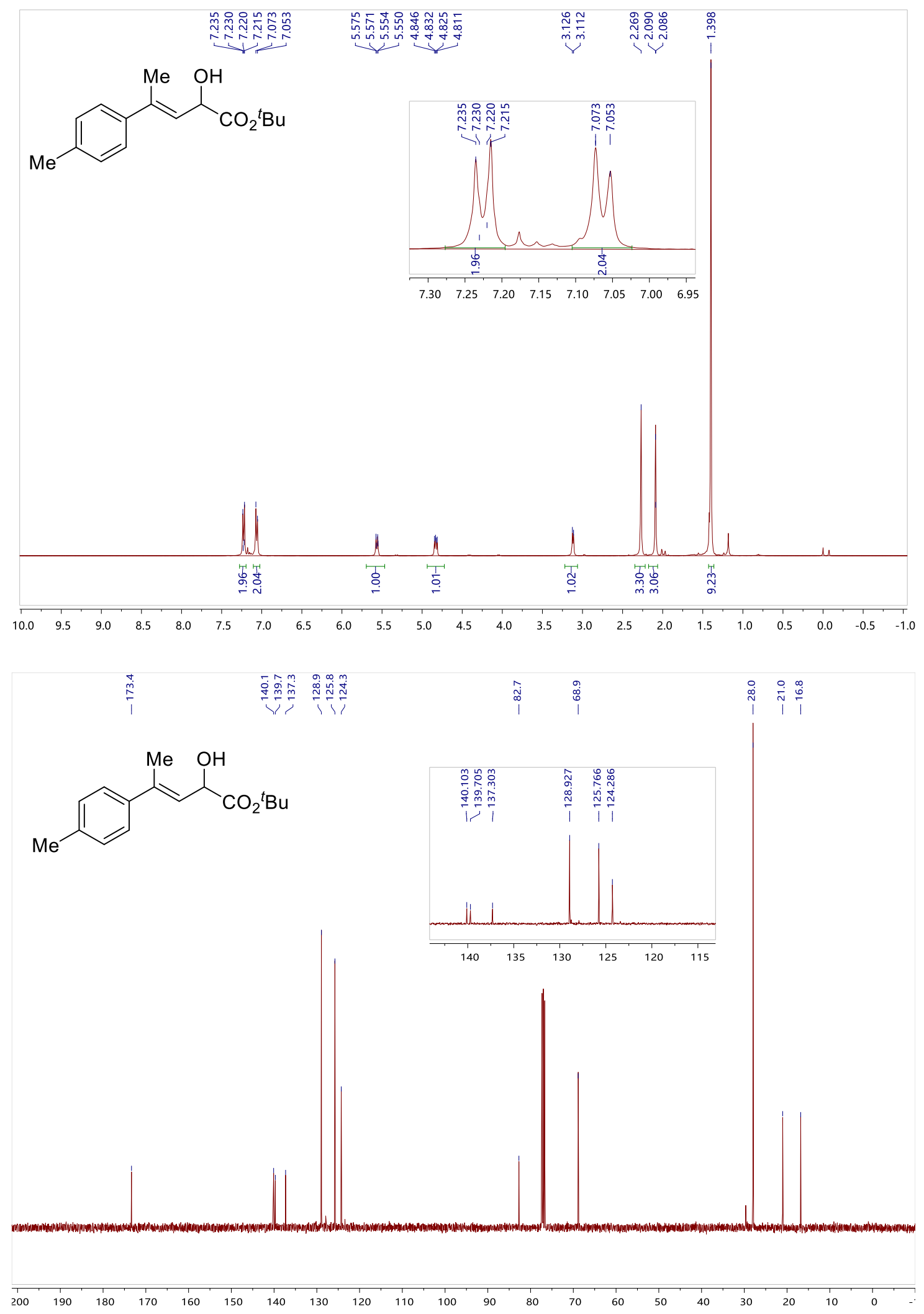
(E)-tert-Butyl 2-hydroxy-4-(4-methoxyphenyl)pent-3-enoate (7d)
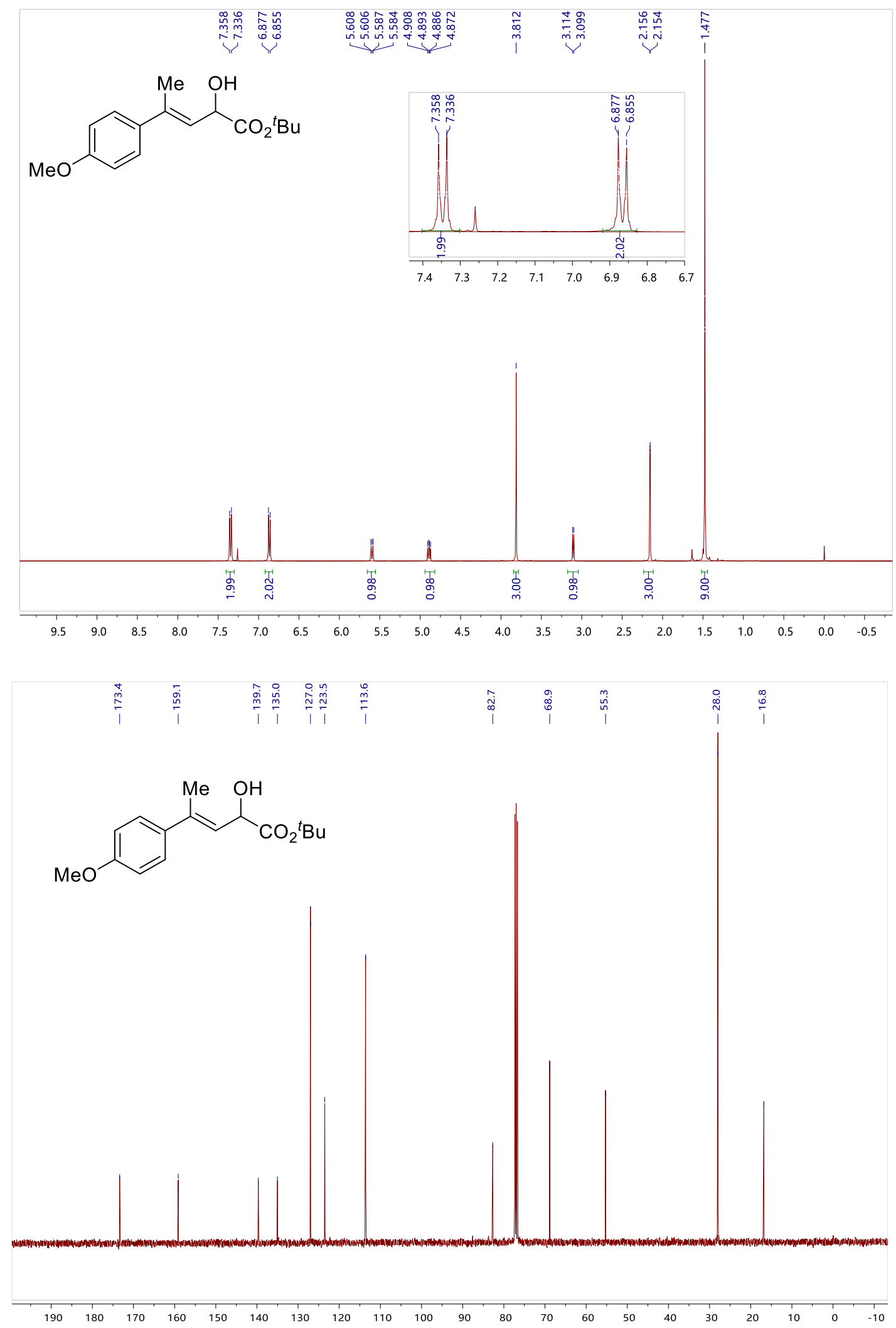
(E)-tert-Butyl 2-hydroxy-4-(4-fluorophenyl)pent-3-enoate (7e)
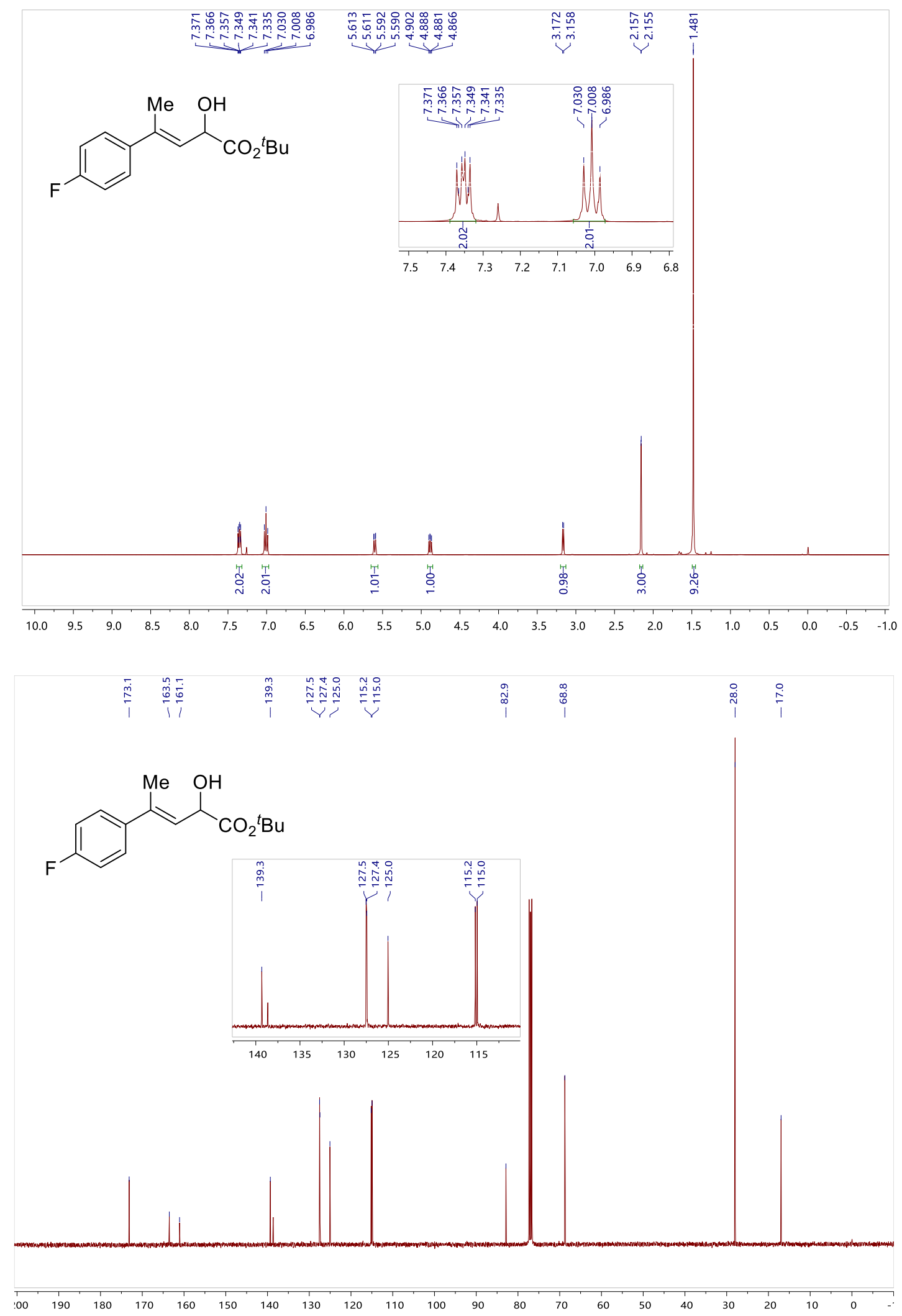
(E)-tert-Butyl 2-hydroxy-4-(2-chlorophenyl)pent-3-enoate (7f)
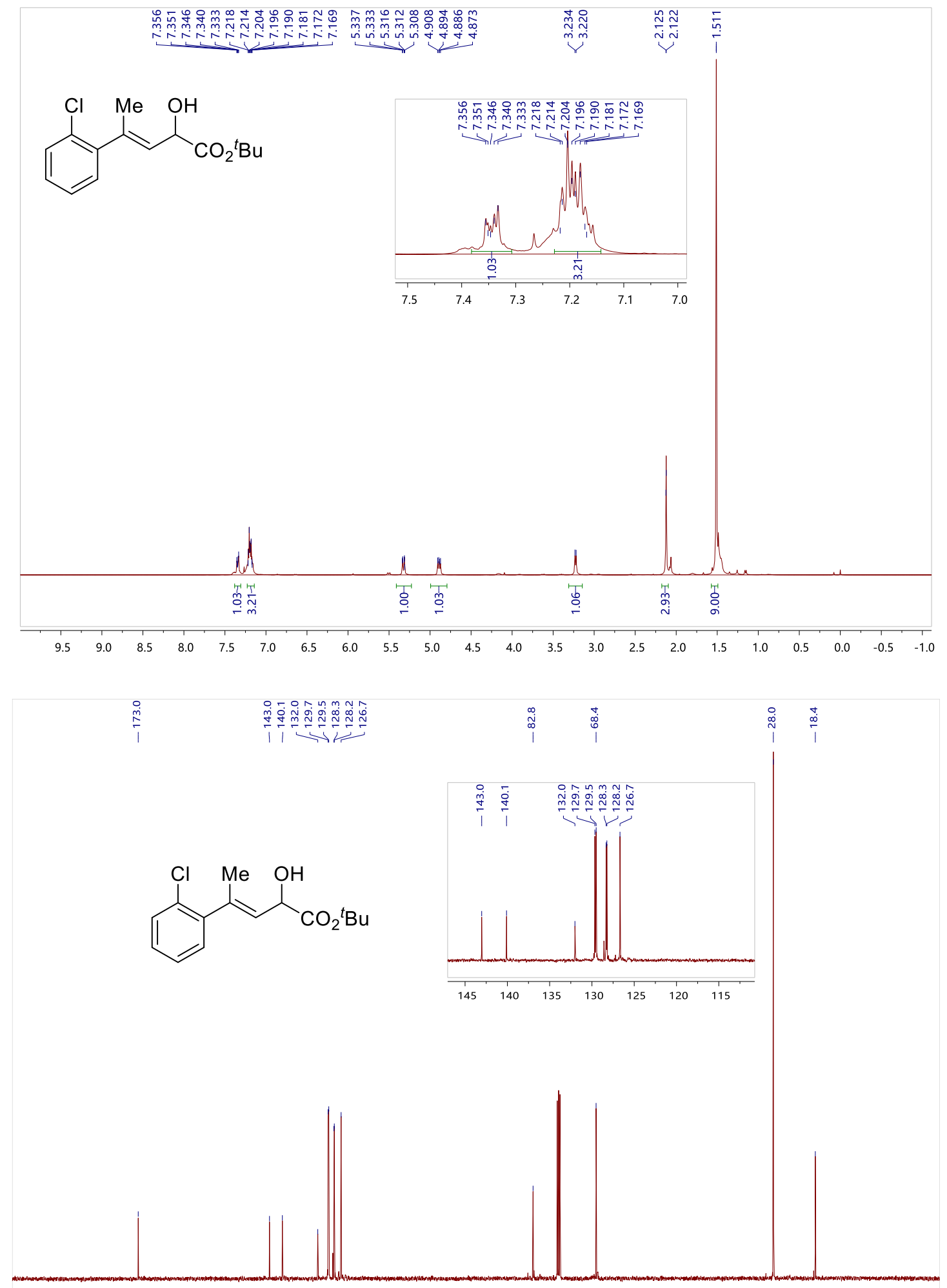

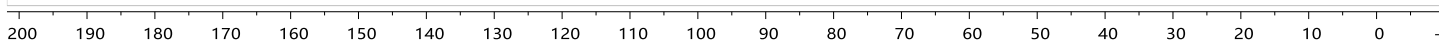


(E)-tert-Butyl 2-hydroxy-4-(3-chlorophenyl)pent-3-enoate (7g)
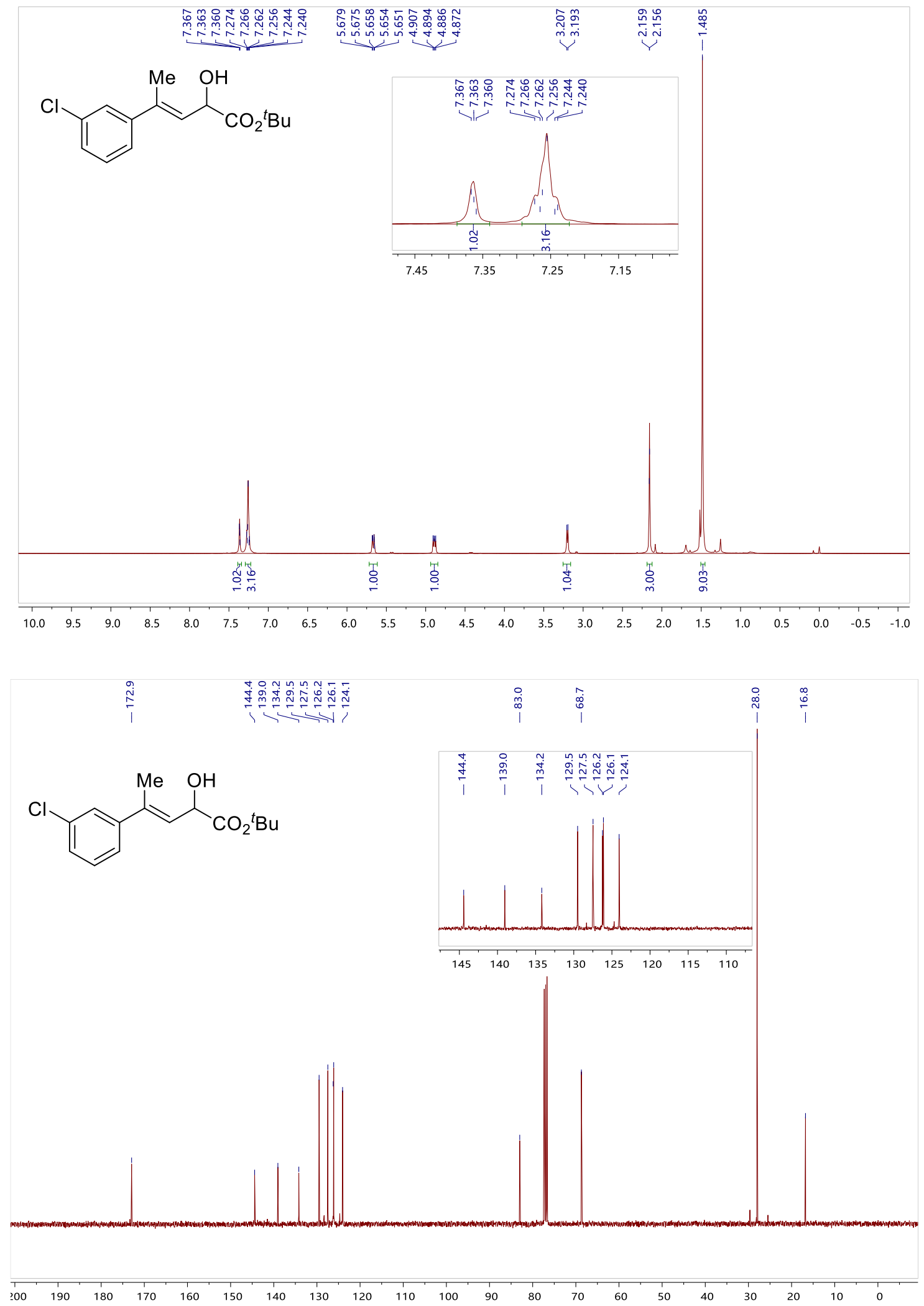
(E)-tert-Butyl 2-hydroxy-4-(4-chlorophenyl)pent-3-enoate (7h)
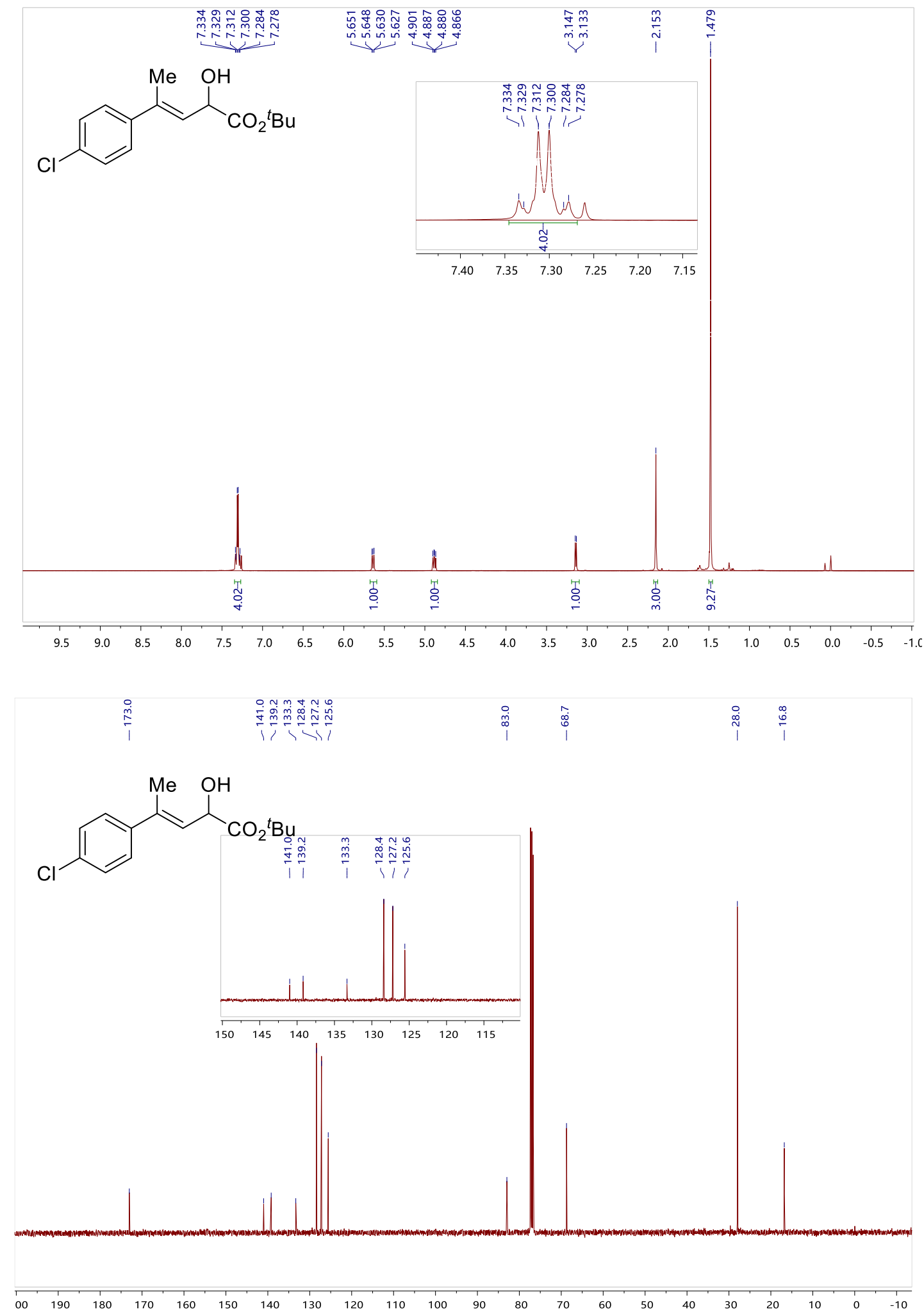
(E)-tert-Butyl 4-(3,4-dimethylphenyl)-2-hydroxypent-3-enoate (7i)
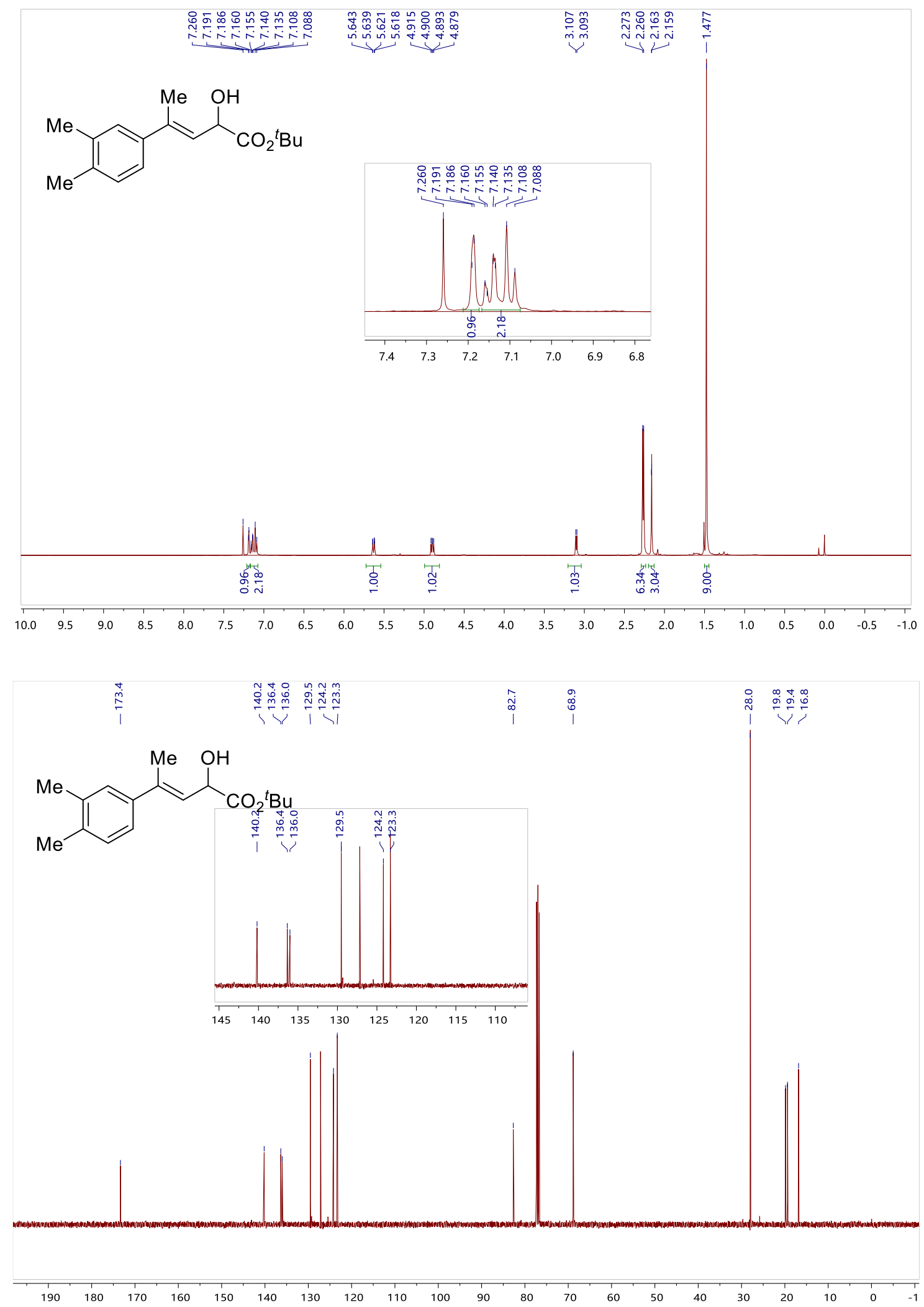
(E)-tert-Butyl 4-(benzo[d][1,3]dioxol-5-yl)-2-hydroxypent-3-enoate (7j)
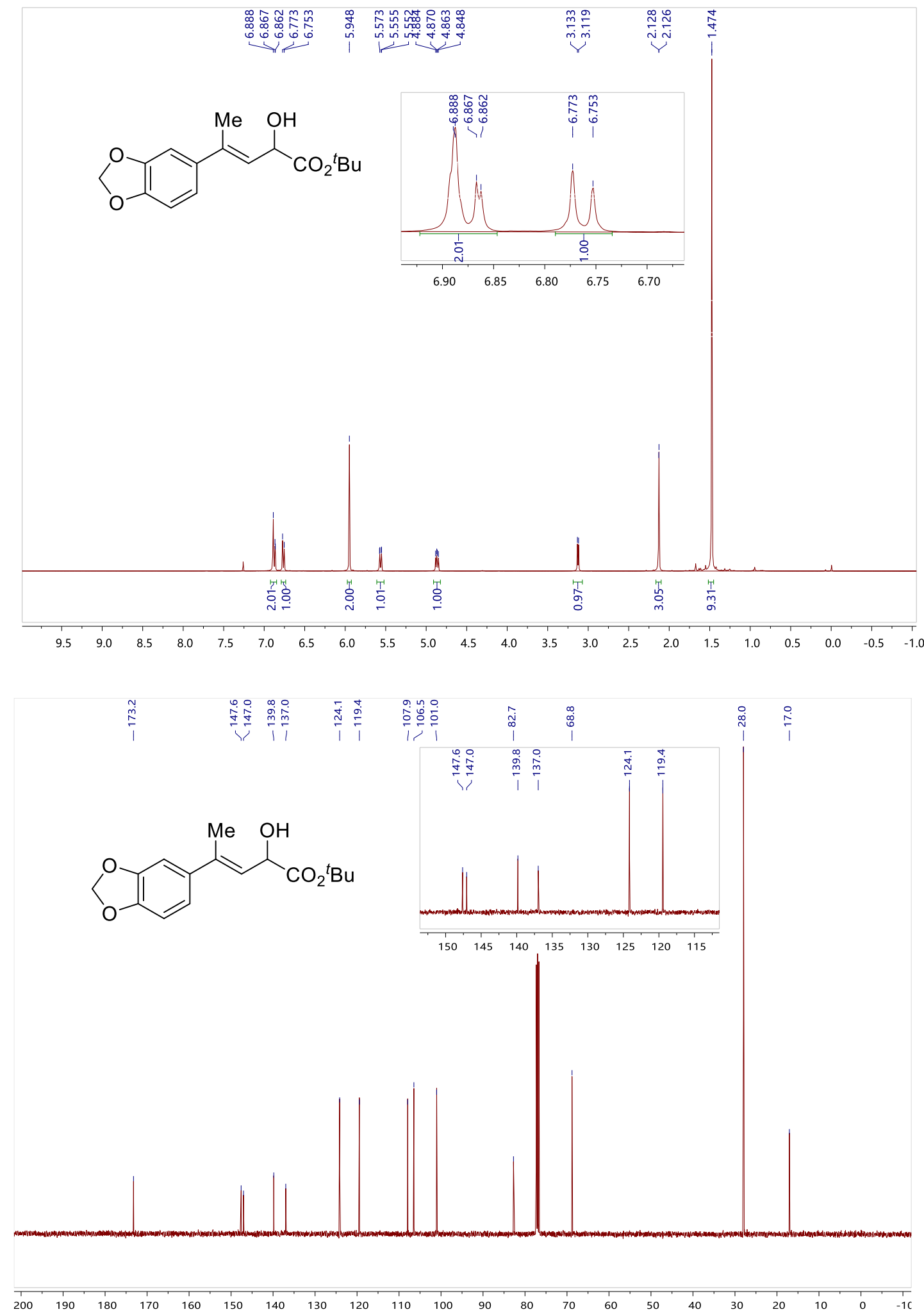
(E)-tert-Butyl 2-hydroxy-4-(naphthalen-2-yl)pent-3-enoate (7k)
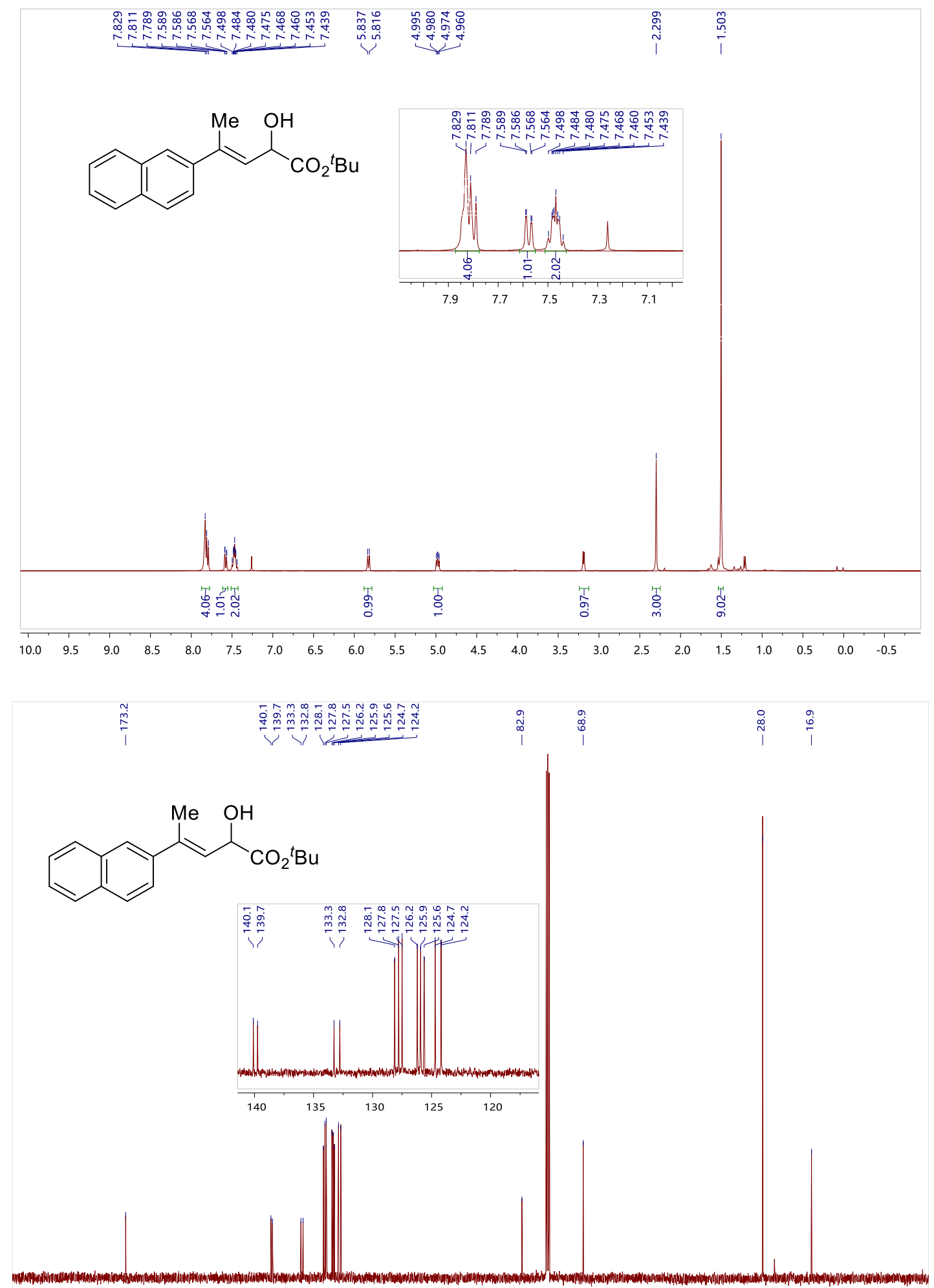

$\begin{array}{llllllllll}190 & 180 & 170 & 160 & 150 & 140 & 130 & 120 & 110 & 100\end{array}$ 
(E)-tert-Butyl 2-hydroxy-4-(thiophen-2-yl)pent-3-enoate (7l)
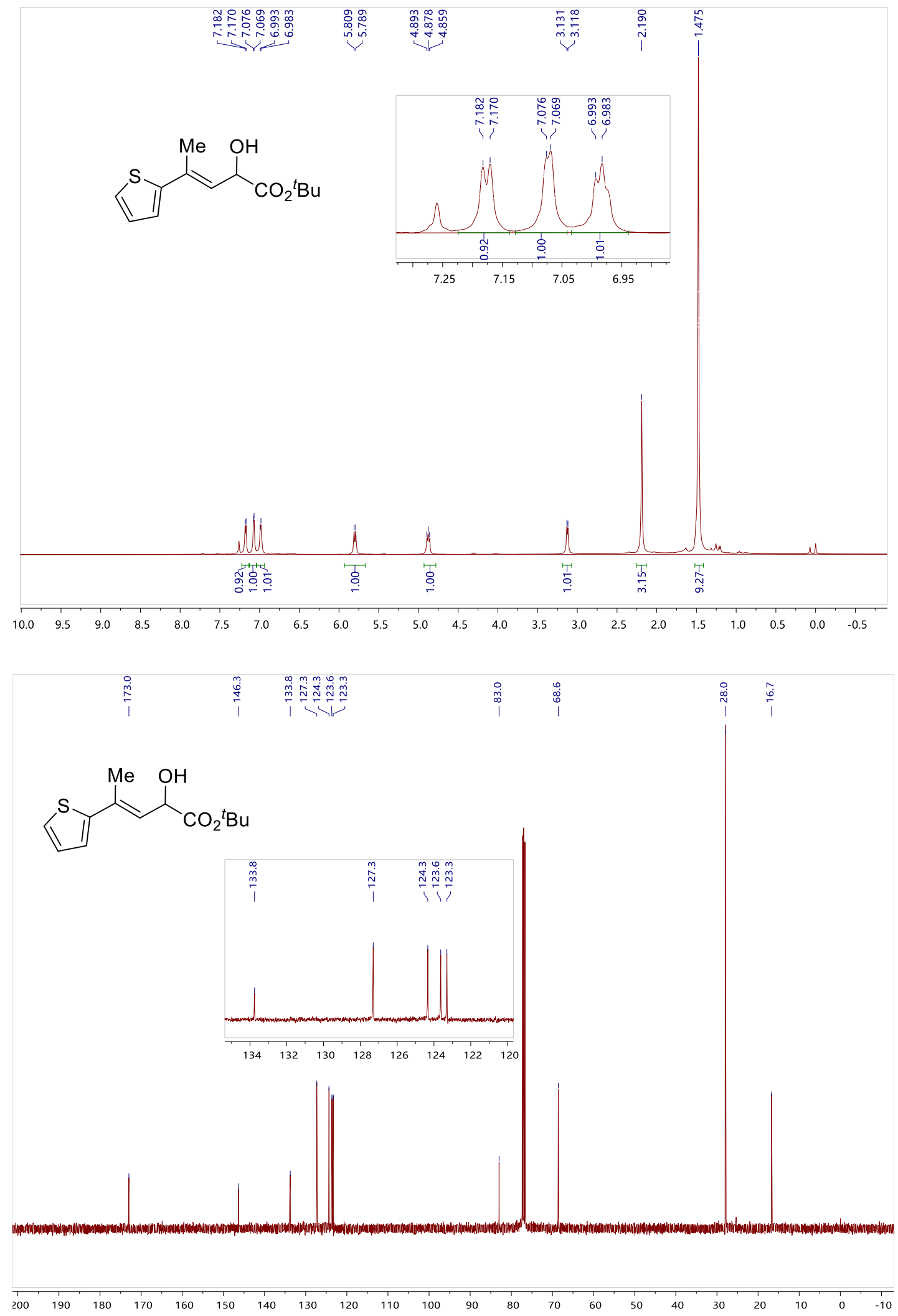
tert-Butyl 2-hydroxy-4,4-diphenylbut-3-enoate (7m)
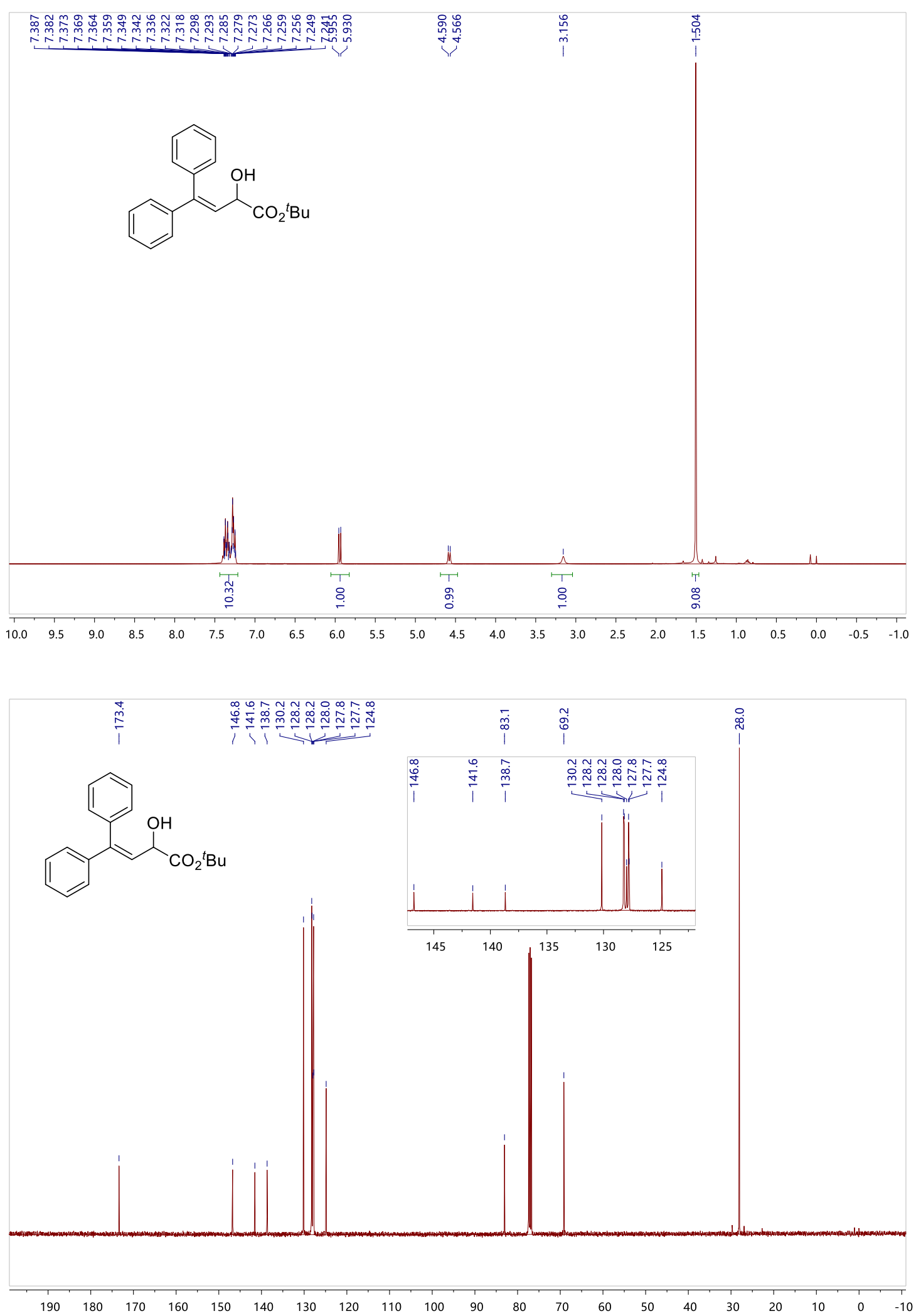
(E)-tert-Butyl 2-hydroxy-4-phenylbut-3-enoate (7n)
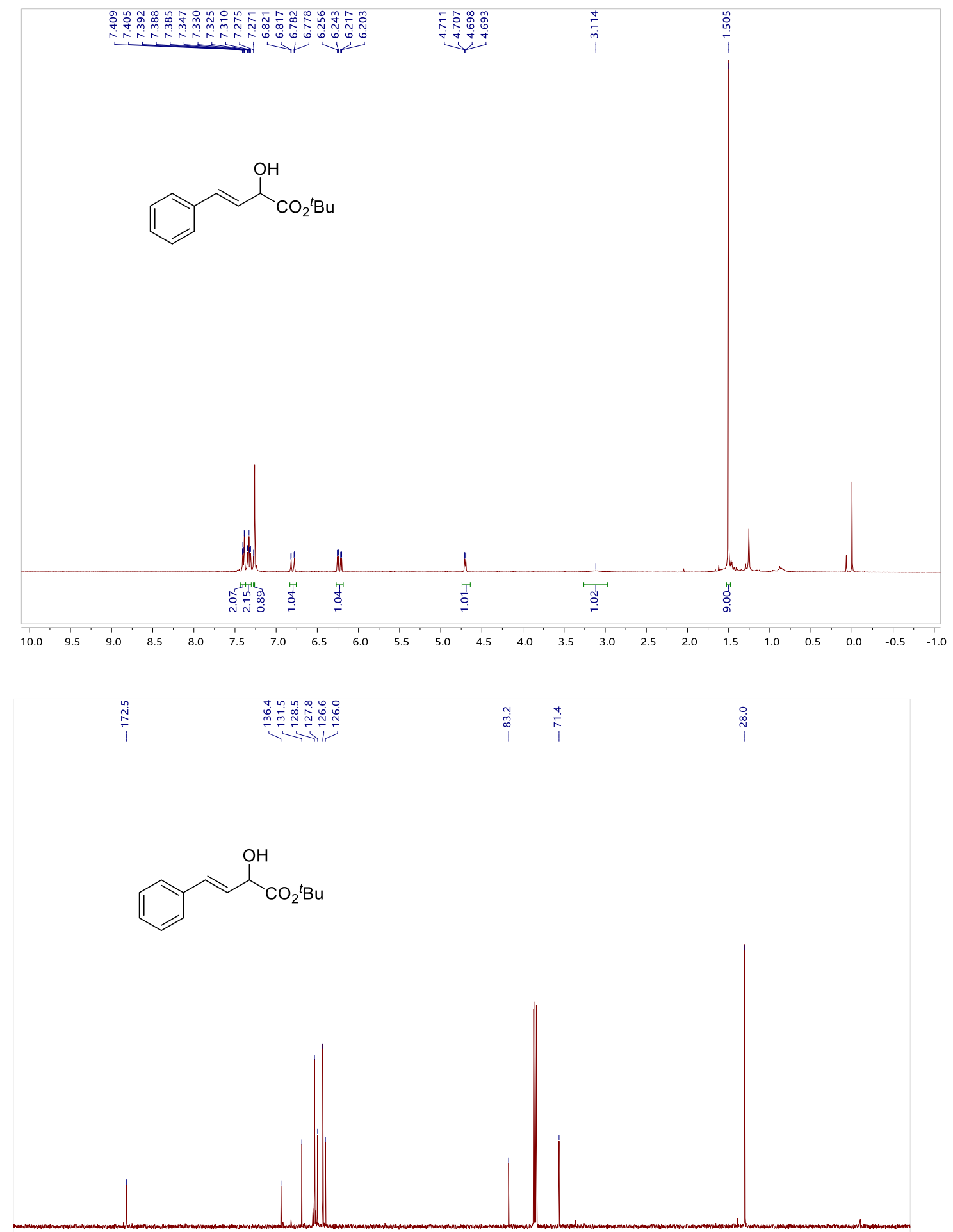

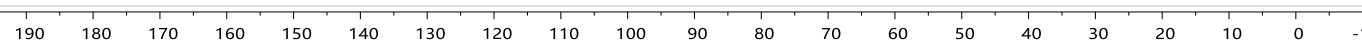


Benzhydryl (E)-2-hydroxypent-3-enoate (7o)
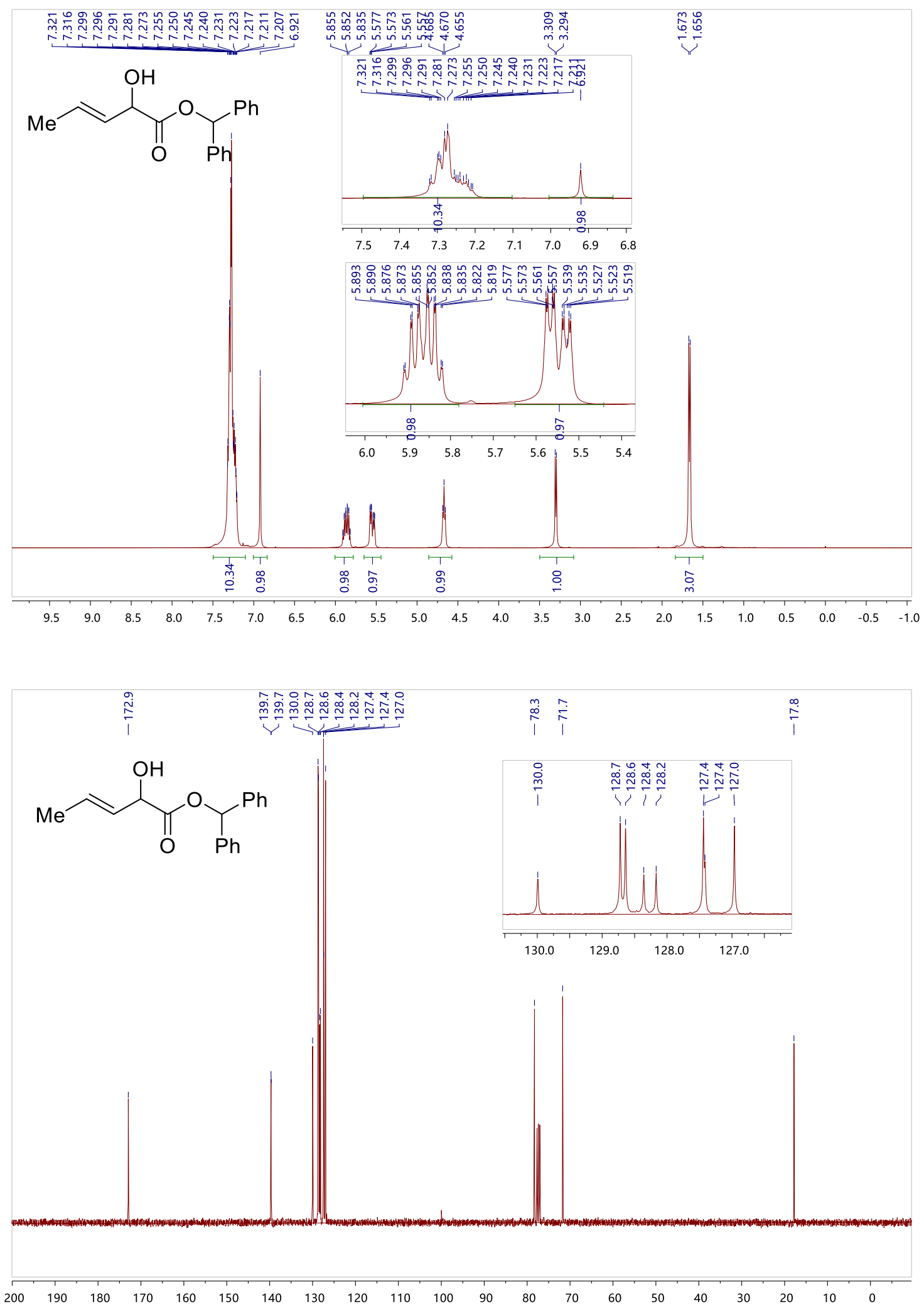
Benzhydryl (E)-2-hydroxyhex-3-enoate (7p)

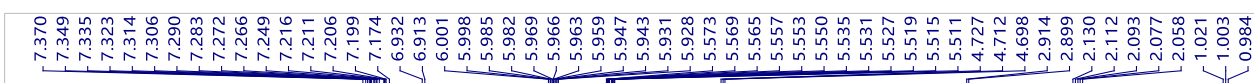

$\left.\overbrace{\prod_{\mathrm{O}}}^{\mathrm{OH}}\right|_{\mathrm{Ph}} ^{\mathrm{O}}$

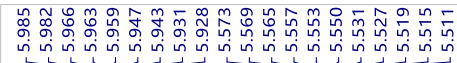
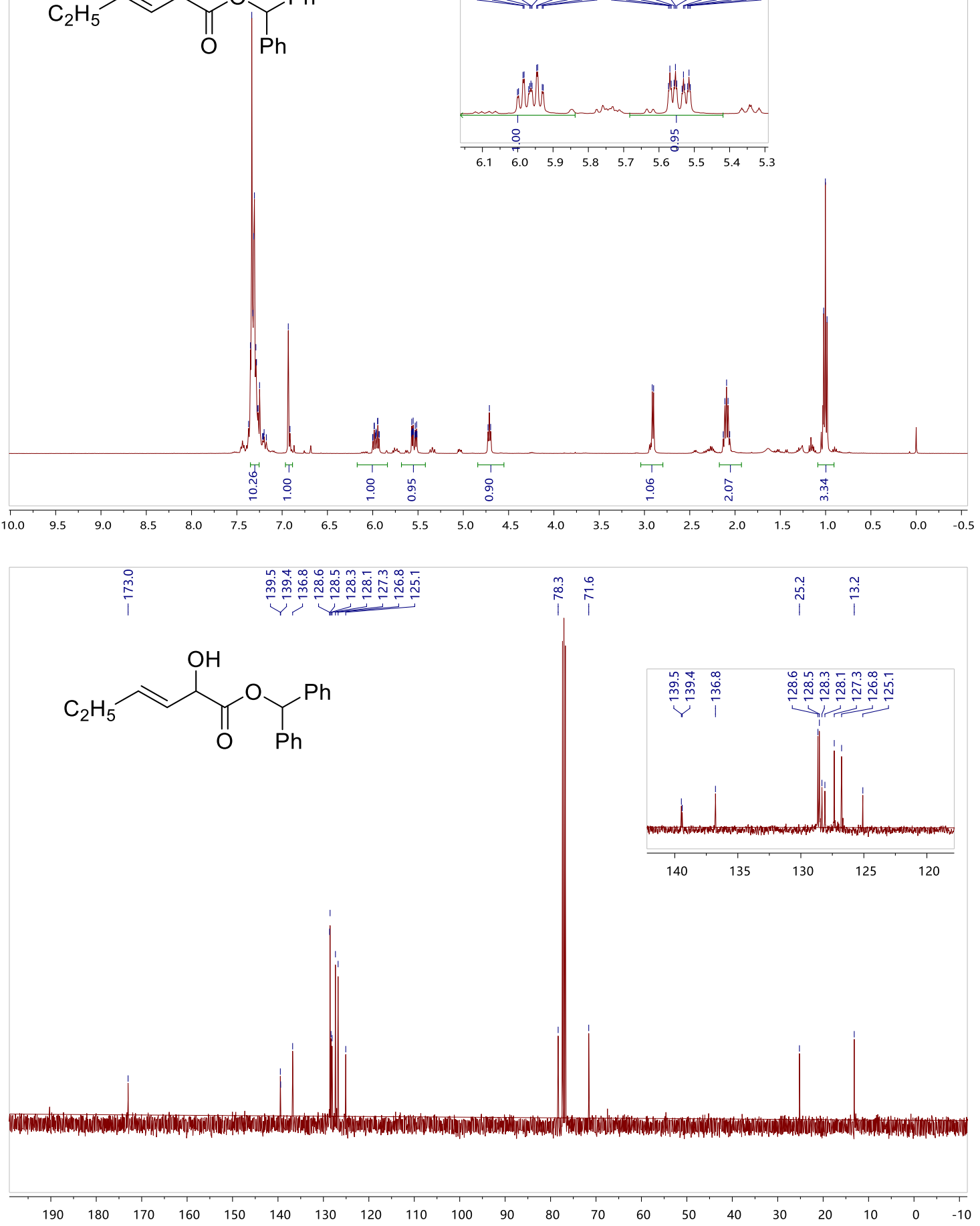
Benzhydryl (E)-2-hydroxyhept-3-enoate (7q)

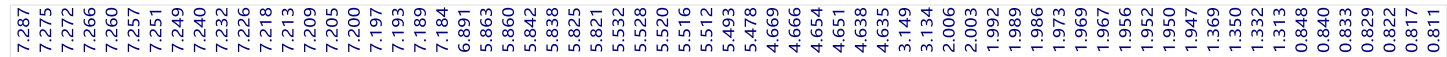

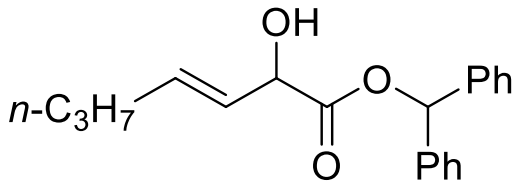
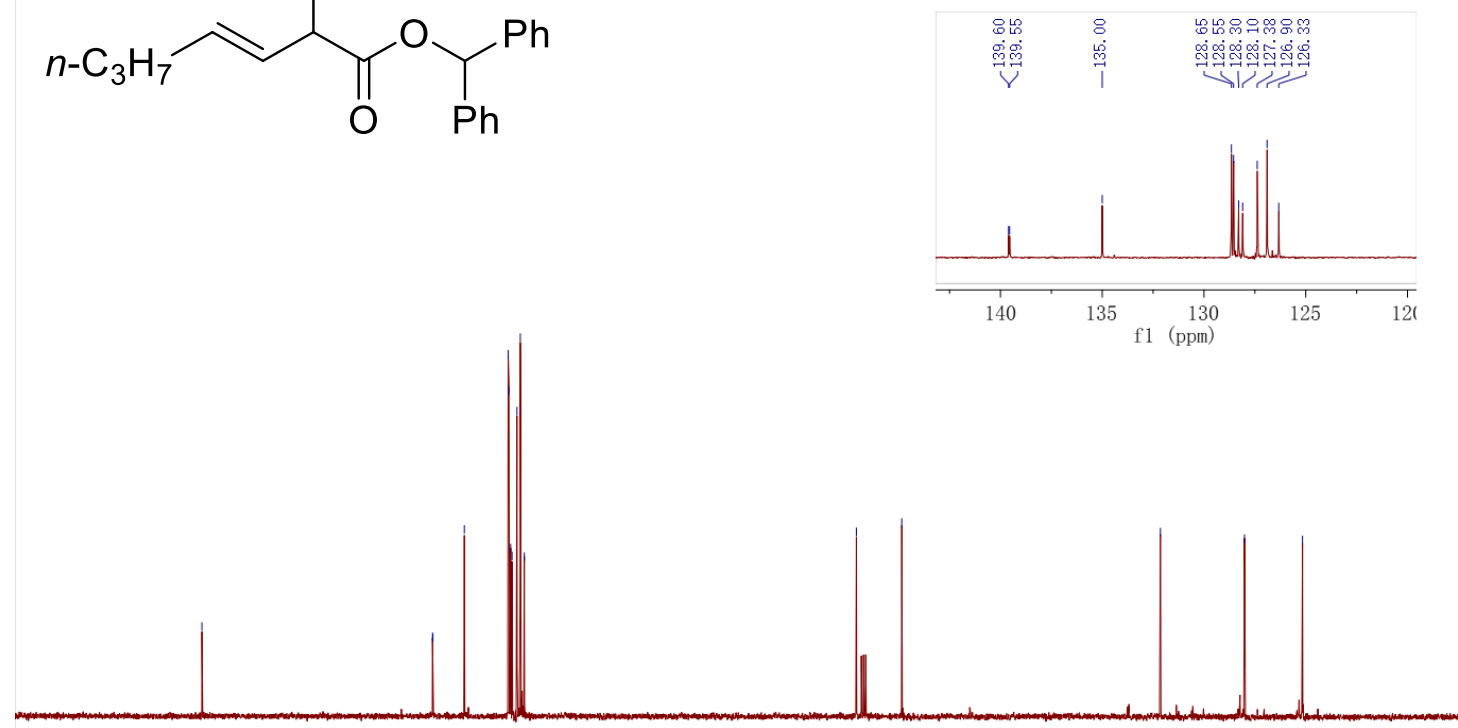

\begin{tabular}{llllllllllll}
\hline 0 & 190 & 180 & 170 & 160 & 150 & 140 & 130 & 120 & 110 & 100 & 90
\end{tabular}

1 (ppm) 
Benzhydryl (E)-2-hydroxydec-3-enoate (7r)
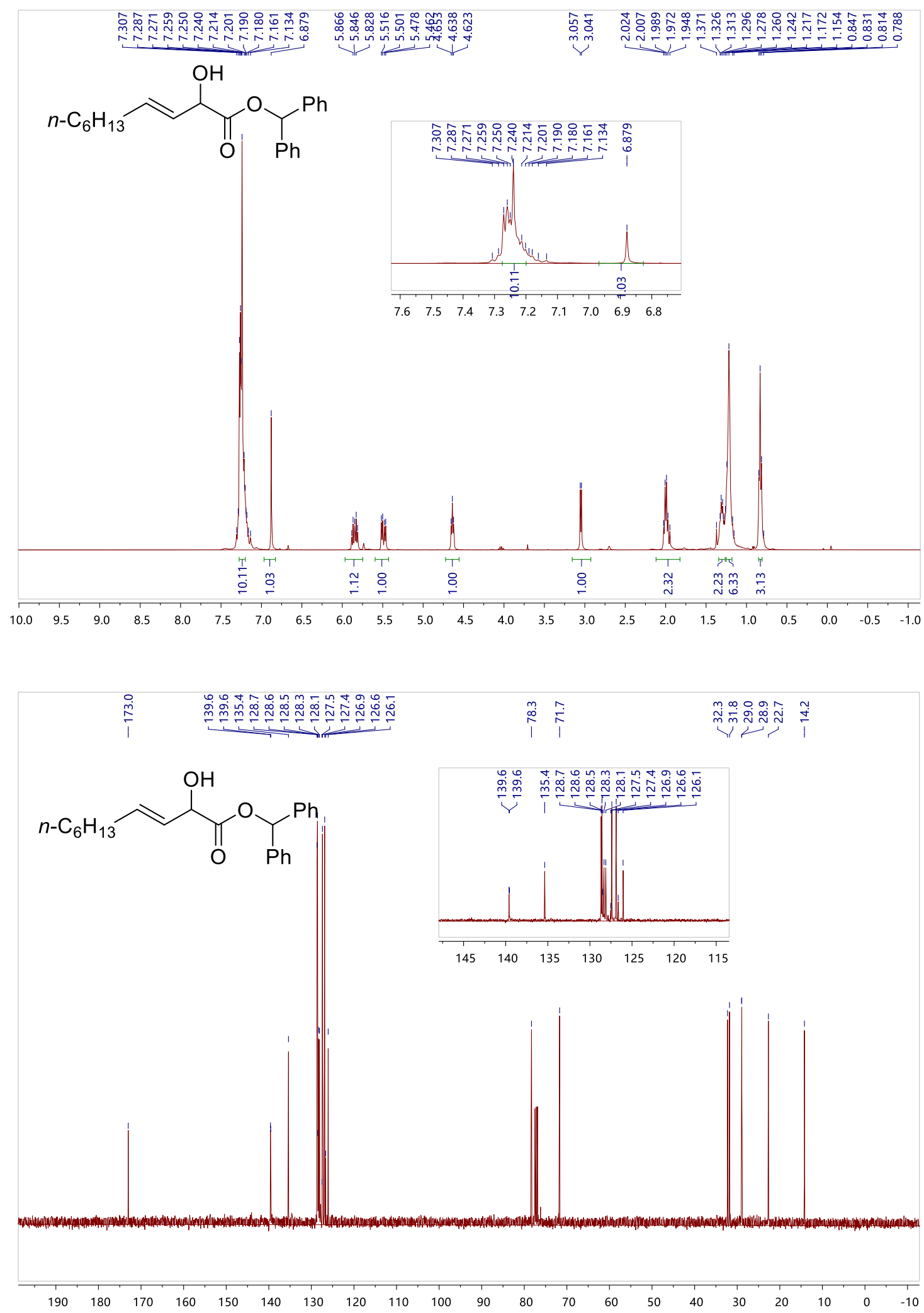
Benzhydryl (E)-2-hydroxy-5-phenylpent-3-enoate (7s)
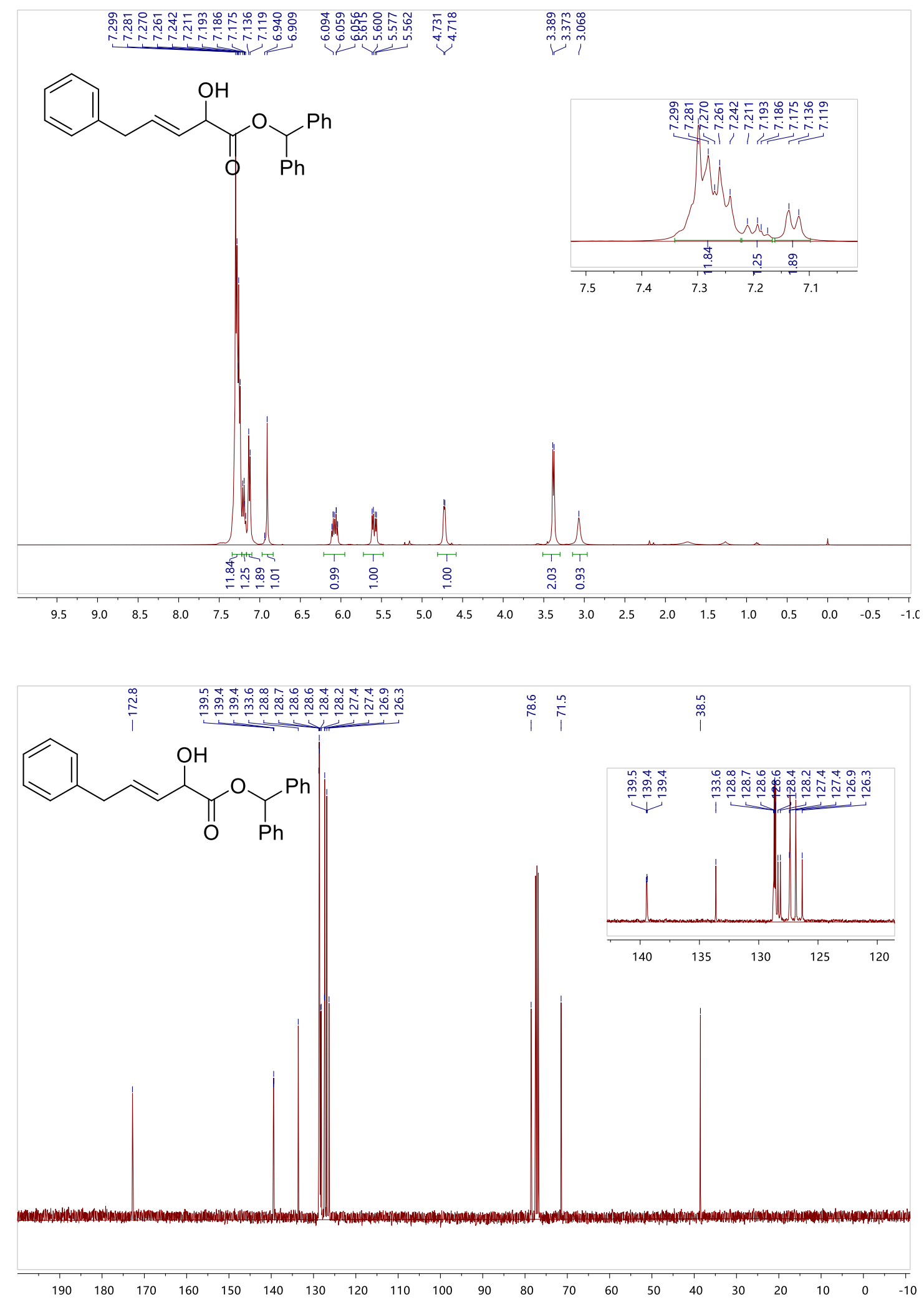
Methyl 2-hydroxybutanoate
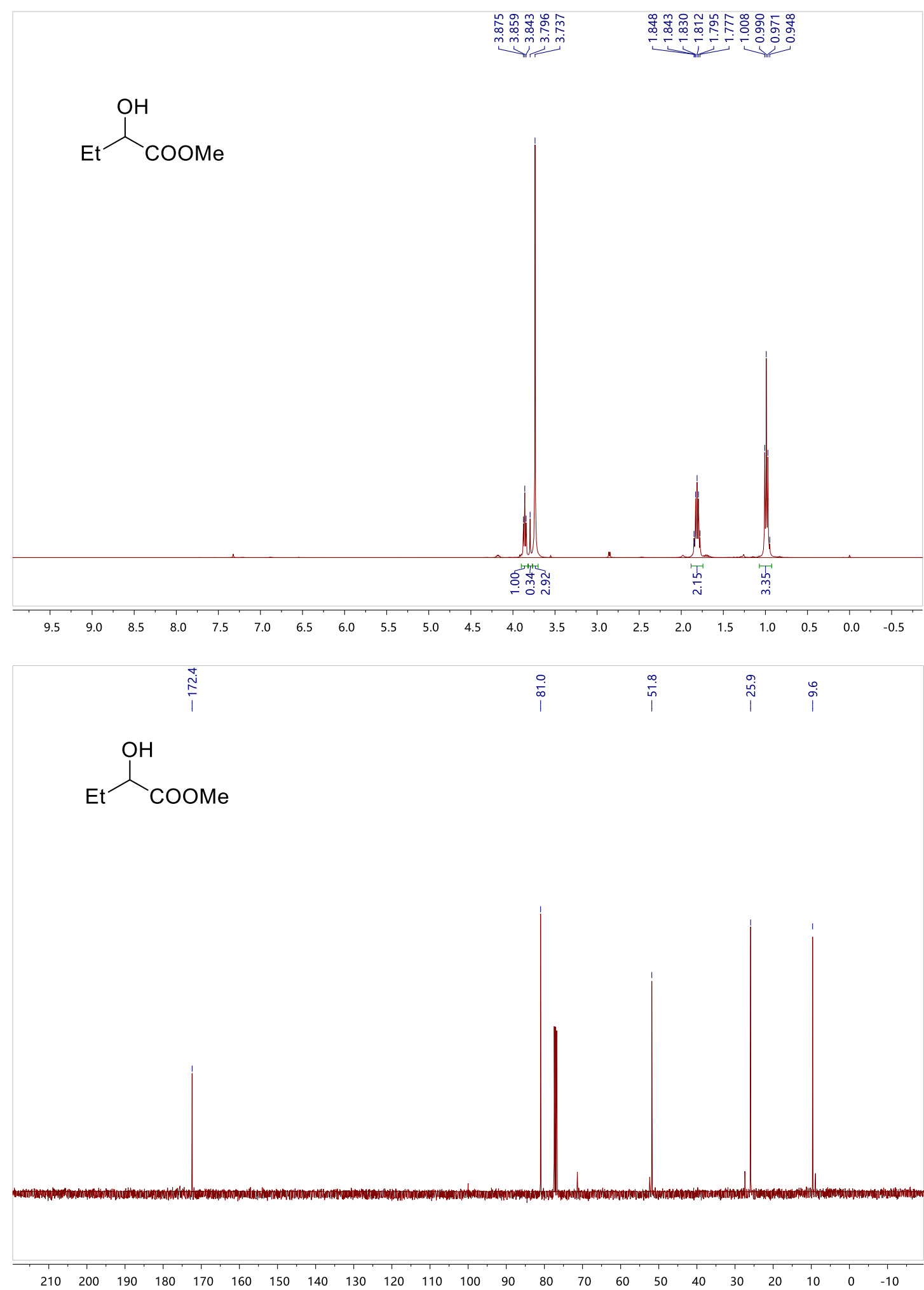


\section{Methyl 2-hydroxyoctanoate}

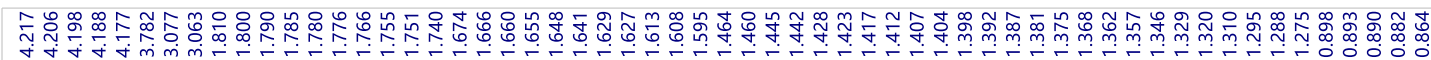
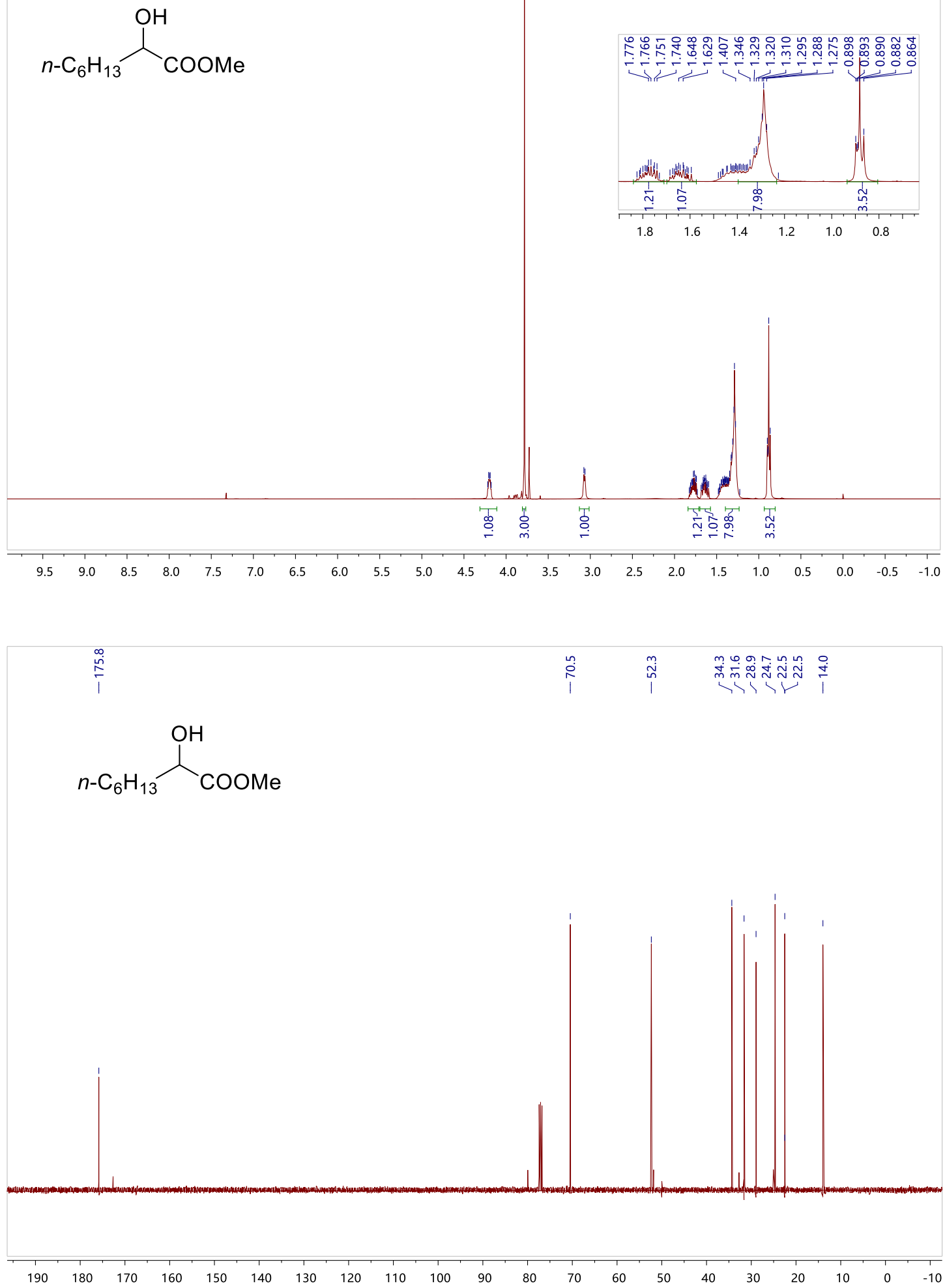
Methyl ( $Z$ )-oct-2-enoate (3a)
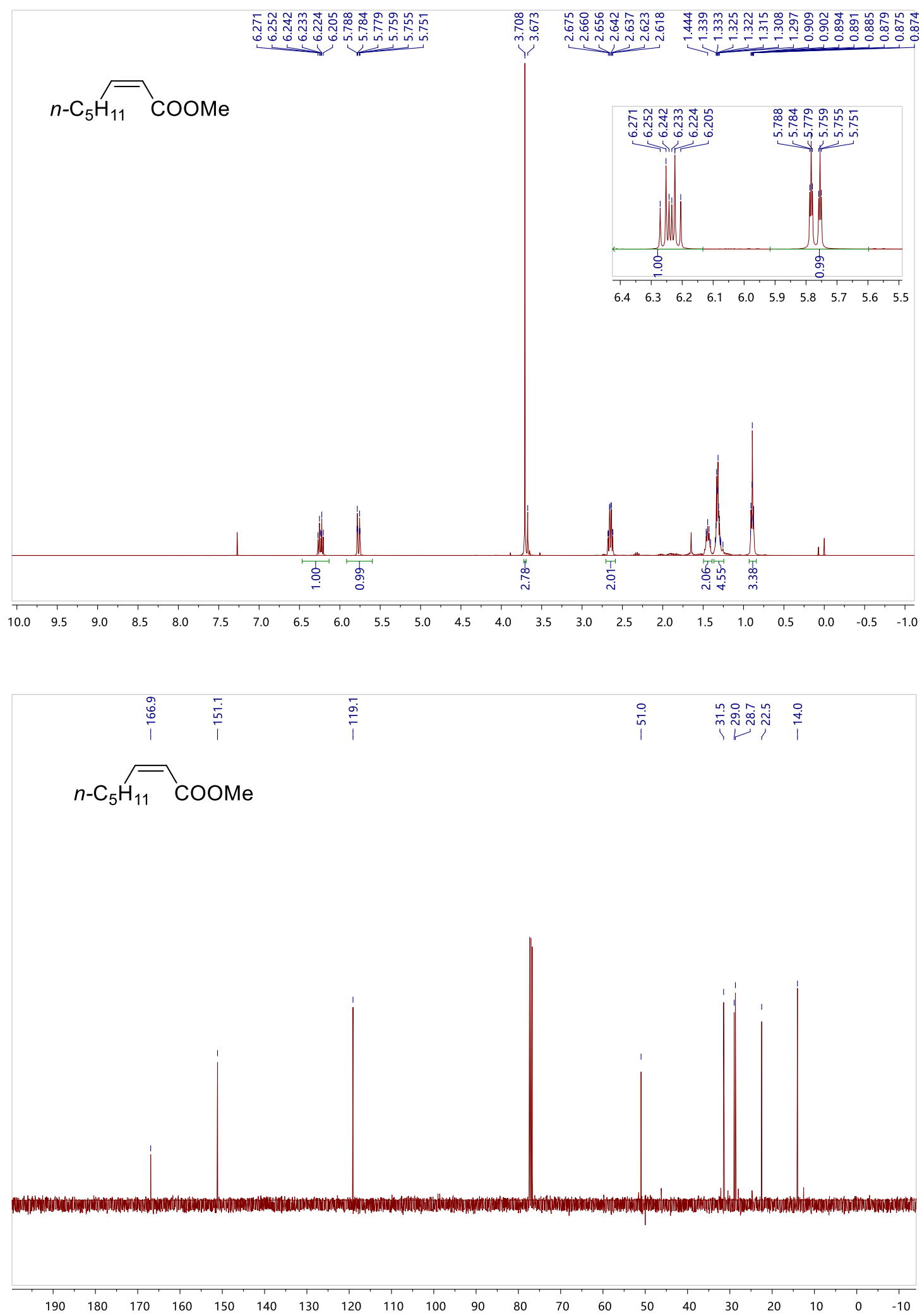
Methyl (E)-oct-2-enoate (3a')
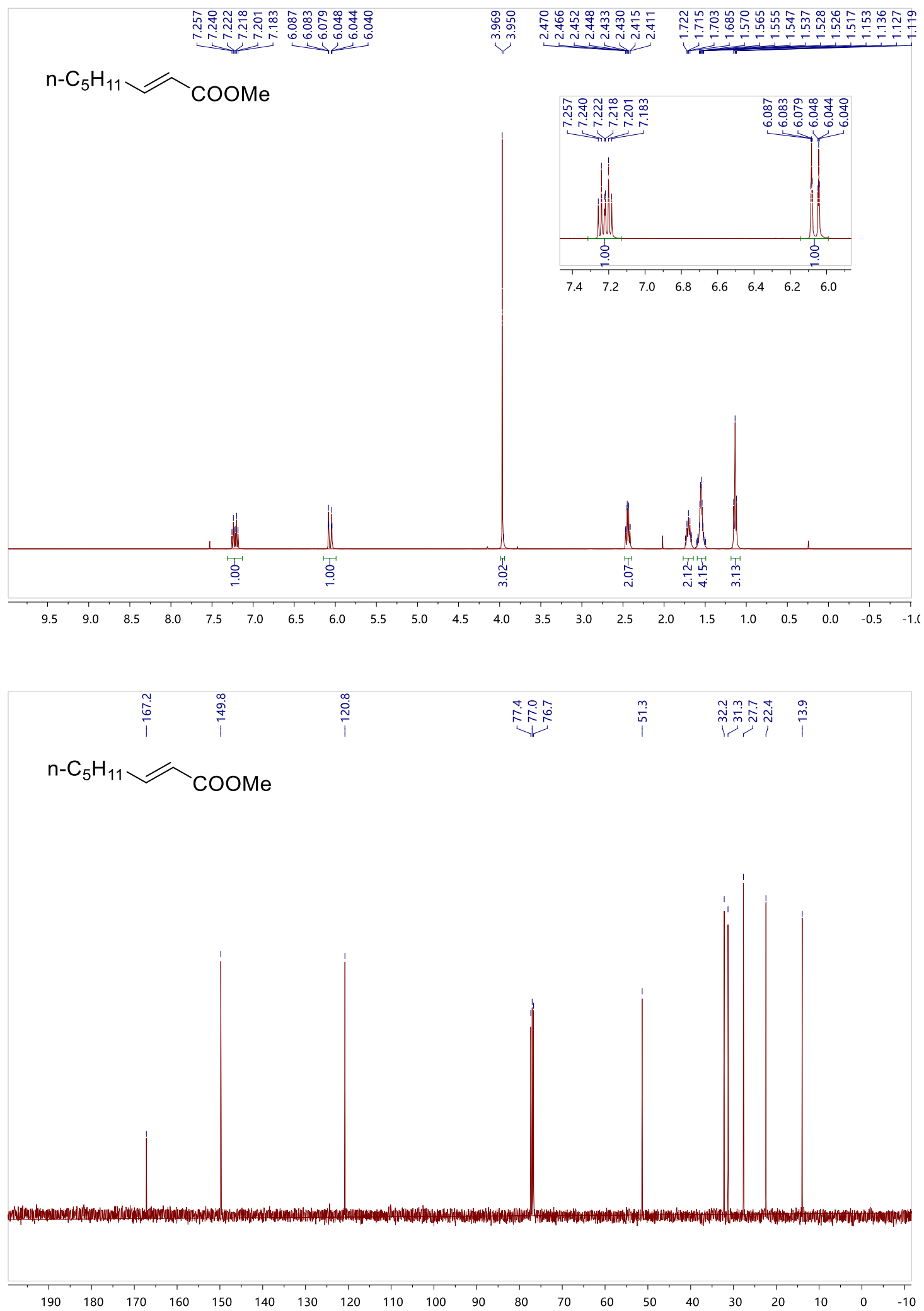
Dimethyl 2,3-dihexylbut-2-enedioate

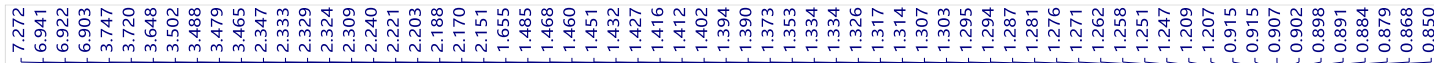
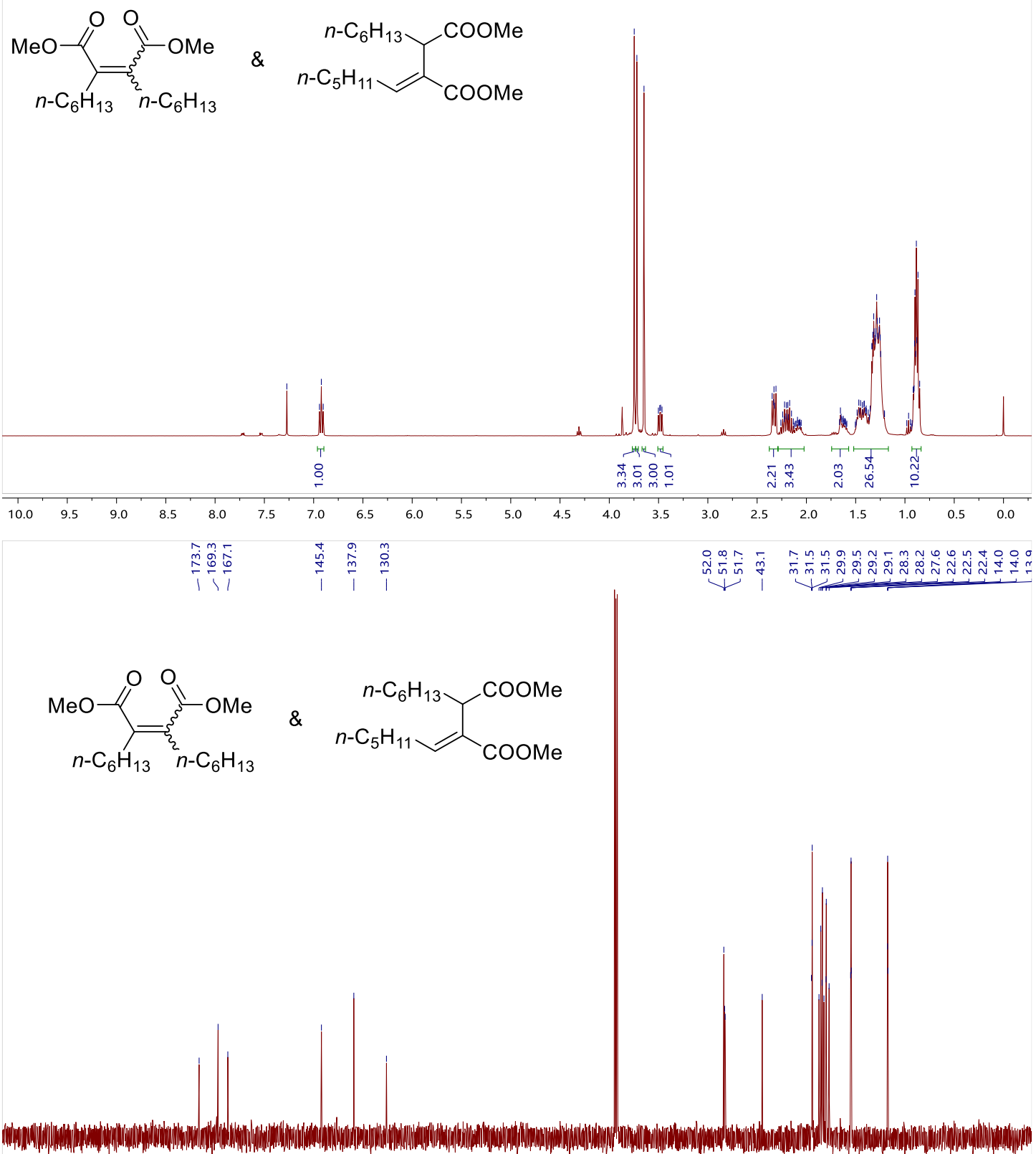

$\begin{array}{lllllllllllllllllllllll}210 & 200 & 190 & 180 & 170 & 160 & 150 & 140 & 130 & 120 & 110 & 100 & 90 & 80 & 70 & 60 & 50 & 40 & 30 & 20 & 10 & 0 & -10\end{array}$ 
Dimethyl 2,2'-(hydrazine-1,2-diylidene)-dioctanoat

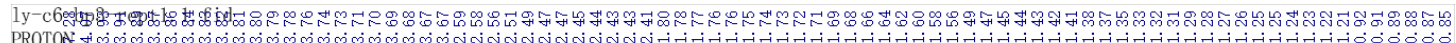

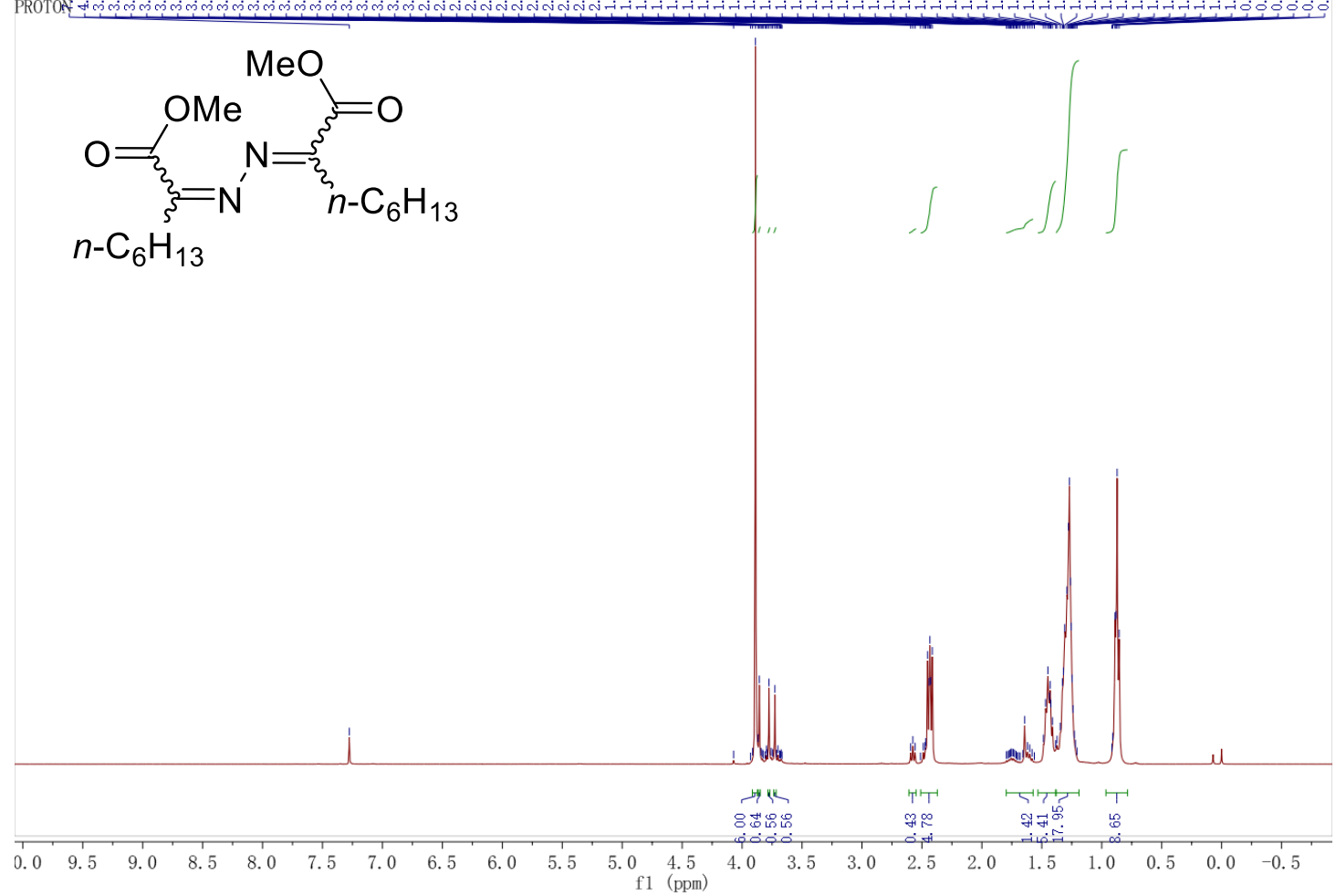

$\stackrel{-j}{i} \frac{5}{i}$

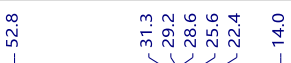<smiles>COC(=O)C(=N/N=C(\C)C(=O)OC)C(=O)OC</smiles>

$n-\mathrm{C}_{6} \mathrm{H}_{13}$

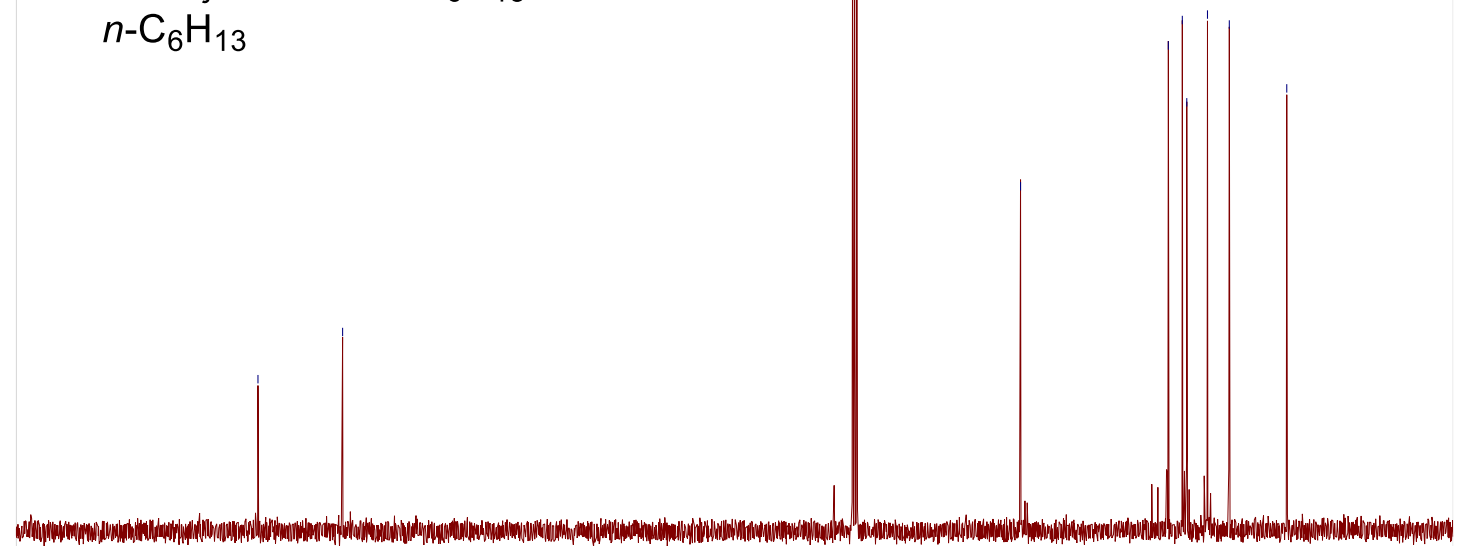

190

$180 \quad 170$

160

o 140

120

100 


\section{$N$-Benzyl-2-hydroxybutanamide (9)}
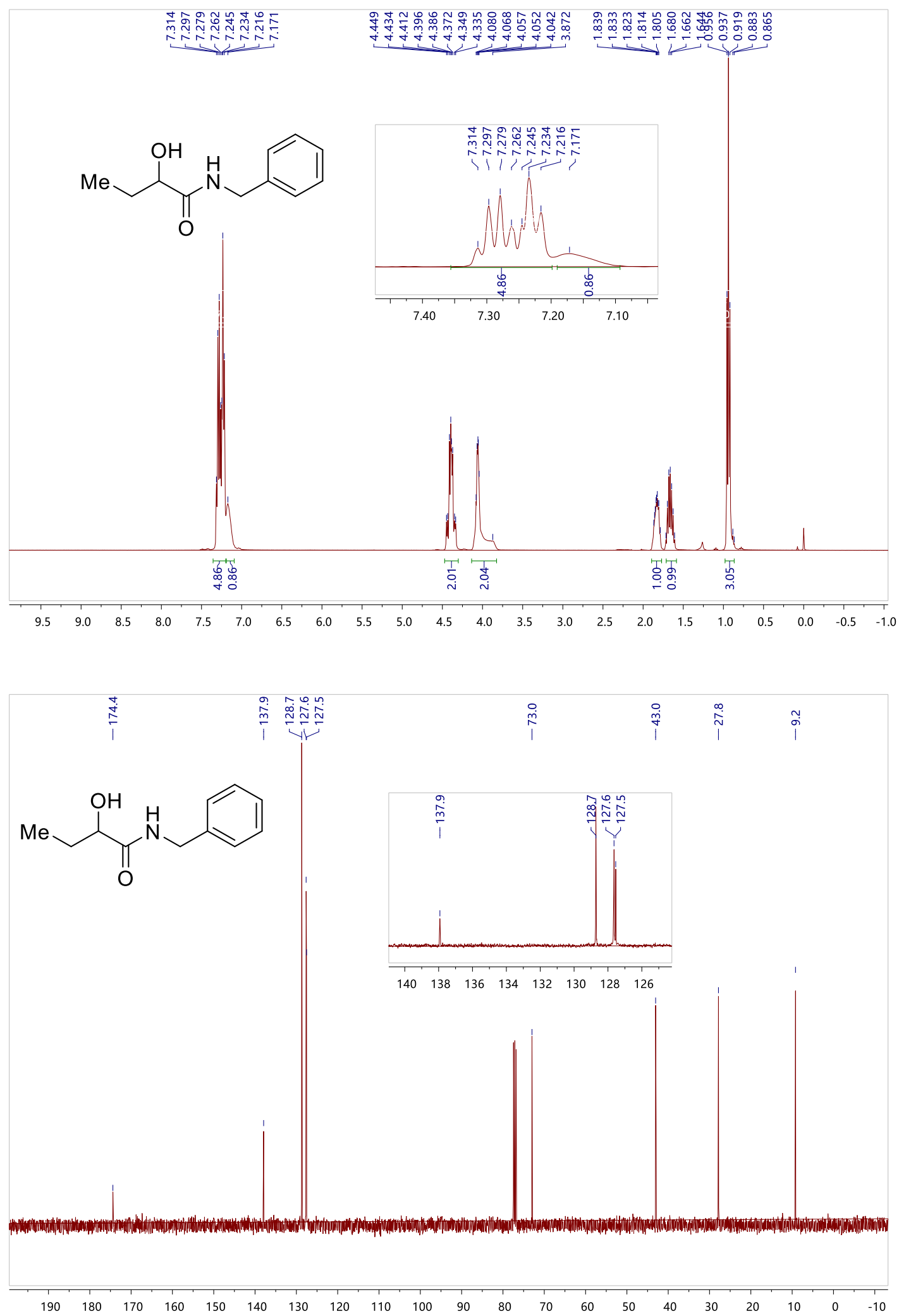
Butane-1,2-diol (10)
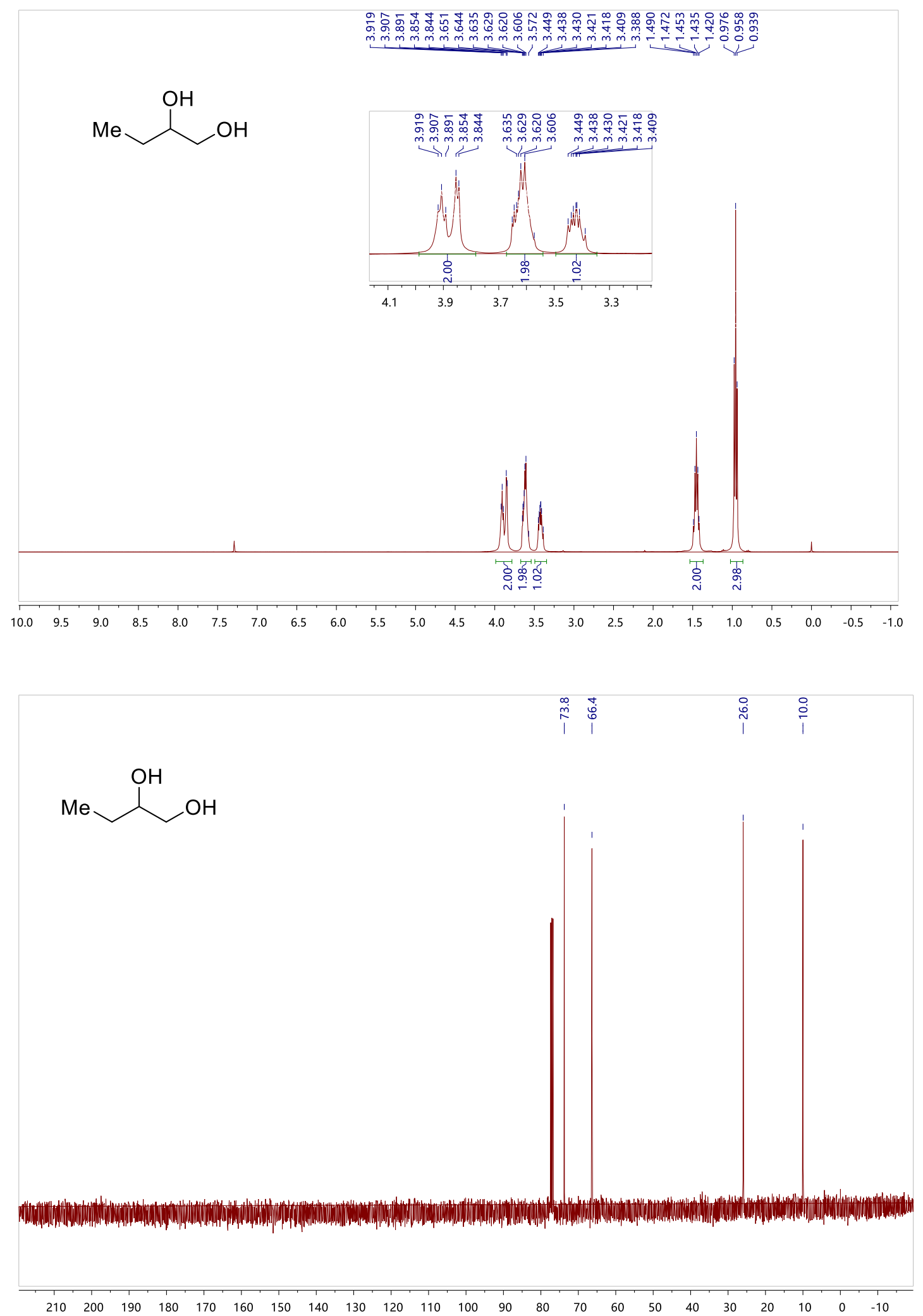
2-Phenylpropan-2-yl 2-((tert-butyldimethylsilyl)oxy)butanoate (2ba)
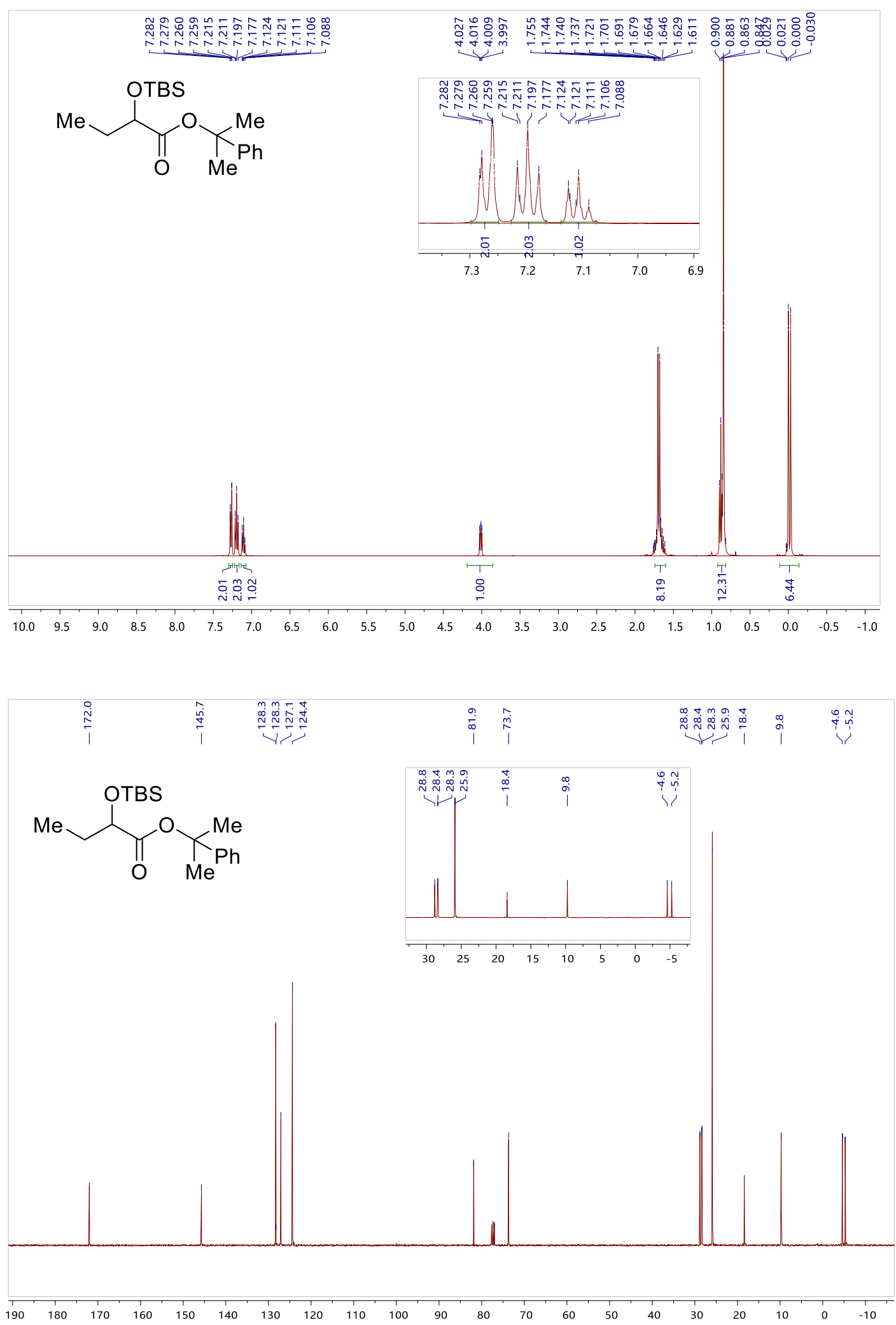
2-((tert-Butyldimethylsilyl)oxy)butan-1-ol (11)
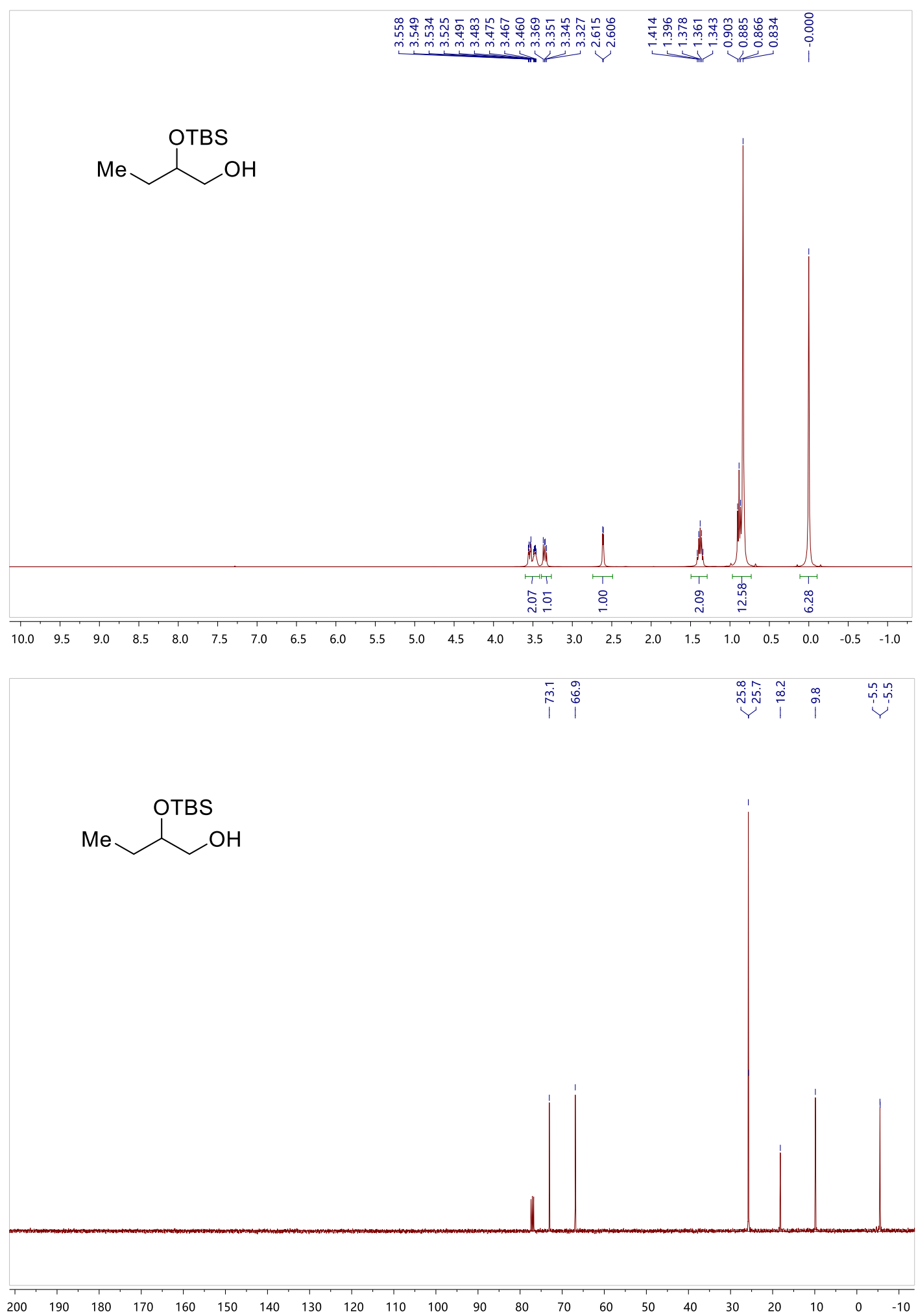


\section{2-Phenylpropan-2-yl 2-fluorobutanoate (12)}

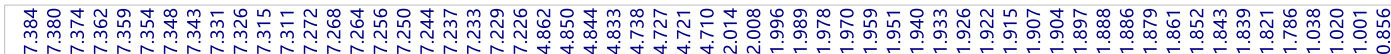<smiles>CCC(F)C(=O)OC(C)(C)c1ccccc1</smiles>
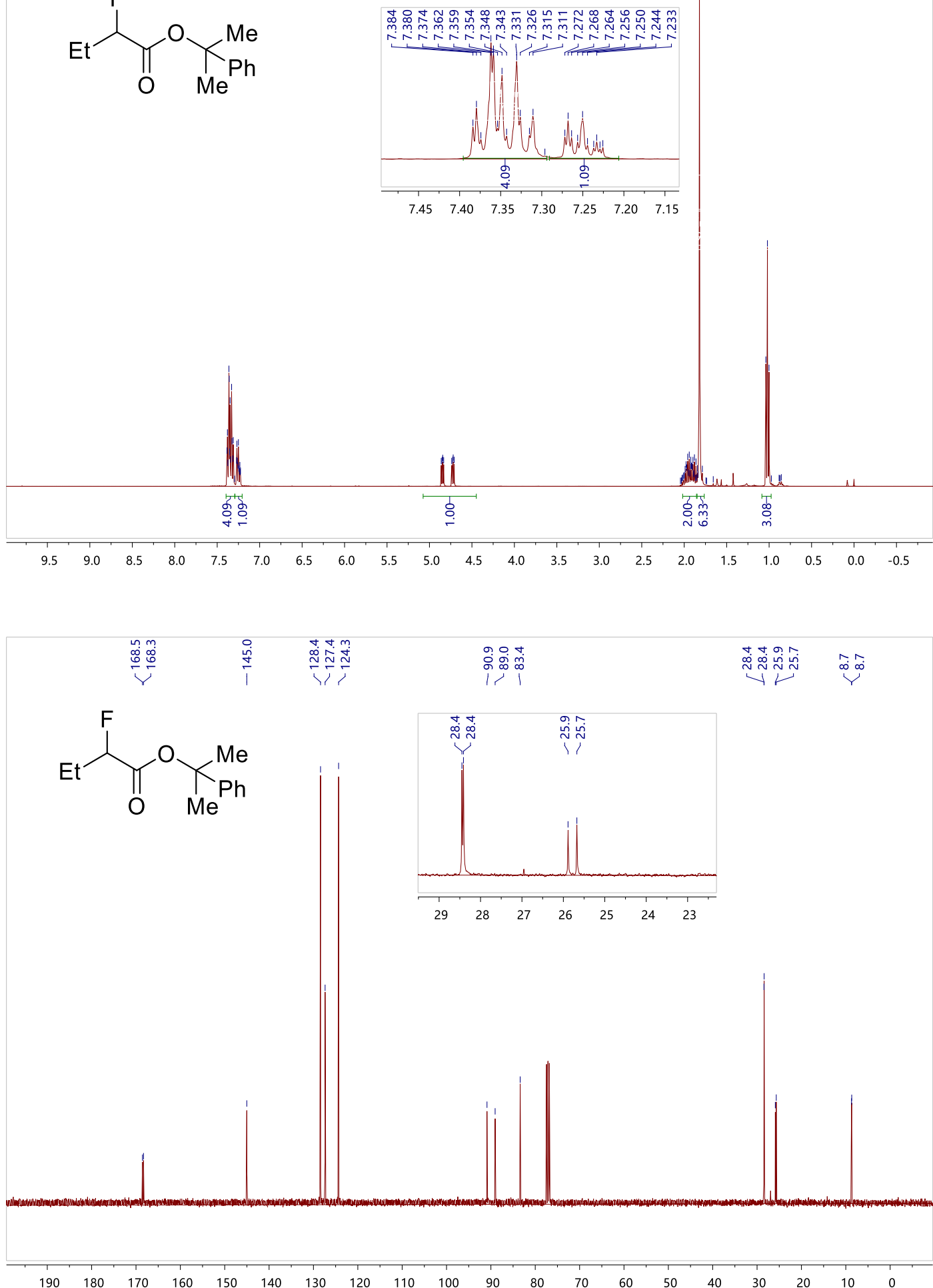


\section{(E)-4-Phenylpent-3-ene-1,2-diol (14)}
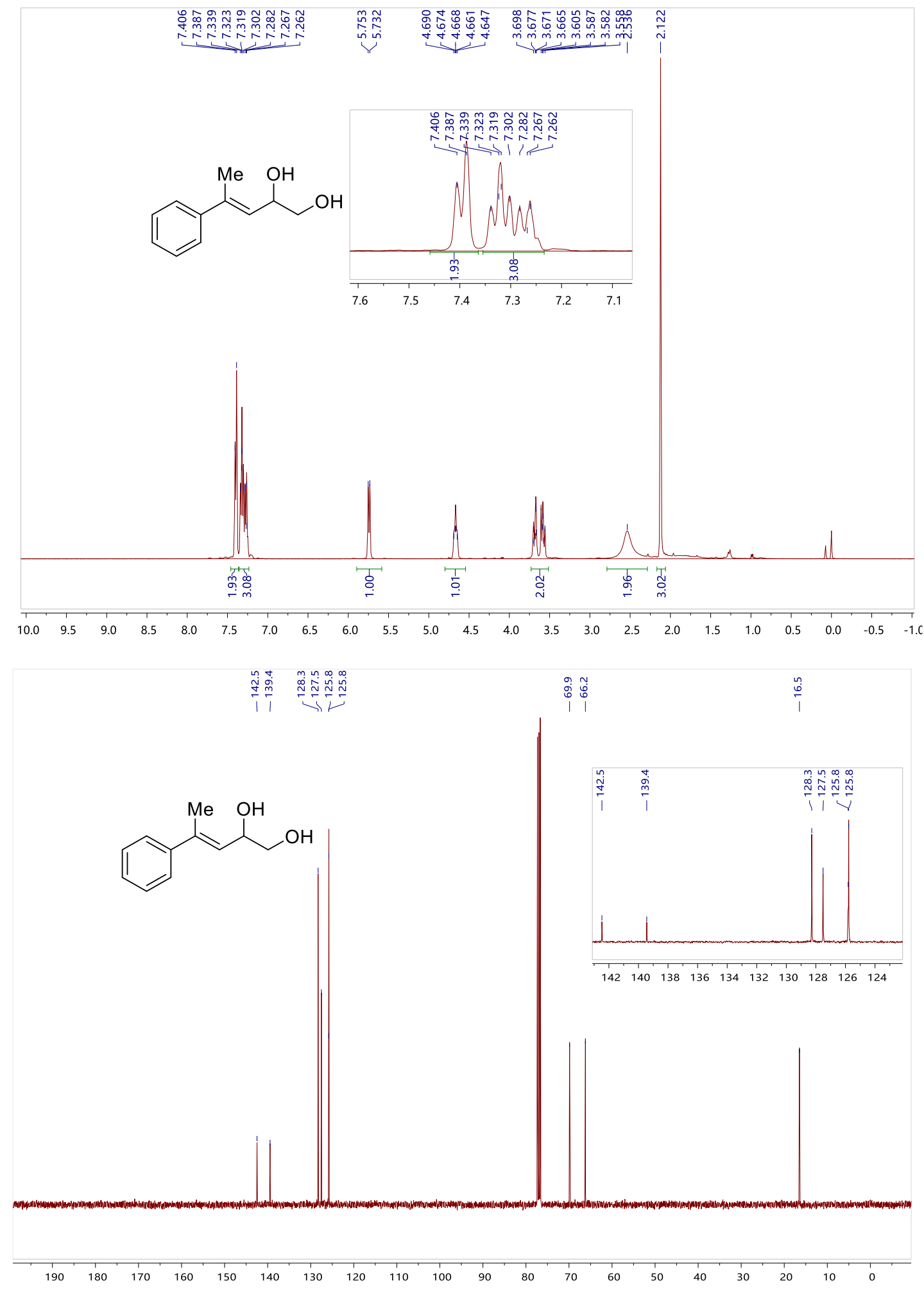
(E)-tert-Butyl 6-(dimethylamino)-4-methyl-6-oxo-4-phenylhex-2-enoate (16)
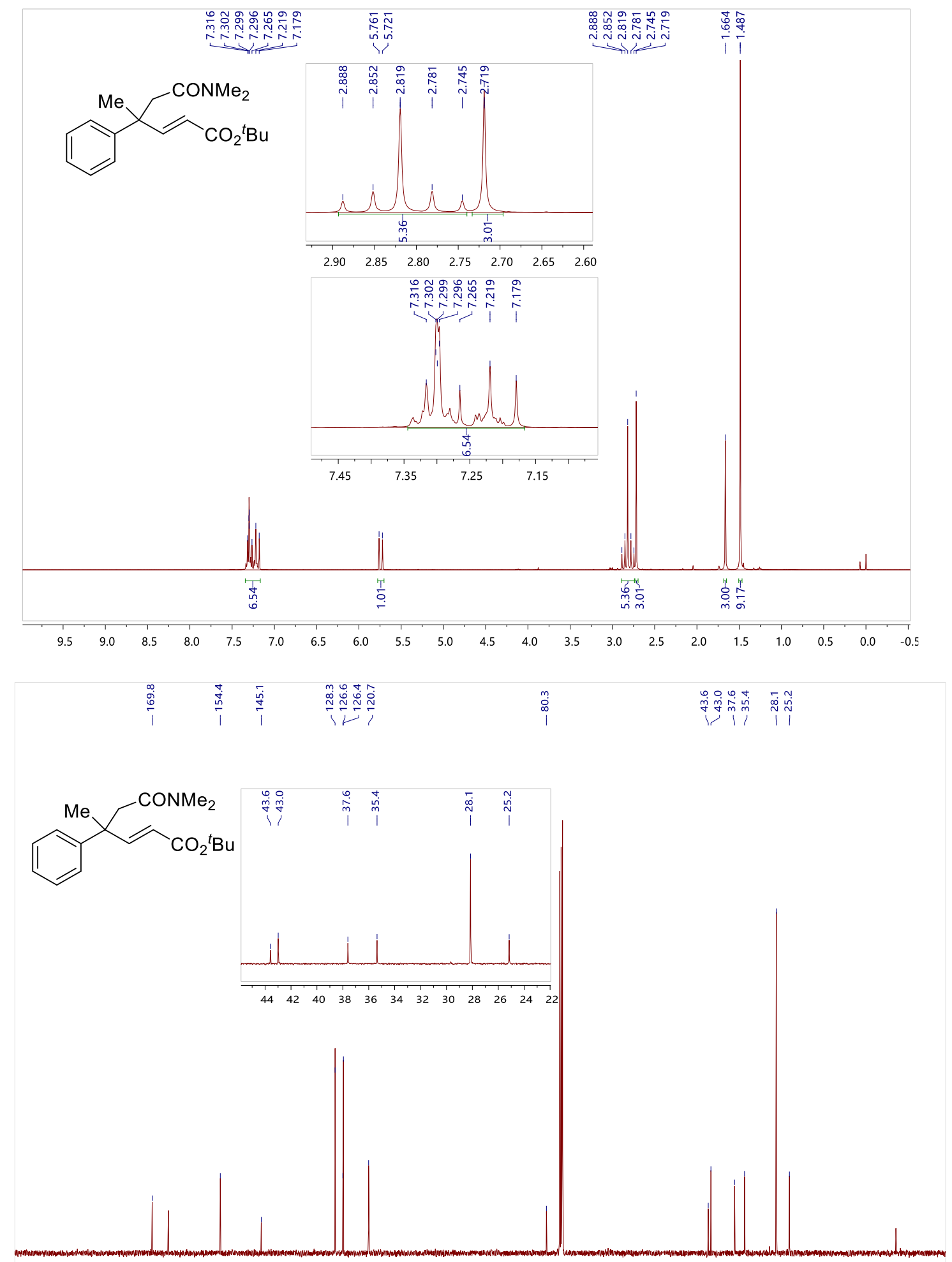

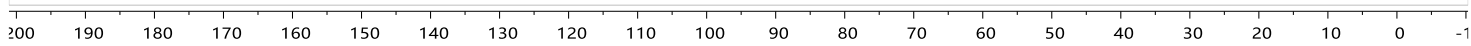


tert-Butyl (E)-4-((4-(4-chlorophenoxy)phenyl)amino)-4-phenylbut-2-enoate (17)
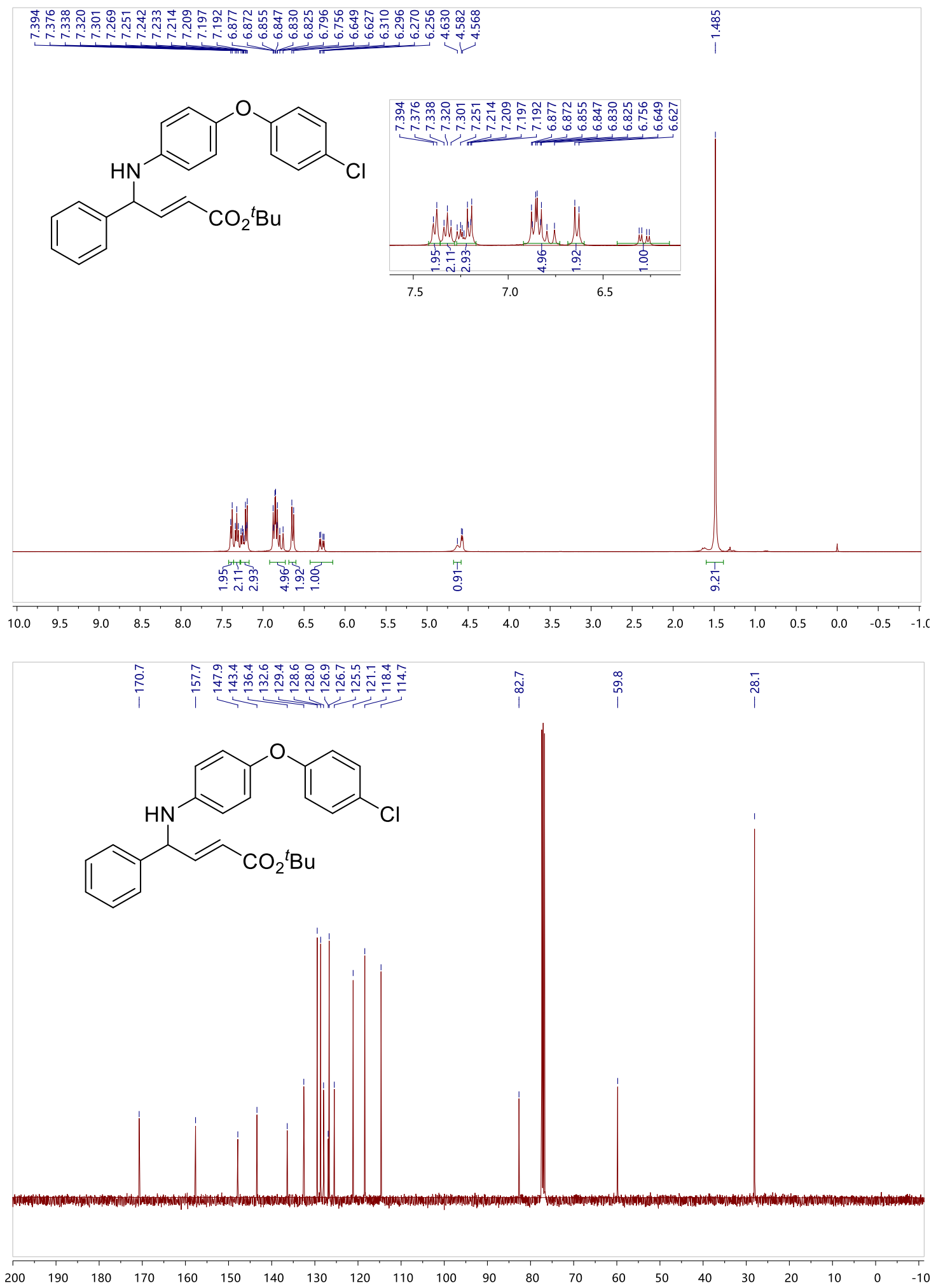
tert-Butyl (E)-4-(3,4-dichlorophenyl)-4-phenylbut-2-enoate (19)
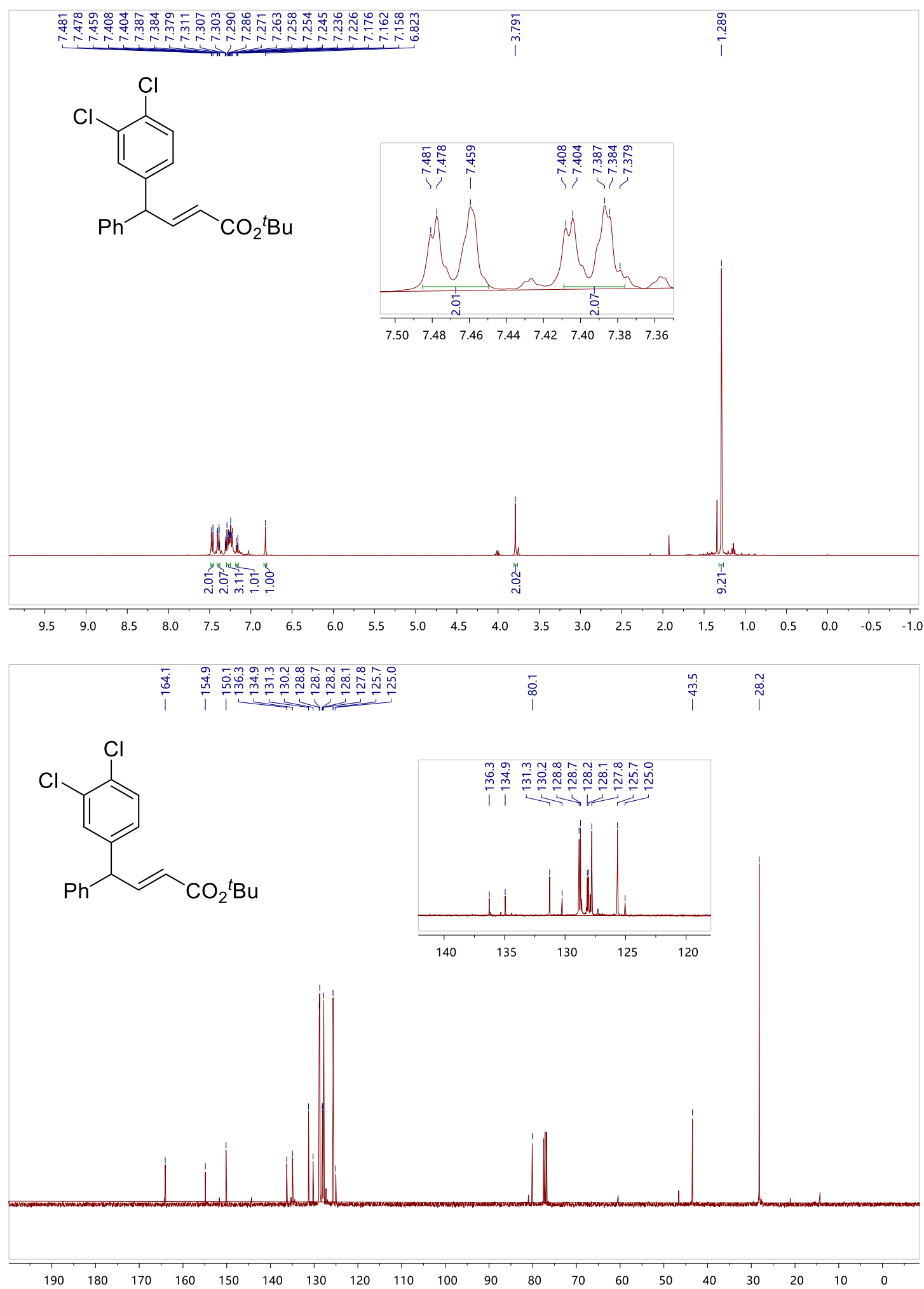


\section{HPLC Charts for Chiral Compounds}

2-Phenylpropan-2-yl 2-hydroxyoctanoate (2a)
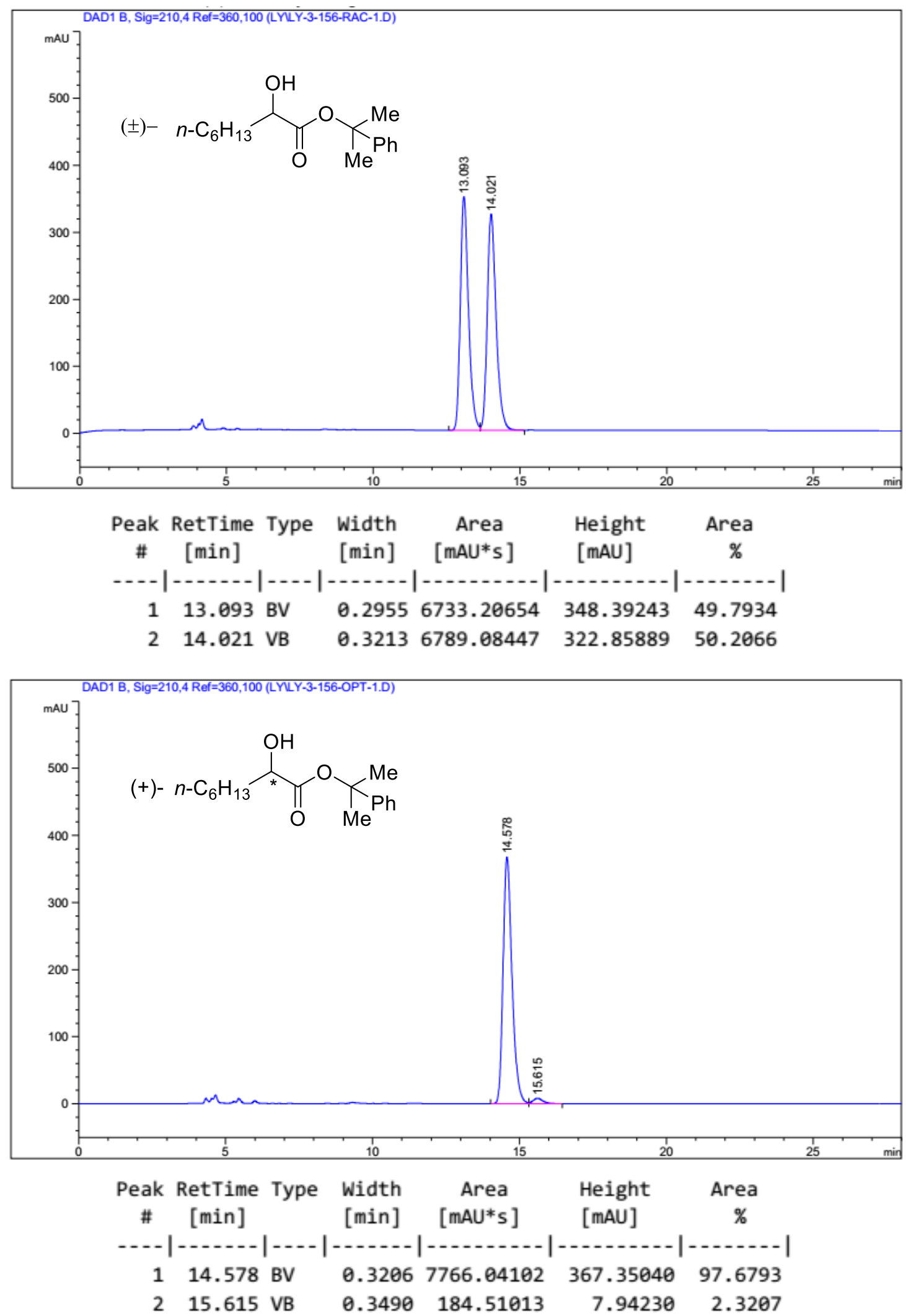
2-Phenylpropan-2-yl 2-hydroxybutanoate (2b)
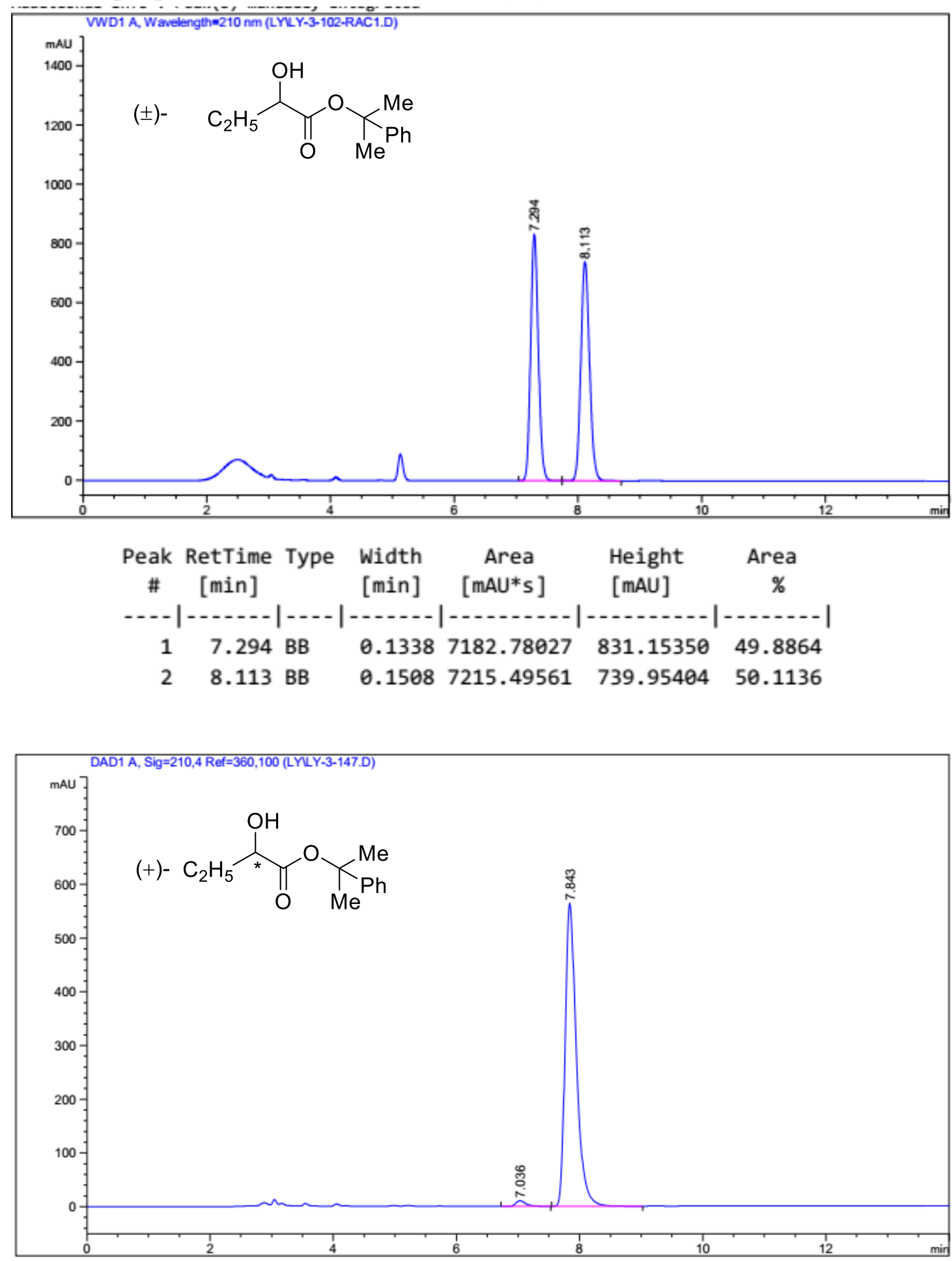

\begin{tabular}{cccccc}
$\begin{array}{c}\text { Peak RetTime Type } \\
\text { [min] }\end{array}$ & $\begin{array}{c}\text { Width } \\
\text { [min] }\end{array}$ & $\begin{array}{c}\text { Area } \\
\text { [mAU*s] }\end{array}$ & $\begin{array}{c}\text { Height } \\
\text { [mAU] }\end{array}$ & $\begin{array}{c}\text { Area } \\
\%\end{array}$ \\
\hdashline 1 & 7.036 BB & 0.1686 & 115.24558 & 10.29761 & 1.5816 \\
2 & 7.843 BB & 0.1945 & 7171.26221 & 563.39392 & 98.4184
\end{tabular}


2-Phenylpropan-2-yl 2-hydroxyhexanoate (2c)

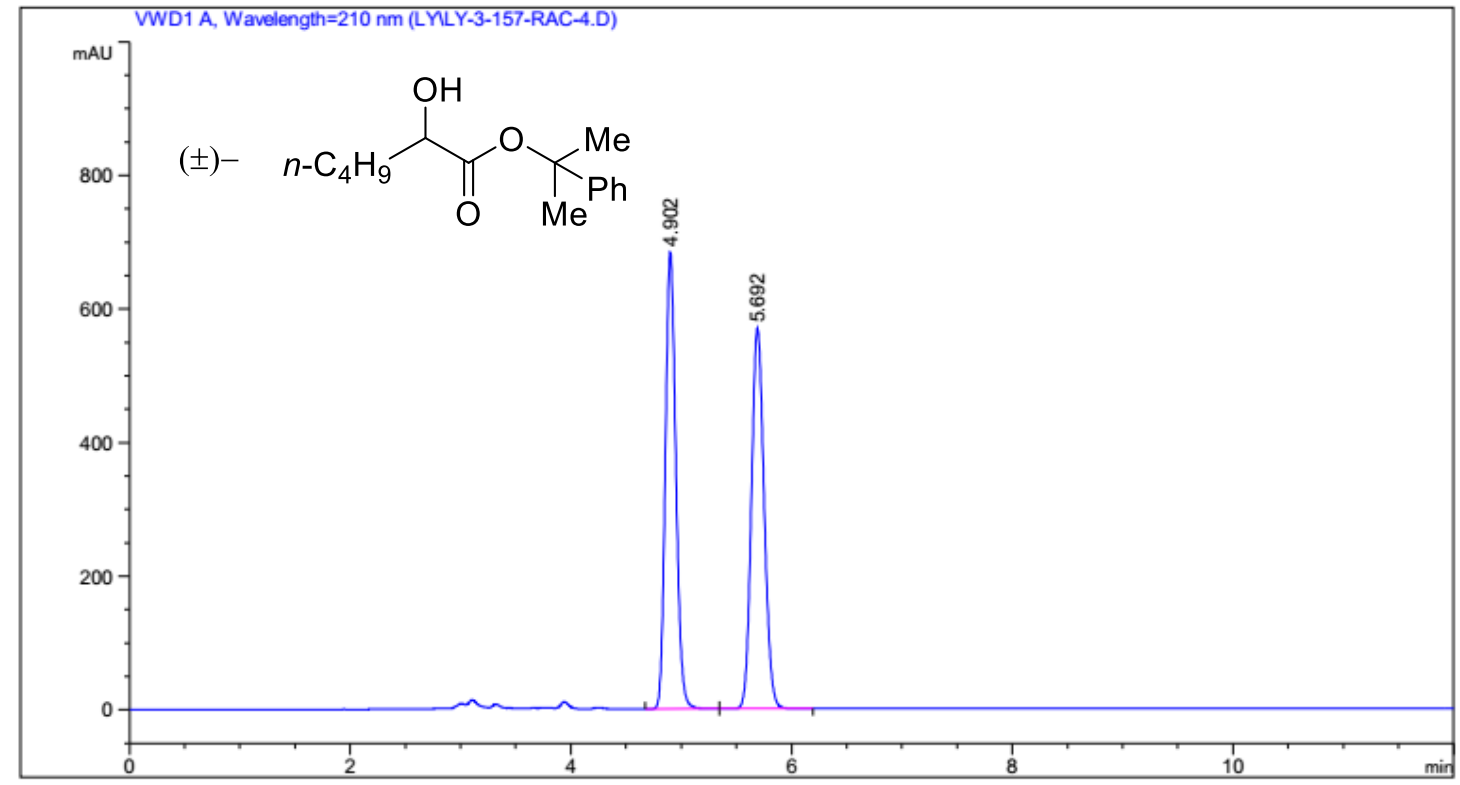

\begin{tabular}{cccccc}
$\begin{array}{c}\text { Peak RetTime Type Width } \\
\text { [min] }\end{array}$ & $\begin{array}{c}\text { Area } \\
\text { [min] }\end{array}\left[\begin{array}{c}\text { Height } \\
\text { [mAU*s] }\end{array}\right.$ & $\begin{array}{c}\text { Area } \\
\text { [mAU] }\end{array}$ & $\begin{array}{c}\text { \% } \\
\text { [min }\end{array}$ \\
\hline 1 & 4.902 BB & 0.1019 & 4513.50586 & 684.31030 & 49.7073 \\
2 & 5.692 BB & 0.1245 & 4566.66943 & 569.51746 & 50.2927
\end{tabular}

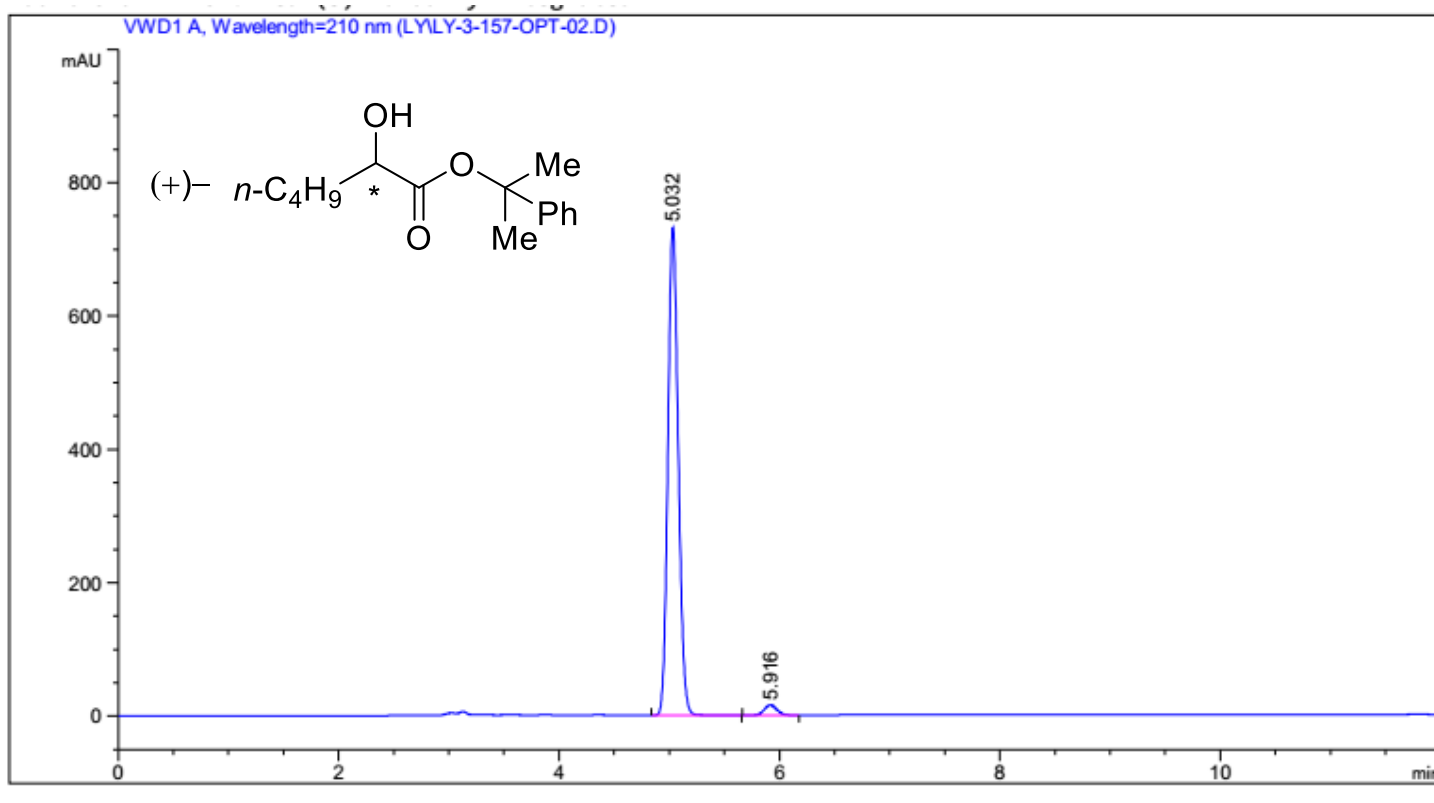

\begin{tabular}{|c|c|c|c|c|c|}
\hline $\begin{array}{c}\text { Peak } \\
\#\end{array}$ & $\begin{array}{l}\text { RetTime Type } \\
\text { [min] }\end{array}$ & $\begin{array}{l}\text { Width } \\
\text { [min] }\end{array}$ & $\begin{array}{c}\text { Area } \\
{[\mathrm{mAU} * \mathrm{~s}]}\end{array}$ & $\begin{array}{l}\text { Height } \\
{[\mathrm{mAU}]}\end{array}$ & $\begin{array}{c}\text { Area } \\
\%\end{array}$ \\
\hline & & & & 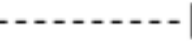 & \\
\hline 1 & $5.032 \mathrm{BB}$ & 0.1015 & 4791.91260 & 731.05127 & 97.4935 \\
\hline 2 & $5.916 \mathrm{BB}$ & 0.1242 & 123.19856 & 15.42472 & 2.5065 \\
\hline
\end{tabular}


2-Phenylpropan-2-yl 2-hydroxytetradecanoate (2d)
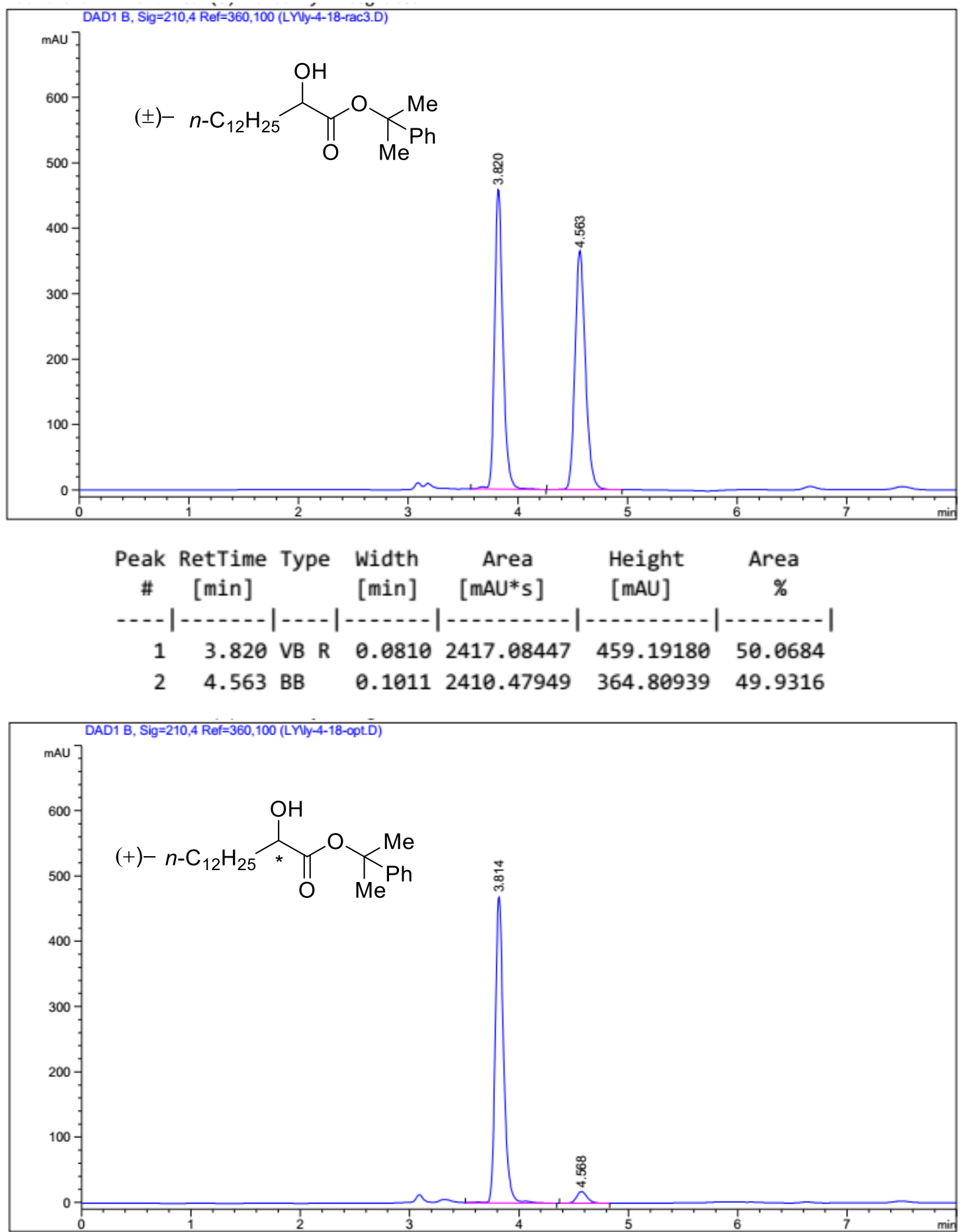

\begin{tabular}{|c|c|c|c|c|c|c|}
\hline $\begin{array}{c}\text { Peak } \\
\#\end{array}$ & $\begin{array}{c}\text { RetTime } \\
\text { [min] }\end{array}$ & Type & $\begin{array}{l}\text { Width } \\
\text { [min] }\end{array}$ & $\begin{array}{c}\text { Area } \\
{\left[\mathrm{mAU}^{*} \mathrm{~s}\right]}\end{array}$ & $\begin{array}{l}\text { Height } \\
\text { [mAU] }\end{array}$ & $\begin{array}{c}\text { Area } \\
\%\end{array}$ \\
\hline & & & & -1 & & \\
\hline 1 & 3.814 & VV R & 0.0810 & 2470.33789 & 469.64230 & 95.3282 \\
\hline 2 & 4.568 & BB & 0.1019 & 121.06552 & 18.12089 & 4.6718 \\
\hline
\end{tabular}


2-Phenylpropan-2-yl 2-hydroxy-5-methylhexanoate (2e)
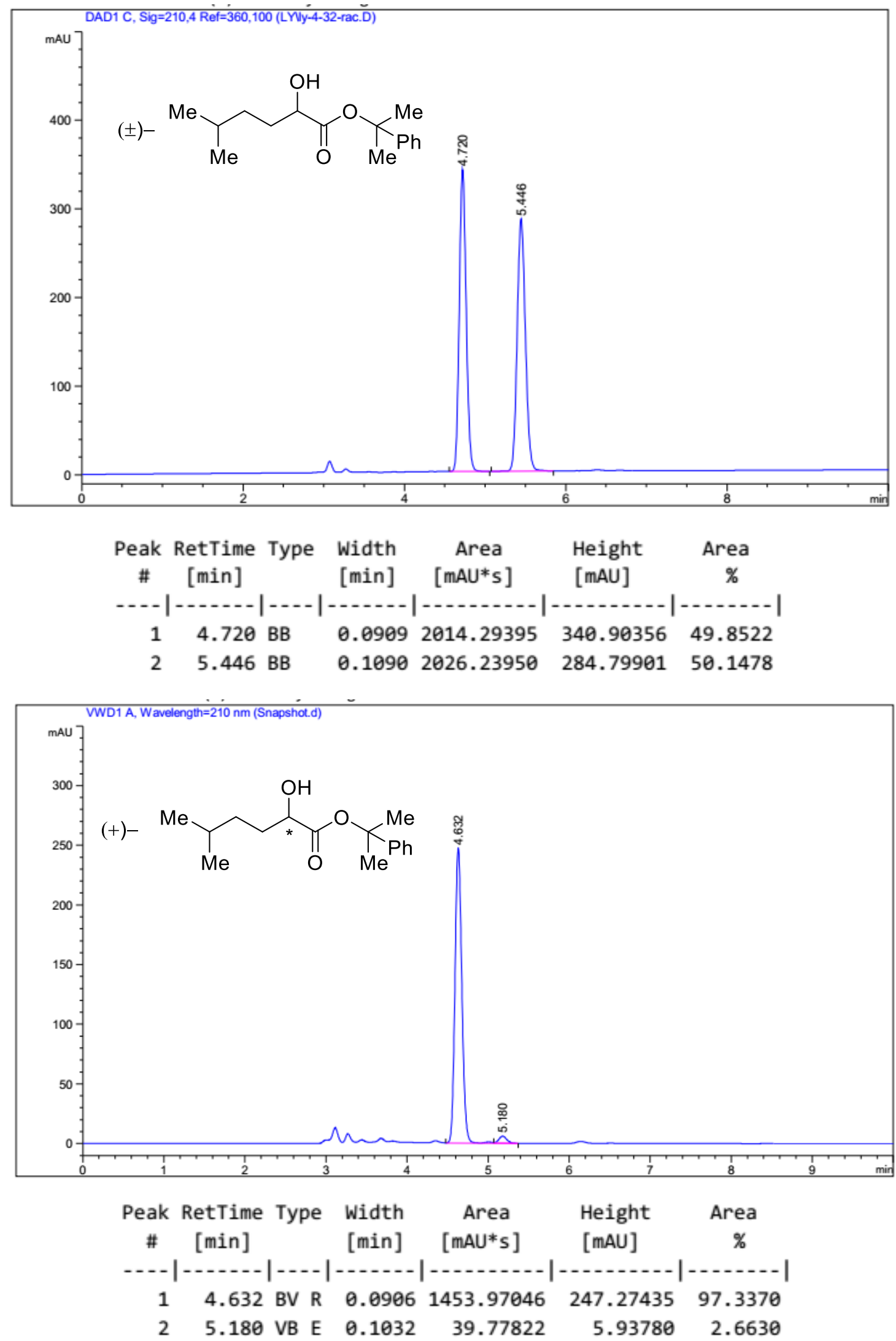
2-Phenylpropan-2-yl 2-hydroxy-5,5-dimethylhexanoate (2f)

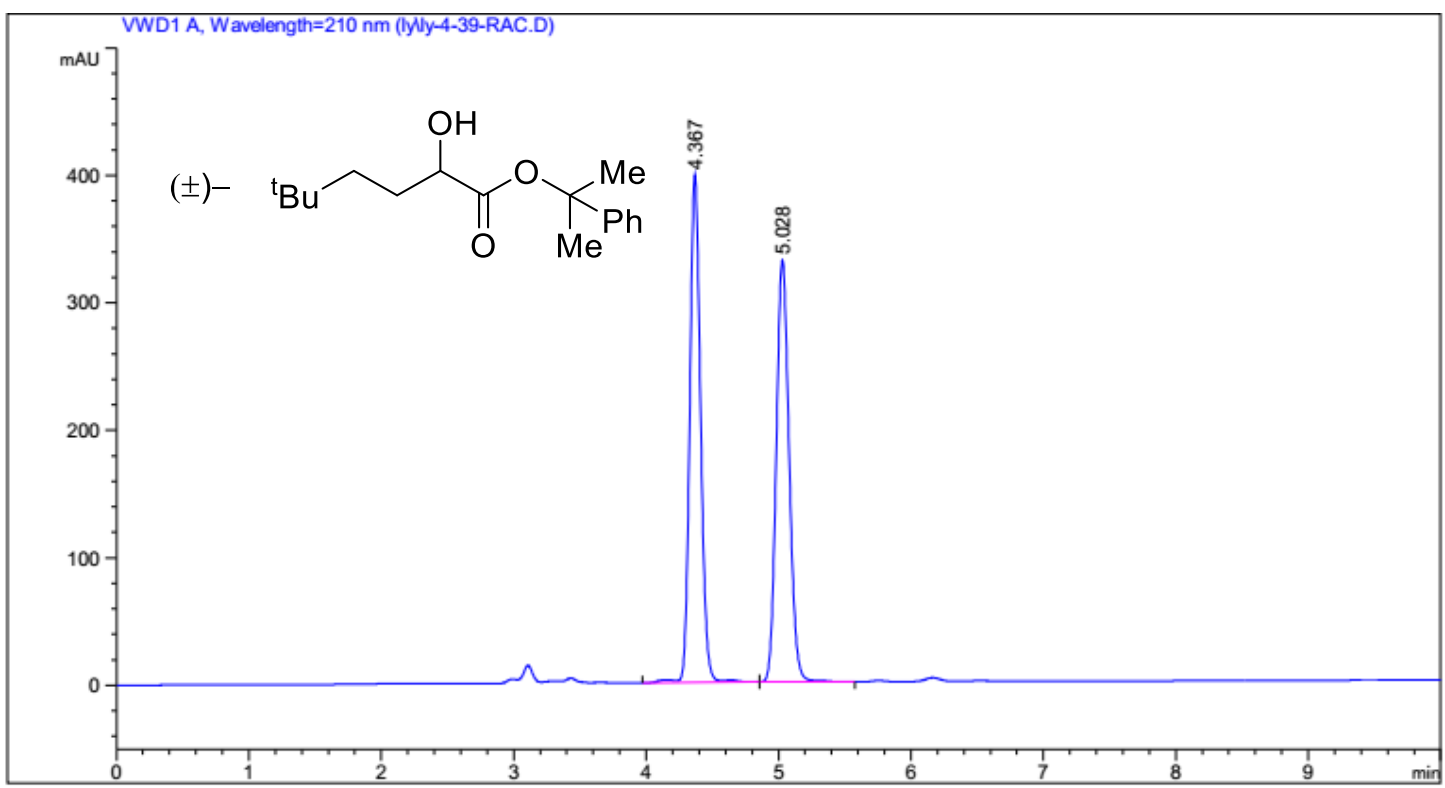

\begin{tabular}{cccccc}
$\begin{array}{c}\text { Peak RetTime Type } \\
\text { \# } \begin{array}{l}\text { Width } \\
\text { [min] }\end{array}\end{array} \begin{array}{c}\text { Area } \\
\text { [mAU*s] }\end{array}$ & $\begin{array}{c}\text { Height } \\
\text { [mAU] }\end{array}$ & $\begin{array}{c}\text { Area } \\
\%\end{array}$ \\
\hline 1 & 4.367 V V R & 0.0875 & 2268.21387 & 398.41989 & 50.4053 \\
2 & 5.028 BV R & 0.1047 & 2231.73340 & 330.88580 & 49.5947
\end{tabular}

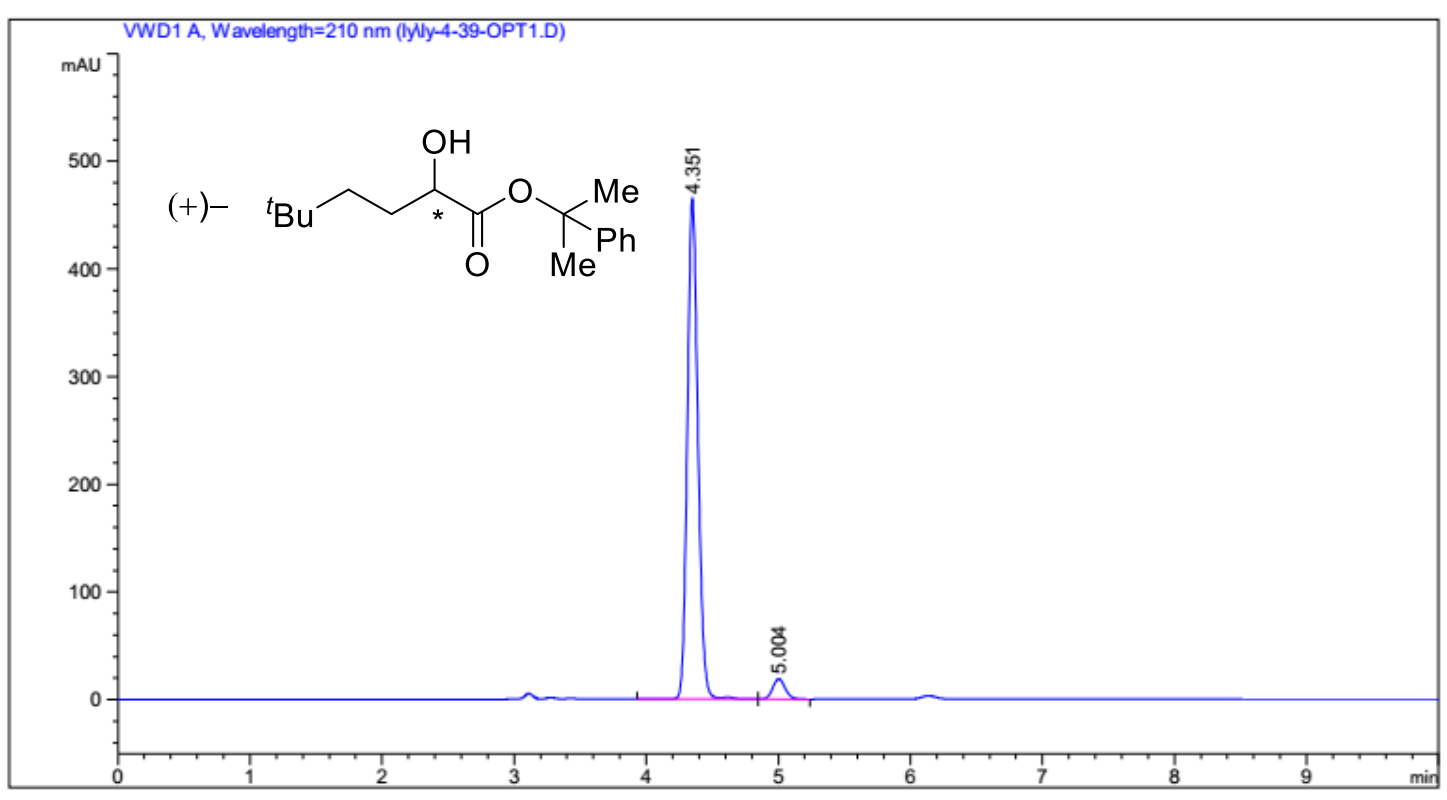

\begin{tabular}{|c|c|c|c|c|c|}
\hline eak & $\begin{array}{l}\text { RetTime Type } \\
\text { [min] }\end{array}$ & $\begin{array}{l}\text { Width } \\
\text { [min] }\end{array}$ & $\begin{array}{c}\text { Area } \\
{[\mathrm{mAU} * \mathrm{~s}]}\end{array}$ & $\begin{array}{l}\text { Height } \\
\text { [mAU] }\end{array}$ & $\begin{array}{c}\text { Area } \\
\%\end{array}$ \\
\hline & & & $6 a$ & & \\
\hline & & & & & \\
\hline
\end{tabular}


2-Phenylpropan-2-yl 4-cyclohexyl-2-hydroxybutanoate (2g)
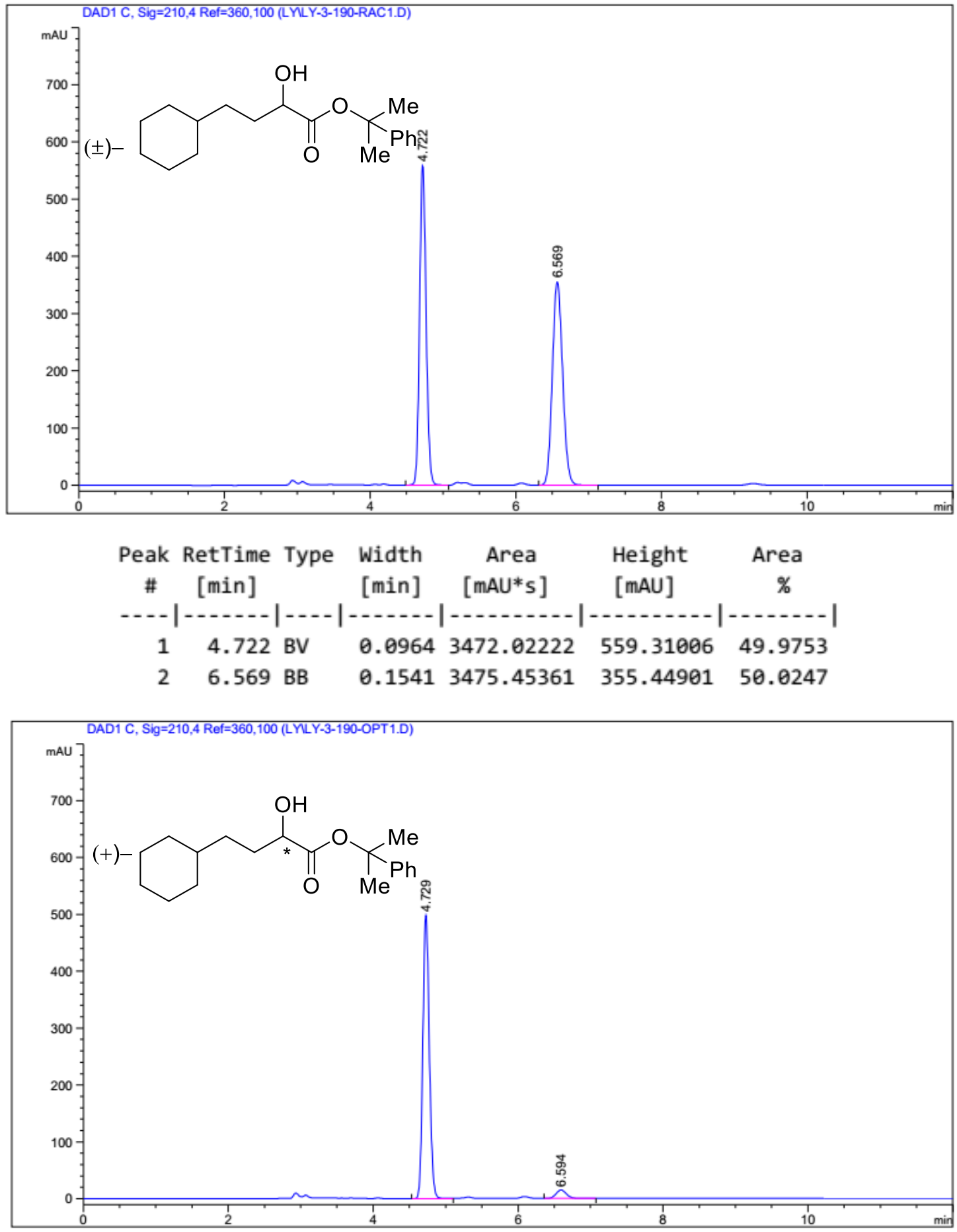

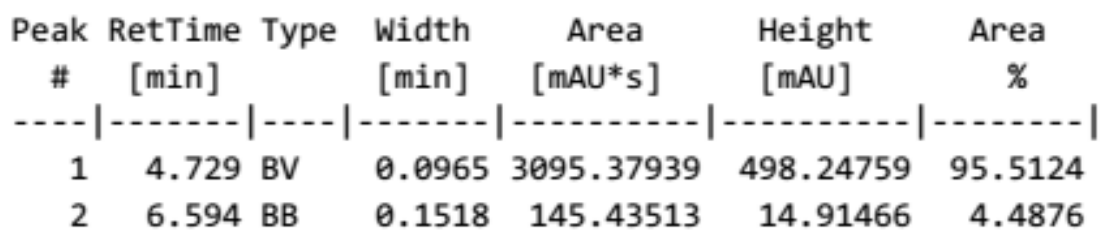


2-Phenylpropan-2-yl 3-cyclopropyl-2-hydroxypropanoate (2h)
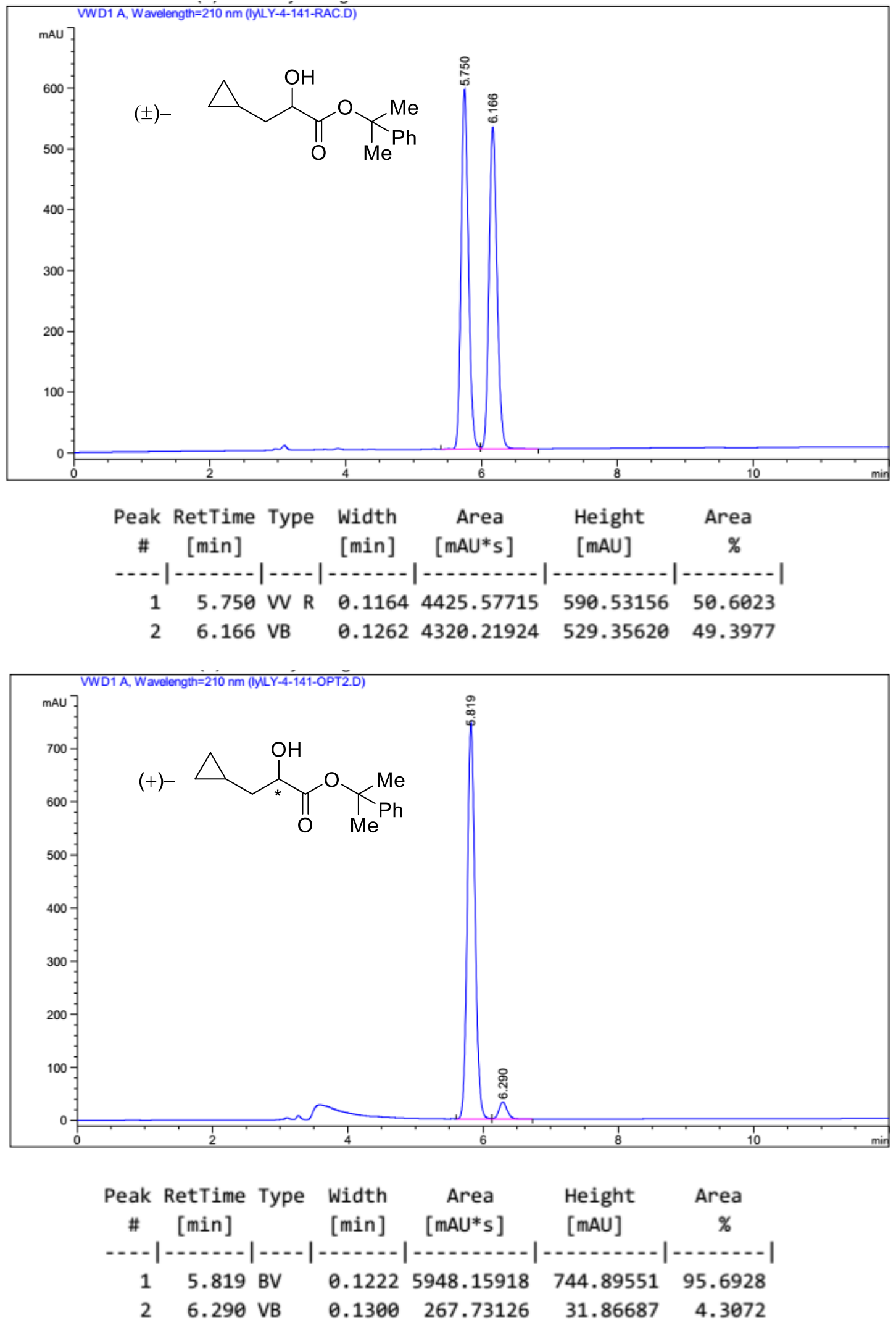
2-Phenylpropan-2-yl 2-hydroxy-4-methylpentanoate (2i)
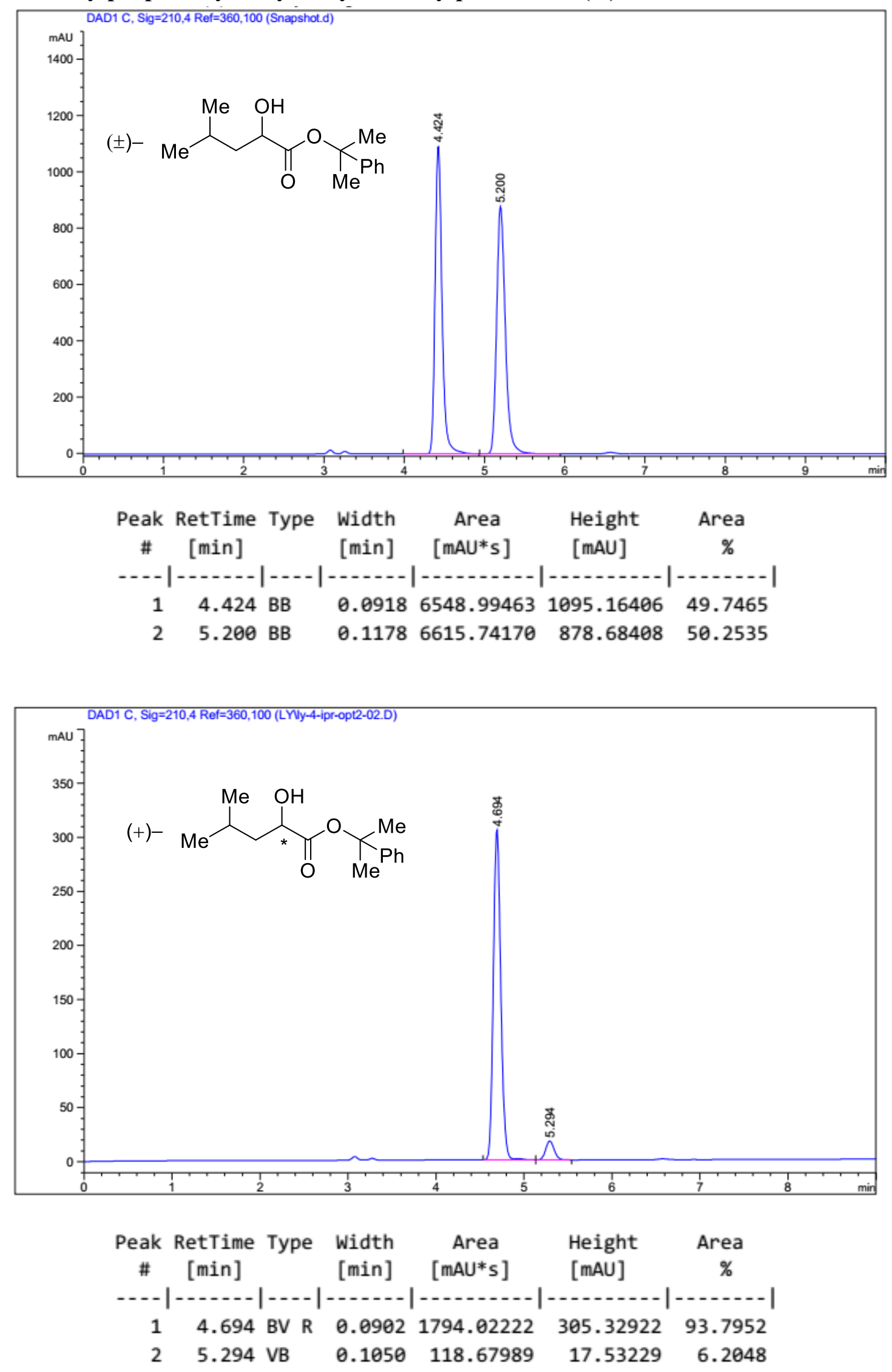
2-Phenylpropan-2-yl 2-hydroxy-3-phenylpropanoate (2j)
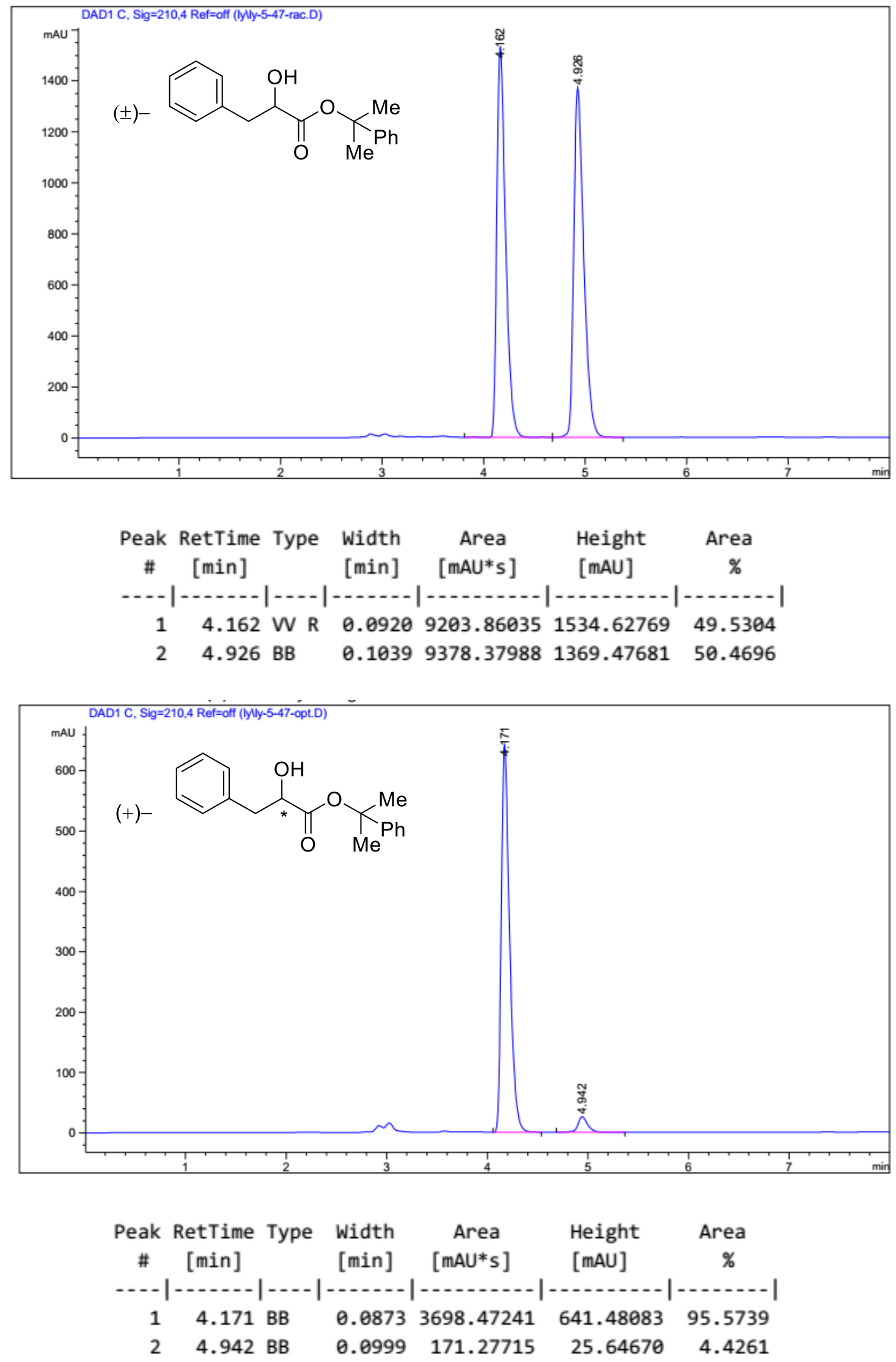
2-Phenylpropan-2-yl 2-hydroxy-4-phenylbutanoate (2k)
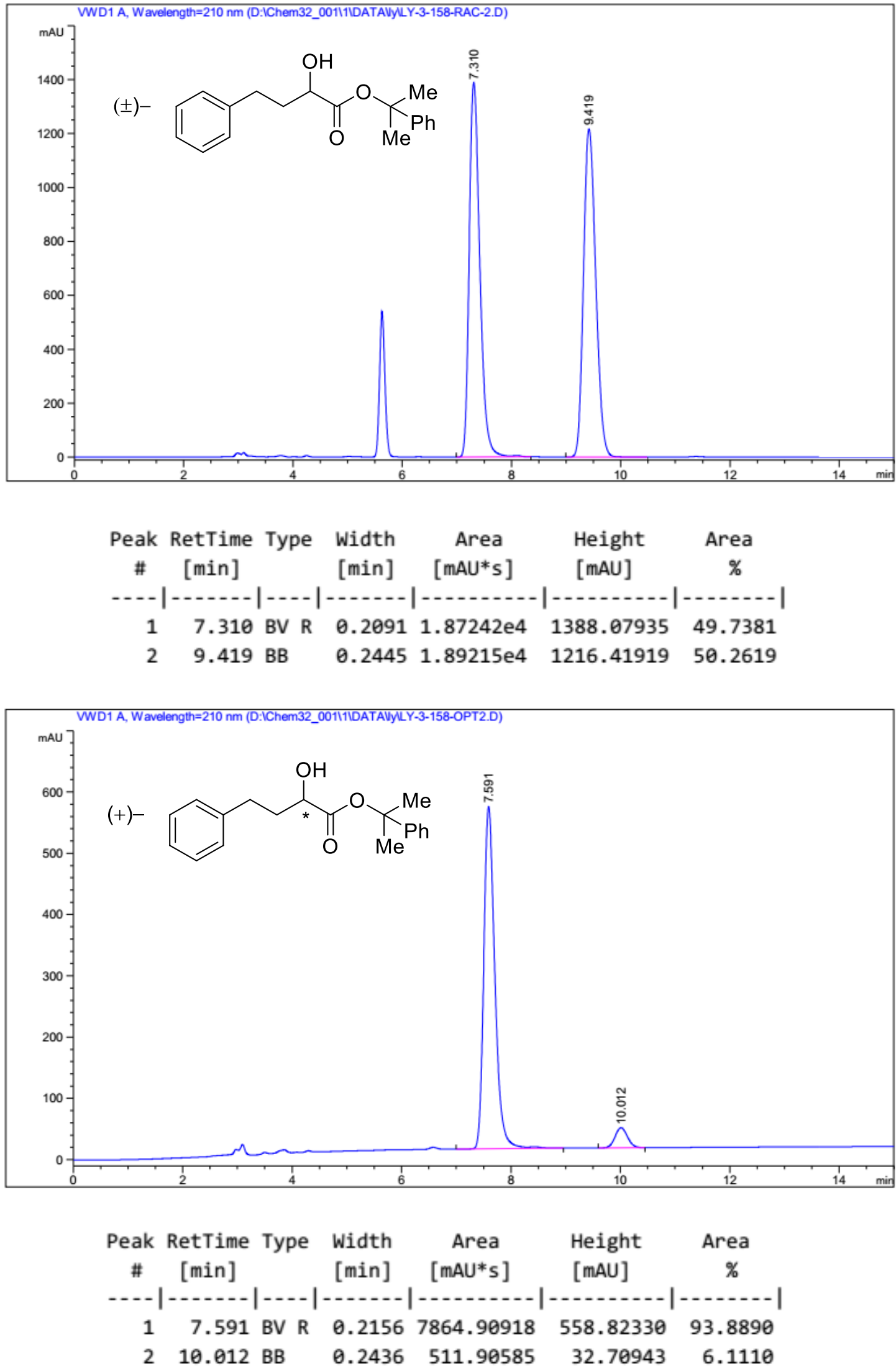
2-Phenylpropan-2-yl 2-hydroxy-5-phenylpentanoate (2l)
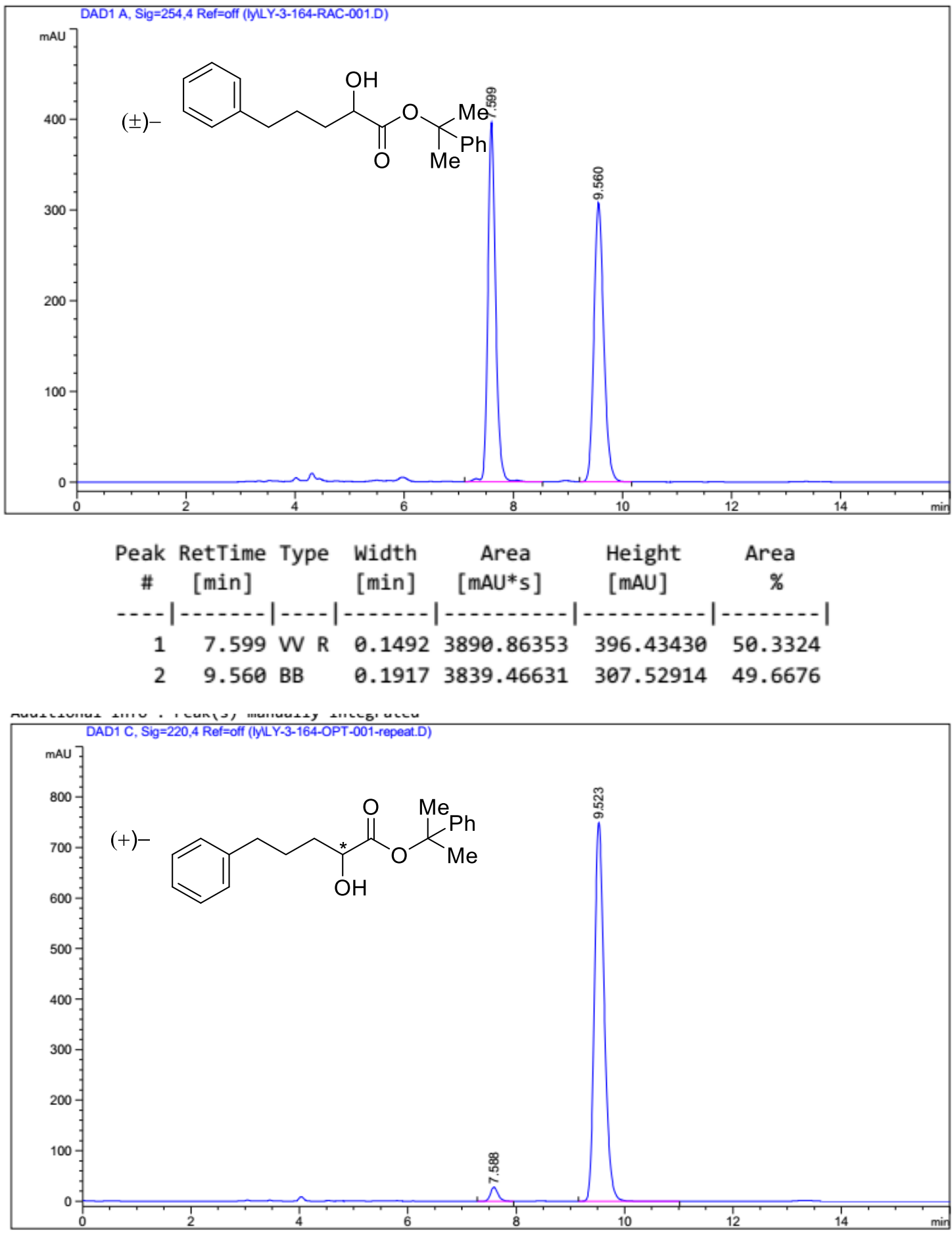

\begin{tabular}{cccccc}
$\begin{array}{c}\text { Peak RetTime Type } \\
\text { [min] }\end{array}$ & $\begin{array}{c}\text { Width } \\
\text { [min] }\end{array}$ & $\begin{array}{c}\text { Area } \\
{\left[\mathrm{mAU}^{*} \mathrm{~s}\right]}\end{array}$ & $\begin{array}{c}\text { Height } \\
\text { [mAU] }\end{array}$ & \multicolumn{1}{c}{$\begin{array}{c}\text { Area } \\
\%\end{array}$} \\
\hline 1 & 7.588 BB & 0.1484 & 268.04352 & 27.81922 & 2.7564 \\
2 & 9.523 BB & 0.1932 & 9456.25000 & 749.33789 & 97.2436
\end{tabular}


2-Phenylpropan-2-yl 2-hydroxypent-4-enoate (2m)
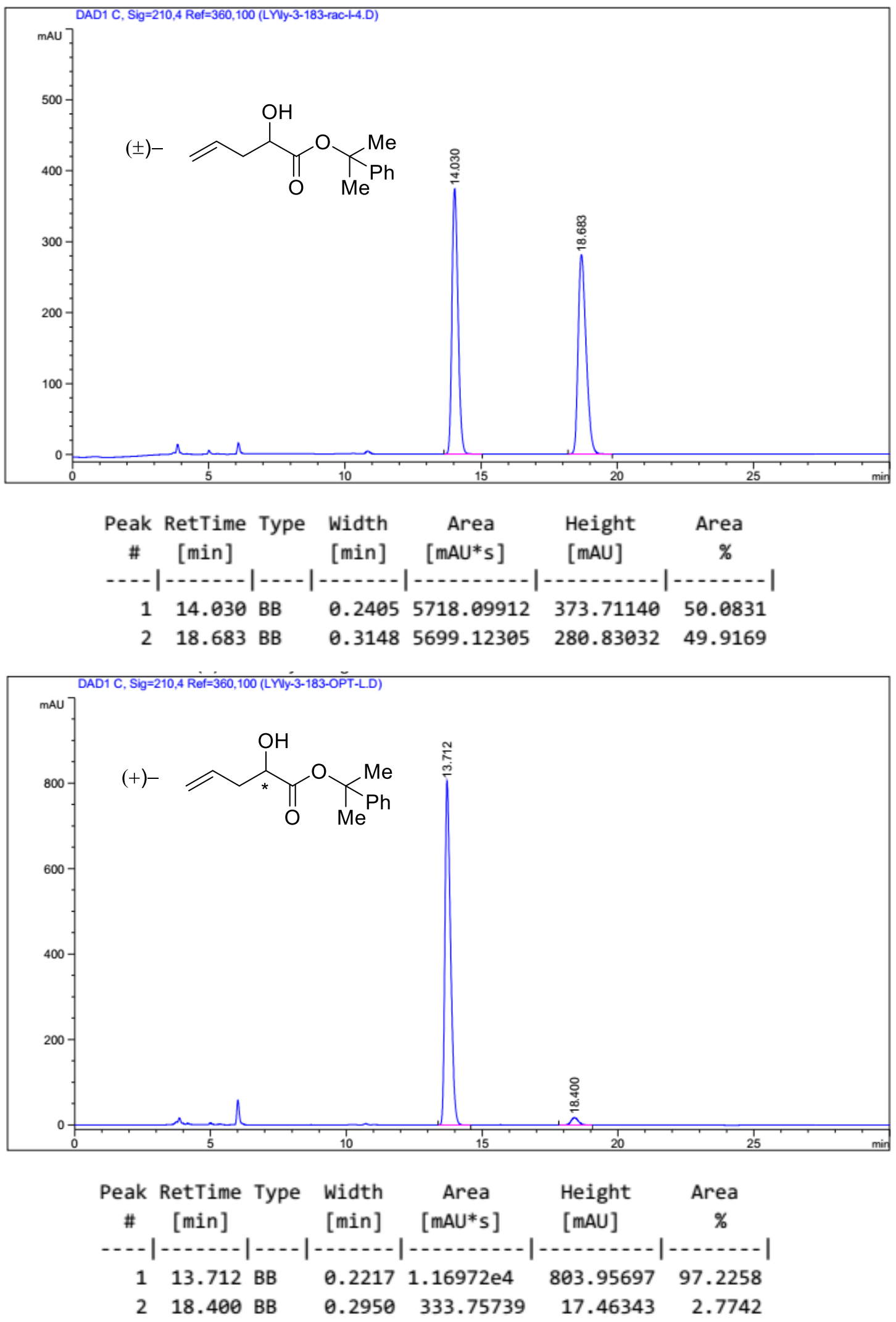
2-Phenylpropan-2-yl 2-hydroxyhex-5-enoate (2n)
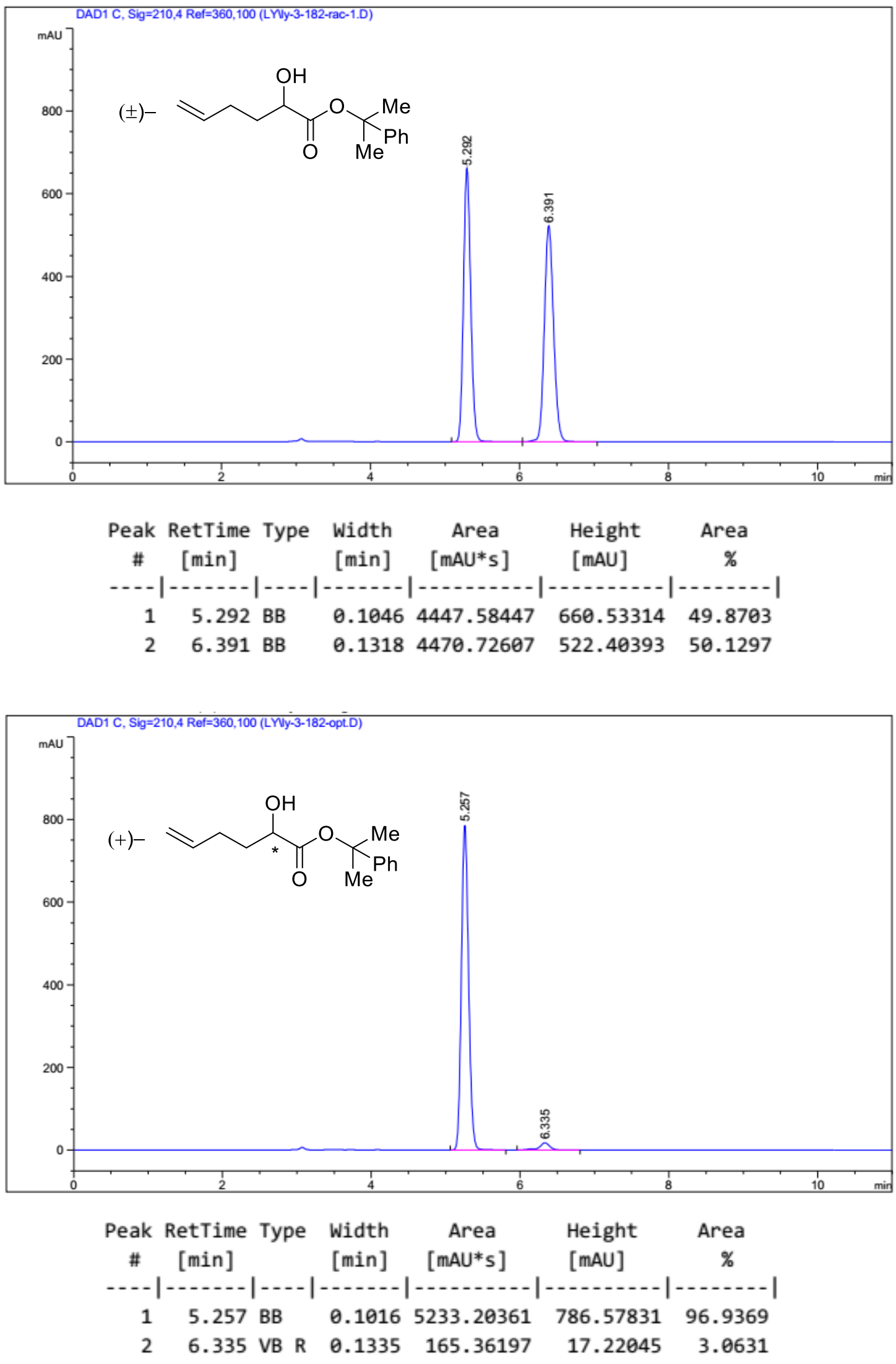
2-Phenylpropan-2-yl 2-hydroxyoct-7-enoate (2o)
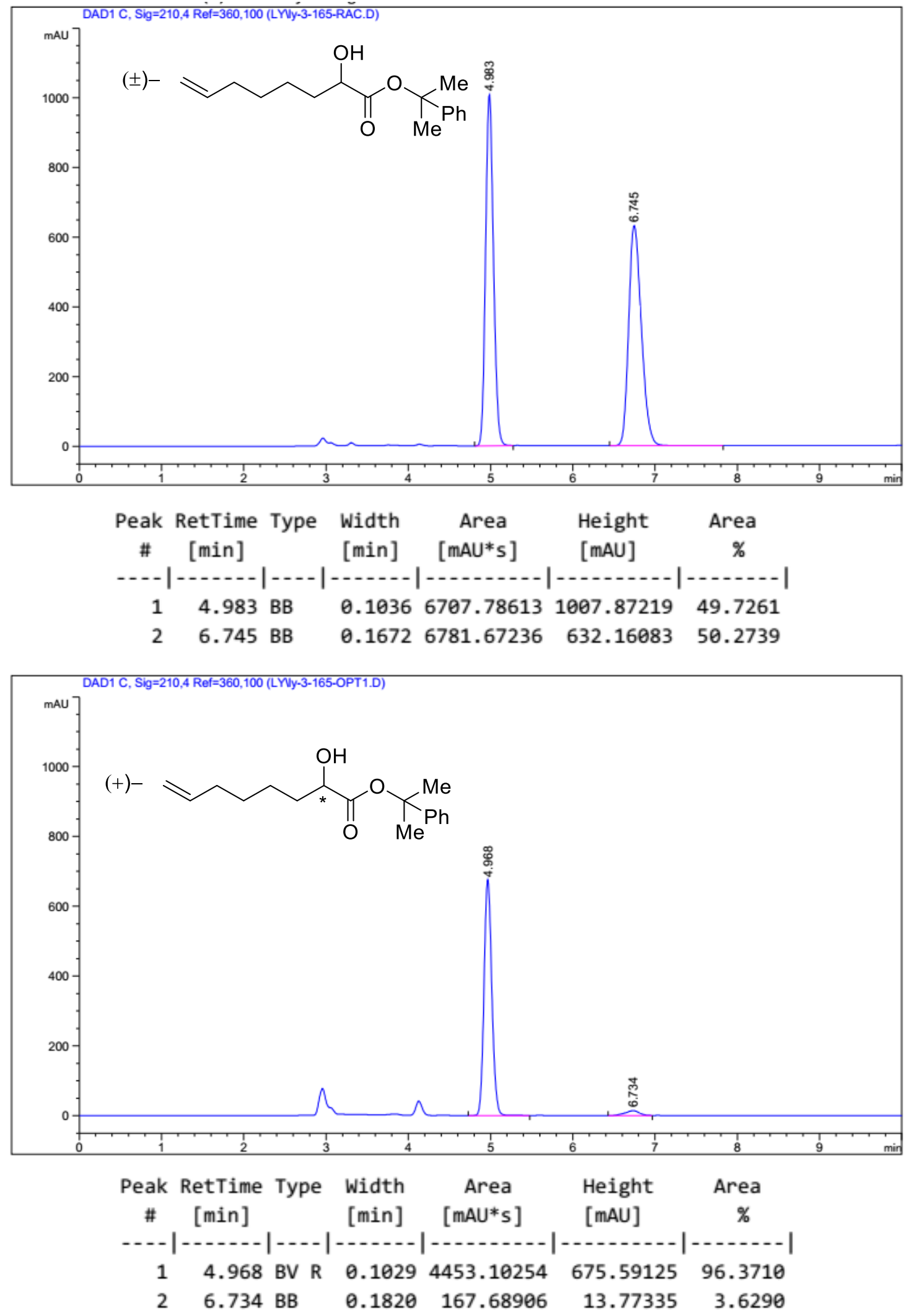
2-Phenylpropan-2-yl 2-hydroxyhex-5-ynoate (2p)

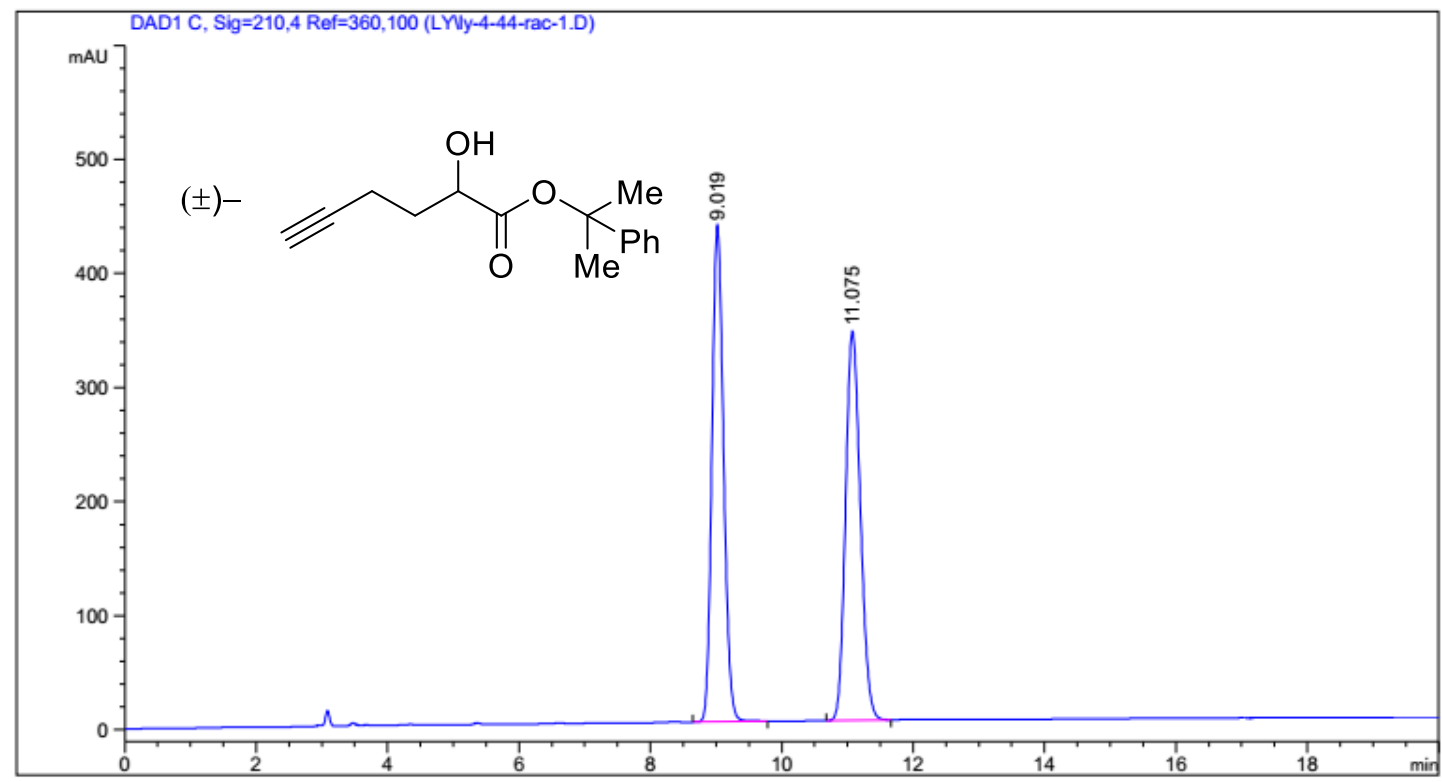

\begin{tabular}{|c|c|c|c|c|c|c|}
\hline $\begin{array}{c}\text { Peak } \\
\#\end{array}$ & $\begin{array}{l}\text { RetTime } \\
\text { [min] }\end{array}$ & Type & $\begin{array}{l}\text { Width } \\
\text { [min] }\end{array}$ & $\begin{array}{c}\text { Area } \\
{[\mathrm{mAU} * \mathrm{~s}]}\end{array}$ & $\begin{array}{l}\text { Height } \\
{[\mathrm{mAU}]}\end{array}$ & $\begin{array}{c}\text { Area } \\
\%\end{array}$ \\
\hline & 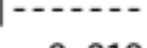 & & & - & ตับ & --1 \\
\hline 1 & 9.019 & BB & 0.1913 & 5341.94580 & 434.91522 & 50.0581 \\
\hline 2 & 11.075 & BB & 0.2443 & 5329.54980 & 341.02975 & 49.9419 \\
\hline
\end{tabular}

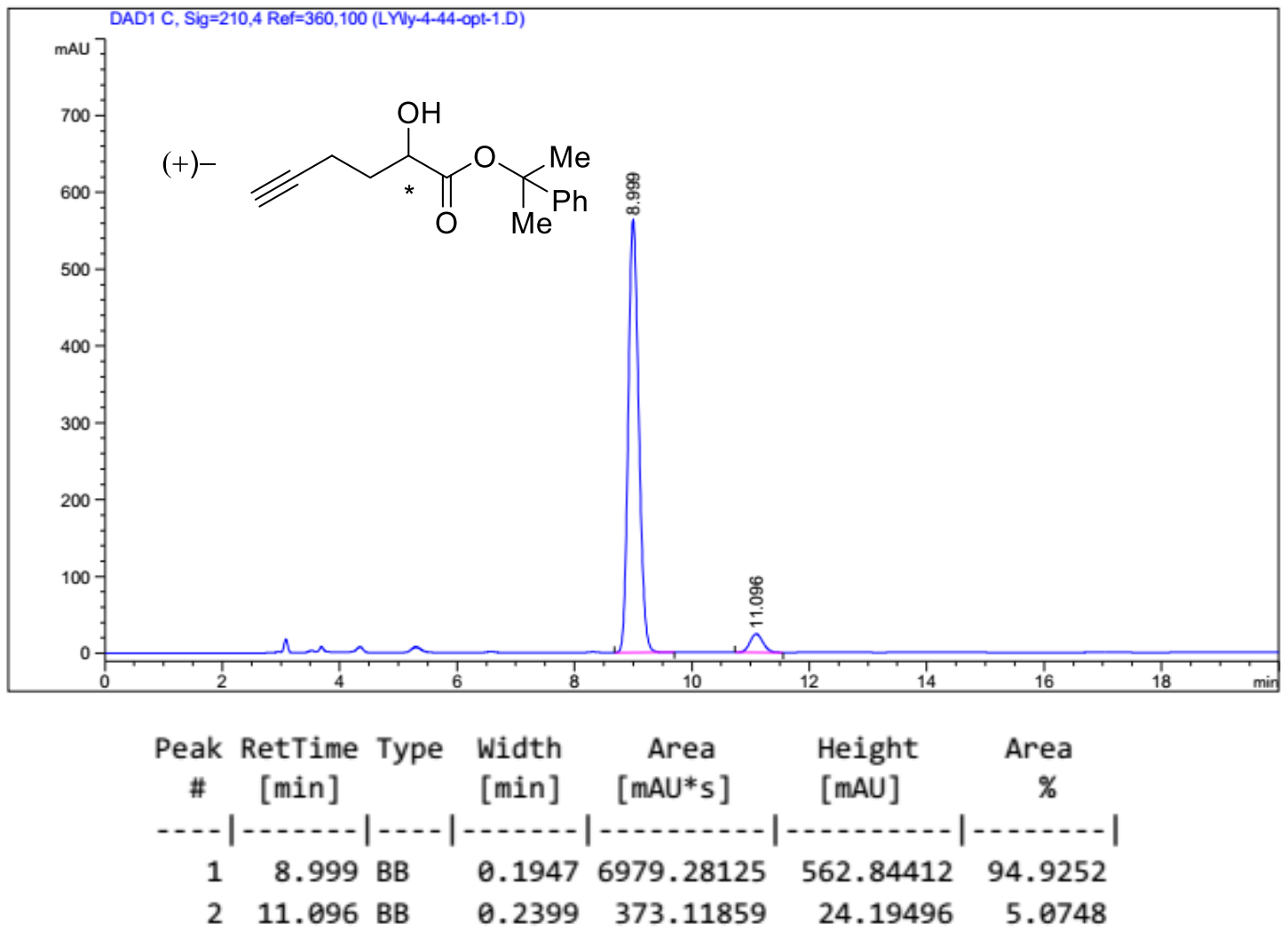


2-Phenylpropan-2-yl 2-hydroxy-5-methoxypentanoate (2q)
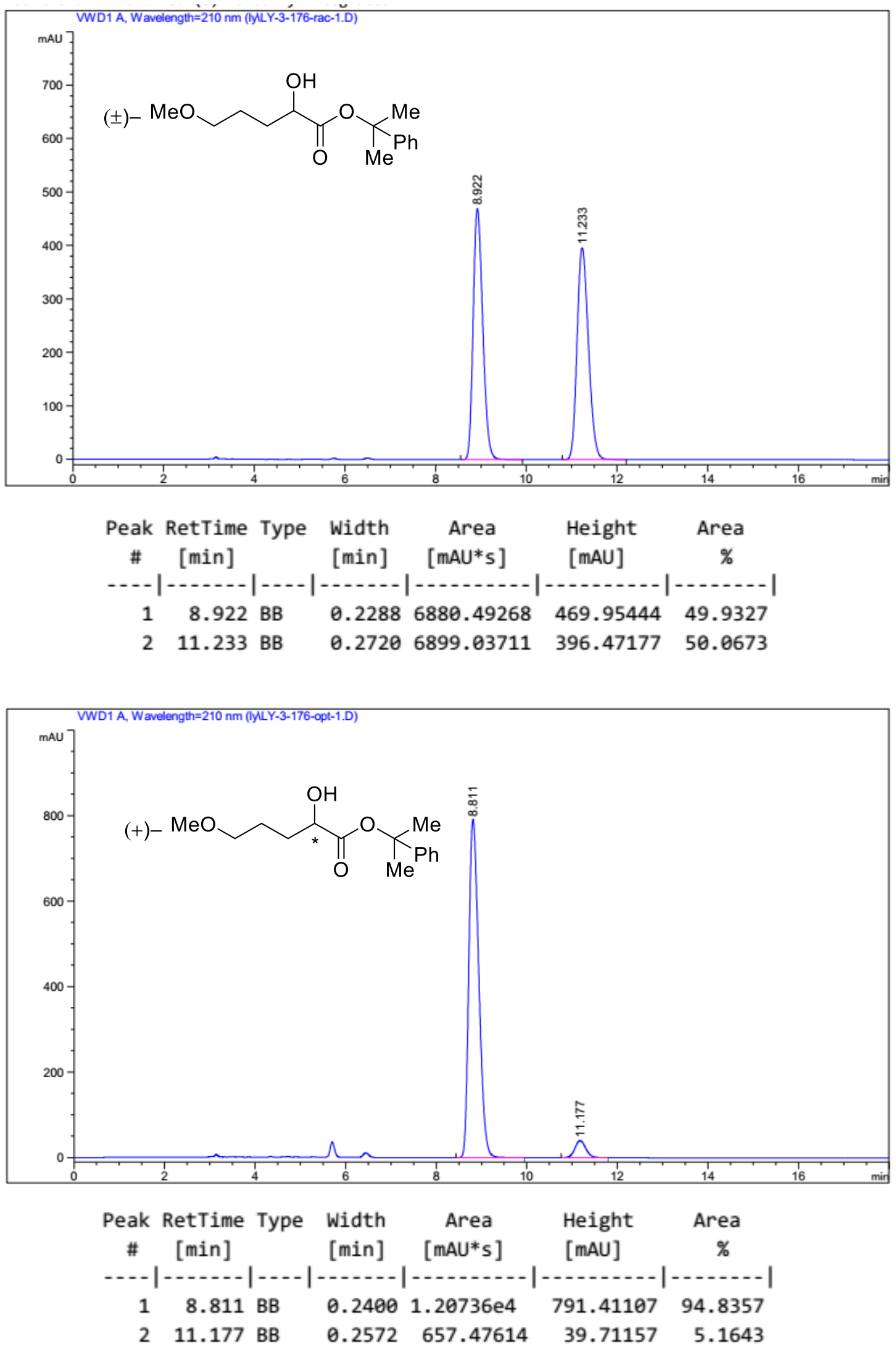
2-Phenylpropan-2-yl 6-((tert-butyldimethylsilyl)oxy)-2-hydroxyhexanoate (2r)
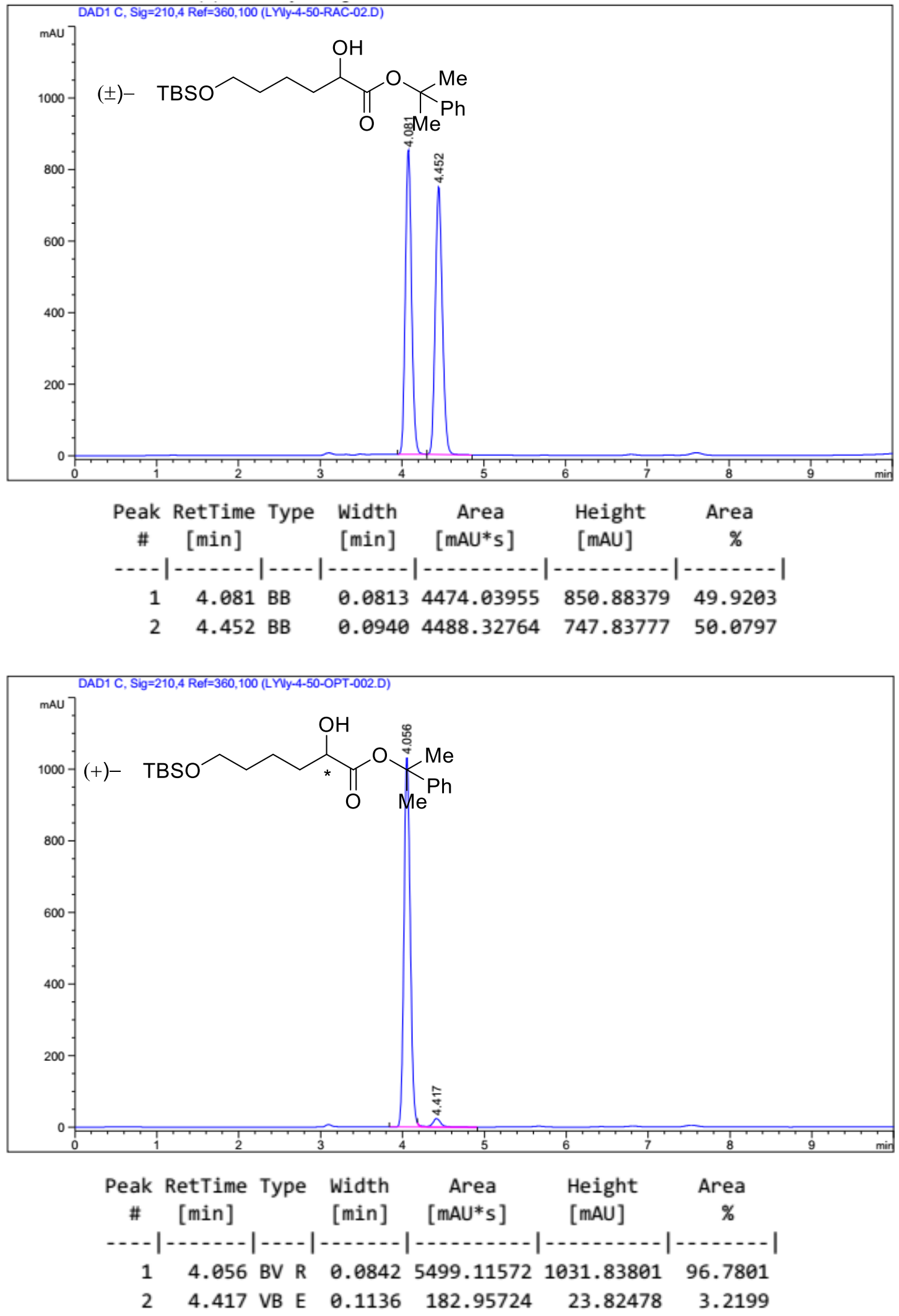
6-Ethyl 1-(2-phenylpropan-2-yl) 2-hydroxyhexanedioate (2s)
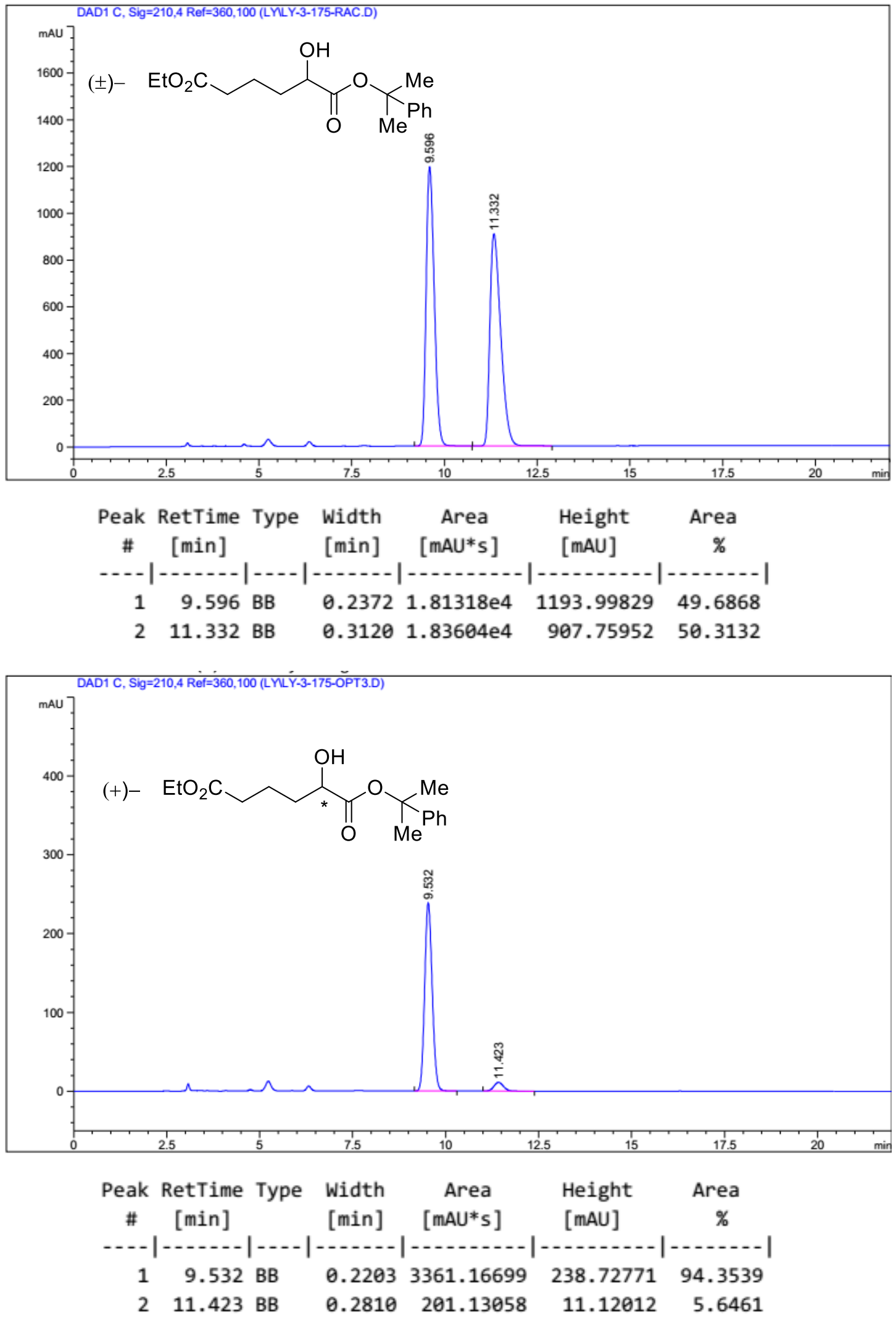
2-Phenylpropan-2-yl 5-cyano-2-hydroxypentanoate (2t)
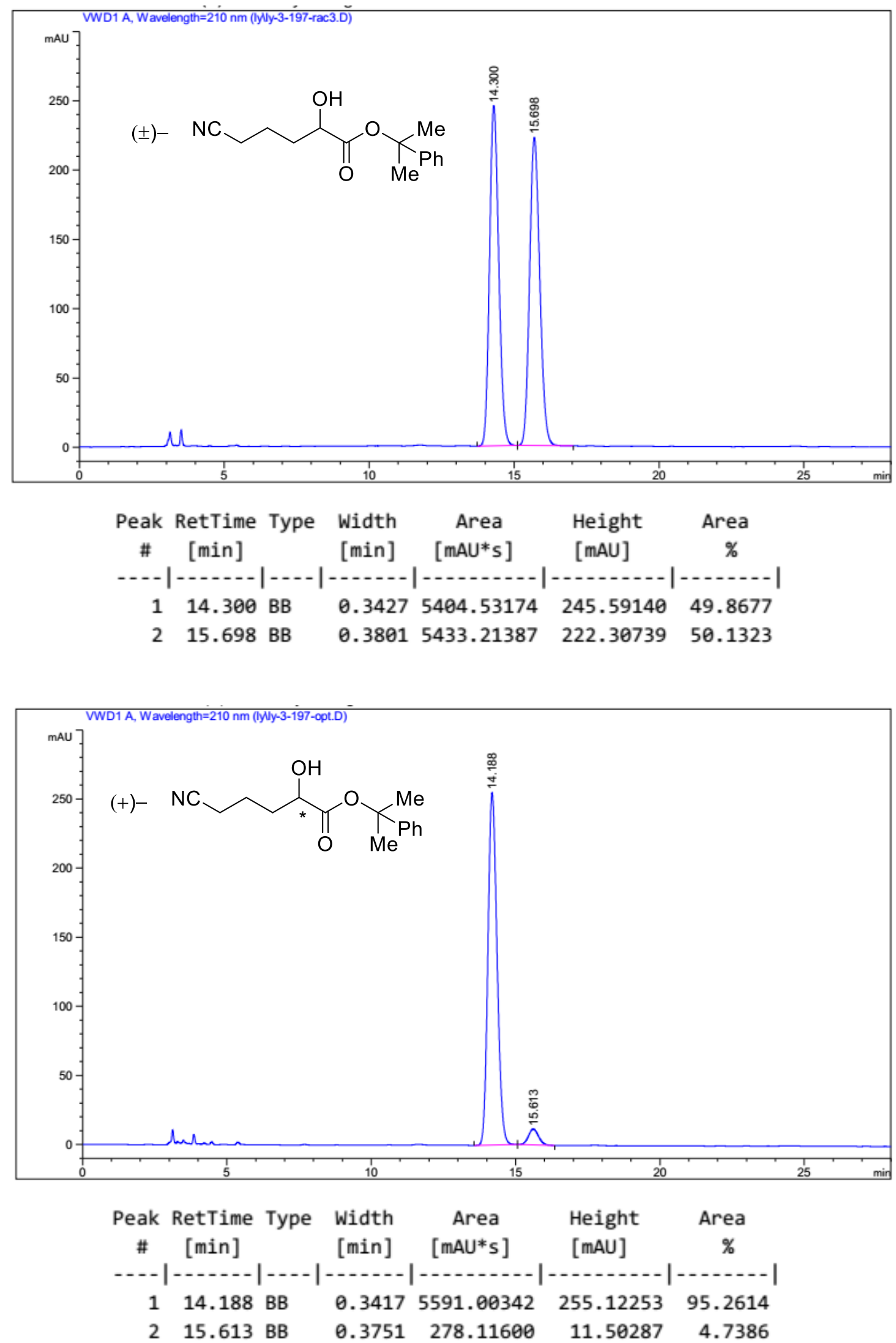
(E)-tert-Butyl 2-hydroxy-4-phenylpent-3-enoate (7a)
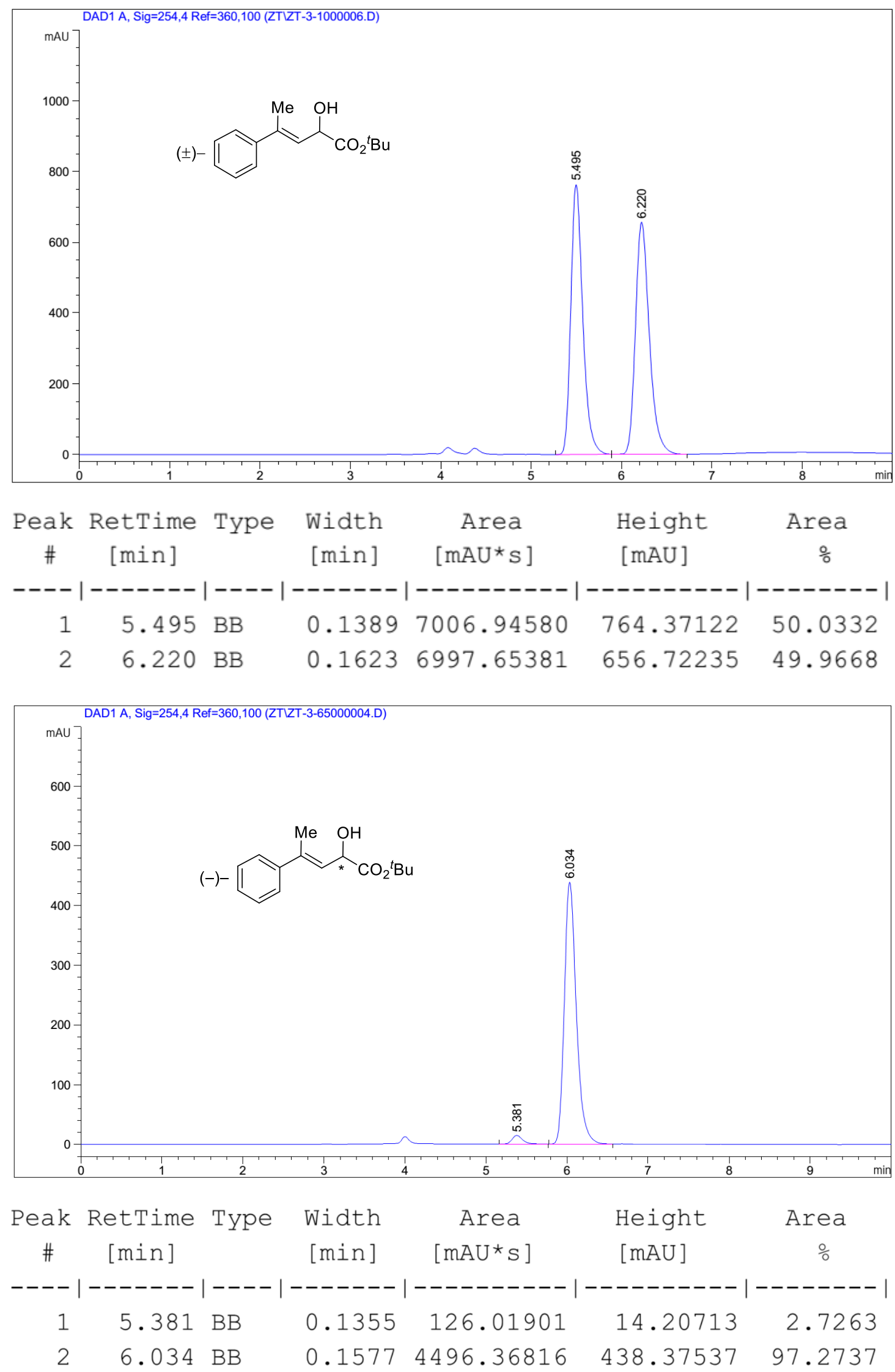


\section{(E)-tert-Butyl 2-hydroxy-4-(m-tolyl)pent-3-enoate (7b)}
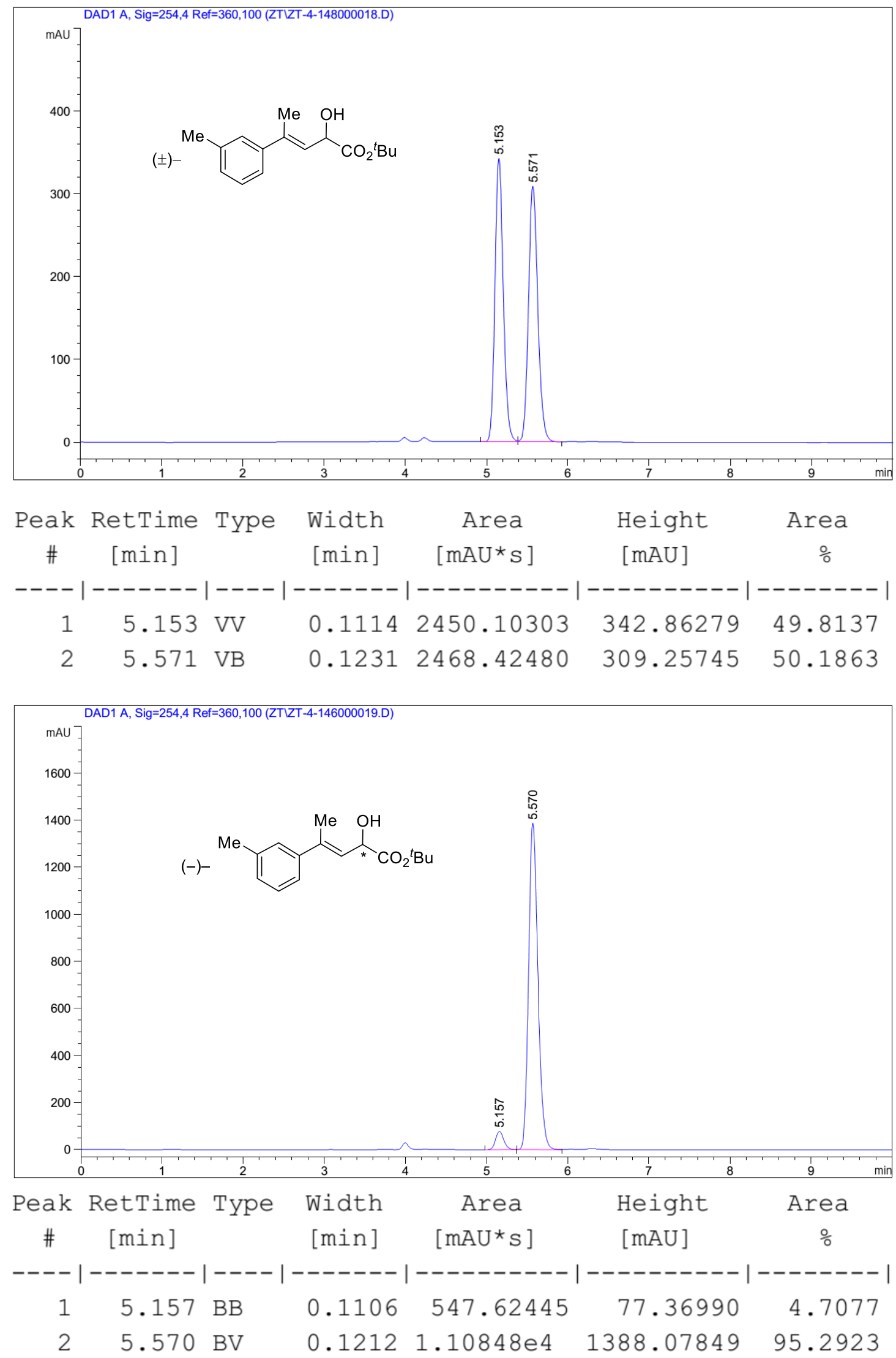


\section{(E)-tert-Butyl 2-hydroxy-4-(p-tolyl)pent-3-enoate (7c)}
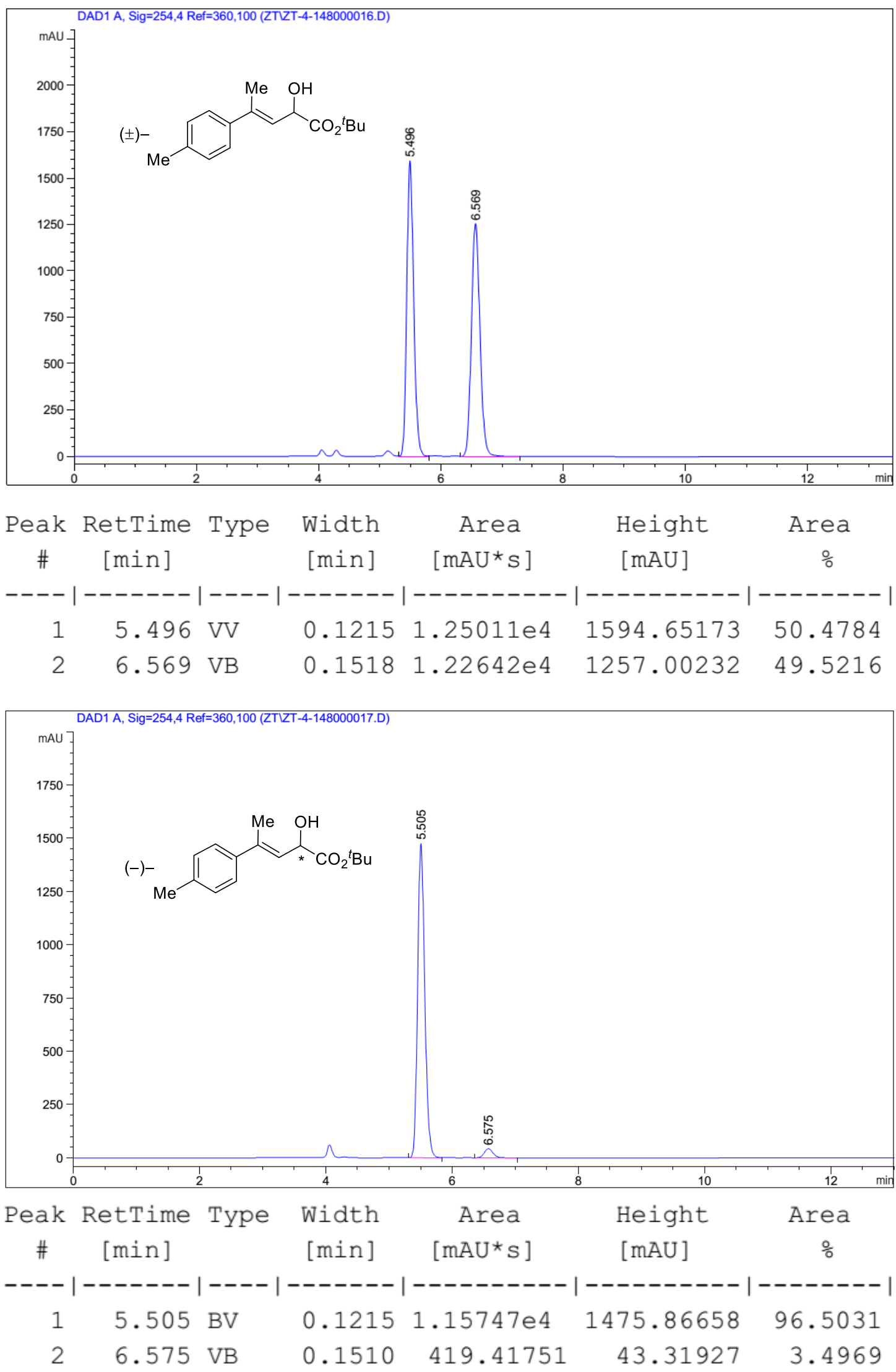


\section{(E)-tert-Butyl 2-hydroxy-4-(4-methoxyphenyl)pent-3-enoate (7d)}

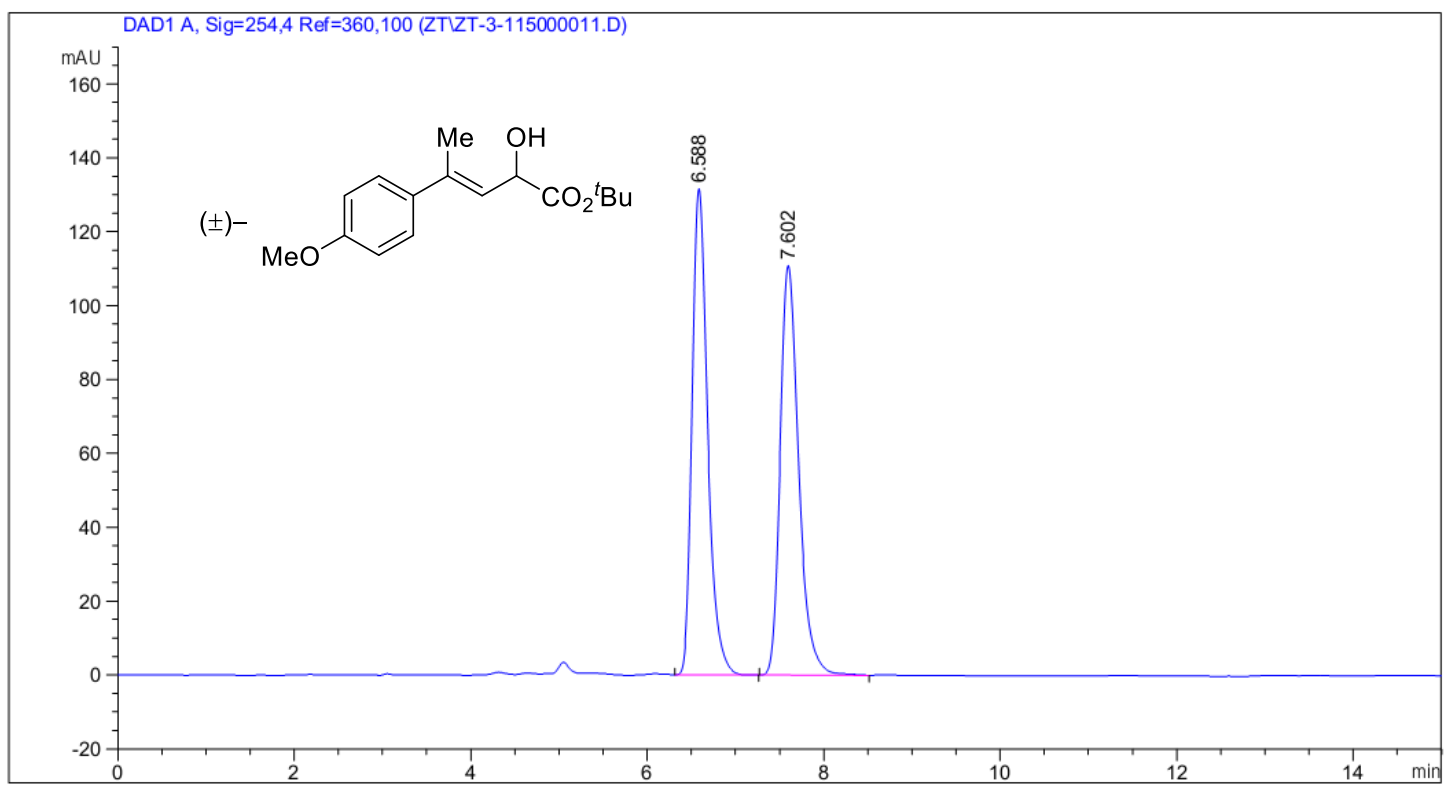

\begin{tabular}{|c|c|c|c|c|c|c|}
\hline $\begin{array}{l}\text { eak } \\
\text { \# }\end{array}$ & $\begin{array}{c}\text { RetTime } \\
\text { [min] }\end{array}$ & Type & $\begin{array}{l}\text { Width } \\
\text { [min] }\end{array}$ & $\begin{array}{c}\text { Area } \\
{\left[\mathrm{mAU}^{\star} \mathrm{s}\right]}\end{array}$ & $\begin{array}{l}\text { Height } \\
{[\mathrm{mAU}]}\end{array}$ & $\begin{array}{c}\text { Area } \\
\%\end{array}$ \\
\hline & & & & ---- & 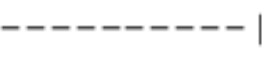 & \\
\hline 1 & 6.588 & & & 1601.73083 & 131.56334 & \\
\hline 2 & 7.602 & 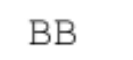 & 215 & 1611.28796 & 110.87523 & 487 \\
\hline
\end{tabular}

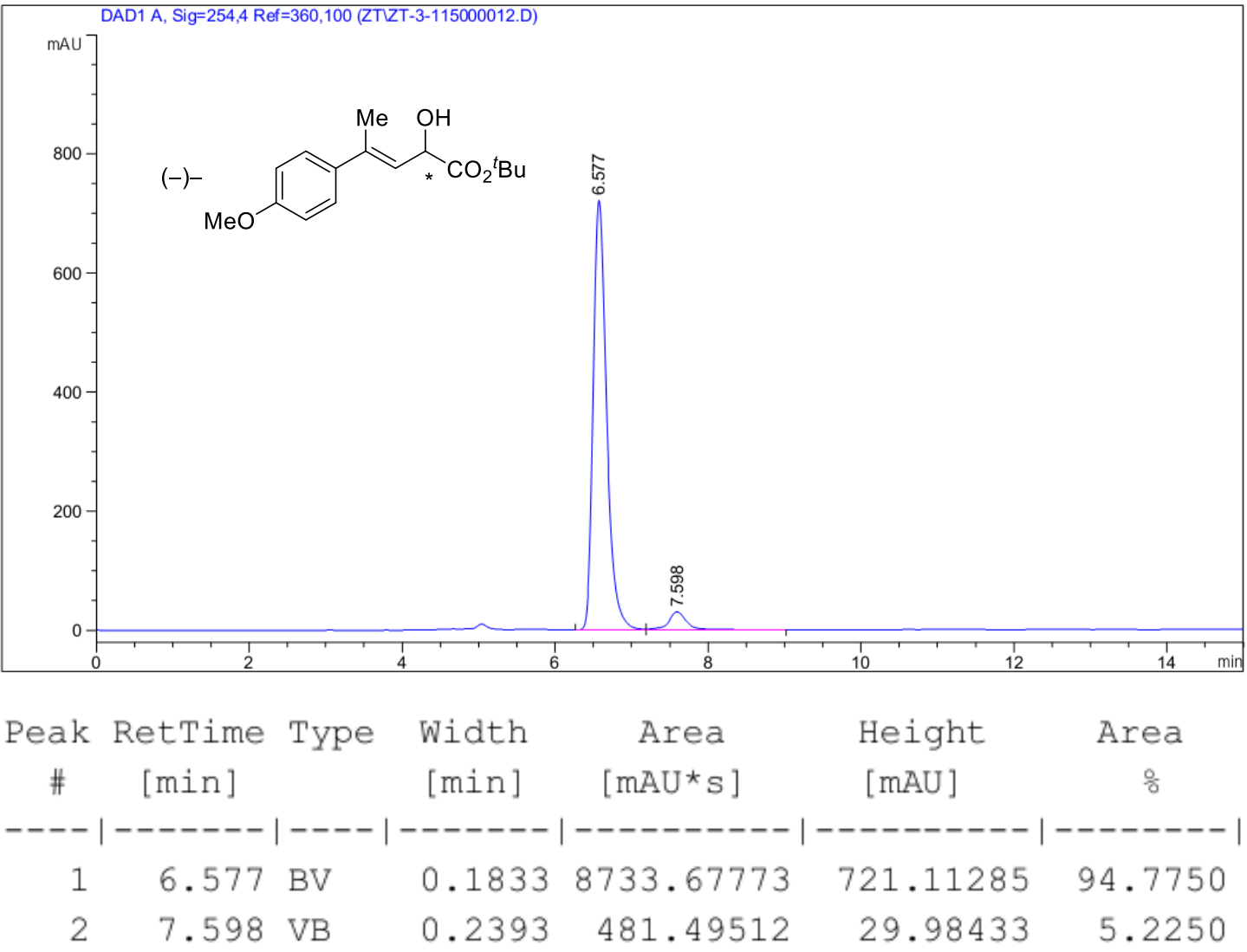




\section{(E)-tert-Butyl 2-hydroxy-4-(4-fluorophenyl)pent-3-enoate (7e)}
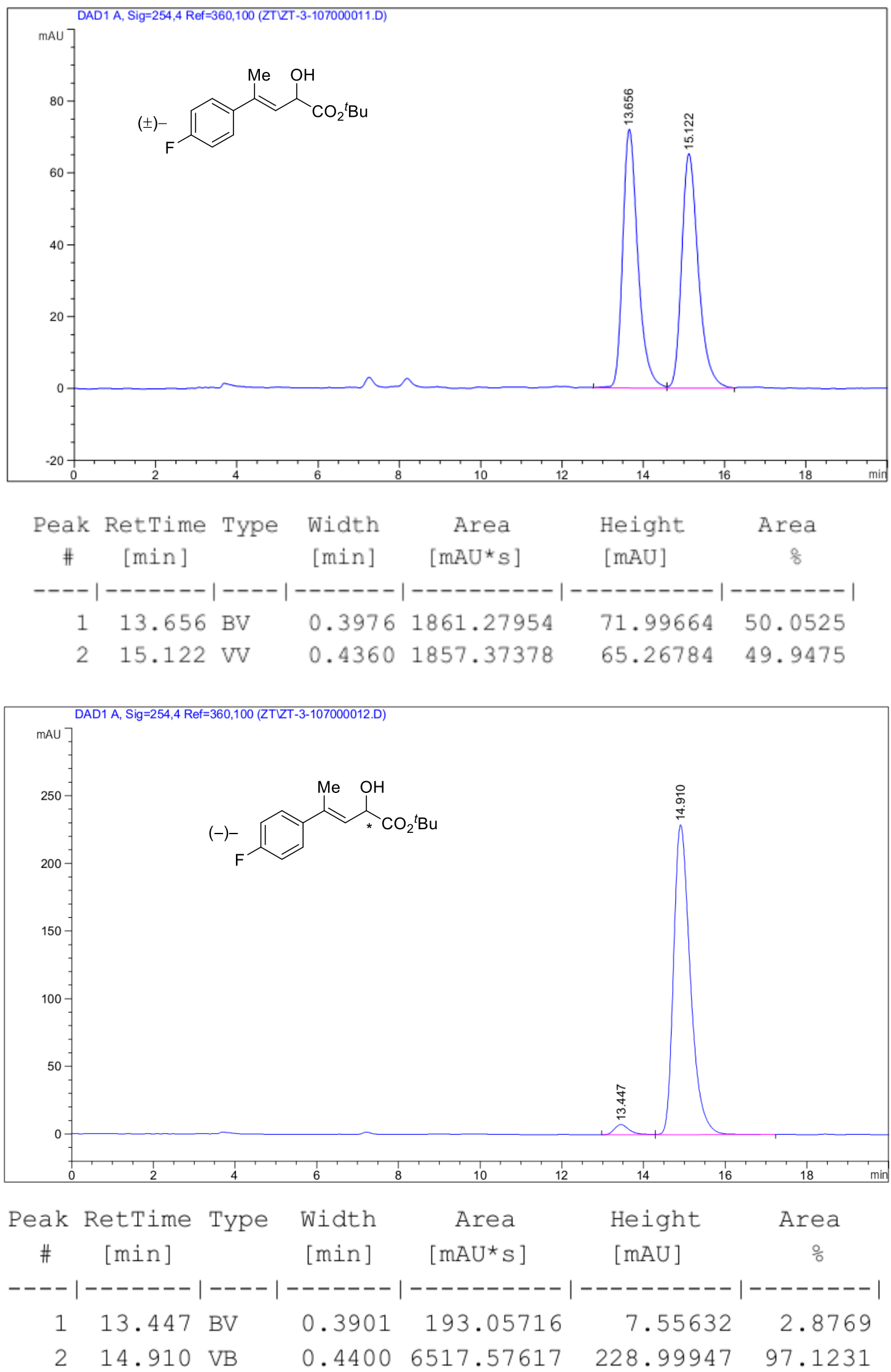


\section{(E)-tert-Butyl 2-hydroxy-4-(2-chlorophenyl)pent-3-enoate (7f)}
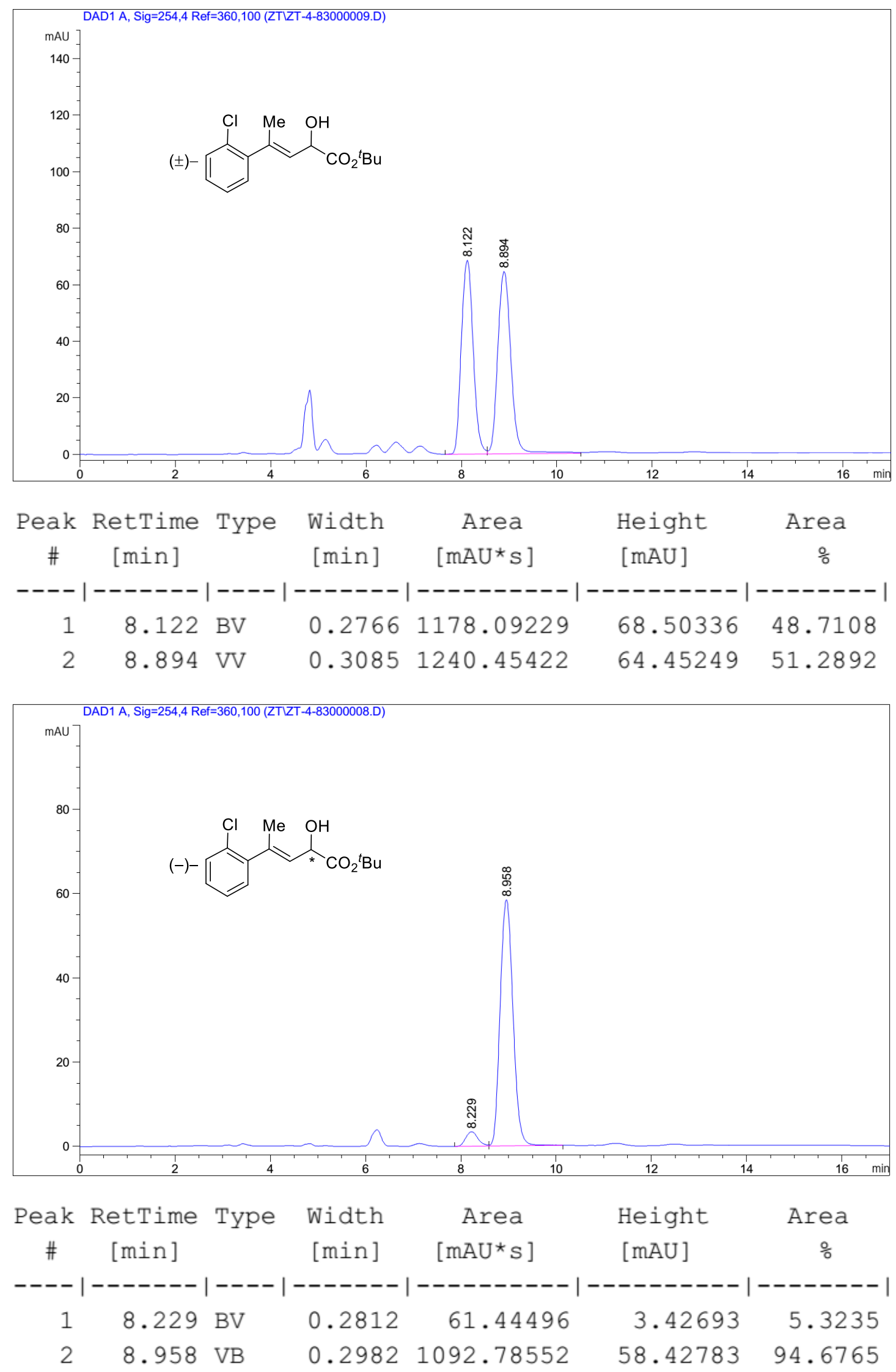


\section{(E)-tert-Butyl 2-hydroxy-4-(3-chlorophenyl)pent-3-enoate (7g)}
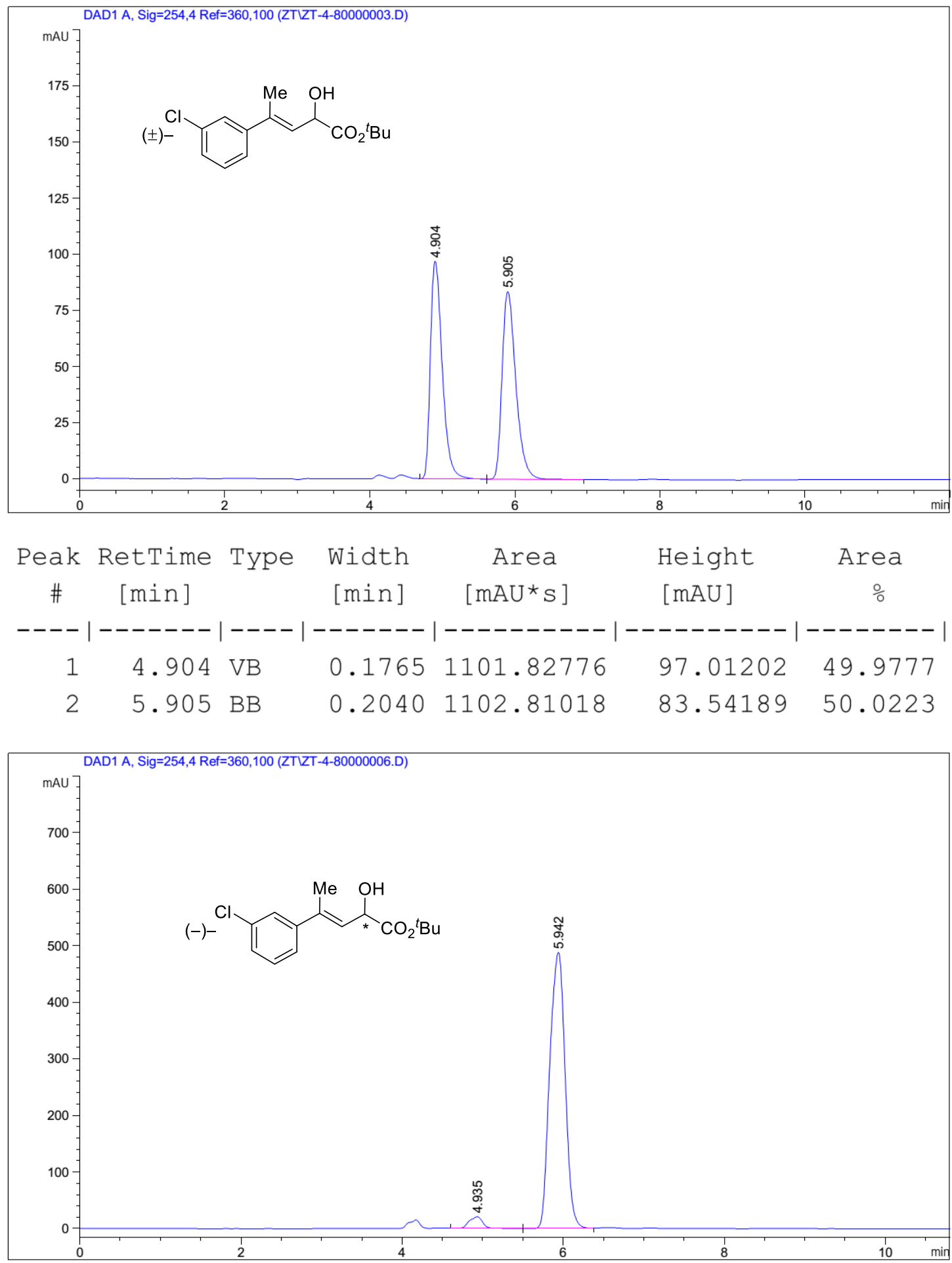

\begin{tabular}{|c|c|c|c|c|c|c|}
\hline eak & $\begin{array}{c}\text { RetTime } \\
\text { [min] }\end{array}$ & YF & $\begin{array}{l}\text { Width } \\
\text { [min] }\end{array}$ & $\begin{array}{c}\text { Area } \\
{\left[\mathrm{mAU}^{*} \mathrm{~s}\right]}\end{array}$ & $\begin{array}{l}\text { Height } \\
{[\mathrm{mAU}]}\end{array}$ & $\begin{array}{c}\text { Area } \\
\quad \%\end{array}$ \\
\hline & & & & ----1 & 1 & \\
\hline 1 & & & & 258.60818 & 28 & 569 \\
\hline 2 & & RV & 60 & 6446.54980 & 488.23526 & 1431 \\
\hline
\end{tabular}




\section{(E)-tert-Butyl 2-hydroxy-4-(4-chlorophenyl)pent-3-enoate (7h)}

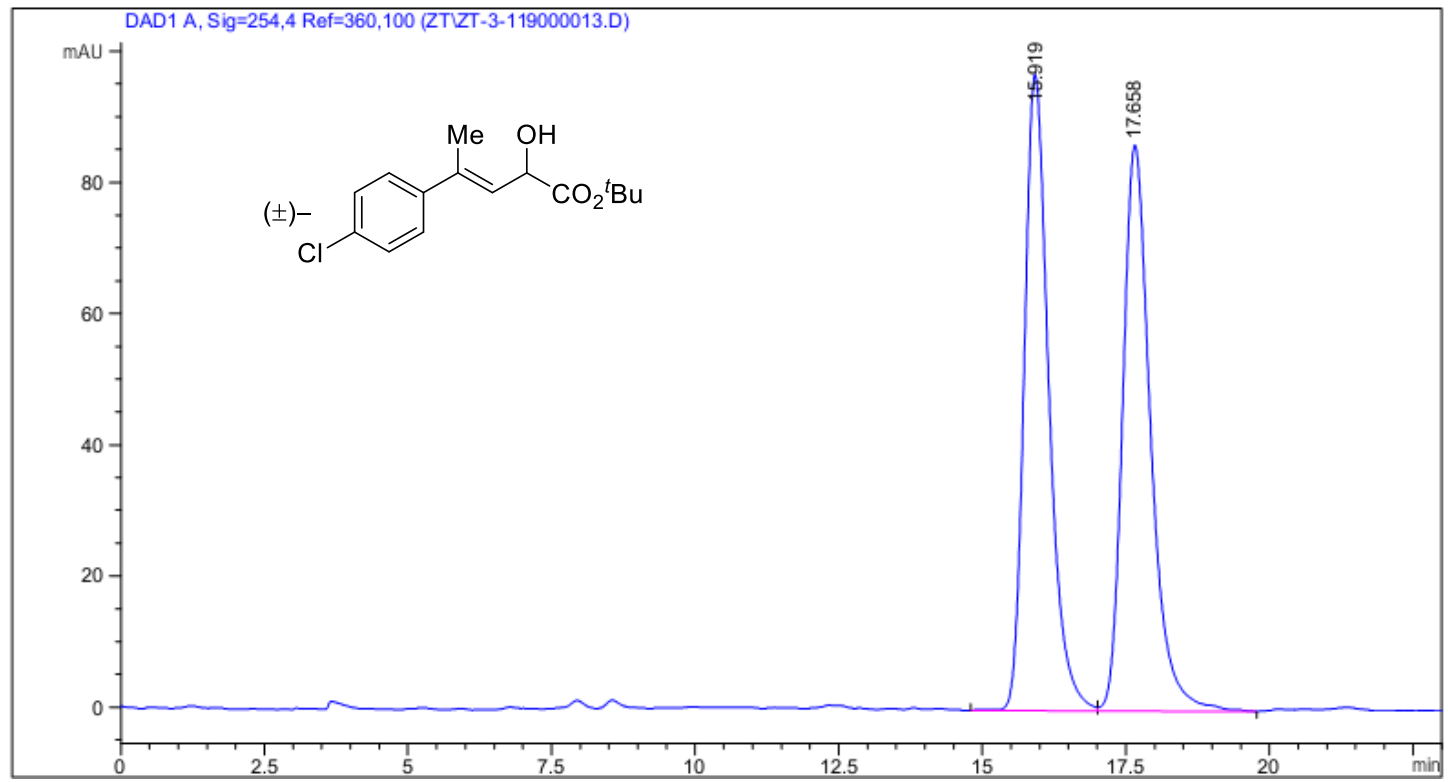

\begin{tabular}{|c|c|c|c|c|c|c|}
\hline eak & $\begin{array}{c}\text { RetTime } \\
\text { [min] }\end{array}$ & Type & $\begin{array}{l}\text { Width } \\
\text { [min] }\end{array}$ & $\begin{array}{c}\text { Area } \\
{\left[\mathrm{mAU}^{\star} \mathrm{S}\right]}\end{array}$ & $\begin{array}{l}\text { Height } \\
{[\mathrm{mAU}]}\end{array}$ & $\begin{array}{c}\text { Area } \\
\frac{\circ}{\circ}\end{array}$ \\
\hline & & & & --------- & --- & \\
\hline 1 & & $\mathrm{~V}$ & & 2876.87134 & 383 & \\
\hline 2 & 17.658 & $\mathrm{IB}$ & 136 & 2908.30029 & 6.20994 & 2716 \\
\hline
\end{tabular}

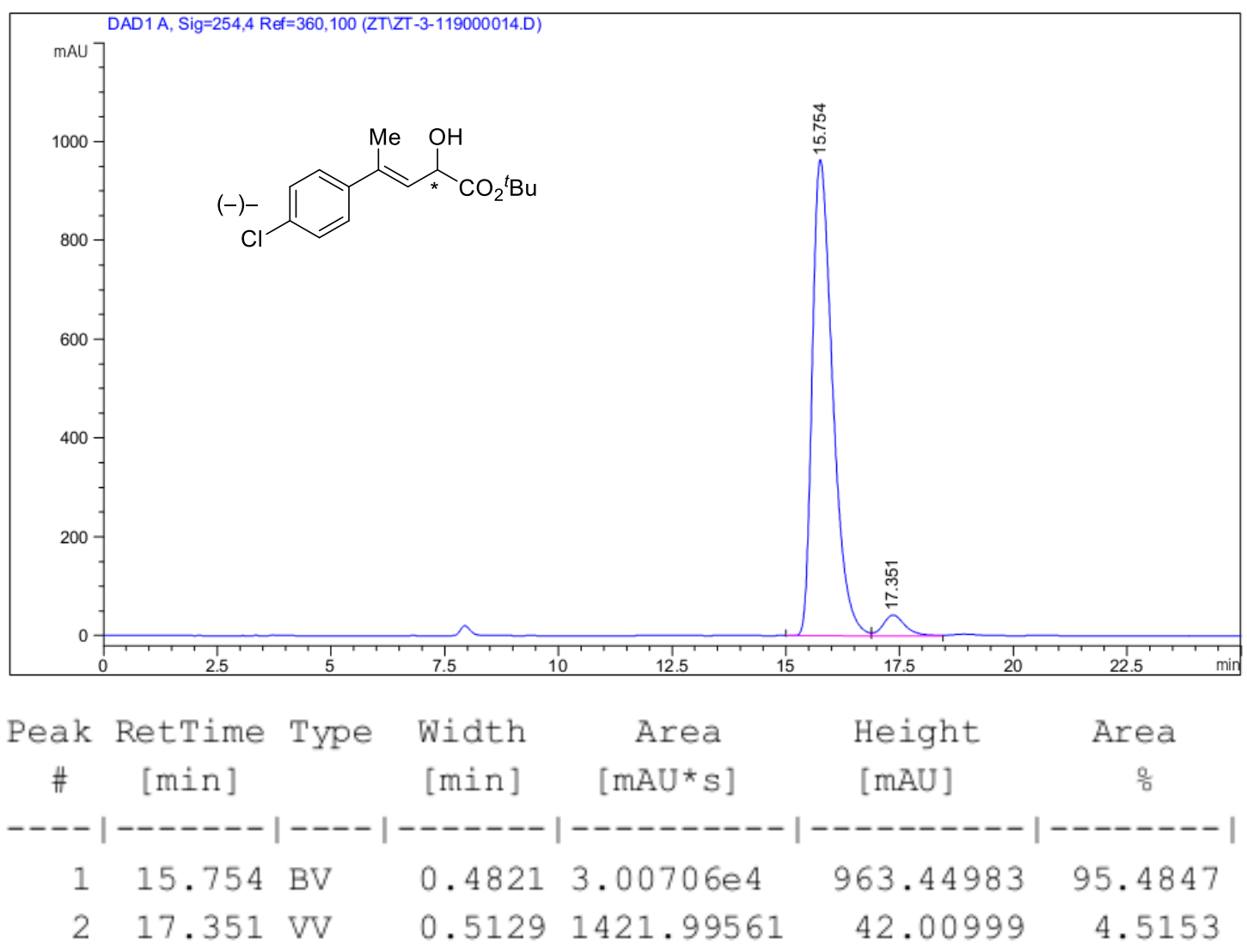




\section{(E)-tert-Butyl 4-(3,4-dimethylphenyl)-2-hydroxypent-3-enoate (7i)}

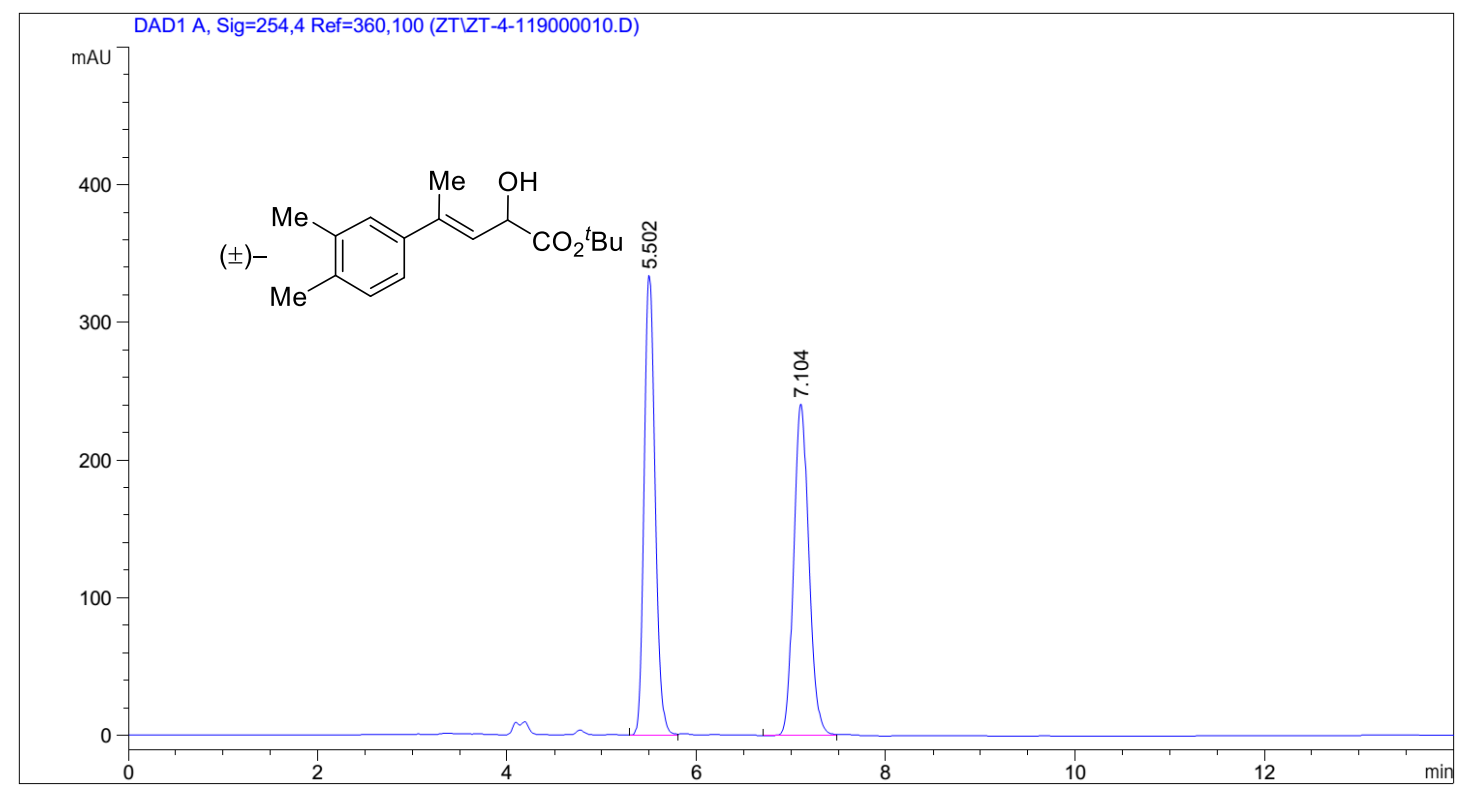

\begin{tabular}{|c|c|c|c|c|c|c|}
\hline $\begin{array}{c}\text { Peak } \\
\#\end{array}$ & $\begin{array}{c}\text { RetTime } \\
\text { [min] }\end{array}$ & Type & $\begin{array}{l}\text { Width } \\
\text { [min] }\end{array}$ & $\begin{array}{c}\text { Area } \\
{\left[\mathrm{mAU}{ }^{*} \mathrm{~S}\right]}\end{array}$ & $\begin{array}{l}\text { Height } \\
{[\mathrm{mAU}]}\end{array}$ & $\begin{array}{c}\text { Area } \\
\quad \%\end{array}$ \\
\hline & & & & & & \\
\hline & & & & 66 & 85 & 58 \\
\hline 0 & & & & 2696.52295 & 7211 & 84 \\
\hline
\end{tabular}

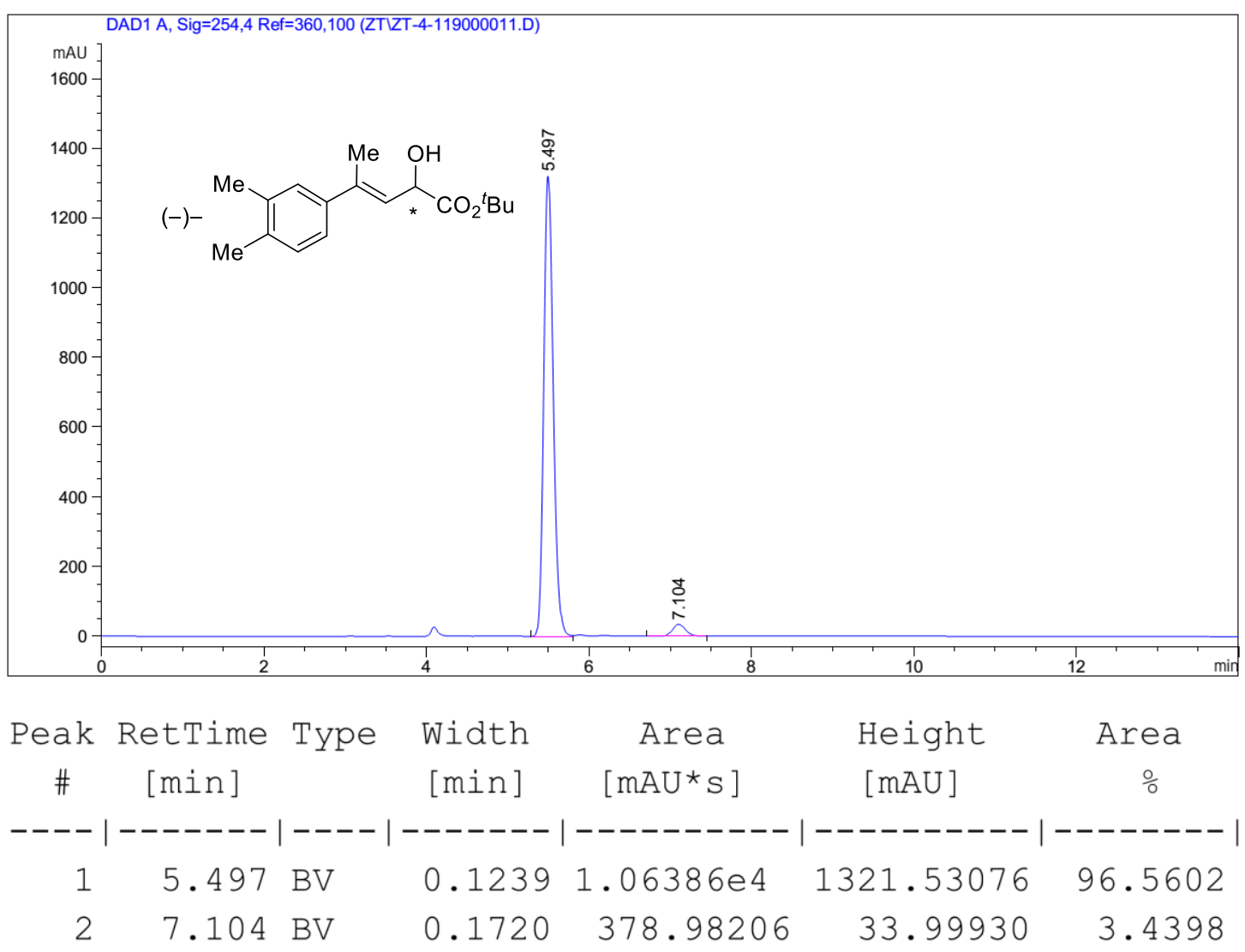


(E)-tert-Butyl 4-(benzo[d][1,3]dioxol-5-yl)-2-hydroxypent-3-enoate (7j)
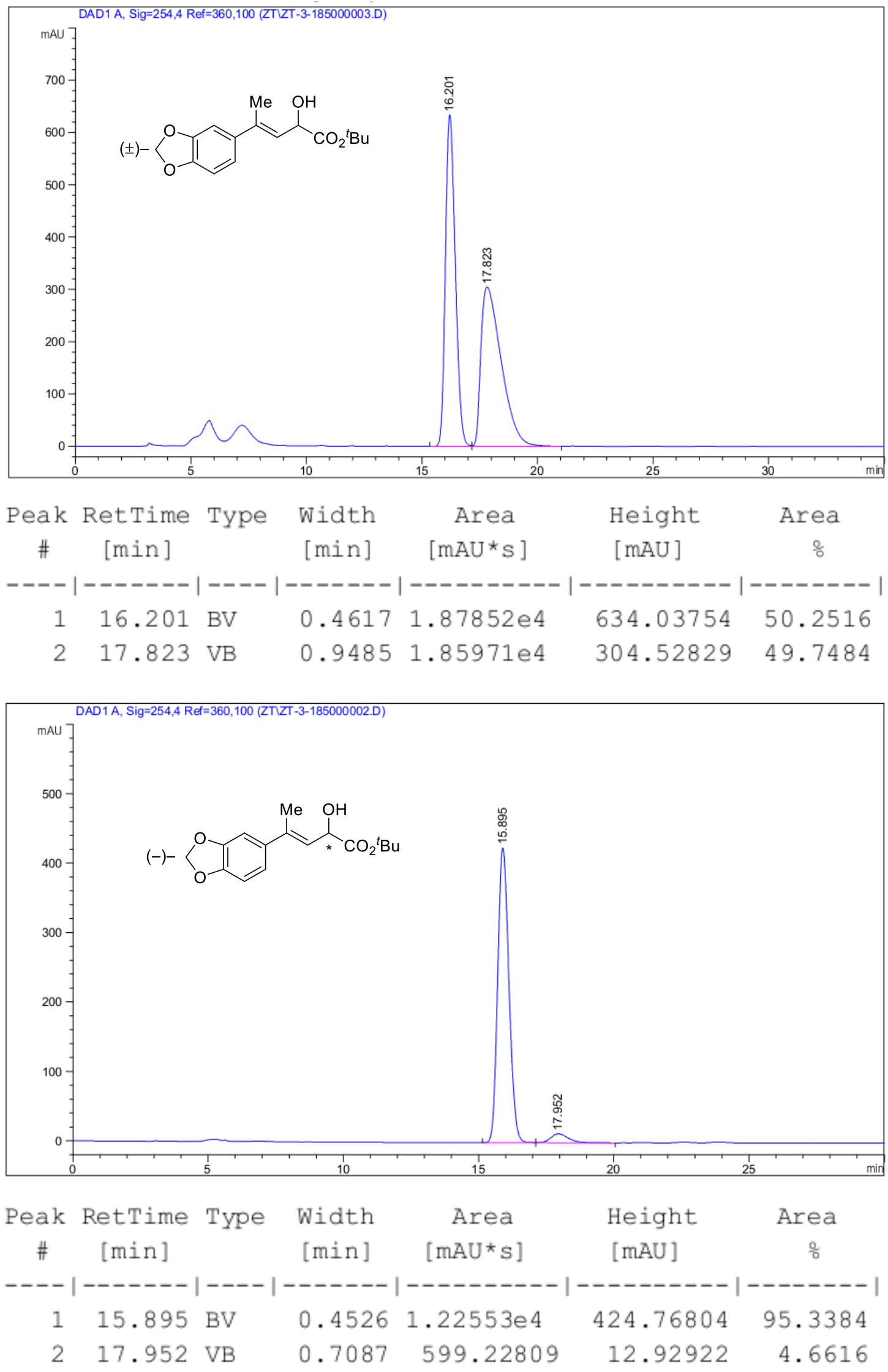
(E)-tert-Butyl 2-hydroxy-4-(naphthalen-2-yl)pent-3-enoate (7k)
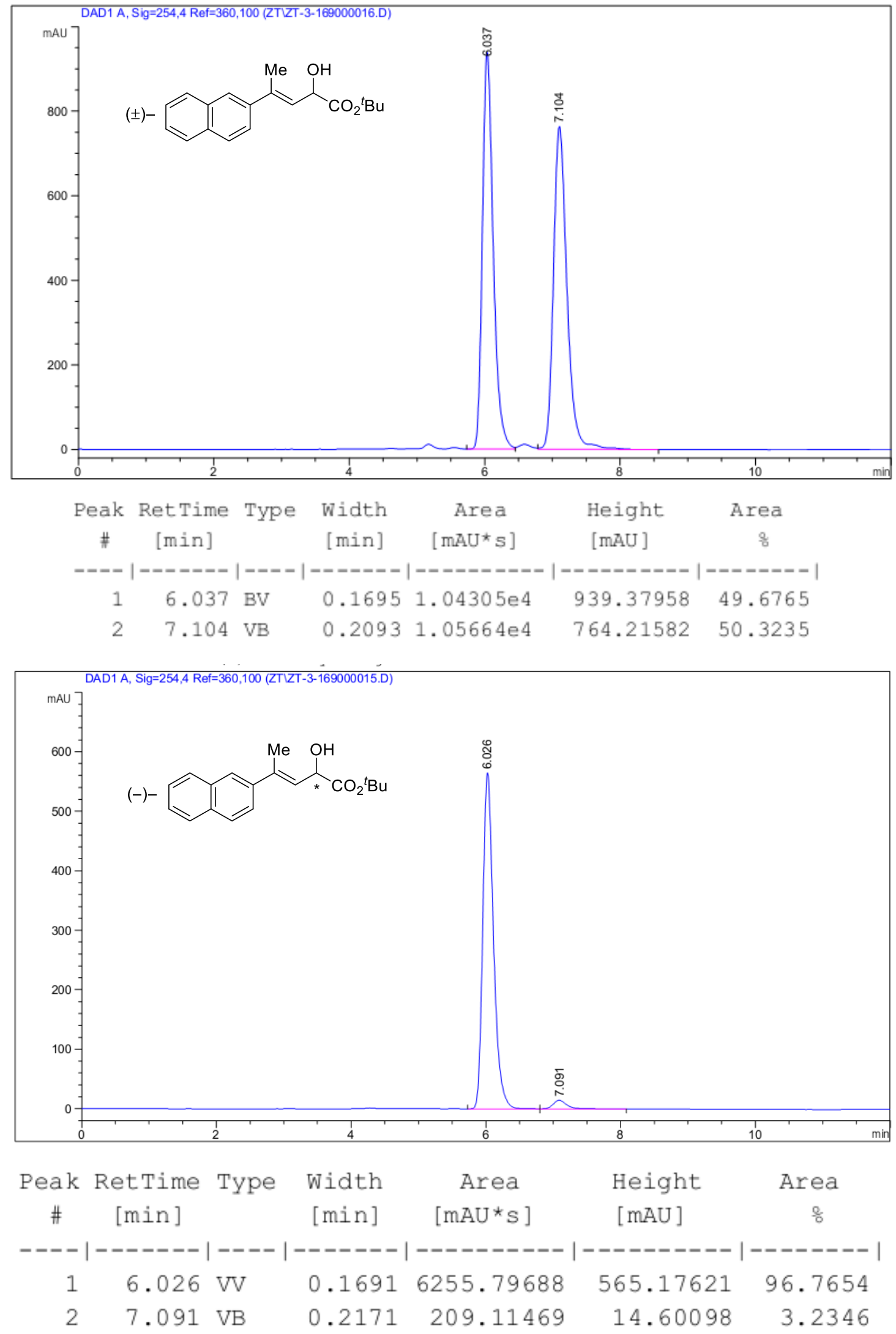


\section{(E)-tert-Butyl 2-hydroxy-4-(thiophen-2-yl)pent-3-enoate (7l)}
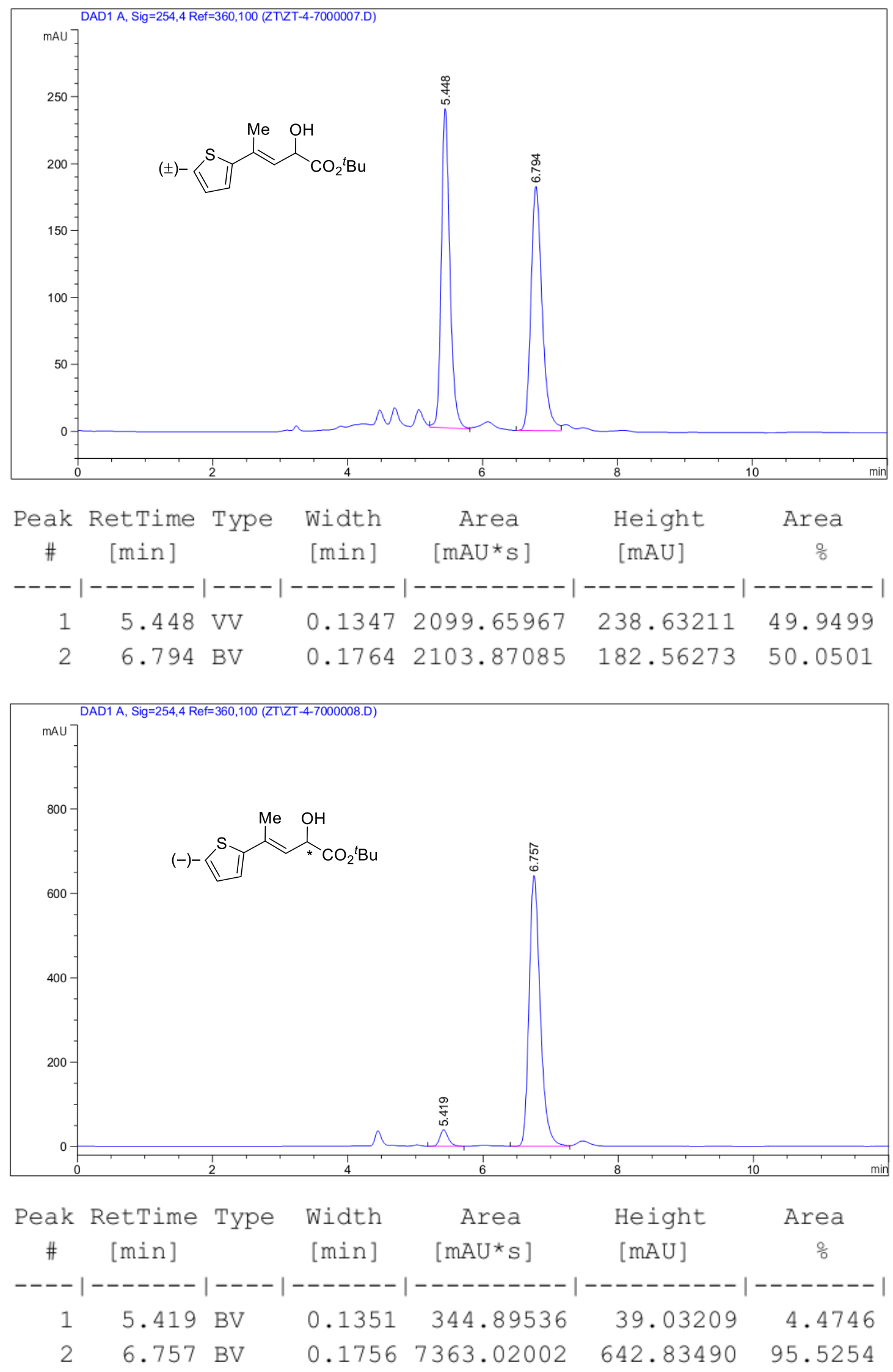
tert-Butyl 2-hydroxy-4,4-diphenylbut-3-enoate (7m)
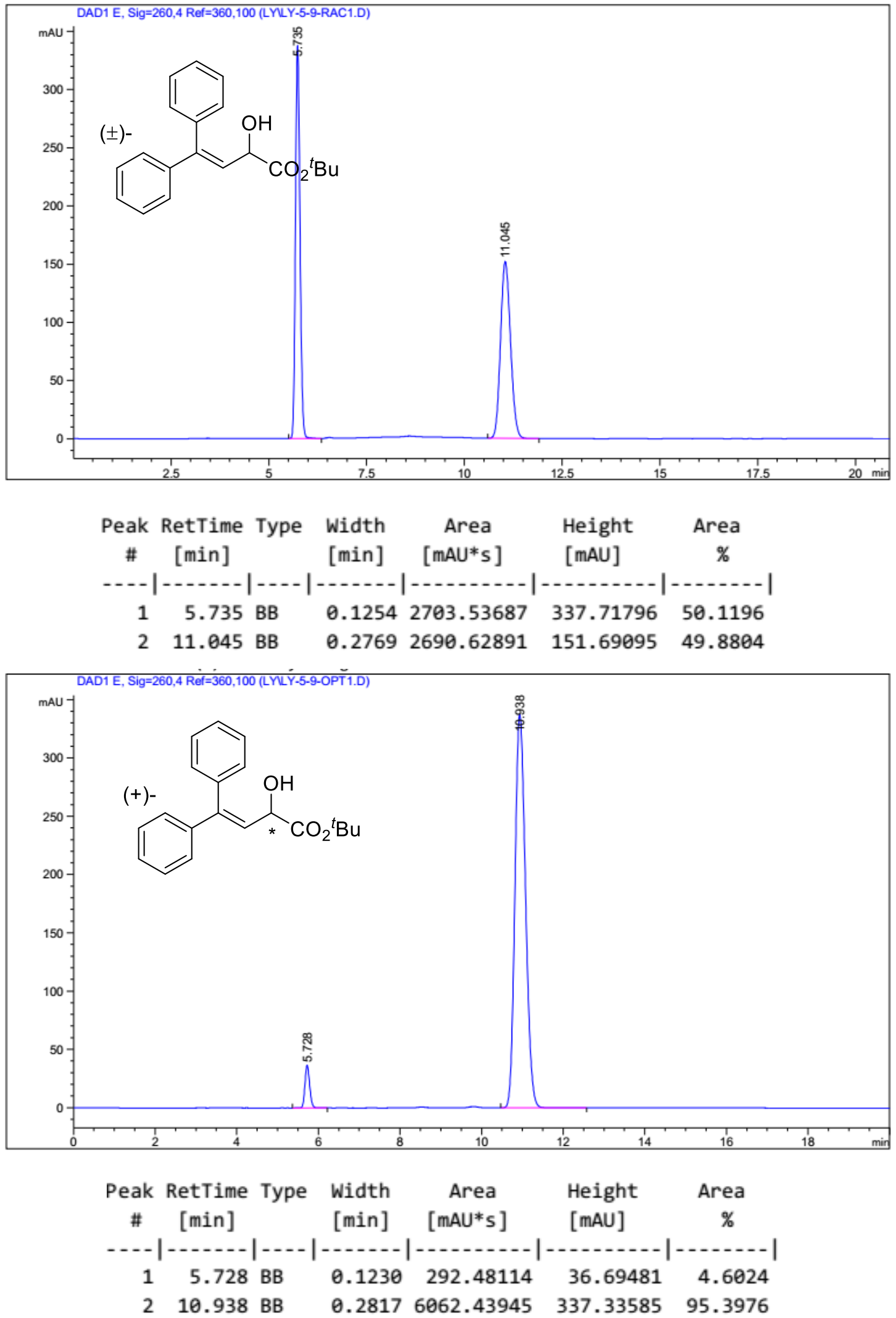
tert-Butyl (E)-2-hydroxy-4-phenylbut-3-enoate (7n)
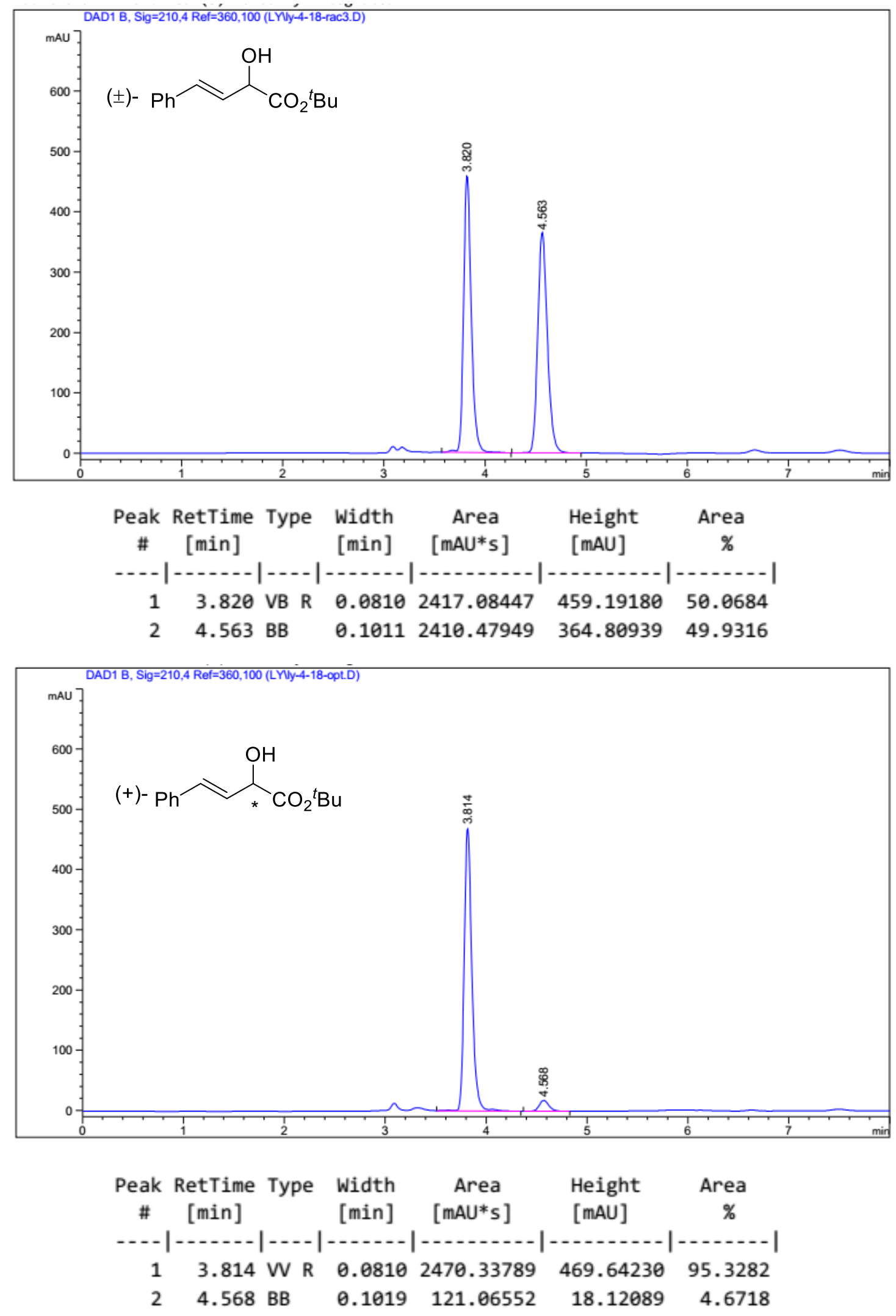
Benzhydryl (E)-2-hydroxypent-3-enoate (7o)
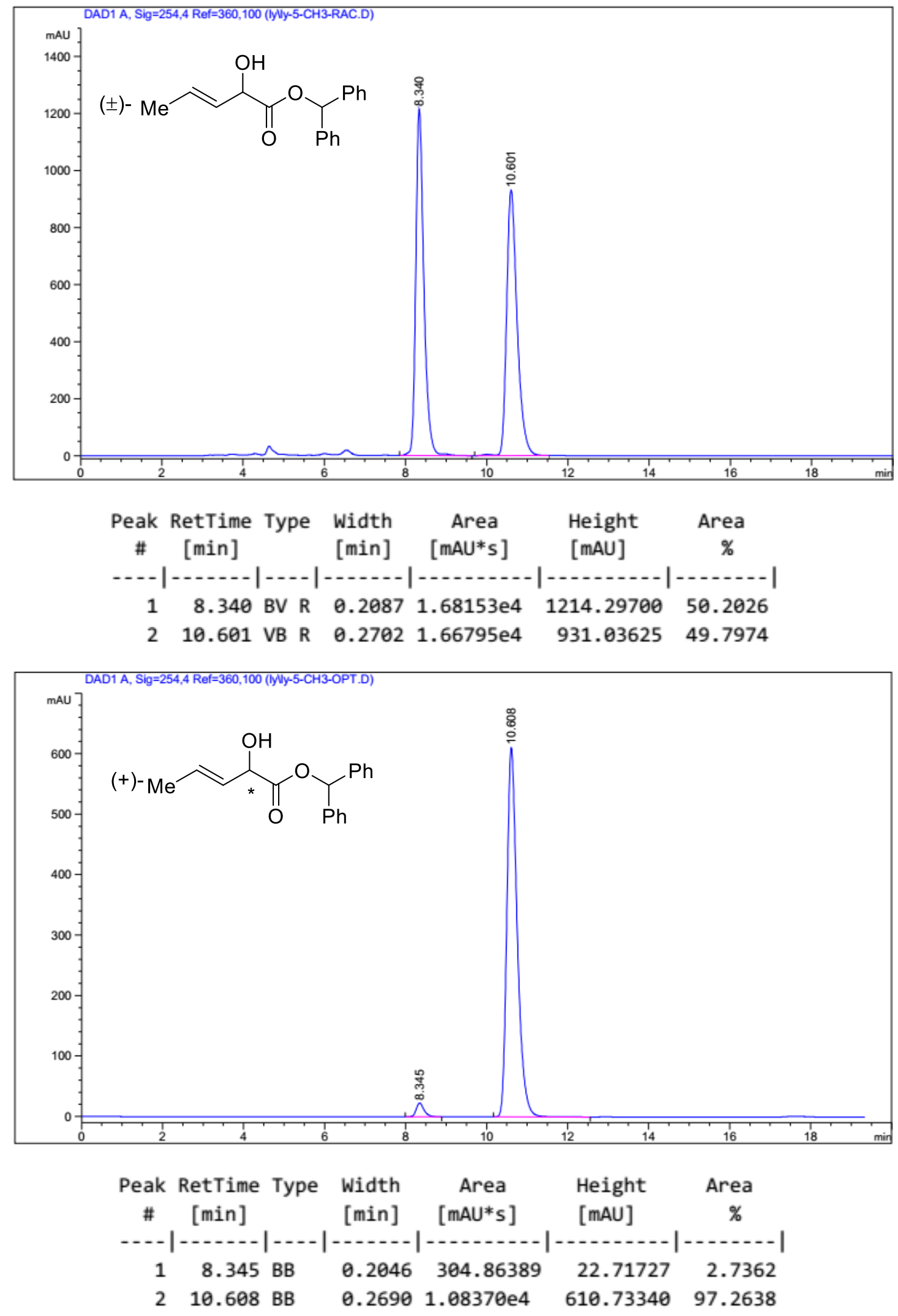
Benzhydryl (E)-2-hydroxyhex-3-enoate (7p)
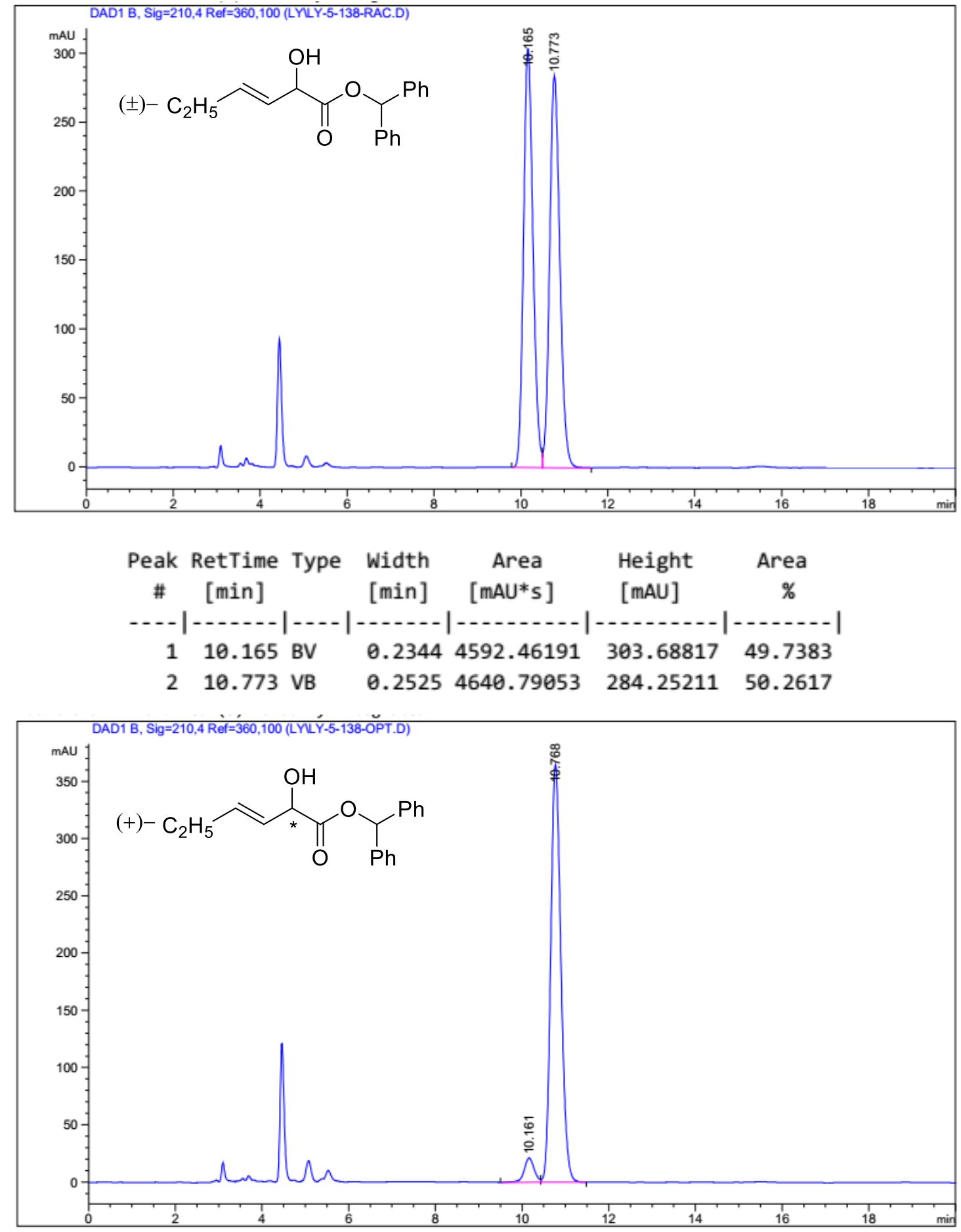

\begin{tabular}{|c|c|c|c|c|c|}
\hline $\begin{array}{c}\text { Peak } \\
\#\end{array}$ & $\begin{array}{l}\text { RetTime Type } \\
\text { [min] }\end{array}$ & $\begin{array}{l}\text { Width } \\
\text { [min] }\end{array}$ & $\begin{array}{c}\text { Area } \\
{[\mathrm{mAU} * \mathrm{~s}]}\end{array}$ & $\begin{array}{l}\text { Height } \\
{[\mathrm{mAU}]}\end{array}$ & $\begin{array}{c}\text { Area } \\
\%\end{array}$ \\
\hline & & & & & \\
\hline 1 & $10.161 \mathrm{BV}$ & 0.2470 & 343.86563 & 21.22395 & 5.4581 \\
\hline 2 & 10.768 VB & 0.2544 & 5956.17480 & 365.03354 & 94.5419 \\
\hline
\end{tabular}


Benzhydryl (E)-2-hydroxyhept-3-enoate (7q)
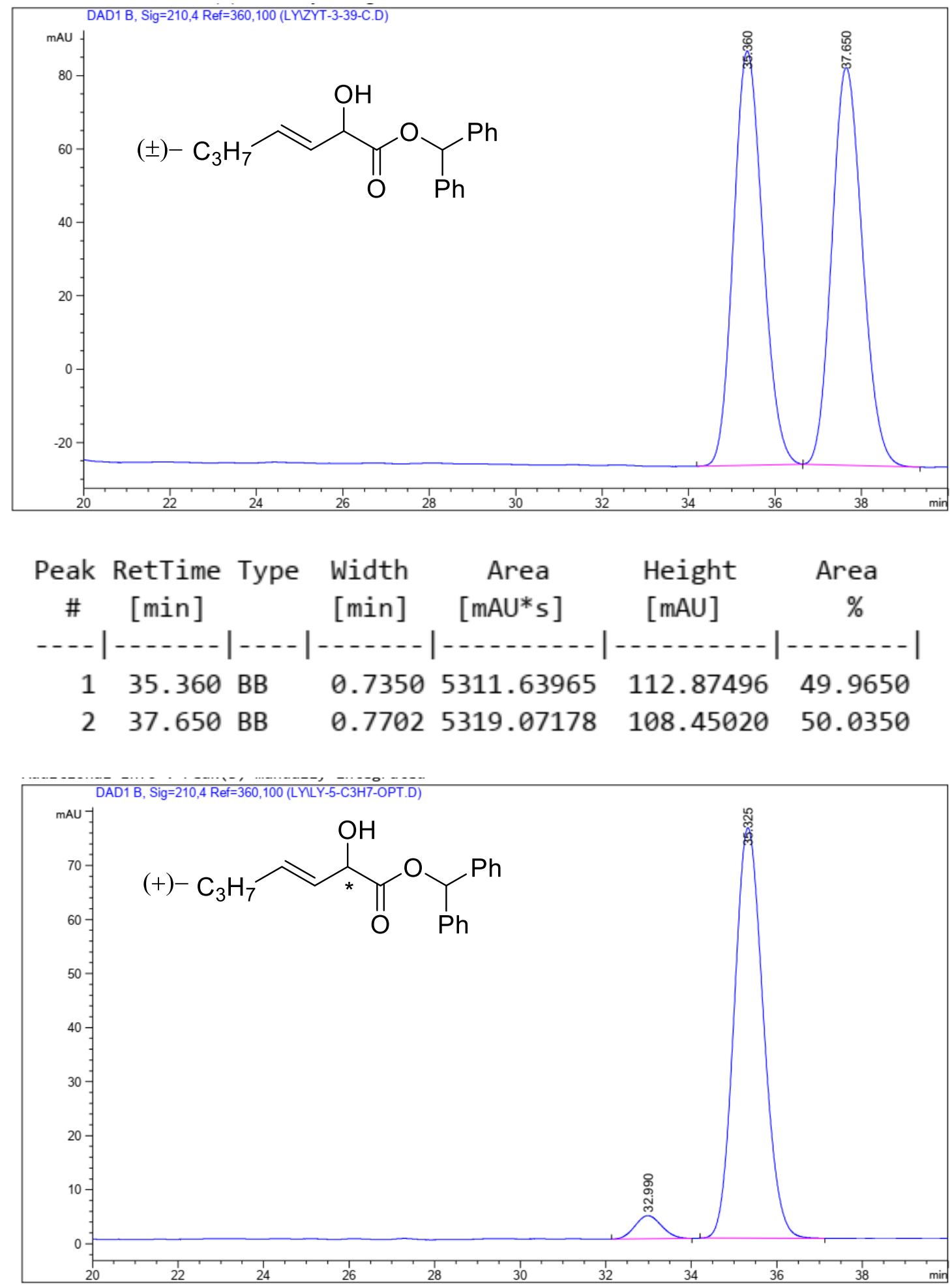

\begin{tabular}{cccccc}
$\begin{array}{c}\text { Peak RetTime Type } \\
\#\end{array}$ & $\begin{array}{c}\text { Width } \\
\text { [min] }\end{array}$ & $\begin{array}{c}\text { Area } \\
\text { [mAU*s] }\end{array}$ & \multicolumn{1}{c}{$\begin{array}{c}\text { Height } \\
\text { [mAU] }\end{array}$} & $\begin{array}{c}\text { Area } \\
\%\end{array}$ \\
\hline 1 & 32.990 BB & 0.5771 & 183.99850 & 4.26713 & 4.8826 \\
2 & 35.325 BB & 0.7346 & 3584.47876 & 75.95245 & 95.1174
\end{tabular}


Benzhydryl (E)-2-hydroxydec-3-enoate (7r)
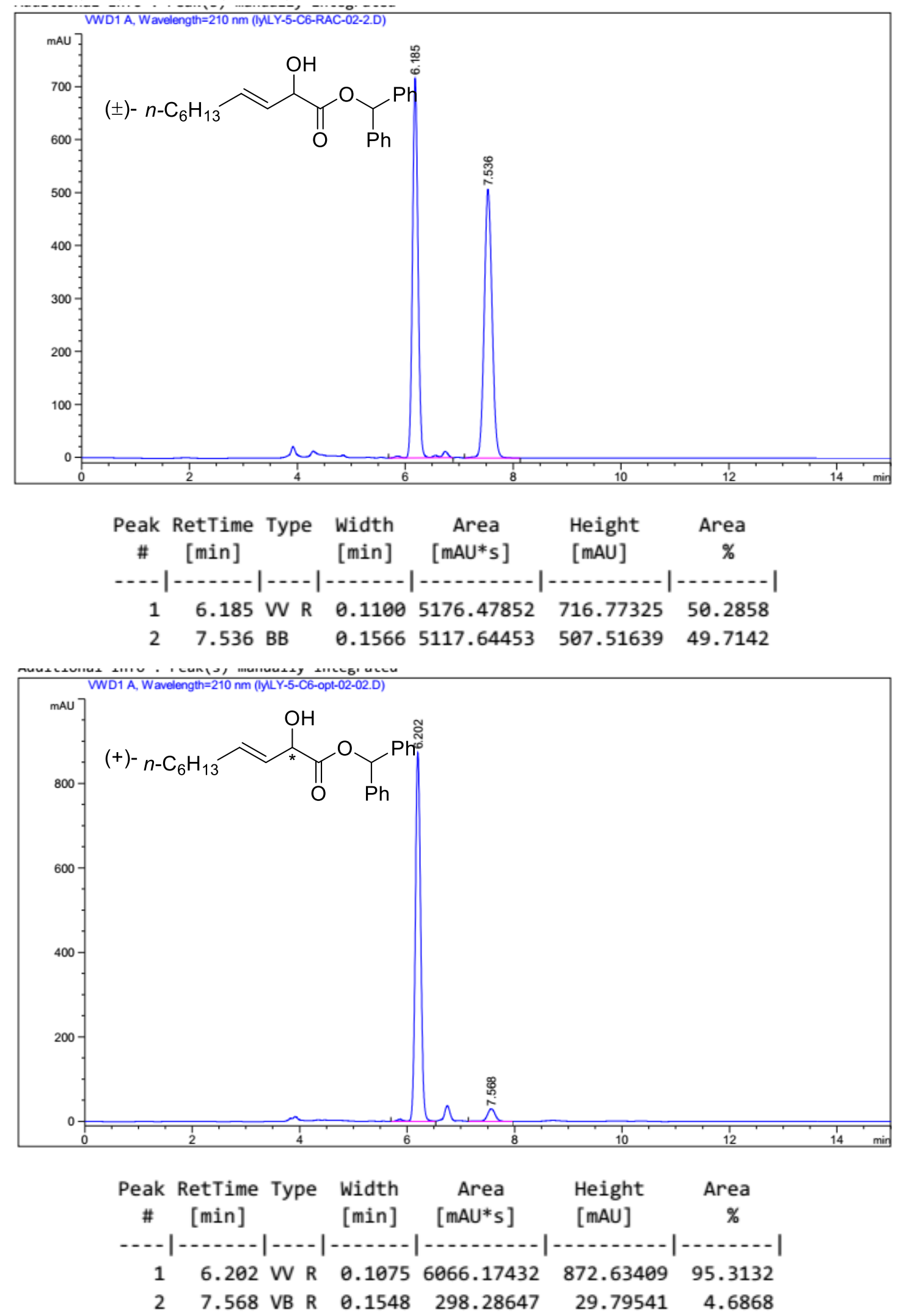
Benzhydryl (E)-2-hydroxy-5-phenylpent-3-enoate (7s)
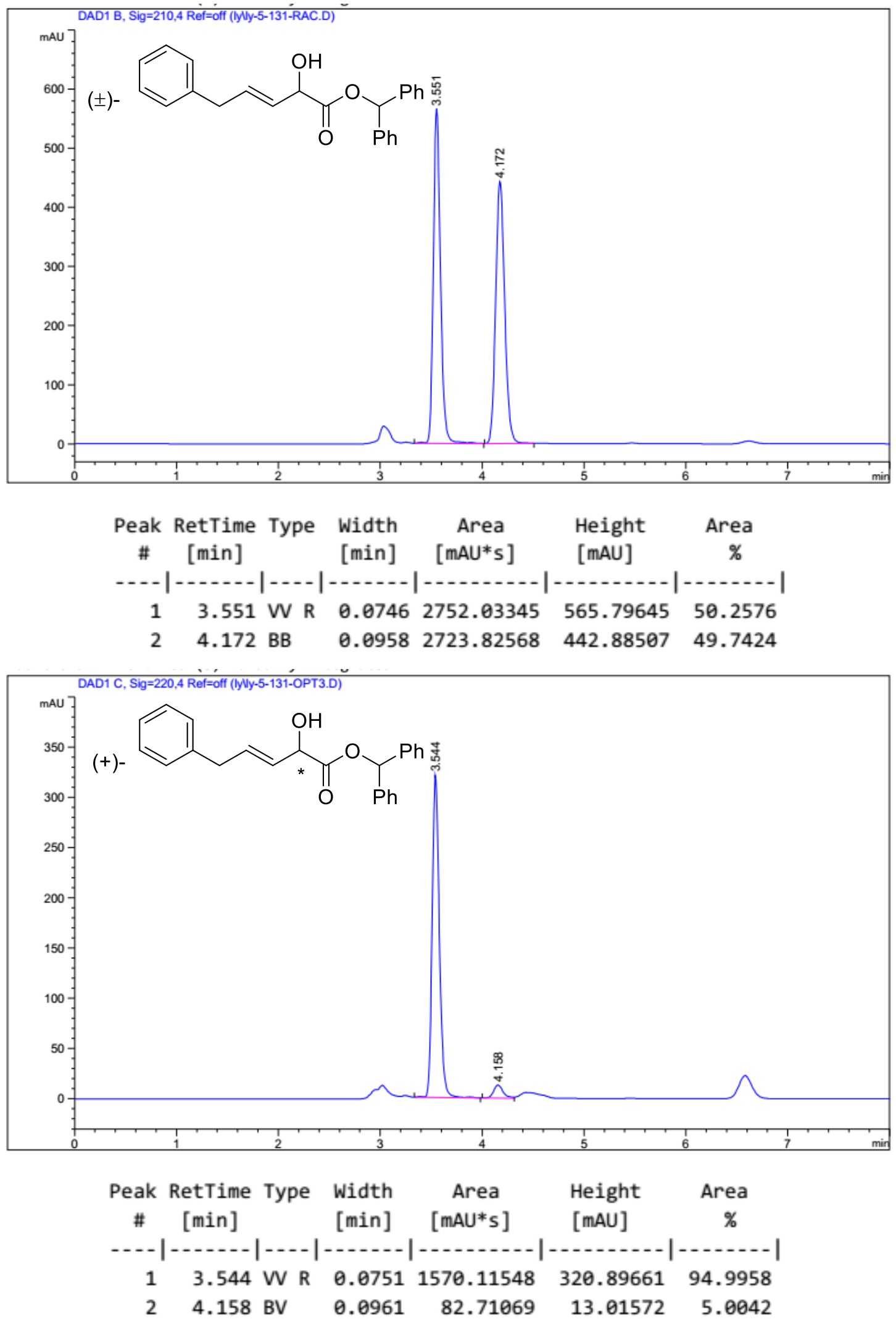
2-Phenylpropan-2-yl 2-fluorobutanoate (12)
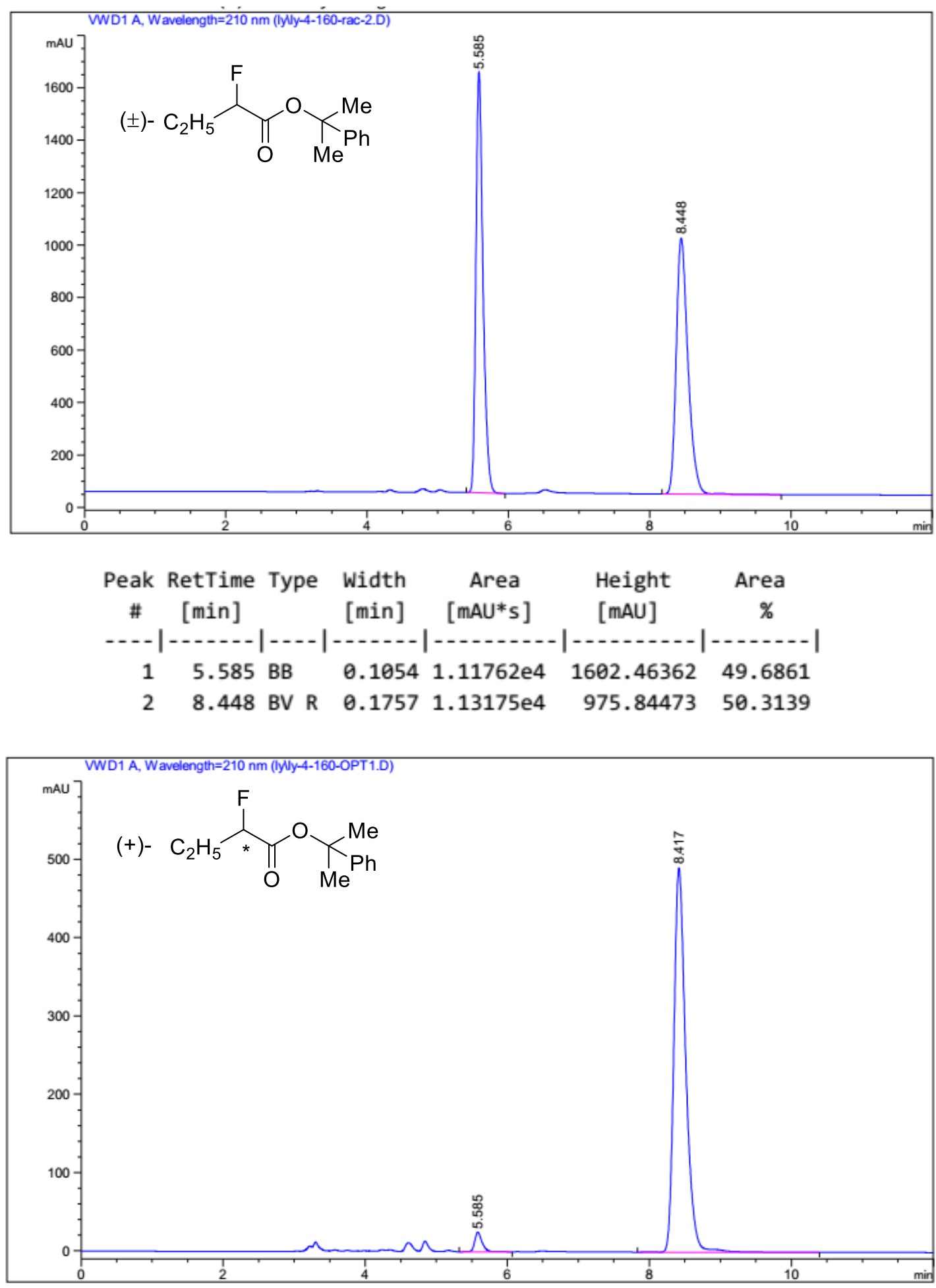

\begin{tabular}{|c|c|c|c|c|c|}
\hline ak & $\begin{array}{l}\text { RetTime Type } \\
\text { [min] }\end{array}$ & $\begin{array}{l}\text { Width } \\
\text { [min] }\end{array}$ & $\begin{array}{c}\text { Area } \\
{[\mathrm{mAU} * \mathrm{~s}]}\end{array}$ & $\begin{array}{l}\text { Height } \\
\text { [mAU] }\end{array}$ & $\begin{array}{c}\text { Area } \\
\%\end{array}$ \\
\hline & & & 17 & & 218 \\
\hline & & & 5703. & 90.46396 & 978 \\
\hline
\end{tabular}




\section{(E)-4-Phenylpent-3-ene-1,2-diol (14)}
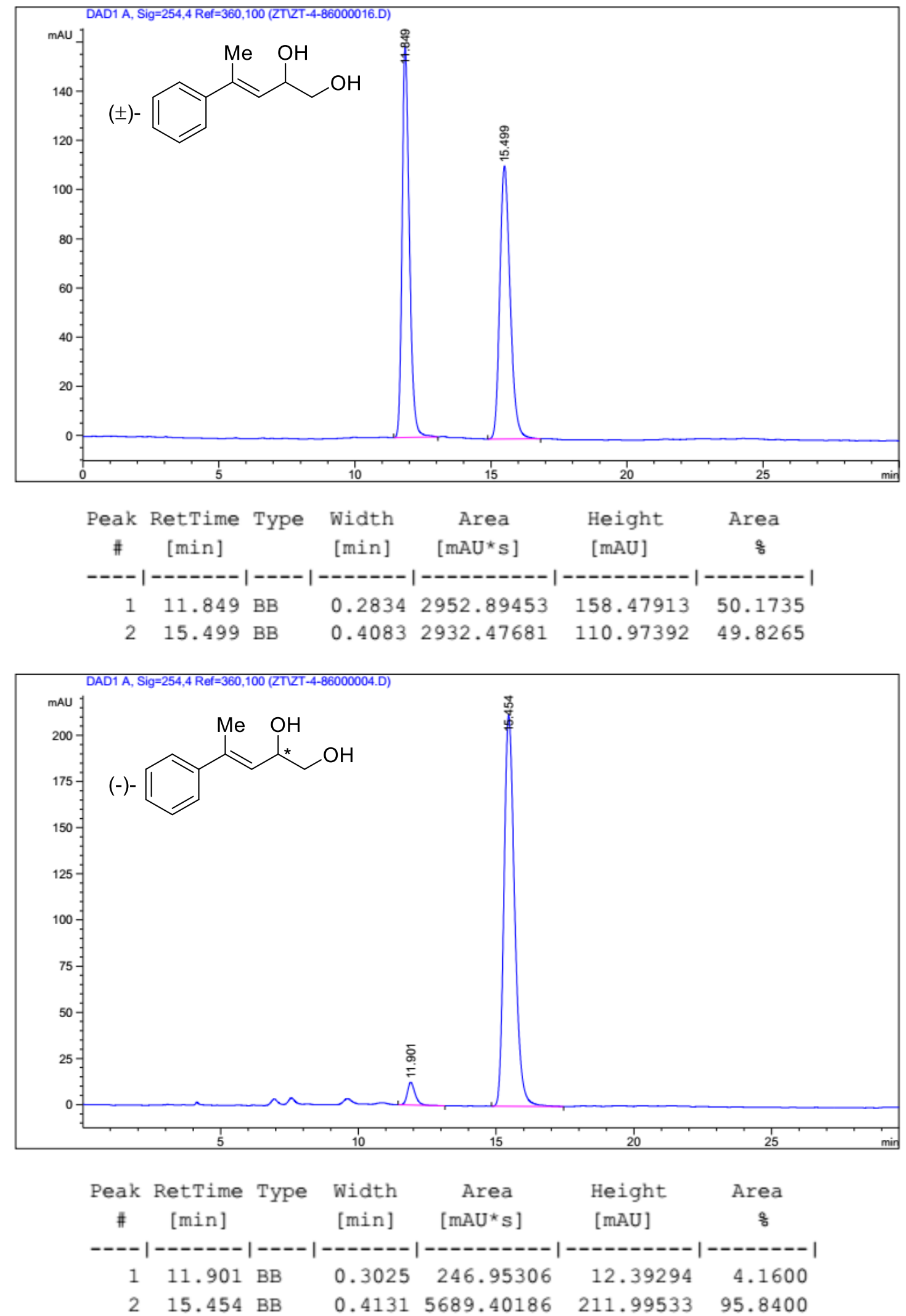
tert-Butyl (E)-2-chloro-4-phenylpent-3-enoate (15)
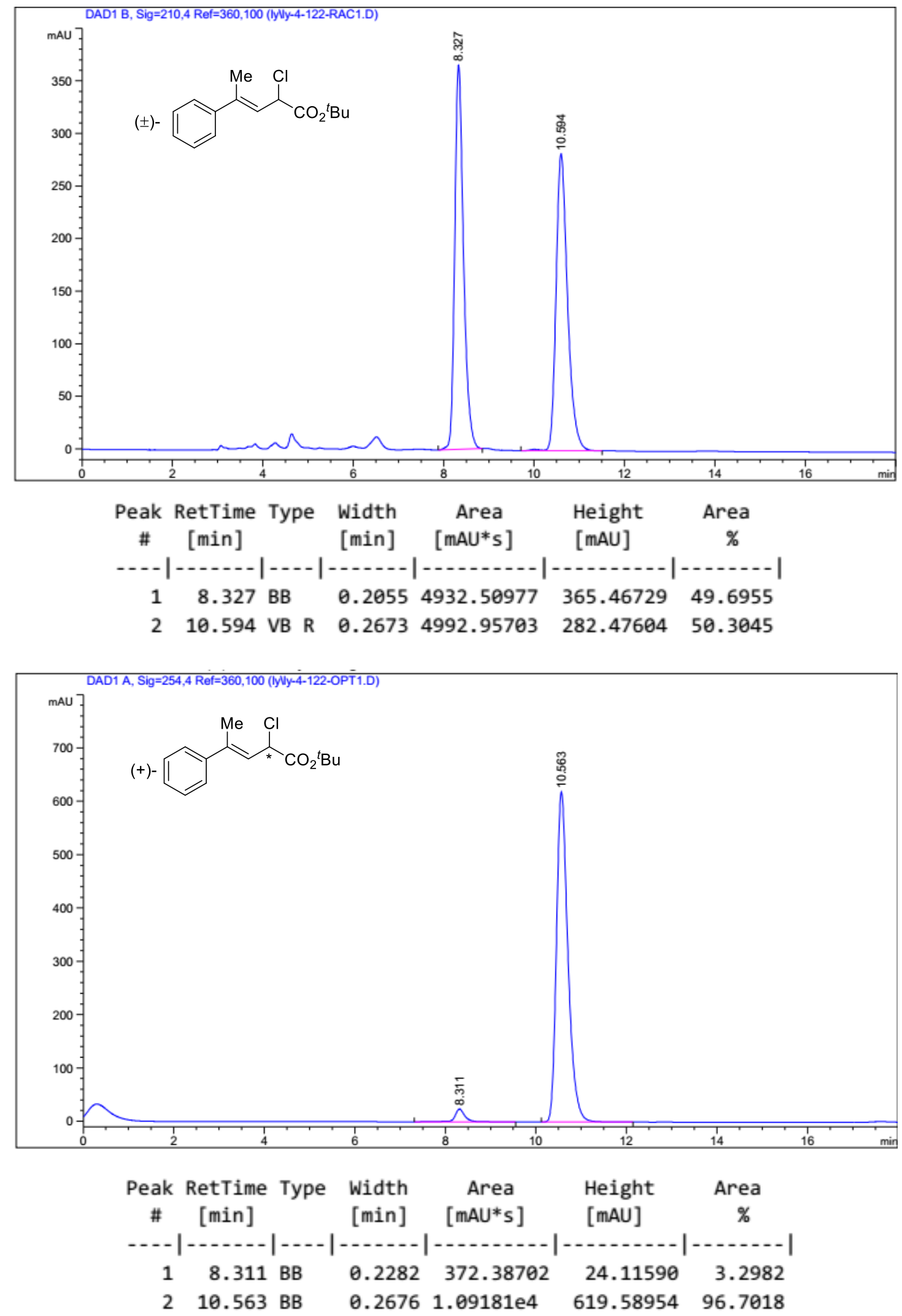
tert-Butyl (E)-6-(dimethylamino)-4-methyl-6-0xo-4-phenylhex-2-enoate (16)
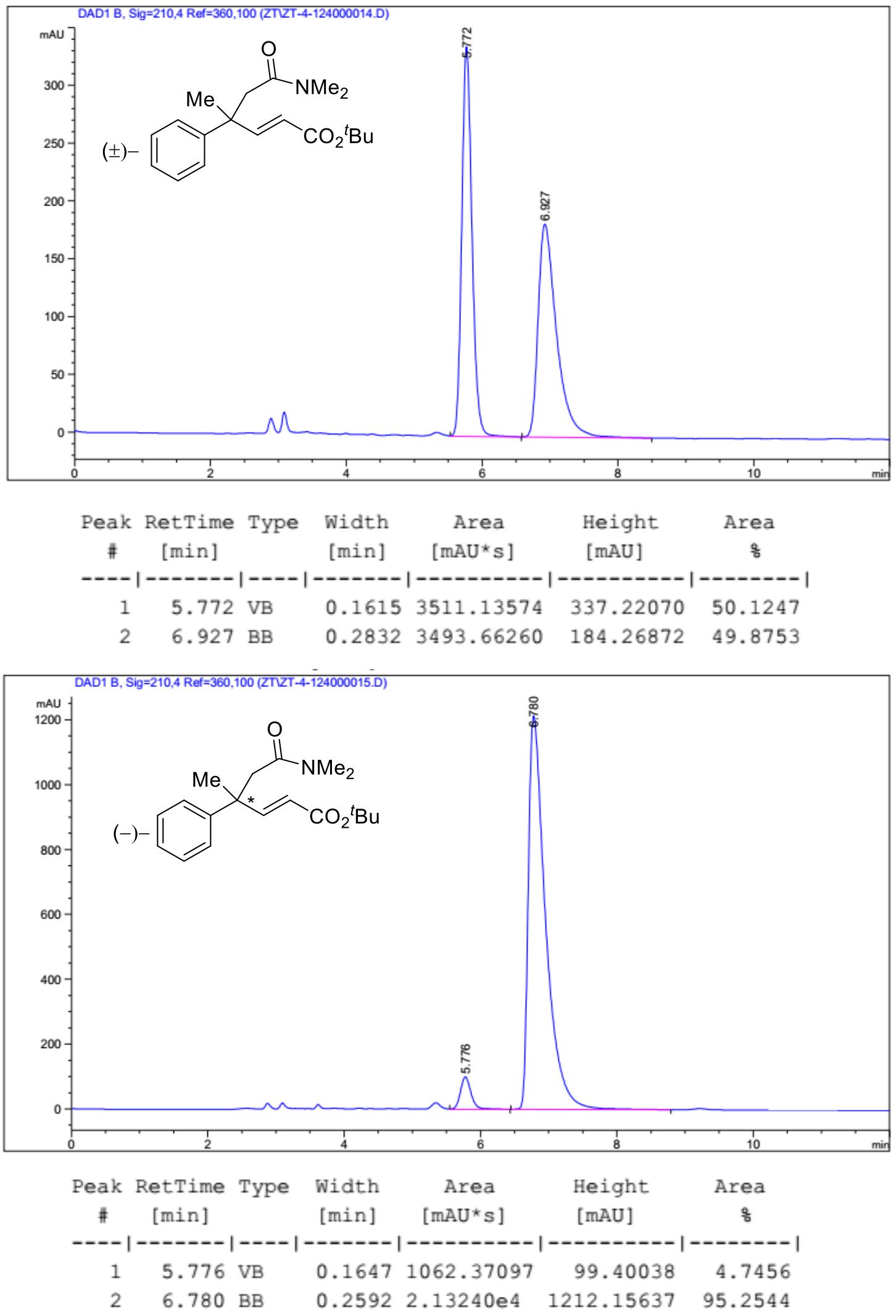
tert-Butyl (E)-4-((4-(4-chlorophenoxy)phenyl)amino)-4-phenylbut-2-enoate (17)
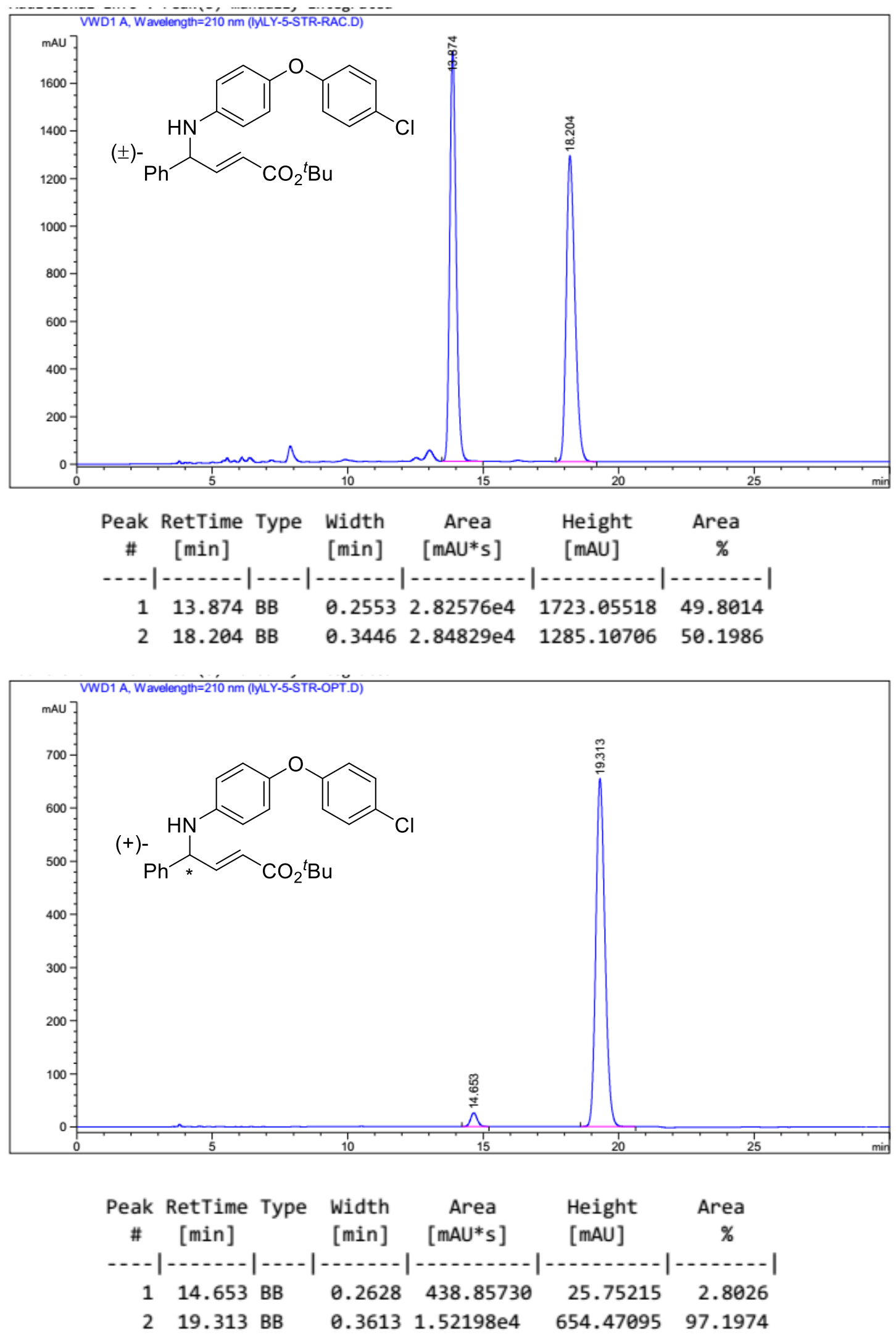
tert-Butyl (E)-4-(3,4-dichlorophenyl)-4-phenylbut-2-enoate (19)
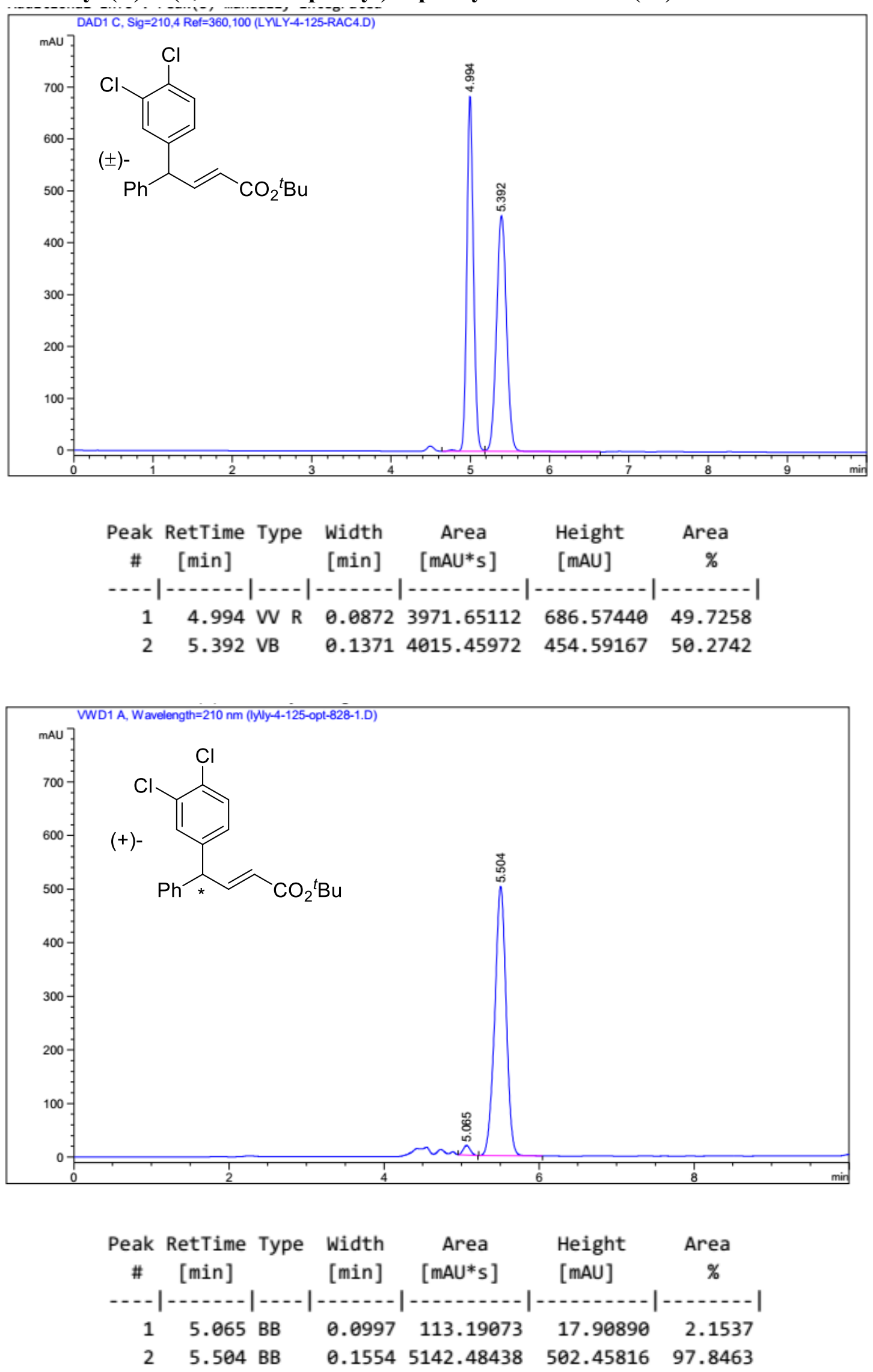


\section{Compound 20}
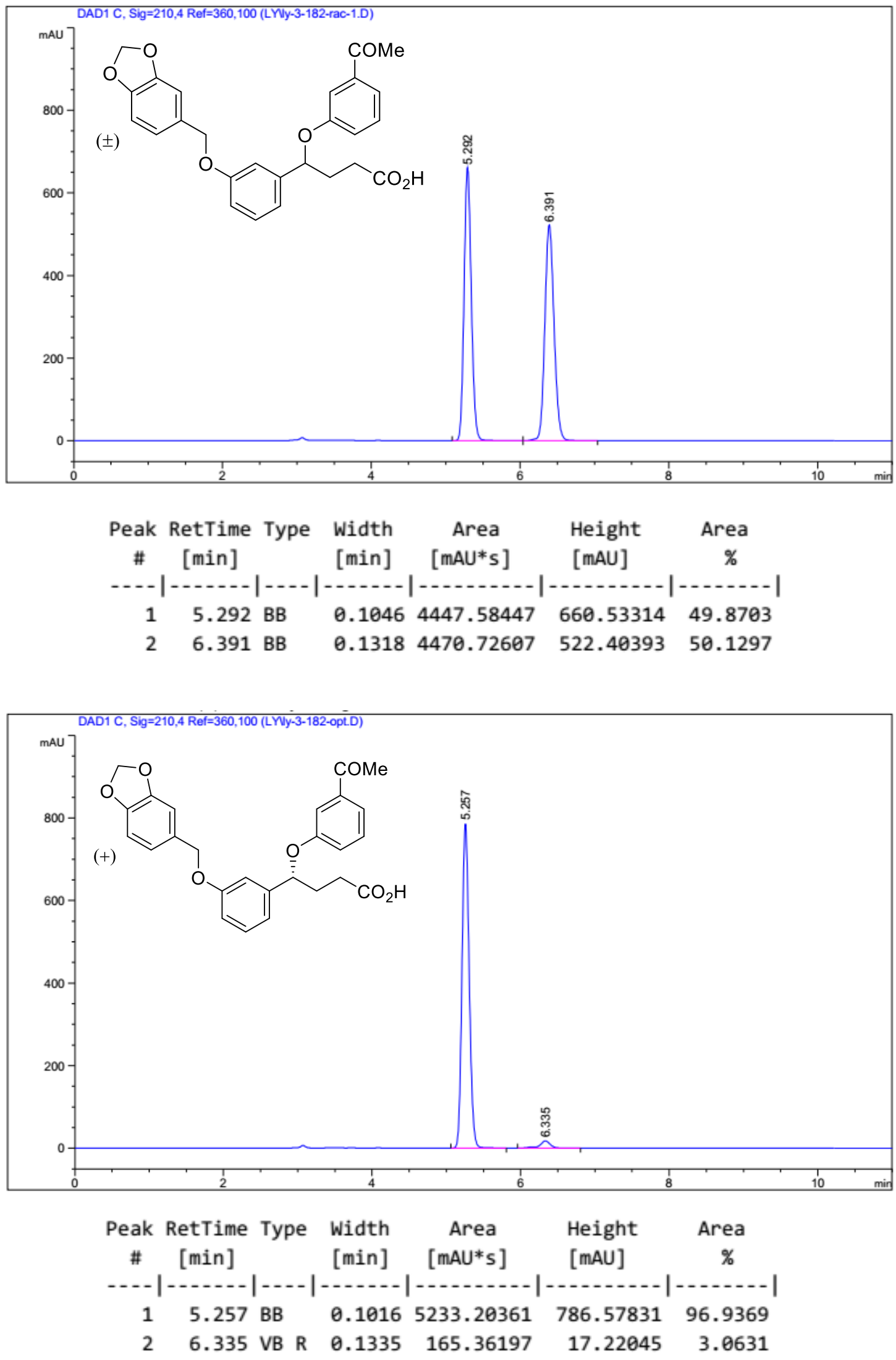


\section{References}

1 Armarego, W. L. F.; Chai, C. L. L. Purification of Laboratory Chemicals-Six Edition; Elsevier Inc.: London, 2009.

2 Xu, B.; Zhu, S.-F.; Xie, X.-L.; Shen, J.-J.; Zhou, Q.-L. Asymmetric N-H Insertion Reaction Cooperatively Catalyzed by Rhodium and Chiral Spiro Phosphoric Acids. Angew. Chem. Int. Ed. 2011, 50, 11483.

3 Zhu, S.-F.; Xu, B.; Wang, G.-P.; Zhou, Q.-L. Well-Defined Binuclear Chiral Spiro Copper Catalysts for Enantioselective N-H Insertion. J. Am. Chem. Soc. 2012, 134, 436.

4 (a) Bulugahapitiya, P.; Landais, Y.; Parra-Rapado, L.; Planchenault, D.; Weber, V. A Stereospecific Access to Allylic Systems Using Rhodium(II)-Vinyl Carbenoid Insertion into Si-H, O-H, and N-H Bonds. J. Org. Chem. 1997, 62, 1630. (b) Doyle, M. P.; Yan, M.; Hu, W.-H.; Gronenberg, L. S. Highly Selective Catalyst-Directed Pathways to Dihydropyrroles from Vinyldiazoacetates and Imines. J. Am. Chem. Soc. 2003, 125, 4692. (c) Schwartz, B. D.; Denton, J. R.; Lian, Y.; Davies, H. M. L.; Williams, C. M. Asymmetric [4 + 3] Cycloadditions between Vinylcarbenoids and Dienes: Application to the Total Synthesis of the Natural Product (-)-5-epi-Vibsanin E. J. Am. Chem. Soc. 2009, 131, 8329.

5 Richmond, E.; Moran, J. Ligand Control of E/Z Selectivity in Nickel-Catalyzed Transfer Hydrogenative Alkyne Semireduction. J. Org. Chem. 2015, 80, 6922.

6 (a) Hegedus, L. S.; Williams, R. E.; McGuire, M. A.; Hayashi, T. Palladium-Assisted Alkylation of Olefins. J. Am. Chem. Soc. 1980, 102, 4973. (b) Maryanoff, C. A.; Sorgi, K. L.; Zientek, A. M. Ketene and Carbenoid Behavior of $\alpha$-Halo Ester Enolates. J. Org. Chem. 1994, 59, 237.

7 Yao, P.; Cui, Y.; Yu, S.; Du, Y.; Feng, J.; Wu, Q.; Zhu, D. Efficient Biosynthesis of $(R)$ - or $(S)$-2-Hydroxybutyrate from L-Threonine through a Synthetic Biology Approach. Adv. Synth. Catal. 2016, 358, 2923.

8 (a) Yamashita, R.; Sakakura, A.; Ishihara, K. Primary Alkylboronic Acids as Highly Active Catalysts for the Dehydrative Amide Condensation of $\alpha$-Hydroxycarboxylic Acids. Org. Lett. 2013, 15, 3654. (b) King, A. M.; Ryck, M. D.; Kaminski, R.; Valade, A.; Stables, J. P.; Kohn, H. Defining the Structural Parameters that Confer 
Anticonvulsant Activity by the Site-by-site Modification of $(R)-N^{\prime}$-Benzyl 2-Amino3-methylbutanamide. J. Med. Chem. 2011, 54, 6432.

9 (a) Wu, W.; Xie, Y.; Li, P.; Li, X.; Liu, Y.; Dong, X.-Q.; Zhang, X. Asymmetric Hydrogenation of $\alpha$-Hydroxy Ketones with an Iridium/f-amphox Catalyst: Efficient Access to Chiral 1,2-Diols. Org. Chem. Front. 2017, 4, 555. (b) Okada, M.; Ito, S.; Matsubara, A.; Iwakura, I.; Egoshia, S.; Ueda, M. Total Syntheses of Coronatines by Exo-selective Stomatal Opening. Org. Biomol. Chem. 2009, 7, 3065.

10 Jaunzeme, I.; Jirgensons, A. Ether-directed Diastereoselectivity in Catalyzed Overman Rearrangement: Comparative Studies of Metal Catalysts. Tetrahedron 2008, 64, 5794.

11 Nagasawa, K.; Georgieva, A.; Koshino, H.; Nakata, T.; Kita, T.; Hashimoto, Y. Total Synthesis of Crambescidin 359. Org. Lett. 2002, 4, 177.

12 Baskakis, C.; Magrioti,V.; Cotton, N.; Stephens, D.; Constantinou-Kokotou, V.; Dennis, E. A.; Kokotos, G. Synthesis of Polyfluoro Ketones for Selective Inhibition of Human Phospholipase $\mathrm{A}_{2}$ Enzymes. J. Med. Chem. 2008, 51, 8027.

13 Igarashi, T.; Tayama, E.; Iwamoto, H.; Hasegawa. E. Asymmetric $\alpha-2-$ Tosylvinylation of in situ Generated $\mathrm{N}$-2-tosylvinyl Proline-derived Ammonium Ylides. Tetrahedron Lett. 2011, 52, 1819.

14 Dieter, R. K.; Picado, R. Regio- and Stereocontrol in the Reactions of $\alpha$-Halo- $\beta, \gamma-$ enoates and $\alpha$-O-Phosphono- $\beta, \gamma$-enenitriles with Organocuprates. J. Org. Chem. 2014, 79, 11125.

15 Findlay, A. D.; Banwell, M. D. A Chemoenzymatic Total Synthesis of $(+)-$ Amabiline. Org. Lett. 2009, 11, 3160.

16 (a) Xia, C.; Shen, J. Liu, D. Zhang, W. Synthesis of Chiral $\alpha, \beta$-Unsaturated $\gamma$-Amino Esters via Pd-Catalyzed Asymmetric Allylic Amination. Org. Lett. 2017, 19, 4251. (b) Ohmiya, H.; Makida, Y.; Li, D.; Tanabe, M.; Sawamura M. Palladium-Catalyzed $\gamma$-Selective and Stereospecific Allyl-Aryl Coupling between Acyclic Allylic Esters and Arylboronic Acids. J. Am. Chem. Soc. 2010, 132, 879.

17 Discolo, C. A.; Graves, A. C.; Deardorff, D. R. Regio- and Stereospecific C- and O- Allylation of Phenols via $\pi$-Allyl Pd Complex Derived from Allylic Ester Carbonates. J. Org. Chem. 2017, 82, 1034. 